WHOI-97-10

1997

Copyl

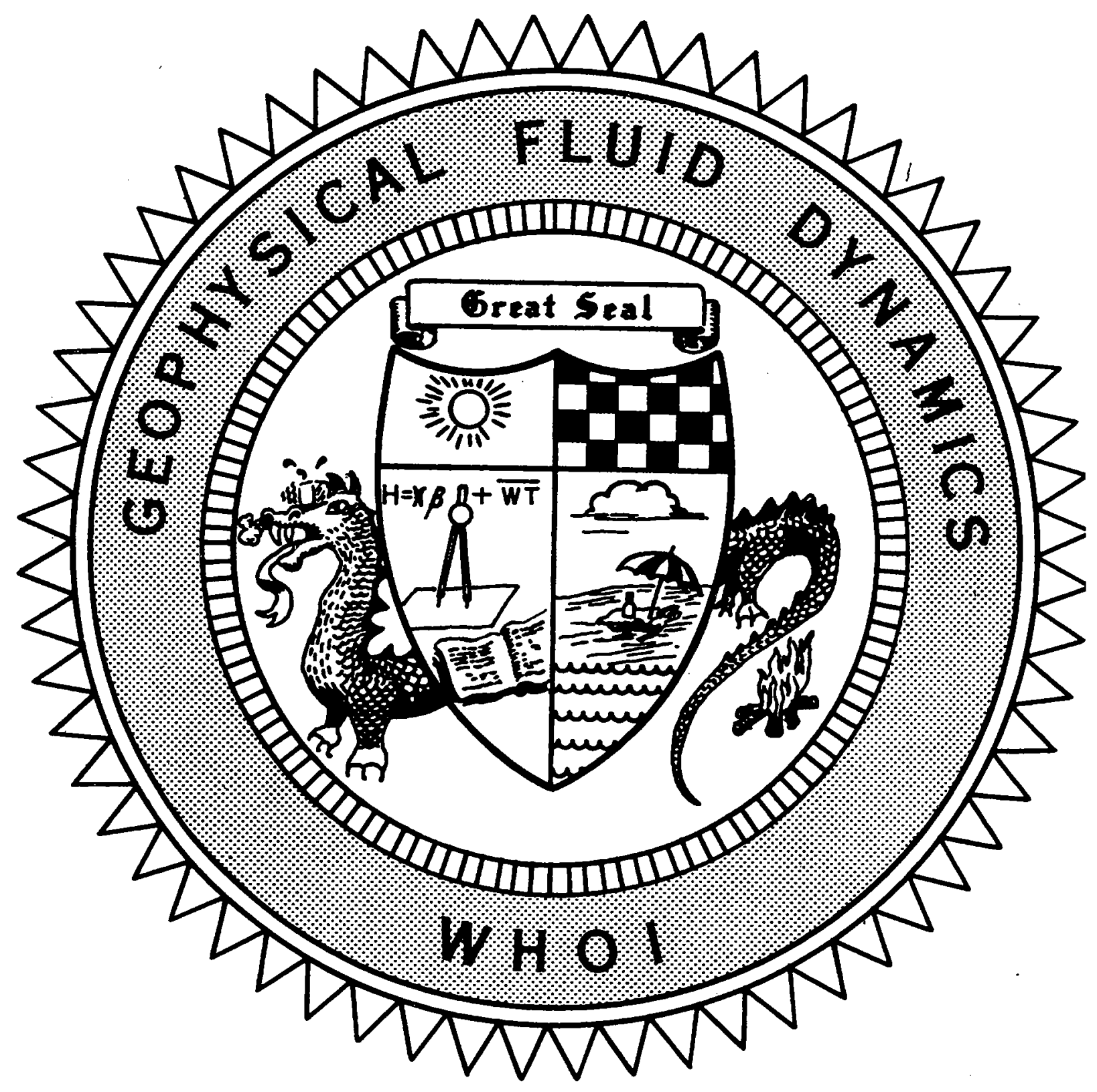

Course Lectures

Abstracts of Participants Fellows Project Reports 


\title{
Double-Diffusive Processes \\ 1996 Summer Study Program in Geophysical Fluid Dynamics
}

\author{
by \\ Steve Meacham, Director \\ Dacia Tucholke, Staff Assistant \\ Woods Hole Oceanographic Institution \\ Woods Hole, Massachusetts 02543
}

July 1997

\section{Technical Report}

Funding was provided by the National Science Foundation through Grant No. OCE-9314484

Reproduction in whole or in part is permitted for any purpose of the United States Government. This report should be cited as Woods Hole Oceanog. Inst. Tech. Rept., WHOI-97-10.

Approved for public release; distribution unlimited.

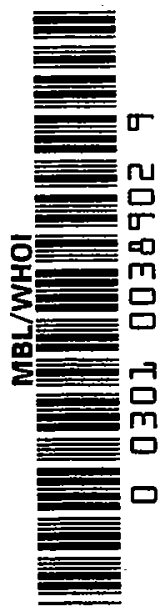

Approved for Distribution:

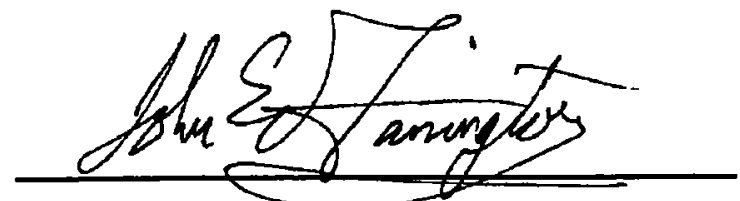

John W. Farrington

Associate Director for Education and Dean of Graduate Studies 



\section{Preface}

The physics of double diffusion and the role that it plays in the ocean provided the central theme for the 1996 summer program in Geophysical Fluid Dynamics. Barry Ruddick gave a broad, yet penetrating survey of experimental studies of double diffusion in the laboratory and followed that up with an overview of oceanic observations in which the presence of double diffusion is manifested in vertical profiles and in horizontal interleaving. Ruddick's involvement in laboratory studies, oceanic observations and analyses of the effects of double diffusion vs those of turbulent exchanges made his lectures particularly enlightening. Ray Schmitt's account of the problems that the observer faces in recording the presence of double diffusion in the ocean made us aware of the subtle and mysterious obstacles that nature puts in the way of the observer. Eric Kunze contributed several semi-empirical analyses (he calls them guesses) and George Veronis provided some background material for the students.

Although Ruddick, Schmitt, Kunze and Veronis gave the formal lecture course summarized in the following pages, there were many seminars that could have served the same purpose. Andy Woods demonstrated some geological applications and Jack Whitehead provided more laboratory examples, some of them quite different from the usual double diffusive experiments. Stewart Turner arrived and showed us an entralling film history of double diffusion as the field developed via laboratory experiments. Oliver Kerr continued the consideration of double diffusion outside the ocean by introducing us to some of the subtle influences of boundaries.

The tutorial component of the summer continued with an excellent pair of lectures by Keith Julien illustrating the use of small amplitude perturbation analysis and bifurcation theory to unfold the dynamics of nonlinear convection. Paul Kolodner and Hermann Riecke introduced us to binary fluid convection, a topic closely related to double diffusion. Kolodner's array of fascinating experiments with binary fluids was especially appealing to the mathematicains because the interesting behavior is at small Rayleigh numbers. Riecke presented mathematical analyses of several of the phenomena observed by Kolodner. Paul Kolodner then described experimental work in viscoelastic convection. The techniques introduced in Julien's lectures and applied by Riecke were further exploited by Edgar Knobloch and Michael Proctor. Knobloch examined travelling waves and patterns in binary fluid convection while Proctor discussed magnetoconvection. Julien, Knobloch and Proctor supported their weakly nonlinear analyses with numerical simulations.

A range of topics was addressed in seminars given by staff and visitors. Abstracts of some of these are included in this volume. A complete list of seminars may be found at the back of this volume.

The Fellows concluded the summer by presenting reports on their summer research that ranged from experiments with double diffusion in a slot, work on the formation and evolution of staircases, double diffusion in stars and the interaction between convection and radiation. Lastly, a question on competition between the oscillatory and stationary instabilities in double 
diffusive convection prompted Keith Julien and Neil Balmforth to study the Takens-Bogdanov bifurcation in an extended system. Their report is included in this volume.

We had hoped that the ten fellows of 1996 could transfer the Charlie Brown trophy to some other team in the softball league but this year, too, they were forced to vent their frustrations on the faculty team on the last day of the program.

We are grateful to the Woods Hole Oceanographic Institution, which has been so supportive of the GFD Program over all of the years. We thank Jake Peirson who, with the staff of the Education Office, has shouldered much of the administrative burden of the GFD program over the past two decades and whose efforts made our stay at Walsh Cottage particularly trouble-free. Dacia Tucholke, with the help of Lee Campbell, was very effective as our local administrator. We are grateful to the National Science Foundation and the Office of Naval Research for supporting this 38th GFD summer program.

George Veronis and Steve Meacham 


\section{Contents}

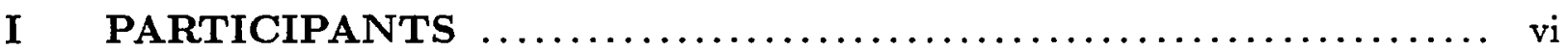

II PHOTOGRAPHS $\ldots \ldots \ldots \ldots \ldots \ldots \ldots \ldots \ldots \ldots \ldots \ldots \ldots \ldots \ldots$ viii

\section{PRINCIPAL LECTURES}

$1 \&$ 6. Double diffusion: Laboratory experiments

Barry Ruddick ......................................... $\quad 1$

2. Double diffusion: An introduction. Pt. I

Barry Ruddick and George Veronis $\ldots \ldots \ldots \ldots \ldots \ldots \ldots \ldots \ldots \ldots \ldots, 22$

3. Double diffusion: An introduction. Pt. II

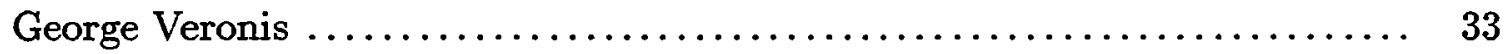

4. Macro-scale signatures of double diffusion in the ocean

Ray Schmitt $\ldots \ldots \ldots \ldots \ldots \ldots \ldots \ldots \ldots \ldots \ldots \ldots \ldots \ldots \ldots \ldots \ldots \ldots \ldots, 41$

5. A model for salt fingers

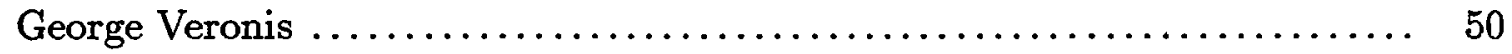

7. Thermohaline fronts

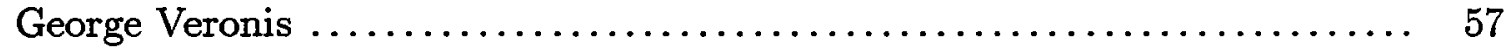

8. Microscale signatures of salt fingers in the ocean

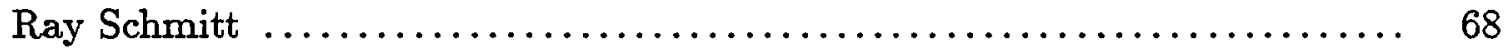

9. Limits on growing, finite length fingers: A gradient Froude number constraint

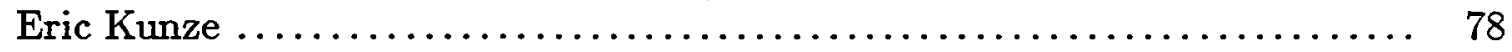

10. Effect of vertical shear on salt fingers

Eric Kunze $\ldots \ldots \ldots \ldots \ldots \ldots \ldots \ldots \ldots \ldots \ldots \ldots \ldots \ldots \ldots \ldots \ldots, 87$

\section{LECTURES}

The criteria for the onset of double-diffusive instabilities at a vertical boundary

Oliver Kerr $\ldots \ldots \ldots \ldots \ldots \ldots \ldots \ldots \ldots \ldots \ldots \ldots \ldots \ldots \ldots \ldots \ldots \ldots, 93$

Laboratory models of double-diffusive processes

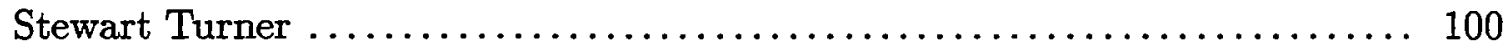

Stratification and circulation produced by double-diffusive sources in closed regions

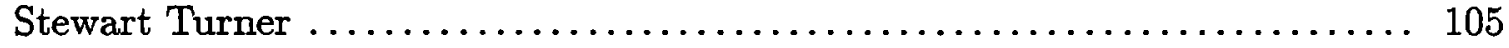

Weakly nonlinear convection I \& II

Keith Julien $\ldots \ldots \ldots \ldots \ldots \ldots \ldots \ldots \ldots \ldots \ldots \ldots \ldots \ldots \ldots \ldots \ldots, 110$

Convection in binary mixtures I-IV

Paul Kolodner and Hermann Riecke

Simmering the primordial soup: Traveling-wave convection in viscoelastic DNA suspensions

Paul Kolodner 
Transition to chaos in doubly diffusive systems

Edgar Knobloch

Magnetoconvection I \& II

Michael Proctor

\section{FELLOWS REPORTS}

Wave propagation in granular flows

Jonathan Wylie

The finger regime of double-diffusive convection with equal diffusion coefficients and mixed boundary conditions

Naftali Tsitverblit

An analog of double-diffusive instabilities in magma chambers

Tonushree Kundu

Semi-convection in stars

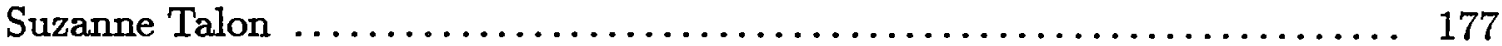

Aspects of double diffusion in a thin slot

Joseph Biello .............................................. 196

Convection with radiation: the fixed flux case

Sebastien Aumaitre ............................................. 216

A few steps toward staircases

Francesco Paparella ........................................ 232

Double diffusion with a density interface

Alexander Casti ........................................... 248

Long-wave instability in double-diffusive Marangoni convection

Jean-Luc Thiffeault ...................................... 269

Linear stability analysis of a gravity current over topography

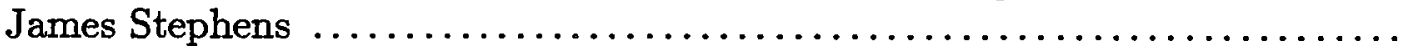

\section{ABSTRACTS OF SEMINARS}

The Takens-Bogdanov bifurcation in an extended system

Neil Balmforth and Keith Julien

Double-diffusive intrusions across the Arctic Ocean

Eddy Carmack

Horizontal variability of thermohaline staircases in the Western Mediterranean

Gerd Krahmann

The statistical stability of convection dynamos

Willem Malkus

Anomalous effects of distant boundaries on traveling-wave instabilities

Michael Proctor

What determines salt finger amplitude in unbounded T-S gradient layers?

Melvin Stern 
Multiplicity of the equilibrium states in laterally heated thermosolutal systems with and without the difference in the diffusivity coefficients

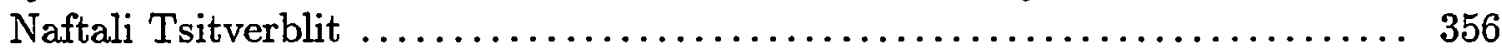

A laboratory study of the effect of bottom topography on ocean circulation

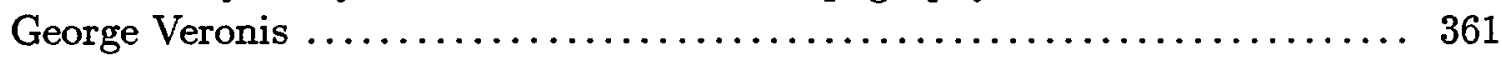

Double diffusive effects in magmas

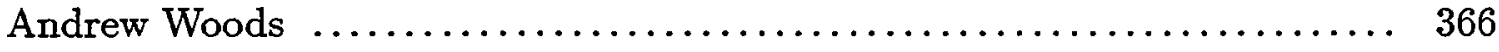

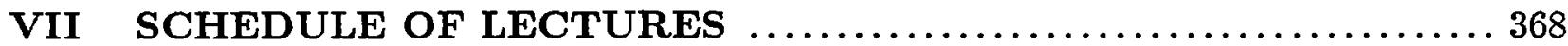

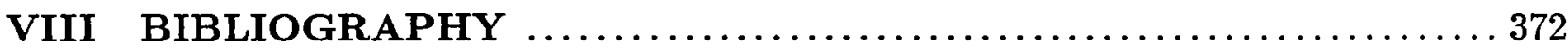




\section{GFD Fellows, Staff and Visitors}

\section{The Fellows}

Sebastien Aumaitre

Joseph Biello

Alexander Casti

Tonushree Kundu

Francesco Paparella

James Stephens

Suzanne Talon

Jean-Luc Thiffeault

Naftali Tsitverblit

Jonathan Wylie

\section{The Staff and Visitors}

James Anderson

Neil Balmforth

Ricardo Becerril

Eddy Carmack

Paola Cessi

Eric Chassignet

Paul Dellar

Charles Doering

Raffaele Ferrari

Isom Herron

Louis Howard

Keith Julien

Joseph B. Keller

Oliver Kerr

Edgar Knobloch

Paul Kolodner

Gerd Krahmann

Eric Kunze

Norman Lebovitz

Willem Malkus

Steven Meacham

Philip J. Morrison

Thomas J. Noyes, Jr.

Tamay Ozgokmen

Michael Proctor

Hermann Riecke
Ecole Normal Superieur de Lyon, France

University of Chicago

Columbia University

University of California at Berkeley

Torino University, Italy

Reading University, UK

University of Paris, France

University of Texas at Austin

Lamont-Doherty Earth Observatory, Columbia University

University of Cambridge, UK

Stevens Insitute of Technology

University of Nottingham, UK

University of Texas at Austin

Inst. of Ocean Sciences, Sidney, British Columbia, Canada

Scripps Inst. of Oceanography, U. California at San Diego

University of Miami

University of Cambridge, UK

Los Alamos National Laboratory

Scripps Inst. of Oceanography, U. California at San Diego

Rensselaer Polytechnic Institute

Florida State University

University of Colorado

Stanford University

City University, London, UK

University of California at Berkeley

ATT Bell Laboratories

Institute for Oceanography, University of Kiel, Germany

University of Washington

University of Chicago

Massachusetts Institute of Technology

Florida State University

University of Texas at Austin

Scripps Inst. of Oceanography, U. California at San Diego

University of Miami

University of Cambridge, UK

Northwestern University 
Claes Rooth

Barry Ruddick

Ray Schmitt

Michael Shelley

Colin Shen

Edward A. Speigel

Melvin Stern

Stewart Turner

George Veronis

Jack Whitehead

Andy Woods

William Young
University of Miami

Dalhousie University, Canada

Woods Hole Oceanographic Institution

Courant Institute, New York University

Naval Research Laboratory

Columbia University

Florida State University

Australian National University, Australia

Yale University

Woods Hole Oceanographic Institution

University of Bristol, UK

Scripps Inst. of Oceanography, U. California at San Diego 


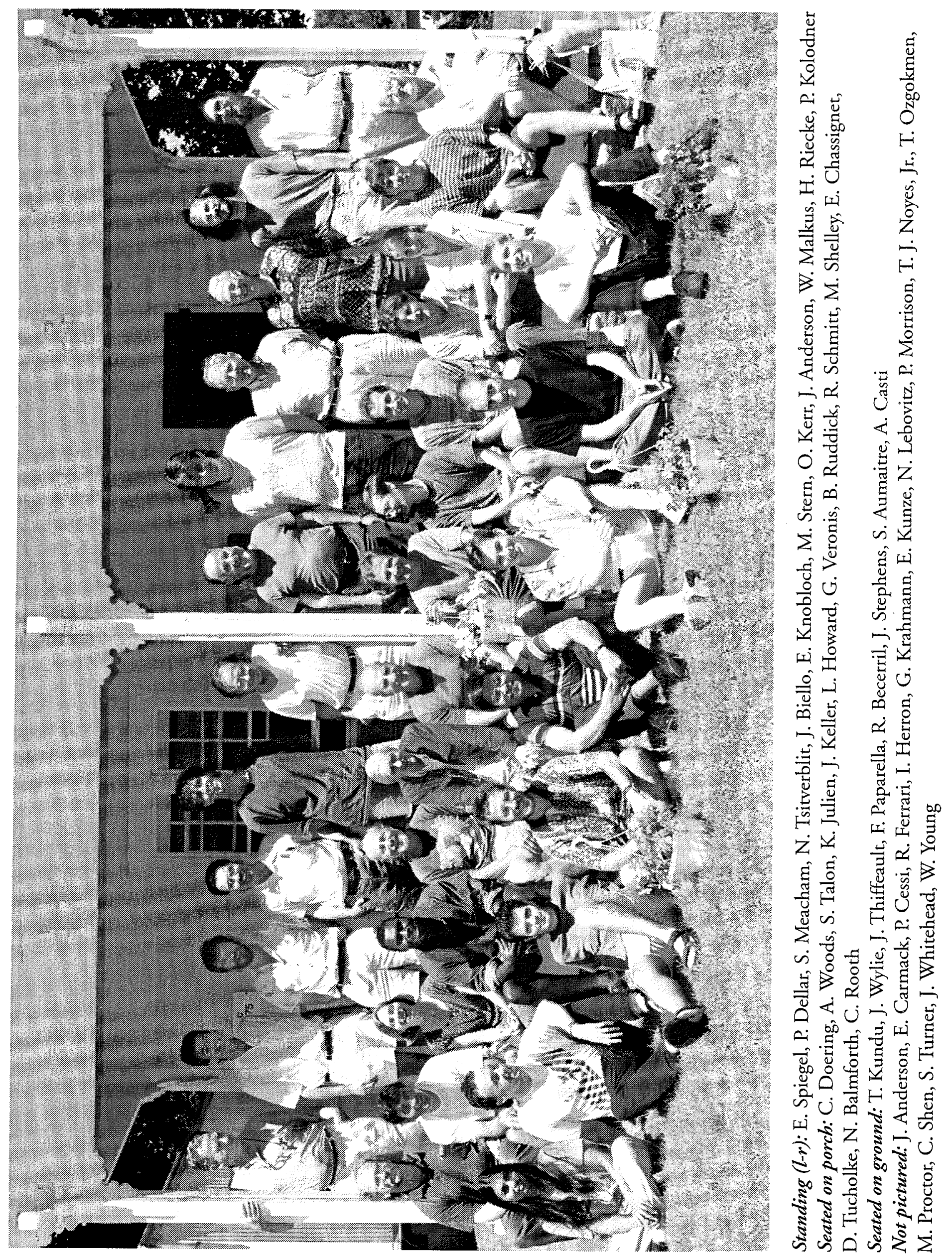



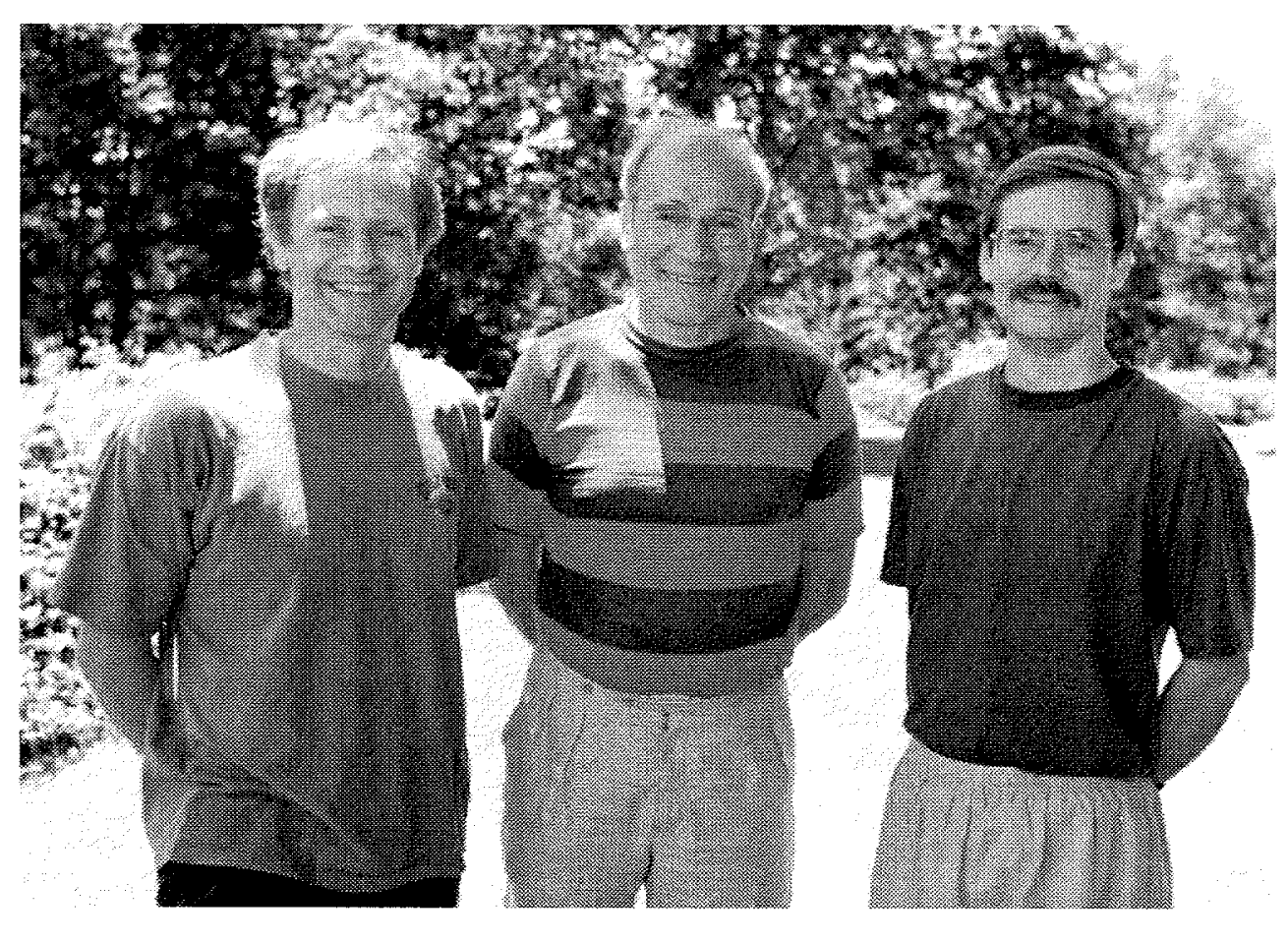

Pictured left to right are 1996 GFD Principal Lecturers Barry Ruddick, George Veronis, and Ray Schmitt.

\section{Principal Lecturers}

Pictured left to right are 1996 GFD Principal Lecturers Keith Julien, Edgar Knobloch, Paul Kolodner, and Hermann Riecke.

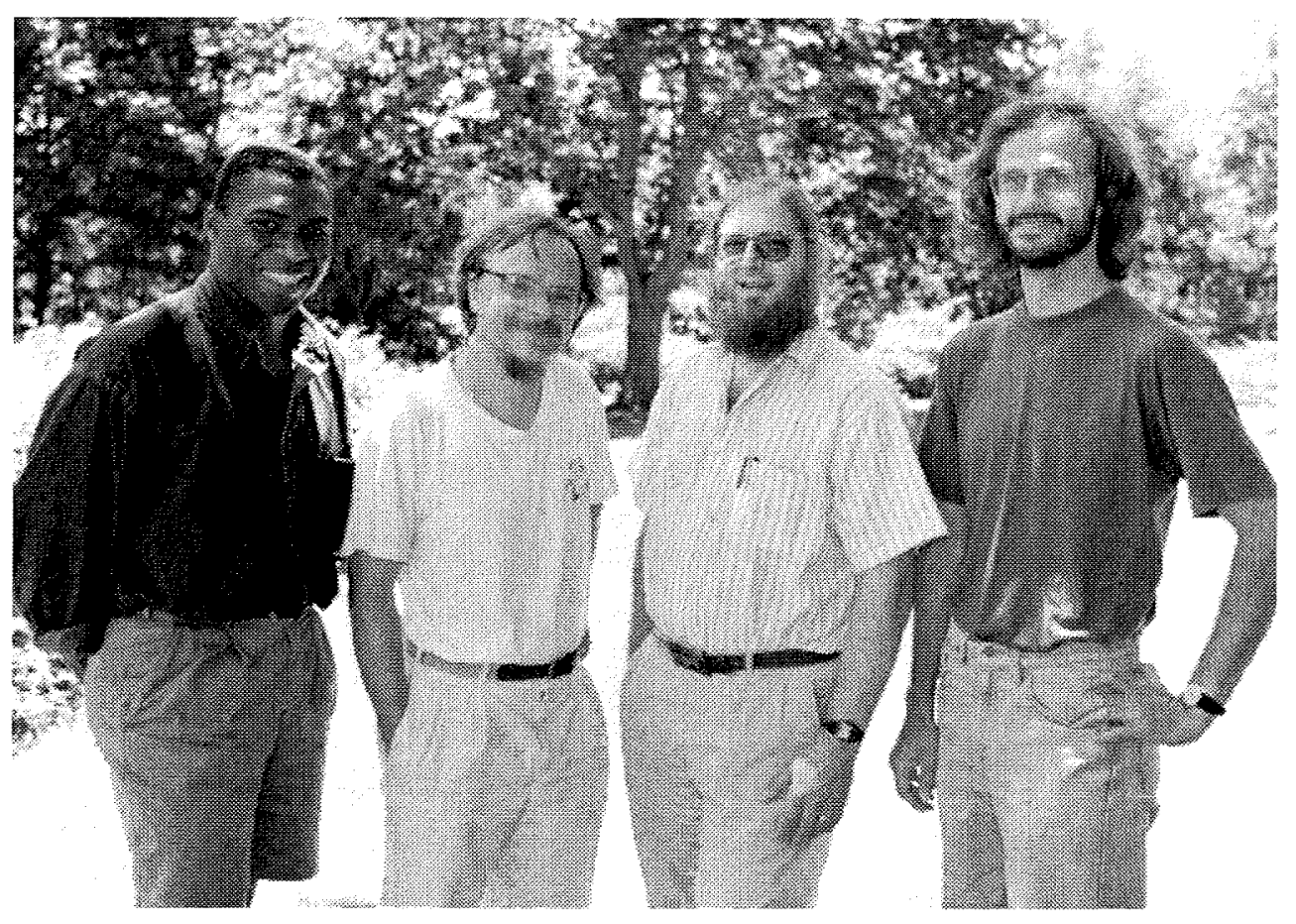




\title{
Double Diffusion: Laboratory Experiments
}

\author{
Barry Ruddick, \\ Dalhousie University
}

June 17 and 24, 1996

\section{Salt Fingers-Qualitative Experiments}

\subsection{The Salt Fountain}

Much of the ocean is stratified with warm, salty water on top of cool, fresh water as shown in Figure 1. This is especially true at lower latitudes where rainfall is less abundant and evaporation makes the top water saltier. In 1956 Stommel, Arons, and Blanchard [1] performed a thought experiment: they imagined a long thin pipe made of a heat conducting material like copper immersed in a fluid with concentration properties akin to the ocean (Figure 2). A cold, fresh fluid element at the bottom of the pipe displaced upwards heats up by diffusion through the pipe, but its salinity remains constant. The fluid element is then at the same temperature but less salty than the surrounding fluid, making it buoyant. The pipe will then act as a perpetual salt fountain. The effect can also be reversed to build a perpetual salt pump. The important thing to note here is that the fluid is stably stratified in density (since the destabilizing effect of the salt gradient is less that the stabilization due the heat gradient) so that one does not expect a convective instability to develop.

\subsection{Salt Fingers}

The perpetual salt fountain was an 'oceanographic curiosity' until Stern [2] showed via linear stability analysis that since salt diffuses more slowly than heat (the ratio of their diffusivities is $\tau=\kappa_{S} / \kappa_{T} \sim 10^{-2}$ ) the pipe isn't needed. Figure 3 shows schematically the mechanism involved. The horizontal scale of the fastest growing fingers, $L$, is given by

$$
L \sim \pi\left(\frac{g \alpha T_{z}}{4 \nu \kappa_{T}}\right)^{-1 / 4},
$$

where $g$ is the acceleration due to gravity, $\alpha$ the coefficient of thermal expansion of water, $T_{z}$ the temperature gradient, $\nu$ the viscosity, and $\kappa_{T}$ the heat diffusivity.

In 1967 Turner [4] performed what is called a 'two-layer rundown' experiment. In this setup a layer of warm, salty water is placed on top of cool, fresh water (Figure 5a). 

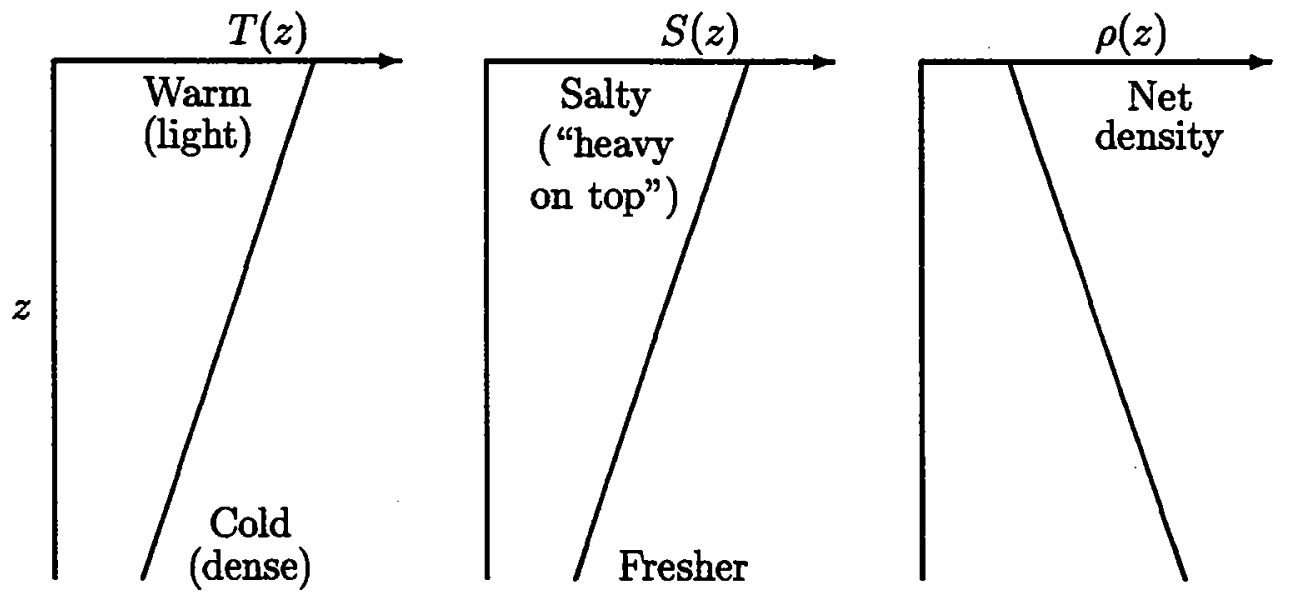
P.E. required to make $T$ uniform
P.E. available in salt field
Statically stable

Figure 1: Temperature, salinity, and density stratification of the ocean.

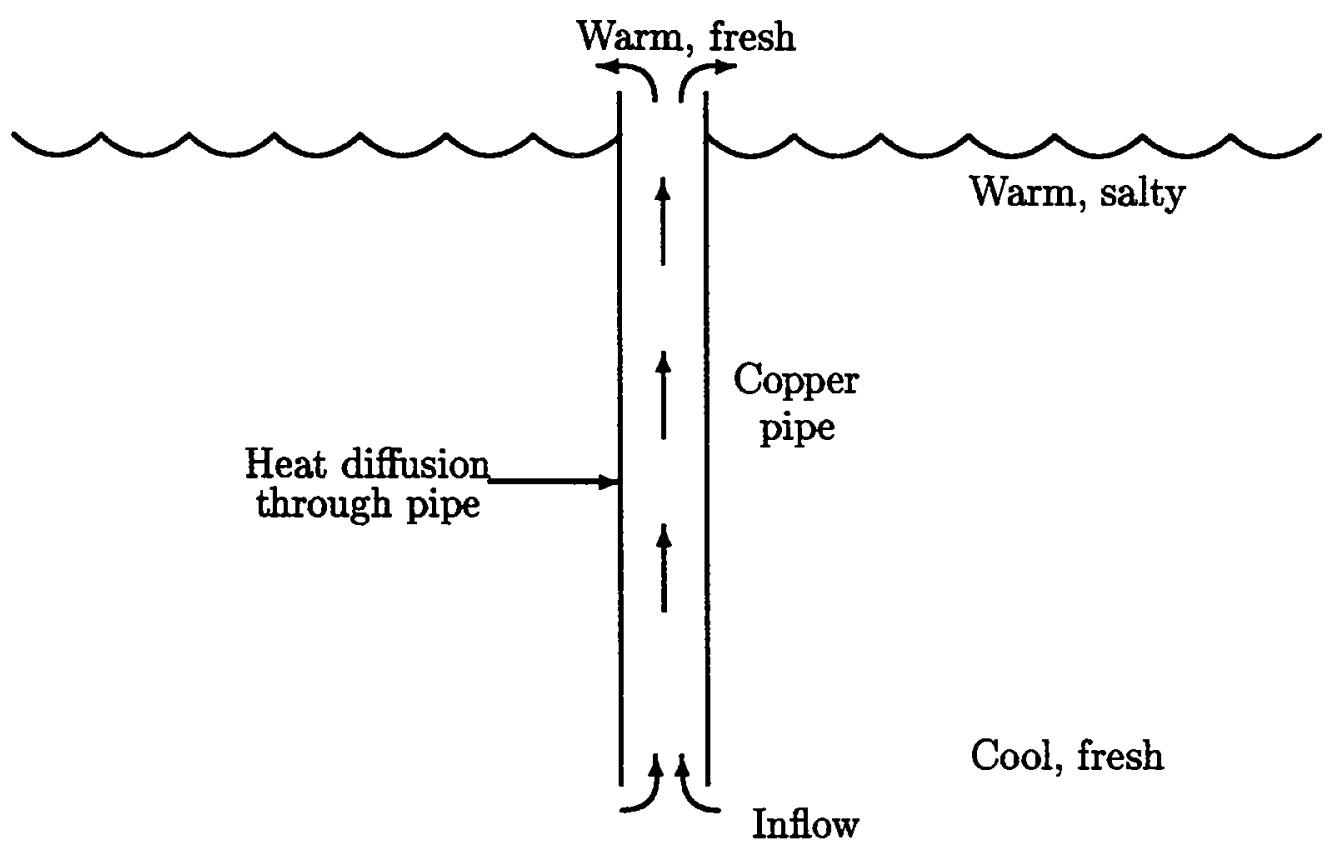

Figure 2: The perpetual salt fountain. 


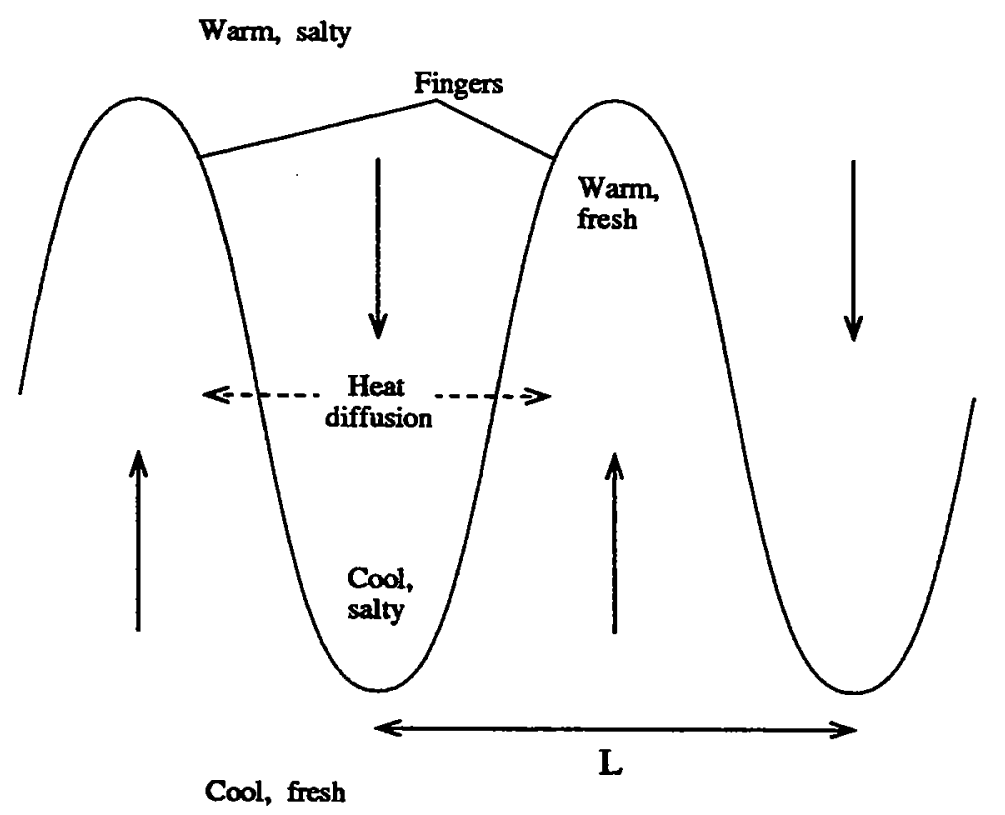

Figure 3: A schematic of the salt fingering instability.

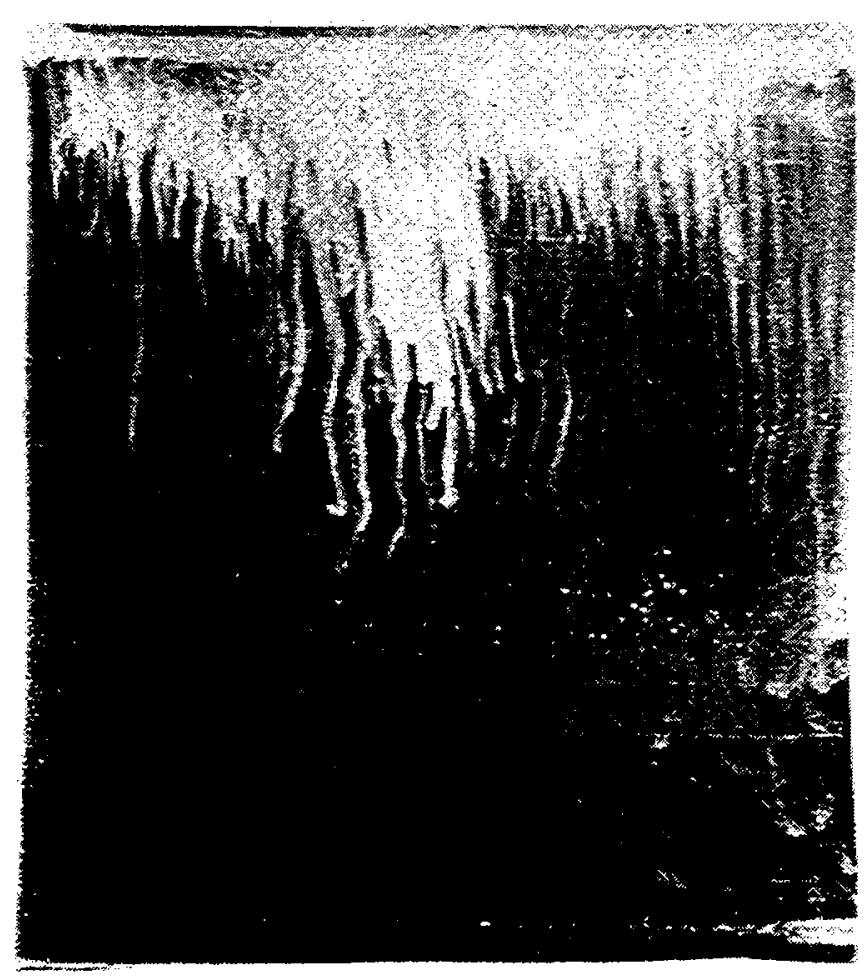

Figure 4: Vertical cross-section of salt fingers from an experiment by Stern and Turner [3]. 


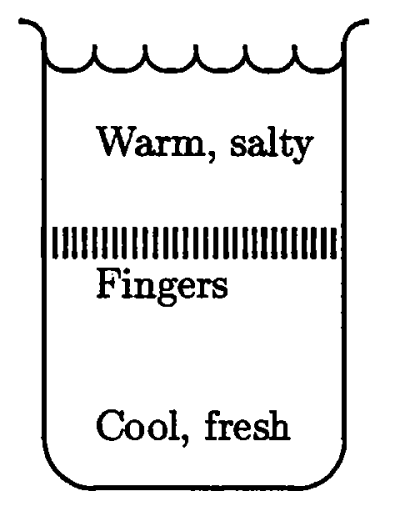

(a)

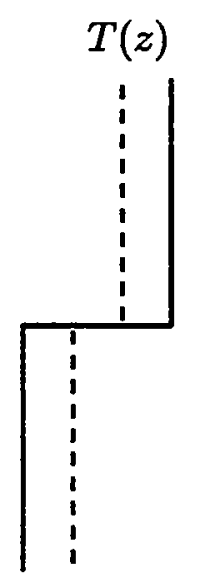

(b)

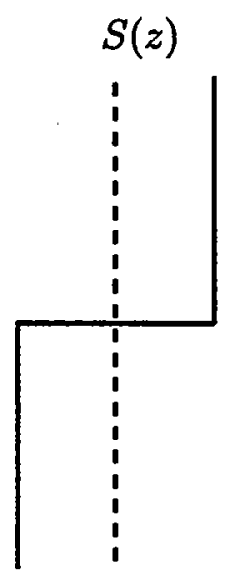

(c)

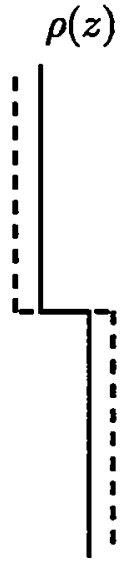

(d)

Figure 5: Two-layer rundown experiment of Turner [4]: (a) Experimental setup, (b) Heat gradient, (c) Salt gradient, (d) Density gradient. The solid line is the initial gradient and the dashed line is the final one.

The experiment is allowed to proceed with no additional heat or salt supply. The density is initially stably stratified, and so the fluid cannot undergo convection. However, "fingers" grow from the interface of the two layers, consisting of alternating columns of ascending and descending fluid. The descending fingers are carrying warm, salty water down and the ascending ones transport cool, fresh water upwards. More potential energy (P.E.) must be lost from the salinity field than is gained by the temperature field in order for the system not to gain energy. The net result is thus to increase the density gradient. This is counter intuitive because both the salinity and temperature are flowing down gradient, whereas the mass flux is flowing up gradient, giving us a negative eddy diffusivity $\kappa_{\rho}$ (see Figures $5 \mathrm{~b}-\mathrm{d}$ ). In addition to showing that the salt field provides the energy for the fingers, Turner also:

1. Showed how the downward mass flux drove convection in the mixed layers (see Figure 6);

2. Showed that convection sweeps away the fingers and sharpens (thins) the interface. The layer thus reaches an "equilibirum" thickness;

3. Measured the salt and heat fluxes-more about this in my lecture on flux measurements.

Shirtcliffe and Turner [5] studied the salt finger pattern in the horizontal plane. They found that the fingers tend to arrange themselves in a square lattice over small domains with irregular orientations, as shown in Figure 7. Their experiment also allowed for direct confirmation of Eq. 1, i.e. that $\mathrm{L} \sim h^{1 / 4}$. 


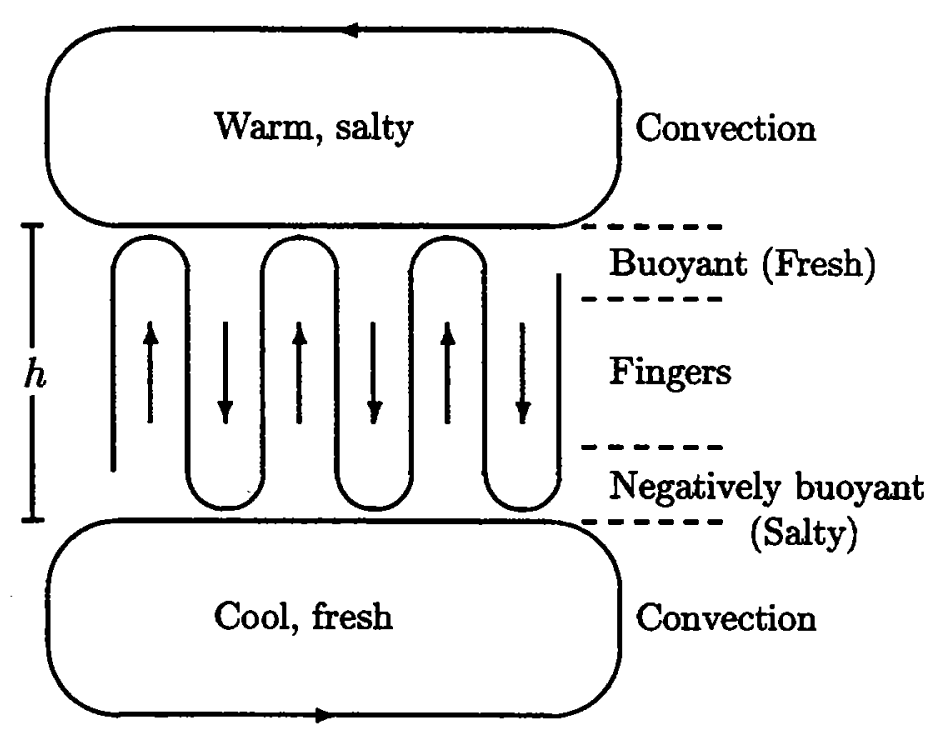

(a)

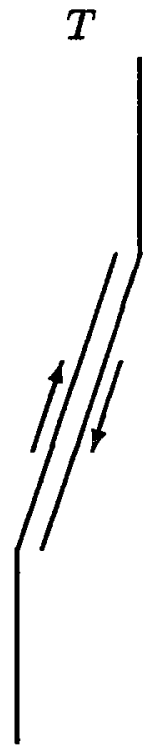

(b)

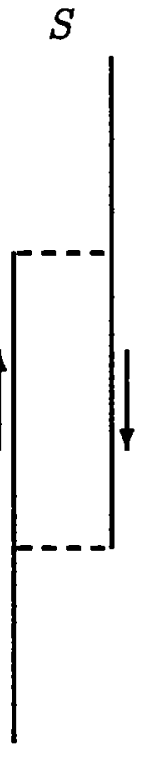

(c)

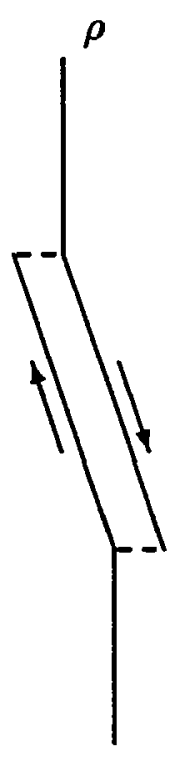

(d)

Figure 6: Salt fingers driving convection in the mixed layers.

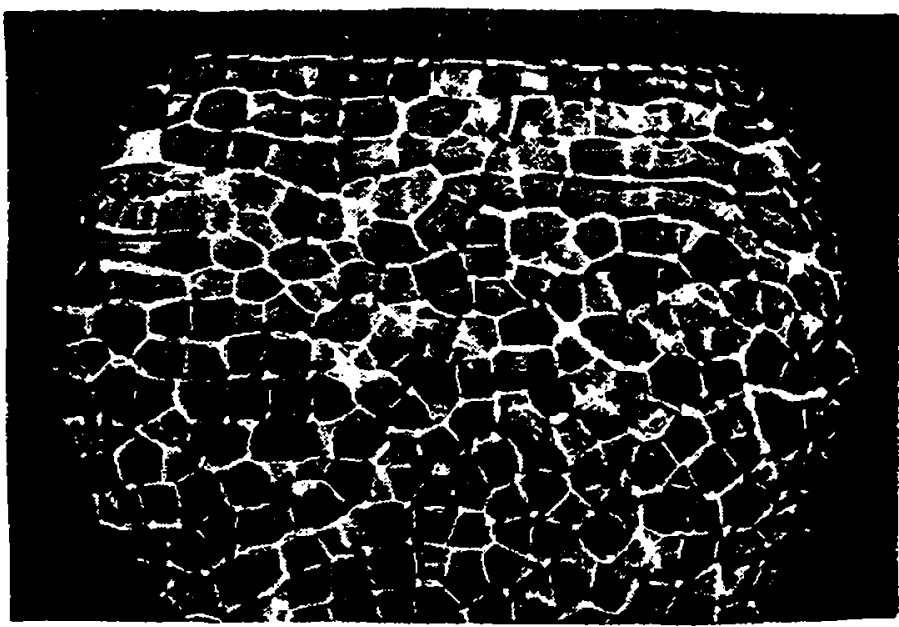

Figure 7: Planview of sugar-salt fingers obtained by the shadowgraph method by Shirtcliffe and Turner [5]. 


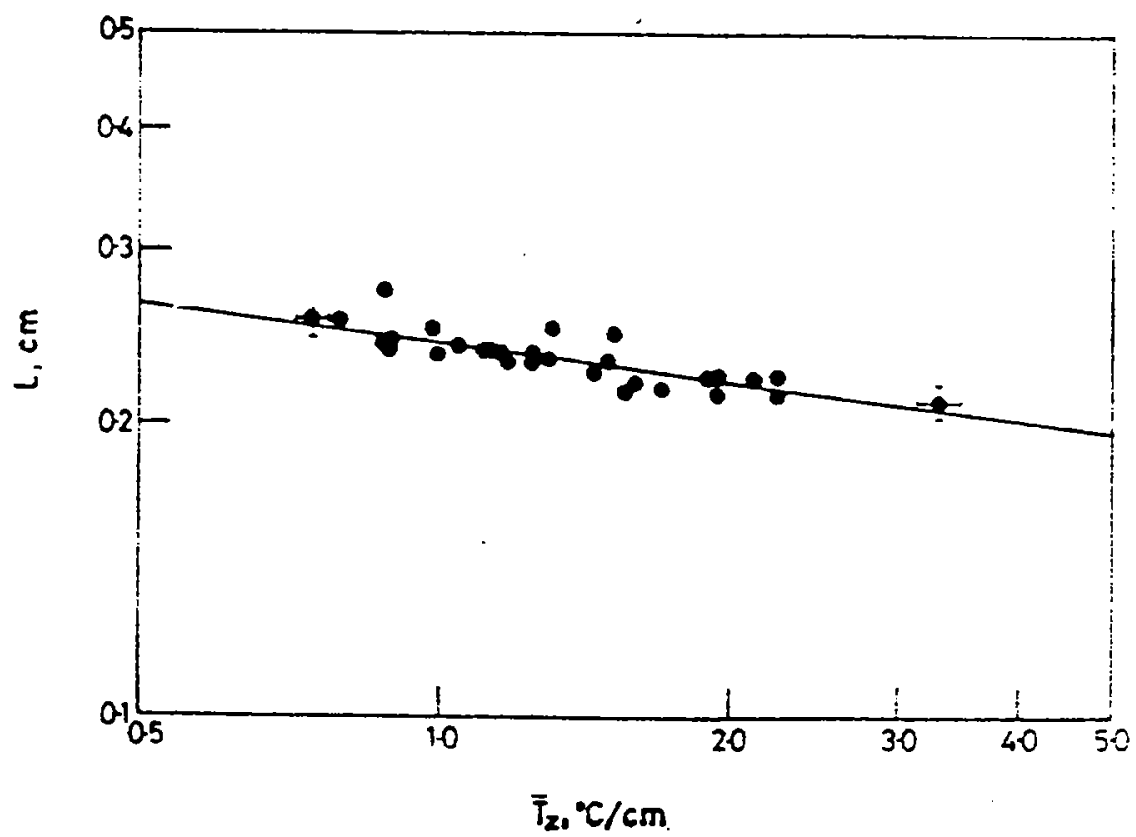

Figure 8: The width of the fingers, $L$, plotted against the mean temperature gradient in the fingers, $\bar{T}_{z}$. The line is a fit to the data with slope $-1 / 4$. From Linden [6].

Double-diffusive experiments are often performed with a variety of solute pairs having unequal diffusivities. For example, sugar diffuses about $1 / 3$ as quickly as salt, and when a solution of sugar is floated over a solution of salt, "sugar" fingers form. Many people think of the classic case of heat and salt when trying to understand the physics of such phenomena, and so experimenters have developed the following terminology: Call the faster-diffusing component " $T$-stuff", and its concentration by $T$. Similarly, call the slower-diffusing component " $S$-stuff", with concentration $S$. For the sugar/salt system, $S$ is the sugar concentration and $T$ is the salt concentration, $\tau \simeq 1 / 3$, and the Prandtl number is $\nu / \kappa_{\text {Salt }} \simeq 500$.

Linden [6] was able to make direct measurements of $w$, the vertical velocity, and the finger width $L$. The former is used to estimate flux. He used heat and sugar $\left(\tau=\kappa_{\text {Sugar }} / \kappa_{T}=.005\right)$ rather than heat and salt $\left(\tau=\kappa_{S} / \kappa_{T}=1 / 70\right)$. This allowed the use of the thymol blue dye technique to trace the flow. He verified Eq. 1 more accurately, as shown in Figure 8.

Linden, upon extrapolating his results to the ocean, predicted salt finger length scales $h \sim 20 \mathrm{~cm}$, widths $L \sim .5 \mathrm{~cm}$, vertical velocities $w \sim .02 \mathrm{~cm} / \mathrm{s}$, and a convecting layer thickness, $H$, of order $40 \mathrm{~m}$. Oceanographic measurements have confirmed the value of $H$, but found $h$ to be of the order of several meters. Linden also made the first detailed salt and temperature profile observations, reproduced in Figure 9. The profile suggests a situation similar to Figure 6 , since the salt concentration is roughly constant through the "core" (fingering) layer, i.e. $S$ is mostly advected in the fingers. Figures 10 and 11 show two shadowgraph pictures of the different regions for high and low density 


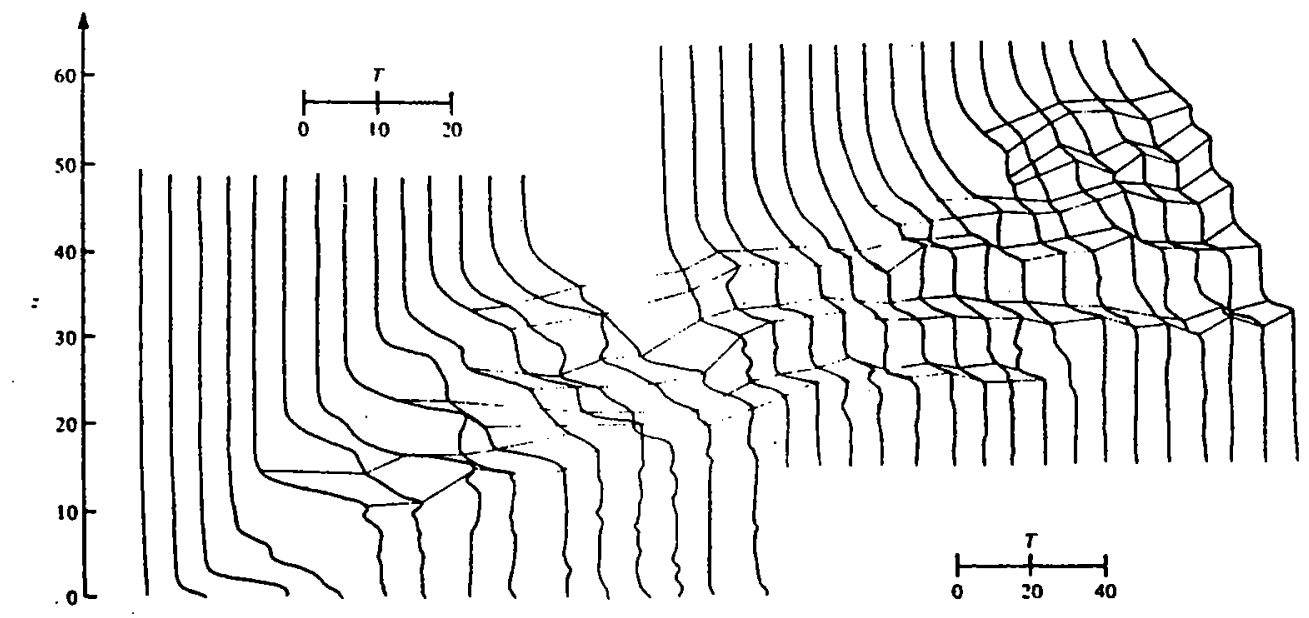

Figure 9: Salt and temperature profiles as a function of depth, from Linden [6].

ratios $R_{\rho}$, respectively (from Griffiths and Ruddick [7]). The density ratio is defined as

$$
\begin{aligned}
R_{\rho} & =\frac{\text { Stabilizing density gradient }}{\text { Destabilizing density gradient }} \\
& =\frac{\alpha|\Delta T|}{\beta|\Delta S|}, \quad \text { in the fingering regime, } \\
& =\frac{\beta|\Delta S|}{\alpha|\Delta T|}, \quad \text { in the diffusive regime. }
\end{aligned}
$$

Turner [4] and Shirtcliffe and Turner [5] introduced the idea of an "equilibrium" interface thickness: in the absence of convection, the fingers would grow vertically and thicken the interface. This is balanced by the tendency of the convective motions to entrain the fingertips. Since the convection is driven by the finger buoyancy flux, a balance between the thickening and thinning effects is expected.

In order to study the breakdown of salt fingering regions into layers, Linden [8] set up a sugar-salt experiment with initial gradients as shown in Figure 12. There is a gradient in the middle providing weak fingers and a sharp $S-T$ interface at the top and bottom of this gradient region. This initial setup leads to the breakdown of the central gradient region into two mixed layers, as shown in Figure 13. 


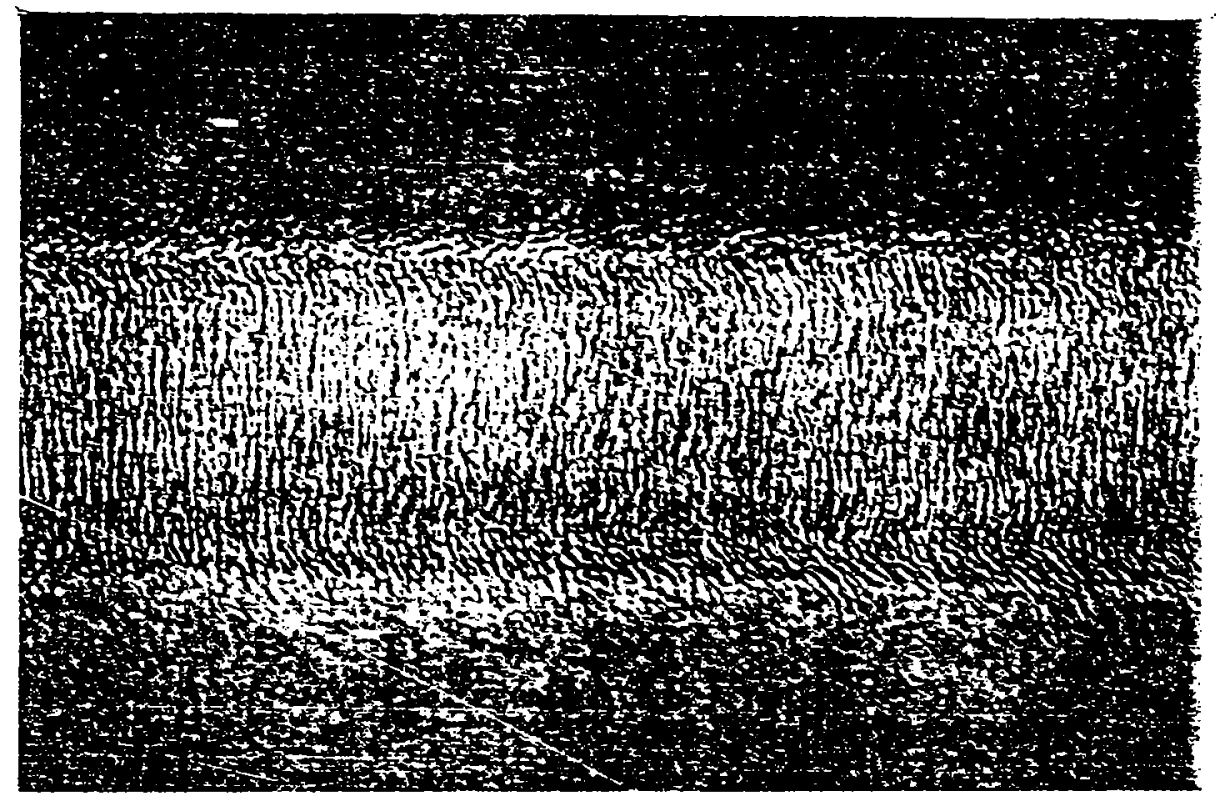

Figure 10: Sugar-salt fingers at high density ratio, $R_{\rho}$. A sharp core zone with wellorganized vertically oriented fingers is clearly visible. There is convection in the well mixed upper and lower regions with transition zones between those regions and the fingers in which buoyant finger fluid is swept into the convection region (from Griffiths and Ruddick [7]).

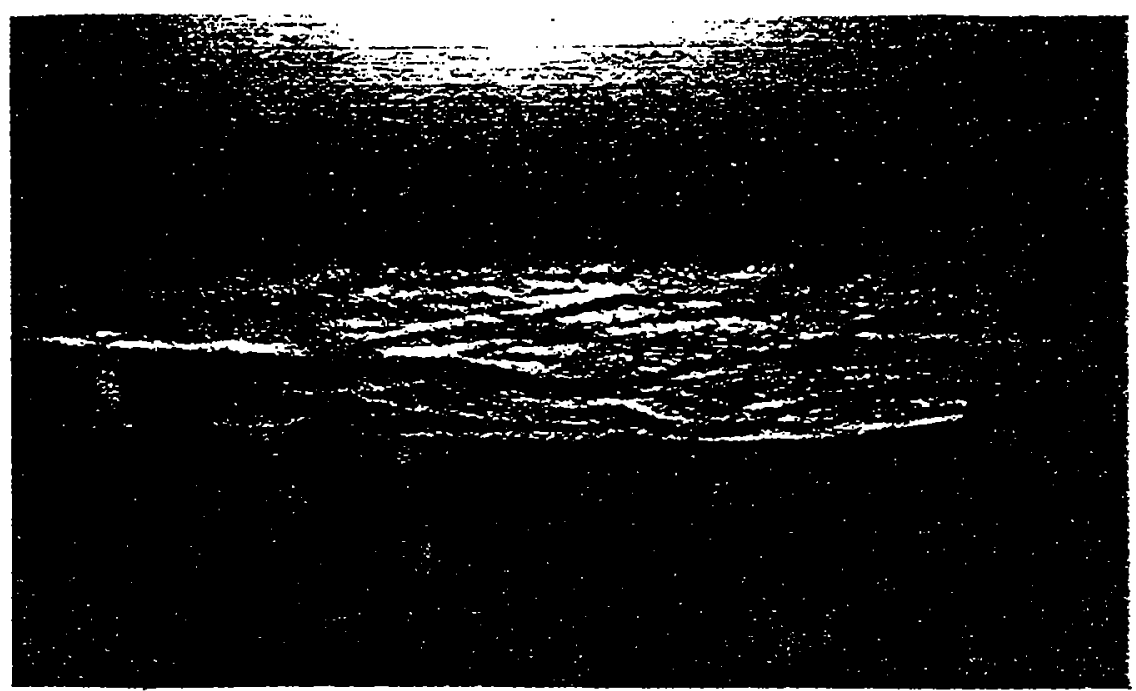

Figure 11: Oblique view of sugar-salt finger interface at low density ratio $R_{\rho}$. Individual fingers are not seen. Fluid is rising from the finger zone into the convecting upper layer in the form of continuous lines of buoyant plumes (from Griffiths and Ruddick [7]). 


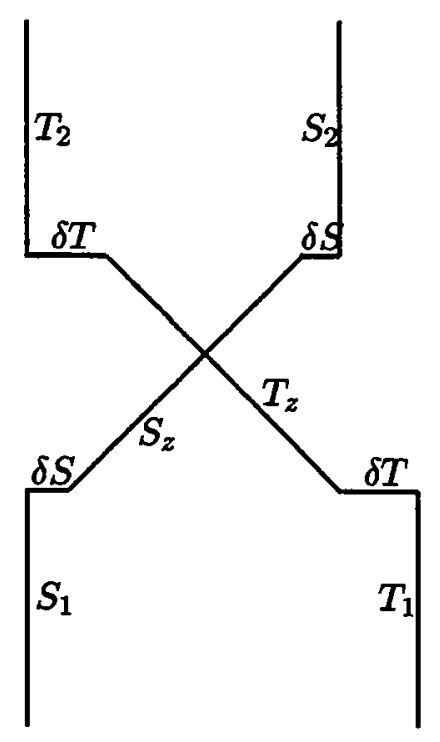

Figure 12: The intended initial vertical profiles of $T$-stuff (salt) and $S$-stuff (sugar) in the experiment by Linden [8].

\section{Two-dimensional Aspects of Double-diffusive Con- vection}

\subsection{Laboratory Experiments}

Recall that the weird and interesting property of double-diffusion is that, while the temperature and salinity fluxes are always downgradient (i.e., warm, salty layers always become cooler and fresher), the flux of density is always upgradient. the density contrast between layers increases. This is of course a release of potential energy, and is the source of energy that drives the convective motions. (Contrast this to Rayleigh-Bénard convection, with temperature and density fluxes downgradient, so that an external source of energy (heating from below and/or cooling from above) is required to drive the convection.)

Double-diffusion is interesting because of its self-driven nature, but it may be important because:

1. The fluxes are enhanced over the molecular values,

2. Double-diffusion causes a stratification to break down into layers, and this can also enhance fluxes.

We were reminded of experiments by Huppert and Linden [9] described in an earlier lecture (Section 1) showing how imposed vertical fluxes of heat or salt can cause 1dimensional layer formation. 


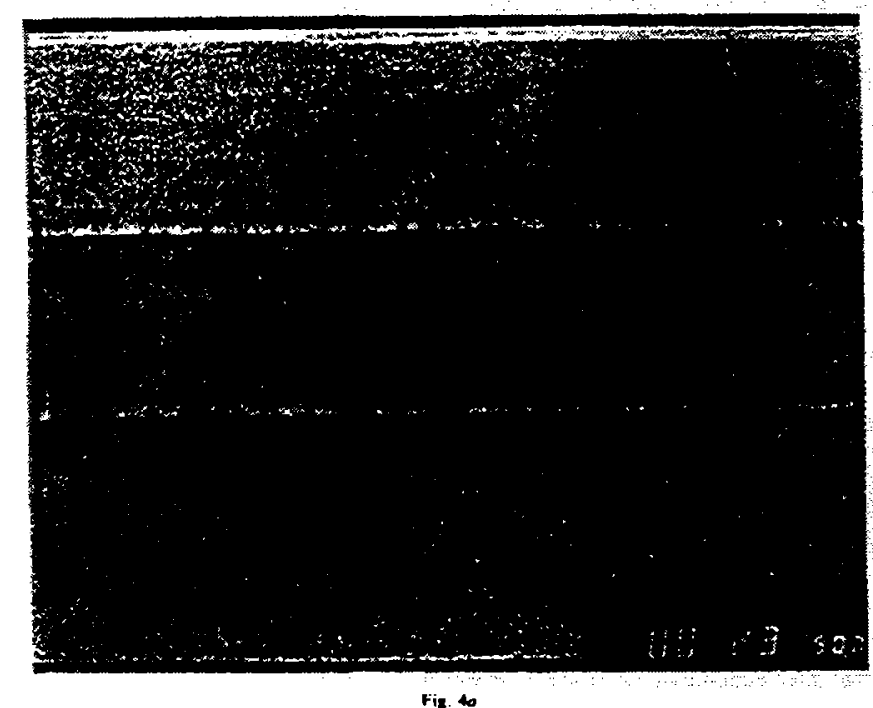

Fiz. 40

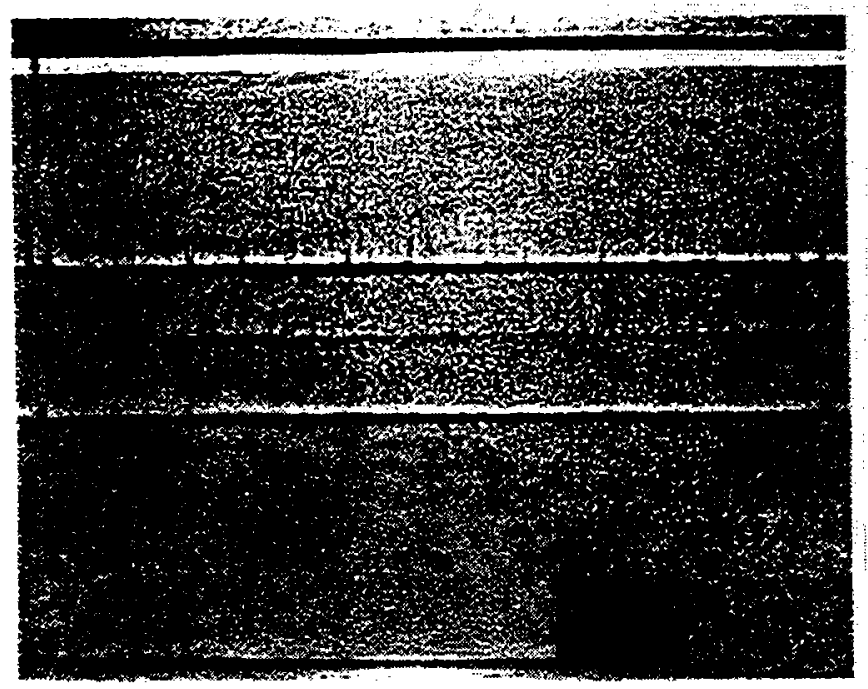

$1 \ldots 1$.

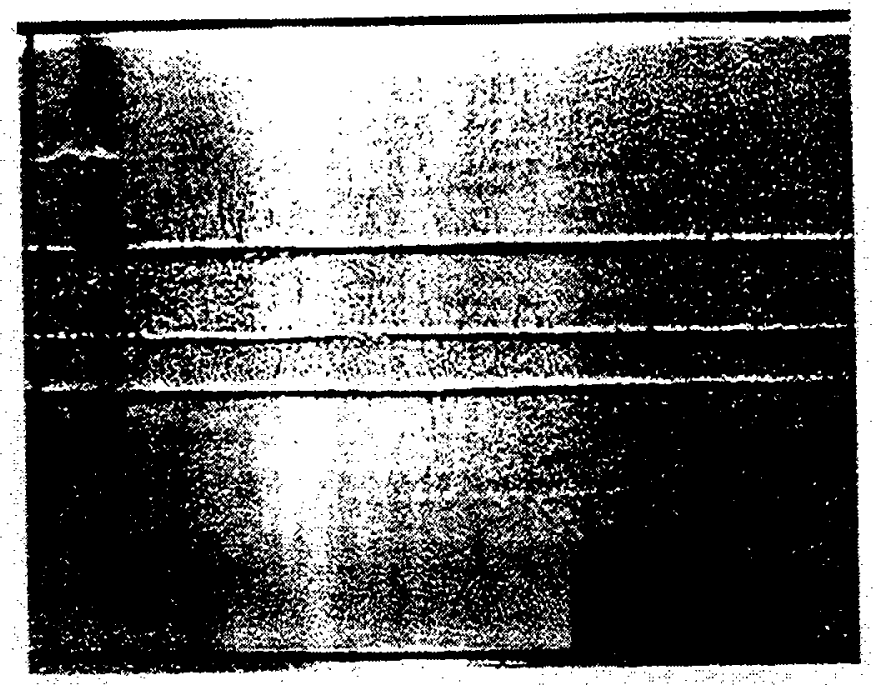

$1+1$

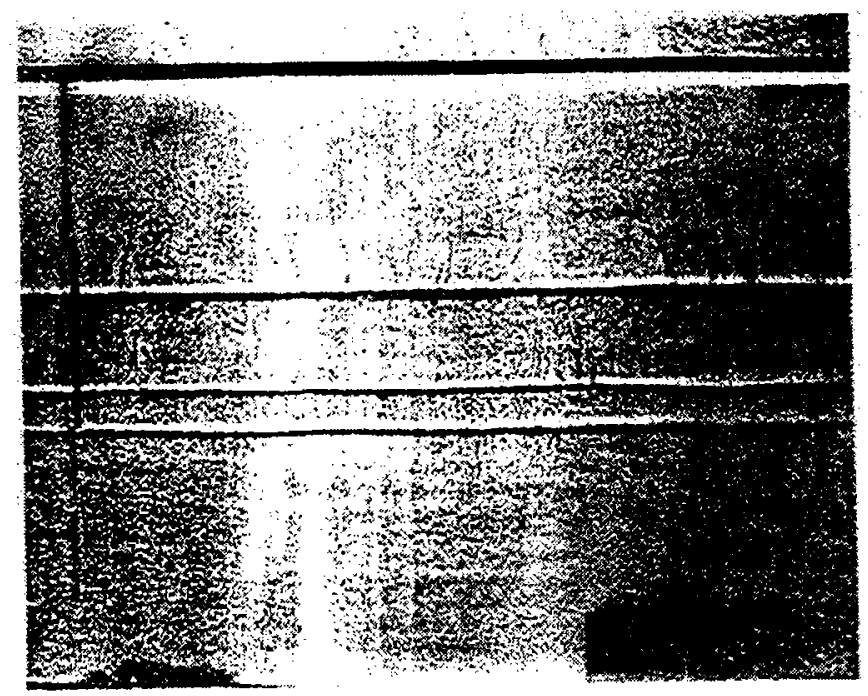

Figure 13: A sequence of photographs, starting from the initial profile in Figure 12, showing the breakdown of the fingers in the interface (from Linden [8]). 


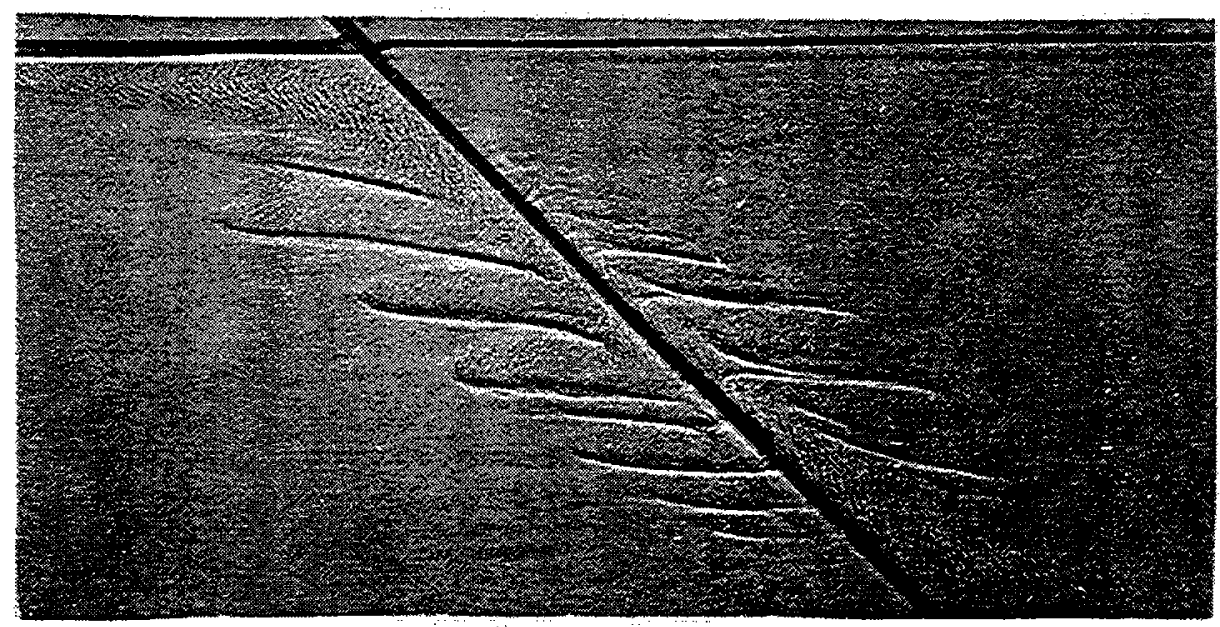

Figure 14: Breakdown of layers due to double diffusive boundary layers (from Turner and Chen [11]).

The classic demonstration of lateral effects was by Thorpe, Hutt and Soulsby [10]. A salt-stratified solution of uniform temperature is heated from one vertical side wall. The thermal boundary layer is buoyant and flows upward, advecting salt solution with it. This vertical boundary layer becomes unstable to a regular series of lateral disturbances, and eventually a regular series of laterally intruding layers grows outward from the heated wall. The important thing is that these layers carry the heat laterally away from the wall far faster than molecular heat diffusion.

Turner and Chen [11] showed that lateral differences of temperature and salinity caused by a great variety of effects can also cause formation of layers, even when they can't form by vertical processes. Figure 14 shows a diffusive salt/sugar stratification into which a sloping impermeable boundary was inserted. The zero-flux boundary condition forces the initially vertical $T$ - and $S$ - concentration gradients to be normal to the sloping boundary. This forces a complex double-diffusive buoyancy layer and causes the eventual breakdown into the layers seen here.

Building on the experiments of Turner [12], Ruddick and Turner [13] designed an experiment to model a thermohaline oceanic front, across which there are lateral differences of $T$ and $S$, equal and opposite in their contribution to the density difference. A long tank with a removable vertical barrier in the middle was filled with density stratified fluid on both sides of the barrier. On the left, the stratification was fresh at the top and sugar water at the bottom, varying linearly in between. On the right, the stratification was fresh at the top and salt water at the bottom. The densities were equal on either side of the barrier at the top and at the bottom, and varied linearly in between. A time-lapse movie showed what happened when the barrier was withdrawn: some initial small-scale internal wave motions generated by the withdrawal died away. This left horizontal isopycnals but fluid properties that varied rapidly across a narrow frontal region in the middle of the tank (the "front"). The contrast in sugar concentra- 

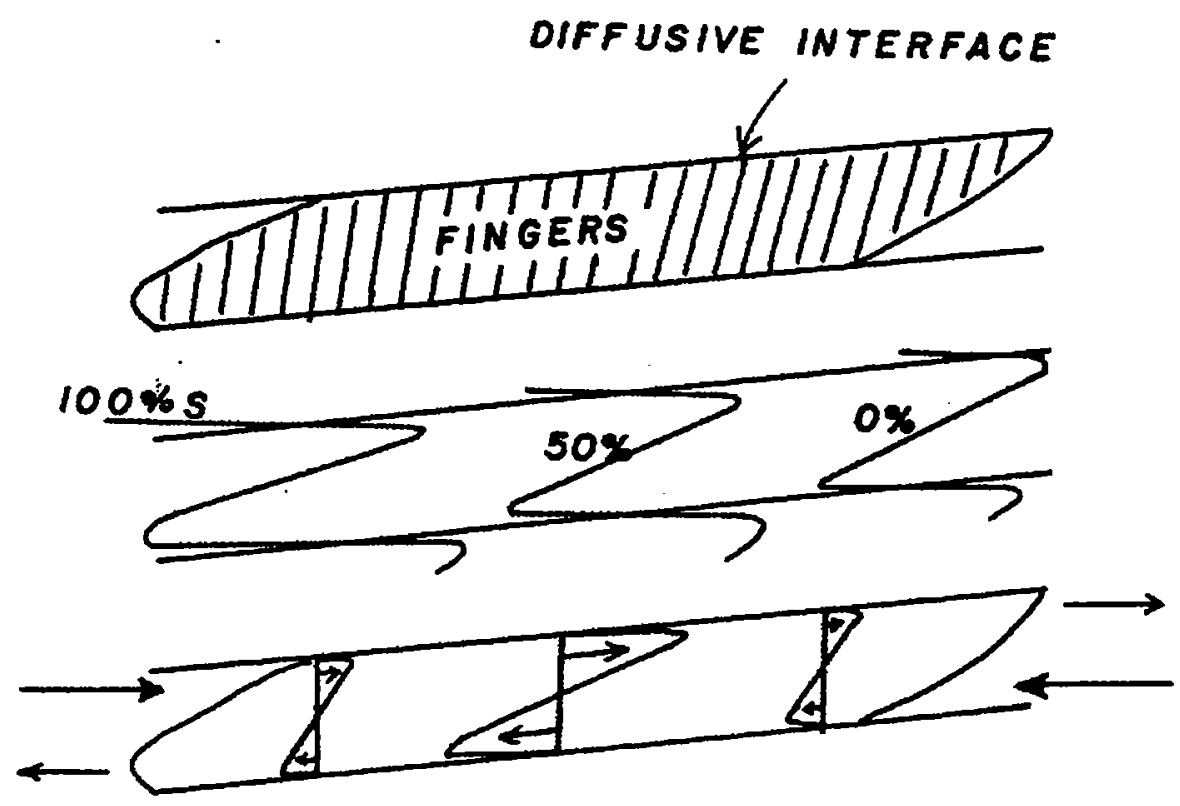

Figure 15: Alternating layers of high- $S$ and high $-T$ water in the central region.

tion, $\beta \Delta S$, varied linearly from zero at the top to a maximum at the bottom. The highsugar side of the front mimics the warm, salty side of an oceanic thermohaline front. The frontal zone appears to have a lot of small-scale turbulent motion, with moderately strong up-and downflows, but no visible organization.

After about ten minutes real time, some organized layering gradually appears, and after about twenty minutes or so, the vertical scale of the layers and the rate of lateral spread becomes visible. The front develops into a slowly broadening "Christmas tree" shape consisting of many intruding layers. The layers near the bottom, where $\beta \Delta S$ is largest, are thicker and intrude faster. Each layer consists of a region of "sugar" fingers bounded above and below by a diffusive interface. The movie also showed that the layers sloped upwards from the "warm and salty" (high-sugar) side.

How do these lateral intrusions work? There is no horizontal difference in density, only in $T-S$ fluid properties. There is a feedback loop involving vertical double-diffusive fluxes as the key link. Suppose a small-amplitude periodic motion is started, consisting of sinusoidally-varying horizontal current:

1. Lateral advection acts to bring high- $S$ water from the left and high- $T$ water from the right, resulting in alternating layers of high- $S$ and high- $T$ water in the central region. If we consider that in the frontal region, contours of $S$ and $T$ are nearly vertical, then lateral advection causes them to become "wiggly" (see Figure 15).

2. The differences between the high $-S$ and high- $T$ layers create alternating bands 


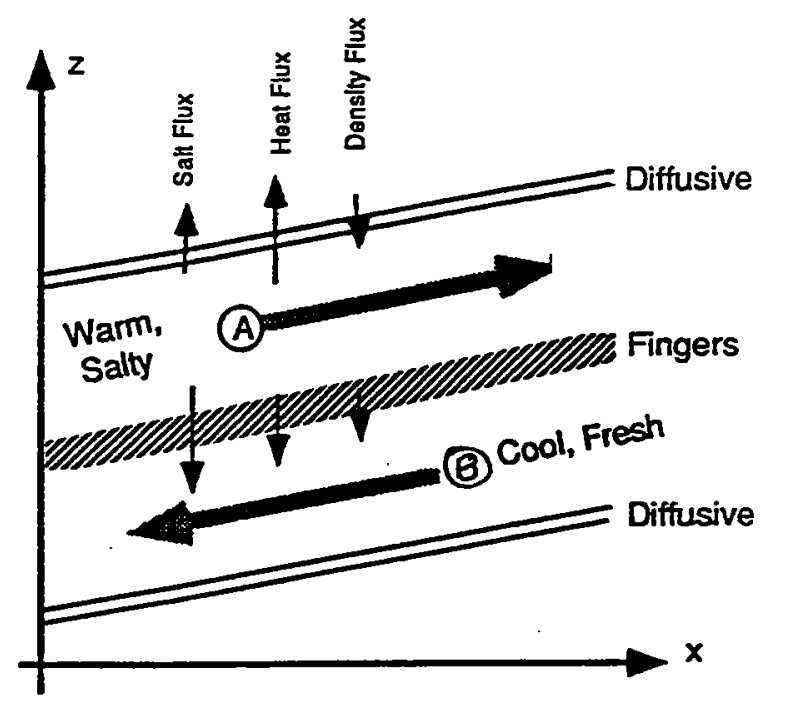

Figure 16: Movement of fluid parcels in the layers.

of finger and diffusive sense vertical gradients in the central region.

3. The vertical finger fluxes cause water parcel $A$, in the upper half of the finger region, to lose $S$, gain $T$, and to become less dense. The opposite occurs to parcel $B$ in the lower half of the fingers (Figure 16).

4. As parcel $A$ moves from left to right, it becomes less dense due to the finger fluxes and so rises as it moves. Parcel $B$ becomes more dense and sinks as it moves from right to left. This explains the slope of the intrusions.

5. Parcel $A$ is slightly lighter than the surrounding waters, and so is caused to move up the slope (i.e., to the right) by its bouyancy. Similarly, parcel $B$ is slightly more dense than its surroundings and "slides" down the slope to the left.

The motions in step 5 reinforce the initial advections in step 1, so the feedback loop is closed.

But what about the diffusive interface, you ask? It also causes $A$ to lose $S$ and gain $T$, but since $A$ lies below the diffusive interface, the diffusive fluxes cause $A$ to become more dense. This alone would cause the layers to slope in the opposite sense, and in combination with the finger fluxes, it is the difference in the finger and diffusive buoyancy fluxes that determines the sign of the layer slopes. Keep this in mind when we look at the Meddy intrusions.

(Melvin Stern noted that the fingers are sheared by the intrusive motions, as evidenced by their tilt. He wondered why they didn't form as sheets, as Linden had found.)

Layer scale: Why are the layers thickest near the bottom and thinnest near the top? Ruddick and Turner [13] presented a simple argument based on the release of potential energy by the fingers. Let's consider a region of height $H$ prior to the formation of an intrusion. It has the same linear density stratification $d \rho / d z=\gamma$ on the left and right of the front, and the "initial" potential energy can easily be calculated. Suppose 
that an intrusion forms, so that in the center of the tank the upper half of the intrusive region is composed of high-S water with concentration excess $\beta \Delta S$, and the lower half is composed of high $-T$ water of concentration $\alpha \Delta T=\beta \Delta S$. When the finger fluxes "run down" the $S$ and $T$ distribution, the $S$ will be nearly uniform from top to bottom of the intrusion. So half of the $S$ has been carried from the top half to the bottom half of the intrusion by the fingers. Since the $T$-flux and $S$-flux are linked by the "flux ratio" $n$ we know the amount of $T$ that has been carried upwards by the fingers, and hence can predict the final density distribution. It is then a simple matter to estimate the "final" potential energy in terms of the cross-frontal $S$-difference and the (unknown) intrusion height. If we require that the final potential energy be less than the initial (i.e., that the fingers have caused a release of potential energy), then a relatively simple expression is obtained for the maximum vertical scale of a double-diffusive intrusion:

$$
H \leq \frac{3}{2}(1-n) \frac{\beta \Delta S}{\frac{1}{\rho} \frac{d \rho}{d z}}
$$

Figure 17 shows the observed layer depth $H$ vs the "depth below fresh water", $d$, from several experiments. the central straight line is the equation above, and the other two lines result from slightly modifying the assumptions in the arguments above. Although the observational scatter is moderately large, the agreement is good. In the next lecture, George Veronis will go through a linear instability theory [14] that predicts the scale of the fastest-growing intrusions, and has some points of agreement with this simple estimate. The width of each intrusion was monitored over time, and the rates of advance of the noses were calculated. The nose velocity $U_{\text {nose }}$ was also found to be proportional to $D=(\beta \Delta S) /\left(\rho^{-1} d \rho / d z\right)$, as can be seen in Figure 18. Combining the results for $H$ and for $U_{\text {nose }}$ gives $U_{\text {nose }} \simeq 0.005 N H$, where $N^{2}=-g \rho^{-1} d \rho / d z$. The reason the nose velocity is of interest is that it is proportional to the lateral flux of $S$ and of $T$ in finiteamplitude intrusions. It is this horizontal flux, driven by vertical fluxes, that makes lateral intrusions potentially important in the ocean. Intrusions are incredibly common, occuring at virtually all oceanic fronts, and probably represent a major mechanism for mixing salt and heat across the fronts.

\subsection{Ocean Observations}

Thermohaline intrusions are found at almost all oceanic fronts - relatively sharp boundaries between'two water masses with different temperature and salinity characteristics. During one of the earliest uses of the STD (Salinity, Temperature, Depth), Stommel and Fedorov [15] found intrusions, deduced that lateral mixing was occuring, and speculated that the intrusion would blend into the surrounding waters via vertical mixing. Prior to this, the point measurements offered by bottle casts gave too coarse resolution to allow intrusions to be observed clearly. Toole [16] showed that intrusions in the Antarctic Circumpolar Front migrated vertically across isopycnal surfaces as they crossed the front, in the same manner as the laboratory intrusions described earlier, and 


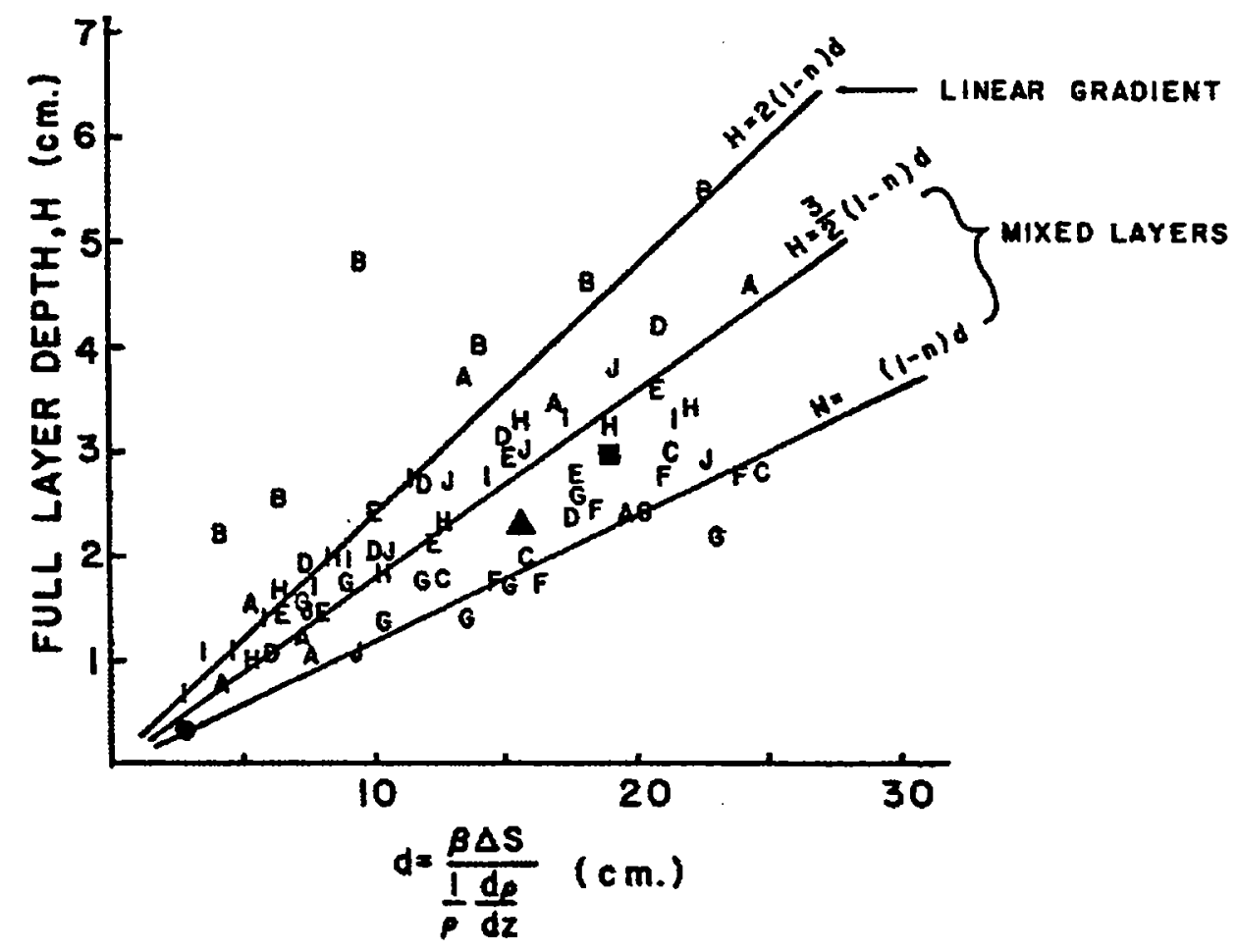

Figure 17: Observed layer depth $H$ vs the "depth below fresh water", $d$, from several experiments.

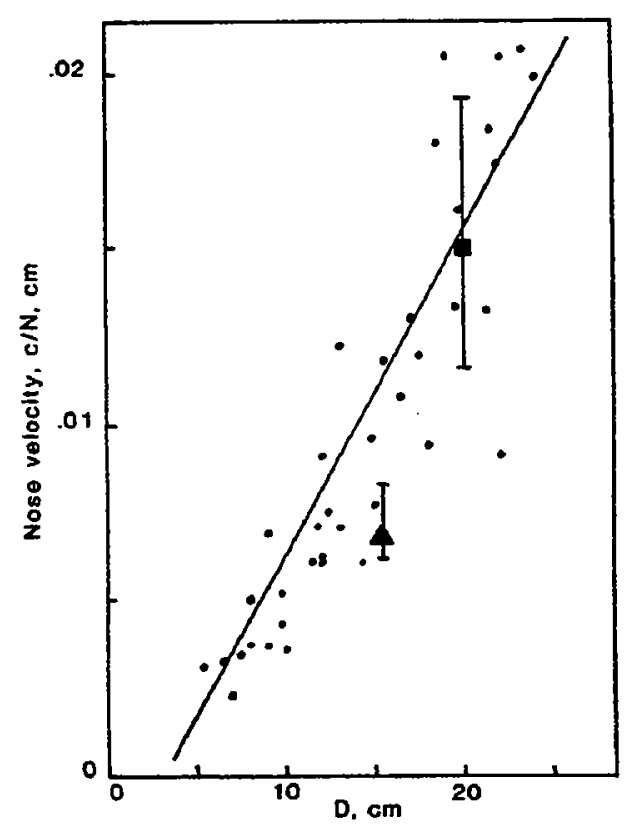

Figure 18: Nose velocity of intrusions as a functions of $D$. 


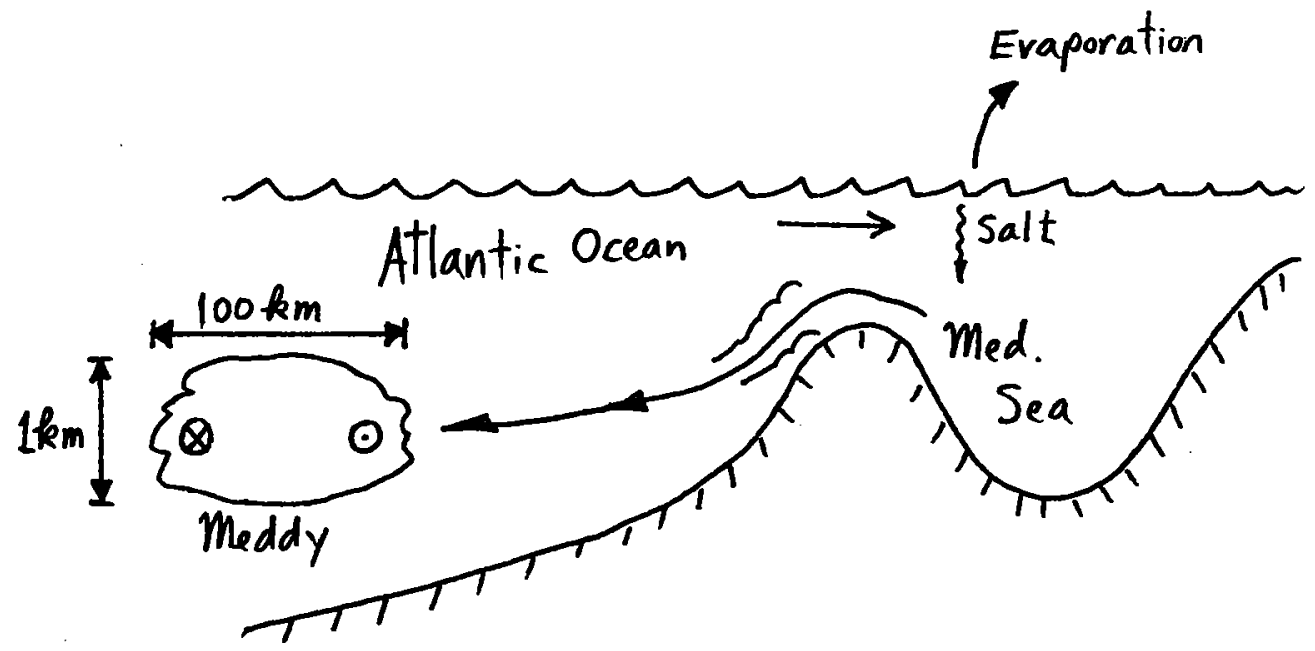

Figure 19: Mechanism of Meddy formation.

deduced that the lateral fluxes of salt and heat were large - about the same as lateral fluxes by instablity of the frontal current. Other examples of intrusion observations by Gregg [17] and Anderson and Pinkel (1996) were shown.

The fact that intrusions migrate across isopycnals suggests that they are driven by vertical double-diffusive mixing, and appear to cause strong lateral fluxes. But are they in fact strong? Observations of a Mediterranean salt lens (a "Meddy") showed how strong the effects of intrusions can be.

The Mediterranean Sea is in a warm, dry climate, and so more water evaporates ifrom its surface than falls as rain. The net loss of water leaves the salt behind, causing the Med to become more salty than the Atlantic. The salty water flows out through the Straits of Gibraltar, and is replaced by inflowing slightly fresher Atlantic water. This exchange of salty water for fresher keeps the salinity from becoming too large (see Figure 19).

The Med outflow water is quite dense compared to the Atlantic water, and tumbles down the Continental slope as a gravity gurrent, mixing as it goes (about 2 parts Atlantic to 1 part Mediterranean). The gravity current is pretty much at it's neutral density level when it reaches $1 \mathrm{~km}$, and the current then goes unstable and forms Meddies. These are $300 \mathrm{~km}$ diameter by $1500 \mathrm{~m}$ thick, composed of the mixture of Med and Atlantic water from the current. They rotate anticyclonically with about a 6day period and drift around the Eastern North Atlantic for years, sometimes travelling thousands of kilometers. They are very warm and salty compared to Atlantic water at the same depth and density, so their travels and eventual decay should affect where the 
salt goes.

Larry Armi of Scripps began an interesting experiment: he found a Meddy (named "Sharon"), surveyed it with a CTD (Conductivity Temperature Depth profiler - the modern digital equivalent of the STD), and put SOFAR floats inside it's core. These could be tracked acoustically so that we could find the Meddy later on.

Figure 20 shows two views of the salinity structure of Meddy Sharon, taken a year apart. The $\mathrm{x}$-axis is horizontal distance, the $\mathrm{y}$-axis is depth in metres, and the $z$-axis is salinity. The meshplot can be regarded as a succession of $S(z)$ traces in the $y-z$ plane, displaced successively in the $x$-direction as the ship moves through the Meddy. Note that in (a) Meddy has the appearance of a mountain with steep, jagged sides and a smooth, sloping top. The jagged sides are intrusions, which show up as wiggles in salinity. The smooth top shows that intrusions haven't reached the centre yet, and the slope means that the core is stably stratified in salt - no fingers can form in the core without intrusions! Note also that you can see evidence of layering underneath the core. This is probably due to salt fingers, but we concluded that the flux of salt out the bottom by salt fingers was unimportant. In (b), taken a year later, we see that the top of the mountain is no longer flat, and that the Meddy has eroded considerably it's visibly smaller. The main point is that the intrusions have worked their way into the centre over the course of a year, a rate of advance of about $1 \mathrm{~mm} / \mathrm{s}$. This is what caused the erosion and the loss of salt. (The $T$ and $S$ didn't change until the intrusions got there.) The steps at the bottom have become much more pronounced, too, so salt fingers seem to be working there, even though their salt flux doesn't have much effect on the Meddy.

Let's compare the Meddy intrusions with the laboratory ones:

Vertical scale: The Ruddick-Turner formula predicts $H$ to be hundreds of metres, when it's really $25 \mathrm{~m}$. This difference is resolved by appealing to Niino's theory (see tomorrow's lecture by George Veronis), and noting that the "Niino number" is large enough that the front can be considered wide, while the laboratory front is narrow, and the layer scales should (and do) obey the Toole and Georgi [18] theory.

Horizontal advance speed: The lab experiment found $U$ to be about $0.005 \mathrm{NH}$, where $\mathrm{N}$ is the buoyancy frequency and $\mathrm{H}$ the full layer thickness. As long as we use the observed $\mathrm{H}$ and not the predicted, this predicts an advance speed of about $1 \mathrm{~mm} / \mathrm{s}$, in agreement with observations.

Intrusion slopes: One set of closely spaced stations gave particularly useful information, since individual intrusions could be followed from one profile to the next. We followed the maxima of salinity from each intrusion, and computed the density at that point. In the lower half of the Meddy that warm and salty intrusions became lighter as they moved outwards, as we found in the lab experiment. However, in the upper part of the Meddy, the exact opposite occured! Warm and salty intrusions moved down as they moved outwards in the upper half. This was surprising until I remembered one laboratory experiment I conducted in which the overall stratification was diffusive on both sides of the barrier. In that experiment, the diffusive stratification inhibited the fingers and gave a boost to the diffusive interfaces, with the result that the layers 

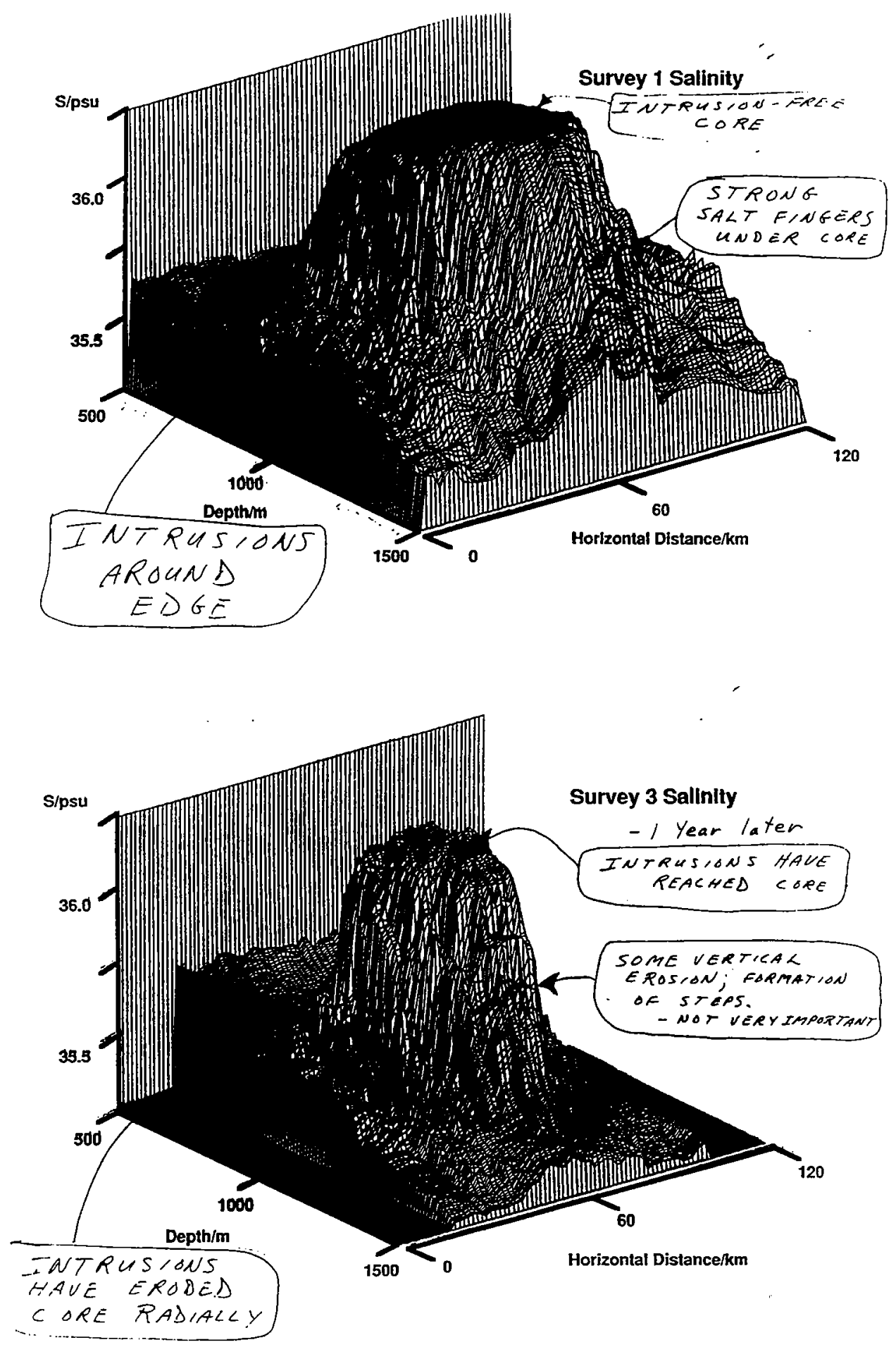

Figure 20: Two views of the salinity structure of Meddy Sharon, taken a year apart. The vertical axis is the salinity. 


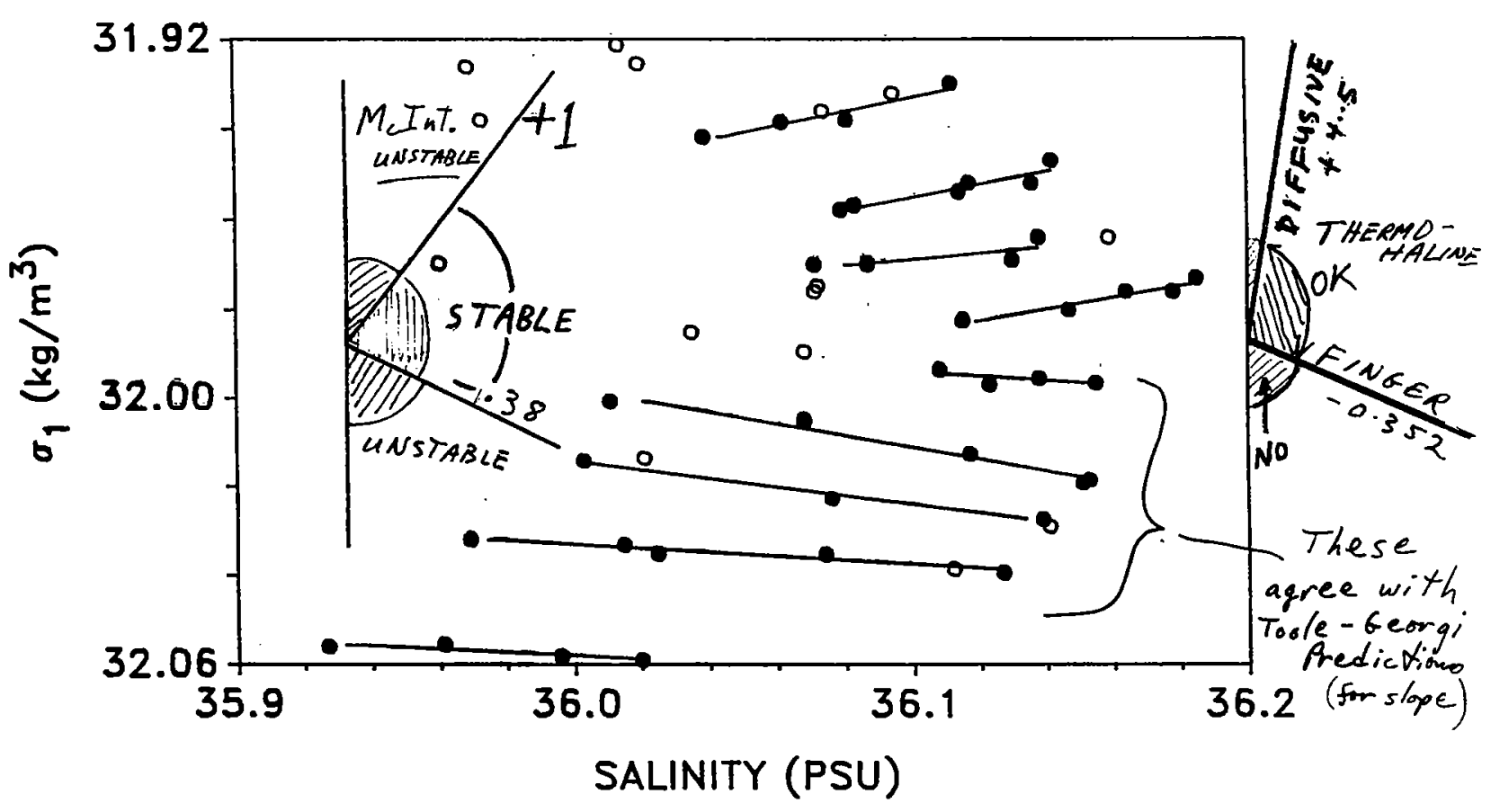

Figure 21: Plot of $S$ at local $S$-maximum (horizontal axis) against density (vertical axis is decreasing density). The solid lines connect maxima found on successive traces, and indicate the migration across isopycnals. The "angle" indicators show the ranges of acceptable slopes for the McIntyre instability [19], and for double-diffusively driven thermohaline intrusions.

sloped with the opposite sense. This makes sense when considering the combined effect of fluxes on a water parcel: it's the difference of the buoyancy fluxes from the diffusive interface above and the fingers below that determines whether a warm, salty fluid parcel becomes lighter or heavier.

So, the slopes seem to make sense in terms of the fluxes across the intrusion boundaries, as long as we don't forget about the diffusive fluxes. A close examination of the slopes allows us to compare the $T / S$ changes along the layers with the expected ratios of fluxes. The slopes are well within the range expected from our knowledge of finger and diffusive flux ratios. More interestingly, there is an alternative theory of layer formation due to McIntyre [19] that does not involve double-diffusion of heat and salt, but instead relies on a Prandtl number different from 1 to destabilize a baroclinic shear flow. This is a type of double-diffusion since the mechanism uses differential diffusion of momentum and of mass to extract either KE of the shear or PE of the sloping isopycnals. It turns out that the layer slopes are completely out of the range in which they can extract either KE or PE of the basic flow (Figure 21), so the McIntyre mechanism is not at work here. Hence the $T / S$ differences are necessary to drive the intrusions, and the energy from the shear flow and sloping isopycnals is not driving them. 
Notes written by: Barry Ruddick, Joseph Biello, Jean-Luc Thiffeault

\section{References}

[1] H. Stommel, A. B. Arons, and D. Blanchard, "An oceanographic curiosity: the perpetual salt fountain," Deep-Sea Research 3, 152 (1956).

[2] M. E. Stern, "The 'salt fountain' and thermohaline convection," Tellus 12, 172 (1960).

[3] M. E. Stern and J. S. Turner, "Salt fingers and convecting layers," Deep-Sea Research 16, 497 (1969).

[4] J. S. Turner, "Salt fingers across a density interface," Deep-Sea Research 14, 599 (1967).

[5] T. G. L. Shirtcliffe and J. S. Turner, "Observations of the cell structure of salt fingers," Journal of Fluid Mechanics 41, 707 (1970).

[6] P. F. Linden, "On the structure of salt fingers," Deep-Sea Research 20, 325 (1973).

[7] R. W. Griffiths and B. R. Ruddick, "Accurate fluxes across a salt-sugar finger interface deduced from direct density measurements," Journal of Fluid Mechanics 99, 85 (1980).

[8] P. F. Linden, "The formation of banded salt finger structure," Journal of Geophysical Research 83, 2902 (1978).

[9] H. E. Huppert and P. F. Linden, "On heating a stable salinity gradient from below," Journal of Fluid Mechanics 95, 431 (1979).

[10] S. A. Thorpe, P. K. Hutt, and R. Soulsby, "The effect of horizontal gradients on thermohaline convection," Journal of Fluid Mechanics 38, 375 (1969).

[11] J. S. Turner and C. F. Chen, "Two-dimensional effects in double-diffusive convection. journal of fluid," Journal of Fluid Mechanics 63, 577 (1974).

[12] J. S. Turner, "Double-diffusive intrusions into a density gradient," Journal of Geophysical Research 83, 2887 (1978).

[13] B. R. Ruddick and J. S. Turner, "The vertical length scale of double-diffusive intrusions," Deep-Sea Research 26A, 903 (1979).

[14] H. Niino, "A linear stability theory of double-diffusive horizontal intrusions in a temperature-salinity front," Journal of Fluid Mechanics 171, 71 (1986). 
[15] H. Stommel and K. N. Fedorov, "Small scale structure in temperature and salinity near timor and mindanao," Tellus 19, 306 (1967).

[16] J. M. Toole, "Intrusion characteristics in the antarctic polar front," Journal pf Physical Oceanography 11, 780 (1981).

[17] M. C. Gregg, "The three-dimensional mapping of a small thermohaline intrusion," Journal pf Physical Oceanography 10, 1468 (1980).

[18] J. M. Toole and D. T. Georgi, "On the dynamics and effects of double-diffusively driven intrusions," Progress in Oceanography 10, 123 (1981).

[19] M. E. McIntyre, "Diffusive destabilization of the baroclinic vortex," Geophysical Fluid Dynamics 1, 19 (1970). 


\section{Lecture 2.}

\section{Barry Ruddick and George Veronis}

Two simple experiments were set up to demonstrate salt fingers (this regime was discussed in detail during the previous lecture). In the first experiment, the solution of a few crystals of pottasium permanganate, a reddish dye, at a high temperature was cautiously poured at the top of fresh, cold water, as can be seen from fig. 1. A clear horizontal interface between the colored (hot and salty) fluid in the top portion of the tank $(20 \times 10 \times 5 \mathrm{~cm})$ and the transparent one in the bottom portion was initially observed. After a relatively long period of time, however, a multitude of thin vertical columns, spreading down from the interface, could clearly be distinguished. Eventually, these columns got mixed with the surrounding fluid, as a result of the lateral diffusion and possibly intrusions, and the whole tank became uniformly reddish. In this experiment the few crystals of permanganate provided the salt concentration. As a consequence, it took about 10-20 minutes until the manifestation of the fingers became clearly discernible, since their growth rate was small for such a low supercriticality. In the second experiment, the salt (sodium-chloride) was deliberately added to the hot blue dye to reach a higher degree of supercriticality. As a result of this, smaller fingers were observed within a few minutes after the clear interface had formed.

There are several locations in the ocean where a sequence of well-mixed layers separated by relatively sharp interfaces (in $S, T$, and density) is observed. If the fluxes of heat and salt could be predicted on the basis of individual observations of $\Delta T$ and $\Delta S$ across the steps, the large-scale effects of thermohaline fluxes could be assessed. Turner (1965) adapted dimensional arguments from thermal convection literature (the " $4 / 3$ law") to make this possible. But beware! It does not seem to work for salt fingers. To understand the fluxes in the double-component fluid, it is first worth considering the simplified case of purely thermal convection (Rayleigh-Benard) from the standpoint

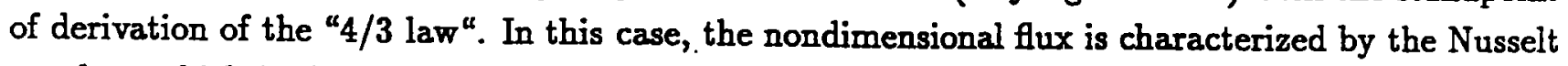
number, which is the ratio of the dimensional temperature flux, $F_{T}\left[\frac{m K}{a}\right]$, and the flux due to pure conduction,

$$
N u=\frac{F_{T} H}{\kappa_{T} \Delta T}
$$

The other nondimensional parameters on which the Nusselt number could depend can be composed of the following:

$$
\begin{array}{ll}
g \alpha \Delta T\left[\frac{m}{s^{2}}\right], & \text { buoyancy contrast due to thermal boundary forcing, } \\
H[m], & \text { fluid depth (the effects of finite width are ignored), } \\
\kappa_{T}\left[\frac{m^{2}}{s}\right], & \text { molecular diffusivity of heat, } \\
\nu\left[\frac{m^{2}}{s}\right] & \text { molecular (kinematic) viscosity. }
\end{array}
$$

Since the motion is driven by buoyancy forces, $g \alpha \Delta T$ should appear as a group. The nondimensional parameters are the Prandtl and the Rayleigh numbers, $\operatorname{Pr}=\frac{\nu}{\kappa \dot{x}}$ and $R a=\frac{g a \Delta T H^{3}}{\kappa_{T^{\nu}}}$, 


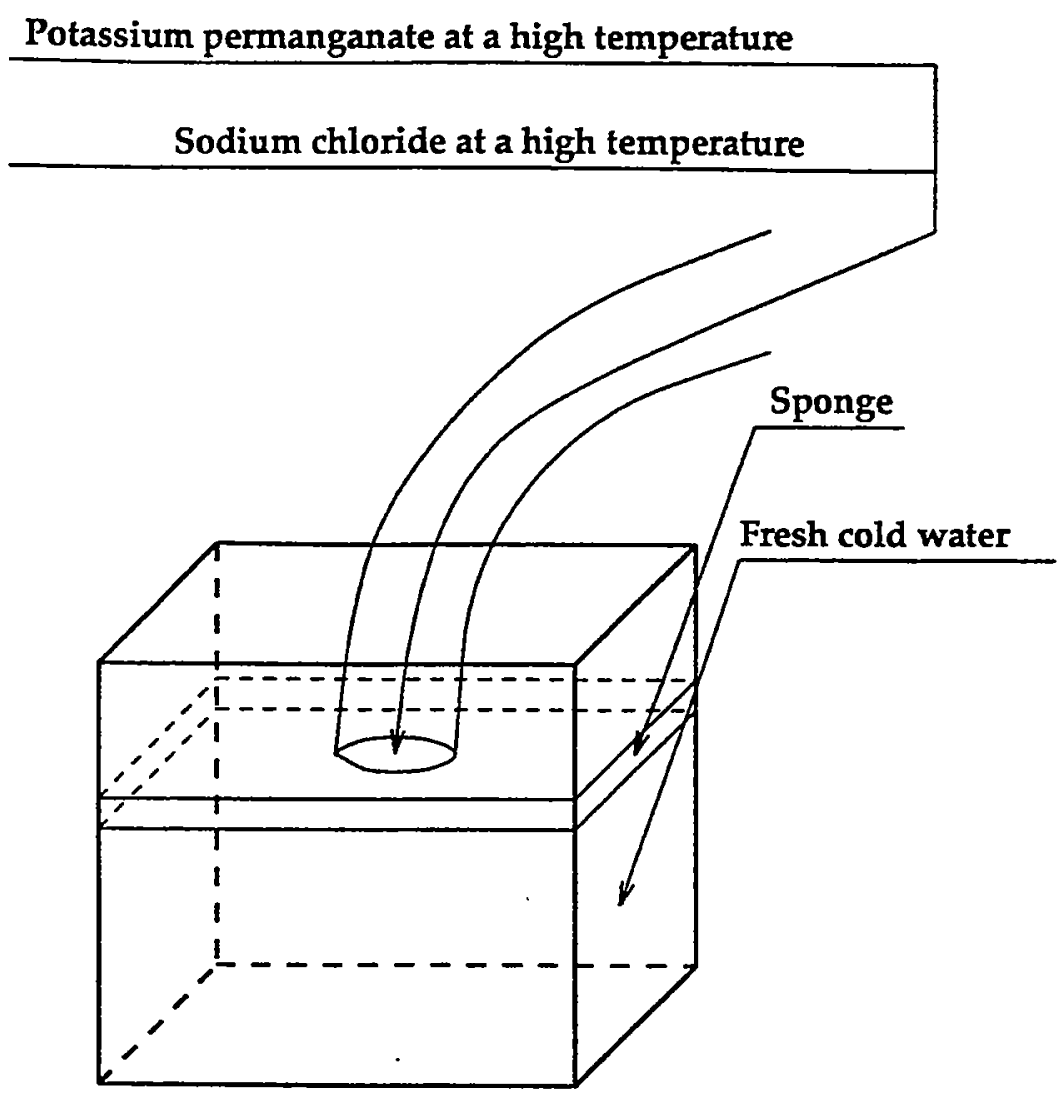

Figure 1: A schematic of the salt-finger experiment. 
respectively. For example, for water $\operatorname{Pr} \approx 7$, for air $\operatorname{Pr} \approx 1$. Thus, one would expect that

$$
N u=f(P r, R a) \text {. }
$$

When the Prandtl number is fixed, we postulate the relationship

$$
\frac{F_{T} H}{\kappa_{T} \Delta T} \propto\left(\frac{g \alpha \Delta T H^{3}}{\kappa_{T} \nu}\right)^{n}
$$

It can be argued that as $H \rightarrow \infty$ the distance between boundaries becomes unimportant in its effect on the heat flux. Demanding that $F_{T}$ be independent of $H$ in (7) yields

$$
n=1 / 3 \text {, }
$$

which has limited support from laboratory measurements (although more precise measurements show powers slightly less than $1 / 3$ ). As it turns out, (8) allows us to write (7) as

$$
\alpha F_{T}=C\left(\frac{g \kappa_{T}^{2}}{\nu}\right)^{1 / 3}(\alpha \Delta T)^{4 / 3}
$$

where $C$ is an empirical flux coefficient.

In the more complex diffusive regime of double-diffusive convection, $C$ is a function of the density ratio,

$$
R_{\rho}=\frac{\beta \Delta S}{\alpha \Delta T}=\frac{\text { stabilizing } S}{\text { destabilizing } T}>1,
$$

where $\tau=\frac{\kappa_{x}}{\kappa_{S}}$, and of $\operatorname{Pr}$ (here $\kappa_{S}$ is the solute diffusivity). Different investigators have used slightly different versions of equation (9): i.e., the factor $\left(\frac{g \kappa_{r}^{2}}{\nu}\right)^{1 / 3}$ has been replaced by similar factors with the same dimensions; for example, $(g \nu)^{1 / 3}$. The salinity flux in turn is commonly expressed as

$$
\beta F_{S}=\alpha F_{T} R_{F},
$$

where the flux ratio, $R_{F}=\frac{\beta F_{S}}{\alpha F_{T}}=\frac{S-\text { flux }}{T-f l u x}$, would also depend mainly on $R_{\rho}$ and the fluid properties.

\section{Flux measurements: Diffusive interface}

Beginning with Turner (1965), several investigators have measured the fluxes across a single diffusive interface. They set up a well-mixed warm, salty layer below a cool, fresh layer, and monitored the temperature and salinity in each layer as a function of time, taking care to allow for the heat losses from the tank walls. Turner found that the heat and salt fluxes were consistent with the " $4 / 3$ flux law" (eqs. 9 and 11). This reduces the experimental problem from a two-dimensional exploration in $\left(R a_{T}, R a_{S}\right)$ space to dependence on one parameter $\left(R_{p}=\frac{R a_{S}}{R a_{r}}\right)$. (This dimensional argument has also been used on flux measurements in the salt finger system, but has not been tested over a wide range of Rayleigh numbers.) Turner found that the heat flux coefficient (eq. 9) is a rapidly decreasing function of $R_{\rho}$, while the flux ratio (eq. 10) is about 0.15 for $R_{\rho}>2$, increasing rapidly towards 1 as $R_{\rho} \rightarrow 1$. These and later measurements have been collated and summarized by Kelley (1990) (fig. 2,3). 


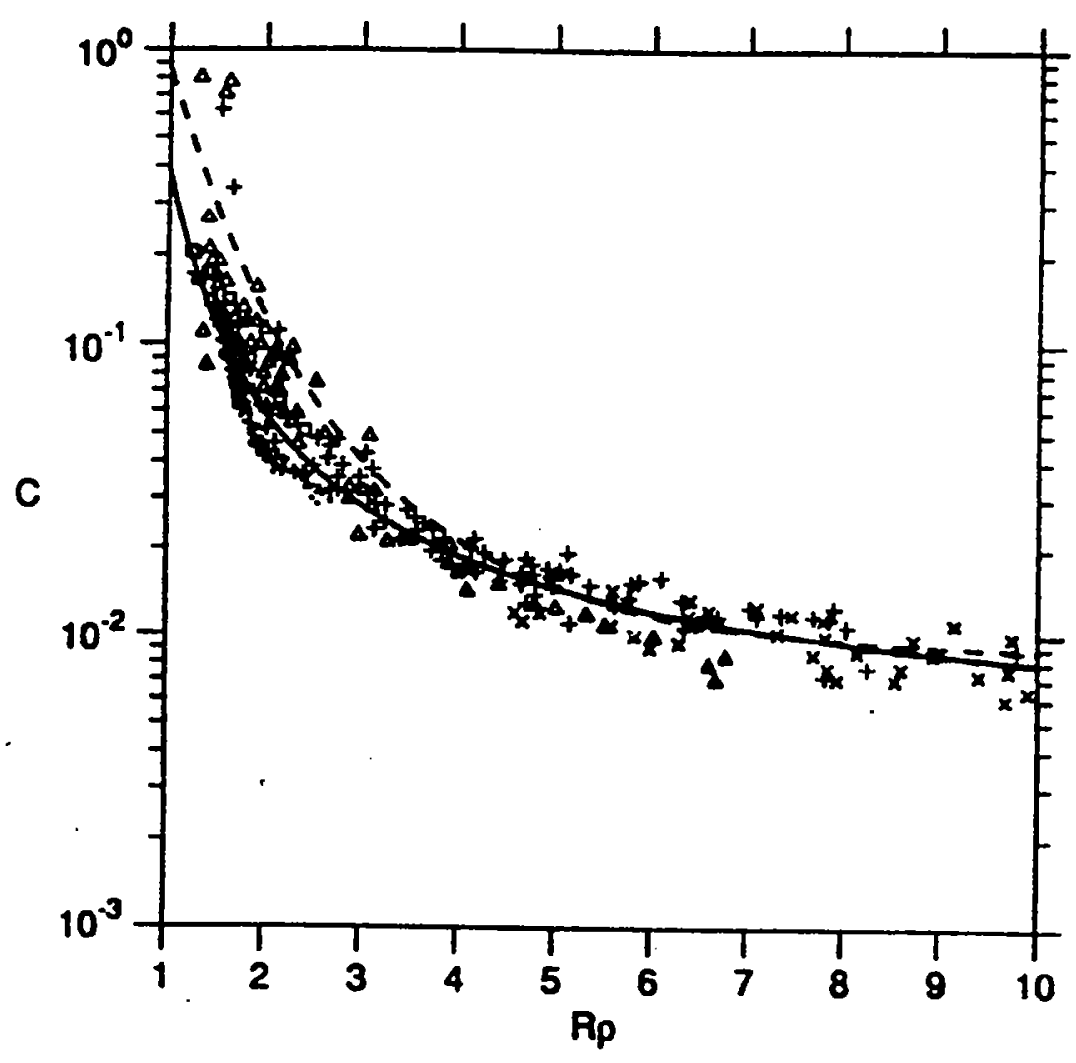

Fic. 2 Scale factor $C\left(R_{p}\right)$ in (1) for diflusive convection in the laboratory. Datz cource are indicated follows: $\Delta$ Turaer [1965], - Crepper [1975], + Mermorino and Caldwell [1976], $x$ Newell [1984], and $\square$ Toylor [1089]. Only dats with $R_{p}<10$ are plotted here and used in the analyais. The solid line is the ompirical fit (4) preaented here, the dacthed line an alternative fit (5) given by Mermerino and Caldwell [1976]. 


\section{KeLLEY: ACCURACI}

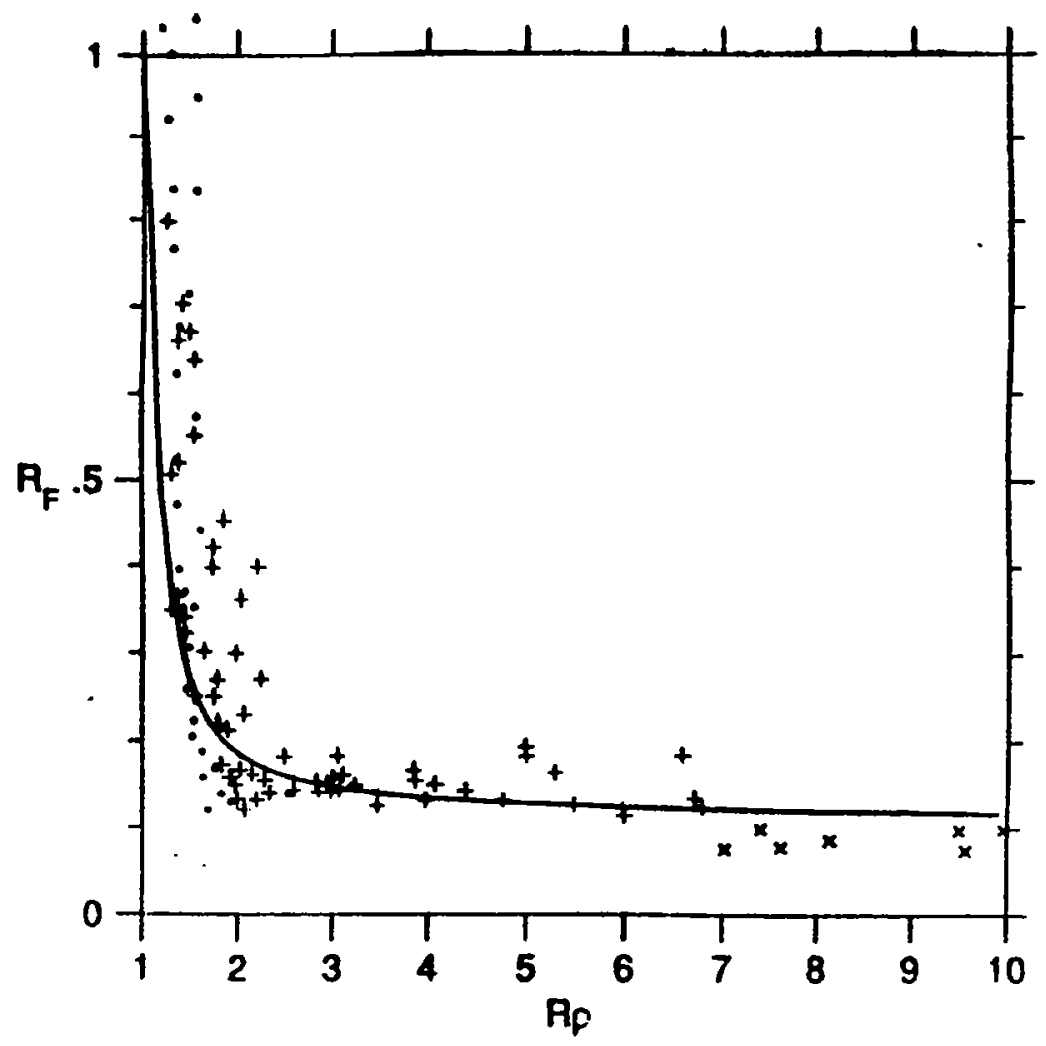

Fig. 3. Bupynncy flux ratio $R_{F}\left(T_{1,1}\right)$ for diffusive convertion: i!! the laborniory. Drin source are indicated as follows: $\bullet$ Crapper [1975], + Turner [1963], and $x$ [Newell, 1981]. Only data with $R_{p}<10$ are plotted here and userl in the analysis. The solid line is an mmpirical fit (6) to the functional form suggested by Linden [1974]. 


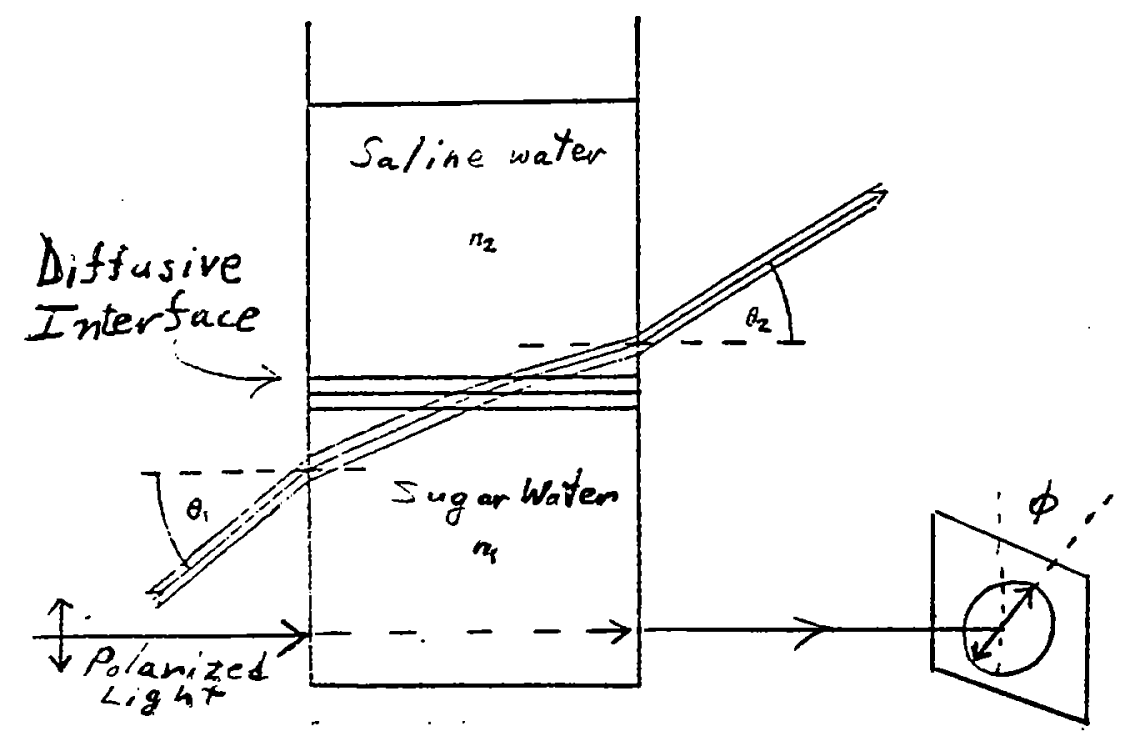

Figure 4: The difference in refractive index between the layers was found by measuring the deviation of a light beam passing obliquely through the interface.

Shirtcliffe (1973) measured the fluxes across the analogous sugar/salt diffusive interface ${ }^{1}$, using elegant optical methods. He measured the deflection of a beam of light passing through the interface (fig. 4) to deduce the difference in refractive indicies of the two layers. He also passed a beam of polarized light through each layer and measured the angle $(\phi)$ through which the polarization was rotated, which is proportional to sugar concentration. His measurements of $\beta \Delta S(t)$ vs $\alpha \Delta T(t)$ (fig. 5) show that the sugar/salt flux ratio is constant at $0.6 \pm 0.02$.

The observation that the flux ratio is approximately constant with the value $R_{F} \approx\left(\kappa_{S} / \kappa_{T}\right)^{1 / 2}$ at sufficiently large stability ratio lends strong support to a model proposed by Rooth (quoted by Veronis 1968, described in Turner 1973) in which initially sharp gradients of $T$ and $S$ diffuse at their respective molecular diffusion rates, and are intermittently swept away to the point of static stability. The observed increase in flux ratio towards 1 at low stability can be explained by the effect of direct turbulent entrainment becoming increasingly strong with the turbulent intensity, a process that can be qualitatively observed in the laboratory experiments.

\footnotetext{
${ }^{1}$ A layer of saline water floated on top of a layer of sugar water. Since the diffusivity for sugar is about $1 / 3$ of that for salt, the sugar water is often denoted as " $S$-stuff" and the salt water (analogous to cold) is called " $T-$ stuff".
} 


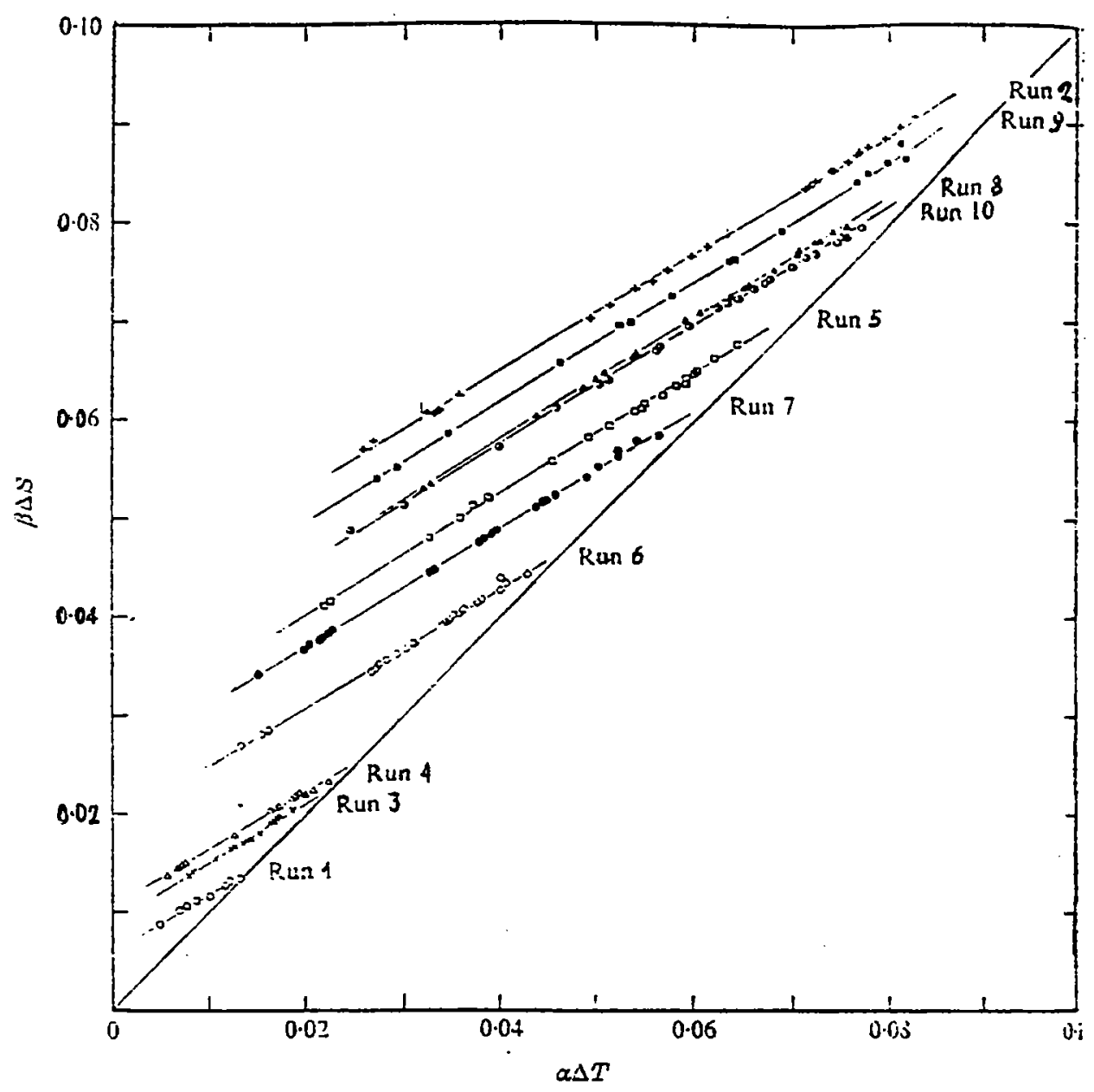

Figure 5: Graphs of $\beta \Delta S$ versus $\alpha \Delta T$ for all ten runs. The linear form of these graphs indicates that the ratio $R_{F}$ of the $S$ flux (sugar) to the $T$ flux (salt) was constant throughout each run. The run number is indicated by each set of data, and the same symbols are used in figures 3-6 from Shirtcliffe (1973). 


\section{Flux measurements: Finger interface}

Several authors have performed "run-down" experiments to measure $T$ and $S$ fluxes across a finger interface bounded by convecting layers. These were not described due to lack of time in the lecture, but results similar to those in the diffusive case obtain: the flux coefficient is a decreasing function of density ratio (now defined as $\frac{\alpha \Delta T}{\beta \Delta S}$ ), as is the flux ratio. The flux ratio is roughly 0.6 for heat/salt fingers and 0.9 for salt/sugar fingers. It is important to realize, however, that:

1. Considerable variation $(20 \div 30 \%)$ in the flux coefficient and/or flux ratio has been found between different experiments. Whether this is due to variations in Rayleigh number, initial conditions, interface thickness, or some other experimental factor has not been determined.

2. Very little systematic checking of the " $4 / 3$ law" has been done for the salt finger case.

3. The "4/3 law" assumes the existence of an "equilibrium interface thickness" for each set of macroscopic conditions towards which deviations will relax on a short time scale.

4. Oceanic observations (to come in future lectures) find that salt finger interfaces are thicker than extrapolations from lab experiments suggest, and that the fluxes are more than an order of magnitude lower.

\section{The finger regime}

\section{The perturbation equations}

The finger regime of double-diffusive convection is characterized by a stabilizing vertical gradient of a more rapidly diffusing component (say temperature) and a destabilizing gradient of the slower diffusing component (say salt). This situation is depicted in fig. 6 , where the temperature and salinity gradients are specified by the difference in these components, $\Delta T$ and $\Delta S$, over the height of the gap between two horizontal plates, $d$. The background state in this case would be characterized by the pure diffusion of both components with the velocities equal to zero. The (linearized) Boussinesq approximation of the Navier-Stokes and continuity equations as well as the salinity and temperature diffusion equations for the perturbation would look as follows:

$$
\begin{aligned}
& \frac{\partial \vec{v}}{\partial t}=-\frac{1}{\rho_{m}} \nabla p+g(\alpha T-\beta S) \vec{k}+\nu \nabla^{2} \vec{v} \\
& \nabla \cdot \vec{v}=0 \\
& \frac{\partial T}{\partial t}+\overline{\left(\frac{\partial T}{\partial z}\right) w}=\kappa_{T} \nabla^{2} T ; \\
& \frac{\partial S}{\partial t}+\overline{\left(\frac{\partial S}{\partial z}\right) w}=\kappa_{S} \nabla^{2} S
\end{aligned}
$$

In the above equations, $\vec{v}=(u, v, w)$ is the velocity vector, $T$ is the temperature, $S$ is the salinity, $\overline{\left(\frac{\partial T}{\partial z}\right)}, \overline{\left(\frac{\partial S}{\partial z}\right)}$ are the mean temperature and salinity vertical gradients, respectively, $\nu$ is the kinematic viscosity, $\rho_{m}$ is the mean density, $p$ is the pressure, $\alpha=-\frac{1}{\rho_{m}} \frac{\partial p}{\partial T}$ is the coefficient of thermal expansion, and $\beta=\frac{1}{\rho} \frac{\partial \rho}{\partial S}$ is the coefficient of salinity contraction. 


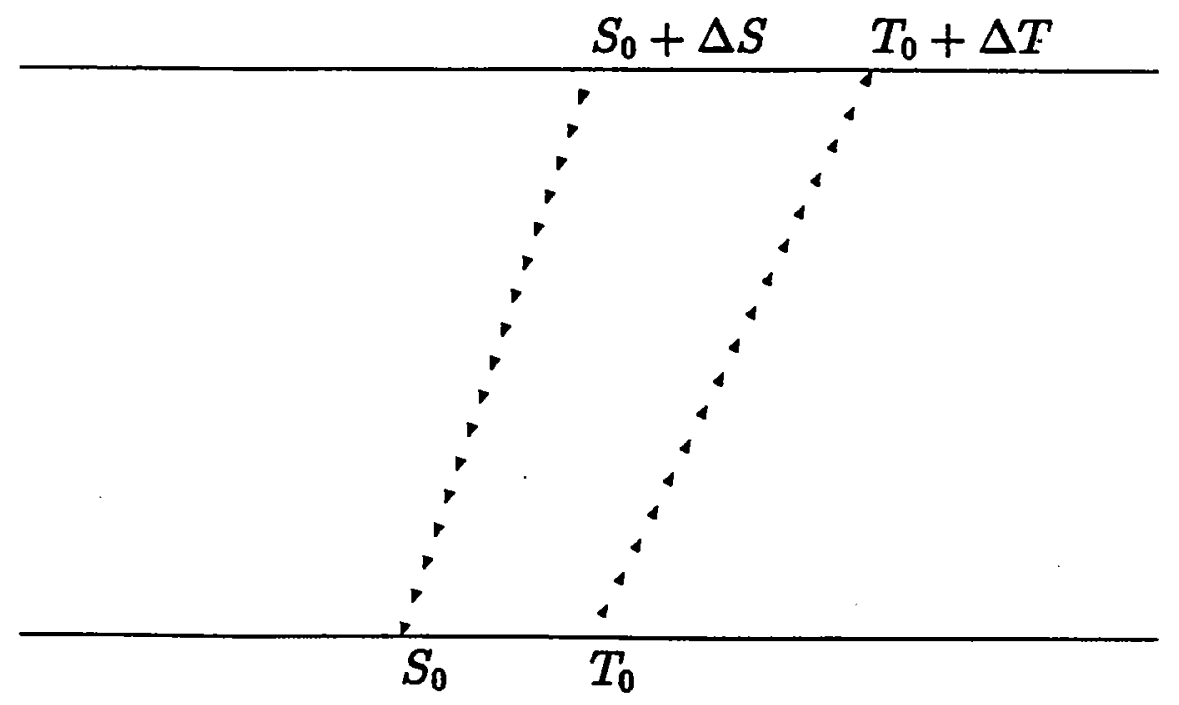

Figure 6: The finger regime of double-diffusive convection.

After differentiating the momentum equation for $u$ with respect to $x$ and $z$, the equation for $v$ with respect to $y$ and $z$, adding the results and subtracting from the resulting equation the equation for $w$, on applying horizontal Laplacian $\nabla_{1}^{2}=\frac{\partial^{2}}{\partial x^{2}}+\frac{\partial^{2}}{\partial y^{2}}$ to the latter, one obtains (with the use of the continuity equation)

$$
-\partial_{t} \nabla^{2} w=-g\left(\alpha \nabla_{1}^{2} T-\beta \nabla_{1}^{2} S\right)-\nu \nabla^{4} w
$$

\section{The thermal convection problem}

First we are going to restrict our attention to the thermal convection formulation alone. That is, a configuration where fluid between two infinite parallel horizontal plates is differentially heated from below, i.e., the upper plate has temperature $T_{0}$ while the temperature of the lower one would be $T_{0}+\Delta T$. In this case, therefore, it is the temperature that plays the destabilizing role. Once the results of this problem are known, it is easier to approach the more complex configuration comprising also the presence of the vertical salinity gradient (the finger regime of double-diffusive convection). In the presence of the temperature alone, equation (16) would look as follows:

$$
\left(\partial_{t}-\nu \nabla^{2}\right) \nabla^{2} w=g \alpha \nabla_{1}^{2} T
$$

We are interested in solving this equation coupled with the equation for the temperature perturbation,

$$
\left(\partial_{t}-\kappa_{T} \nabla^{2}\right) T=-\overline{\left(\frac{\partial T}{\partial z}\right) w},
$$

and the following boundary conditions (stress-free boundaries are assumed for the velocities):

$$
w=\frac{\partial^{2} w}{\partial z^{2}}=0, T=0 ; z=0, d
$$


Because the coefficients in equations (17),(18) are constants, their solution can be sought in the following form:

$$
(w, T) \sim(\tilde{w}, \tilde{T}) \exp \left(\frac{\kappa_{T} p}{d^{2}} t+i \frac{\pi}{d}(k x+l y)\right) \sin \left(\frac{n \pi z}{d}\right) ; n= \pm 1, \pm 2, \pm 3, \ldots,
$$

where $\tilde{w}, \tilde{T}$ are some constant amplitudes. Having introduced (20) into (17),(18), and allowing for $\overline{\left(\frac{\partial T}{\partial z}\right)}=-\frac{\Delta T}{d}$, one gets

$$
\begin{aligned}
\left(\frac{p}{\sigma}+K^{2}\right) K^{2} \tilde{w} & =\frac{g \alpha}{\nu} a^{2} d^{4} \tilde{T} ; \\
\left(p+K^{2}\right) \tilde{T} & =\frac{\Delta T}{d \kappa_{T}} \tilde{w} .
\end{aligned}
$$

On nondimensionalizing the above equations $\left(w \sim \frac{\kappa_{r}}{d}, T \sim \Delta T\right)$,

$$
\begin{aligned}
\left(\frac{p}{\sigma}+K^{2}\right) K^{2} \bar{w} & =R a^{2} \bar{T}, \\
\left(p+K^{2}\right) \bar{T} & =\bar{w},
\end{aligned}
$$

where $\sigma=\frac{\nu}{\kappa_{T}}, R=\frac{g a \Delta T d^{\infty}}{\nu \kappa_{T}}, a^{2}=\pi^{2}\left(k^{2}+l^{2}\right), K^{2}=a^{2}+n^{2} \pi^{2}$, and $\bar{w}, \bar{T}$ are nondimensional amplitudes of temperature and velocity, respectively.

After substituting $\bar{T}$ from (24) into (23) and canceling $\bar{w}$, one obtains

$$
\left(p+K^{2}\right)\left(\frac{p}{\sigma}+K^{2}\right) K^{2}=R a^{2} .
$$

Since $p$ is a complex number, one needs to consider its real and imaginary parts independently, i.e., when $p=p_{\mathrm{r}}+i p_{i}$,

$$
\begin{gathered}
p_{\mathrm{r}}^{2}+p_{i}^{2}+(1+\sigma) K^{2} p_{\mathrm{r}}+\sigma\left(K^{4}-\frac{R a^{2}}{k^{2}}\right)=0, \\
i\left(2 p_{\mathrm{r}} p_{i}+(1+\sigma) K^{2} p_{i}\right)=0 .
\end{gathered}
$$

Let us first assume that $p_{i}=0$. Since we are interested only in the growing modes, it is the positive root alone of the quadratic (equation 26) that should be considered:

$$
p_{r}=\frac{1}{2}\left(-(1+\sigma) K^{2}+\sqrt{(1+\sigma)^{2} K^{4}-4 \sigma\left(K^{4}-\frac{R a^{2}}{K^{2}}\right)}\right) .
$$

The requirement that the expression for $p_{r}$ not be negative, is equivalent to

$$
R \geq \frac{K^{6}}{a^{2}}=\frac{\pi^{6}\left(k^{2}+l^{2}+n^{2}\right)^{3}}{\pi^{2}\left(k^{2}+l^{2}\right)}=\frac{\pi^{4}\left(\alpha^{2}+n^{2}\right)^{3}}{\alpha^{2}}
$$

where $\alpha^{2}=k^{2}+l^{2}$. The rightmost expression above takes on its minimum value when $n=1$ and $\boldsymbol{\alpha}^{2}=1 / 2$. Therefore,

$$
R_{\min }=\frac{27}{4} \pi^{4}
$$

It is important to emphasize that only a cell of a certain size (i.e., of an appropriate wave number) could form at the onset of the instability. If the wave number is larger than the critical one (i.e., a thinner cell), the temperature will diffuse and dissipate horizontally. For a small wave number 
(a wide cell), on the other hand, the release of the potential energy of the fluid is too slow. The damping effect of the thermal diffusion and viscous dissipation within the Rayleigh number can be seen when $R$ is rewritten in the following way:

$$
R=\frac{g \alpha \Delta T / d}{\frac{k_{r}}{d^{2}} \frac{\nu}{d^{2}}},
$$

where $\frac{\kappa_{x}}{d^{2}}$ and $\frac{\nu}{d^{2}}$ are measures of the diffusion and viscous dissipation times, respectively.

If $p_{i} \neq 0$, equation (27) gives

$$
p_{r}=-\frac{(1+\sigma) K^{2}}{2}
$$

which means that the corresponding oscillations are always damped. By substituting the above expression for $p_{r}$ into (26), one can also see that $p_{i}^{2}$ can be positive only if $R<0$, which means that oscillatory motions are possible (but damped) for a stably stratified fluid.

\section{References}

[1] Crapper, P. F., (1975) Measurements across a diffusive interface. Deep-Sea Research, 22, 537-545.

[2] Kelley, D. E., (1990) Fluxes through diffusive staircases: A new formulation. Journal of Geophysical Research 95, 3365-3371.

[3] Linden, P. F. (1974) A note on the transport across a diffusive interface. Deep-Sea Research 21, 283-287.

[4] Marmorine, G. J., Caldwell, D. R. (1976) Heat and salt transport through a diffusive thermohaline interface, Deep-Sea Res 23, 59-67.

[5] Newell, T. A. (1984) Characteristics of a double-diffusive interface at high density stability ratios. Journal of Fluid Mechanics 149, 385-401.

[6] Shirtcliffe, T. G. L., (1973) Transport and profile measurements of the diffusive interface in double-diffusive convection with similar diffusivities. Journal of Fluid Mechanics 57, 27-43.

[7] Taylor, J. (1988) The fluxes across a diffusive interface at low values of the density ratio. Deep Sea Research 35, 555-567.

[8] Turner, J. S., (1965) The coupled turbulent transports of salt and heat across a sharp density interface. International Journal of Heat and Mass Transfer 8, 759-767.

[9] Turner, J. S., (1973) Buoyancy Effects in Fluids, Cambridge University Press Chapter 8, pp. 251-287.

[10] Veronis, G., (1968) Effect of a stabilizing gradient of solute on thermal convection. Journal of Fluid Mechanics 34, 315-336. 


\section{Lecture 3}

\section{Instability with $\Delta T$ and $\Delta S$ (heat and salt from above)}

We will now solve the problem of salt fingers. We scale quantities as

$$
T \sim \Delta T ; S \sim \Delta S ; w \sim \frac{\kappa_{T}}{d} ; \partial_{t} \sim \frac{\kappa_{S}}{d^{2}} .
$$

The non-dimensional vorticity equation is then

$$
\left(\eta \partial_{t}-\nabla^{2}\right) \nabla^{2} w=R_{T} \nabla_{1}^{2} T-R_{S} \nabla_{1}^{2} S \quad \text { with } \nabla_{1}^{2}=\frac{\partial^{2}}{\partial x^{2}}+\frac{\partial^{2}}{\partial y^{2}}
$$

and the equations for heat and salt are

$$
\begin{aligned}
& \left(\tau \partial_{t}-\nabla^{2}\right) T=-w \\
& \left(\partial_{t}-\nabla^{2}\right) S=-\frac{w}{\tau}
\end{aligned}
$$

where we use the non-dimensionnal parameters

$$
\tau=\frac{\kappa_{S}}{\kappa_{T}} ; \sigma=\frac{\nu}{\kappa_{T}} ; \eta=\frac{\kappa_{S}}{\nu} ; R_{T}=\frac{g \alpha \Delta T d^{3}}{\nu \kappa_{T}} ; R_{S}=\frac{g \beta \Delta S d^{3}}{\nu \kappa_{T}} .
$$

Eliminating $S$ and $T$ from this system of equations yields

$$
\left(\tau \partial_{t}-\nabla^{2}\right)\left(\partial_{t}-\nabla^{2}\right)\left(\eta \partial_{t}-\nabla^{2}\right) \nabla^{2} w=-R_{T}\left(\partial_{t}-\nabla^{2}\right) \nabla_{1}^{2} w+\frac{R_{S}}{\tau}\left(\tau \partial_{t}-\nabla^{2}\right) \nabla_{1}^{2} w
$$

Let us first find the stationary solution $\left(\partial_{t}=0\right)$. Equation $(5)$ is then reduced to

$$
\nabla^{6} w=\left(-R_{T}+\frac{R_{S}}{\tau}\right) \nabla_{1}^{2} w
$$

As for the Rayleigh-Bénard problem, we have solutions of the form

$$
(T, S, w) \sim(\tilde{T}, \tilde{S}, \tilde{w}) e^{p t+\pi i(k x+l y)} \sin (n \pi z)
$$

and the boundary conditions

$$
w=w_{z z}=0 \quad S, T=0 \quad \text { at } \quad z=0,1
$$

Thus,

$$
\frac{\pi^{4}\left(\alpha^{2}+n^{2}\right)^{3}}{\alpha^{2}}=-R_{T}+\frac{R_{S}}{\tau} \quad \text { and } \quad \alpha^{2}=k^{2}+l^{2}
$$

The minimum value the left hand side term can take (for $n=1$ ) is $\frac{27}{4} \pi^{4}$. Since normally $R_{T} \gg \frac{27}{4} \pi^{4}$, the condition for the real part of $p$ to be positive (and thus, for instability) is simply

$$
\beta \Delta S>\frac{\kappa_{S}}{\kappa_{T}} \alpha \Delta T
$$

The interpretation of this condition is that the salinity gradient has to contribute only $\kappa_{S} / \kappa_{T}=1 / 70$ of the fractional change in density over a vertical distance that temperature does in order for the instability to appear, i.e. for the potential energy of the salt stratification to be released. 


\section{Stern(1967)'s maximum growth rate analysis}

We now wish to consider the wavenumber of maximum growth rate. Experiments by Linden(1973) have determined that the order of magnitude of the width of salt fingers is directly related to this. Qualitatively, we require thin fingers to diffuse heat efficiently from the advecting salt fingers, but not so thin that salt also diffuses. We thus anticipate a preferential scale which is small in the horizontal compared with the vertical. In his analysis Stern chose the particular case of our system where

$$
\sigma \gg 1 ; \partial_{t} \neq 0 ; \tau \ll 1 ; \eta \ll 1 ; K^{2}=\pi^{2}\left(\alpha^{2}+n^{2}\right) .
$$

We consider $n=1$ which corresponds to the most unstable mode in all cases of $R_{T}$ and $R_{S}$ (Turner 1973, p258). Crossing off $\eta \partial_{t}$ and $\tau \partial_{t}$ in equation (5), we obtain

$$
\left(p+K^{2}\right) \frac{K^{6}}{\pi^{2} \alpha^{2}}=-R_{T}\left(p+K^{2}\right)+R_{S} \frac{K_{S}}{\tau} .
$$

Rearranging,

$$
p+K^{2}=\frac{\frac{R_{S}}{T} K^{2}}{\frac{K^{6}}{\pi^{2} \alpha^{2}}+R_{T}} .
$$

To find the maximum growth rate we now take $\frac{\partial p}{\partial K^{2}}=0$ which gives

$$
p+K^{2}=\frac{\left(-R_{T}+\frac{R_{S}}{\tau}\right) \pi^{2} \alpha^{2}+\frac{R_{S}}{\tau} K^{2}-K^{6}}{3 K^{4}+R_{T}} .
$$

Equating the right hand sides of equations (13) and (14) gives

$$
\pi^{8} \alpha^{8}+\left(\frac{R_{S}}{\tau}+2 R_{T}\right) \pi^{4} \alpha^{4}-\frac{R_{S}}{\tau} R_{T}+R_{T}^{2}=0
$$

This is a quadratic for $\pi^{4} \alpha^{4}$ which we solve, and taking the first two terms in a binomial expansion we obtain

$$
\pi^{4} \alpha^{4}=\frac{\left(\frac{R_{S}}{\tau}-1\right) R_{T}}{\frac{R_{S}}{\tau}+2 R_{T}}
$$

Denoting $\frac{R_{S}}{\tau}=C R_{T}$ we obtain

$$
\pi^{4} \alpha^{4}=\frac{(C-1)}{(C+2)} R_{T}
$$

For $C=2$,

$$
\pi^{4} \alpha^{4}=\frac{N^{2} d^{4}}{4 \nu K_{T}}
$$

With $L=\frac{\pi}{\alpha}$ we have

$$
L^{4}=\frac{4 \nu K_{T} \pi^{4}}{g \alpha \bar{T}_{Z}}
$$




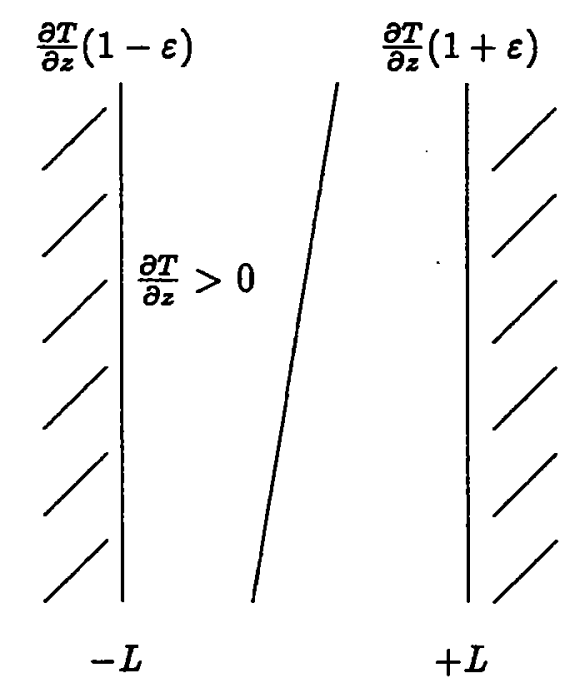

Figure 1: Initial setup to make a buoyancy layer

hence

$$
L \propto\left(\alpha \bar{T}_{Z}\right)^{-\frac{1}{4}}
$$

Thus $L$ increases as $\alpha \bar{T}_{Z}$ decreases and vice versa.

\section{Prandtl's Buoyancy Layer(1952)}

Prandtl considered a 2-D system in the $X-Z$ plane, between horizontal bounds

$$
-L \leq X \leq L
$$

but disregarding vertical bounds. He imposed a constant vertical temperature gradient with heating/cooling at opposite boundaries such that

$$
T=T_{0}+\frac{\Delta T}{d} Z(1 \pm \epsilon) \quad \text { at } \quad x= \pm L
$$

where $d$ is the vertical distance over which $T$ changes by $\Delta T$. He assumed $\frac{\partial P^{\prime}}{\partial Z}=0$ (which may be confirmed a posteriori), and since the $Z$-dependence is linear our equations reduce to

$$
g \alpha T+\nu w_{x x}=0
$$

for the vertical momentum equation and

$$
\frac{\Delta T}{d} w=\kappa_{T} T_{x x}
$$


for the heat equation. We scale

$$
w \sim \frac{\kappa_{T}}{d} ; T \sim \Delta T ; x \sim d
$$

and obtain

$$
\begin{gathered}
w_{x x}=-R_{T} T, \\
w=T_{x x} .
\end{gathered}
$$

Introducing boundary conditions $w=0$ at $x= \pm \frac{L}{d}=a$ and defining $\theta=R_{T}^{\frac{1}{2}} T$ to lend symmetry to the problem we obtain

$$
\begin{gathered}
w_{x x}=-R_{T}^{\frac{1}{2}} \theta \\
\theta_{x x}=R_{T}^{\frac{1}{2}} w .
\end{gathered}
$$

We also specify the boundary conditions

$$
\theta= \pm \epsilon R^{\frac{1}{2}} z \text { and } w=0 \text { at } x= \pm \frac{L}{d} .
$$

Defining $\phi=w+i \theta$, we obtain

$$
\phi_{x x}=i R_{T}^{\frac{1}{2}} \phi
$$

such that (28) and (29) (once rearranged), are respectively the real and imaginary parts of this equation which has the solution

$$
\phi=\phi_{0} \exp \left[\frac{R_{T}^{\frac{1}{4}}}{\sqrt{ } 2}(1+i)(x \mp a)\right] \text { near } x= \pm a ; a=\frac{L}{d} .
$$

We apply the boundary condition for $\theta$ to obtain

$$
\phi= \pm i R^{\frac{1}{2}} \epsilon z \exp \left[\frac{R_{T}^{\frac{1}{2}}}{\sqrt{ } 2}(1+i)(x \mp a)\right] \text { near } x= \pm a .
$$

The solutions for $w$ and $T$ are

$$
\begin{gathered}
w= \pm R^{\frac{1}{2}} \epsilon z \exp \left[\frac{R_{T}^{\frac{1}{4}}}{\sqrt{ } 2}(x \mp a)\right] \sin \left[\frac{R_{T}^{\frac{1}{4}}}{\sqrt{ } 2} a x\right] \\
T= \pm \epsilon z \exp \left[\frac{R_{T}^{\frac{1}{4}}}{\sqrt{ } 2}(x \mp a)\right] \cos \left[\frac{R_{T}^{\frac{1}{4}}}{\sqrt{ } 2} a \mp x\right]
\end{gathered}
$$

We thus obtain a boundary layer of thickness

$$
L^{*} \sim \frac{\sqrt{ } 2 d}{R^{\frac{1}{4}}}=\left[\frac{4 \nu \kappa_{T}}{g \alpha T_{z}}\right]^{\frac{1}{4}}
$$




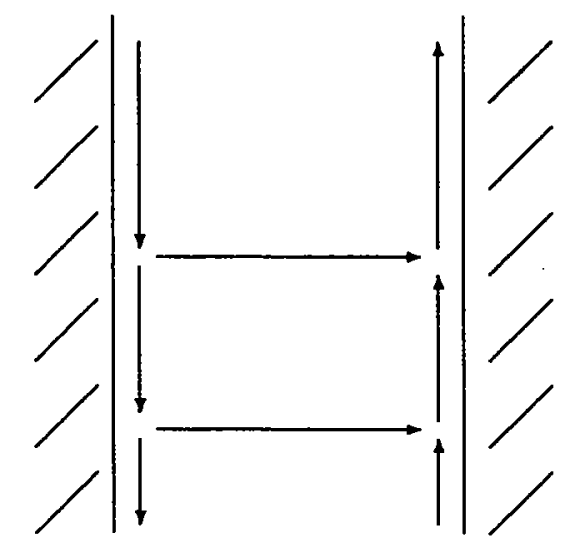

Figure 2: Prandtl's buoyancy layer

which is called the buoyancy layer. $L^{*}$ is the same as the scale of maximum growth rate (19). It gives the thickness of the boundary layer in which $w$ and $T$ depart from their interior values, 0 and $\frac{\Delta T}{d} z$ repectively in order to satisfy the boundary conditions (30) at the sides. We also have to introduce a velocity in the $x$ direction since we must have $\nabla \cdot \vec{v}=0$.

$$
u=-\int_{-\infty}^{a} \frac{\partial w}{\partial z} \mathrm{~d} z=\frac{\varepsilon R^{\frac{1}{4}}}{\sqrt{2}}
$$

The resulting flow pattern in illustrated in fig. 2.

\section{Reversed mean gradients - heat and salt from below}

We will now address the problem of heating from below; the fluid is stabilized by salt. Introducing the stream function $\Psi$ defined by $w=-\Psi_{x}$ and $u=\Psi_{z}$, we get

$$
\begin{gathered}
\left(\partial_{t}-\nu \nabla^{2}\right) \nabla^{2} \Psi=-g \alpha T_{x}+g \beta S_{x} \\
\left(\partial_{t}-\kappa_{T} \nabla^{2}\right) T+\frac{\Delta T}{d} \Psi_{x}=0 \\
\left(\partial_{t}-\kappa_{S} \nabla^{2}\right) S+\frac{\Delta S}{d} \Psi_{x}=0
\end{gathered}
$$


Scaling quantities as $\Psi \sim \kappa_{T} ; t \sim \frac{d^{2}}{\kappa_{T}} ; x \sim d ; T \sim \Delta T ; S \sim \Delta S$, the set of equations becomes

$$
\begin{gathered}
\left(\frac{1}{\sigma} \partial_{t}-\nabla^{2}\right) \nabla^{2} \Psi=-R_{T} T_{x}+R_{S} S_{x} \\
\left(\partial_{t}-\nabla^{2}\right) T=-\Psi_{x} \\
\left(\partial_{t}-\tau \nabla^{2}\right) S=-\Psi_{x}
\end{gathered}
$$

The boundary conditions are, as before,

$$
\Psi=\Psi_{x x}=T=S=0 \quad \text { at } z=0,1 .
$$

The form of the solution is then

$$
\begin{array}{r}
\Psi \sim e^{p t} \sin (\pi \alpha x) \sin (n \pi z) \\
T, S \sim e^{p t} \cos (\pi \alpha x) \sin (n \pi z)
\end{array}
$$

The three previous equations can be combined to give the dispertion relation

$$
\begin{array}{r}
\frac{p^{3}}{\sigma}+\left(1+\frac{1+\tau}{\sigma}\right) K^{2} p^{2}+\left[\left(\frac{\tau}{\sigma}+\tau+1\right) K^{4}-\left(R_{T}-R_{S}\right) \frac{\pi^{2} \alpha^{2}}{K^{2}}\right] p+ \\
\tau K^{6}+\left(R_{S}-\tau R_{T}\right) \pi^{2} \alpha^{2}=0
\end{array}
$$

where $p$ contains a real and an imaginary part $p=p_{r}+i p_{i}$.

\subsection{The zero growth rate solution}

Here, we search for the solution given that $p_{r}=0$. We then split equation (45) into two equations (for the real and imaginary parts)

$$
\begin{aligned}
& {\left[-\frac{p_{i}^{2}}{\sigma}+\left(\frac{\tau}{\sigma}+\tau+1\right) K^{4}-\left(R_{T}-R_{S}\right) \frac{\pi^{2} \alpha^{2}}{K^{2}}\right] p_{i}=0} \\
& -k^{2}\left(1+\frac{1+\tau}{\sigma}\right) p_{i}^{2}+\tau K^{6}+\left(R_{S}-\tau R_{T}\right) \pi^{2} \alpha^{2}=0
\end{aligned}
$$

If $p_{i}=0$ (a nonoscillating, convective mode), we get the critical Rayleigh number $\left(R_{T}^{c}\right)$

$$
R_{T}^{c}=\frac{K^{6}}{\pi^{2} \alpha^{2}}+\frac{R_{S}}{\tau}
$$

This requires an unrealistically large destabilizing temperature gradient. For $p_{i} \neq 0$, we obtain an oscillatory mode with

$$
p_{i}^{2}=\sigma\left(\frac{\tau}{\sigma}+\tau+1\right) K^{4}-\sigma\left(R_{T}-R_{S}\right) \frac{\pi^{2} \alpha^{2}}{K^{2}}
$$




$$
R_{T}=\frac{\sigma+\tau}{1+\sigma} R_{S}+\frac{(\sigma+1+\tau)\left(\frac{\tau}{\sigma}+\tau+1\right)-\tau}{\sigma+1} \frac{K^{6}}{\pi^{2} \alpha^{2}}
$$

For the salt/heat system, we have $\tau \sim 10^{-2} ; \sigma \sim 7$ and if $R_{S} \gg \frac{27}{4} \pi^{4}$, the oscillatory instability criterion becomes

$$
\begin{gathered}
R_{T} \sim \frac{\sigma}{1+\sigma} R_{S} \\
\alpha \Delta T \sim \frac{\nu}{\nu+\kappa_{T}} \beta \Delta S
\end{gathered}
$$

Thus, overstability can occur when the fluid is mildly stable. In contrast, for salt fingers instability can occur when the destabilizing salt gradient is only $\tau$ times the stabilizing temperature gradient.

Baines \& Gill (1969) have calculated the onset of these instabilities (overstability and salt fingers) for various combinations of Rayleigh numbers. That is illustrated in fig. 3.

\section{References}

[1] Baines, P.G. and Gill, A.E. (1969) On thermohaline convection with linear gradients, J. Fluid Mech., 37, 289.

[2] Linden, P.F. (1973) On the structure of salt fingers, Deep-sea research, 20, 325.

[3] Prandtl, L. (1952) Essentials of fluid dynamics, London, Blackie.

[4] Stern, M.E. (1967) Lateral mixing of water masses, Deep-sea research, 14, 747.

[5] Turner, J.S. (1973) Buoyancy effects in fluids, Cambridge monographs on mechanics \& applied mathematics, Cambridge at the university press. 


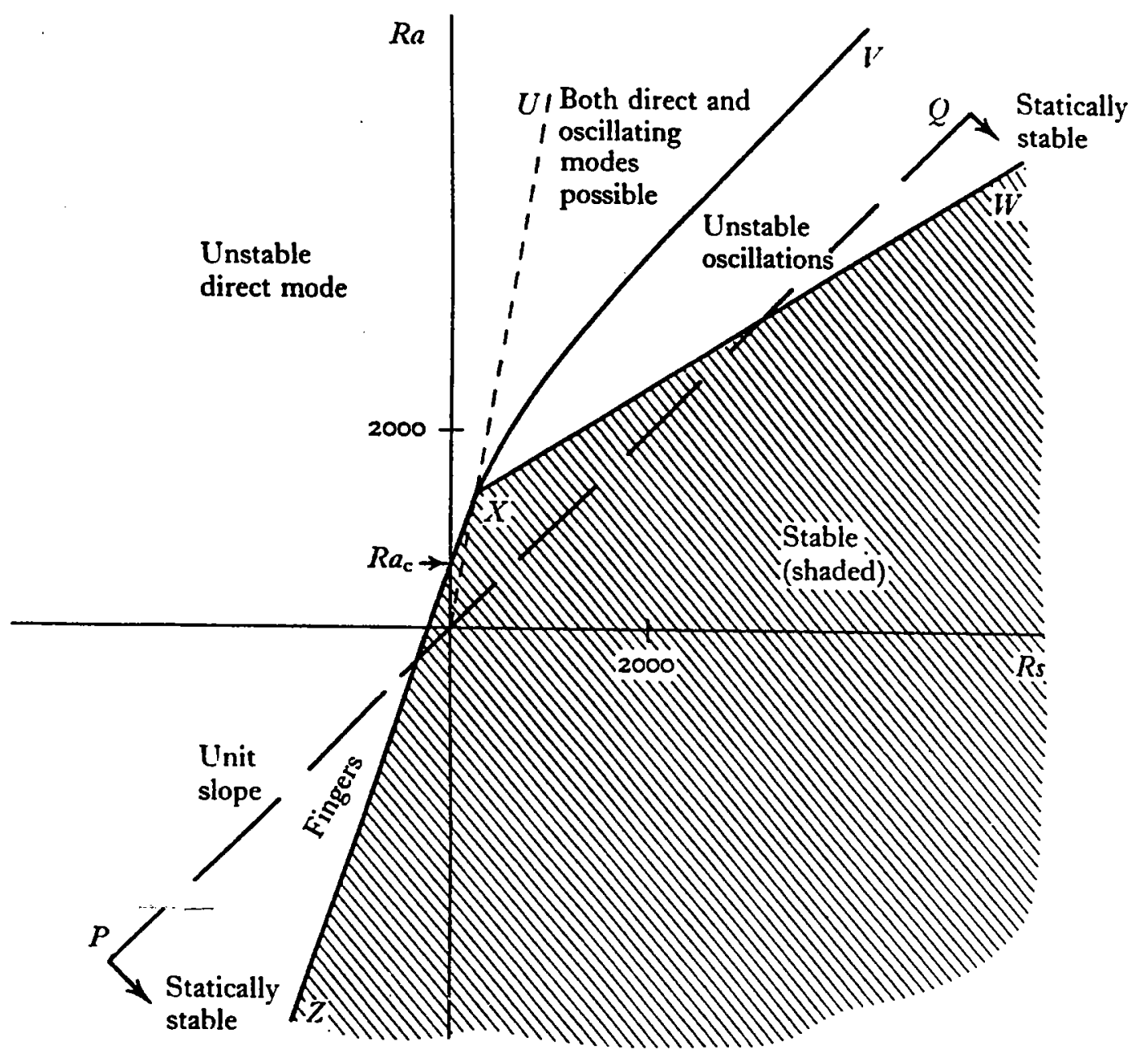

Figure 3: Diagram of the various convection regimes. The sign convention is that negative $R_{\alpha}\left(\equiv R_{T}\right)$ and positive $R_{S}$ represent stabilizing gradients of the respective components. 


\title{
Macro-Scale Signatures of Double Diffusion in the Ocean
}

\author{
Raymond W. Schmitt
}

\section{Introduction}

An experiment to model double-diffusive processes in the ocean was shown. A layer of sugar water was placed on top of a stably stratified salt solution. Altough this setup would be statically stable if considering only the density profile, a double-diffusive instability develops that eventually leads to the formation of staircases in the vertical profiles of concentration of sugar and salt as shown in Figure 1. Here the salt plays the role of heat in the oceanic water, and the sugar, having a diffusivity much less than that of salt, mimics the oceanic salt.

These staircases are expected to be the most easily recognizable signature of doublediffusive processes in the ocean. In fact, in several surveys such structures have been found. Some examples are reported in Figure 2. It is important to note that the density ratio $R_{\rho}$ (defined below) seems to play a crucial role in the ocean. In particular, it appears that the condition $R_{p}<1.9$ is necessary, although not sufficient, for the formation of staircases.

\section{The linear, depth-independent theory}

The linear (but not linearized) equations for a double-diffusive system in a region of constant vertical gradients are:

$$
\begin{aligned}
\frac{\partial w^{\prime}}{\partial t}+g\left(\beta S^{\prime}-\alpha T^{\prime}\right) & =\nu \nabla_{2}^{2} w^{\prime} \\
\frac{\partial \alpha T^{\prime}}{\partial t}+w^{\prime} \alpha \bar{T}_{z} & =\kappa_{T} \nabla_{2}^{2} \alpha T^{\prime} \\
\frac{\partial \beta S^{\prime}}{\partial t}+w^{\prime} \beta \bar{S}_{z} & =\kappa_{S} \nabla_{2}^{2} \beta S^{\prime}
\end{aligned}
$$

where the thermal expansion and haline contraction coefficients $\alpha=-\frac{1}{\rho} \frac{\partial \rho}{\partial T}$ and $\beta=\frac{1}{\rho} \frac{\partial \rho}{\partial S}$ are assumed to be constant, $\kappa_{T}$ and $\kappa_{S}$ are the molecular diffusivities for heat and salt, and $\nu$ is 


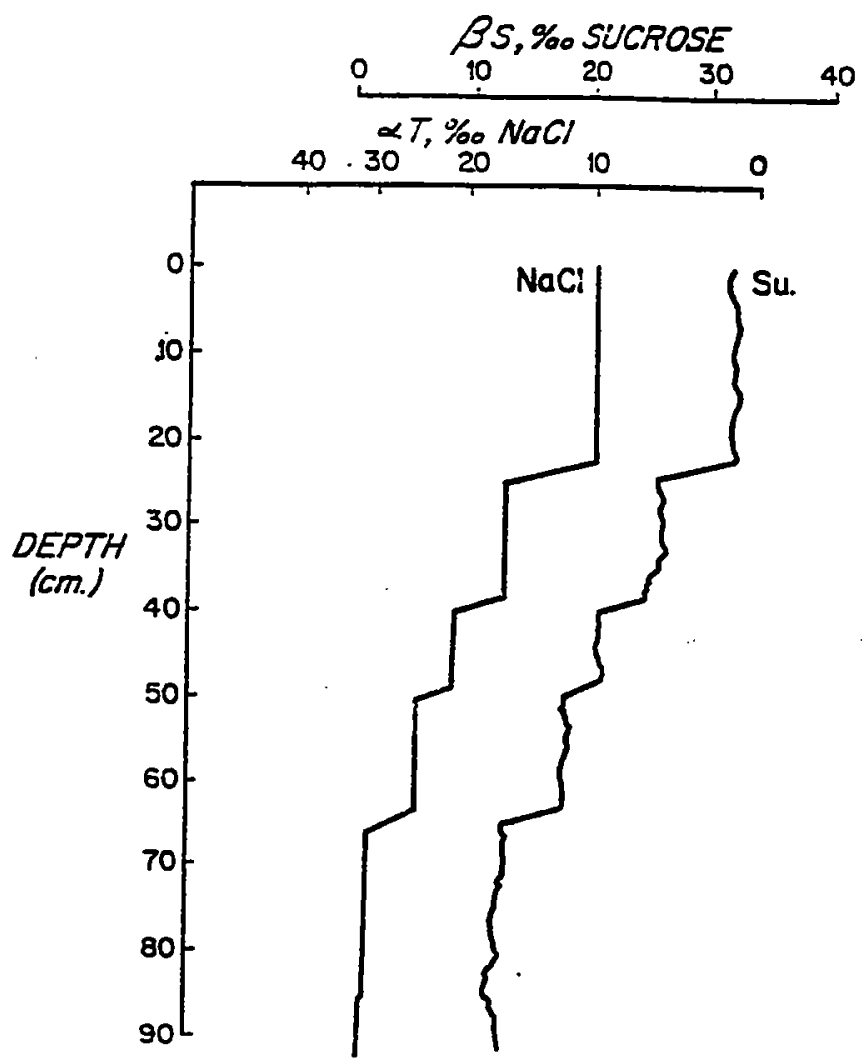

Figure 1: Staircases in the horizontally averaged salt and sugar concentration profiles.
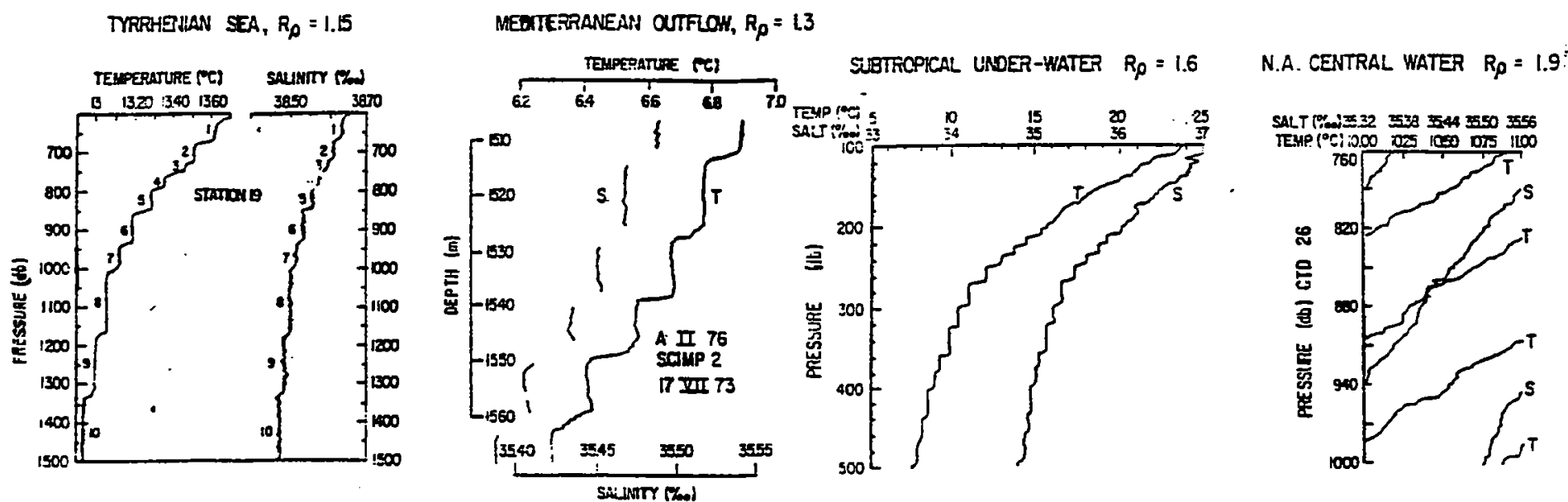

Figure 2: Staircases from the Tyrrhenian Sea, Mediterranean Outflow, and the Subtropical Under-water. Irregular steppiness from the North Atlantic Central Water is also shown. 
the kinematic viscosity. Quantities with a prime (') represent the perturbations away from the horizontal averages, denoted by a bar $\left(^{-}\right)$. The subscript $z$ indicates a derivative with respect to $z$.

The requirement for double-diffusive instability is that the density ratio $R_{p}=\frac{\alpha \bar{T}_{z}}{\beta \bar{S}_{z}}$ be less than the diffusivity ratio $\tau=\frac{\kappa \pi}{\kappa_{s}}$. On the other hand, for $R_{p}<1$ the fluid is statically unstable and double diffusion plays no role. A value of $R_{\rho}$ between these two bounds is observed over vast regions of the ocean.

These equations admit solutions of the form:

$$
\left(w^{\prime}, T^{\prime}, S^{\prime}\right) \propto \exp (\lambda t) \phi(x, y)
$$

where $\lambda$ is the growth rate and $\phi(x, y)$ is a horizontal planform function which obeys the Helmholtz equation. Substituting this solution in the linearized equations one gets the following expression for the growth rate:

$$
\lambda=\left(g \alpha \bar{T}_{z}\right)^{1 / 2} G
$$

where the nondimensional growth rate $G$ is

$$
G=\frac{M^{2}}{\sigma^{1 / 2}} \frac{\left(\gamma-R_{\rho} / \tau\right)}{\left(R_{p}-\gamma\right)}
$$

, $\sigma$ is the Prandtl number, and $M=m\left(g \alpha \bar{T}_{z} / \nu \kappa_{T}\right)^{-1 / 4}$ where $\mathrm{m}$ is the total horizontal wavenumber. The parameter $\gamma=\frac{\alpha T^{\prime}}{\beta S^{\prime}}$ is the flux ratio. Note that $0<\gamma<1$ is necessary for the formation of salt fingers.

The growth rate versus the flux ratio is given in Figure 3 for several density ratios. The growth rate decreases with increasing density ratio. In particular, the value $R_{p}=2$ is typical of the ocean, while the fastest growing fingers occur for $R_{p}=1$. For $R_{p}=100$ no fingers can grow, and all the initial perturbations will die away.

The dependence of the growth rate on the wave number is shown in Figure 4 for several buoyancy periods with $R_{p}=2$. It is evident that there is a single fastest-growing mode which selects a preferred horizontal length scale. Temperature microstructure observations show features that agree with the theoretical prediction (see the next report).

\section{Observations}

The possibility of finding double-diffusive phenomena in the oceans depends mainly on the value of $R_{p}$. Figure 5 displays a map of the values of $R_{\rho}$ over the main thermocline. The areas where a low value of the density ratio is found are shadowed. The Pacific ocean doesen't seem to be a favorable place to look for double-diffusive processes. On the other hand, values lower than 1.9 seem quite common both in the Atlantic and the Indian ocean. Why does 


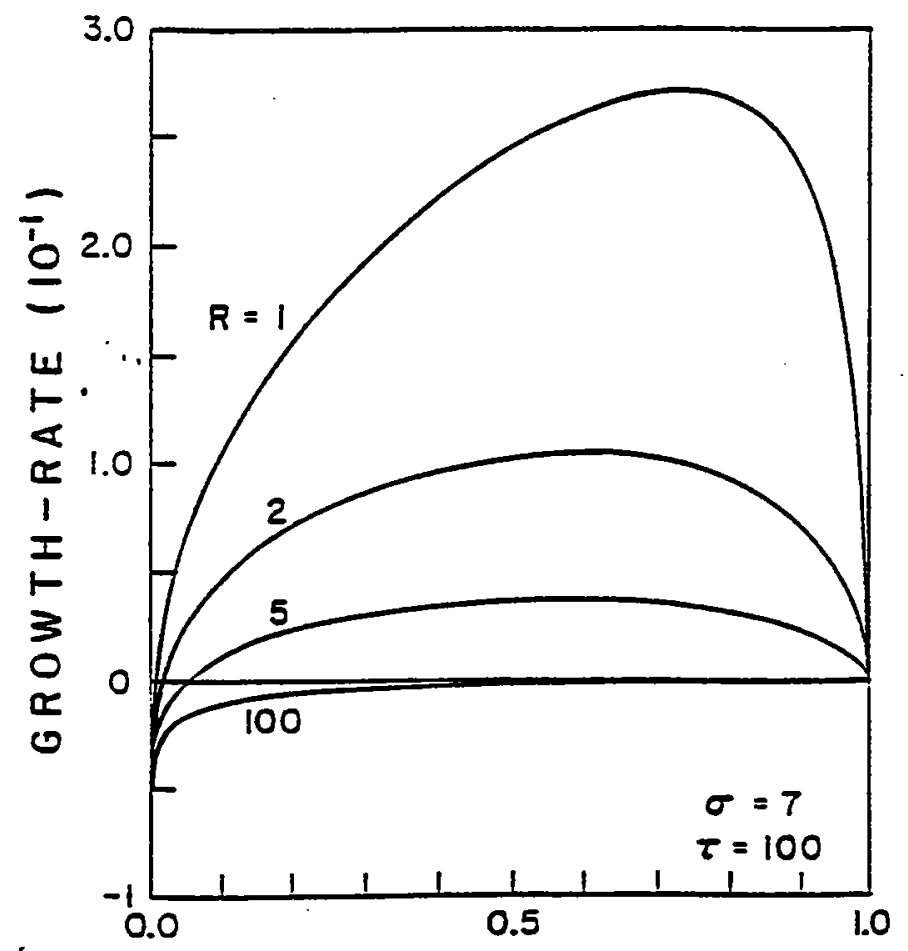

FLUX-RATIO

Figure 3: Growth-rate of the maximum unstable mode as a function of the flux-ratio. The four lines refer to four different density ratios.

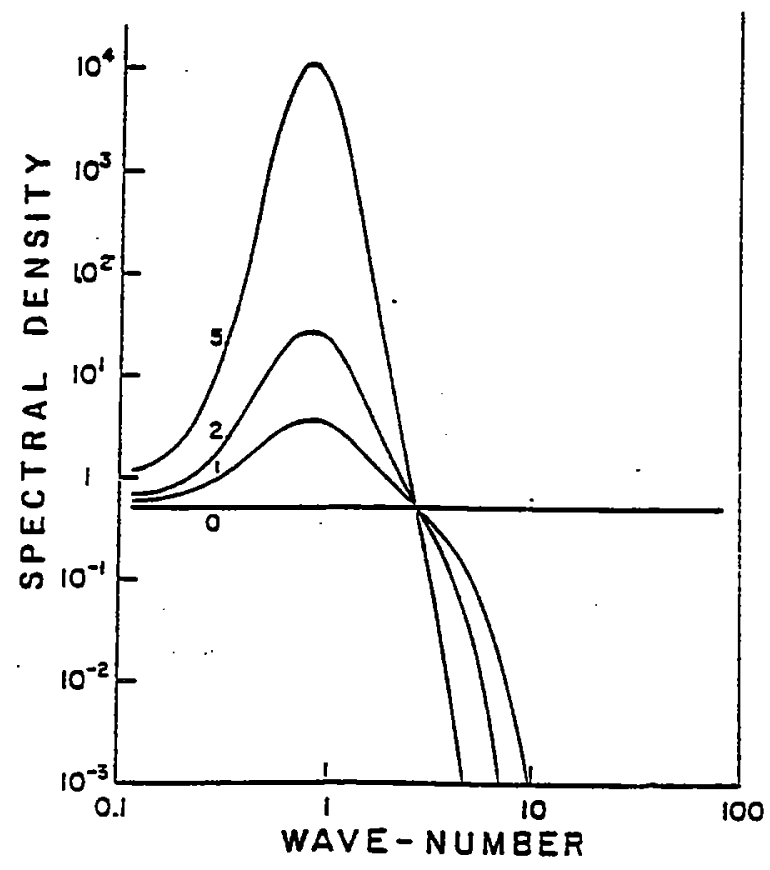

Figure 4: Evolution of a white noise perturbation. The curves are the spectrum calculated after 1,2 and 5 buoyancy periods. Here $R_{p}=2$ 


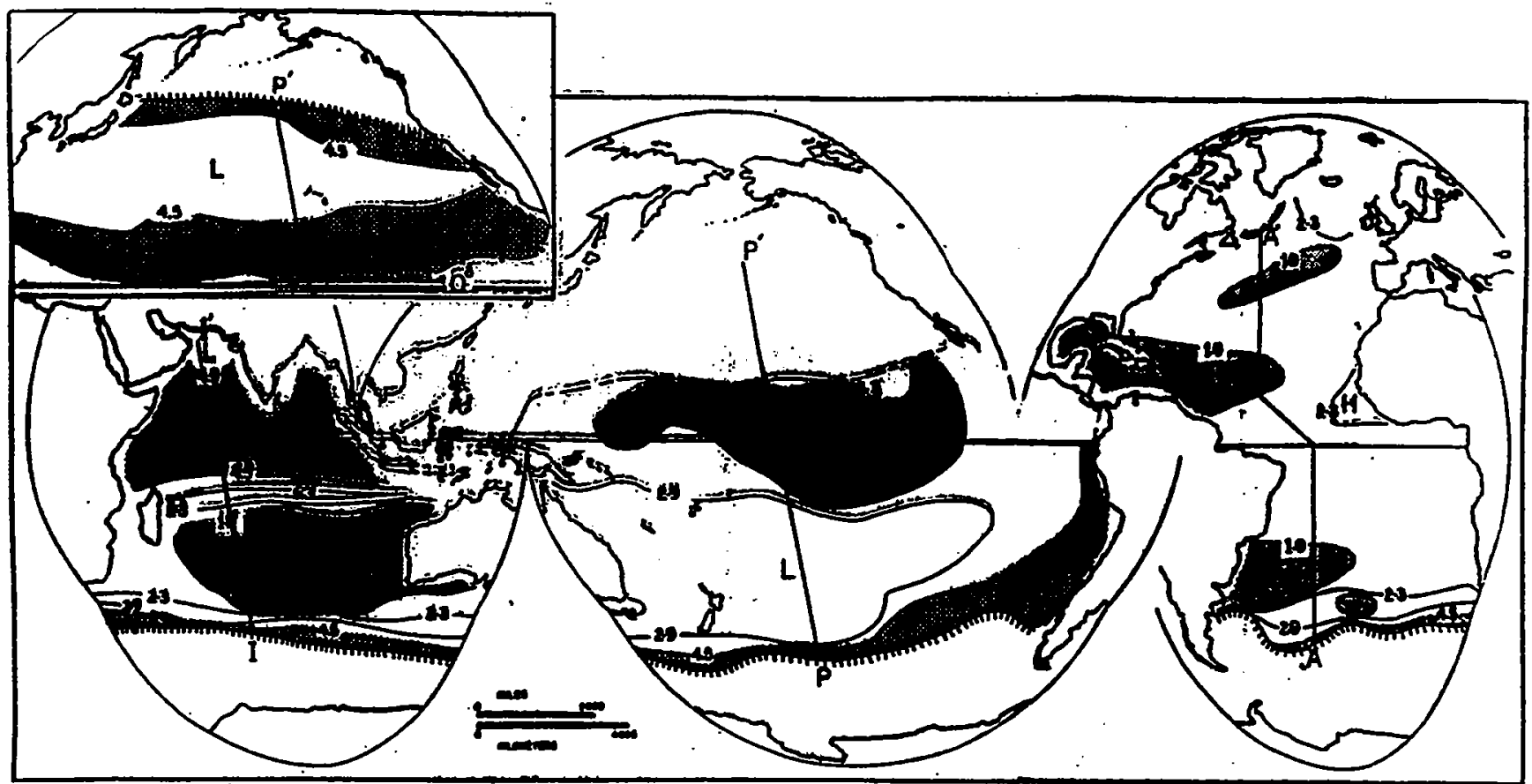

Chart 14. Slope ratio (B) of the T-8 curve for the upper kllometer of depth Ia regiona showing a neEa-

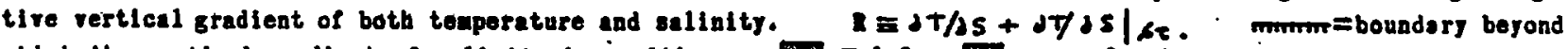
which the rertical gradient of salinity le positive. $₹$ 1.9. 54.5 . Iaeet mp obon distribution sbove North Pacifle dininui salinity surfaos. $B=$ reletively high slope retio. $L=$ reletively lor siope ratio.

Figure 5:

the ocean have such low density ratios? In the case of the Atlantic, low $R_{p}$ is attributed to the mid-latitude circulation coupled with strong evaporation.

Furthermore, it is possible to argue that double-diffusive processes are important in determining the vertical large-scale structure of vast regions of the ocean. Figure 6 shows the temperature vs. salinity profile in the main thermocline of the South Atlantic. Taking into account the full equation of state for salt water, the curve of constant density ratio fits the observed data very well. This is in agreement with the Schmitt(1981) model which predicts an effective eddy diffusivity for the vertical transport of $R_{\rho}$ much higher than the eddy diffusivity of the the salt or the temperature alone. In that model a salt flux convergence or divergence occurs at points away from the constant $R_{p}$ profile so that $R_{p}$ perturbations die away.

Much more detailed evidence of the presence of double-diffusive phenomena in the oceans come from experiments such as the Caribbean-Sheets and Layers Transects (C-SALT). This experiment detected very sharp temperature and salinity steps in the main Barbados thermocline over an area of 1 million square kilometers (see Figure 7). Figure 8 displays a typical staircase where well-mixed layers 5-30 meters thick are separated by sharp interfaces with temperature contrasts of about $1^{\circ} \mathrm{C}$. Note that when $R_{p}>1.8$ the staircase disappears. It is possible to track the interfaces across the staircases (see Figure 9) and we see that the layers are fairly coherent in the horizontal direction. The heaving up and down of the layers is attributed to internal waves, and at the top some of the mixed layers disappear.

Figure 10 displays a scatter plot of observed temperatures from a mooring in the C-SALT experiment. The observed temperatures tended to cluster at layer values. The evolving temperature was tracked over a 8-month period, and advection was responsible for horizontal 


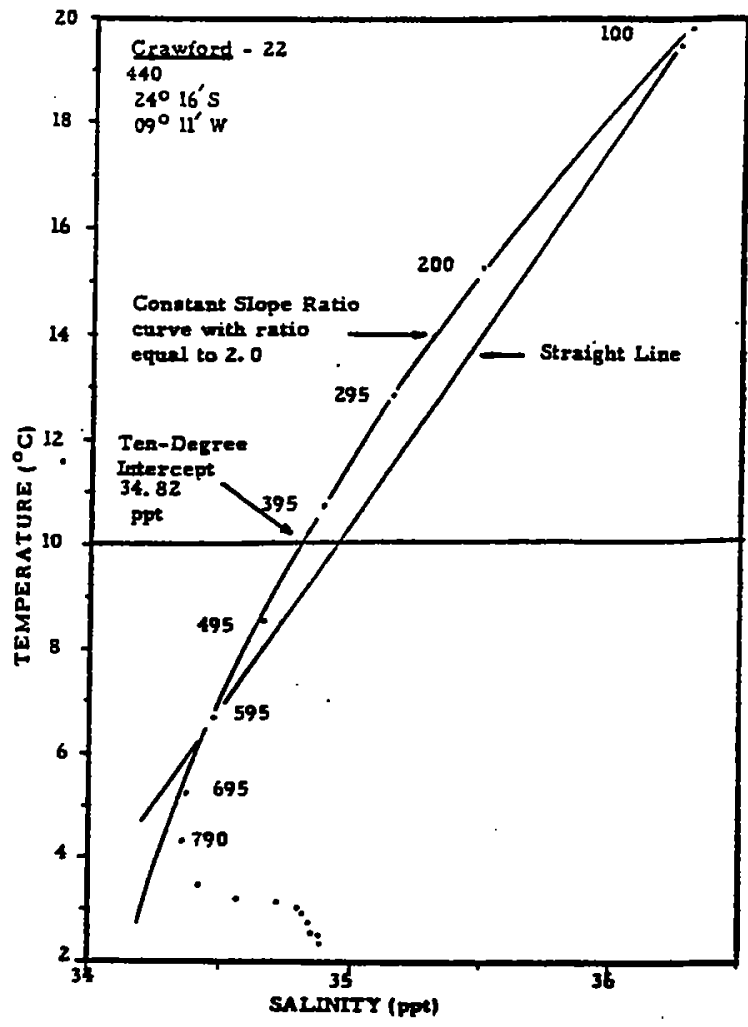

Figure 6: Sample T-S curve showing close coincidence of the observed T-S distribution with a constant slope ratio curve. Numbers near points are depths in meters.

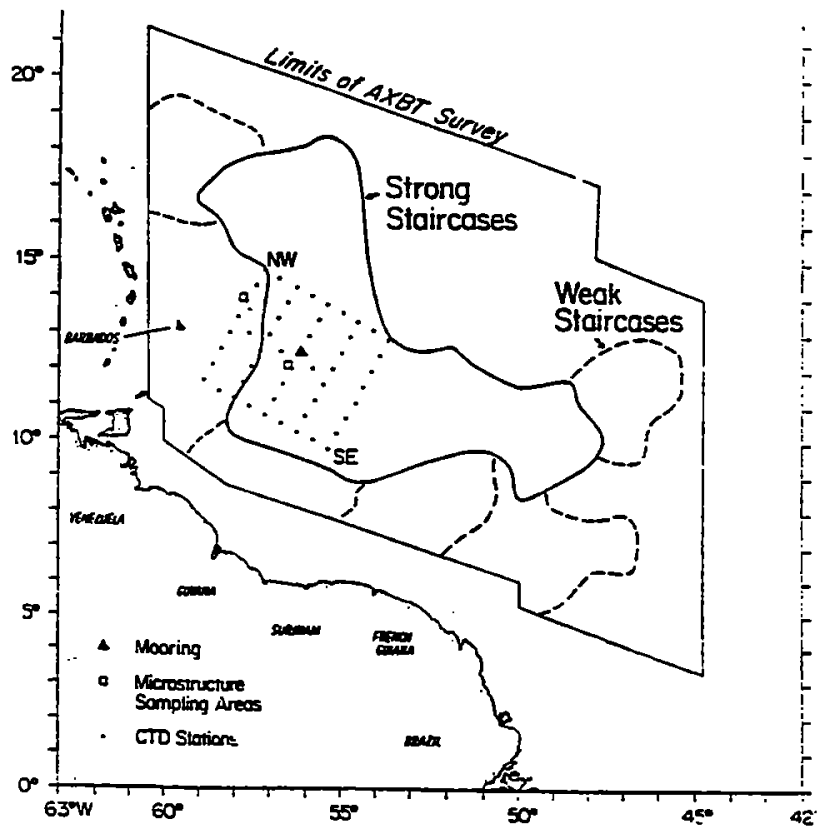

Figure 7: The region of the western tropical North Atlantic surveyed during the C-SALT field program. The AXBT is an Air deployable eXpendible Bathy Thermograph, a device used to obtain ocean temperature profiles from an airplane. An area of over $10^{6} \mathrm{~km}^{2}$ was found to have significant thermohaline layering in the main thermocline. 


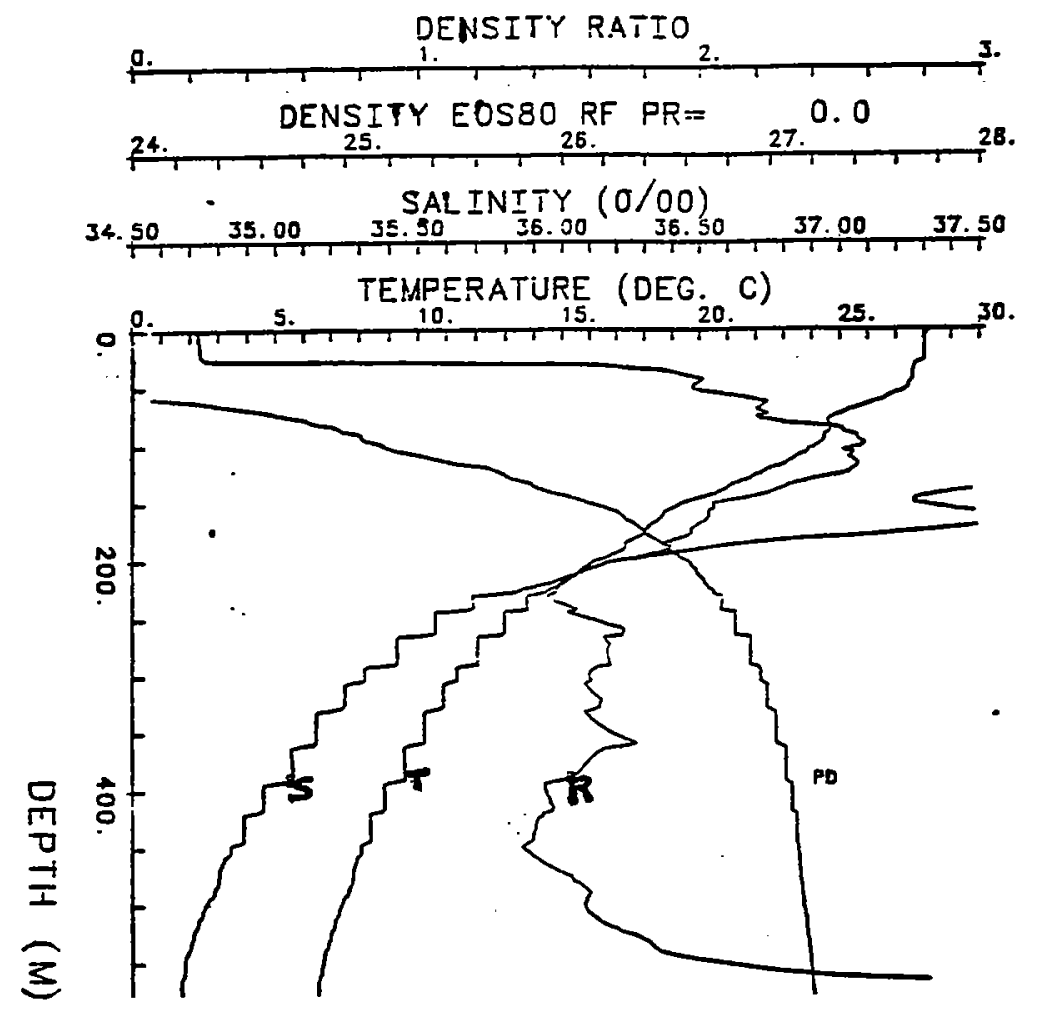

Figure 8: The vertical profiles of temperature, salinity, potential density anomaly and $\boldsymbol{R}_{\boldsymbol{\rho}}$ from a station in the C-SALT area. Temperature contrasts across the steps are typically $0.5-1.0^{\circ} \mathrm{C}$. Mixed layers are 5-30 meters thick. The layered structure in the 300-600 m depth range has $1.5<R_{\rho}<1.8$.

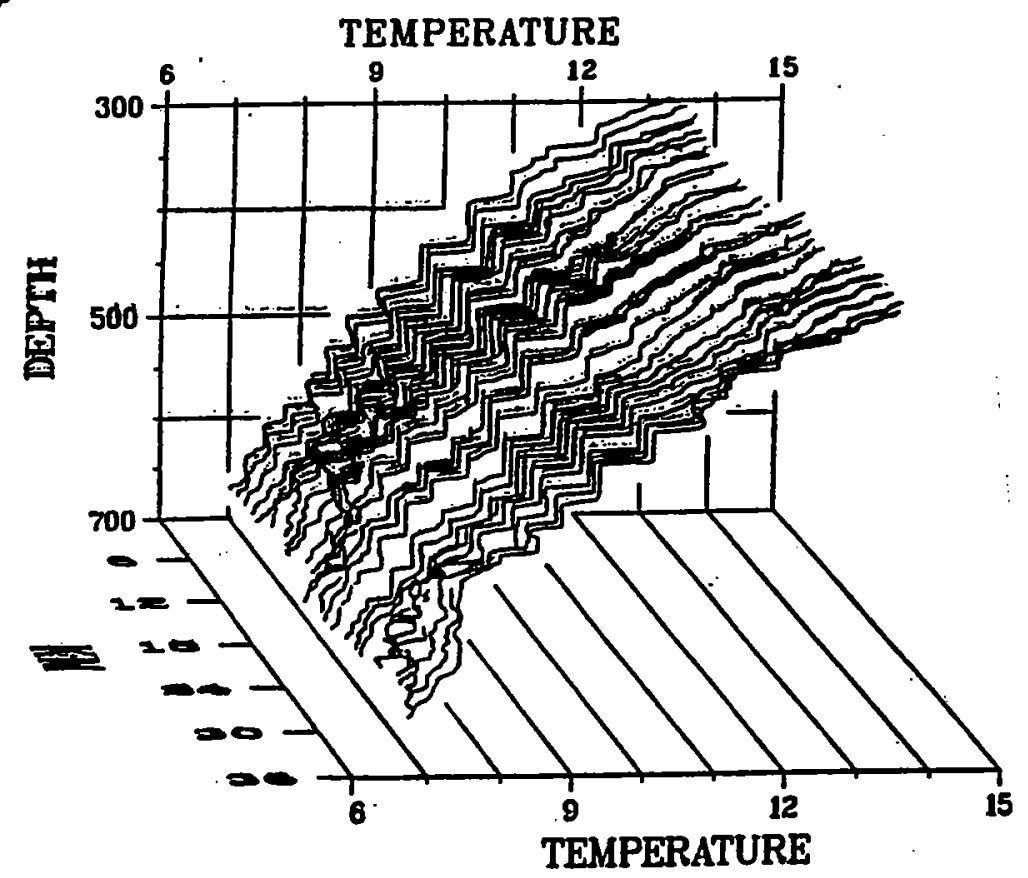

Figure 9: Temperature staircases observed in the C-SALT experiment, spanning a length of about $30 \mathrm{~km}$. The horizontal structures are fairly coherent. 


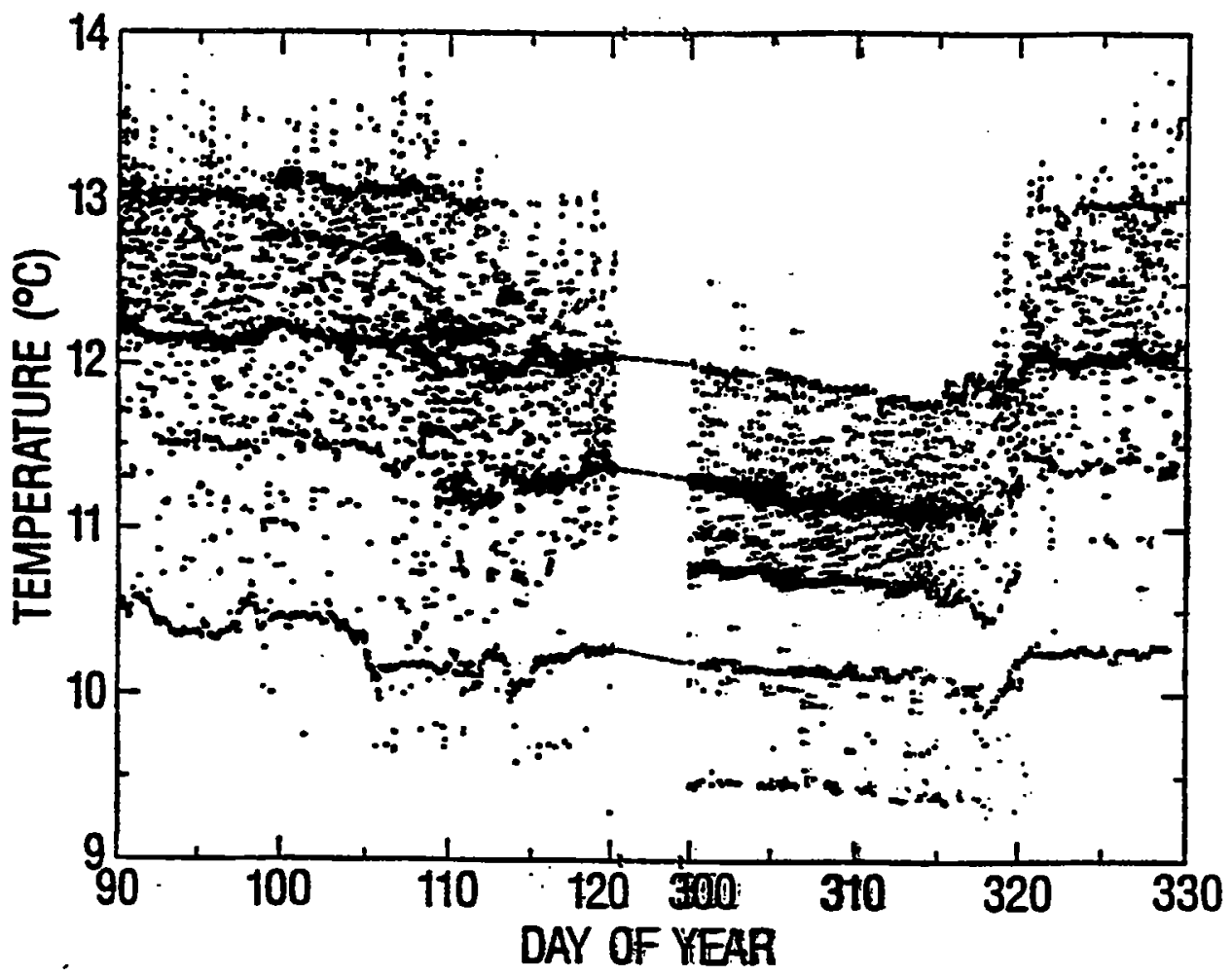

Figure 10: Time evolution of a temperature staircase. This structure is fairly coherent in time, too.

gradients of temperature and salinity. Eddies and internal waves tended to mix properties around.

The horizontal structure of these layers was characterized by a remarkably constant lateral density ratio of $\frac{\alpha T_{s}}{\beta S_{o}}=0.85 \pm 0.03$. This observation excludes the turbulence as the dominant mechanism for small-scale mixing, which would require the same density ratio in both the vertical and the horizontal directions. Even assuming purely isopycnal processes, one would expect a lateral density ratio of about 1.0. The theoretical and laboratory lateral density ratios for salt fingers vary between 0.6 and 0.7 . Therefore double diffusion must be the main factor in the horizontal mixing, altough some contribution from turbulence cannot be excluded.

\section{Conclusion}

Although the theoretical instability requirement is merely $R_{\rho}<\tau$, only weak evidence for double diffusive phenomena have been found when $R_{p} \geq 2$. Nevertheless, it seems that the maximum allowable density for the formation of staircases decreases with latitude. Kunze (1990) suggested that the shear due to inertial oscillations (which frequency is inversely proportional to the Coriolis parameter) may disrupt the fingers. His model may account for the relationship between the allowable density ratio to find staircases and the latitude.

In some cases the C-SALT surveys didn't find staircases even under favorable conditions. In Figure 11 a stratification similar to that of Figure 8 is shown, but no staircase is found. A possible intrerpretation is to attribute the disruption of the staircase to patches of turbulence, 


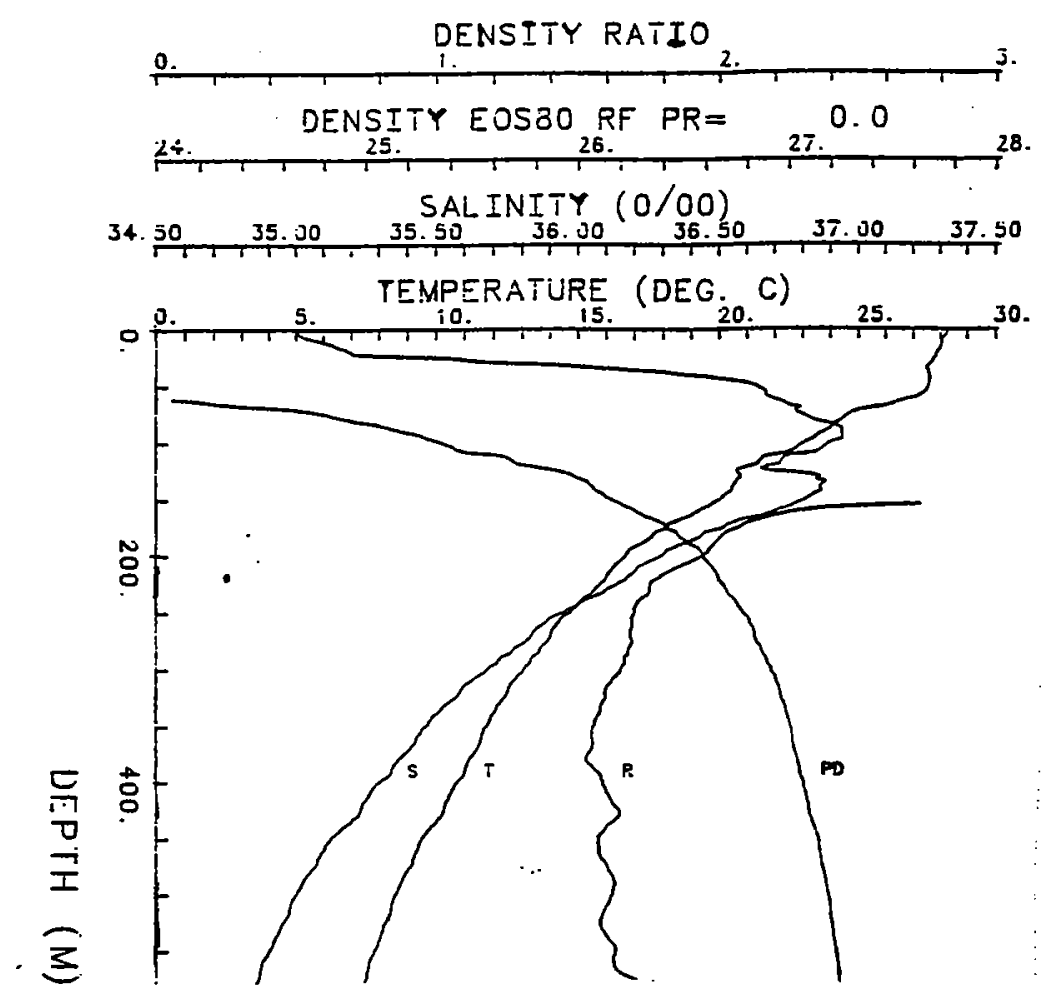

Figure 11: Salinity, temperature, potential density and density ratio profiles similar to fig. 8. Although the conditions were favorable to the formation of staircases, no strong vertical structure is observed in this case.

but there is no experimental or theoretical evidence that this is the right answer.

Finally, it is important to stress that the size of the staircases themselves is very puzzling. Laboratory experiments have only shown mixed layers with thicknesses of tens of centimeters, separated by salt finger layers no more than a few centimeters thick. Furthermore, it seems possible that there can be a finer structure in the steps of the oceanic staircases, not observed until recently because of the limited resolution of the soundings.

\section{References}

[1] Kunze, E. 1990. The evolution of salt fingers in inertial wave shear. J. Mar. Res 48 471-504

[2] Schmitt, R. W. 1981. Form of the temperature-salinity relationship in the Central Water: evidence for double-diffusive mixing. J. Phys Ocean. 11 1015-1026.

[3] Schmitt, R. W. 1994. Double diffusion in oceanography. Anru. Rev. Fluid Mech. 26 255-285

[4] Stern, M. E. 1975. Ocean Circulation Physics. New York; Academic. 


\section{A Model for Salt Fingers}

In this section we focus on the finite amplitute behavior of saltfingering motions. We examine two ditinct models. The first, and most commonly used was developed by Stern. He made the assumptions that the fingers do not depend on the vertical coordinate. An alternative model developed by Howard \& Veronis takes a slightly different approach. They assume that the salt diffuses much more slowly than heat and then at leading order are left with a simpler problem. They then obtain corrections for the small salt diffusivity. The analysis therefore is applicable in the salt-heat case where the ratio of diffusivities is approximately 70 , but not for the case of sugar-salt where the ratio is only approximately 3.

\section{Stern's Model}

We assume that $\partial_{z} \equiv 0$ and $u \equiv v \equiv 0$. This implies that $p_{x}=p_{y}=0$.

For simplicity we consider only two dimensional solutions $\left(\partial_{y} \equiv 0\right)$. Then the equations of momentum, heat and salt become

$$
\begin{gathered}
w_{t}=g \alpha T-g \beta S+\nu w_{x x} \\
T_{t}+w \bar{T}_{z}=\kappa_{T} T_{x x} \\
S_{t}+w \bar{S}_{z}=\kappa_{S} S_{x x}
\end{gathered}
$$

We look for modes $w, T, S \sim \exp (\lambda t) \sin (k x)$. Writing $\sigma=\nu / \kappa_{T}, \tau=\kappa_{S} / \kappa_{T}$, $\lambda=\kappa_{T} k^{2} \Lambda$,

$$
\frac{R_{T}=g \alpha \bar{T}_{z}}{\nu \kappa_{T} k^{4}}
$$


and

$$
\frac{R_{S}=g \beta \bar{S}_{z}}{\nu \kappa_{T} k^{4}}
$$

we obtain the dispersion relation

$$
(\Lambda+1)(\Lambda+\tau)(\Lambda / \sigma+1)-(\Lambda+1) R_{S}+(\Lambda+\tau) R_{T}=0 .
$$

Then for steady modes we obtain $R_{S}=\tau\left(R_{T}+1\right)$ which implies a wavenumber for steady fingers. Alternatively, we can consider nonsteady modes and look for the solutions with maximium growth rate.

\section{Howard \& Veronis' Model}

We remove the assumption of infinitely long fingers and replace it with the less restrictive assumption that $\partial_{z} \ll \partial_{x}, \partial_{y}$. We consider fingers between two reservoirs, the upper one hot and salty and the lower one cold and fresh, seperated by a finite distance.

We consider the case where $\kappa_{S} \ll \kappa_{T}$ so at zeroth order we take $\kappa_{S}=0$. We take the salt distribution to be $\Delta S / 2$ in descending fingers and $-\Delta S / 2$ in ascending fingers. We assume that the temperature distribution between the reservoirs has a constant gradient $\bar{T}_{z}$.

For simplicity we consider $\partial_{y} \equiv 0$. We anticipate a buoyancy layer scale and take

$$
x \sim L \equiv\left(\frac{4 \nu \kappa_{T}}{g \alpha \bar{T}_{z}}\right)^{1 / 4}
$$

We nondimensionalise $t=L^{2} t^{\prime} / \kappa_{T}, w=\kappa_{T} w^{\prime} / L, T=L \bar{T}_{z} \theta / 2$ and drop primes. Since $\kappa_{S}=0$ at leading order, $S$ defined above satisfies the salt equation and the equations of momentum and heat become

$$
\begin{gathered}
w_{t} / \sigma-2 \theta-w_{x x}=2 Q \\
\theta_{t}+2 w-\theta_{x x}=0
\end{gathered}
$$

where

$$
Q \equiv \pm \frac{\beta \Delta S}{\alpha L \bar{T}_{z}}
$$


where the \pm refers to ascending and descending fingers respectively.

We consider a horizontal array of fingers, at lateral boundaries $w, \theta=0$ at $x=0 . \pi b$. We have $w, \theta$ in ascending fingers and $-w,-\theta$ in descending fingers. It is easy to show that this only has steady states as time goes to infinity. The steady equations are the thermal analagies of Ekman or buoyancy layer eqautions. So the steady solutions can be expressed in terms of trigonometric and hyperbolic functions.

For $b>2$ the solution posesses the property that the downward velocity is negetive in the centre of a finger. This violates the assumptions of the model, since we implicitly assume that salt is being fluxed in a given direction in any given finger.

The convective heat, salt and buoyancy fluxes are

$$
\begin{aligned}
& F_{T}=\frac{1}{\pi b L} \int_{0}^{\pi b L} w T d x, \\
& F_{S}=\frac{1}{\pi b L} \int_{0}^{\pi b L} w S d x
\end{aligned}
$$

and

$$
B=\alpha F_{T}-\beta F_{s}
$$

If we make the assumption that the fingers behave in such a way that they maximise the buoyancy flux, we find that this occurs when $\pi b L \approx 1.7 L$ and

$$
R_{f} \equiv \frac{\left|\alpha F_{T}\right|}{\left|\beta F_{S}\right|} \approx 0.251 .
$$

These values are close, but not identical with Stern's result for maximal growth rate. If we consider a square array of fingers rather than the sheets discussed above, this only gives rise to a small change in the value to the flux ratio. However, it should be noted that salt-heat experiments by Turner, Linden and Schmit exhibit much larger values of $R_{f}$ than those predicted by the theory.

Now we consider a boundary layer between two finger where the diffusivity of salt can't be neglected. we still take $\kappa_{S} \ll \kappa_{T}$ but now $\kappa_{S} \neq 0$. So we must resolve an equation for the salinity

$$
w S_{z}=\kappa_{S} S_{x x}
$$


Near the boundary of a finger we can consider $w$ proportionnal to $x$ where the coefficient of propotionnality is deducted from the our previous estimation of $w$. We choose the scaling

$$
z=h \cdot z^{\prime} ; x=H \cdot x^{\prime}
$$

where $h$ is the length of the finger and

$$
H^{3}=\frac{\cos (\pi b)+\cosh (\pi b)}{\sin (\pi b)+\sinh (\pi b)} \cdot \tau \cdot \frac{\alpha \overline{T_{z}} h}{\beta \Delta S} \cdot\left(\frac{4 \nu \kappa_{T}}{9 \alpha \overline{T_{z}}}\right)^{3 / 4}
$$

in a such manner that the previous equation become

$$
x \cdot S_{z}=S_{x x}
$$

First for $x>0$ and $z>0$ we take $S=0$ on $z=0$ and $S=f(z)$ on $x=0$.This equation are similarity solution having $x^{3} / y$ fixed. For $S(x, z)=0$ on $z=0$ for $x>0$ we have

$$
S_{o}(x, y)=\int_{x / z^{1 / 3}}^{\infty} \exp \left(-\zeta^{3} / q\right) d \zeta
$$

where

$S_{0}=\Gamma(1 / 3) \cdot 3^{-1 / 3}$ in $x=0$ for $z>0$. So a multiple of $S_{0}$ satisfy this problem in the special case where $f(z)=0$. When it isn't a constante $f(z)$ can be treated by take a Laplace Transform of our previous solution to get

$$
S(x, y)=\frac{3^{1 / 3}}{\Gamma(1 / 3)} \int_{x / z^{1 / 3}}^{\infty} \exp \left(-\zeta^{3} / q\right) \cdot f\left(z-x^{3} / \zeta^{3}\right) d \zeta .
$$

solution of $x \cdot S_{z}=S_{x x}$ and which satisfy the boundary condition.

We can solve the same problem but with $S_{x}=g(z)$ on $x=0$ and the require that $S(0, z)$ satisfies 


$$
f(z)=-\frac{\Gamma(1 / 3)}{3^{1 / 3} \Gamma(2 / 3)} \cdot I^{1 / 3}(g)
$$

where

$$
I^{n}(g)=\frac{1}{\Gamma(n)} \int_{0}^{z}(z-\zeta)^{n-1} \cdot g(\zeta) d \zeta
$$

is a Riemann-Louville fractional intergral.

We now match the salt field and its gradint between adjacent fingers and after eliminating $f$ we obtain

$$
\int_{0}^{1}|z-\zeta|^{-2 / 3} g(\zeta) d \zeta=-3^{1 / 3} \cdot \Gamma(2 / 3)
$$

Or if we take $G=-\frac{g}{3^{1 / 3 \Gamma(2 / 3)}}$

$$
\int_{0}^{1}|z-\zeta|^{-2 / 3} G(\zeta) d \zeta=1
$$

To conclude, we must first specify that all these results are valid only if we have $H x<1 / 2 \pi b_{n} L$ so $x<\frac{0.94}{\left(\tau R_{\rho}\right)^{1 / s}}$ with $1<R_{p}<(32 \tau)^{-1}$. We must also examine the result of numerical simulation of the finger dynamics. Figures 1 and 2 give the contour plot of $T, S, \rho$ and $\psi$ where $\psi$ is the vorticity for a fully nonlinear numerical model by Veronis et al. The results shown in figure 2 are for a lower densty ratio and higher $\Delta S$ than the results for figure 1 and the temperature of the reservoirs penetrates deeply into the finger and the salinity contours flare out as fluid accumulates near the finger tip. The salinity in the reservoir extends all the way to the tips of the fingers and curve back into the finger along the sides. This phenomena creates a mixing layer where other finger instabilities can grow: 

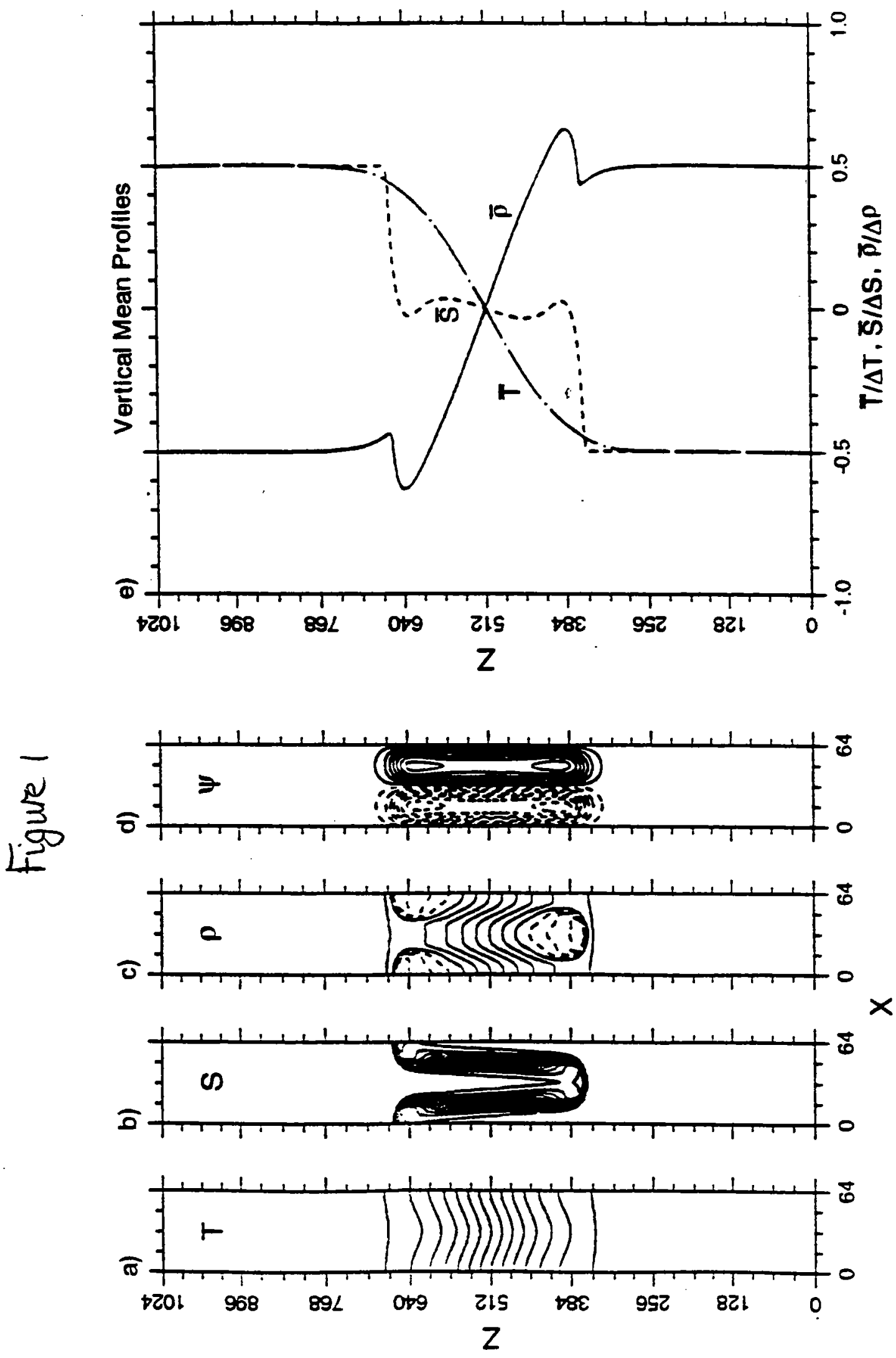


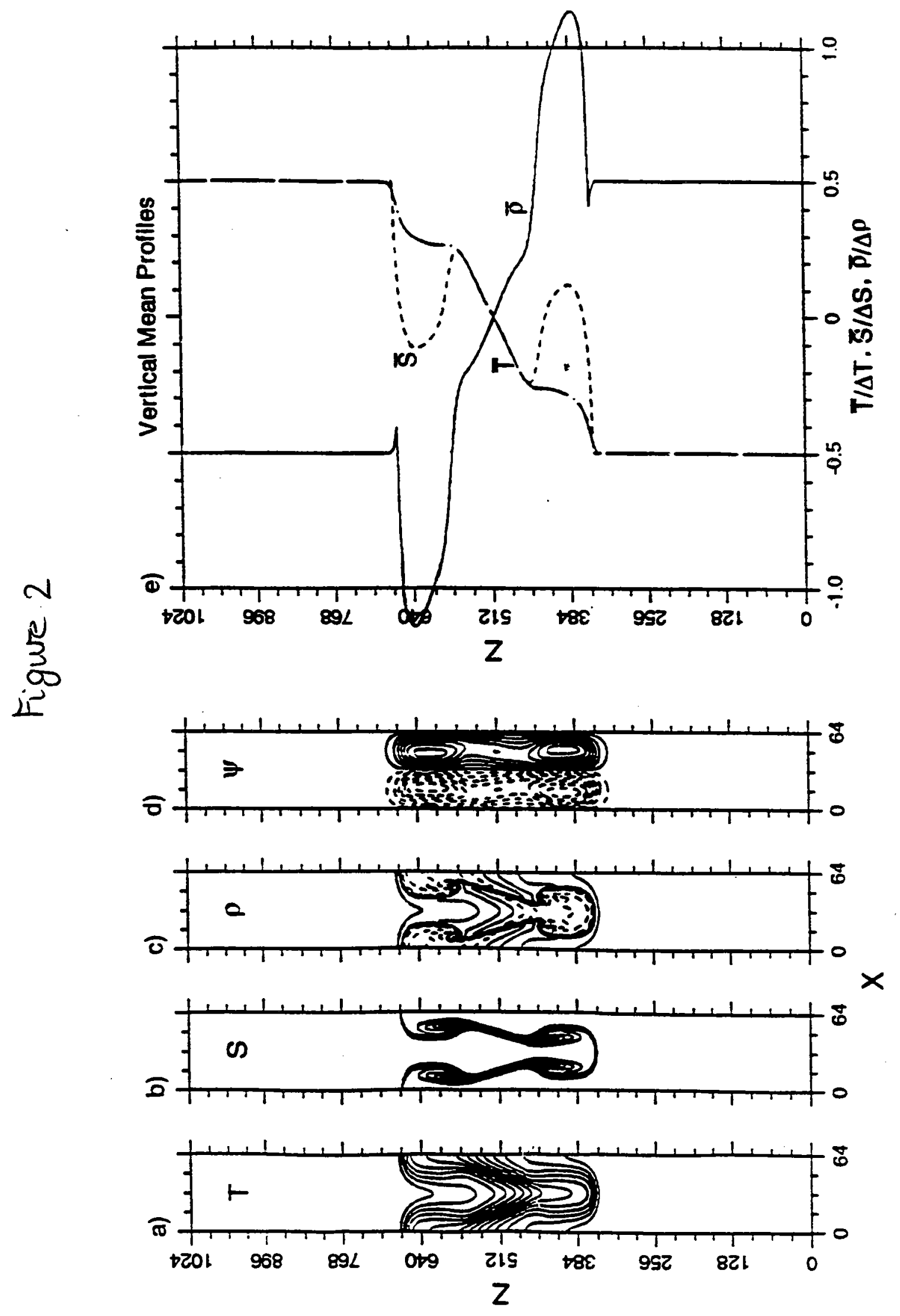




\section{Lecture 7, Part 1}

George Veronis opened the morning festivities with some caveats about salt-finger patterns. The salt-finger scenario has cold and fresh fluid below, while the fluid near the top, like a lascivious longshoreman, is hot and salty. The typical setup has been outlined in the previous lectures, but the basic physics is as follows: the relatively rapid diffusion of heat allows thermal equilibration to occur at any given height much more quickly than salt field equilibration. Consequently, any fluid in an upward moving finger can increase its temperature through lateral diffusion, thereby reducing its density and allowing its upward romp to continue. An analagous argument holds for the downward travellers. However, it is a bit risky to cling to classical stereotypes regarding the fundamental balances involved in the Boussinesq equations (this is elucidated below). A common notion is that salt fingers become tall and thin once they have settled upon their preferred scale as described by Stern (1960). This scenario is realized only in the cases for which the basic state density profile is stable enough, which occurs when the density ratio

$$
R_{\rho}=\frac{\alpha \Delta T}{\beta \Delta S}
$$

satisfies $R_{\rho} \gg 1$. For smaller $R_{\rho}$ (never less than 1, for otherwise the layer is gravitationally unstable), the fluid at the top of the layer is too heavy and drags the temperature field downward before it has a chance to diffuse laterally as much as it would in the long, thin finger case. Since the stabilizing temperature field is less and less effective as $R_{p} \rightarrow 1^{+}$, larger vertical velocities ensue, and the ends of the fingers contain bulbous tips of salt anomalies. After a while the blobs tend to spread out, and it would be a stretch to call them fingers, human fingers at any rate. The informal of heart might dare say they look like the digits of some sort of space critter, like ET or any of the myriad of aliens cooked up in the brain of Steven Spielberg. In addition to having blobby bottoms, the fingers can also be roughly as wide as they are tall, as is shown in figure(1) and figure(2). Although these structures are still called fingers because the fundamental mechanism for their creation is the same, the crucial message is that salt fingers are not necessarily tall and thin.

Another warning trumpeted by George Veronis is that much of the salt-finger literature refers to tall, thin salt fingers with large flux ratios, as if these two properties are both ubiquitous salt-finger features. The flux ratio is defined by

$$
R_{F}=\frac{\alpha F_{T}}{\beta F_{S}}
$$

where $F_{S}$ and $F_{T}$ denote the horizontally averaged salt and heat fluxes, respectively. For clarity, let us say that $R_{F} \approx 0.75$ typifies large flux ratios, and $R_{F} \approx 0.25$ lies on the low end. Through a series of high resolution numerical experiments, Shen and Veronis (1996) have shown that fluids with small density ratios tend to have relatively large flux ratios. This clashes with the common notions of long and thin fingers with large flux ratios, since the fingers become increasingly distorted as $R_{\rho}$ approaches unity. For example, the bulbous tipped fingers of figure(1) formed in a situation where $R_{\rho}=1.5$, which resulted in a flux ratio $R_{F}=0.74$. In another numerical run of $R_{\rho}=3.0$, the fingers were much taller and thinner, and a typical flux ratio of about 0.2 resulted (note that $R_{F}$ 
does not merely depend upon $R_{\rho}$ alone; it can vary in a non-obvious manner with the chosen $\Delta S$, as larger $\Delta S$ will increase $R_{F}$ ). Thus, if one wanted to attain a higher flux ratio $R_{F}$, one would have to settle for blobby, distorted fingers, not the classic tall and thin fingers. The basic physics underlying this is encompassed by the dominant balances in the momentum, heat, and salt equations. In the classic finger scenario the dominant balances are diffusive:

$$
\begin{array}{r}
\kappa_{x} \frac{\partial^{2} T}{\partial x^{2}}-w \overline{T_{z}}=0 \\
\kappa_{s} \frac{\partial^{2} S}{\partial x^{2}}-w \overline{S_{z}}=0 \\
\nu \frac{\partial^{2} w}{\partial x^{2}}=g \beta S-g \alpha T
\end{array}
$$

where $\kappa_{T}$ is the thermal diffusivity, $\kappa_{s}$ the salt diffusivity, $g$ the gravitational acceleration, $\nu$ the molecular viscosity, and $w$ the vertical velocity. These are the classical balances for a diffusively dominated buoyancy layer. When $R_{p}$ is near unity the balances can be different. In this case the dominant terms in the heat and salt equations are advective ones:

$$
\begin{aligned}
& \frac{\partial T}{\partial t} \sim-u \frac{\partial T}{\partial x} \\
& \frac{\partial S}{\partial t} \sim-u \frac{\partial S}{\partial x}
\end{aligned}
$$

where $u$ is the horizontal velocity. An important point is that the advective balance holds near regions of clustering isohalines. The diffusive balance can still hold near mid- layer where the fingers retain more regular, sinusoidal structures. Since the fundamental balances can vary with height, any analysis that concentrates on assuming one or the other is likely to get the physics wrong. The highly unstable blobs associated with a vast surplus or deficit of salinity can lead to convective overturning, and eventually the diffusive regions and the advective regions will interact in a highly nonlinear manner. The issue as to how they interact remains a challenge to any salt-finger theory, as a variety of spatial scales seem to be involved.

\section{Lecture 7, Part 2}

Many oceanographers speculate that one manifestation of double diffusion in the ocean is the existence of oceanic fronts, the characteristic features of which are strong horizontal temperature and salinity gradients. The classic model proposed by Stern (1967) is one in which interleaving layers ("intrusions") of hot, salty water and cold, fresh water overlie each other, resulting in alternating layers of salt fingers and convection. The bare bones of the physical scenario are sketched in figure(3).

Horizontal intrusions have been realized through a variety of laboratory experiments. Two of the most famous are the experiment of Chen, Briggs, and Wirtz (1971), in which stably stratified salt water was heated at one vertical sidewall and cooled at the other (which creates a basic state with nonzero $\frac{\partial T}{\partial x}$ ), and the experiment of Ruddick and Turner (1979), where a stably stratified sugar solution and a stably stratified salt solution (with zero horizontal density gradient), initially separated by a vertical plate, were allowed to interact. In the latter experiment Ruddick and Turner 


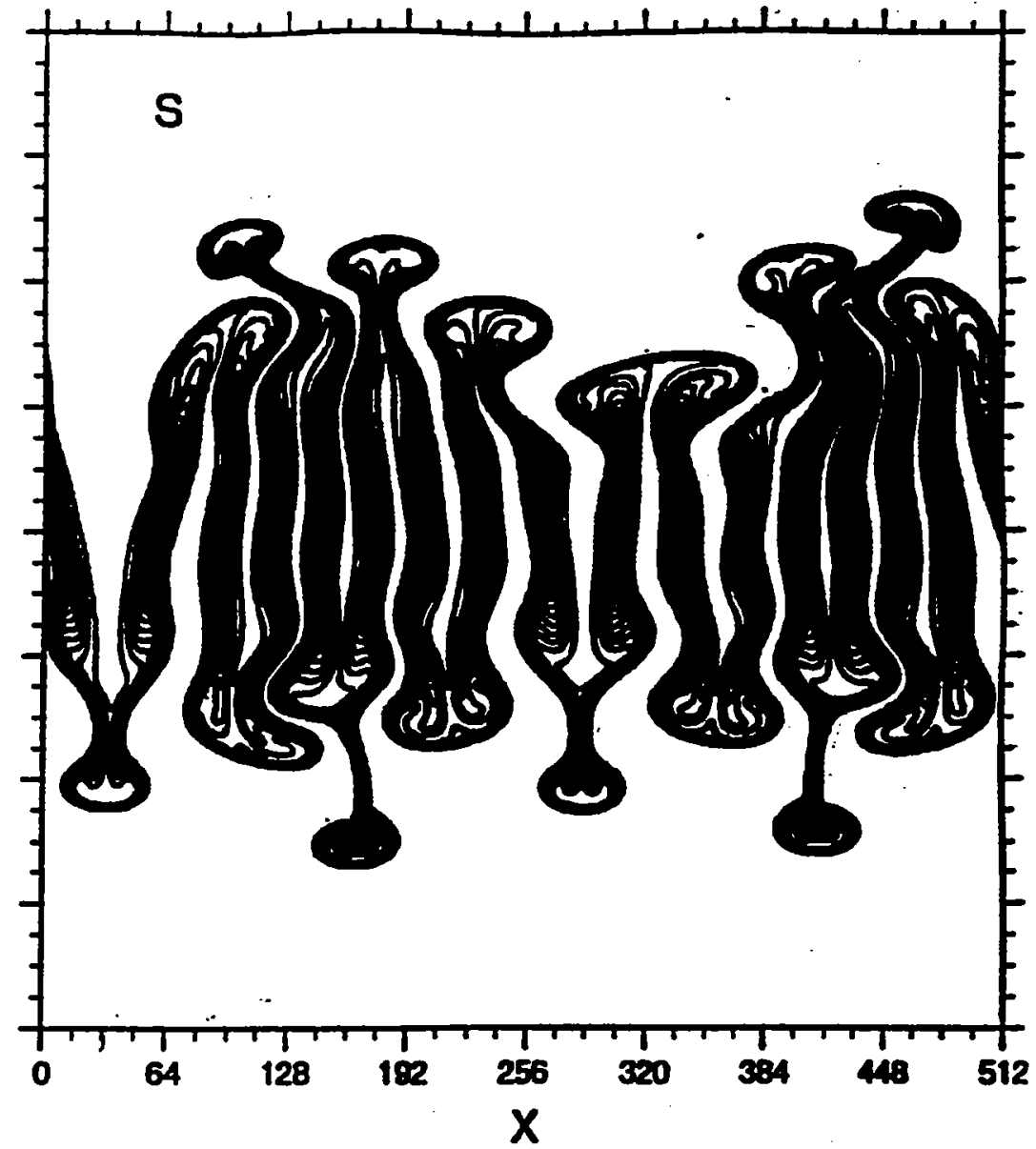

Figure 1: Salt Fingers before imminent destruction, $R_{p}=1.5$ (Shen and Veronis, 1996)
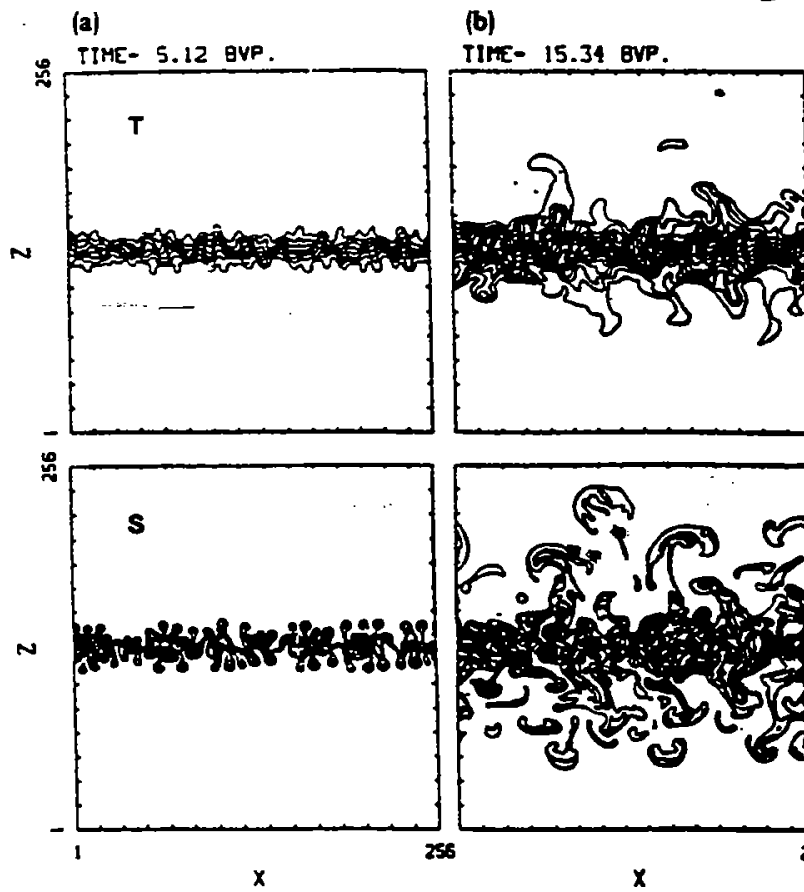

(c)

TIKE- 30.67 BVP.
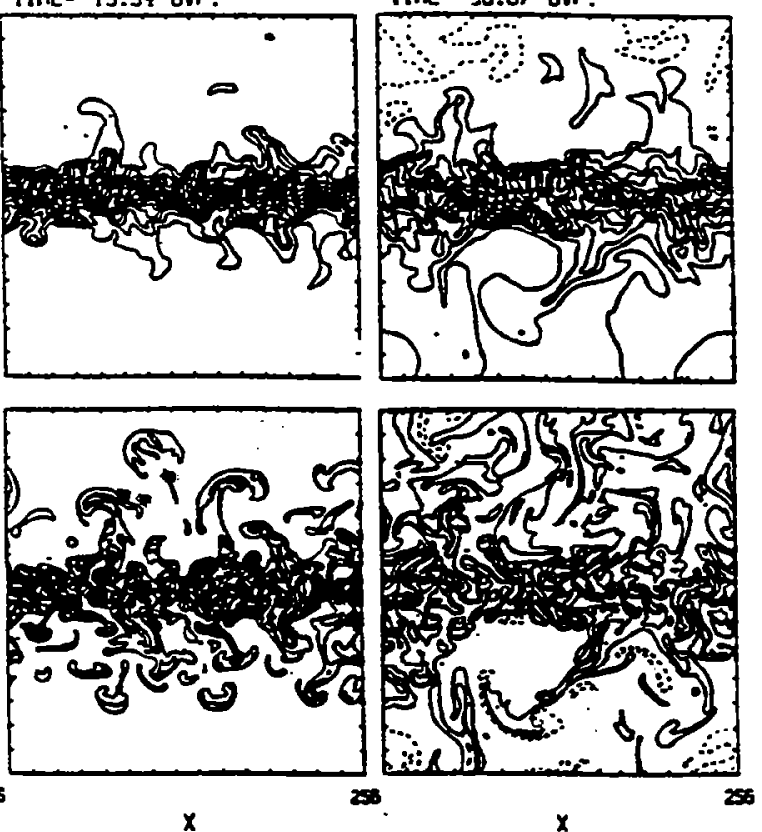

Figure 2: Breakup of salt fingers (Shen, 1989) 


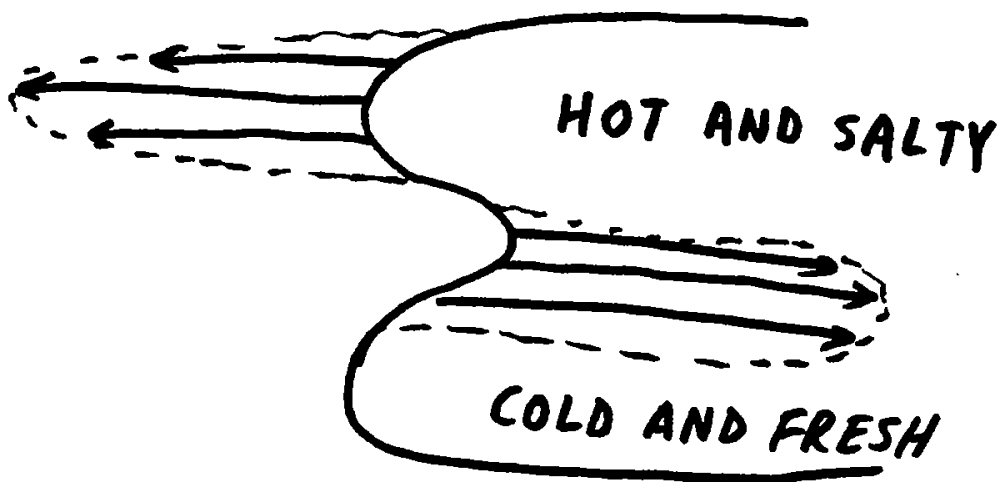

Figure 3: Interleaving Layers Due To Horizontal Intrusions

observed a transient period of internal gravity waves, then followed by sugar and salt clustering that resulted in a front across which there were concentration differences. A regular series of intrusions typically appeared after a few minutes.

An interesting question regards the stability of these fronts. Niino (1986) explored this issue by analyzing the stability of a basic state for which both $\mathrm{T}$ and $\mathrm{S}$ had horizontal variation, but such that their individual contributions to the basic state horizontal density gradient exactly cancelled. The latter assumption allows one to perturb about a basic state of zero velocity. Toole and Georgi (1981) examined this sort of scenario, but they chose to look at a front of infinite extent. Niino examined the case of a front of arbitrary width. In addition to other details of the flow pattern, one of the goals of this analysis is to predict the vertical length scale of the observed intrusions, a feature that the analysis of Toole and Georgi unsuccessfully attempted to reproduce.

Niino chose to perturb about the following basic state, employing the Boussinesq, thermohaline equations as described in the previous lectures:

$$
\begin{array}{r}
\bar{S}=\overline{S_{z}} z+\Delta S f(x) \\
\bar{T}=\bar{T}_{z} z+\frac{\beta \Delta S}{\alpha} f(x) \\
\bar{\rho}=\rho_{0}\left(1-\alpha\left(\bar{T}-T_{0}\right)+\beta\left(\bar{S}-S_{0}\right)\right) \\
f(x)= \begin{cases}1, & x>a \\
\frac{x}{a}, & |x|<a \\
-1, & x<-a\end{cases}
\end{array}
$$

where $\overline{S_{z}}>0$ and $\overline{T_{z}}>0$ are the vertically imposed salinity and temperature gradients, respectively. Note that equations (10) and (11) imply $\frac{\partial \bar{p}}{\partial x} \doteq 0$, and that the vertical stratification is in the saltfinger sense.

Niino assumes that the contribution of the diffusive interface to the vertical heat and salt fluxes is unimportant, and linearizes the 2D Boussinesq equations in the usual manner to obtain

$$
\begin{array}{r}
\frac{\partial u}{\partial t}=-\frac{\partial p}{\partial x}+\epsilon \kappa_{e} \frac{\partial^{2} u}{\partial z^{2}} \\
0=-\frac{\partial p}{\partial z}+g(\alpha T-\beta S) \\
0=\frac{\partial u}{\partial x}+\frac{\partial w}{\partial z}
\end{array}
$$




$$
\begin{array}{r}
\frac{\partial T}{\partial t}+u \bar{T}_{x}+w \bar{T}_{z}=\left(\frac{\beta}{\alpha}\right) \gamma \kappa_{e} \frac{\partial^{2} S}{\partial z^{2}} \\
\frac{\partial S}{\partial t}+u \bar{S}_{x}+w \overline{S_{z}}=\kappa_{e} \frac{\partial^{2} S}{\partial z^{2}}
\end{array}
$$

where $\kappa_{e}$ is a salt eddy diffusivity associated with the salt fingers, $\nu$ an eddy viscosity, $\epsilon=\frac{\nu}{\kappa_{e}}$ is the Schmidt number, and $\gamma$ the flux ratio. Some additional comments about the assumptions used are warranted. First, since the aspect ratio of the observed intrusions in the experiments of Ruddick and Turner is seen to be small, the hydrostatic approximation is employed. Second, the temperature flux in equation (15) is assumed proportional to the salinity flux with a proportionality constant $\left(\begin{array}{l}\beta \\ \alpha\end{array}\right) \gamma$. The latter is justified by run down experiments, although one should be warned that $\gamma$ seems to depend on the chosen density ratio, whereas Niino assumes it to be constant. Third, the eddy diffusion term of equation (12) is assumed to give a measure of the horizontal momentum transferred vertically by the salt fingers.

One can now play the familiar game of Fourier decomposition to ascertain which disturbances are most unstable. For any field variable, $u$ for example, write

$$
u(x, z, t)=\hat{u}(x) e^{i m z+\sigma t}+\text { c.c. }
$$

After a heap of algebraic manipulations, and with the variables no longer wearing their hats, the front dynamics can be reduced to a single, nondimensional differential equation for the pressure:

$$
\frac{d^{2} p}{d x^{2}}-i L \frac{d f}{d x} \frac{d p}{d x}-k^{2} p=0
$$

where $x \rightarrow a x, m \rightarrow \frac{m}{d}$, and $\sigma \rightarrow \frac{k_{e}}{d z} \sigma$ have been used to nondimensionalize the equations. The various parameters are

$$
\begin{array}{r}
L=\frac{m^{3}}{\sigma+(1+\mu) m^{2}} \\
k^{2}=\frac{\sigma m^{2}\left(\sigma+\epsilon m^{2}\right)\left(\sigma+m^{2}\right)}{G\left(\sigma+[1+\mu] m^{2}\right)} \\
d=\frac{g \beta \Delta S\left(1-R_{F}\right)}{N^{2}}=\frac{\mu \Delta S}{\overline{S_{z}}} \\
\mu=\frac{1-R_{F}}{R_{\rho}-1} \\
G=\frac{N^{2} d^{6}}{\kappa_{e}^{2} a^{2}}
\end{array}
$$

where $R_{F}$ is the flux ratio, $R_{\rho}$ the density ratio, $\mathrm{k}$ a spatial decay length for the front, $\mathrm{N}$ the BruntVaisala frequency, and G a measure of the Rayleigh number. The parameter $\mu$ gives a measure of the ratio of the vertical density stratification due to salinity to the overall vertical density stratification. Note that the apparant singularity at $\mu=0$ is in fact taken care of by the time scaling; there is no salt stratification if $\mu=0$, and the instability will take infinite time to develop. 
Using (11), we can solve (18) for $p(x)$ and obtain

$$
\begin{gathered}
p(x)= \begin{cases}A e^{-k x}, & x>1 \\
B e^{k x}, & x<-1 \\
C e^{i n_{1} x}+D e^{i n_{2} x}, & |x|<1\end{cases} \\
n_{1,2}=\frac{1}{2}\left(L \pm\left[L^{2}-k^{2}\right]\right)^{\frac{1}{2}}
\end{gathered}
$$

The four constants $A, B, C, D$ are determined by enforcing the continuity of $p$ and $\frac{d p}{d x}$ at $x= \pm 1$. The latter matching condition is in fact equivalent to demanding that the horizontal velocity be continuous, and also follows from integrating (18) through the front on either side. Once the smoke clears from another tour de force of algebra, we obtain the dispersion relation

$\tan \left[\frac{m^{6}}{\left(\sigma+[1+\mu] m^{2}\right)^{2}}-\frac{4 m^{2} \sigma\left(\sigma+m^{2}\right)\left(\sigma+\epsilon m^{2}\right)}{G\left(\sigma+[1+\mu] m^{2}\right)}\right]=-\left[\frac{G m^{4}}{4 \sigma\left(\sigma+m^{2}\right)\left(\sigma+\epsilon m^{2}\right)\left(\sigma+[1+\mu] m^{2}\right)}-1\right]^{\frac{1}{2}}$

This dispersion relation is fairly gruesome and must in general be solved numerically. However, Niino derives some asymptotic results for large $m$ in the case when the eigenvalue $\sigma$ conspires to make the argument of the tangent term equal to $\frac{n \pi}{2}(n=1,2, \ldots)$. Denote this discrete set of growth rates by $\left\{\sigma_{n}\right\}$. The primary result is

$$
\sigma_{n} \rightarrow \begin{cases}{\left[\frac{G}{4\left(\frac{1}{G}+\mu\right)}\right]^{\frac{1}{2}}-\frac{G(2+\mu)}{2\left(1+\mu^{2}\right)} m^{-2}+\mathcal{O}\left(m^{-4}\right),} & \epsilon=0 \\ \frac{G \epsilon(1+\mu)}{m^{-2}}+\mathcal{O}\left(m^{-4}\right), & \epsilon \neq 0\end{cases}
$$

Thus, we see that in the inviscid case the growth rate for $m \rightarrow \infty$ asymptotes to the constant value $\left[\frac{G}{4(1+\mu)}\right]^{\frac{1}{2}}$. In the viscous case, the growth rate becomes small for large $\mathrm{m}$. An interesting feature in either case is that the growth rate becomes smaller for larger $\mu$. That is, the linearly increasing basic state salinity profile has a stabilizing effect for small scale disturbances.

In the general case, Niino solves (26) iteratively using Newton's method. The resulting dispersion curves for typical cases are shown in figure $(4)(\epsilon=0)$ and figure $(5)(\epsilon \neq 0)$. The most interesting feature is that the front is always unstable, even in the presence of viscosity. Consequently, there is no marginal stability curve. The dotted lines in these figures are for the non-hydrostatic case, and they clearly show that non-hydrostatic effects are unimportant for this model. A few of the other interesting results for $\mu=0$ are summarized below:

1) The growth rate increases with $G$ and is always unstable for some $\mathrm{m}$.

2) There is a low wavenumber cutoff for which eigenvalues no longer exist. In dimensional terms, no disturbances with a vertical wavelength larger than $4 \mathrm{~d}$ can grow. This will turn out to be consistent with the experiments of Ruddick and Turner.

3) For $G<10^{5}$, more than half of the asymptotic value of the growth rate is realized for $m<10$. This verifies the previous asymptotic results and shows that high wavenumber modes are eventually damped.

4) The wavenumber of the fastest growing mode decreases if either G decreases or $\epsilon$ increases. Niino derives an asymptotic result for the minimum, maximally growing wavenumber for all combinations 


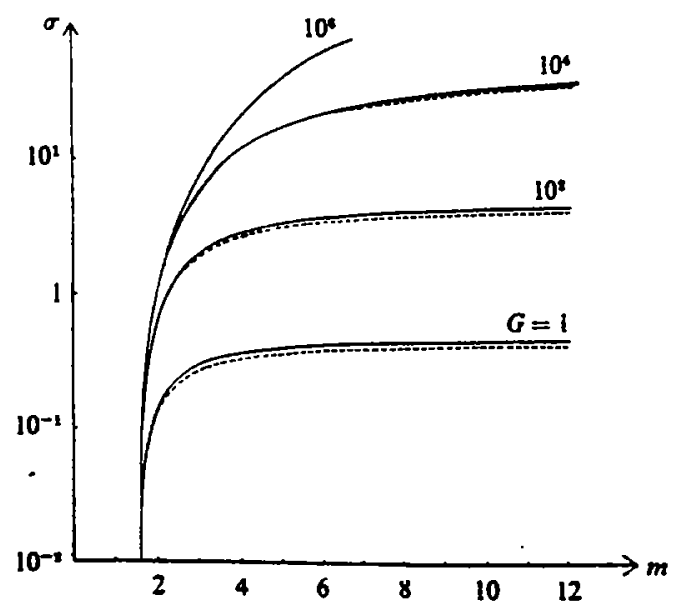

Figure 4: Growth Rate $\sigma$ vs. vertical wavenumber m, $\epsilon=0$ (Niino, 1986)

of the stability parameters $G$ and $\epsilon$ in the particular case $\mu=0$. This asymptotic wavenumber is 2.88, and corresponds to the growth rate $\sigma \sim 0.04719\left(\frac{G}{4 c}\right)$. This suggests that the important stability parameter for $\epsilon \neq 0$ is $\frac{G}{\epsilon}$ and not $G$ alone. This is evident in figure6. Hereafter, this parameter will be denoted by $\mathrm{R}=\frac{\mathrm{G}}{\epsilon}$.

5) For small $R<40$, the fastest growing mode (FGM) asymptotes to a constant value corresponding to the vertical wavelength $2.2 \mathrm{~d}$. For large $\mathrm{R}>2 \cdot 10^{5}$, the dimensional wavenumber scales as $m \sim d^{-1} R^{\frac{1}{4}}$. The growth rate of the FGM for large $\mathrm{R}$ thus scales as $\sigma \sim R^{\frac{1}{2}}$.

6) The flow pattern consists of tilted rolls, with hot, salty fluid rising and cold, fresh fluid sinking. The angle of tilt is in the same sense as the isotherms of the basic state temperature profile. The rolls have roughly the same scale in the horizontal and vertical directions. A point of disagreement between Niino's theory and the Ruddick/Turner experiments is that Niino's rolls rotate in opposite directions as one moves through the layer, whereas in the experiments they are co-rotating. Niino attributes this to his neglect of the contribution of the diffusive interfaces to the vertical fluxes, likely a finite amplitude effect. Niino's bias is built in through his assumption that the fluid is stratified in the salt-finger sense from the outset. However, some other basic features, such as sinking cold/fresh water and rising hot/salty water, a signature of salt-finger driven intrusions, are in agreement with experiment. The streamlines and other field variables are shown in figure7.

When $\mu \neq 0$, the same asymptotic relations in extreme $\mathrm{R}$ limits between $\sigma$ and $\mathrm{R}$ hold as in the case $\mu=0$. The essential $\mu$ dependence is that the wavenumber of the FGM increases with increasing $\mu$ when $\mathrm{R}$ is small, and decreases with increasing $\mu$ when $\mathrm{R}$ is large. As a result, the growth rate for the FGM decreases as $\mu$ is increased (when $\mathrm{R}$ is smaller, the decrease in the growth rate is more drastic). A simple argument in terms of the buoyancy dynamics illustrates why this is true. Upon giving a suitable massage to equations (15) and (16), one can obtain the buoyancy equation

$$
g \frac{\partial}{\partial t}(\alpha T-\beta S)=-N^{2} w-\beta\left(1-R_{F}\right) \kappa_{e} \frac{\partial^{2} S}{\partial z^{2}}
$$

Since $\mu$ is directly proportional to $N^{2}$, we see that for upward moving fluid equation (16) predicts a decrease in $\mathrm{S}$ when $\overline{S_{z}}>0$; therefore, $\mathrm{S}$ decreases with increasing $\mu$. The diffusive term in (28) is 


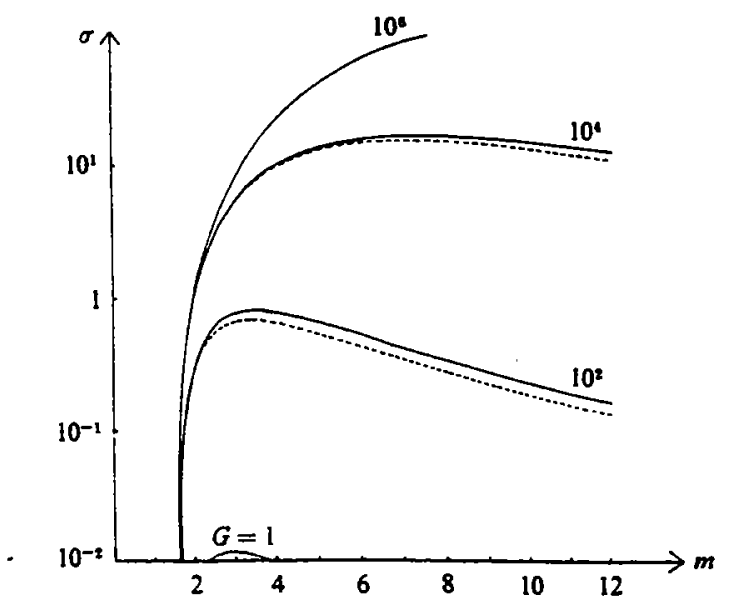

Figure 5: Growth Rate $\sigma$ vs. vertical wavenumber $\mathrm{m}, \epsilon=1$ (Niino, 1986)

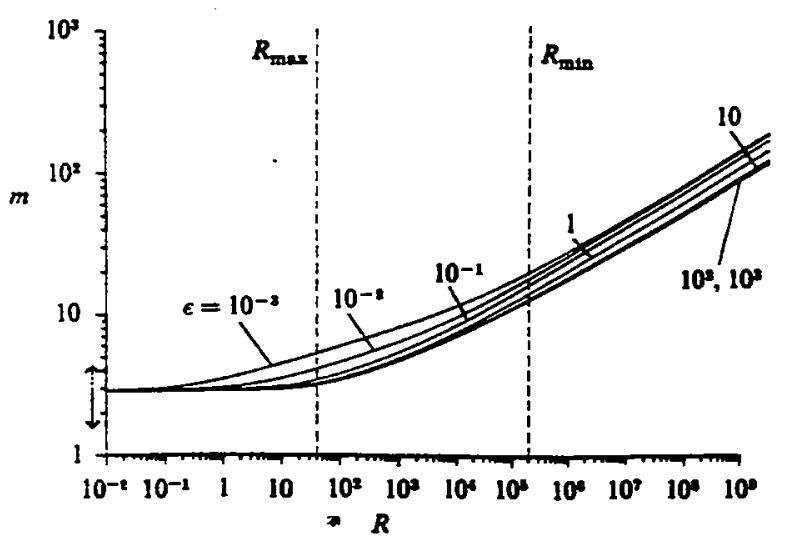

Figure 6: Fastest growing wavenumber vs. $\mathrm{R}$, various values of $\epsilon$ (Niino, 1986). 
proportional to $m^{2} S$, so when $\mathrm{S}$ decreases with increasing $\mu$ and fixed $N^{2}$, the time rate of change of buoyancy also decreases. Thus, the growth rate of disturbances decreases with increasing $\mu$. In fact, Niino's results recover the results of Toole and Georgi and of Ruddick and Turner in certain R limits. For $R<40(1+\mu)$, the limit of a narrow front, the vertical scale of the fastest growing mode is $\mathrm{d}$. This is the same scale that Ruddick and Turner observed. In the limit $R>2 \cdot 10^{5}(1+\mu)$, the scaling for a wide front is given by that used by Toole and Georgi.

Niino then goes on to compare the vertical scales of the FGM in his theory to those observed in the Ruddick/Turner experiments. Since Niino's theory assumes a vertical stratification in the saltfinger sense, his theory is not directly comparable to the experiments for which the fluid is initially stratified in the diffusive sense. Despite this fact, Niino boldly makes comparisons with all of the Ruddick/Turner experiments. It is a bit ironic that the best agreement between experiment and theory occurs in the case for which Niino's theory should not be valid, the diffusive case. Niino's theory predicts $m=3.4$, which jibes well with the experimentally observed $m=3.1$. In the salt-finger stratified case for which the theory is built, Niino's prediction is $m=7.9$, whereas the experiments showed $m=2.7$. There is a variety of possible explanations for the discrepancy. First, it is possible, even likely, that nonlinear effects play an important role in the evolution of the rolls to their final steady state. If nonlinearities are considered, it is quite reasonable that the wavelength might be modified by a factor of two or three. Another possible problem is that Niino assumed a constant eddy diffusivity $\kappa_{e}$. This is the Stern paramaterization (Stern, 1967), which assumes that the heat and salt fluxes are dominated by salt-finger interfaces. It is possible that diffusive interfaces would effectively cause $\kappa_{e}$ to vary in some regions. This latter consideration has received little theoretical attention, at least at the time of Niino's work. 
(a)

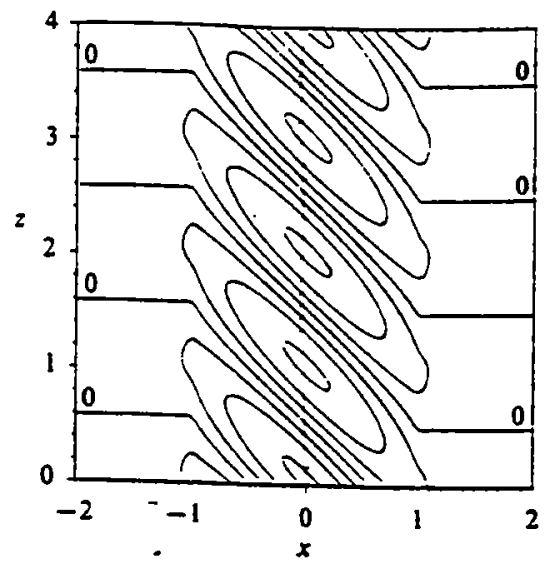

(c)

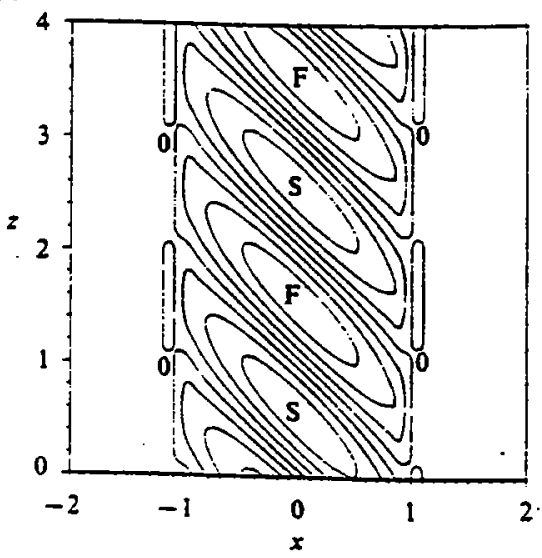

(e)

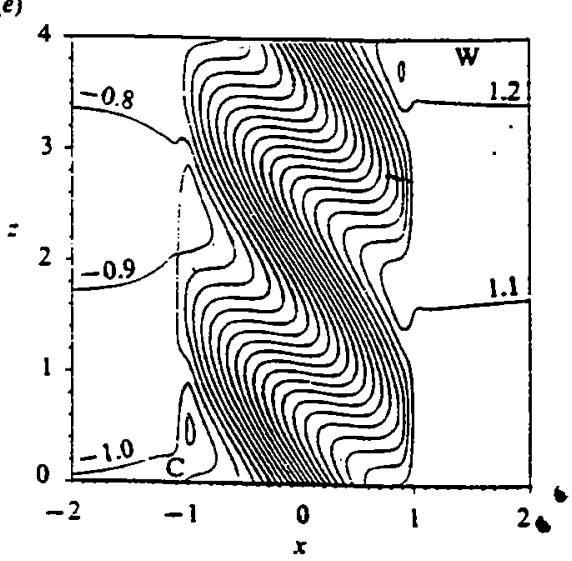

(b)

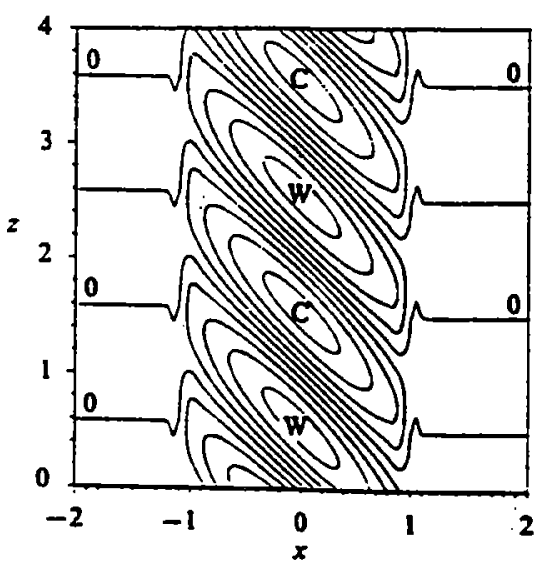

(d)

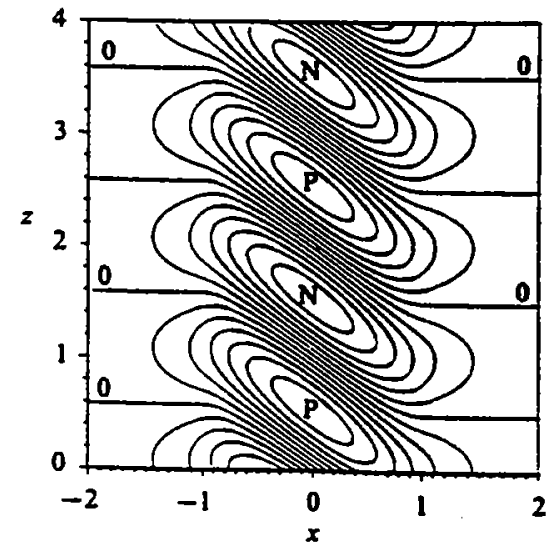

O

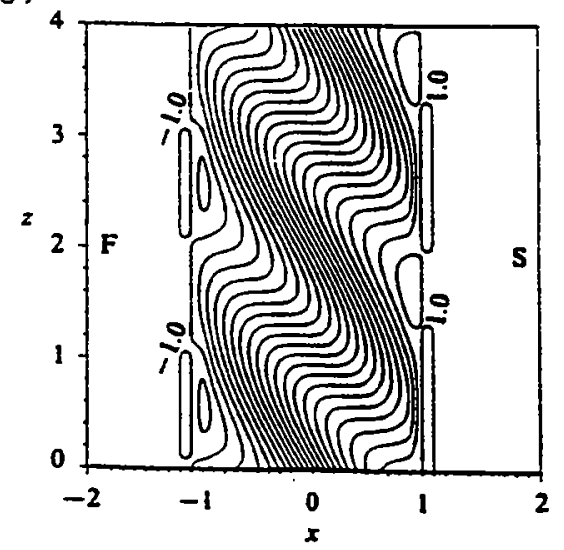

The stream function $(a)$, temperature $(b)$, salinity $(c)$ and buoyancy (d) fields of the fastest-growing mode for $\epsilon=2.0 . \mu=0, G=10^{+}$and $m=6.4$, and the corresponding total temperature $(t)$ and total salinity field $(f)$, in which the amplitude of the temperature perturbation is taken to be $) .4+\beta \Delta S / x$. The vertical coordinate is scaled by the wavelength $2 \pi / m$. Warm salty water is in the right-hand side. C. IF, F, S, N and $P$ stand for cold. warm, fregh. salty. negative and positive respectively.

Figure 7: Flow pattern for the frontal intrusions (Niino, 1986) 


\section{References}

Chen, C.F., Briggs, D.G., and Wirtz, R.A., (1971), "Stability of thermal convection in a salinity gradient due to lateral heating," Int.J.Heat.Mass. Transfer 14, pp.57-65.

Niino, H. (1986), “A linear stability theory of double-diffusive horizontal intrusions in a temperaturesalinity front," J.Fluid.Mech. 171, pp.71-100.

Ruddick, B.R. and Turner, J.S., (1979), "The vertical length scale of double diffusive intrusions," Deep-Sea Res. 26A, pp.747-753.

Shen, C.Y. (1989), "The Evolution of Double-Diffusive Instability: Salt Fingers," Phys.Fluids A 1 (5), pp.829-844

Shen, C.Y., and Veronis, G. (1996), "Numerical Simulation of Two-Dimensional Salt Fingers," submitted to Journal of Geophysical Research

Stern, M.E., (1960), "The "Salt-Fountain" and thermohaline convection," Tellus 12, pp.172-175.

Stern, M.E., (1967), “Lateral Mixing of Water Masses," Deep-Sea Res. 14, pp.747-753.

Toole,J.M., and Georgi, D.T., (1981), "On the Dynamics and Effects of Double-Diffusively Driven Intrusions," Prog.Oceanogr. 10, pp.121-145. 


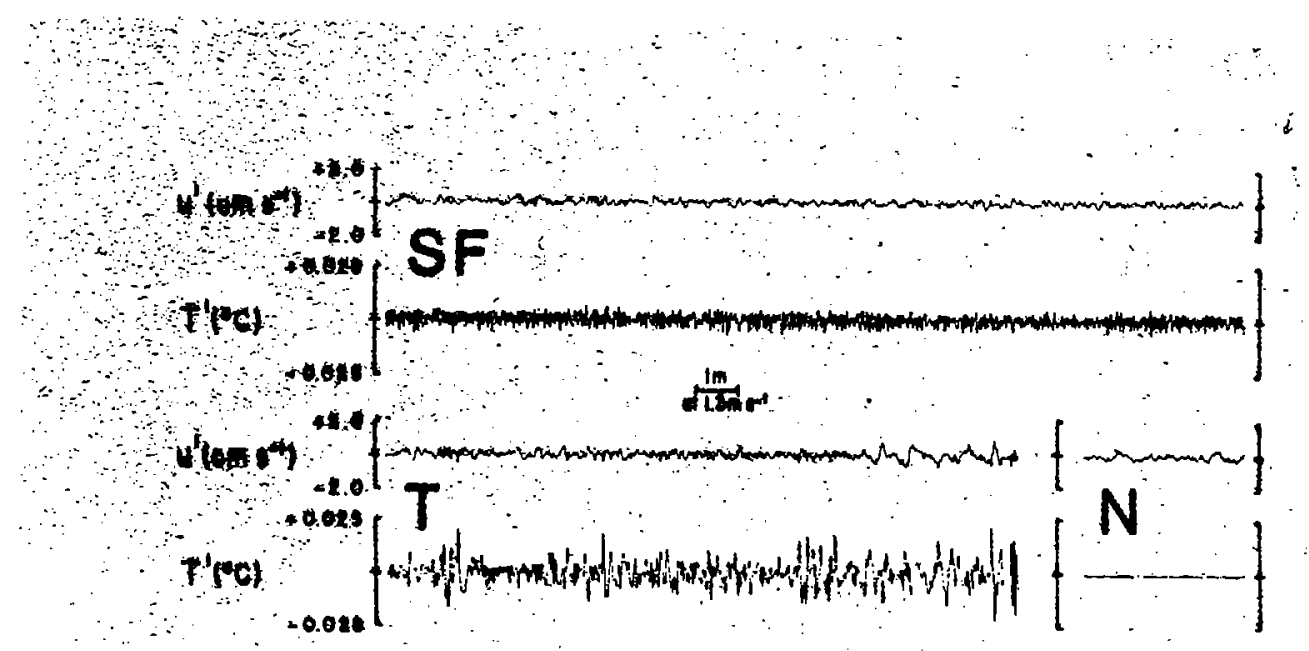

Figure 1: Small scale structure of the temperature field $(T)$ and the velocity field $\left(u^{\prime}\right)$.

\section{Microscale signatures of salt fingers in the ocean}

As well as producing staircases, we expect to be able to diagnose the presence of salt fingers by some other signatures. In Figure 1, we show high frequency measurements of temperature and velocity. The abscissa is the horizontal displacement.

The figure illustrates three different types of behavior: (SF) shows the salt finger behavior, where $T^{\prime}$ fluctuates rapidly with a rather constant amplitude. (T) is characteristic of a turbulent region, where $T^{\prime}$ now varies with a more variable amplitude. (N) just shows the natural background noise level.

We may also plot the amplitude of the temperature fluctuations as a function of the wavelength (see Figure 2). We then see that the observed largest temperature fluctuations have a wavelength which corresponds to the fingers developing under the fastest growing mode (calculated using the Stern(1960) model). Furthermore, it is even possible to reproduce the whole spectrum using a more refined model (Schmitt 1979).

Another illustration of the small scale structure is seen in detailed isotemperature curves (Figure 3) from a thermohaline staircase. These isotemperature curves are grouped together in the interfaces where the salt fingers are present, showing that the gradients there are the largest.

Furthermore, as may be seen from the bottom of the Figure, the variance in small scale temperature fluctuations increases whenever the microstructure probe enters an interface. When the spectra of small scale horizontal temperature gradients is calculated, it is found to have a slope of +2 at wavenumbers less than the fastest growing finger. This is in contrast to turbulence, which has a slope of 1 . 


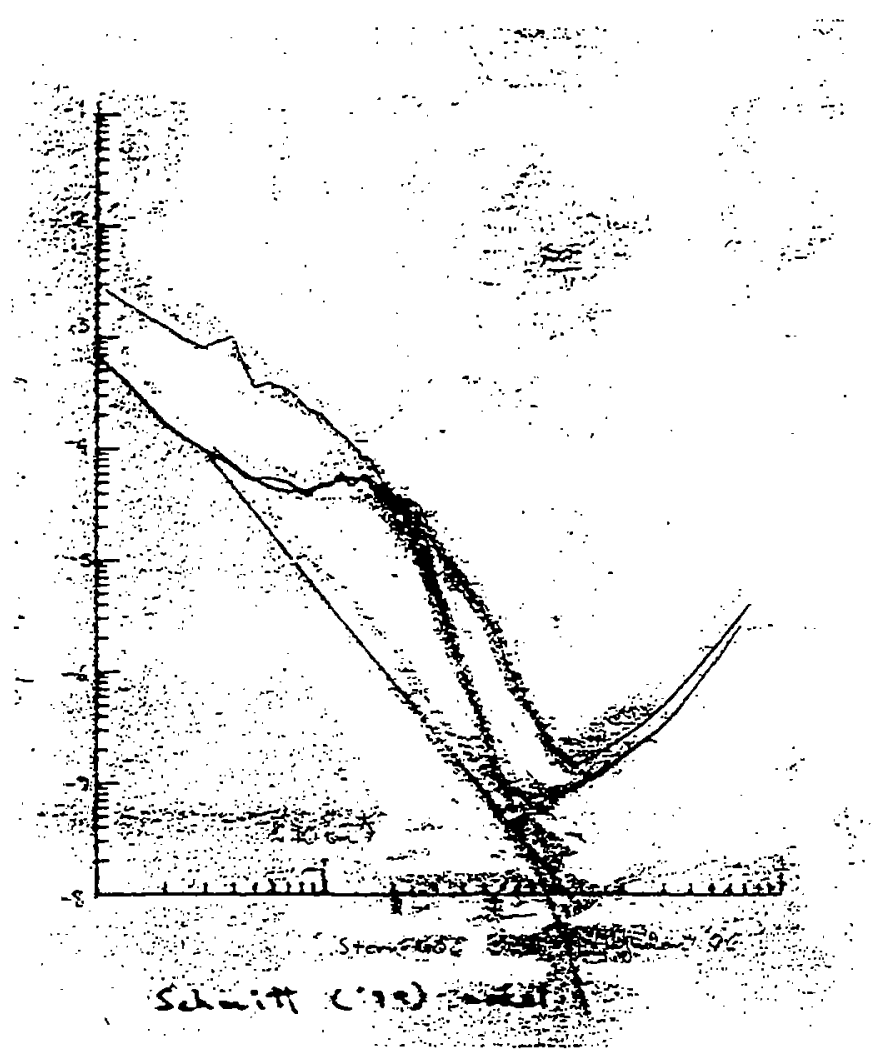

Figure 2: Amplitude of temperature fluctuations as a function of wavelength for salt fingers and turbulence. Superimposed on the salt finger curve is the spectrum obtained with the Schmitt(1979) model.

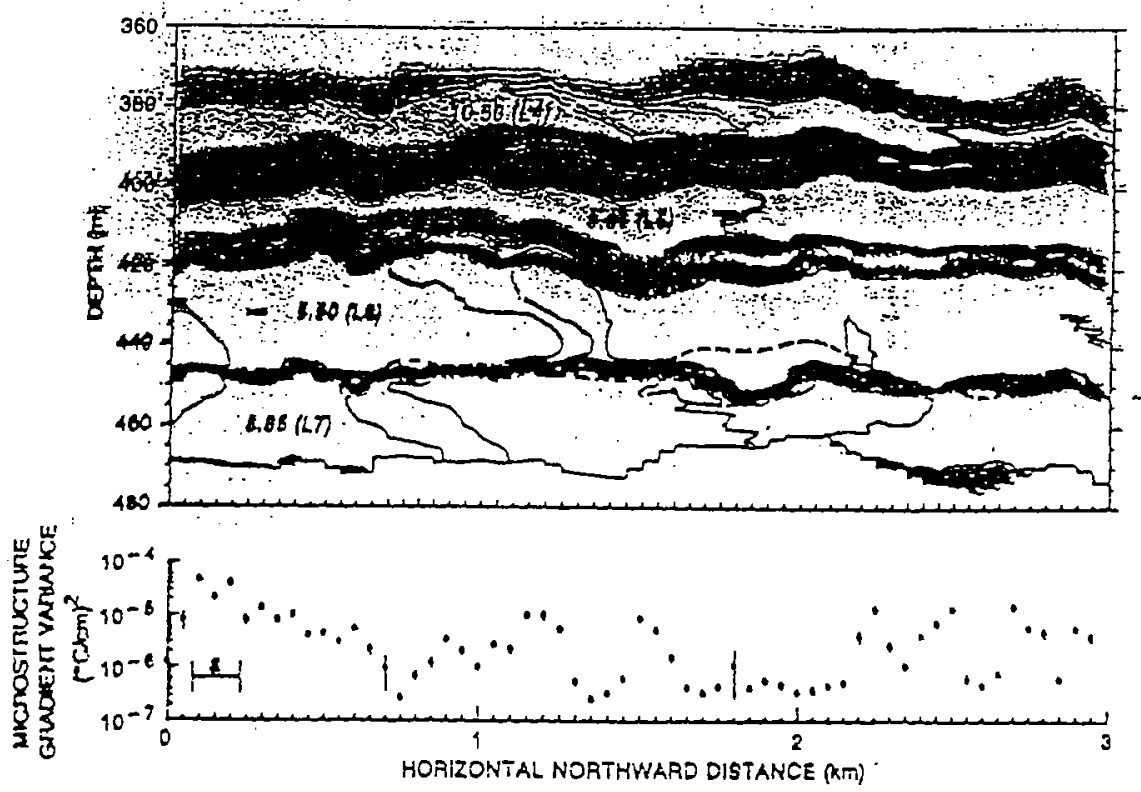

Figure 3: Staircase structure seen in iso-temperature curves. 


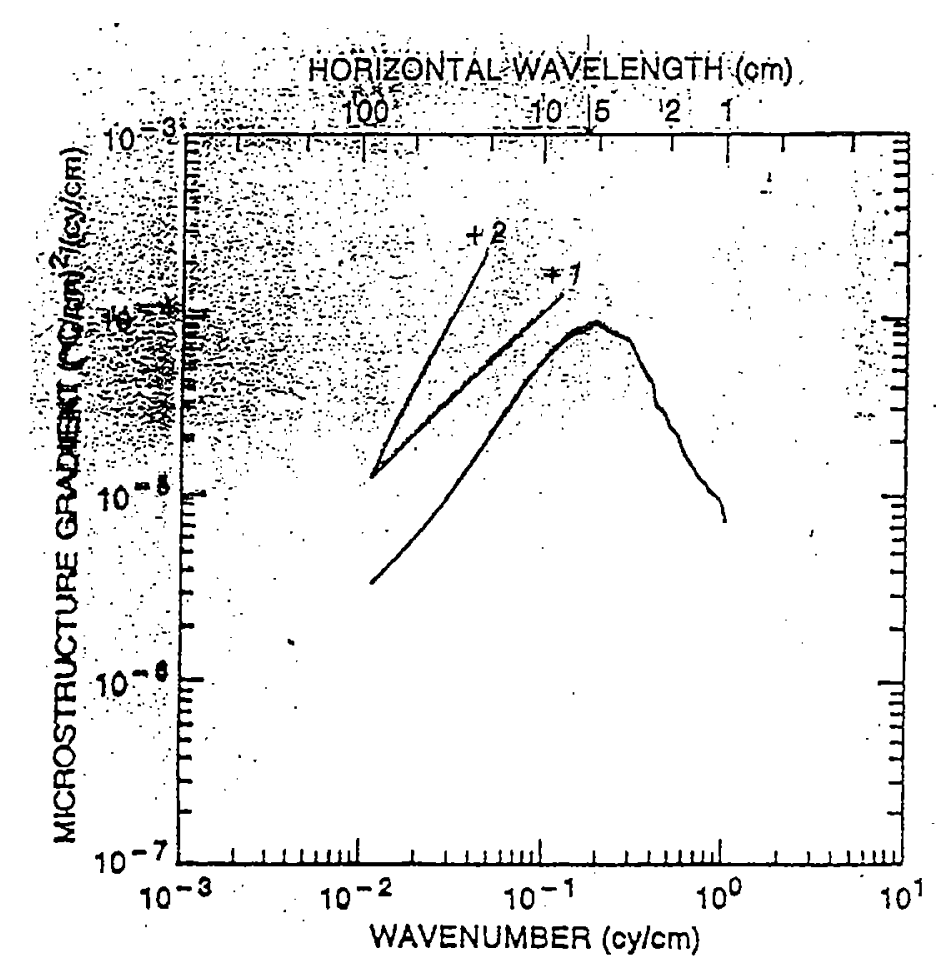

Figure 4: Microstructure gradient sectrum for salt fingers.

Another quantity which is useful for discriminating turbulence from salt fingers is the Kurtosis, which is defined as the normalized 4th moment of the variance:

$$
\text { Kurtosis }=\frac{\left\langle T^{\prime 4}\right\rangle}{\left\langle T^{\prime 2}\right\rangle^{2}}
$$

This quantity is low for salt fingers, since the small scale fluctuations are more even (Holloway and Gargett, 1987). The kurtosis is higher for turbulence. This is illustrated in Figure 5.

Mack and Schoeberlein (1993) use both Kurtosis and spectral slope to distinguish fingers (high slope, low Kurtosis) from turbulence (low slope, high Kurtosis).

We can illustrate the success of the model by overlaying a map of the small scale structure along with a map of the density ratios (see Figure 6).

On Figure 7, we see an histogram relating the number of observations of microscale turbulence as a function of density ratio. For $R_{p}$ between 1 and 3, we have a maximum, corresponding to the salt finger region. 

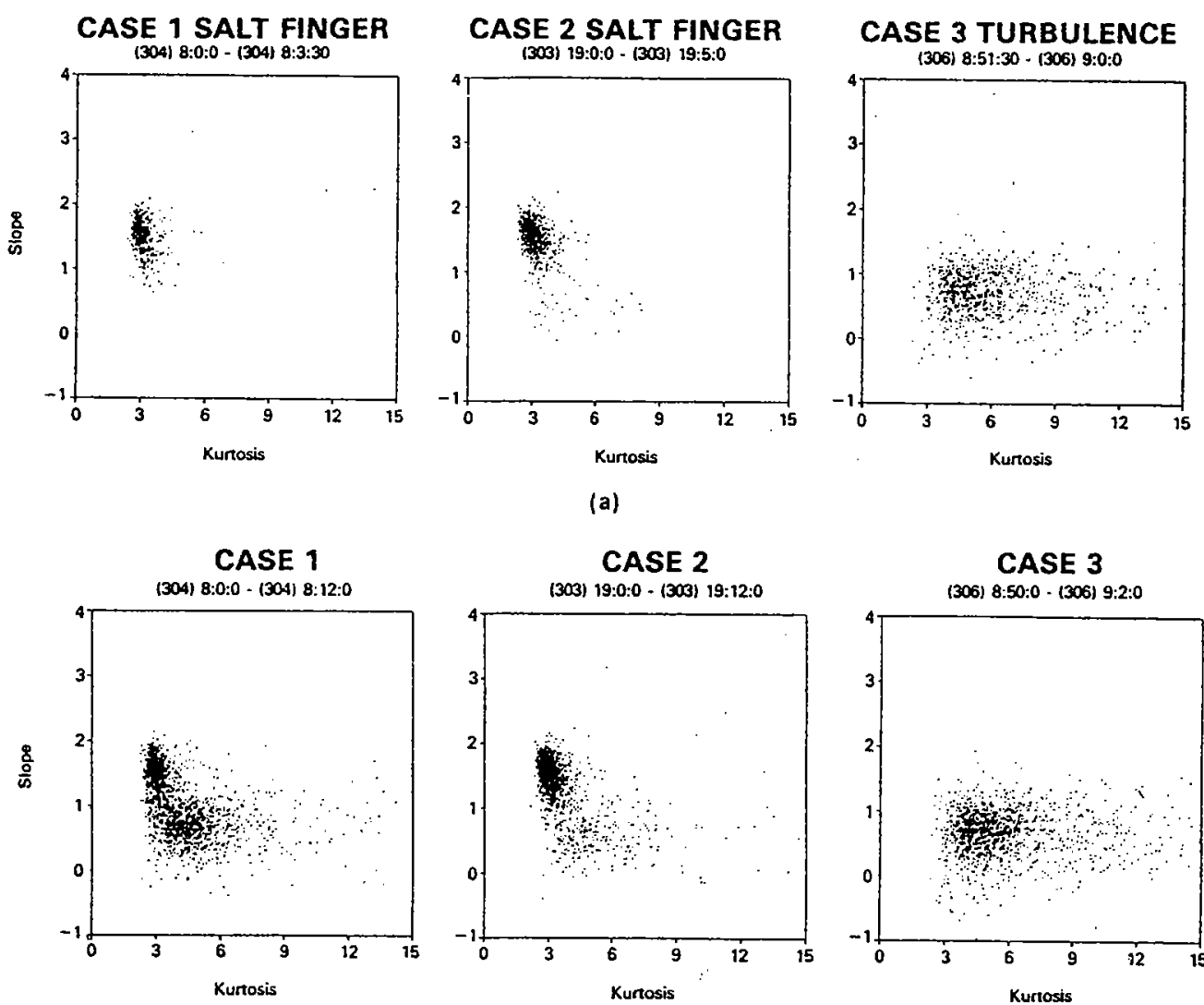

(a)
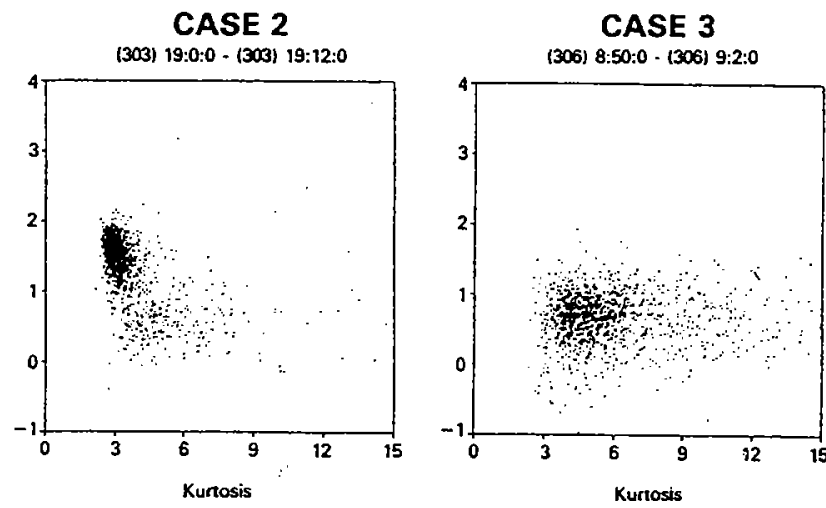

(b)

Figure 5: Measures of the Kurtosis for salt finger regions compared to a turbulent region.

\section{The Shen-Schmitt model}

In this new model (Shen and Schmitt 1995), we will use the height $(h)$ of the interface to determine the finger strength. However, we do not specify how the $h$ is fixed.

Consider the displacement $(\eta)$ of particles in a fingering interface of thickness $h$

$$
\eta(t)=\left(z-z_{0}\right) \phi(x, y)
$$

The finger velocity field is given by

$$
w^{\prime}=w_{0} e^{\lambda t} \phi(x, y)
$$

which also defines the particle speed

$$
\frac{\partial \eta}{\partial t}=w^{\prime}
$$

Integrating over time, we see that $w$ is proportional to the particle displacement

$$
\eta(t)=\frac{w_{0} e^{\lambda t}}{\lambda}
$$

or

$$
w^{\prime}=\lambda\left(z-z_{0}\right)
$$




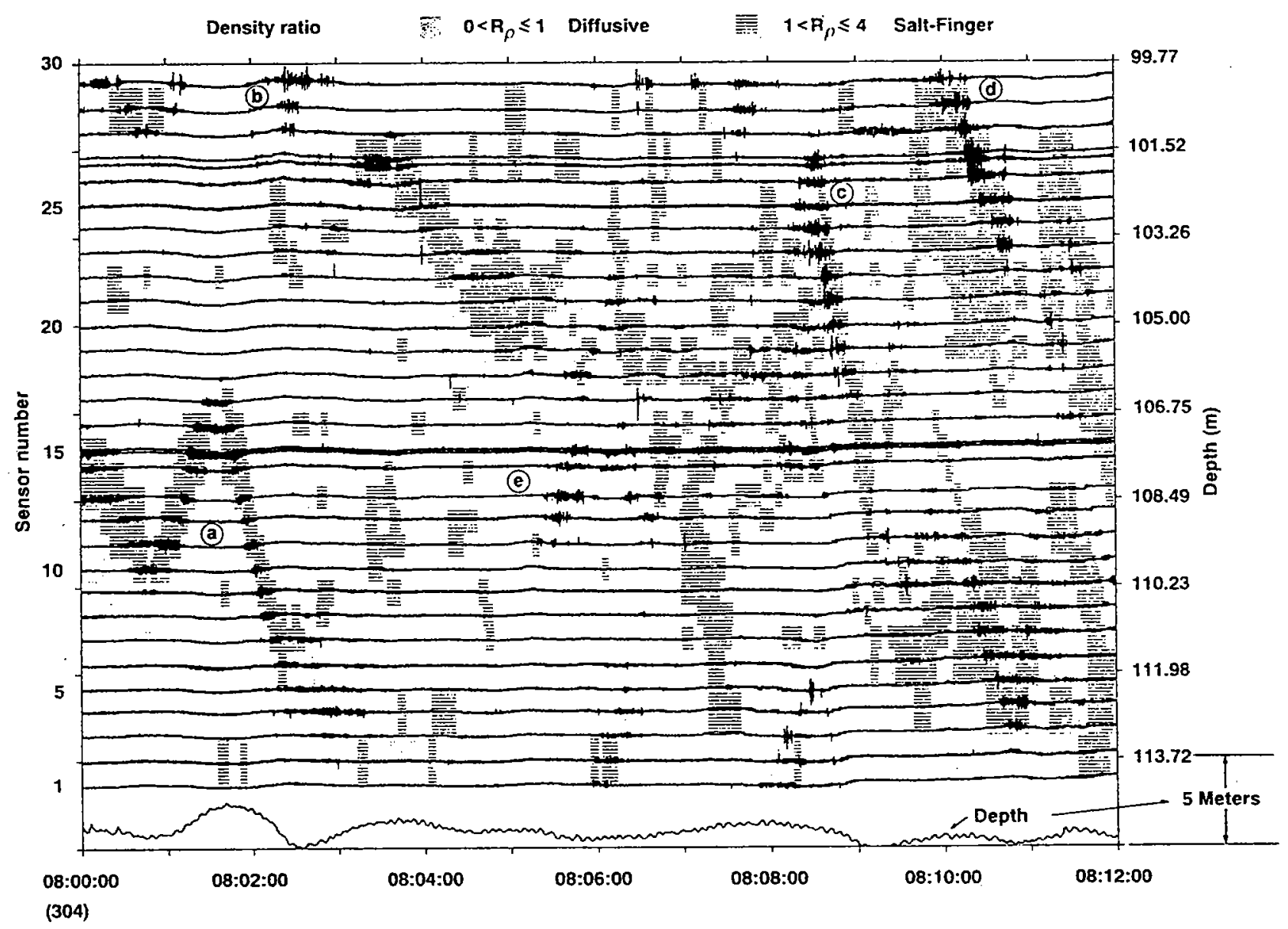

Figure 6: Small scale turbulence amplitude and density ratios as a function of depth and longitude from Mack and Schoeberlein (1993). 

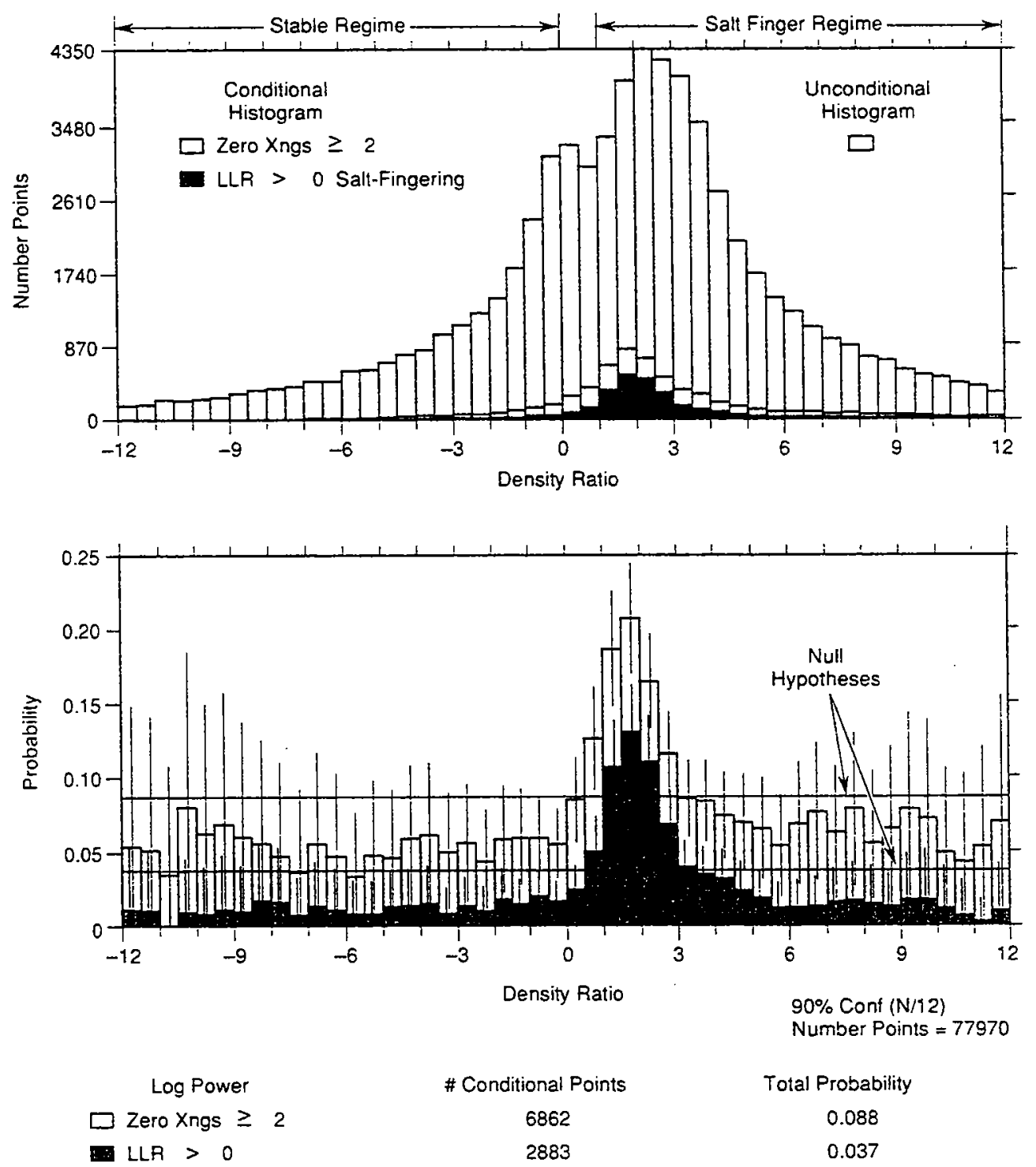

TOW 5 (304) 01:07

Figure 7: Density ratio histogram and microstructure probability from Mack and Schoeberlein (1993). 
Since maximum displacement is given by $h$, the maximum velocity is simply $\hat{w}=\lambda h$. This can be used in the heat equation to define the amplitude of the temperature perturbations within the fingers.

We now use the heat equation

$$
\frac{\partial T^{\prime}}{\partial t}+w^{\prime} \bar{T}_{z}=\kappa_{T} \nabla^{2} T^{\prime}
$$

with a solution of the form $T^{\prime}=\hat{T} e^{\lambda t} \phi(x, y)$ and we get

$$
\hat{T}\left(\lambda+\kappa_{T} m^{2}\right)=-\hat{w} \bar{T}_{z}
$$

where $m^{2}$ represents the total of the squares of the horizontal wavenumbers. Substituting the value of $\hat{w}$

$$
\hat{T} \simeq-\frac{\lambda h \bar{T}_{z}}{\left(\lambda+\kappa_{T} m^{2}\right)} \simeq-\frac{\lambda}{\left(\lambda+\kappa_{T} m^{2}\right)} \frac{\Delta T}{2}
$$

For a given salt finger dispersion relation $\lambda(m)$ we can then construct a salt finger wavenumber spectrum

$$
\hat{T}(m)^{2} \simeq\left(\frac{\lambda(m)}{\lambda(m)+\kappa_{T} m^{2}}\right)^{2} \frac{(\Delta T)^{2}}{4}
$$

Introducing the scaling

$$
m=\left(\frac{g \alpha \bar{T}_{z}}{\nu \kappa_{T}}\right)^{\frac{1}{4}} M \quad \text { and } \quad \lambda=\left(g \alpha \bar{T}_{z}\right)^{\frac{1}{2}} G
$$

where $M$ is now the non-dimensional wavenumber and $G$ the non-dimensional growth rate, and the definitions

$$
\gamma=\frac{\alpha T^{\prime}}{\beta S^{\prime}} ; R_{\rho}=\frac{\alpha \bar{T}_{z}}{\beta \bar{S}_{z}} ; \sigma=\frac{\nu}{\kappa_{T}} ; \tau=\frac{\kappa_{T}}{\kappa_{S}}
$$

for the heat to salt flux ratio $(\gamma)$, the density ratio $\left(R_{\rho}\right)$, the Prandtl number $(\sigma)$ and the ratio of diffusivities $(\tau)$.

The dispersion relation is then

$$
G(M)=\frac{M^{2}\left(\gamma-\frac{R_{\rho}}{\tau}\right)}{\sigma^{\frac{1}{2}}\left(R_{\rho}-\gamma\right)} .
$$

A good approximation (Schmitt 1994) is obtained by using 


$$
\gamma(M)=\frac{1}{2}\left[b-\left(b^{2}-4 R_{\rho}\right)^{\frac{1}{2}}\right] \text { with } \quad b=R_{\rho}\left(1+M^{4}\right)+1
$$

This yields a spectrum

$$
T^{\prime 2}(m) \simeq\left(\frac{\gamma(m)-\frac{R_{\rho}}{\tau}}{R_{\rho}}\right)^{2} \frac{(\Delta T)^{2}}{4}
$$

One can also use the dispersion relation of Stern(1975) (assuming $\kappa_{S} \ll \kappa_{T} \ll \nu$ ). In this case all wavenumbers grow and we get

$$
T^{\prime 2}(m) \simeq\left[\frac{1}{R_{\rho}\left(m^{2}+1\right)}\right]^{2} \frac{(\Delta T)^{2}}{4}
$$

i.e. we have a white spectrum until $M \simeq 1$. That corresponds to a gradient spectrum with a slope of +2 . It is found that the new model agrees well with results from numerical simulations, laboratory experiments and oceanic observations.

\section{Models Of Mixing}

Vertical profiling techniques allow estimates of the dissipation rates of turbulent kinetic energy $(\epsilon)$ and thermal variance $(\chi)$ to be made. These measurements can be combined with information on background gradients to provide estimates of the vertical eddy diffusivity.

In the case of ordinary turbulence, such as that caused by breaking internal waves, the effective vertical eddy diffusivity is given by the relations

$$
\kappa_{T}=\frac{\chi}{2\left(\bar{T}_{z}\right)^{2}}
$$

due to Osborn and Cox (1972) and

$$
\kappa_{\rho}=\left[\frac{R_{f}}{\left(1-R_{f}\right)}\right] \frac{\epsilon}{N^{2}}=\Gamma^{t} \frac{\epsilon}{N^{2}}
$$

due to Osborn (1980), where $R_{f}$ is the effiency of conversion of kinetic to potential energy, and $\Gamma^{t}$ is the 'scaled dissipation ratio' or 'mixing efficiency' of turbulence. Laboratory data suggest that $R_{f}$ is about $0.15-0.2$ which gives an expected $\Gamma^{t}$ of about $0.18-0.25$. 
For salt fingers, the relation between diffusivity and $\epsilon / N^{2}$ is much different. McDougall (1988), Schmitt(1988), and Hamilton et al $(1989,1993)$ point out that the appropriate relation for salt fingers is

$$
\kappa_{s}=\Gamma^{f} \frac{\epsilon}{N^{2}}
$$

where $\Gamma^{f}=\frac{\gamma\left(R_{\rho}-1\right)}{R_{\rho}(1-\gamma)}, r$ is the flux ratio and $R_{\rho}$ is the density ratio.

This expression can give a diffusivity over ten times the Osborn formula for the same dissipation rate due to the high efficiency of salt fingers in converting haline to thermal potential energy. Turbulence on the other hand dissipates most of its kinetic energy, converting only a small fraction to potential energy.

The above relation is very sensitive to the value of the flux ratio, leading to some uncertainty in the effective diffusivity expected in salt finger regions. This is due in part to the differences in theoretical models, and also because $\Gamma^{f}$ is a particularly difficult variable to estimate: There are questions about the isotropy of microstructure, both $\chi$ and $\epsilon$ have non-Gaussian distributions, and there is much internal variability to contend with. Recent measurements with the High Resolution Profiler (Schmitt et al 1989) show a variation of observed $\Gamma$ in Richardson number and density ratio space which suggests that salt fingers dominate the mixing at high Richardson number and low density ratio. Turbulence tends to dominate when the Richardson number is low, regardless of $R_{\rho}$.

\section{References}

[1] Hamilton, J.M., Lewis, M.R., Ruddick, B.R., Vetical fluxex of nitrate associated with salt fingers in the world's oceans, J. Geophys. Res., 94(C2), 2137, 1989

[2] Hamilton, J.M., Oakey, N.S., Kelley, D.E., Salt finger signatures in micro-structure measurements, J. Geophys. Res., 98(C2), 2453, 1993

[3] Mack, S.A.,\& Schoeberlein, H.C., Discriminating salt fingering from turbulenceinduced microstructure: Analysis of towed temperature-conductivity chain data, J. Phys. Oceanogr., 23, 2073, 1993

[4] McDougall, T.J., Small-scale Turbulence and Mixing in the Ocean, Some implications of ocean mixing for ocean modelling, Elsevier Oceanogr. Ser. Vol. 46, p.21, New York, Elsevier, 1988

[5] Osborn, T. \& Cox, C.S., Oceanic fine structure, Geophys. Fluid Dyn. 3, 321, 1972 
[6] Osborn, T.R., Estimates of the local rate of vertical diffusion from dissipation measurements, J. Phys. Ocean. 10, 83, 1980

[7] Schmitt, R.W., The growth rate of supercritical salt fingers, Deep Sea Research, 26A, 23,1979

[8] Schmitt, R.W., Small-scale Turbulence and Mixing in the Ocean, Mixing in a thermohaline staircase, Elsevier Oceanogr. Ser. Vol. 46, p.435, New York, Elsevier, 1988

[9] Schmitt, R.W., Double-diffusion in oceanography, Annual Review of Fluid Mechanics, $26,255,1994$

[10] Stern, M.E., The salt-fountain and thermhaline convection, Tellus, 12, 172, 1960

[11] Stern, M.E., Ocean circulation physics, 246 pp., Academic, New York, 1975 


\title{
Limits on Growing, Finite-Length Fingers: A Gradient Froude Number Constraint
}

\author{
Eric Kunze
}

\section{Introduction}

The role of salt-finger fluxes is crucial to ocean-mixing and water-mass modification. The C-SALT experiment showed that those fluxes are 30 times smaller than the laboratory $\Delta S^{4 / 3}$ flux law predictions.

A new model for fastest-growing fingers is formulated to try to reconcile the predictions of the $\Delta S^{4 / 3}$ flux law with the recent dissipation measurements. We shall assume that the fastest-growing fingers dominate the fluxes, that the fingers grow in a background gradient of salinity and temperature (and not on a thin interface, like in most of the laboratory experiments), and that the finger tips are not important to fluxes. In our model, a finger gradient Froude number constraint $F r_{f}=\frac{|\nabla w|}{N} \leq 2$ is applied to fastest-growing fingers. This constraint is identical to Stern's collective instability criterion. For typical ocean staircase interface thicknesses, the gradient Froude number constraint leads to fluxes well below the $\Delta S^{4 / 3}$ parameterization predictions. The $\Delta S^{4 / 3}$ flux law can be reproduced if the maximum finger length is identical to the interface thickness.

The expressions developed here are cast in terms of readily-measured quantities. Numerical examples use values (summarized in Table 1) typical of thermohaline staircases east of Barbados for density ratio $R_{p}(1.6)$, salinity step $\Delta S(0.1 \%)$, and interface thickness $l_{i}(2 \mathrm{~m})$. This interface thickness is much larger than what would be extrapolated from laboratory studies, but is not atypical of the ocean.

\section{The equations of motion}

The basic features of the model are sketched in Figure 1. Figure 1a shows a vertical slice through a fingering zone. Figure $1 \mathrm{~b}$ displays temperature and salinity profiles through upand down-going fingers as well as the contrast between fingers. Sinusoidal horizontal structure is assumed (Figure 1c), either in square planform $\sin \left(k_{x} x\right) \sin \left(k_{y} y\right)$ corresponding to 

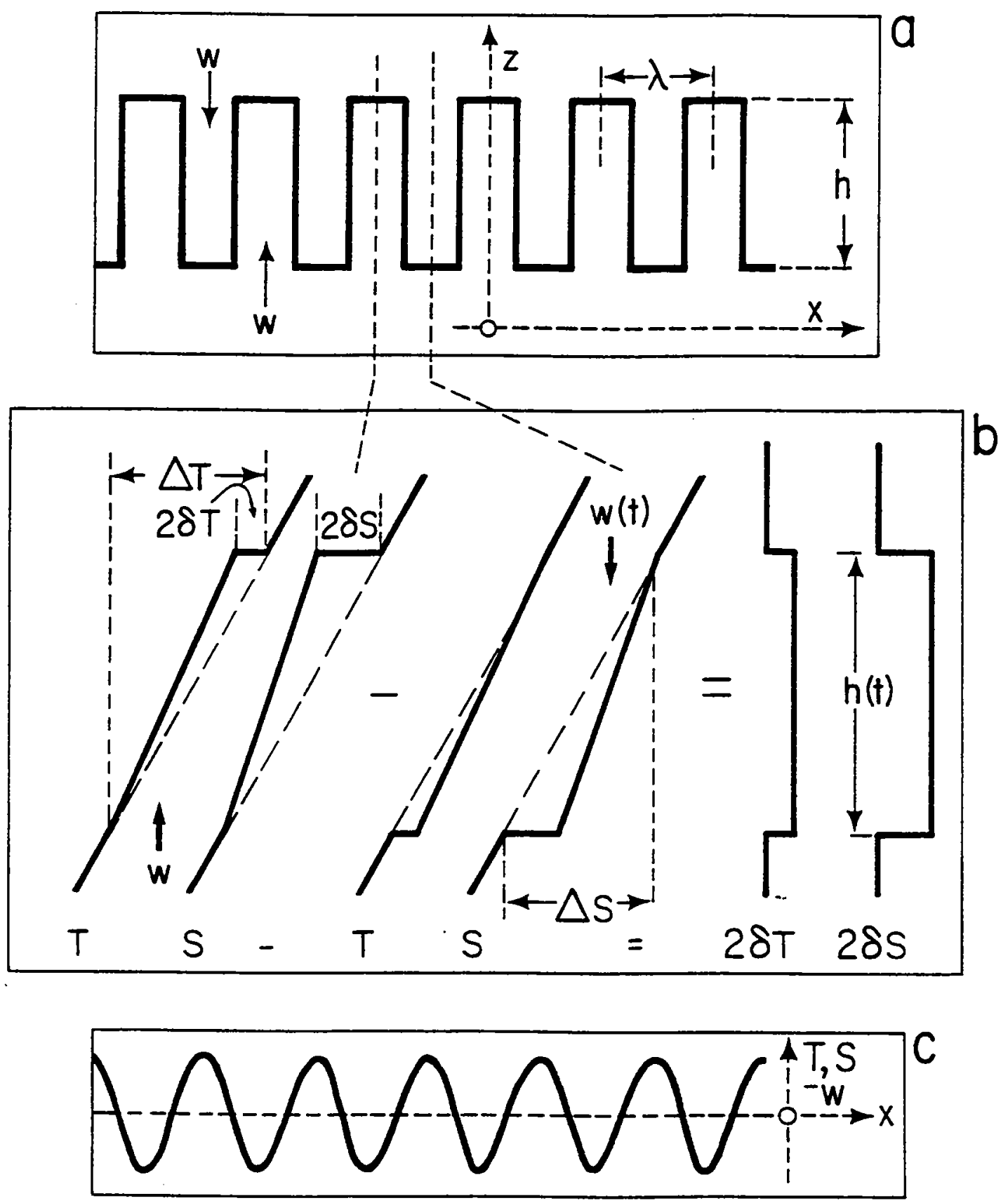

Figure 1: A schematic describing the major features of the growing finger model 


\begin{tabular}{ll}
\multicolumn{1}{c}{ Variable } & \multicolumn{1}{c}{ Value } \\
$\nu$ & $10^{-6} \mathrm{~m}^{2} / \mathrm{s}$ \\
$\kappa_{T}$ & $1.4 \times 10^{-7} \mathrm{~m}^{2} / \mathrm{s}$ \\
$\kappa_{S}$ & $1.1 \times 10^{-9} \mathrm{~m}^{2} / \mathrm{s}$ \\
$g$ & $9.8 \mathrm{~m} / \mathrm{s}^{2}$ \\
$\alpha$ & $2 \times 10^{-4} \circ \mathrm{C}^{-1}$ \\
$\beta$ & $7.5 \times 10^{-4} \% 0^{-1}$ \\
$L_{o}$ & $20 \mathrm{~m}$ \\
$l_{i}$ & $2 \mathrm{~m}$ \\
$\bar{T}_{z}$ & $0.3{ }^{\circ} \mathrm{C} / \mathrm{m}$ \\
$\bar{S}_{z}$ & $0.05 \% / \mathrm{m}$ \\
$N$ & $1.5 \times 10^{-2} / \mathrm{s}$ \\
$P_{N}=2 \pi / N$ & $7 \mathrm{~min}$ \\
$R_{\rho}$ & 1.6 \\
$\lambda_{H f_{s}}$ & $3.1 \mathrm{~cm}$ \\
$U_{z}$ & $6.3 \times 10^{-3} / \mathrm{s}$ \\
$f$ & $3.5 \times 10^{-5} / \mathrm{s}$ \\
$P_{f}=2 \pi / f$ & $2 \mathrm{days}$ \\
$R i$ & 6
\end{tabular}

Table 1: Parameter values used for figures. Values are typical of the thermohaline staircases east of Barbados.

unsheared conditions, or in vertical sheets $\sin \left(k_{x} x+k_{y} y\right)$ aligned to the internal wave shear $\frac{\partial U}{\partial z}$.

Mathematical expressions for temperature and salinity reflecting the above descriptions are:

$$
\begin{aligned}
& T=T_{0}+\frac{\partial T}{\partial z} z+\delta T(x, y, t) \\
& S=S_{0}+\frac{\partial S}{\partial z} z+\delta S(x, y, t)
\end{aligned}
$$

where $\delta T$ and $\delta S$ are the contrasts between adjacent columns relative to the horizontal average. The equation of motions for time-varying salt fingers are:

$$
\begin{array}{r}
\frac{\partial w}{\partial t}-\nu \nabla^{2} w=b=g(\alpha \delta T-\beta \delta S) \\
\frac{\partial \delta T}{\partial t}-\kappa_{T} \nabla^{2} \delta T+w \frac{\partial T}{\partial z}=0 \\
\frac{\partial \delta S}{\partial t}-\kappa_{S} \nabla^{2} \delta S+w \frac{\partial S}{\partial z}=0
\end{array}
$$

where $\nu>>\kappa_{T}>>\kappa_{S}$ (which implies $\sigma<<N<<k^{2}$ ) and $\nabla^{2} \approx \frac{\partial^{2}}{\partial x^{2}}+\frac{\partial^{2}}{\partial y^{2}}$ for tall, narrow fingers. Continuity implies that finger length $h$ and vertical velocity $w$ are related by

$$
w=\frac{\sigma h}{2}
$$




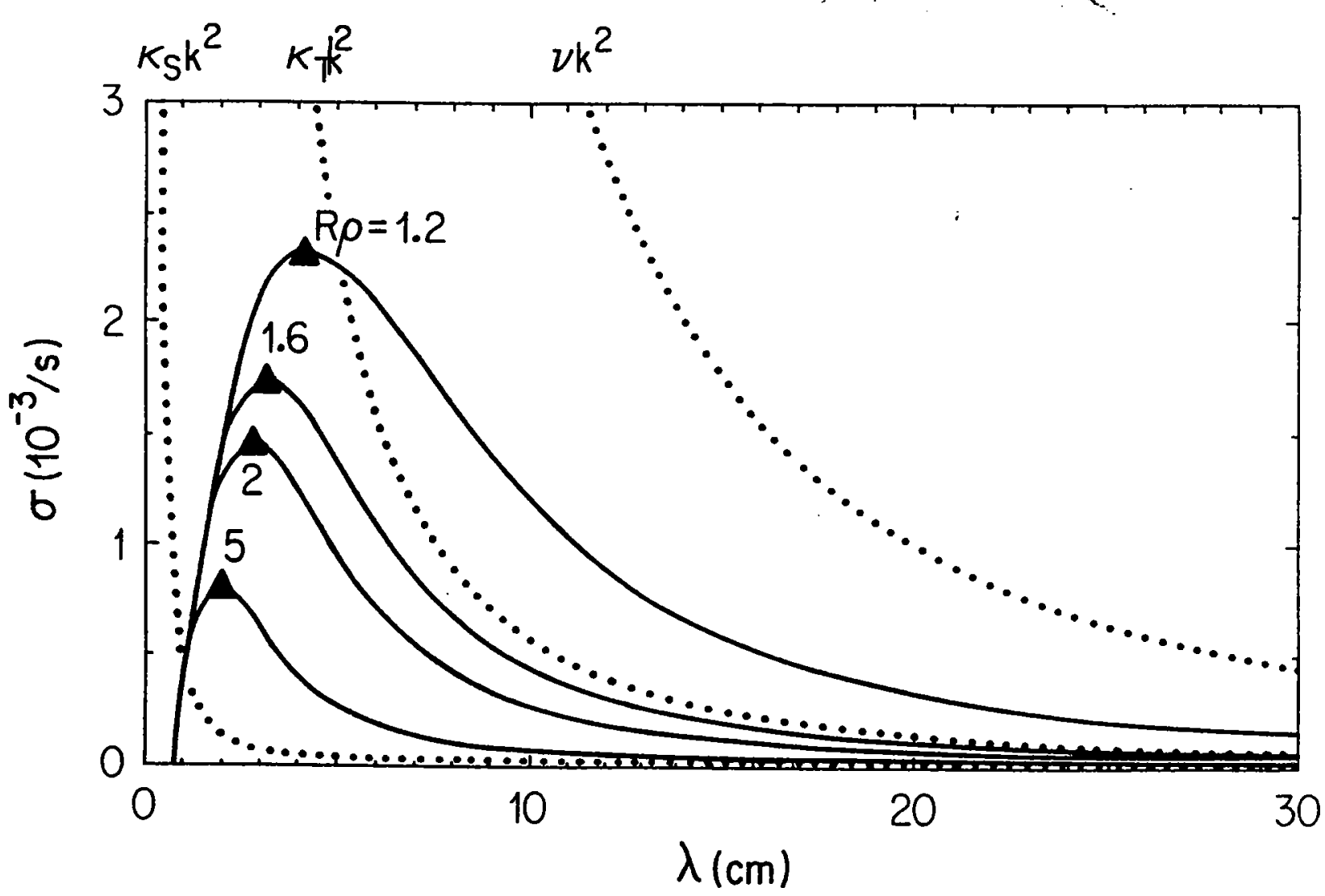

Figure 2: Growth rate $\sigma$ vs. horizontal wavelength $\lambda=2 \pi / k$ for four different values of $\boldsymbol{R}_{\boldsymbol{p}}$. The triangles indicate the maximum growth rate at its corresponding wavelength. The dotted curves correspond to molecular decay scales $\nu k^{2}, \kappa_{T} k^{2}$, and $\kappa_{S} k^{2}$.

where $\sigma$ is the growth rate of the fingers. From the geometry of Figure $1 \mathrm{~b}$ the contrasts between adjacent fingers are given by:

$$
\begin{aligned}
& \delta T=-\frac{\bar{T}_{z} h \delta_{T}}{2} \\
& \delta S=-\frac{\bar{S}_{z} h \delta_{S}}{2}
\end{aligned}
$$

Within the fingers the gradients are diminished because of the contrast:

$$
\begin{gathered}
\delta T=\bar{T}_{z}\left(1-\delta_{T}\right) \\
\delta S=\bar{S}_{z}\left(1-\delta_{S}\right)
\end{gathered}
$$

\section{Fastest-growing fingers}

We now focus on the fastest-growing fingers which will ultimately dominate the field. The growth rate is plotted versus wavelength $\lambda$ in Figure 2 at several density ratios. The wave number band of large growth rate is very narrow, so considering only fastest-growing fingers is justified. Maximum growth occurs for

$$
k_{M A X}^{4} \approx \frac{g \beta \bar{S}_{z}}{\nu \kappa_{T}}\left(R_{\rho}-1\right)=\frac{N_{0}^{2}}{\nu \kappa_{T}}
$$




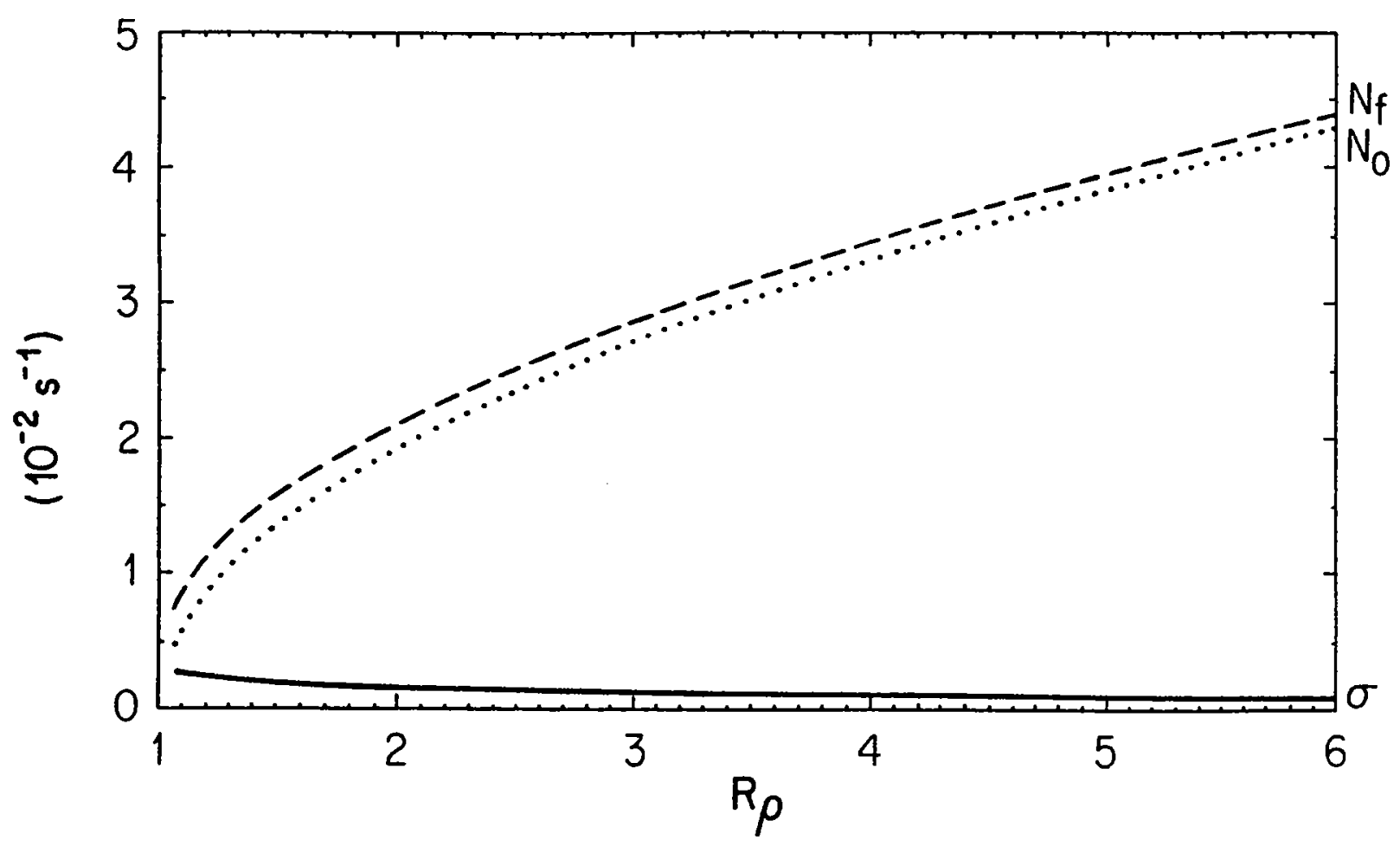

Figure 3: Maximum growth rate $\sigma_{M A X}$, unperturbed buoyancy frequency $N_{0}$ and finger buoyancy frequency $N_{f}$ vs. density ratio for parameter values typical of the staircase east of Barbados.

where $N_{0}$ is the unperturbed buoyancy frequency and $R_{p}=\frac{\alpha \bar{T}_{x}}{\beta \bar{S}_{z}}$. As $R_{p}$ increases the wavelength decreases because more diffusion is needed to produce the buoyancy required to drive fingering at higher $N_{0}$.

The maximum growth rate is plotted in Figure 3 and is given by

$$
\sigma_{M A X} \approx \frac{1}{2} \sqrt{\frac{\left(\kappa_{T}-R_{\rho} \kappa_{S}\right) g \beta \bar{S}_{z}}{\nu}}\left(\sqrt{R_{\rho}}-\sqrt{R_{\rho}-1}\right)
$$

which decreases with increasing $R_{\rho}$ because the stronger temperature gradients at high $R_{\rho}$ retard acceleration. Also plotted in Figure 3 are the buoyancy frequencies of the background, $N_{0}$, and inside the fingers, $N_{f}$. The growth rate $\sigma$ is smaller than either the buoyancy frequency by a factor of $2-3$ at $R_{\rho}=1.1$, a factor of $\approx 12$ at $R_{\rho}=2$.

The relative temperature and salinity contrast for the fastest growing fingers are functions only of $R_{\rho}$ (Figure 4). The temperature contrast $\delta_{T}$ decreases with density ratio because the smaller wavelengths at higher $R_{p}$ allow more molecular diffusion. Because its molecular diffusivity is so much lower, salinity is virtually unaffected; it remains approximately constant at $\frac{1}{2}$.

The ratio of heat flux to the salt flux is given by

$$
R_{F} \approx \sqrt{R_{\rho}}\left(\sqrt{R_{\rho}}-\sqrt{R_{\rho}}-1\right)
$$

In Figure 4 the flux ratio's dependence on density ratio is compared to various laboratory estimates. Over the range $R_{p}=1$ to 6 shown, the model agrees with the laboratory ex- 


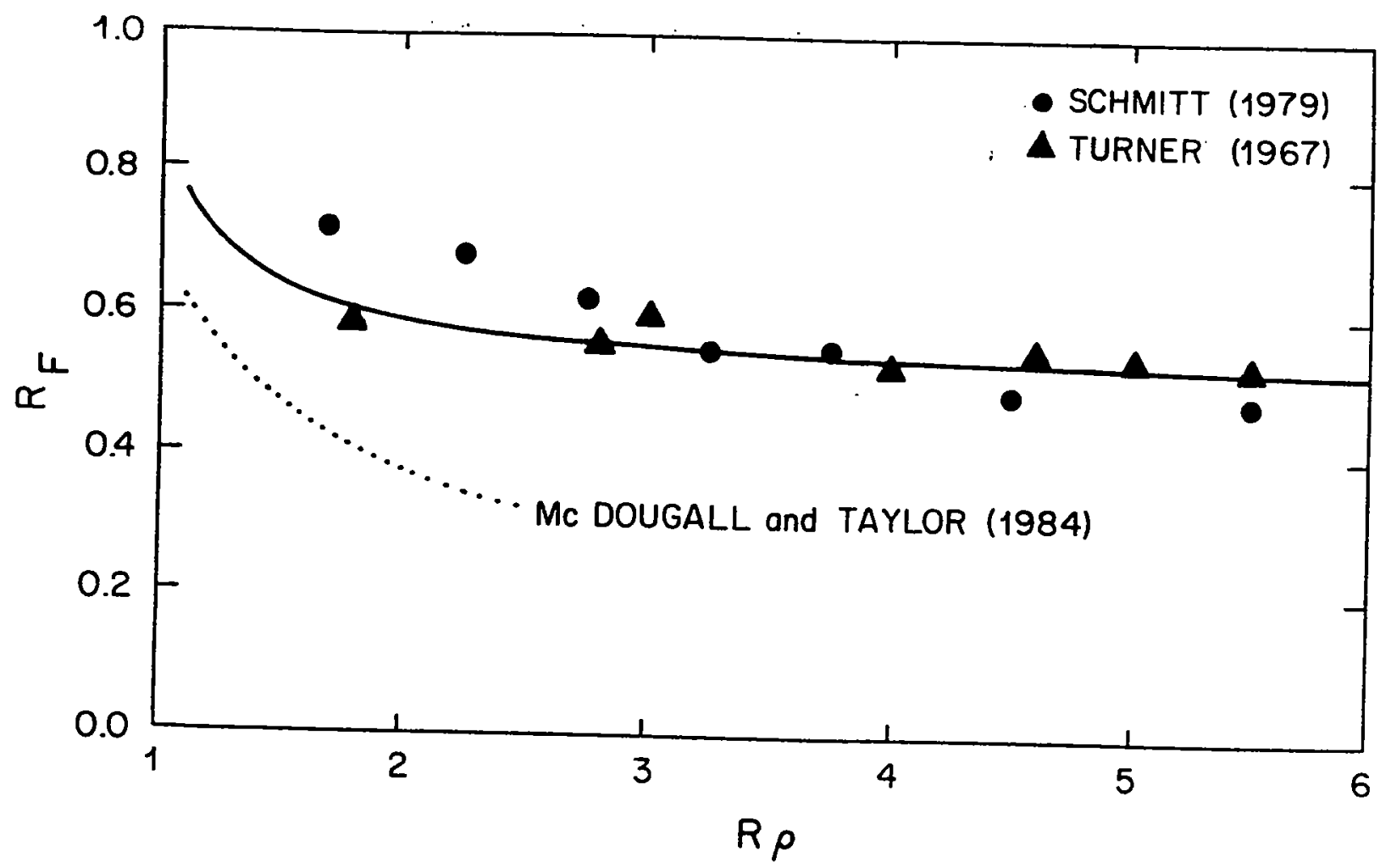

Figure 4: A comparison of the flux ratio's dependence on density ratio $R_{\rho}$ for the fastestgrowing model with various laboratory estimates.

periments of Turner (1967) and Schmitt (1979a), but not those of McDougall and Taylor (1984).

\section{The finger gradient Froude number}

The finger height is unbounded in a purely linear theory, so we need a supplementary constraint to limit their growth. Larger vertical velocities occur in longer fingers. Therefore, the shear between adjacent fingers will disrupt those fingers which have grown beyond a certain critical height. Comparing the vertical shear $|\nabla w|$ with the bouyancy frequency $N$, we introduce the finger gradient Froude number

$$
F r_{f}=\frac{|\nabla w|}{N}
$$

We shall assume that the fingers cannot grow above a critical finger Froude number $F r_{f}=2$. For critical $F r_{f}$ the maximum finger length is (Figure 5)

$$
h_{M A X}=\frac{2 \sqrt{2} C_{w}^{-1 / 2} F_{f} \nu^{3 / 4}}{\left(\kappa_{T} g \beta \bar{S}_{z}\right)^{1 / 4}}\left(\sqrt{R_{\rho}}+\sqrt{R_{\rho}-1}\right)^{3 / 2}
$$

The coefficient $C_{w}$ depends on the horizontal structure of $w(1 / 2$ for single sinusoid and $1 / 4$ for a double sinusoid). For typical density ratios $(<2)$ this is approximately $30 \mathrm{~cm}$. 


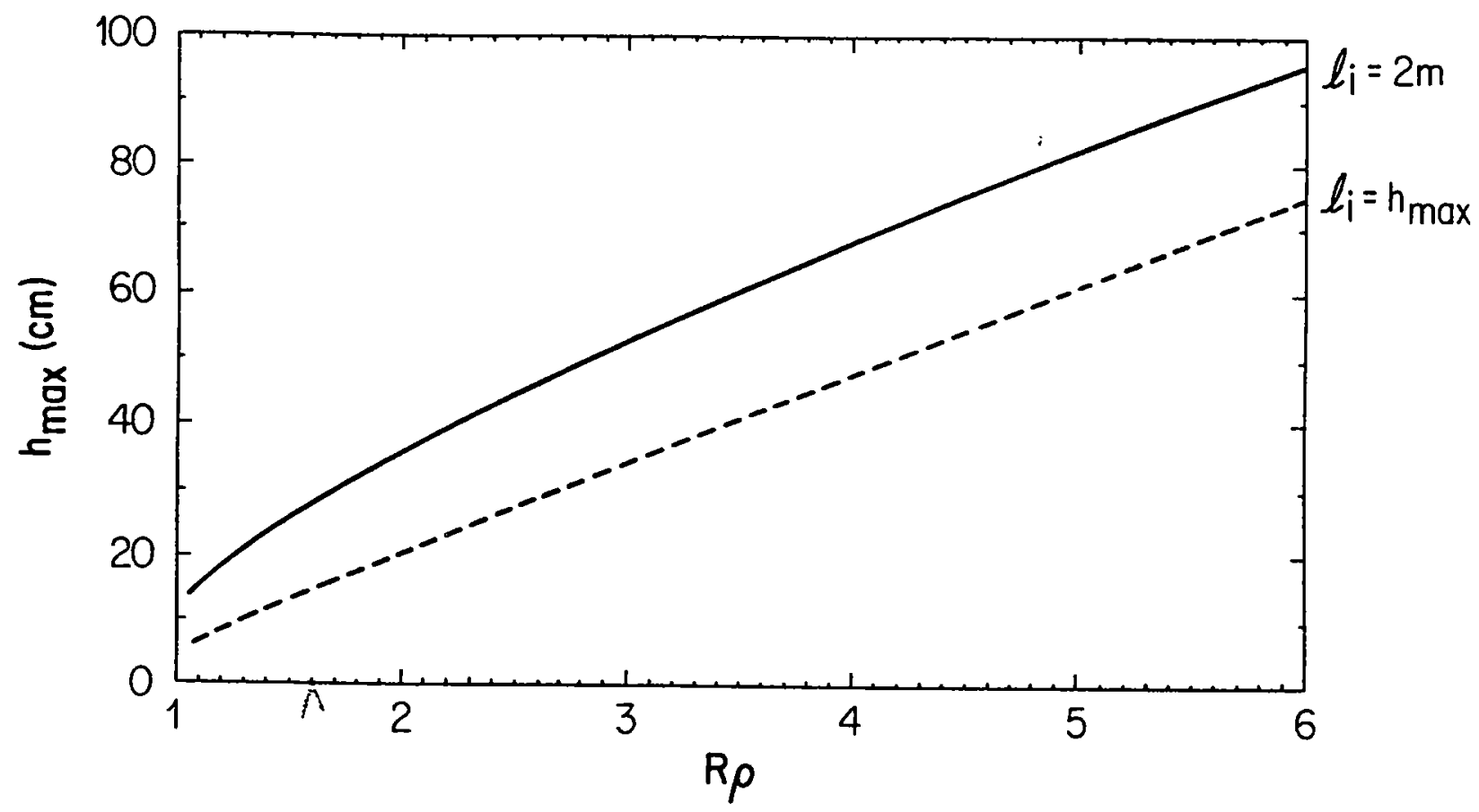

Figure 5: Maximum finger length $h_{M A X}$ plotted against density ratio for fingers growing within an interface with salinity step $\Delta S=0.1 \%, F r_{f}=2$. The solid curve corresponds to an interface $2 \mathrm{~m}$ thick $\left(l_{i}=2 \mathrm{~m}\right)$ so that the backround salinity gradient $\bar{S}_{z}$ is $0.05 \% / \mathrm{m}$. For the dashed curve, the fingers are assumed to fill the interface, that is, $l_{i}=h_{M A X}$.

\section{Fluxes}

The average fluxes are given by

$$
\begin{gathered}
g \alpha\left\langle F_{T}\right\rangle=I \frac{\sqrt{C_{T}} f r_{c}^{2}}{2 \sqrt{C_{w}}} \nu g \beta \bar{S}_{z}\left[\sqrt{R_{\rho}}\left(\sqrt{R_{\rho}}+\sqrt{R_{\rho}-1}\right)\right] \\
g \beta\left\langle F_{T}\right\rangle=I \frac{\sqrt{C_{T}} f r_{c}^{2}}{2 \sqrt{C_{w}}} \nu g \beta \bar{S}_{z}\left(\sqrt{R_{\rho}}+\sqrt{R_{\rho}-1}\right)^{2}
\end{gathered}
$$

where the time-average integral

$$
I^{-1}=2 \sigma t_{M A X}=2 \ln \left(k h_{M A X}\right)=\ln \left[\frac{8 \nu F r_{c}^{2} \sqrt{R_{\rho}-1}\left(\sqrt{R_{p}}+\sqrt{R_{\rho}-1}\right)^{3}}{C_{w} \kappa_{T}}\right]
$$

and $C_{T}=C_{a}=1$ for a square wave, $1 / 2$ for a sinusoid, and $1 / 4$ for a double sinusoid. The average fluxes and the total buoyancy flux $\left\langle F_{b}>=g\left(\beta<F_{S}>-\alpha<F_{T}>\right)\right.$ are plotted in Figure 6a. If $h_{M A X}=l_{i}$,

$$
g \alpha<F_{T}>=\frac{I C_{T}}{8}\left(\frac{F r_{c}^{2}}{C_{w}}\right)^{1 / 3} \kappa_{T}^{1 / 3}(g \beta \Delta S)^{4 / 3} \sqrt{R_{p}}\left(\sqrt{R_{\rho}}+\sqrt{R_{\rho}-1}\right)
$$



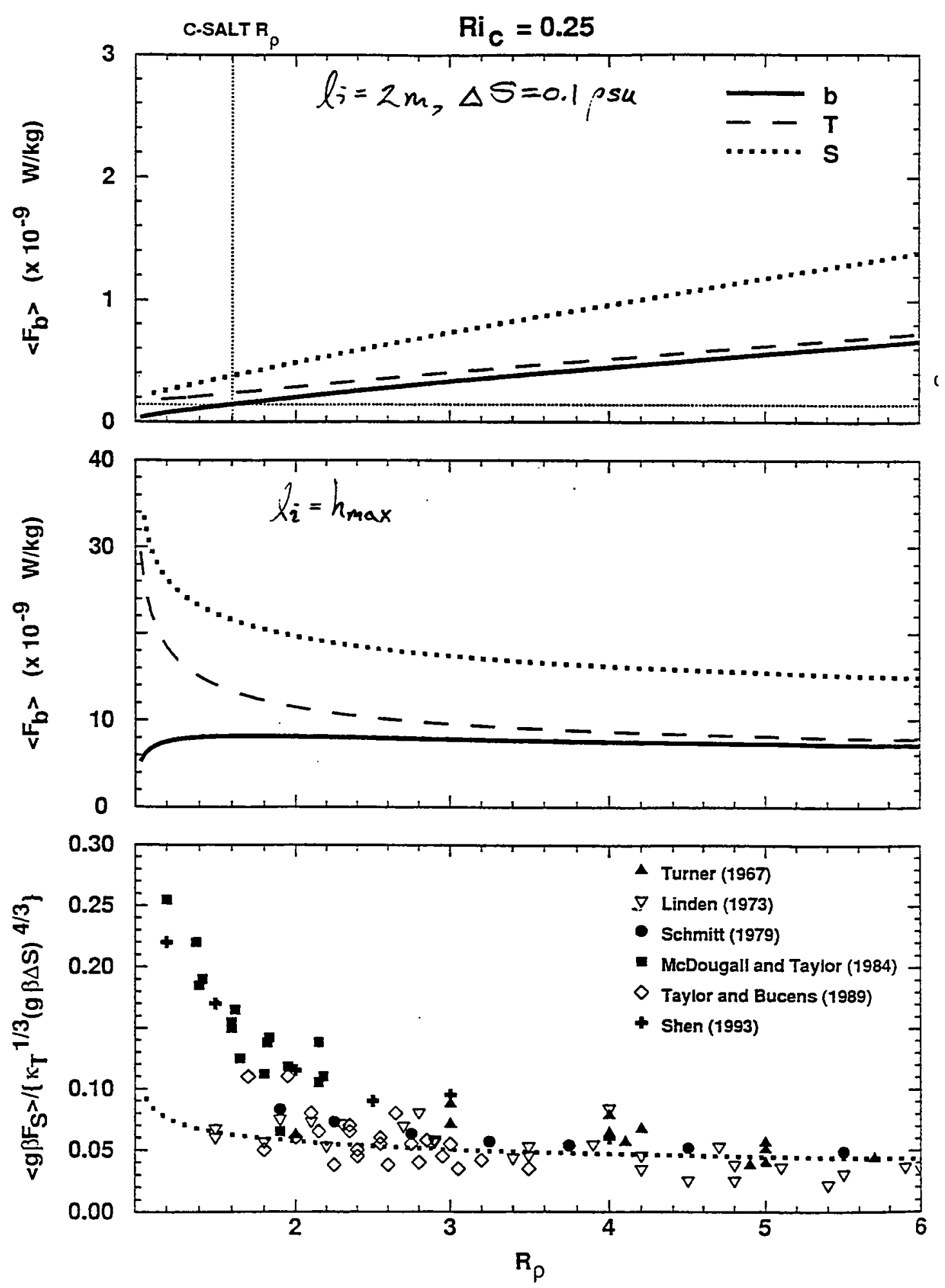

Figure 6: Average buoyancy fluxes vs. density ratio for fingers growing in an interface with $\Delta S=0.1 \%, F r_{f}=2$. The upper panel (a) corresponds to a $2 \mathrm{~m}$ thick interface $\left(l_{f}=2 \mathrm{~m}\right)$ in which, according to Figure 5, individual fingers do not fill the interface.In the midpanel (b) the thickness of the interface is identical to the maximum finger length $\left(l_{i}=h_{M A X}\right)$. In (c), the case shown in (b) has been scaled by $\kappa_{T}^{1 / 3}(g \beta \Delta S)^{4 / 3}$ and is compared to laboratory estimates of the salt buoyancy-flux. 


$$
g \beta<F_{S}>=\frac{I C_{S}}{8}\left(\frac{F r_{c}^{2}}{C_{w}}\right)^{1 / 3} \kappa_{T}^{1 / 3}(g \beta \Delta S)^{4 / 3}
$$

reproducing the laboratory $\Delta S^{4 / 3}$ laws.

\section{Conclusions}

A model for fastest-growing fingers in an interface has been described with a finger gradient Froude number constraint limiting the magnitude of the fingers. The dependence of the flux ratio is identical to the nonequilibrium models of Stern(1975) and Schmitt (1979b) and the laboratory measurements of Turner (1967) and Schmitt (1979a). If a finger fills the interface, the laboratory behavior, in particular, the $\Delta S^{4 / 3}$ flux law, can be reproduced. However, for the staircase region east of Barbados, the majority of interfaces are much thicker $(2-5 \mathrm{~m})$ than the maximum finger lengths allowed by this model $(30 \mathrm{~cm})$. Under these conditions, the model fluxes depend on the interface gradients and are $\approx 3 \cdot 10^{-10} \mathrm{~W} / \mathrm{kg}$ or less, more than thirty times weaker than the $\approx 80 \cdot 10^{-10} \mathrm{~W} / \mathrm{kg}$ predicted by the $\Delta S^{4 / 3}$ flux law. This is in qualitative agreement with the microstructure measurements collected east of Barbados during the C-SALT cruise of November 1985.

\section{References}

[1] Kunze E. 1987. Limits on growing, finite-lenght salt fingers: a Richardson number constraint. J. Mar. Res. 45 533-556

[2] McDougall, T. J. and J. R. Taylor. 1984. Flux measurements across a finger interface at low values of the stability ratio. J. Mar. Res. 42 1-14.

[3] Schmitt, R. W. 1979a. Flux measurements at an interface. J. Mar. Res. 37 419-436.

[4] Schmitt, R. W. 1979b. The growth rate of supercritical salt fingers. Deep-Sea Res. 26A 23-44.

[5] Schmitt, R. W., H. Perkins, J. D. Boyd and M.C. Stalcup. 1987. C-SALT: An investigation of the thermohaline staircase in the western tropical North Atlantic. Deep-Sea Res.

[6] Stern, M. E. 1975. Ocean Circulation Physics. New York; Academic.

[7] Turner J. S. 1967. Salt fingers across a density interface. Deep-Sea Res. 14 599-611. 


\section{Effect of vertical shear on salt fingers}

\section{Motivation}

The shadowgraph profiles of the thermohaline staircase near Barbados show a $3 \mathrm{~m}$ depth fingering favorable rone with a vertical wavelength of $1 \mathrm{~cm}$ where one would expect a horizontal structure. Figure 1 shows these observed structures. The visualization technique shows the Laplacian of the index of refraction. The variation seen here is almost entirely due to variation in salinity.

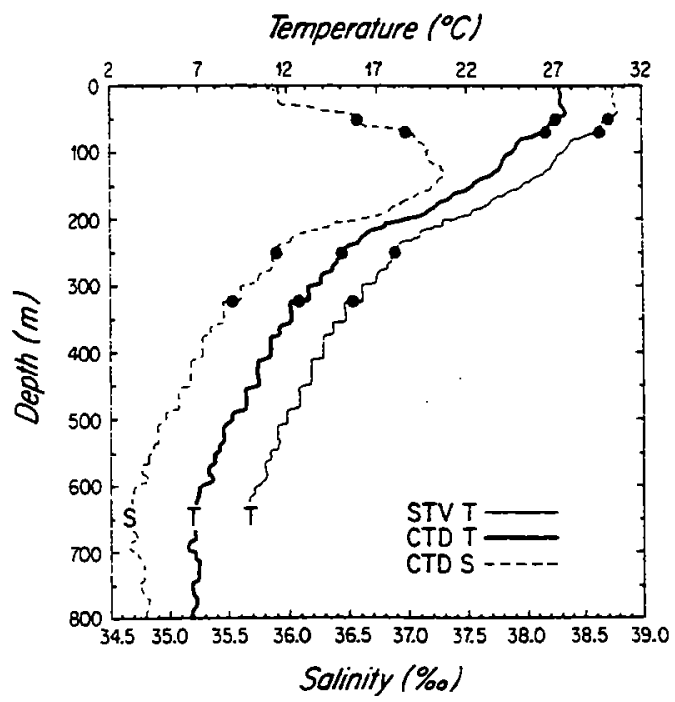

Fig. 1. Vertical profiles of temperature from the shadowgraph protiler offset by $3^{\circ} \mathrm{C}$ (STV T), CTD temperature (T) anj CTD salinity (S). Between 40 and $130 \mathrm{~m}$. the water column is stable to double diffusion (temperature decreasing and salinity increasing with depth). Below $130 \mathrm{~m}$, the thermohaline structure is largely favorable to sait fingering (temperature and salinity both decreasing with depth). A well-ordered staircase occupies $300-600 \mathrm{~m}$. The dots indicate the depths of the images 
A model of this phenomena should also explain the following observations :

- a dominant vertical wavelength in temperature field of $\sim 6 \mathrm{~cm}$

- a dominant horizontal wavelength in the temperature field of $5-7 \mathrm{~cm}$ which is consistent with the fastest-growing wavelengthlatex

- an average turbulent dissipation rate of $\sim 10^{-10} \mathrm{~W} / \mathrm{kg}$. A factor of 30 below those deduced by an extrapolation of laboratory experimental data

- an average interface dissipation rate of $\sim 5 \cdot 10^{-10} \mathrm{~W} / \mathrm{kg}$

- a median interface Cox number $\left(C_{x}=\frac{(\nabla T)^{2}}{\bar{T}_{x}}\right)$ linearly dependent on the interface thickness $l_{i}$.

It seems reasonable that these observations may result from the presence of vertical shear.

\section{Equations of motion}

Our previous model should be changed to include a vertical shear as shown figure 2 .

The equations of motion become :

$$
\begin{gathered}
\partial_{t} u+\underline{U \partial_{x} u}-\nu \nabla^{2} u+U_{z} w=\partial_{x} p \\
\partial_{t} w+\underline{U \partial_{x}}-\nu \nabla^{2} w=-\partial_{z}+g(\alpha \delta T-\beta \delta S) \\
\partial_{t} \delta T+\underline{U \partial_{x} \delta T}-\kappa_{T} \nabla^{2} \delta T+\overline{T_{z}} w=0 \\
\partial_{t} \delta S+\frac{U \partial_{x} \delta S}{\partial_{x} u}-\kappa_{S} \nabla^{2} \delta S+\overline{S_{z}} w=0 \\
\partial_{z} w=0
\end{gathered}
$$

where the presence of the uniform shear $\left(U_{z}\right)$ adds the underlined terms to the unsheared equations. Note that $U,{U_{z}}_{z}, \overline{T_{z}}, \overline{S_{z}}$ are not necessarily constant.

We look for the numerical solution proportional to $\exp \left[i\left(k_{x} x+k_{y} y+k_{z} z\right)\right]$ giving rise to the substitution :

$$
\begin{aligned}
\partial_{x} & \longrightarrow i k_{x} \\
\partial_{z} & \longrightarrow i k_{z} \\
\nabla^{2} & \longrightarrow-k^{2}
\end{aligned}
$$

where $k^{2}=k_{x}^{2}+k_{y}^{2}+k_{x}^{2}$. 


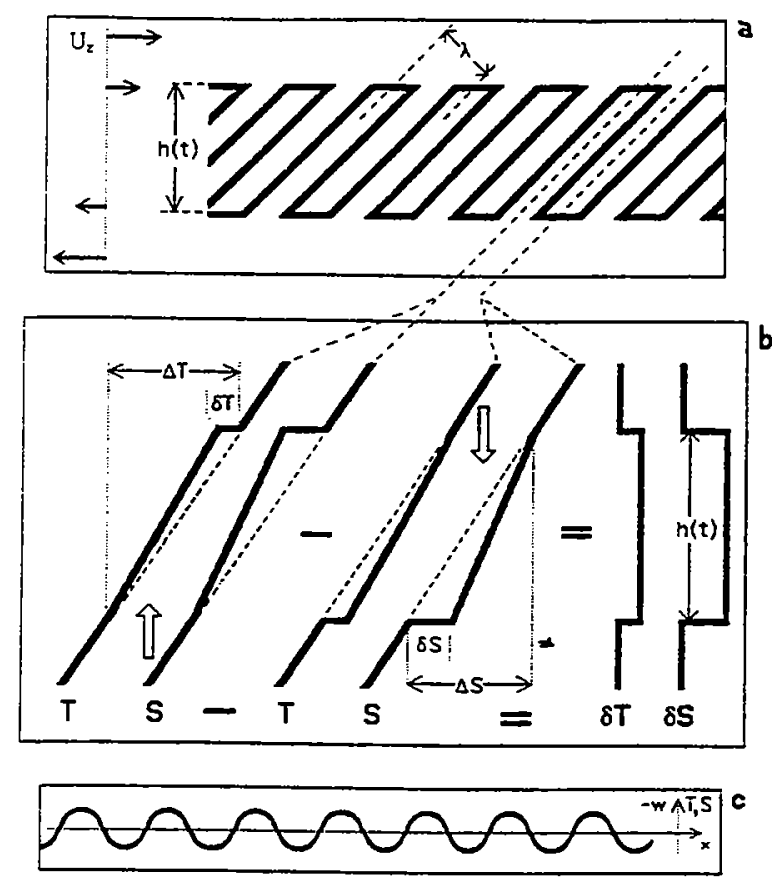

Figure 2. A schematic defining the parameters of the sheared finger model. In the upper panel (a), a vertical cross-section through a zone of tilted fingers is shown. The thick solid lines demark the strong gradients between adjacent fingers and at their intruding tips. The central panel (b) displays temperature and salinity profiles along the dashed lines in (a) for up- and downgoing fingers (left and center) and the contrasts between fingers (right). Temperature and salinity are continuous at the inlet to a finger, vary smoothly over its length and are discontinuous at the intruding tip. Upgoing fingers are lighter than the surrounding fluid, downgoing fingers heavier. Gradients inside the fingers (solid lines) are weaker than in the unperturbed fluid (dashed lines). The finger height $h$ lengthens in time. Sinusoidal structure for finger temperature, salinity and vertical velocity is assumed (c).

\section{Numerical results}

We use parameters values for this simulation which are typical for the thermohaline stairecase east of Barbados where the observations were made. Two horizontal configurations will be considered. In the first we look for a square planform where $k_{x}=k_{y}$, with a steady shear parallel to the $x$-axis. In the second case we consider a sheet form where the initially aligned shear rotates with time. 


\section{Steady shear}

The numerical results for a square planform of salt fingers in steady shear are plotted figure 3 .

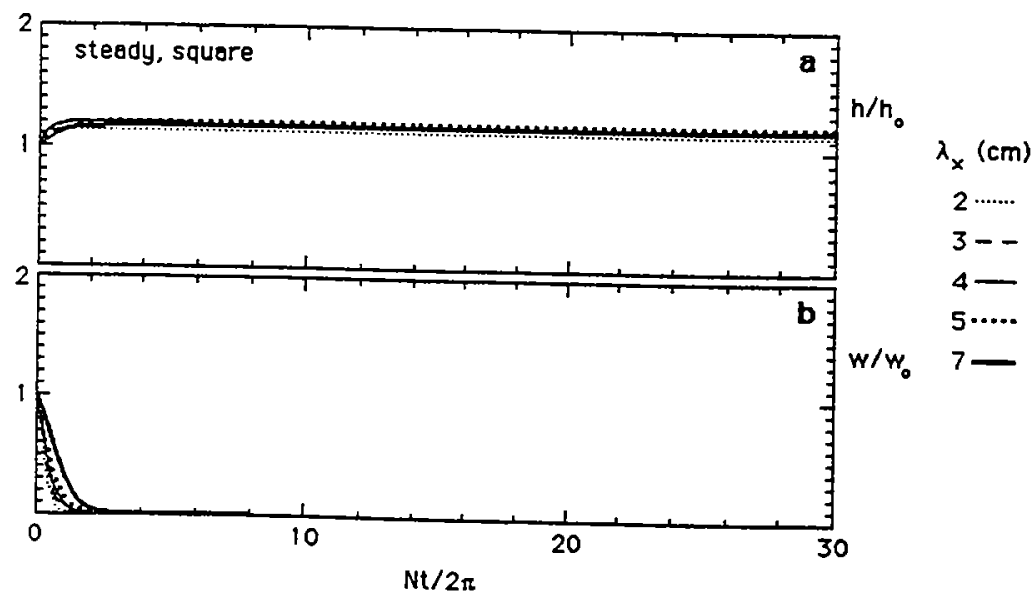

Figure 3 The time evolution of normalized (by their initial values) finger height $h / h_{o}$ (upper panel) and vertical velocity $w / w_{o}$ (lower panel) for square planform fingers with horizontal wavelengths ranging from $2-7 \mathrm{~cm}$ in a shear of $R i=6$. The vertical velocity decays without growing. As a consequence, the finger height does not increase significantly.

We can see that the finger size does not grow with time and the vertical velocity decreases swiftly to zero. Therefore fingers form sheets aligned with the shear. They are titled over and then become molecularly damped too rapidly to contribute significant fluxes. Equations of motion become essentially the same as the case in which there is no shear if $\partial_{z}=0$. 


\section{Rotating shear}

Results for fingers size and their vertical velocity are plotted figure 4.

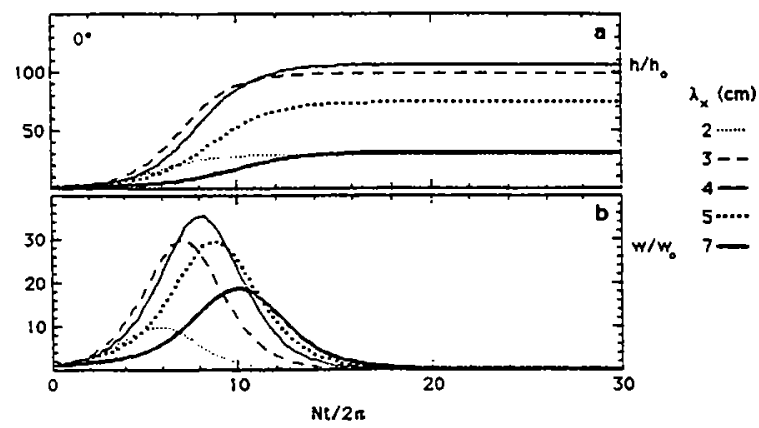

Figure 4. The time-evolution of sait sheets with wavelengths ranging from 2-7 cm in inertially-rotating shear of $R i=6$. The normalized finger height $h / h_{o}$ is shown in the upper panel and vertical velocity $w / w_{0}$ in the lower panel. The sheets are initially aligned with the shear (Fig. 6a). They tilt over and are damped more slowly than square planform fingers (Fig. 5) which feel the full effect of shear. The vertical velocities grow for $\sim 7$ buoyancy periods, attaining magnitudes up to 35 times their initial value before decaying. The finger heights reach up to 100 times their initial value for horizontal wavelengths similar to the unsheared fastest-growing wavelength of $3 \mathrm{~cm}$.

It seems possible that sheets could grow significantly before being tilted and damped, yet be significantly tilted before becoming unstable.

If we plot the normalized vertical gradients of temperature $\left(\frac{\delta T_{t}}{\nabla \circ T_{0}}\right)$ and salinity $\left(\frac{\delta S_{s}}{\nabla_{0} S_{0}}\right)$ against the vertical wavelength we find a maximum of these quantities at $\lambda_{z} \sim 1 \mathrm{~cm}$ for the salinity and $\sim 3 \mathrm{~cm}$ for temperature. The wave length of the salinity field agrees well with oceanographic observations, supporting the hypothesis that the observed layers are due to salt. The vertical wave length for the temperature field is smaller than the observed value. This may be as a result of instability in the sheets, which will be discussed below.

\section{Comparision for fluxes}

\section{unconstrained fluxes}

A model must give a microstucture in agreement with observations, but to be relevent must also give flux estimates which are consistent with measurements. However, if we consider the case where sheetgrowth is unrestricted except by the tilting, numerical results give values of : 
- the average flux ratio $R_{F}=\alpha\left\langle F_{T}\right\rangle / \beta\left\langle F_{S}\right\rangle$

- the dissipation rate $\langle\epsilon\rangle$

- the buoyancy-flux $\left\langle F_{b}\right\rangle$

- and the Cox number $\left\langle C_{x}\right\rangle$

which are inconsistent with observations. For example the dissipation rate and the buoyancy-flux are 2 orders of magnitude larger in the simulations than in those measured in the thermohaline staircase near Barbados.

\section{Constrained fluxes}

As in the previous chapter we impose a constraint on the Stern number. Considering the vertical shear we can assume that

$$
R i_{f}^{-1}=\frac{(\nabla \times v)^{2}}{N^{2}}=\frac{F_{b}}{\nu N^{2}}\left(1+s^{2}\right)^{2}=A \leq 2 .
$$

This assumption allow us to obtain results in better agreement with observations as shown figure 5.

\section{Conclusion}

We have considered a model describing the interaction of salt fingers and the oceanic shear. This provides an extension to previous models by allowing the tilting of salt fingers by both staedy and unsteady shear. The results for steady shear are consistent with the experiments of Linden which show that fingers preferentially occur as sheets aligned with the shear.

However, shear in the ocean is not steady, but rotates to produce a component of the shear across the initially aligned sheets. The process of rotation occurs slowly enough that the sheets have time to grow before being significantly tilted. If the sheets are allowed to freely evolve in the shear the values of the fluxes predicted is significantly higher than those observed. However, if we assume that the growth is halted when the Stern number exceeds a critical value the the fluxes become consistent with observations. The results strongly suggest that salt fingering is responsible for the observed layering. 


\section{The Criteria for the Onset of Double-Diffusive Instabilities at a Vertical Boundary Oliver S. Kerr \\ City University, Northampton Square, London EClV OHB, U.K.}

Over the years there have been a number of reports in the literature of experimental investigations into the onset of instabilities when a salt stratified body of water is heated at a single vertical wall. These include Thorpe, Hutt \& Soulsby (1969), Chen, Briggs \& Wirtz (1971), Narusawa \& Suzukawa (1981), Tanny \& Tsinober (1988) and Schladow, Thomas \& Koseff (1992). The first of these concentrated on the onset of convection in a vertical slot, while the last focussed on the effects of having a vertical temperature stratification in addition to the salt stratification. We will focus on the other three papers. For a more general review of this whole field see Kerr (1995)

These experimental works have come up with various criteria for the initial onset of instabilities. In particular Chen, Briggs \& Wirtz (1971) proposed the criterion

$$
R a=\frac{g \alpha \Delta T H^{3}}{\nu \kappa_{T}}=15000 \pm 2500 .
$$

This is a Rayleigh number based on the Chen scale, $H=\alpha \Delta T /\left(-\beta \bar{S}_{z}\right)$ where $\Delta T$ is the wall temperature, $\bar{S}_{z}$, the vertical salinity gradient, $g$ the acceleration due to gravity, $\nu$ the kinematic viscosity, $\kappa_{T}$ the thermal diffusivity, $\alpha$ the coefficient of thermal expansion and $\beta$ the coefficient of density increase with respect to the addition of salt. The Chen scale is a natural vertical scale that arises in side wall heating, representing the distance an element of fluid would rise if its temperature were increased by $\Delta T$. Narusawa \& Suzukawa (1981) proposed the criterion

$$
\pi_{3}=\frac{\alpha q / k}{-\beta \bar{S}_{z}}=0.28
$$

where $q$ is the heat flux at the wall and $k$ is the thermal conductivity. This is essentially the nondimensional ratio of the horizontal temperature gradient at 
the wall to the vertical salinity gradient. Tanny \& Tsinober (1988) proposed

$$
R a_{\xi}=\frac{g \alpha \Delta T \xi^{3}}{\nu \kappa_{S}}=53.8 \pm 12,
$$

where $\xi=\left(\nu \kappa_{S} /\left(-g \beta \bar{S}_{z}\right)\right)^{1 / 4}$. This is a Rayleigh number based on the thin salt boundary layer scale, $\xi$. None of these are the same, nor do they agree with the theoretical result of $\operatorname{Kerr}(1989)$ where the criterion of

$$
Q=\frac{(1-\tau)^{6} g(\alpha \Delta T) H^{5}}{\nu \kappa_{S} L^{2}}=147700
$$

was derived. This is like a Rayleigh number but involves two different length scales, the vertical scale $H$ and the horizontal scale $L=\sqrt{\kappa_{T} t}$ where $t$ is the time since the onset of wall heating. A physical interpretation of this nondimensional number can be found in Kerr (1995). Here we will examine these various stability criteria. We will show that they are all compatible with the observations, but are based on different interpretations of the experimental results.

One important feature of the problem of heating a salinity gradient from a single side wall is that the background state is always changing. The length horizontal scale, $L=\sqrt{\kappa_{T} t}$, is steadily increasing. In addition the wall temperature, $\Delta T$, is also usually increasing. This could either be because the heat is applied as constant flux, implying $\Delta T \propto \sqrt{t}$, or because the wall takes a finite time to reach its final temperature (about 3 minutes for Chen et $a$ al.). In real experiments the nondimensional numbers $R a, R a_{\xi}$ and $Q$ if evaluated at the instantaneous value of the wall temperature are all functions of time. In the experiments of Tanny \& Tsinober the evolution of the wall temperature was recorded along with time when instabilities first appeared. From this information they were able to calculate the evolution of the values of the salt and thermal Rayleigh numbers based on the horizontal length $L$ defined by

$$
R a_{T}=\frac{g \alpha \Delta T L^{3}}{\nu \kappa_{T}}, \quad R a_{S}=\frac{-g \beta \bar{S}_{z} L^{4}}{\nu \kappa_{T}}
$$

as well as the the points in the $R a_{T}-R a_{S}$ plane corresponding to the onset of instabilities. These are shown in Figure 1. Tanny \& Tsinober found a line of best fit through their data points for larger values of $R a_{S}$, finding that 


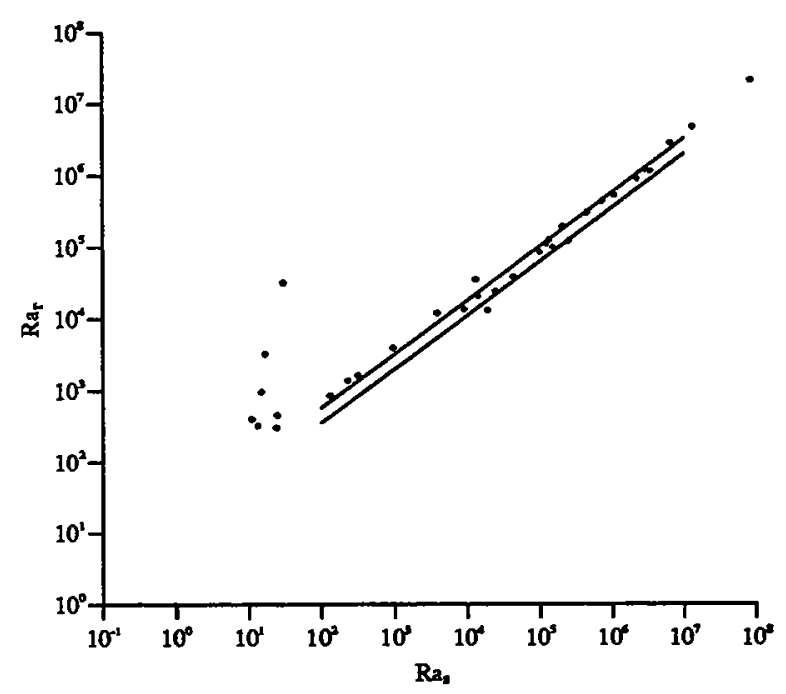

(a)

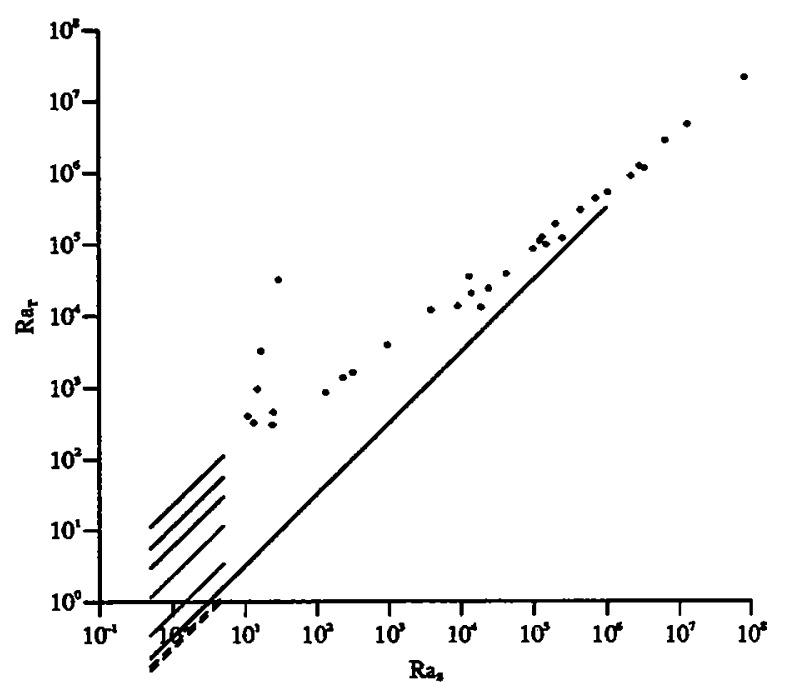

(b)

Figure 1: Points indicating the values of $R a_{T}$ and $R a_{S}$ at the onset of instability form the heat-salt experiments of Tanny \& Tsinober (1988) along with lines indicating (a) the stability criteria of Chen, Wirtz \& Briggs (1971) (lower line) and Tanny \& Tsinober (upper line), and (b) the stability criterion of Narusawa \& Suzukawa (1981) (long line) along with the initial trajectories of their experiments using common salt (short lines).

the slope was almost exactly $3 / 4$ (upper line in Figure 1(a)). This slope is the same as that for the criterion of Chen et al. (lower line in Figure 1(a)) when expressed as a relationship between $R a_{S}$ and $R a_{T}$. However, if Tanny \& Tsinober's condition was converted into the Rayleigh number of Chen et al. the constant would be 83700 and not $15000 \pm 2500$. Neither of these results are incompatible with the experimental results shown in the figure. They represent two ways that could be used to interpret the experimental information. Tanny \& Tsinober gave the formula for the line of best fit through the data points, while the line of Chen et al. is a good choice for a line of slope $3 / 4$ that represents a lower bound for the region of instability. If one assumes that all points should lie inside some region of instability then this could be the more appropriate approach. Instabilities are only visible when they have grown to a finite amplitude and so the values of the Rayleigh 
numbers recorded when instabilities are first observed may not be those that were applicable when heated salinity gradient first became unstable. The second approach may be a more meaningful way of looking at the problem when considering marginal stability, while the line of Tanny \& Tsinober gives a good description of what will be observed.

The experimental results of Narusawa \& Suzukawa (1981) are harder to fit into this picture as they did not report information about the time at which instabilities were first observed. Hence it is not possible to calculate the corresponding instantaneous salt and thermal Rayleigh numbers. However, it is possible to calculate the lines of slope 1 in the $R a_{T}-R a_{S}$ plane along which their experiments using common salt would evolve. Their marginal stability criterion corresponds to the long line shown in Figure 1(b) and separates those trajectories corresponding to experiments where instabilities arose (short solid lines) and those where they did not (short dashed lines). It would appear that all the trajectories would cross the stability boundaries found by the others and so should become unstable. However, they terminated their experiments after a finite time determined by the rise in temperature at the centre of their tank. This put an upper bound on the Rayleigh numbers that could be observed which corresponds approximately to the upper end of the marginal stability line shown. Since this point lies close to the region of marginal stability found by the others, their results are compatible with the other criteria if the time constraint is applied. If there were no time limit on the experiments caused by the finite extent of the tank then it is likely that instabilities would appear for all values of $\pi_{3}$.

In Figure 2 the experimental results of Tanny \& Tsinober are repeated. Superimposed on these are (a) the linear stability criterion for the onset of steady convection in an infinite vertical slot of width $L$, and (b) the linear stability result of Kerr (1989) for an evolving wall temperature of the same form as the experiments of both Chen, Briggs \& Wirtz and of Tanny \& Tsinober. The former example shows many of the features of the observed experimental results and would seem to give a reasonable boundary for the observations. In particular it gives a lower limit for possible values of $R a_{T}$ and an almost vertical boundary for smaller $R a_{S}$. But the match is not perfect, for example the smallest value of $R a_{T}$ for which instabilities were observed differs by a factor of 10 from the prediction of the analysis of the slot. In addition, the vertical wavenumber for marginal stability in a slot is 0 near the bottom of this curve. This is not observed in single side wall experiments. 


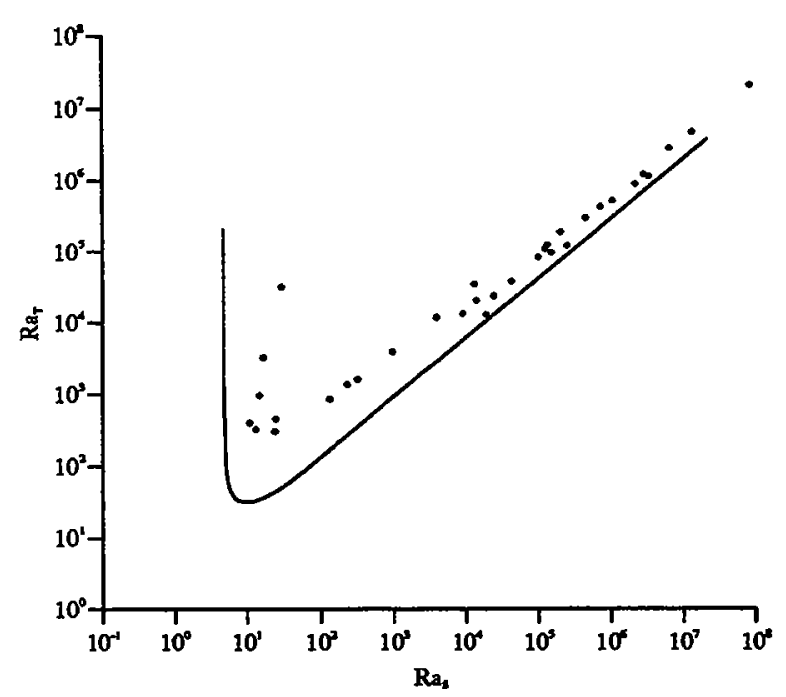

(a)

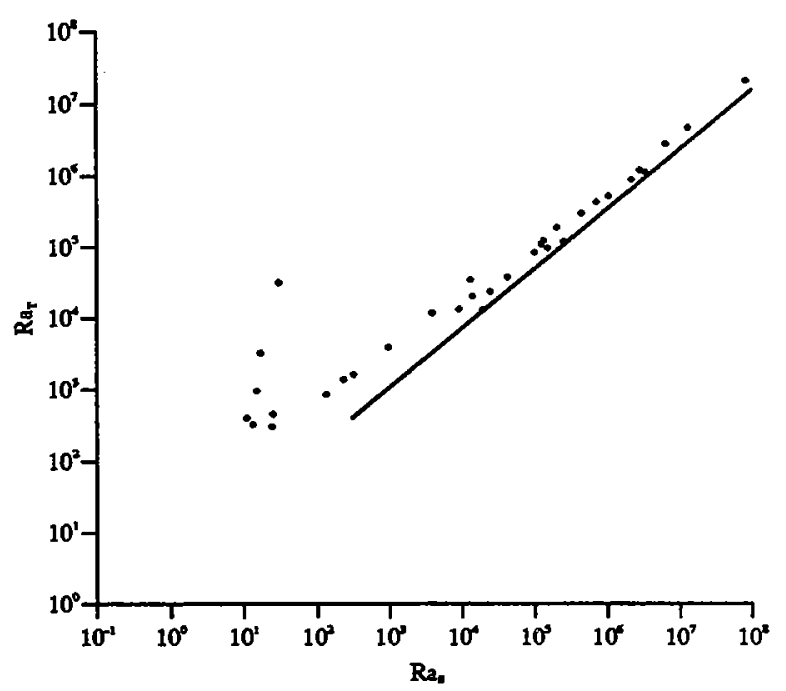

(b)

Figure 2: Comparison of the experimental results of Tanny \& Tsinober with (a) the linear stability of a slot of width $L$, and (b) the linear stability criterion of Kerr (1989).

The structure of the corresponding instabilities in a slot relies on the presence of two walls, and would not be appropriate for the single wall case.

The criterion from the linear stability analysis of Kerr (1989) is based on the assumption that $(H / L)^{2} \ll 1$. This approximation is appropriate for the experiments of Chen et al. and many of those of Tsinober \& Tanny, but only a few of those of Narusawa \& Suzukawa. It provides a good lower bound for the data points, especially for larger values of $R a_{S}$ where the above condition is most appropriate. The nonlinear energy stability analysis of Kerr (1990) shows that if the vertical periodicity of the intrusions is limited by the Chen scale then arbitrary disturbances will always decay if $Q<19585(1-\tau)^{4}$. This gives a line of slope $5 / 6$ parallel to and just below the line shown. The stability criteria of both Chen et al. and Tanny \& Tsinober both intersect and pass below this line of absolute stability, and so cannot hold for arbitrarily large values of $R a_{S}$.

All the experimentally derived stability criteria listed earlier give good descriptions of the experiments in which they were derived, and when consid- 
eration is given to the conditions of the experiments the results would appear to be compatible with each other. Similarly the experimental results can be interpreted as supporting the theoretical prediction in its region of validity. How can all this be true given the disparate nature of the stability criteria? There are several areas where these differences arise, one is the difficulty of conducting these experiments and making precise measurements. This can lead to a significant scatter of the data points. Tanny \& Tsinober reported that this scatter was more pronounced for their experiments with lower $R a_{S}$. This could account for the reduced slope of their line of best fit through their data points when compared with the theoretical slope if the true stability boundary provides a lower bound for the observations. Thus, by using a line of best fit, they will get a different form for their answer from that predicted by theory. Another problem is the finite extent of the experimental tanks used. If you have to terminate your experiment after a finite time does this mean that instabilities would not have formed for the same salinity gradients and wall heating if the tank had been larger? Clearly this is something that cannot be guaranteed without further experimentation or some theoretical justification. In the situation examined by Narusawa \& Suzukawa it is probable that instabilities would always appear eventually. One difficulty that arises in the problem of side wall heating is the variety of different length scales that are present and that can be used in the derivation of nondimensional parameters. We have seen three here, $L, H$ and $\xi$. But there are others that can appear in this problem which may be important such as the amplitude of the excursions experienced by the internal waves generated by the wall heating (suggested by Schladow, Thomas \& Koseff, 1992). In different combinations these can lead to a variety of nondimensional parameters that may be hard to differentiate by experimentation alone.

Examination of the experimental results of Tanny \& Tsinober, and comparison with the stability of a vertical slot, indicates that there is no single stability criterion for the onset of instabilities in the heating of a salinity gradient from a single side wall. In different parts of the stability boundary different combinations of length scales will be important. This is clearly the case in the linear stability analysis of a vertical slot. The stability parameter $Q$ seems to be the appropriate one for larger values of $R a_{S}$, but for lower values it is clearly not. It is not known what other combinations of length scales are important for different regimes of the onset of instability in a salinity gradient heated from a single side wall. However it is clear that 
attempts to find these purely by the interpretation of experiments is fraught with difficulty, and a solid theoretical basis will also be required.

\section{References}

Chen, C.F., Briggs, R.A. \& Wirtz, D.G. 1971 Stability of thermal convection in a salinity gradient due to lateral heating. Intl. J. Heat Mass Transfer 14, 57-65.

Kerr, O.S. 1989 Heating a salinity gradient from a vertical sidewall: linear theory. J. Fluid Mech. 207, 323-352.

Kerr, O.S. 1990 Heating a salinity gradient from a vertical sidewall: nonlinear theory. J. Fluid Mech. 217, 529-546.

Kerr, O.S. 1996 Double-diffusive instabilities at a vertical boundary. In Double Diffusive Convection (ed. A. Brandt \& J. Fernando) American Geophysical Union, 105-113.

Narusawa, U. \& Suzukawa, Y. 1981 Experimental study of double-diffusive cellular convection due to a uniform lateral heat flux. J. Fluid Mech. 113, $387-405$.

Schladow, S.G., Thomas, E. \& Koseff, J.R. 1992 The dynamics of intrusions into a thermohaline stratification. J. Fluid Mech. 236, 127-165.

Tanny, J. \& Tsinober, A.B. 1988 The dynamics and structure of doublediffusive layers in sidewall-heating experiments. J. Fluid Mech. 196, 135-156. 


\title{
Laboratory models of double-diffusive processes
}

\author{
by J. Stewart Turner \\ Research School of Earth Sciences \\ Australian National University, Canberra ACT 0200, Australia
}

\section{Introduction}

The study of the physical processes now included under the heading of double-diffusive convection has expanded considerably over the past thirty five years or so. It now embraces applications to many different fields, though the major early contributions were made in the context of oceanography (and many of these in Woods Hole). I first heard about these novel phenomena here, and began to study them through laboratory experiments which were continued in Cambridge and Canberra.

In this first seminar I presented a very personal historical review of the laboratory side of the subject, using many slides and movies made at the three major institutions in which I have worked WHOI, the University of Cambridge and the Australian National University. It is clear in retrospect that as the subject has evolved, too little attention has been paid to twodimensional processes, compared to one-dimensional transports through interfaces, and in this and my second seminar I attempted to redress the balance. I have also discussed the application and transfer of ideas to another field of interest to me personally, the geology of igneous rocks.

Two major themes are emphasized here:

1) The importance of simple laboratory experiments in understanding the basic physics of double-diffusive processes.

2) The influence of interactions between people with different skills - observers, laboratory experimenters and theoreticians.

\section{Fundamentals of the subject}

The basic requirements for double-diffusive or multicomponent convection to occur the presence of two or more physical properties in a fluid, with different molecular diffusivities, and distributed in such a way that they have opposing effects on the density - will be taken for granted following the introductory lectures, and they have been covered in many reviews [1], [2]. A whole range of unexpected phenomena can arise, and our physical intuition based on ordinary thermal convection is of little help. For example dyed salt solution flowing slowly into a homogeneous solution of the same solute (say $\mathrm{NaCl}$ ) forms a laminar plume which gently rises or falls without mixing. A plume of sugar solution of the same density, released at the same rate into salt solution (the latter having about three times the molecular diffusivity) leads on the other hand to vigorous upward and downward convection, and light and dense layers flowing out along the top and bottom of the experimental tank.

Laboratory experiments immediately produced two counter-intuitive surprises: many convective phenomena are observed in systems which are "hydrostatically stable" and the vertical density difference actually increases during an experiment; and the rates of vertical transport are much faster with double-diffusion than in a simple fluid containing a single solute. An early series of experiments at WHOI (illustrated with a movie) used a dam-break technique to place fluid layers with different properties on top of one another. Hot water flowing over cold water led to intruding noses with little mixing. Dyed hot salty water flowing over cold fresh water of nearly the same density, however, produced vigorous salt fingers, with the dye and salt dropping out rapidly as the layers advanced. The opposite case of slightly denser hot salty water flowing under cold fresh water produced a sharp diffusive interface with vigorous thermal convection on each side, but a much slower transfer of salt and dye. 
It was also demonstrated at a relatively early stage [3] that similar phenomena can be produce using sugar and salt solutions. This system has since provided a convenient and much-used analogue for the salt-heat case, with the more rapidly diffusing salt now being the equivalent of heat (i.e. a layer of salt solution over denser sugar solution is analogous to a cold fresh layer on top of a hot salty layer of water). A movie made by Tim Shirtcliffe in 1969 using a colour-schlieren visualization of both finger and diffusive interfaces - the results from which were never published in detail - was also shown. The finger interface was revealed as having a hydrostatically stable core through which fingers provide the transport mechanism, with thin unstable edges which break down and drive large scale convection in the layers on each side. Diffusive interfaces break down intermittently in a remarkably symmetrical manner (with upward and downward patterns of convection appearing like mirror images of one another ). At a later stage, persistent solitary-wave-like motions develop on the interface, and these couple with the large-scale convection in the layers on each side. (Only recently have these motions been studied in the detail they deserve.)

It is worth pointing out, in support of the overall theme of this seminar, that the laboratory experiments on transport across heat-salt interfaces in both the diffusive [4] and finger [5] senses (which were stimulated by theoretical predictions of these phenomena) were completed before there were any field data with which to compare them. Observations of layering consistent with these processes soon followed, but it was not until much later that Sandy Williams [6] directly detected salt fingers in the ocean in regions of strong gradients, using a sophisticated variant of the shadowgraph technique (to the great relief of several theoreticians and laboratory experimenters in this audience!).

\section{Two-dimensional effects}

The strongest layering in the ocean is observed near intrusions and fronts, and yet there have been relatively few laboratory experiments which explicitly consider horizontal gradients and two-dimensional effects. The dam-break experiments described above did start with horizontal flows, and the waves on a salt/sugar diffusive interface set up two-dimensional convection, but these effects were peripheral to the original purpose of the investigations.

The third movie presented during the seminar showed the behaviour of line sources of fluid released continuously into a density gradient. When a dyed source of salt solution is released into a salinity gradient at its own density level, it just spreads as a thin layer, displacing its surroundings upwards and downwards and also ahead of the intrusion to produce an "upstream wake". However, when a source of sugar solution having the same density and flowrate as the previous salt source is injected into the same salinity gradient at its neutral buoyancy level strikingly different effects are observed [7]. There is strong vertical doubledifusive convection near the source (as in the case of a homogeneous salty environment) but the vertical spread is now limited by the stratification. Several intrusions spread out at levels above and below the source; each spreading 'nose' contains an excess of sugar, so conditions are favourable for the formation of a diffusive interface above and fingers below each intrusion. There is a slight upward tilt to each layer as it extends, implying that the net density flux due to fingers is larger than that across diffusive interfaces, so that the layer becomes

lighter as it expands. Intrusions driven by temperature and salinity differences, and showing the same features as the salt/sugar analogue system, have been observed in both the laboratory and the ocean.

Note a remarkable general implication of these (and other oceanic measurements) which has emerged as a result of the laboratory work. Not only can differential molecular diffusion across interfaces affect the convective motions on the layer scale (of the order of tens of meters in the vertical in the ocean) but it can also, by driving intrusions across fronts, influence the large-scale mixing between water masses, and hence the whole ocean circulation. 


\section{Horizontal property gradients}

When the side-wall boundary conditions do not match the conditions in the interior of the fluid containing several diffusing properties, instabilities can develop rapidly without the need to inject fluid from a source. The simplest example is a linear salinity gradient heated through a vertical side wall, which produces a series of equally-spaced extending layers. Similar effects can be produced when one wall of a tank is composed of a solid block of a soluble salt, and this dissolves into a gradient of another salt with a different molecular diffusivity. This has geological implications, as a mechanism for layering in magma chambers.

The equivalent process in a salt-sugar system can be investigated by setting up a stable density gradient composed of smooth opposing gradients of salt and sugar solutions, with a maximum of salt concentration at the top and a maximum of sugar at the bottom [8]. With vertical side walls this system was stable for the gradients chosen; the surfaces of constant concentration were normal to the boundaries, and so the no-flux condition for both properties was automatically satisfied. Whe a sloping boundary was inserted, however, the concentration and density surfaces were distorted by diffusion so that they could remain normal to the slope. This upset the hydrostatic equilibrium, and flows parallel to the slope developed, with a thin downflow just above the boundary, and above that a thicker upslope counterflow. As shown in a time-lapse movie, these flows could not remain steady; the fluid rose until it reached its level of neutral buoyancy and then turned outwards to form a series of layers propagating into the interior of the fluid.

Experiments using discrete sources or solid boundaries do not properly model conditions at an oceanic front, where there are horizontal property gradients but no physical barriers. A short movie sequence was also shown of Barry Ruddick's more realistic experiments [9]. He set up horizontal sugar and salt gradients (with no net horizontal density gradient), using a dam break technique described in more detail in his introductory lectures.

An example in which both the temperature and salinity are unmatched to the interior properties at a side wall is a block of ice melting into a tank of salt water, either homogeneous or stratified. This problem arose in the practical context of "harvesting" fresh water from icebergs, which had been proposed as a potential source of drinking water for arid regions. As the ice melts in the homogeneous case, a boundary layer of fresh cold water rises, but it mixes with a great deal of its surroundings and reaches the surface far from fresh. When the surroundings are stratified, a series of extending layers forms along the whole depth of the vertical ice wall [10] and the melt water does not reach the surface at all. Systematic circulations are set up which bring warm water against the block at the top of each layer and form scallops on the melting surface. Thus whatever the stratification of the surrounding seawater the collection of fresh meltwater from icebergs is likely to be much more difficult than was envisaged, even if it turns out to be technically feasible to tow icebergs from the Antarctic to coastal cities in need of water.

\section{Geological applications}

Many of the early laboratory experiments related to geological phenomena (other than sedimentation, which has a long history) dealt with double-diffusive processes, and were suggested by analogous observations previously studied in an oceanographic context. Turner and Gustafson [11] reviewed the various phenomena that can result from the efflux of hot, salty water from vents in the sea floor; they were motivated by suggestions that this could be the mechanism of formation of ancient massive sulfide ore deposits. (This was before active "black smokers" were observed on the present-day sea floor, and the discovery of these has led to an acceleration of interest in these processes, including further laboratory work). That early paper [11] also pointed out that double-diffusive effects can be significant not only in aqueous solutions, but also in liquid rocks. Magmas and lavas have a wide range of chemical 
constituents with different molecular diffusivities, as well as large temperature variations. It is natural to ask if the layers observed in igneous rocks could have formed in the liquid state, and then been preserved during cooling and solidification.

This approach to the problem has led to a whole new range of experiments which have influenced current thinking about many igneous processes, as described in recent reviews [12], [13]. Clearly crystallization needs to be added to the convective processes previously investigated; again it has proved possible to use aqueous solutions to model dynamical phenomena in solidifying magmas. In particular, the density changes in the residual fluid resulting from crystallization can lead to "compositional convection" in both systems, and the effect of this have been studied in many different geometries.

When crystallization occurs at a cooled vertical boundary in a tank of finite size containing, say, sodium carbonate solution, the upflowing residual fluid is cold but light (because crystallization causes much larger changes in the density of this solution than the associated temperature changes). The upwelling cold depleted fluid causes stratification of temperature and composition in the "diffusive" sense, and this can produce layering. When there is a stable compositional gradient in the tank initially, a series of convecting layers forms, extending away from the boundary, and the growth of crystals is in turn affected by the position of these layers. The dynamical processes of stratification and layer formation are exactly the same as those occurring at a melting iceblock - cold, light fluid is produced in each case. This is another good example of the influence of laboratory experiments in producing unforeseen cross-fertilization between two apparently very different fields of application.

Cooling a crystallizing fluid through an inclined roof of a closed tank, with the other boundaries insulated, produces effects more closely related to what happens in a magma chamber. Compositional convection leads to an upflow along the roof which ponds at the highest point, producing a stable region stratified in temperature and composition; if several chemical components are present and crystallize at different rates "differentiation", or the production of vertical gradients of composition, can be modelled too. As some crystals fall from the roof to the floor, they grow and cause strong compositional convection and turbulent mixing in the lower part of the chamber, but this region remains quite distinct from the stratified region above. The implication for stratified magma chambers is that eruptions from vents tapping the upper and lower layers could produce silicic and basaltic magmas simultaneously or in rapid sequence from the same chamber.

A wide range of dynamical processes can be identified when a magma chamber is replenished from below with magma which is compositionally denser (and usually hotter) than the resident magma, and many of these have been studied using laboratory experiments. With a rapid turbulent inflow a "fountain" forms, which rises and mixes vigorously with its surroundings before falling back. If the input is hot as well as dense, a series of doublediffusive layers forms, each of which is convecting and well-mixed. Lower rates of input give rise to very different phenomena. When a hot, saturated layer of, say, potassium nitrate is inserted carefully under cold, less-dense sodium nitrate solution, a double-diffusive interface forms, through which heat is transferred faster than the solutes. The lower layer cools much more rapidly than it could just by conduction through the side walls, and crystallizes as it does so. The density of the residual fluid in contact with the crystals decreases because of the strong dependence of the density of the saturated solution on temperature. The lower layer evolves towards a state where its density approaches that of the upper layer, but the interface remains sharp and there is little transfer of composition between the layers. As the densities become equal the interface suddenly breaks down and the two layers mix intimately together, leaving a layer of potassium nitrate crystals on the floor. 
Further variations on these experiments have been carried out with magma mixing in mind. If the upper layer is stratified rather than homogeneous, when overturning occurs the rise of the lower-layer fluid is confined by the gradient to the lower part of the chamber rather than mixing through the depth. The exsolution of gas following quenching and crystallization of water-rich magma in the lower layer as it overturns and mixes with colder magma above can be modelled using a chemical reaction to release gas. The detailed mechanism is different, but the dynamical processes occurring in the magma chamber and the model are closely analogous. Both these cases were illustrated using movies of the laboratory experiments.

\section{Summary and conclusions}

It should be clear from this review that the identification and physical understanding of double-diffusive phenomena has been strongly influenced by laboratory experiments. These are a very effective way to isolate and study individual physical and dynamical processes. Associated analytical models based on simple scaling arguments can then lead to the formulation of principles which can be realistically transferred to the geophysical contexts of interest. In the oceanographic field, the interaction of observers, laboratory experimenters and theoreticians in close and frequent contact with one another, particularly at Woods Hole, led to very efficient communication and rapid progress in the early years. There is, however, a large element of chance in the spread of ideas across disciplines [14]. It helps to work in a broadbased department or group, and to attend interdisciplinary Programs such as this one, but ulimately the most exciting and fruitful interactions are those which develop from personal contacts. I believe they must grow naturally from 'the bottom up' and cannot be imposed by administrators from the top down.

\section{References}

[1] J.S. Turner, Buoyancy Effects in Fluids. Cambridge Univ. Press, 367pp., 1973.

[2] J.S. Turner, Multicomponent convection. Ann. Rev. Fluid Mech., 17, 11-44, 1985.

[3] M.E. Stem and J.S. Turner, Salt fingers and convecting layers. Deep-Sea Res. 34, 95-110, 1969.

[4] J.S. Turner, The coupled turbulent transports of salt and heat across a sharp density interface. Int. J. Heat Mass Transfer, 38, 375-400, 1965.

[5] J.S. Turner, Salt fingers across a density interface. Deep-Sea Res. 14, 599-611, 1967.

[6] A.J. Williams, Images of ocean microstructure. Deep-Sea Res. 22, 811-829, 1975.

[7] J.S. Turner, Double-diffusive intrusions into a density gradient. J. Geophys. Res. 83, 2887-2901, 1978.

[8] J.S. Turner and C.F. Chen, Two-dimensional effects in double-diffusive convection. J. Fluid Mech. 63, 577-592, 1974

[9] B.R. Ruddick and J.S. Turner, The vertical lengthscale of double-diffusive intrusions. Deep-Sea Res. 26A, 903-913, 1979.

[10] H.E. Huppert and J.S. Turner, Ice blocks melting into a salinity gradient. J. Fluid Mech. 100, 367-384, 1980.

[11] J.S. Turner and L.B. Gustafson, The flow of hot saline solutions from vents in the sea floor: some implications for exhalative sulfide and other ore deposits. Econ. Geol. 73, 1082-1100, 1978.

[12] H.E. Huppert, The intrusion of fluid mechanics into geology. J. Fluid Mech. 173, 557-594, 1986.

[13] J.S. Turner and I.H. Campbell, Convection and mixing in magma chambers. Earth-Science Reviews, 23, 255-352, 1986.

[14] H.E. Huppert and J.S. Turner, Double-diffusive convection. J. Fluid Mech. 106, 299-329, 1981. 


\title{
Stratification and circulation produced by double-diffusive sources in closed regions
}

\author{
J. Stewart Turner \\ Research School of Eath Sciences \\ Australian National University, Canberra ACT 0200, Australia
}

\section{Introduction}

The starting point of the laboratory investigations which are the focus of my second seminar is the fact that both the stratification of the ocean and the thermohaline circulations within it are ultimately determined by the action of localized and distributed sources and sinks of heat and salt near the surface. The bottom water in the world's oceans is formed by the sources having the largest buoyancy flux, and the mean stratification adjusts to this influx, with the effects of upwelling being balanced by distributed downward fluxes from the surface. Sources having a smaller buoyancy flus (or more mixing) cannot penetrate to the bottom of this density gradient set up by the major sources of bottom water, but instead spread out as mid-depth intrusions.

Little detailed work has been done to investigate the effects of multiple, spatially separated buoyancy sources in closed regions, even in the simplest case without diffusion. Oceanic sources, however, are inherently doúble-diffusive, because of their different temperatures and salinities, and the effect of this can be explored using the salt/sugar analogue system in various geometries.

\section{Previous two-dimensional experiments}

As an introduction to the new experiments, I will follow up one of the themes of my first seminar by surveying various previously neglected two-dimensional studies of my own, which were conducted with some specific, different application in mind. Many of these were unpublished at the time (though summaries were given in the reports of the 1974 and 1979 GFD Summer Programs), and the one subsequent publication [1] was not pursued in detail. In retrospect they clearly demonstrate some of the dynamical processes which are relevant in the present context. The theme of [1] was the behaviour of sources of fluids with different properties intruding into a stratified environment with the same or a different composition. The experiments reported in that paper showed the strong influence of double-diffusion, as seen in the film shown during the first seminar. I also discussed then the layers spreading out from a melting iceblock [2] and across a front at which there are large property differences [3] (a gradient of salt solution on one side and sugar solution on the other) but no horizontal density difference. These two studies also demonstrate the importance of double-diffusion in driving quasi-horizontal flows.

Earlier experiments carried out in Cambridge with Tony Chen [4] also exhibit strong two-dimensional effects. One sequence of a time-lapse movie made at that time (the effect of an inclined boundary) was shown during my first seminar, and further sequences with different boundary conditions are relevant now. A long tank filled in the finger sense (with interleaved gradients, sugar above salt solution) exhibited strong shearing motions distorting the fingers. These motions were driven by accidental horizontal anomalies caused by filling through three floats at slightly different rates. When a disturbance was introduced at one end during or shortly after the filling (for example by raising a flap at the end wall) the fingers broke down locally, and a well-mixed convecting layer propagated rapidly along the whole length of the tank - at the wave speed, not as an intrusion. If, on the other hand, the gradients were allowed to "run down" for a while due to the vertical transports by the fingers, even a vigorous disturbance could no longer cause a convective overturn. 
Counter gradients of salt and sugar solutions set up in the diffusive sense (salt above sugar ), with a small net stable density gradient, also become unstable to small disturbances. A little heating through the side walls produced incipient layering with a scale of about $1 \mathrm{~cm}$, and then stronger convection developed, now evidently driven by the potential energy in the salt field. Layers bounded by diffusive interfaces grew at different rates at different horizontal positions, so that complicated boundaries and junctions between layers were observed. The layers merged in pairs, twice, so that after a day they were four times the original depth. There were strong persisting convective motions and finally a series of horizontal diffusive interfaces.

\section{Unpublished experimental work at WHOI}

Other attempts were made to introduce localized double-diffusive fluxes in simple geometries. A second movie shown during the lecture documented a series of experiments carried out in the basement of Walsh Cottage in 1974. A tank was set up containing three compartments separated by two horizontal plexiglas inserts, with a gap at the left between the upper and central layers, and a gap at the right between the central and lower layers. The top and bottom compartments, filled with homogeneous salt or sugar solutions, acted as reservoirs through which diagonal gradients of properties were imposed on the central chamber. When a series of layers was set up in the diffusive sense, the fluxes through the gaps produced convection in all the layers and systematic shearing motions, with active, tilted doublediffusive interfaces. When counter-gradients of sugar above salt (in the finger sense) were set up in the central chamber, driving from the sugar reservoir above and the salt below produced large steady shears, which broke the fingers down to give a deep well-mixed convecting layer with a persistent circulation and finger interfaces above and below, much as described in [4].

Another exploratory experiment carried out at WHOI in 1964 has qualitative features which seem very relevant now. A tank $2 \mathrm{~m}$ long was fitted with a foam plastic slope and shelf at one end, with a steep slope decreasing gradually to a shallow portion about $50 \mathrm{~cm}$ long. It was filled with salt solution, S.G. 1.050, and the shallow end was heated with floodlights to produce both heating and evaporation. Fresh water was put in as a plume at the other end and the input rate was controlled by a constant head device, to keep the depth in the tank constant at $18 \mathrm{~cm}$. The vertical density gradient increased over time, and this and the circulation became steady after about three days. A line of dye streaks put down the centre of the tank revealed two distinct regions of circulation and types of layering. In the top $6 \mathrm{~cm}$ there were strong salt fingers driven by a thin hot saline layer spreading away from the shelf right at the surface, with strongly sheared counterflows immediately below. In the bottom $12 \mathrm{~cm}$ there were much slower intrusions spreading into the interior from the slope, with a series of diffusive layers and interfaces evidently driven by much saltier, warm water flowing down the steeper slope.

\section{The "filling box" effect}

So far I have concentrated on the first element of the new experiments, the production of horizontal circulations and intrusions in a stratified fluid using various horizontally nonuniform methods of forcing. The second element is the production of the stable stratification in the first place. The release of a continuous dense plume from a small source into a homogeneous fluid produces a stable density profile whose asymptotic form has been calculated [5]. This "filling box" effect implies that the densest water in a closed region results from the source or sources with the largest buoyancy flux (e.g. in the ocean, the sources of Antarctic bottom water in the Weddell Sea). Lesser buoyancy sources from marginal seas (such as the Mediterranean outflow) entrain lighter water from the density gradient set up by the major sources; they become neutrally buoyant before they reach the bottom and spread out as intrusions at mid-depth. There are also indications from measurements of the oxygen isotope concentrations in shells from benthic fauna that the water at the bottom of the ocean $70 \mathrm{M}$ years ago was warm (and salty), rather than cold as it is at present. This implies that the largest buoyancy fluxes at that time arose from heating and evaporation in marginal seas, and that outflow from these dominated the formation of bottom water at that time. 


\section{Simple buoyancy sources in a long tank}

The most recent experiments to be described in the rest of this seminar were nearly all carried out in the same tank, $75 \mathrm{~cm}$ long and $7.5 \mathrm{~cm}$ wide, filled to a depth of $15 \mathrm{~cm}$. Sources of fluid with different densities and/or compositions were supplied near the two ends, and flowed in at different depths in the various experiments, at constant rates controlled by a peristaltic pump. The tank level was kept steady using a constant head overflow pipe at the centreline of the tank, but with its inlet placed at different depths in different experiments.

The first "control" experiment to be described started with homogeneous salt solution of S.G. 1.11 in the tank, and two sources of salt solution S.G. 1.10 and S.G. 1.12 flowing in near the surface, each at a rate of $5.3 \mathrm{ml} / \mathrm{min}$. Hence there could be no double-diffusive effects in this experiment. The overflow was also placed just below the surface. As the stratification evolved towards a steady state, the fluid at the bottom of the tank became steadily denser. The fluid at the top (and in the overflow) at first became lighter, reflecting the density of the lighter input, and then at a time comparable with the "replacement" timescale (the time at which the volume of the combined inputs equals the volume of fluid in the tank) it began to get denser again. At long time the overflow density tended to the mean density of the inputs. We also note that the density range in the tank never went outside that of the two inputs: in a non double-diffusive system, with ordinary turbulent mixing, there is no mechanism for it to do so.

\section{Double-diffusive sources}

When one salt source and one sugar source, of nearly the same density, are injected into a tank containing a 50:50 mixture of the two fluids, the behaviour is very different. The first experiment of this kind, with both sources and also the overflow tube placed at mid depth, was done at WHOI during the summer of 1972 . The sugar source was slightly light and the salt dense, but near both of them vigorous upward and downward double-diffusive plumes developed. Over a week the vertical density profile gradually evolved to a steady state, with the overall density difference steadily increasing to become very much greater than the original difference between the source densities, as a consequence of the double-diffusive convection. A sharp interface developed at mid-depth, because of the presence of the withdrawal at the centre of the tank at that depth, and the upper and lower layers were weakly stratified. It did not prove possible to measure the separate sugar and salt concentrations in detail (as was done in recent experiments) but the overall pattern was apparent from shadowgraph pictures. The distributions were antisymmetric: in the half of the tank nearest the salt input, fingers appeared above the interface with a series of layers separated by diffusive interfaces below (consistent with a maximum of salt near the central interface). At the other end of the tank near the sugar source, diffusive interfaces formed at the top, and fingers below the interface. A more detailed quantitative analysis of a related recent experiment will be described later.

Another example presented (this time of an experiment in the new $75 \mathrm{~cm}$ tank) had an input of salt solution, S.G. 1.104, feeding in at the bottom at one end, and sugar, S.G. 1.100, at the top, each at a rate near $5.0 \mathrm{ml} / \mathrm{min}$. There was a 50:50 mixture in the tank at the start and withdrawal was at the surface. The density of the bottom fluid increased steadily, and rapidly became greater than that of the input salt solution, due to double diffusion. The fluid at the top at first became lighter for the same reason, and this density decrease continued for 30 hours (about twice the "replacement timescale"). Then as withdrawal of fluid became more significant the overflow density increased again, to approach the mean density of the inputs.

Photos of dye streaks put in at various times revealed vigorous intrusive motions in both directions, with the level of the outflows from the two sources changing systematically with time as the density structure evolved. After two hours the sugar outflow was still at the top and the salt at the bottom; at six hours both inputs were effectively at the bottom, with the sugar outflow starting horizontal and then being taken to the bottom by a double-diffusive plume. At 10 hours both plumes spread out at $5 \mathrm{~cm}$ above the bottom, and at 21 hours they 
were $5 \mathrm{~cm}$ from the top - this happened because at these times the density difference between the sources was small compared with the vertical density difference set up by double diffusion. Finally, after about 45 hours, both sources were feeding in near the top of the tank, where the overflow had the mean of their densities, and all the fluid in the tank had a larger density.

\section{Quantitative double-diffusive experiments using various techniques}

I will now describe the results of a series of experiments in the $75 \mathrm{~cm}$ tank, all carried out with nominally the same sugar and salt input properties and flowrates. However we observed and monitored different features in each run of the experiment: the time history of the plumes and intrusions using photographs and a time-lapse video; the sugar concentration at several points as a function of time using a polarimeter, and the vertical structure of the density and salt and sugar concentrations, deduced by withdrawing and analysing many samples. The tank contained homogeneous salt solution, S.G. 1.110, initially. Both inputs and the overflow were at the top, with each input flowrate $5.0 \mathrm{ml} / \mathrm{min}$. The salt source was dense, S.G. 1.120 , and was ejected through a small nozzle to make it turbulent, and the sugar source, S.G. 1.100, was laminar, but rapidly became double-diffusive and caused strong vertical convection.

During the very early stages the turbulent plume was not double-diffusive, but it spread out along the bottom and began to "fill the box". A sugar intrusion, produced by doublediffusive convection below the sugar source, spread out above the salt outflow, and fingers were observed between them. This is an even better analogy to the production of ocean bottom water by a large buoyancy flux in the Antarctic, and the subsequent intrusion of the warm salty Mediterranean outflow at mid-depth. Each intrusion marked by dye rose as it advanced because of the evolution of the density profile, leading to the increase of density at each depth. In one experiment monitored on the time-lapse video the input salt plume fed out into the interior at a level which rose steadily until it reached the surface. It then dropped rapidly to the bottom again, and rose a second time, the two cycles being completed between 15 and 25 hours into the experiment. The concentrations of sugar and salt were measured in two ways. A polarimeter record (of the total sugar in a light beam shone through the tank) showed fluctuations with a 45-60 min timescale, corresponding to the passage of fronts past the point of measurement. This confirmed that the fronts observed with dye streaks were not a artefact, but represented a real unsteady aspect of the flow. The vertical distributions of both sugar and salt were deduced by withdrawing samples at discrete times and various positions and depths, and measuring their density and refractive index. These values were inverted to give salt and sugar concentrations using the Ruddick and Shirtcliffe [6] results. The differences in properties over the bottom half of the tank at the centre evolved from the finger sense at the start to the diffusive sense after several days, with excursions due to the passage of fronts.

\section{Evolution of three experiments with different initial conditions}

The final three related experiments to be described were carried out with George Veronis while he was on leave in Canberra earlier in 1996. We monitored, by withdrawing and analysing samples over many days, the evolution to a steady state of experiments in which the densities of the original fluid in the standard tank and the salt and sugar inputs were the same (S.G. 1.10). The only difference was that in the three experiments the tank fluid was pure salt, pure sugar and a 50:50 mixture, respectively. The inputs at each end of the tank were at mid depth, each at $5 \mathrm{ml} / \mathrm{min}$, and the constant-head withdrawal was also at mid depth, in the centre.

With homogeneous salt solution initially in the tank, the top to bottom density difference increased steadily over time, reaching an asymptotic maximum after about 100 hours. The stratification developed a clear two-layer structure during the first day. Both the top and bottom salt concentrations (gm/gm of solution) fell continuously as sugar was added, with the top concentration remaining the higher. The sugar concentration in the top rose to its final value, lower than either of the salt concentrations, in about 40 hours, which is twice the replacement time. The bottom sugar concentration rose to a higher value than either salt 
concentration (but of course less than the undiluted input sugar concentration, because of the dilution with water in the environment). The total (depth integrated) values of the contributions of sugar and salt to the density also reached a steady state in which they were nearly equal. With sugar alone in the tank initially the evolution of the density difference was much faster in the early stages, and so were the changes in sugar and salt, but the final asymptotic values were little different from those in the first experiment. The experiment starting with a 50:50 sugar/salt mixture in the tank was monitored in most detail and for the longest time. Again the initial evolution was rapid, but the same asymptotic state as in the other two experiments was achieved in about 100 hours. Some oscillations in sugar and salt values were seen in this case, perhaps reflecting small differences in the properties or input rates of the sources. The top salt concentration rose rapidly, overshot its final value, and fell to just above the initial salt concentration in the tank. The top sugar concentration fell rapidly (in one replacement time) to the final value, with no overshoot. The concentrations at the bottom changed more slowly, in the sense to be expected from the initial concentrations in the tank.

The final distributions in all three experiments corresponded quite closely to the "rundown" state of a two-layer diffusive system i.e. what would be achieved if a layer of salt solution, S.G. 1.10, were placed over a layer of sugar solution having the same density and depth. Interpreting the "asymptotic" profiles in this way leads to a flux ratio of 0.475 , not very different from the values measured for a diffusive interface in one-dimensional experiments, and it could certainly not be achieved by rundown across a finger interface. But it is not clear how this could be the relevant mechanism, in the three experiments that evolved in such different ways. Perhaps the system achieves this state of minimum potential energy by a different mechanism. In subsidiary experiments we observed very strong localized transports of sugar downwards in plumes forming on the central interface near the sugar source, and this could produce the much larger sugar concentration observed at the bottom.

\section{Discussion}

These exploratory experiments have shown how horizontally separated sources and sinks with different double-diffusive properties can produce complex effects in closed regions, even in simple geometries. The evolution to an asymptotic state having the maximum vertical density difference may not be steady or monotonic, and it is certainly sensitive to the boundary conditions. The 'final' state of stratification may have complicated horizontal velocities superimposed on it, with unsteady intrusions and interleaving motions. There are several different timescales in the problem, related to the overall replacement time of the tank fluid, the velocity of intrusions and the vertical property fluxes. Although the final three experiments showed how the same final state can be achieved starting from different initial conditions, other experiments not discussed have suggested that several quasi-equilibrium states can exist for given external boundary conditions. There may be states compatible with given inputs which can not be realized from some starting conditions without a finite perturbation of the system.

\section{References}

[1] J.S. Turner, Double-diffusive intrusions into a density gradient. J. Geophys. Res. 83, 2887-2901, 1978.

[2] H.E. Huppert and J.S. Turner, Ice blocks melting into a salinity gradient. J. Fluid Mech. 100, 367-384. 1980.

[3] B.R. Ruddick and J.S. Turner, The vertical length scale of double-diffusive intrusions. Deep-Sea Res. 26, 903-913, 1979.

[4] J.S. Turner and C.F. Chen, Two-dimensional effects in double-diffusive convection. J. Fluid Mech. 63 577-592, 1974.

[5] W.D. Baines and J.S. Turner, Turbulent buoyant convection from a source in a confined region. J. Fluid Mech. 37, 51-80, 1969.

[6] B.R. Ruddick and T.G.L. Shirtcliffe, Data for double diffusers: Physical properties of aqueous salt-sugar solutions. Deep-Sea Res. 26, 775-787, 1979. 


\section{Weakly nonlinear convection}

\section{Keith Julien}

\section{University of Colorado}

The lecturer presented a two lecture tutorial on the use of small amplitude asymptotic analysis in the study of nonlinear phenomena in convection.

The first lecture used the Rayleigh-Benard problem to illustrate some of the general methods of weakly nonlinear analysis, deriving the Landau equation and discussing some of its properties. The center manifold reduction technique was illustrated using Lorenz' three-component model of convection.

The second lecture focused on bifurcations of higher co-dimension than one and the unfolding of the nonlinear dynamics around points in parameter space where multiple bifurcations occur. Examples of co-dimension 2 bifurcations were discussed in multi-layer convection, and double diffusive convection. 


\title{
Convection in binary mixtures
}

\author{
Paul Kolodner \\ ATGT Bell Laboratories
}

and

Hermann Riecke

Northwestern University

This series of four lectures introduced a variety of phenomena seen in experiments with binary fluid mixtures and went on to discuss theoretical models of several of them.

The lectures reviewed experimental results obtained with binary fluid mixtures in rectangular cells and went on to describe a series of experiments made with an alcohol-water mixture in a narrow annulus. The existence of a thermally driven molecular concentraion flux (the Soret effect) modifies the onset of convection in a binary fluid mixture. Depending on the relative strength of this effect, the initial instability seen can be either an oscillatory instability or steady convection. A variety of different patterns can be seen at low to moderate Rayleigh numbers according to the experimental parameters used. These include traveling waves, stationary rolls, pulses and dispersive chaos.

Several theoretical approaches were discussed including direct numerical simulation, an asymptotic analysis of the basic continuum equations and the study of model equations. Numerical simulations were shown and compared to experimental results. A weakly nonlinear asymptotic analysis of the basic equations, using center-manifold reduction and assuming a wave with slow spatial variation yielded a Ginsburg-Landau equation. The lecturers then went on to use the real and complex GL equations, as well as the NLS equation to model a variety of the nonlinear dynamics seen in the laboratory and numerical experiments. 


\title{
Simmering the primordial soup: Traveling wave convection in viscoelastic DNA suspensions
}

\author{
Paul Kolodner \\ ATET Bell Laboratories
}

The lecturer introduced us to some of the peculiarities of convection in a viscoelastic medium, describing earlier theoretical work in this field and then going on to discuss his own experimetnal work with DNA suspensions.

An obstacle to experimental studies of viscoelastic convection in polymeric viscoelastic fluids is that in general such fluids are too viscous to convect in practical temperature gradients. Kolodner was able to overcome this by using suspensions of DNA, showing that monodisperse, semi-dilute suspensions of DNA can be made with a viscosity comparable to that of water but a viscoelastic relaxation time of several seconds, allowing one to probe the parameter regime where theory predicts the existence of oscillatory convection. He described the practical problems involved, such as avoiding mixtures with short chain fragments of DNA that boost the viscosity of the medium, and the vulnerability of the DNA molecule in sheared flows.

Using a monodisperse DNA suspension in a narrow annulus, Kolodner was able to confirm that, in the appropriate parameter regime, the first convective instability encountered as the Rayleigh number is raised is an oscillatory instability. Nonlinear forms of these oscillations were observed to take several forms including spoatially-localazed traveling waves and stanting waves with very long oscillation periods. 


\title{
Transition to Chaos in Doubly Diffusive Systems
}

\author{
Edgar Knobloch \\ JILA, University of Colorado, Boulder, CO 80309 \\ and \\ Department of Physics, University of California \\ Berkeley, CA 94720
}

Doubly diffusive systems provide numerous instances of chaotic behavior, both in experiments and in numerical simulations. These systems are characterized by the competition between a destabilizing temperature gradient and the stabilizing effect of concentration gradient, Coriolis force or magnetic field. As a result chaotic behavior occurs in these systems already at small to moderate amplitudes, and hence is amenable to interpretation and analysis from a dynamical systems point of view, a fact anticipated by Moore and Spiegel (1966). In this brief review I shall summarize the present status of our understanding of the transition to chaos for both standing and travelling waves that arise as a result of overstability in these systems. I will confine my attention to convection in two spatial dimensions, with one horizontal and one vertical dimension. Because the mechanisms I shall describe are "generic" their details are independent of the specific system considered, and they can be encountered in a wide variety of systems.

Consider a horizontal fluid layer heated from below and suppose that at a critical value $R_{c}$ of the Rayleigh number the conduction state loses stability at a Hopf bifurcation. In the presence of periodic boundary conditions (PBC) in the horizontal one looks for weakly nonlinear solutions in the form

$$
\psi(x, z, t)=A \exp \left\{i \Omega_{0} t+i k x\right\} f_{k}(z)+B \exp \left\{i \Omega_{0} t-i k x\right\} f_{-k}(z)+c . c .+\ldots
$$

corresponding to a (non)linear superposition of left and right-travelling waves. Here $\psi$ is the streamfunction, $\Omega_{0}$ is the Hopf frequency at $R_{c}$ and $k$ the horizontal wavenumber. Abstract theory (eg. Knobloch 1986) shows that the complex amplitudes $A(t)$ and $B(t)$ satisfy the
equations

$$
\begin{aligned}
& A_{t}=\left[\mu+i \omega+a|B|^{2}+b\left(|A|^{2}+|B|^{2}\right)+\ldots\right] A \\
& B_{t}=\left[\mu+i \omega+a|A|^{2}+b\left(|A|^{2}+|B|^{2}\right)+\ldots\right] B .
\end{aligned}
$$

Here $\mu \equiv\left(R-R_{c}\right) / R_{c}$ is the bifurcation parameter and $\omega \propto \mu$ is the change in the linear frequency due to $\mu$. The form of these equations (though not the values of the complex coefficients $a$ and $b$ ) is independent of the boundary conditions at the top and bottom.

Because of invariance under translations and phase shifts in time the phases of the two amplitudes $A, B$ decouple from the equations for the amplitudes $|A|,|B|$ :

$$
|A|_{t}=\left[\mu+a_{r}|B|^{2}+b_{r}\left(|A|^{2}+|B|^{2}\right)+\ldots\right]|A|
$$




$$
|B|_{t}=\left[\mu+a_{r}|A|^{2}+b_{r}\left(|A|^{2}+|B|^{2}\right)+\ldots\right]|B| .
$$

Here the subscript $r$ denotes the real part. Generically, these equations have two nontrivial solutions, travelling waves $(|A|,|B|)=(r, 0)$ or $(0, r)$, and standing waves $(|A|,|B|)=(r, r)$. The stability properties of these solutions are summarized in the $\left(a_{r}, b_{r}\right)$ plane in fig. 1 . Both travelling waves (TW) and standing waves (SW) bifurcate simultaneously; stable solutions are present only if both branches bifurcate supercritically and the stable solution is the one with the largest Nusselt number $\left(\propto|A|^{2}+|B|^{2}\right)$. In the following I will focus on two cases. In the first standing waves are stable at onset; in the second travelling waves are selected. Observe that the TW have one zero eigenvalue (corresponding to translations and/or phase shifts in time) while the SW have two (since translations and phase shifts act differently on SW). Thus the SW form a circle of solutions, parametrized by their spatial phase.

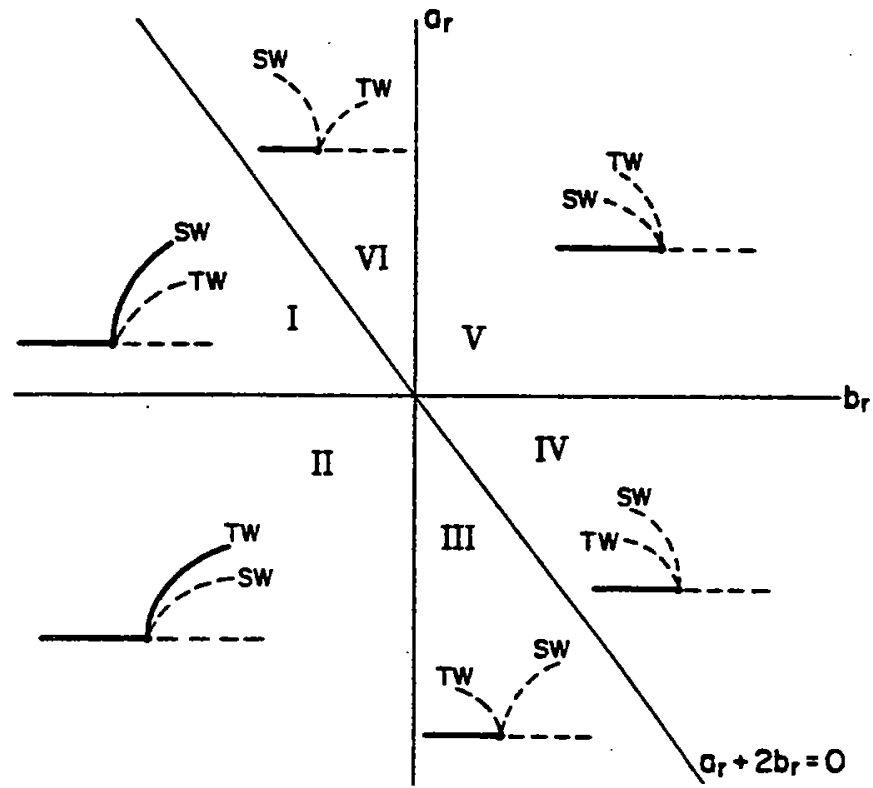

Figure 1. Bifurcation diagrams $\sqrt{|A|^{2}+|\bar{B}|^{2}}$ vs $\mu$ in the $\left(a_{r}, b_{r}\right)$ plane. Stable (unstable) branches are indicated by solid (dashed) lines.

The two mechanisms for transition to chaos (for SW and TW) both involve interaction with steady convection (SS), and are both associated with so-called global bifurcations. To understand the nature of this interaction it is convenient to consider the codimension-two bifurcation that arises when $\Omega_{0}=0$ (the Takens-Bogdanov bifurcation). Analysis of the unfolding of this bifurcation in the presence of PBC (Dangelmayr and Knobloch 1987) shows that with increasing Rayleigh number the TW terminate on the SS branch with their (nonlinear) frequency $\Omega$ approaching zero as the square root of the distance from the termination point. This type of bifurcation is called a parity-breaking bifurcation, and it is a local bifurcation from a circle of equilibria. In contrast, when the SS bifurcate subcritically (as in thermohaline convection) the SW terminate on the unstable SS branch in a global bifurcation involving the formation of a heteroclinic orbit connecting two steady states corresponding to clockwise and counterclockwise rolls (Knobloch and Proctor 1981, Coullet and Spiegel 1983). The formation of this orbit is characterized by a logarithmic divergence of the SW period, but unlike the parity-breaking bifurcation implies no change in stability of the SS . On the other hand, when the SS bifurcate supercritically (as in magnetoconvection), the SW terminate on the SS branch in a local bifurcation (a secondary Hopf bifurcation), as discussed by Knobloch and Proctor (1981). 


\section{Transition to chaotic standing waves}

Suppose first that stable standing waves are selected at onset (region I of fig. 1) and suppose that the Rayleigh number is raised. This problem was explored in detail for the partial differential equations (pdes) describing two-dimensional thermohaline convection by Moore et al (1983) and Knobloch et al (1986b) using idealized boundary conditions. Since for these boundary conditions the SW are always unstable to TW (as in region II) these authors employed no-horizontal-flux boundary conditions to suppress the instability to TW. The results are summarized in fig. 2 . The oscillations start out as symmetric oscillations (in, for example, the vertical velocity $w$ ) and grow in amplitude with increasing $R$. They then undergo what looks like a period-doubling bifurcation in the Nusselt number, but is in fact a bifurcation to asymmetry, as can be verified by examining the time series for $w$. This bifurcation was found already by Huppert and Moore (1976) and is a necessary prerequisite for true period doubling (Swift and Wiesenfeld 1984). With increasing $R$ a period-doubling cascade takes place; beyond the accumulation point of this cascade one finds an interval of chaos with the familiar merging of chaotic bands interspersed with windows of periodic solutions, much as occurs in the logistic map. This resemblence is not accidental but is a consequence of the highly dissipative nature of the pdes. However, in contrast to the logistic map, with increasing $R$ one exits from the chaotic region (via period halving) and recovers a singly periodic oscillation. Knobloch et al refer to this phenomenon as a bifurcation "bubble". With further increase in $R$ this periodic solution suddenly loses stability (at a presumed saddle-node bifurcation) and the system makes a (hysteretic) transition to a "second" branch of oscillatory solutions, also containing an interval of chaos (and periodic windows). This process repeats several times before the final (hysteretic) transition to steady convection. This study still represents the most detailed investigation of chaotic dynamics in partial differential equations.

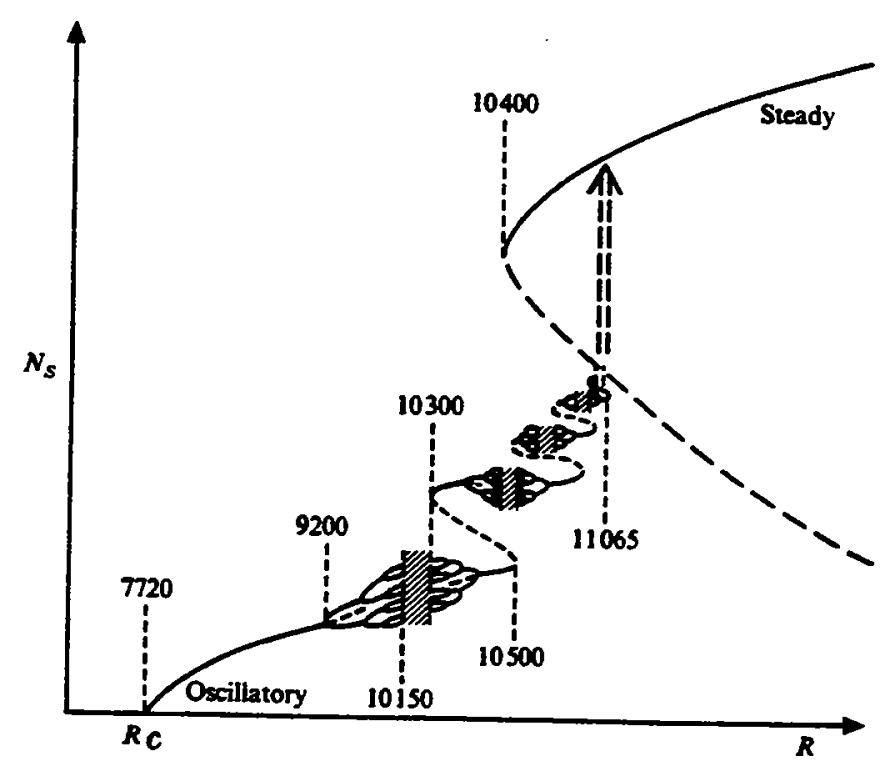

Frounz 2 Steady and oscillatory solutions of the partial differential equations: schematic bifurcation diagram of the solutal Nusselt number $N_{S}$ as a function of $R$. Note the bubbles of period-doubling bifurcations on the first and second oscillatory branches, the hysteresis loop connecting the two branches and subsequent (conjectured) branches. Conjectured unstable
solutions are indicated by broken lines. 
A parallel study of truncated Galerkin models (Da Costa et al 1981, Knobloch et al 1981, Knobloch and Weiss 1983) has proved invaluable in understanding the origin of this complex behavior. The models studied included only the modes necessary to recover the solutions of the partial differential equations to second order in amplitude and no other, i.e., they were constructed to have the correct small amplitude behavior but were otherwise drastically truncated. Such models go back to the work of Veronis (1965). Integration of the resulting ordinary differential equations (odes) revealed that the bifurcation "bubbles" are associated with the formation of a heteroclinic orbit in the phase space of the model of the type identified in the Takens-Bogdanov analysis, but now connecting a pair of saddlefoci instead of a pair of two-dimensional saddles. These (subcritical) steady states are born in a pitchfork bifurcation from the conduction solution that occurs beyond the initial Hopf bifurcation; they gain stability only after a secondary saddle-node bifurcation at which the branch of steady convection turns around (see fig. 2). The integration of these models revealed an even richer wealth of behavior (Knobloch and Weiss 1983) and in particular the presence of "gaps" in the solution branches in which no stable oscillatory solutions could be found. An appealing picture of what is going on was constructed by Glendinning and Sparrow (1984) who extended earlier work of Shil'nikov (1965) on the dynamics associated with a homoclinic orbit connecting a saddle-focus to itself in $\mathrm{R}^{3}$. Shil'nikov showed that under appropriate conditions on the eigenvalues of the saddle-focus, viz. $\delta<1$, where $\delta \equiv \alpha / \beta$, and the eigenvalues are $-\alpha \pm i \omega, \beta(\alpha>0, \beta>0)$, the system contains a countably infinite number of "horseshoes". The horseshoe construction was studied by S. Smale who showed that the invariant set that describes the long time dynamics contains an uncountable number of nonperiodic orbits as well as countably infinite number of period orbits of arbitrarily high periods, all of which are nonstable (i.e., not attracting). This then is the situation when the homoclinic orbit is present. Glendinning and Sparrow (1984) constructed a map to investigate how these orbits come into being as the homoclinic orbit forms. They showed that the formation of the homoclinic orbit is preceded by a countably infinite number of period-doubling "bubbles" with hysteretic transitions among them (see fig. 3). Within these bubbles intervals of chaos are present, much as in the partial differential equations. Glendinning and Sparrow were able to demonstrate, moreover, that the gaps in the solution branches were due to the (pairwise) formation of subsidiary homoclinic orbits. These are orbits that start from the saddle-focus and go round twice before before connecting back to the saddle-focus. Associated with each subsidiary homoclinic orbit is another countably infinite sequence of bifurcation "bubbles" and so on. Closely related behavior takes place when a heteroclinic orbit forms connecting two saddle-foci related by reflection symmetry, as in the case under discussion. Parts of this remarkable complexity were uncovered by painstaking integration of the pdes and of related odes, but in a certain sense these details obscure a very simple explanation: they are an unavoidable consequence of the formation of a homoclinic (or in the present case, heteroclinic) orbit satisfying the Shil'nikov condition.

The formation of such heteroclinic orbits cannot be followed by direct numerical integration when the Shil'nikov condition holds - precisely because of the multiplicity of solutions, hysteretic transitions among them and the presence of "gaps" in the branches containing no stable oscillations. Consequently, the ability to follow the primary solution branch even when it is unstable becomes essential in order to demonstrate that the heteroclinic orbit actually forms. This was first done for a model of magnetoconvection by Bernoff (1985) using the path-following program AUTO. Once the location of the heteroclinic connection is known the checking of the Shil'nikov condition is a simple matter of calculation the three leading 
eigenvalues at the saddle-focus. By leading eigenvalues I mean the eigenvalues with the smallest (in absolute value) real parts. In the examples under discussion there is one positive eigenvalue (due to the subcriticality of the branch) and either two real negative eigenvalues, or a complex conjugate pair with a negative real part. The former occurs when the primary Hopf and steady state bifurcations are close to one another (i.e., near the Takens-Bogdanov bifurcation), but as the Hopf and steady state bifurcations separate the termination point of the oscillatory branch moves up the unstable steady state branch and encounters complex eigenvalues. The connecting heteroclinic orbit now becomes fully three-dimensional and Shil'nikov dynamics becomes possible. While this scenario is well substantiated in the model odes no comparable studies for the pdes are available. In fact the Shil'nikov $\delta$ has never been computed in the pdes so that the scenario described here must remain a (plausible) conjecture. Only in the asymptotic regime in which the convection cells are taken to be very thin can the presence of the Shil'nikov mechanism be demonstrated systematically for the $p d e s$. In this limit the pdes can be reduced to a set of asymptotically exact odes (different from the ode models mentioned above) that are tractable semianalytically, as discussed by Proctor and Weiss (1990) and Knobloch et al $(1992,1993)$.
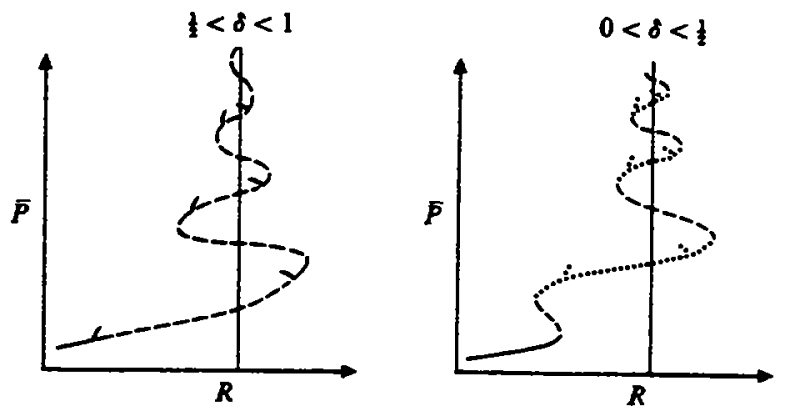

Figure 3. Variation of the normalized period $\tilde{P}$ with the bifurcation parameter $R$ in the neighborhood of a homoclinic bifurcation showing the presence of bifurcation "bubbles". The full, broken and dotted lines represent stable, non-stable and unstable solutions, respectively.

\section{Transition to chaotic travelling waves}

We now turn to region II of fig. 1 and focus on the interaction of travelling waves with steady states away from the Takens-Bogdanov bifurcation. As the primary Hopf frequency is increased (e.g., by increasing the salt gradient) the oscillation amplitude becomes larger and the saddle-node bifurcation of the SS branch comes into view. One can now ask what happens when the termination points of the SW and TW branches interact with this saddlenode bifurcation. This question can be answered in one of two ways. Instead of increasing the primary Hopf frequency one can decrease the degree of subcriticality of the SS branch thereby bringing the saddle-node bifurcation to small amplitude where it can be captured by the unfolding of a degenerate Takens-Bogdanov bifurcation. With no-flux boundary conditions this analysis was performed by Dangelmayr et al (1985) and shows that the transition from the heteroclinic orbit on the unstable SS branch to the Hopf bifurcation that takes place on the upper SS branch requires the presence of a structurally stable heteroclinic orbit. However, away from this degenerate Takens-Bogdanov bifurcation the details of this transition change (because the system becomes higher-dimensional) and can only be described by 
a local analysis near the saddle-node bifurcation (cf. Langford 1983).

The degenerate Takens-Bogdanov bifurcation with PBC has not been fully analyzed. However, the interaction of the TW termination point with the saddle-node bifurcation is amenable to simple analysis (Knobloch and Moore 1990). One finds that before the termination point reaches the saddle-node the TW branch must lose stability in a Hopf bifurcation, creating a secondary branch of modulated TW (hereafter MTW). It is the MTW that are responsible for the global bifurcation that produces chaotic travelling waves. The bifurcation in question involves a collision between the two-torus on which the MTW live and the circle of SS. By itself this is a simple process. But what makes it interesting is what is missing from such a local description, namely the dynamics resulting from the ejection of the trajectory along the unstable manifold of the SS. This process was studied by Knobloch and Moore $(1990,1991)$ using truncated Galerkin expansions of the type already described but this time with PBC, but it remains incompletely understood (Knobloch and Landsberg 1996).

The numerical integrations provide strong evidence that the MTW two-torus becomes heteroclinic to the circle of SS states and the circle of standing waves SW. To understand why this should be so consider an individual trajectory of the two-torus. At heteroclinicity this trajectory trajectory will intersect a particular steady state $\mathrm{SS}_{\phi}$, where $\phi$ denotes its spatial phase. The eigenvalues of this steady state split into two types, those lying in the reflection invariant subspace $\Sigma_{\phi}$ containing $\mathrm{SS}_{\phi}$ and those orthogonal to it (cf. Landsberg and Knobloch 1991). The former describe the stability properties of $\mathrm{SS}_{\phi}$ with respect to perturbations satisfying no-flux boundary conditions in the horizontal. Since the SS branch is subcritical at least one of these eigenvalues is positive. In the following we assume that there is exactly one such eigenvalue. The latter include a zero eigenvalue associated with phase shifts (spatial translations); we assume that the other eigenvalues are all stable. In these circumstances it follows that the trajectory spiralling into $\mathrm{SS}_{\phi}$ along the two-torus will be ejected from it along the one-dimensional unstable manifold $W_{\phi}^{u}$ in $\Sigma_{\phi}$. Generically this trajectory approaches an invariant set, such as the standing waves $\mathrm{SW}_{\phi}$, that is attracting in $\Sigma_{\phi}$. In region II the $\mathrm{SW}_{\phi}$ are unstable with respect to reflection symmetry-breaking perturbations and the $\mathrm{SW}_{\phi}$ decay towards $\mathrm{TW}$. Since the TW are themselves unstable to MTW the trajectory, after it leaves the $\mathrm{SW}_{\phi}$, lies on the original two-torus, and is thus brought back to the SS circle, but now with a different phase due to the the spatial translation undergone during the transition from the $\mathrm{SW}_{\phi}$ back to the SS. The whole process now repeats with a different value of $\phi$. Thus one ends up with a trajectory connecting the two circles of SS and SW. Note that because $\phi$ is constant during "half" of the above cycle the trajectory on the heteroclinic torus corresponds to spatial translation in "fits and starts". field,

The Galerkin equations for two-dimensional convection in an imposed horizontal magnetic

$$
\begin{aligned}
a^{\prime} & =-\sigma a+\sigma r b-\sigma \zeta q d+\sigma \zeta(1-\varpi) q d e \\
b^{\prime} & =-b+a-a c \\
c^{\prime} & =-\varpi c+\frac{1}{2} \varpi(a \bar{b}+\bar{a} b) \\
d^{\prime} & =-\zeta d+a-a e \\
e^{\prime} & =-\zeta \varpi e+\frac{1}{2} \varpi(a \bar{d}+\bar{a} d),
\end{aligned}
$$

are among the simplest exhibiting this behavior. Here $a, \ldots, e$ are amplitudes of appropriate spatial modes, the prime denotes differentiation with respect to a suitably scaled time, and 
$r, q, \sigma, \zeta$ and $\varpi$ are, respectively, the (scaled) Rayleigh number, the (scaled) Chandrasekhar number, the Prandtl number, the ratio of ohmic diffusivity to thermal diffusivity, and a geometrical factor $(0<\varpi<4)$ related to the wavenumber $k$ of the mode that first becomes unstable. The reflection-invariant subspace $\Sigma_{0}$ defined by real $a, \ldots, e$ is invariant under the dynamics, and contains both $\mathrm{SS}_{0}$ and $\mathrm{SW}_{0}$. Conjugate subspaces $\Sigma_{\phi}$ may be defined by spatial translation $x \rightarrow x+\phi / k$. Knobloch and Moore (1991) report results obtained for $q=1.0, \sigma=1.0, \zeta=0.1$ and $\varpi=2.66666666667$. The conduction state $a=\ldots=e=0$ loses stability in a Hopf bifurcation producing stable TW and unstable but supercritical SW. The TW amplitude increases monotonically with $r-r_{c}$ before terminating on the unstable SS branch below the saddle-node (fig. 4). Before doing so it loses stability in a secondary Hopf bifurcation producing a branch of MTW. With increasing $r$ the modulation period increases asymptotically as $-\ln \left(r_{k}-r\right)$ suggesting the presence of a global bifurcation at $r=r_{k}$. Fig. 5 shows $c(t)$ for $r=1.335887484$, a value near $r_{h}$. Since $c$ (like $e$ ) is a mean mode it is singly periodic, with its period equal to the modulation period. The solution spends a significant length of time near $c=0.114154921$, the value of $c$ corresponding to the unstable SS at $r=r_{h}$. In between, however, the solution drops rapidly, executing a number of fast small oscillations, before growing back to the SS state. Fig. 6 shows a blowup of the projection of these oscillations onto the $(c, e)$ plane, showing that the trajectory approaches a limit cycle (i.e. the standing wave $\mathrm{SW}$ ) before slowly spiralling away. This spiralling away is the consequence of a weakly unstable Floquet multiplier of the SW in a direction perpendicular to $\Sigma_{\phi}$ and it takes the trajectory from the SW onto the MTW two-torus. This process is shown in a different projection in fig. 7. In this plane the $\mathrm{SS}_{\phi}$ form a circle of fixed points (not shown) centered on the origin, the TW form a limit cycle (not shown) surrounding the origin and lying inside the $\mathrm{SS}$ circle, and the $\mathrm{SW}_{\phi}$ correspond to oscillations in straight lines through the origin whose orientation is specified by $\phi$. The figure shows clearly the "capture" of the MTW trajectory by the reflection-invariant subspace, the SW part of the trajectory, and the subsequent decay of the SW into a growing TW. Such a trajectory visits in due course all $\mathrm{SS}_{\phi}$ and all $\mathrm{SW}_{\phi}$; in the limit $r=r_{h}$ it provides the promised connection between the two circles of solutions. Note that this trajectory, although complicated, is quasiperiodic and not chaotic. Knobloch and Moore (1991) also find circumstances under which the corresponding trajectory is chaotic, with chaos appearing via a torus-doubling cascade. In their example the SS eigenvalues out of $\Sigma$ are both real. In other problems of this type, such as the triple zero bifurcation with $\mathrm{O}(2)$ symmetry (Knobloch and Landsberg 1996), one finds the formation of chaotic "bubbles" reminiscent of the behavior encountered for SW (fig. 2), and most likely due to Shil'nikov-like dynamics when the two SS eigenvalues out of $\Sigma$ form a complex conjugate pair. Note, finally, that the invariant set responsible for the ejection of the trajectory out of $\Sigma_{\phi}$ may be more complicated than a limit cycle. We have seen in the previous section that the SW in $\Sigma_{\phi}$ can be chaotic and that for other parameter values SW of any period can be found. For the thermohaline problem all of these are unstable to symmetry-breaking perturbations (Knobloch et al. 1986a). It follows that by varying parameters one can locate connections between the SS circle and, for example, a circle of strange "attractors". In particular one should be able to find a codimension-two heteroclinic connection to countably many horseshoes! Such solutions would resemble figs. 5 and 8 but would sample intervals of chaotic standing waves separated by intervals of translation, with both intervals getting longer and longer as $r \rightarrow r_{h}$. A more detailed account of these mechanisms for generating chaotic travelling waves can be found in Knobloch and Landsberg (1996). 


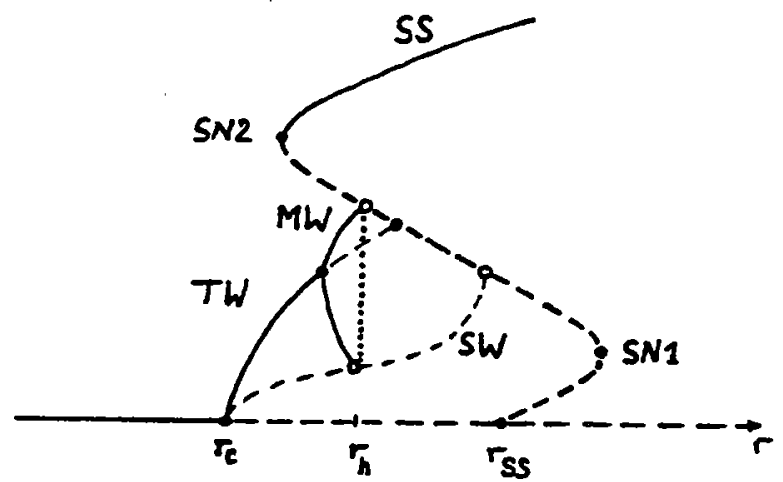

Fig. 4. A typical bifurcation diagram (|a| $\left.\right|^{2}$ vs. $r$ ) for the system (1). The filled (open) circles represent local (global) bifurcations. Solid (dashed) lines indicate stable (unstable) branches. The $T W$ and $M W$ branches terminate between the two saddle-node bifurcations $S N 1, S N 2$. The heteroclinic orbit at $r=r_{h}$ is indicated by a dotted line.

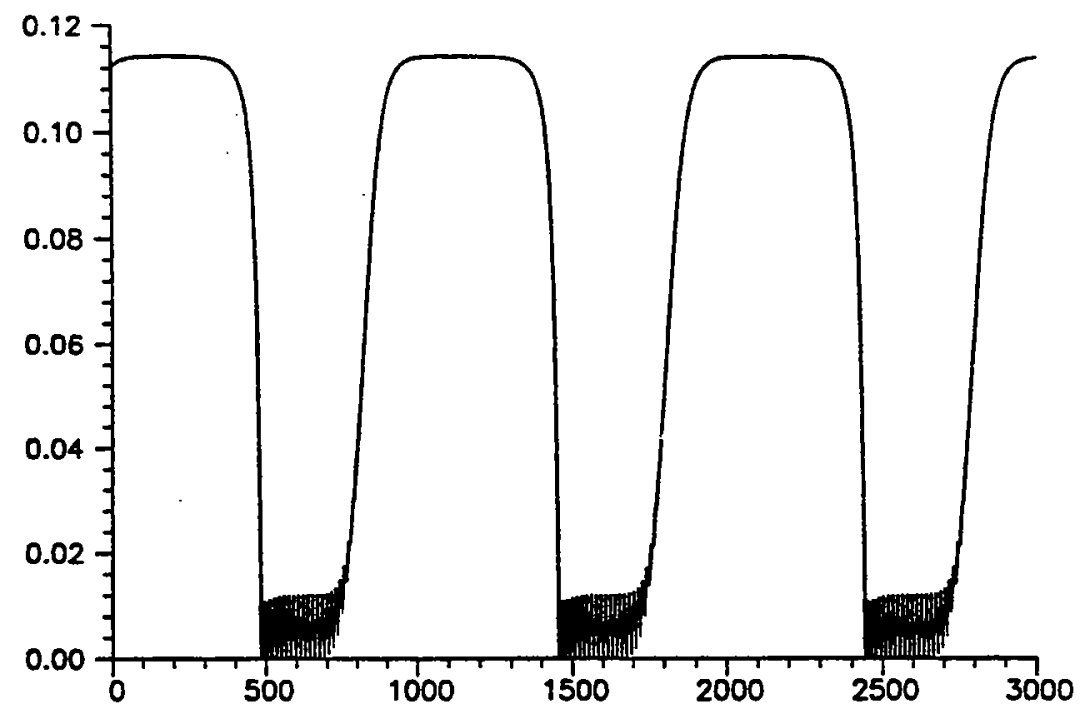

Fig. S. The time series $c(t)$ for $r=1.335887484, q=1.0, \sigma=1.0, \zeta=1.0$ and $\varpi=2.66666667$.

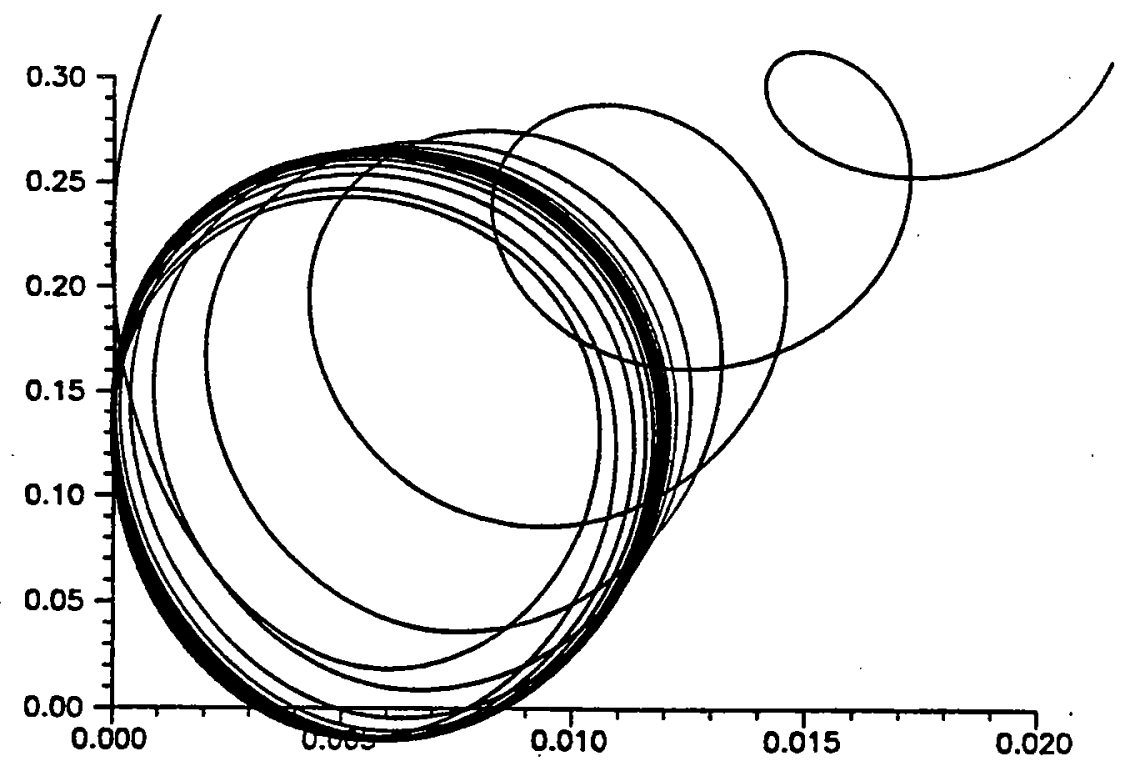

Fig. 6. As for fig. 5 but showing a detail of the $(c, e)$ piane. 
The mechanisms outlined above are not just a dynamical curiosity. A recent paper by Cox et al.(1992) on nonlinear Langmuir circulation reports direct numerical simulations of the associated pdes with PBC, and presents strong evidence for the presence of a global bifurcation of the above type (see fig. 8). The pdes for this problem are closely related to those for thermohaline convection in which chaotic travelling waves have been found (Deane et al. 1987). Whether a connection of the above type is responsible for the existence of these waves is unknown.

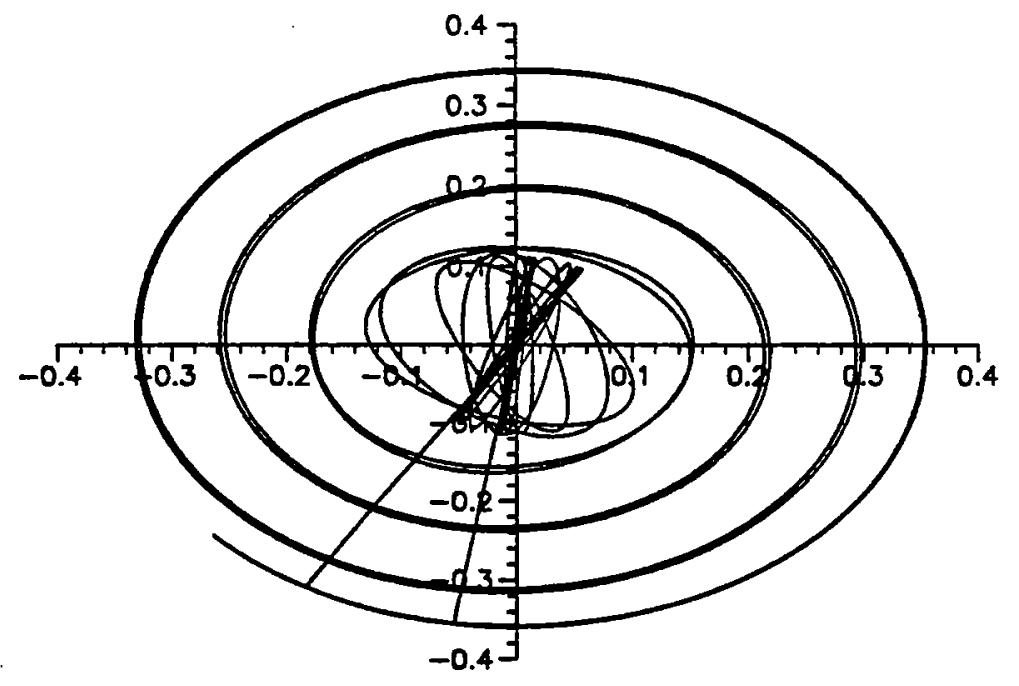

Figure 7. As for fig. 5 but showing the (Re a, Im a) plane.

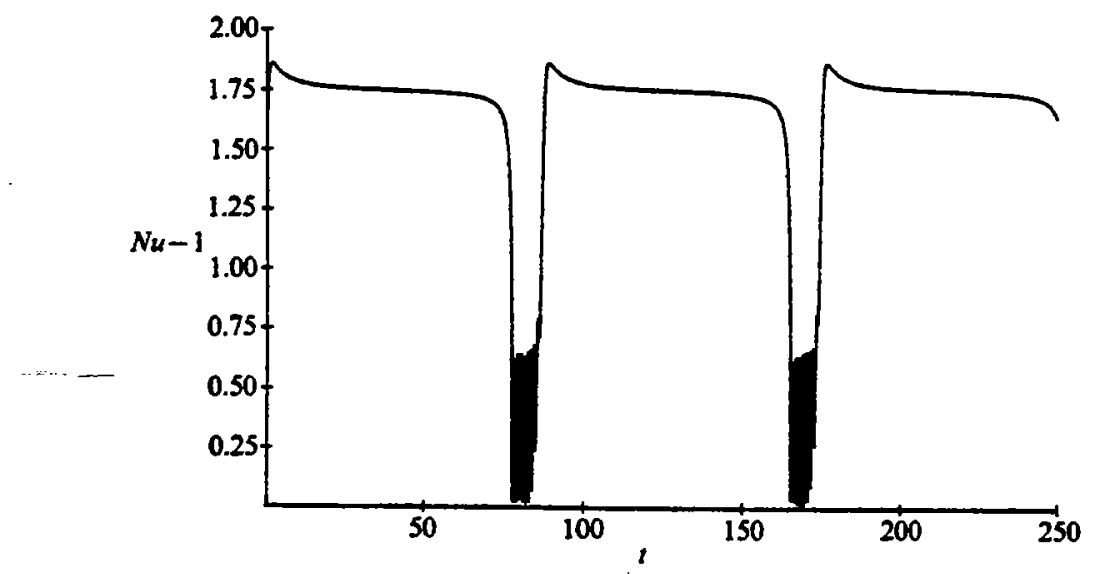

Figure 8. Nusselt number plotted against time, $t$, for the $M W$ solution near its heteroclinic bifurcation with the unstable SS and SW solutions. The unstable SS has $N u-1 \approx 1.76$, which we identify with the long quasi-stesdy portion of the Nusselt-number time trace.

\section{References}

[1] A.J. Bernoff, Ph.D. thesis, University of Cambridge (1985).

[2] S.M. Cox, S. Leibovich, I.M. Moroz and A. Tandon, J. Fluid Mech. 241 (1992) 669-704.

[3] P.H. Coullet and E.A. Spiegel, SIAM J. Appl. Math. 43 (1983) 776-821.

[4] L. N. Da Costa, E. Knobloch and N.O. Weiss, J. Fluid Mech. 109 (1981) 25-43. 
[5] G. Dangelmayr and E. Knobloch, Phil. Trans. R. Soc. London A, 322 (1987) 243-279.

[6] G. Dangelmayr, D. Armbruster and M. Neveling, Z. Phys. B 59 (1985) 365-370.

[7] A.E. Deane, E. Knobloch and J. Toomre, Phys. Rev. A 36 (1987) 2862-2869.

[8] P.A. Glendinning and C.T. Sparrow, J. Stat. Phys. 35 (1984) 645-696.

[9] H.E. Huppert and D.R. Moore, J. Fluid Mech. 78 (1976) 821-854.

[10] E. Knobloch, Phys. Rev. A 34 (1986) 1538-1549.

[11] E. Knobloch, A. Deane, J. Toomre and D.R. Moore, Contemporary Math. 56 (1986a) 203-216.

[12] E. Knobloch and A.S. Landsberg, preprint (1996).

[13] E. Knobloch and D.R. Moore, Phys. Rev. A 42 (1990) 4693-4709.

[14] E. Knobloch and D.R. Moore, European J. Mech. B 10, no. 2-Suppl. (1991) 37-42.

[15] E. Knobloch, D.R. Moore, J. Toomre and N.O. Weiss, J. Fluid Mech. 166 (1986b) 409-448.

[16] E. Knobloch and M.R.E. Proctor, J. Fluid Mech. 108 (1981) 291-316.

[17] E. Knobloch, M.R.E. Proctor and N.O. Weiss, J. Fluid Mech. 239 (1992) 273292.

[18] E. Knobloch, M.R.E. Proctor and N.O. Weiss, in Turbulence in Fluid Flows: A Dynamical Systems Approach, G.R. Sell, C. Foias and R. Temam (eds), SpringerVerlag (1993) pp. 59-72.

[19] E. Knobloch and N.O. Weiss, Physica D 9 (1983) 379-407.

[20] E. Knobloch, N.O. Weiss and L.N. Da Costa, J. Fluid Mech. 113 (1981) 153-186.

[21] A.S. Landsberg and E. Knobloch, Phys. Lett. A 159 (1991) 17-20.

[22] W.F. Langford, in Nonlinear Dynamics and Turbulence, G.I. Barenblatt, G. Iooss and D.D. Joseph (eds), Pitman (1983) pp. 215-237.

[23] D.R. Moore, J. Toomre, E. Knobloch and N.O. Weiss, Nature 303 (1983) 663667.

[24] D.W. Moore and E.A. Spiegel, Ap. J. 143 (1966) 871-887.

[25] M.R.E. Proctor and N.O. Weiss, Nonlinearity 3 (1990) 619-637.

[26] L.P. Shil'nikov, Sov. Math. Dokl. 6 (1965) 163-166.

[27] J.W. Swift and K. Wiesenfeld, Phys. Rev. Lett. 52 (1984) 705-708.

[28] G. Veronis, J. Mar. Res. 23 (1965) 1-17. 


\title{
Magnetoconvection
}

\author{
Michael Proctor \\ University of Cambridge, U.K.
}

In these lectures, I discussed the effects of magnetic fields on thermal convection, a subject motivated by the dynamically important fields in the the solar convection zone. After a brief resume of magnetohydrodynamics, I developed models of incompressible (Boussinesq) and compressible convection in the presence of an imposed magnetic field. Important phenomena discussed included the occurrence of oscillatory convection, the reduction of preferred horizontal scale, and the possibility of instabilities with horizontal streaming.

In the lecture, I showed several videos of simulations of nonlinear convection, and discussed these in relation both to the basic theory and to the behavior of solar magnetic fields. 


\title{
Wave Propagation in Granular Flows
}

\author{
Jonathan Wylie
}

\section{Introduction}

Granular flows are the movement of large numbers of discrete solid particles. In general, the gaps between solid particles will be filled with a fluid (eg. air). However, if the particles are closely packed and more dense than the interstitial fluid then the majority of the momentum transfer occurs via particle-particle interaction, thus we can neglect the effects of the fluid. In this case, there are two extreme cases of granular flows. The first is the so called quasi-static limit. Here frictional forces between grains dominate the dynamics. In this regime granular material can form hills with finite slopes and support loads without yielding. If the load becomes too large the friction between grains can no longer support it and so the particles begin to flow slowly. This regime has been extensively studied by considering Coulomb friction based plasticity models.

The second case is that of rapid granular flow. In this regime particles are highly agitated and collide rapidly with their neighbors. The dynamics are controlled by the collisions between grains. The grains are analagous to molecules in a dense gas; that is, the grains have large apparently random velocities relative to one another. What distinguishes granular media from a perfect dense gas is the fact that collisions between grains are inelastic and so energy is lost during collisions. Examples of rapid granular flows are landslides and rapid sand flows.

We adopt a methodology based on the kinetic theory of dense gases with col- 
lisional loss terms incorporated (Haff 1993). We assume that the particles are all approximately spherical with diameter $d$ and mass $m$. We decompose the velocity of the flow into two distinct components: the mean velocity of the bulk material $\mathbf{u}$ and an apparently random fluctuation from the mean $\mathbf{u}^{\prime}$.

We define the root-mean-square of this random flucuation velocity to be $q$, which we refer to as the agitation,

$$
q \equiv \sqrt{\left\langle\mathbf{u}^{\prime} \cdot \mathbf{u}^{\prime}\right\rangle}
$$

The particles are continuously colliding and thus changing their separation, but we define the mean separation $s$ such that the bulk mass density $\rho$ is given by

$$
\rho=\frac{m}{(s+d)^{3}} .
$$

We restrict our attention to the case of large bulk mass density, where the separation between particles is much smaller than the particle diameter; that is

$$
s \ll d
$$

Thus,

$$
\rho \sim \frac{m}{d^{3}}
$$

and so the media is approximately of constant density.

In writing down the equations governing the conservation of mass, momentum and energy we adopt the approach used in fluid mechanics. Since the density is approximately constant, conservation of mass takes the form

$$
\nabla \cdot \mathbf{u}=\mathbf{0}
$$

Using dimensional arguments (Bagnold 1954) the momentum equation takes the form

$$
\rho \frac{\partial u_{i}}{\partial t}=-\rho \frac{\partial}{\partial x_{k}}\left[\frac{p \delta_{i k}}{\rho}+u_{i} u_{k}-\nu\left(\frac{\partial u_{i}}{\partial x_{k}}+\frac{\partial u_{k}}{\partial x_{i}}\right)\right]+\rho g_{i}
$$


where $g_{i}$ is the component of gravity in the $i$-direction, $p$ is the pressure and $\nu$ is the viscosity. The pressure and the viscosity depend on the agitation of the media and the mean separation between particles. The energy equation takes the form

$\rho \frac{\partial}{\partial t}\left(\frac{u^{2}}{2}+\frac{q^{2}}{2}\right)=-\rho \frac{\partial}{\partial x_{k}}\left[u_{k}\left(\frac{p}{\rho}+\frac{u^{2}}{2}+\frac{q^{2}}{2}\right)-\nu u_{i}\left(\frac{\partial u_{i}}{\partial x_{k}}+\frac{\partial u_{k}}{\partial x_{i}}\right)-\kappa \frac{\partial}{\partial x_{k}}\left(\frac{q^{2}}{2}\right)\right]+\rho u_{i} g_{i}-I$,

where $\kappa$ acts like a diffusivity of agitation and $I$ represents the rate at which energy is lost due to the fact that grain-grain collisions are inelastic. The second term on the right hand side is due to the viscous generation of agitation from the large scale shear, which is equivalent to shear-heating in fluid mechanics. As in the case for $p$ and $\nu$, both $\kappa$ and $I$ also depend on $q$ and $s$.

In order to close the system we must relate the quantities $p, \nu, \kappa, I$ to the agitation and mean seperation. We follow the simple cage model (Hirshfelder, Curtis \& Bird 1964) where each particle is envisaged as being closely surrounded by a 'cage' of other particles. The central particle then vibrates with speed $q$ and collides with the particles which form the cage. The average distance between the central particle and the cage is $s$. Therefore the collision frequency, $f$, scales as

$$
f \sim q / s .
$$

The momentum transfered in a collision scales as $m q$ and because the $s \ll d$ the surface area of the cage scales as $d^{2}$. Hence

$$
p \sim(m q) f \frac{1}{d^{2}}
$$

Rewriting, we obtain

$$
p=\hat{p} d \rho \frac{q^{2}}{s}
$$

where $\hat{p}$ is the dimensionless constant of proportionality.

In deriving the coefficient of viscosity we consider grain collisions between two layers. The upper layer moves with velocity $\Delta u$ relative to the lower layer. The 
collisions provide tangential stress between the two layers. The average momentum transfer per collision is $m \Delta u$ and the collision frequency is $q / s$. Therefore the tangential shear stress exerted by the upper layer on the lower layer is proportional to

$$
\frac{m \Delta u}{d^{2}} \frac{q}{s} .
$$

The shear rate $\partial u / \partial y$ scales as $\Delta / d$ since the two layers are a distance of order $d$ apart. Therefore the stress scales as

$$
d^{2} \rho \frac{q}{s} \frac{\partial u}{\partial y} .
$$

So the viscosity is given by

$$
\nu=\hat{\nu} d^{2} \frac{q}{s},
$$

where $\hat{\nu}$ is the dimensionless constant of proportionality.

By a similar argument the agitation diffusivity can be found as

$$
\kappa=\hat{\kappa} d^{2} \frac{q}{s},
$$

where as before $\hat{\kappa}$ is the constant of proportionality.

To model the collisional energy sink we suppose that the coefficient of restitution for a collision between two grains takes a constant value $e$. Therefore the energy loss per collision scales as

$$
\frac{1}{2} m q^{2}\left(1-e^{2}\right) .
$$

Multiplying by the collision frequency and the number of particles per unit volume we obtain

$$
I=\hat{\gamma} \frac{q^{3}}{s},
$$

where $\hat{\gamma}$ is the dimensionless constant proportional to $1-e^{2}$. 


\section{Long Wavelength approximation}

We consider a layer of particles of depth $h(x, t)$ on a, in general, sloping boundary. Taking the long-wavelength limit (shallow layer approximation) we find that the governing equations become

$$
\begin{gathered}
u_{x}+w_{z}=0 \\
\frac{D u}{D t}=-d \hat{p}\left(\frac{q^{2}}{s}\right)_{x}+g \sin \theta+d^{2} \hat{\nu}\left(\frac{q}{s} u_{z}\right)_{z} \\
0=-d \hat{p}\left(\frac{q^{2}}{s}\right)_{z}-g \cos \theta
\end{gathered}
$$

and

$$
\frac{1}{2} \frac{D q^{2}}{D t}=d^{2} \hat{\nu} \frac{q}{s} u_{z}^{2}+d^{2} \hat{\kappa}\left[\frac{q}{s}\left(\frac{q^{2}}{2}\right)_{z}\right]_{z}-\hat{\gamma} \frac{q^{2}}{s} q .
$$

These equation must be supplemented by suitable boundary conditions. At the surface these conditions are relatively straightforward. The kinematic condition

$$
w=\frac{D h}{D t} \quad \text { at } \quad z=h,
$$

zero tangential surface stress

$$
d^{2} \hat{\nu}\left(\frac{q}{s} u_{z}\right) \rightarrow 0 \quad \text { as } \quad z \rightarrow h
$$

and zero energy flux through the surface

$$
d^{2} \hat{\kappa}\left(\frac{q}{s}\left(\frac{q^{2}}{2}\right)_{z}\right) \rightarrow 0 \quad \text { as } \quad z \rightarrow h .
$$

The boundary conditions that must be applied at the base are more complex and will depend upon the nature of the boundary and any energetic forcing which occurs at the boundary. 
Integrating the vertical momentum equation and applying the boundary condition of zero presure at the free surface we obtain the hydrostatic balance

$$
d \hat{p}\left(\frac{q^{2}}{s}\right)=g(h-z) \cos \theta
$$

Using this relation to eliminate $s$ from the remaining equations we obtain

$$
\begin{gathered}
u_{x}+w_{z}=0 \\
\frac{D u}{D t}=-g h_{x} \cos \theta+g \sin \theta+\frac{d g \cos \theta \hat{\nu}}{\hat{p}}\left(\frac{(h-z)}{q} u_{z}\right)_{z}
\end{gathered}
$$

and

$$
q \frac{D q}{D t}=d \frac{g \cos \theta \hat{\nu}}{\hat{p}} \frac{u_{z}^{2}}{q}+d \frac{g \cos \theta \hat{\kappa}}{\hat{p}}\left((h-z) q_{z}\right)_{z}-\frac{g \cos \theta \hat{\gamma}}{d \hat{p}}(h-z) q .
$$

\section{Model Equations}

In general the only way to solve the above set of equations is by projection onto a truncated set of Galerkin modes. However, we choose a slightly more heuristic approach in order to gain more physical understanding. Averaging across the layer and making use of the surface boundary conditions gives

$$
\begin{gathered}
\partial_{t} h+\partial_{x}\left(\int_{0}^{h} u \mathrm{~d} z\right)=0, \\
\partial_{t}\left(\int_{0}^{h} u \mathrm{~d} z\right)+\partial_{x}\left(\int_{0}^{h} u^{2} \mathrm{~d} z\right)=h g \sin \theta-h h_{x} g \cos \theta-\left.h \frac{d g \cos \theta \hat{\nu}}{\hat{p}} \frac{u_{z}}{q}\right|_{z=0},
\end{gathered}
$$


$\partial_{t}\left(\int_{0}^{h} \frac{q^{2}}{2} \mathrm{~d} z\right)+\partial_{x}\left(\int_{0}^{h} u \frac{q^{2}}{2} \mathrm{~d} z\right)=\frac{g \cos \theta}{\hat{p}}\left[\hat{\nu} d \int_{0}^{h} \frac{(h-z) u_{z}^{2}}{q} \mathrm{~d} z-\left.\hat{\kappa} d h q_{z}\right|_{z=0}-\frac{\hat{\gamma}}{d} \int_{0}^{h}(h-z) q \mathrm{~d} z\right]$

We follow an approach similar in principle to that used in deriving the shallow water equations with viscous drag. The conservation of mass equation becomes

$$
\partial_{t} h+\partial_{x}(h U)=0
$$

For the momentum equatiion we estimate the shear stress at the base as a 'Rayleigh drag' to obtain

$$
\partial_{t}(h U)+\partial_{x}\left(h U^{2}\right)=h g \sin \theta-h h_{x} g \cos \theta-h d g \cos \theta \epsilon\left(\frac{U}{h} \frac{1}{Q}\right),
$$

where $\epsilon$ represents the dimensionless drag coefficient.

When the agitation flux is not given at the base, we should in general examine the exact nature of the boundary condition in effect at the lower boundary. However, following similar arguments for obtaining the drag coefficient, we suppose that the loss of agitation through the lower boundary can be modelled as a 'Newtonian cooling'. Hence

$$
\partial_{t}\left(h Q^{2}\right)+\partial_{x}\left(h U \frac{Q^{2}}{2}\right)=d g \cos \theta\left[\epsilon \frac{h^{2}}{Q} \frac{U^{2}}{h^{2}}+K d h \frac{Q}{h}-\frac{\Gamma h^{2} Q}{d}\right]
$$

where $K$ represents the dimensionless agitation loss parameter and $\Gamma$ represents the collisional loss coefficient.

\section{Landslides and chute flows}

Consider the case where a granular material flows down a slope. Then after subtracting the continuity equation from the other two equations we obtain 


$$
\begin{gathered}
\partial_{t} h+\partial_{x}(h U)=0 \\
U_{t}+U U_{x}=g \sin \theta-h_{x} g \cos \theta-d g \cos \theta \epsilon \frac{U}{h Q} \\
\partial_{t}\left(\frac{Q^{2}}{2}\right)+U \partial_{x}\left(\frac{Q^{2}}{2}\right)=d g \cos \theta\left[\epsilon \frac{U^{2}}{Q h}-\frac{\Gamma}{d^{2}} h Q-\frac{K}{h}\right] .
\end{gathered}
$$

\subsection{Steady Flows}

We first examine the case of spatially homogeneous solutions of the above equations. Then continuity implies that the height of the layer must remain at a constant depth,

$$
\begin{gathered}
h=h_{0}, \\
\frac{1}{g \cos \theta} U_{t}=\tan \theta-\frac{d \epsilon}{h_{0}}\left(\frac{U}{Q}\right), \\
\frac{1}{g \cos \theta} Q_{t}=\frac{d^{2} \epsilon}{h_{0}}\left(\frac{U}{Q}\right)^{2}-\frac{\Gamma h_{0}}{d}-\frac{K d}{h_{0}}
\end{gathered}
$$

It is clear that the above equations do not in general have any steady states. We define a critical slope $\theta_{c}$ by

$$
\tan \theta_{c}=\epsilon \Gamma
$$

If $\theta<\theta_{c}$ (Shallow slopes) there are no steady solutions, whereas for $\theta>\theta_{c}$ (Steep slopes) the only steady flow that exists is for a particular value of the depth of the layer given by

$$
h_{0 c}^{2}=\frac{\epsilon K}{\tan ^{2} \theta-\epsilon \Gamma}
$$


For this value of the height there are is an infinite one-parameter set of steady solutions satisfying

$$
\frac{U}{Q}=\frac{h_{0} \tan \theta}{\epsilon d}
$$

For the shallow slopes and values of $h$ other than the critical depth, the dynamics are unsteady. In order to examine these flows we introduce the variable

$$
R \equiv \frac{U}{Q}
$$

so that the above equations become

$$
\begin{gathered}
\frac{h_{0}}{d g \cos \theta} Q R_{t}=-\epsilon R^{3}+\left(\frac{h_{0}^{2} \Gamma}{d^{2}}+K-\epsilon\right) R+\frac{h_{0} \tan \theta}{d} \equiv f(R) \\
\frac{h_{0}}{d \epsilon g \cos \theta} U_{t}=\frac{h_{0} \tan \theta}{d \epsilon}-R .
\end{gathered}
$$

By definition $Q$ must be positive and we are only interested in the case where $U$ is positive, since $U$ being negative corresponds to flows going up a slope. Therefore we need only consider positive $R$. Plotting $f$ against $R$ we see that there is always a unique positive root. This root is also clearly always stable. We deduce that all trajectories tend to a fixed ratio. The time scale on which trajectories approach the fixed ratio scales as $d \epsilon g \cos \theta / h_{0} Q$. Although all trajectories tend to a fixed ratio, only the special case where the depth of the layer takes its critical value gives steady solutions.

The time dependent behavior is now easily determined as being in one of three cases (See Figure 1). For steep slopes and thick layers $\left(\theta>\theta_{c}\right.$ and $\left.h_{0}>h_{0 c}\right)$ all flows continuously accelerate and become more agitated. The agitation and velocity both increase linearly with time and so the average separation $s$ increases as the square of time. This continuous acceleration can be understood by considering the nature of the frictional drag experienced by the flow. When $U / Q$ is a constant the frictional 
force is also a constant and if this is less than the acceleration due to gravity the layer will experience constant acceleration. Since our assumption in deriving the density equation and the constitutive relations required that $s \ll d$ it is clear that the model must break down at some time when the behavior will depend on the nature of the correction to the constitutive relation which is chosen.

For steep slopes and thin layers $\left(\theta>\theta_{c}\right.$ and $\left.h_{0}<h_{0 c}\right)$ or for shallow slopes $\left(\theta<\theta_{c}\right)$ all flows eventually decelerate and lose agitation until they reach the zero agitation state in finite time. At this point the granular material loses its fluidization and will stop, or at least go into a flow regime governed by the frictional forces between individual grains.

The limiting regime between these two cases is that of steep slope and critical layer depth $\left(\theta>\theta_{c}\right.$ and $\left.h_{0}=h_{0 c}\right)$. In this case all flows will eventually tend to a steady solution with the values of $U$ and $Q$ determined by initial conditions.

\subsection{Fixed Mass Flux Flows}

We now turn our attention to steady spatially developing flows where we input a fixed mass flux at some point on a slope. In this case continuity implies that the mass flux is fixed everywhere along the slope

$$
U h=J .
$$

Then the equations for momentum and agitation become

$$
\begin{gathered}
\left(U-\frac{J g \cos \theta}{U^{2}}\right) U_{x}=g \sin \theta-\frac{d g \cos \theta \epsilon}{J} \frac{U^{2}}{Q} \\
Q_{x}=d g \cos \theta\left(\frac{\epsilon}{J} \frac{U^{2}}{Q^{2}}-\frac{\Gamma J}{d} \frac{1}{U^{2}}-\frac{K}{J}\right) .
\end{gathered}
$$


There are only fully developed solutions for slopes above the critical value $\left(\tan ^{2} \theta>\right.$ $\epsilon \Gamma):$

$$
\begin{aligned}
U_{0}^{2} & =\frac{J^{2}\left(\tan ^{2} \theta-\epsilon \Gamma\right)}{\epsilon d^{2} K} \\
Q_{0} & =\frac{J\left(\tan ^{2} \theta-\epsilon \Gamma\right)}{\epsilon d K}
\end{aligned}
$$

At the point where $U^{3}=J g \cos \theta$ the momentum equation becomes singular. To examine this more closely we write the equation in the form

$$
\left(\frac{U^{2}}{2}+g \cos \theta h\right)_{x}=\text { gravitational source }- \text { viscous sink }
$$

The quantity in the brackets can be thought of as the available mechanical energy of the layer. The constant mass flux implies that this available mechanical energy has a minimum. If we now consider the case where the viscous sink term dominates the gravitational source term then the energy will decrease until it reaches its minimum. At this point a 'hydraulic catastrophe' occurs and time-independent flow cannot continue.

As an example, consider the case where we impose a fixed mass flux and the height of the layer at the source such that the the initial velocity is greater than the gravity wave speed, so that information only propagates downstream. If the viscous term is dominant then the velocity will decrease until it reaches the hydraulic catastrophe. At this point the velocity and the gravity wave speed are equal and so a nonlinear bore is generated which travels upstream and violates the source boundary condition. We note that Johnson et al were able to create sttaionary hydraulic jumps in their granular flow experiments.

We can now examine the behavior of flows as they move down a slope. Ignoring nongeneric examples there are three distinct cases.

1. Steep slope $\left(\theta>\theta_{c}\right)$ and weak conductive loss to the base $U_{0}^{3}>J g \cos \theta$ 
(Figure 2). In this case flows develop into one of three different possibilities:

a) The flow evolves to the uniform state,

b) Flows decelerate and the agitation goes to zero so that the flow loses its fluidisation in a finite distance,

c) The flow ends in a hydraulic catastrophe.

2. Steep slope $\left(\theta>\theta_{c}\right)$ and strong conductive loss to the base $U_{0}^{3}>J g \cos \theta$ (Figure 3). In this case flows develop into one of two different possibilities:

a) Flows decelerate and the agitation goes to zero so that the flow loses its fluidisation in a finite distance,

b) The flows ends in a hydraulic catastrophe.

3. Shallow slopes $\left(\theta>\theta_{c}\right)$. In this case all flows terminate in the same way (Figure 4):

a) Flows end in a hyraulic catastrophe.

We note the important role played by the agitation loss through the base. In the case where $K=0$ there are no fully developed flows except at the critical slope. For slopes greater than critical the flow can continuously accelerate and get thinner as it goes down the slope. The asymptotic behavior is given by $U \sim x^{1 / 4}, h \sim x^{-1 / 4}$ and $Q \sim x^{1 / 2}$. 


\subsection{Finite Mass Release}

We now examine the finite mass release case. In order to do this it is most informative to write the equations in characteristic form:

$$
\begin{array}{ccc}
\frac{d}{d t}\left[U+2(g h \cos \theta)^{1 / 2}\right]=g \sin \theta-\epsilon d g \cos \theta \frac{U}{Q h} \quad \text { on } & \frac{d x}{d t}=U+(g h \cos \theta)^{1 / 2} \\
\frac{d}{d t}\left[U-2(g h \cos \theta)^{1 / 2}\right]=g \sin \theta-\epsilon d g \cos \theta \frac{U}{Q h} \quad \text { on } \quad \frac{d x}{d t}=U-(g h \cos \theta)^{1 / 2} \\
\frac{d Q}{d t}=d g \cos \theta\left(\epsilon \frac{U^{2}}{Q^{2} h}-K \frac{Q}{h}-\frac{\Gamma h Q}{d^{2}}\right) \quad \text { on } \quad \frac{d x}{d t}=U
\end{array}
$$

We can now, in principle, calculate the spatial and temporal evolution of a flow. We consider a flow in which $h=0$ at the front. In this case all the characteristics coincide, but the front is retarded by the frictional force which is infinite at the front (the constitute relations obviously break down as $h$ tends to zero, but the friction can be expected to be large there). Therefore a shock will form at the front of the flow.

There is also the possibility of shock formation, rarefaction and contact discontinuities away from the front. This will be the subject of a future study.

\section{Agitated Flat Plate}

If we consider the parameter range where both mechanical and agitation inertia are negligible then we do not need to resort to the model equations. We can integrate the momentum equation and use the condition of zero tangential stress to obtain 


$$
u_{z}=-\frac{\hat{p}}{d \hat{\nu}} q\left(h_{x}-\tan \theta\right) .
$$

Substitution into the agitation equaion yields

$$
\left((h-z) q_{z}\right)_{z}+\frac{1}{d^{2}}\left(\frac{\hat{p}^{2}}{\hat{\kappa} \hat{\nu}}\left(h_{x}-\tan \theta\right)^{2}-\frac{\hat{\gamma}}{\hat{\kappa}}\right)(h-z) q=0
$$

For the continuum assumption to hold we require that the thermal diffusion length scale is much larger than the diameter of a grain. Hence we must restrict our analysis to the case where collisional loss and shear generated agitation are small.

We consider the case where a layer of grains lays on a flat plate (ie. $\theta=0$ ) and energy is supplied to the layer by collisions with the plate. If the plate moves in a sawtooth motion then a simple argument shows that the rate at which the plate does work on the layer is proportional to the mass of the layer. If we assume that the majority of the work done by the plate goes into agitation then we can impose a boundary condition of constant agitation flux per unit height of the layer: that is,

$$
q_{z}=-\frac{\mathcal{F} \hat{p}}{\hat{\kappa} d g} \quad \text { at } \quad z=0 .
$$

There are two distinct cases. Firstly,

$$
\frac{1}{d^{2}}\left(\frac{\hat{p}^{2}}{\hat{\kappa} \hat{\nu}} h_{x}^{2}-\frac{\hat{\gamma}}{\hat{\kappa}}\right)=-M^{2}<0
$$

in which case the solution can be found in terms of Imaginary Bessel functions:

$$
q=\frac{\mathcal{F} \hat{p}}{\hat{\kappa} d g} \frac{I_{0}[M(h-z)]}{M I_{0}^{\prime}[M h]} .
$$

In this case collisional loss overcomes the shear generation and so agitation is being supplied to the layer through the plate and is then lost via collisions in the interior of the layer. 
Secondly,

$$
\frac{1}{d^{2}}\left(\frac{\hat{p}^{2}}{\hat{\kappa} \hat{\nu}} h_{x}^{2}-\frac{\hat{\gamma}}{\hat{\kappa}}\right)>0
$$

In this case shear generation overcomes collisional losses and so agitation is being supplied to the layer by the forcing and generated in the interior. Agitation cannot be lost through the free surface and so the layer must become continuously more agitated. So there is no steady-state profile and the neglection of inertial terms in the agitation equation is invalid.

However, if we confine our attention to the first case we can examine the evolution of a layer. Integrating the stress profile we get

$$
\int_{0}^{h} u \mathrm{~d} z=\left.h u\right|_{z=0}-\frac{\hat{p}}{\hat{\nu}} h_{x} \int_{0}^{h}(h-z) q \mathrm{~d} z .
$$

If we take a boundary condition of no slip at the base, then continuity together with the kinematic condition at the surface gives

$$
h_{t}=\frac{\hat{p}}{\hat{\nu}} \partial_{x}\left(h_{x} \int_{0}^{h}(h-z) q \mathrm{~d} z\right)
$$

The integral in the above expression can be evaluated by integrating the agitation equation between the plate and the free surface to obtain

$$
\int_{0}^{h}(h-z) q \mathrm{~d} z=\frac{h \mathcal{F} d \hat{p} \hat{\nu}}{g\left(\hat{\gamma} \hat{\nu}-\hat{p}^{2} h_{x}^{2}\right)} .
$$

Hence

$$
h_{t}=\frac{\mathcal{F} \hat{p}^{2} d}{g} \partial_{x}\left[\frac{h h_{x}}{\left(\hat{\gamma} \hat{\nu}-\hat{p}^{2} h_{x}^{2}\right)}\right] .
$$

This is a diffusion equation which can be written as

$$
h_{t}=\frac{\mathcal{F} \hat{p}^{2} d}{g} \frac{h\left(\hat{\gamma} \hat{\nu}+\hat{p}^{2} h_{x}^{2}\right)}{\left(\hat{\gamma} \hat{\nu}-\hat{p}^{2} h_{x}^{2}\right)} h_{x x}+\text { nondiffusive terms }
$$


Hence the diffusion coefficient is always positive and tends to infinity when collisional losses balance shear generation. Consequently if we begin with any surface displacement having the property that $h_{x}^{2}<\hat{\gamma} \hat{\nu} / \hat{p}^{2}$ then the displacement will be diffusively flattened to a uniform layer.

\section{Conclusions}

We considered the kinetic theory of granular flows developed by Haff (1993) and Jenkins \& Savage (1987) and developed a long wavelength approximation of granular flow. Then by heuristically estimating shear stress and agitational loss terms we derived a simple set of model equations which form a framework for examining rapid granular flow. This allowed for the first consideration of unsteady and spatially developing granular flows. The model predicts an interesting set of behavior including granular hydraulic controls and rapid shock formation of finite mass releases. We also showed that if mechanical and thermal inertia are negligible then sufficiently small surface perturbations will diffuse away. Future work involves experimental verification of the results and further investigation of shock dynamics by shock capturing numerical methods. 


\section{Acknowledgements}

Primarily I would like to thank Bill Young and Joe Keller for their expert advice and patience. I really learnt a lot from them both. I would also like to thank the rest of the staff for many useful discussions, especially Neil Balmforth, Stewart Turner, Mike Shelley, Steve Meacham, Lou Howard and Andy Woods. I have had a wonderful time and made a lot of good friends.

\section{References}

Haff, P.K. 1983 Journal of Fluid Dynamics 134401.

Hirshfelder, J.O., Curtis, D.F. \& Bird R.F. 1964 Molecular Theory of Gases and Liquids Wiley.

Savage, S.V. \& Jefferey, J.D. 1981 Journal of Fluid Dynamics 110255. 


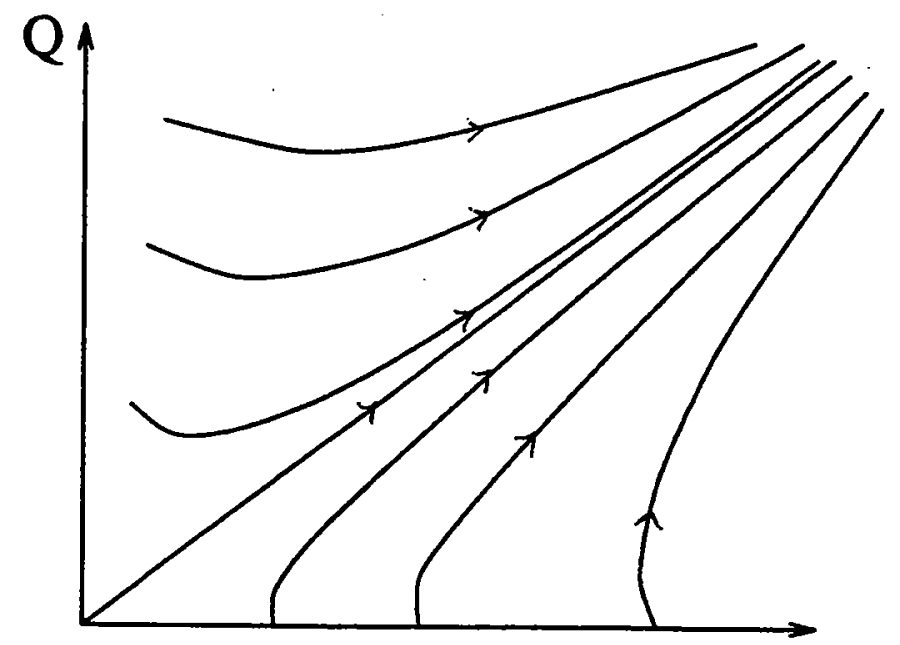

\section{Steep Slope \\ Deep Layer}
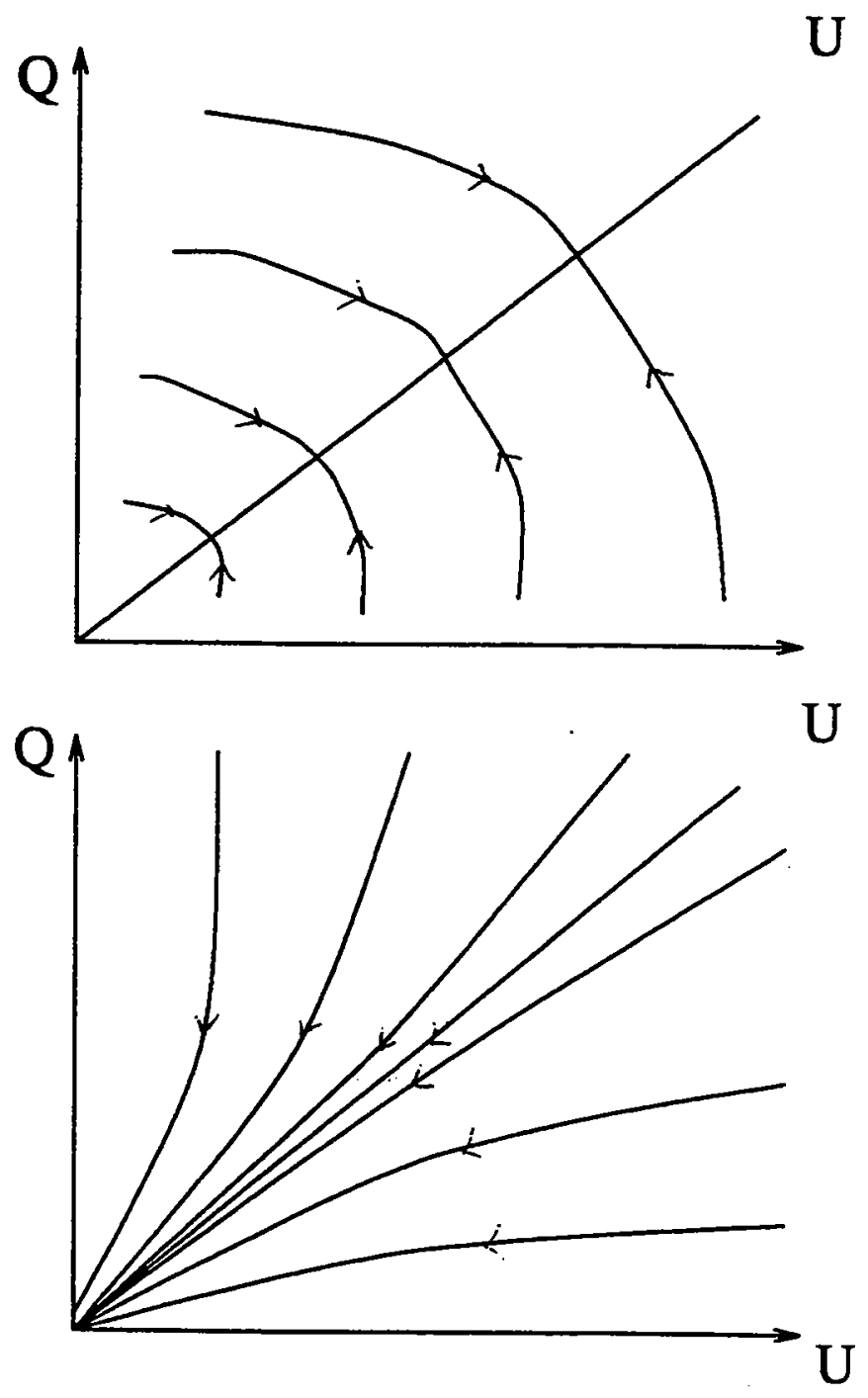

Shallow Slope

OR

Steep Slope

Thin Layer

Figure 1 


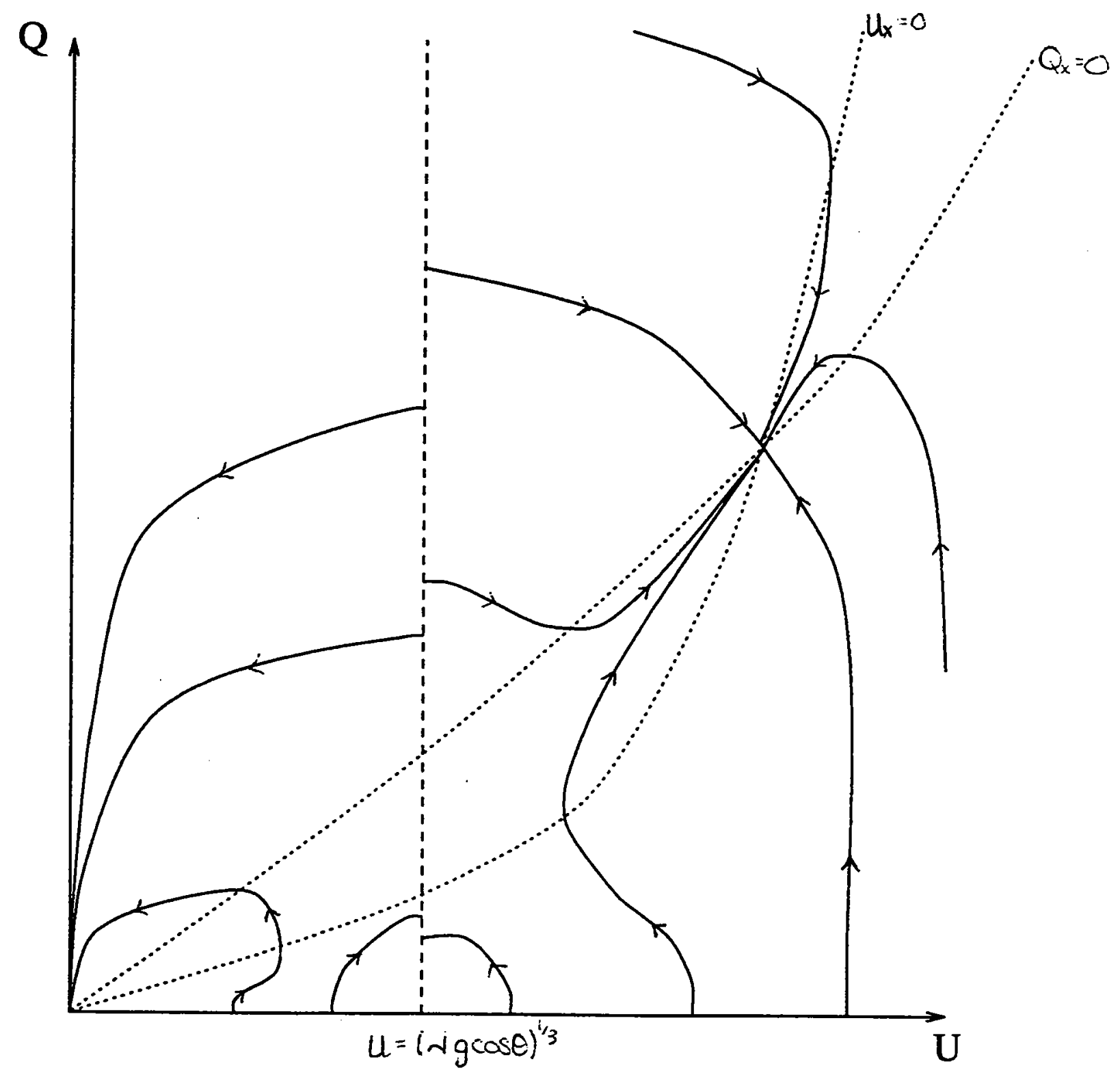

Figure 2 


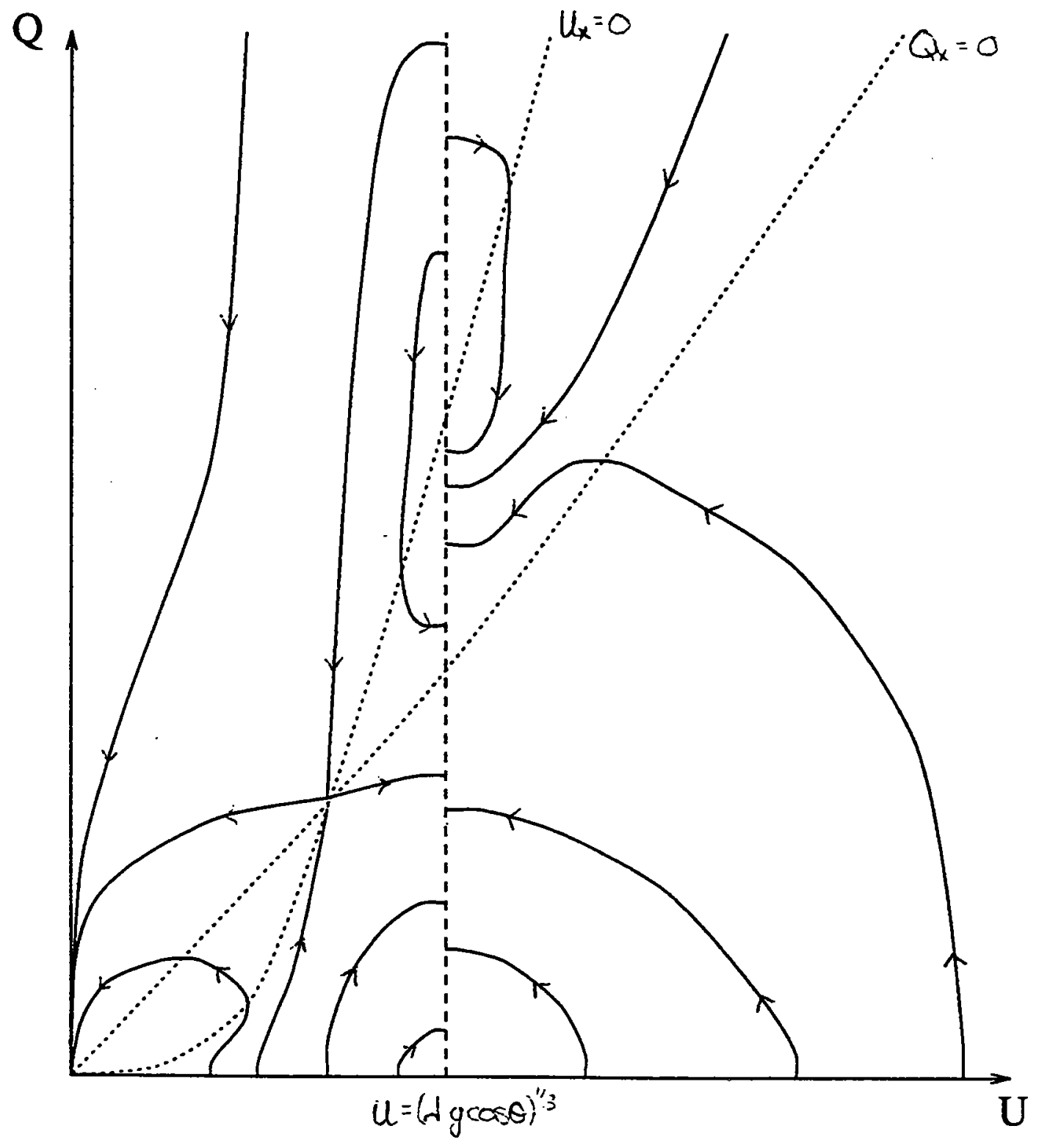

Figure 3 


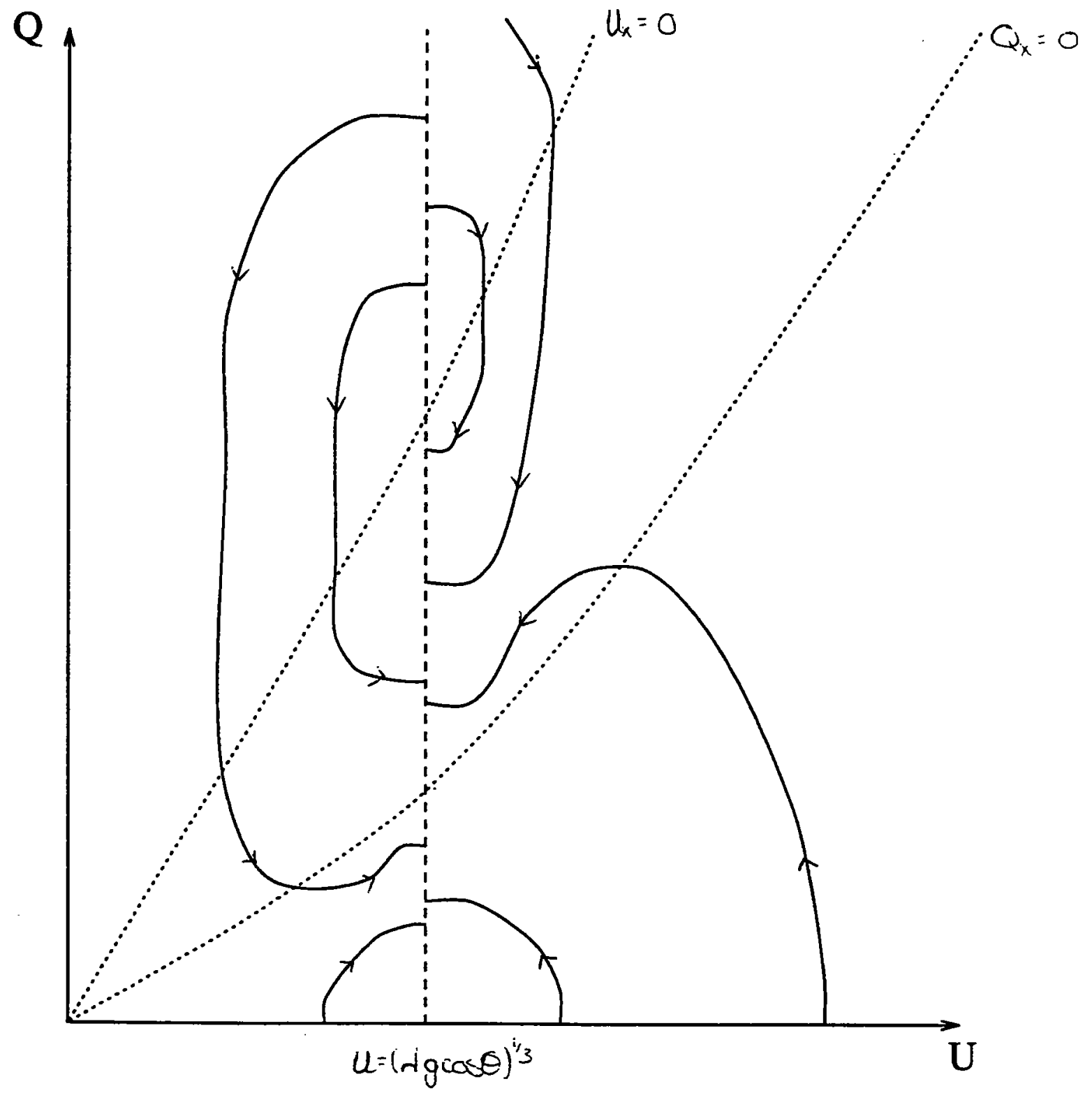

Figure 4 


\title{
The finger regime of double-diffusive convection with the equal diffusion coefficients and mixed boundary conditions
}

\author{
Naftali A. Tsitverblit
}

Oceanography, Lamont-Doherty Earth Observatory of Columbia
University P.O. BOX 1000 / RT 9W/ PALISADES,
NY 10964-8000, USA

Report on the work performed during GFD 1996 WHOI Program

September 8, 1996

\section{Introduction}

Since the "oceanographical curiosity", first observed manifestation of double-diffusive convection, was discovered by Stommel, Arons, \& Blanchard (1956), one of the main questions commonly addressed about this subject has been the effect double-diffusive phenomena have on large scale ocean processes (a recent review of the subject has been provided by Schmitt 1994). Recent works (see Gargett \& Holloway 1991, Zhang, Schmitt, \& Huang 1996, for example), in particular, have been concentrated on implications the presence of the different fluxes of heat and salt, maintained by doublediffusive convection, has for eddy parameterization in a large-scale model.

In most previous studies of double-diffusive convection, it seems to have been assumed that the different rates of diffusion could be due solely to the difference in the diffusivity coefficients between the two involved components. Although instabilities have been long known to occur also in various ocean-related two-component systems described by the equal (eddy) diffusion coefficients and mixed boundary conditions (the idea underlying the physical mechanisms causing these instabilities dates back to the paper by Stommel 1961), it was not until the work by Welander (1989) that the possibility of mixed boundary conditions generating double-diffusive effects was raised. The conceptual framework that suggests itself based on the mechanism analysed by Welander (1989) could potentially be expected to make double-diffusion ideas directly relevant to large-scale ocean phenomena, through eddy-diffusion description.

The type of stratification considered by Welander (1989) is analogous to the diffusive regime of double-diffusive convection. For this reason, the primary instability distinguished in that work was of the oscillatory type. Recent results by Tsitverblit (1996), however, emphasize that a similar mixed-boundary-conditions mechanism could also be very effective in producing numerous steady instabilities in laterally heated thermosolutal systems. Based on the results of this latter work, it was hypothesized by the author that mixed boundary conditions would be capable of generating the instability mechanism of the similar nature also in the finger regime 


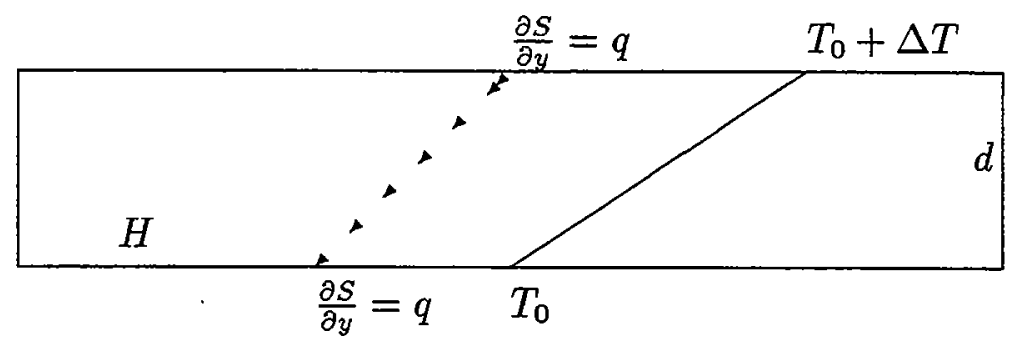

Figure 1: Geometry of the problem.

of double-diffusive convection (i.e., that one could observe a steady instability under stratification of the finger type even when the net gradient is statically stable) even though the diffusion coefficients would be equal. It is the verification of this hypothesis and mark-up of the directions in this problem worth further exploration that has been the primary purpose of the GFD 1996 summer project.

\section{Formulation and methodology}

The primary attention during the project has been devoted to the computations of the bifurcation structure arising in the regime where the destabilizing gradient of one component (the analogue of a solute) is maintained by the flux conditions at the horizontal boundaries with the gradient of the other component (the analogue of temperature) being governed by the difference in its values between these boundaries. It is because the gradient formed by the component expected to diffuse faster is stabilizing while the part of the overall stratification due to the slower diffusing component is destabilizing that the regime is referred to as the analogue of the finger regime. Apart from the presence of the mixed boundary conditions, the principal difference of this configuration from the classical finger regime of double-diffusive convection is that the diffusivity coefficients of the components were set equal.

For the geometry of a horizontal box of width $H$ and height $d$ illustrated in fig. 1 , the Boussinesq approximation of the steady two-dimensional Navier-Stokes equation in the vorticity-streamfunction formulation has been considered together with the energy and solute diffusion equations. These equations were nondimensionalized using the following scales:

length scale $d$ for the horizontal $x$ - and vertical $y$-coordinates;

velocity scale $\nu / d$, where $\nu$ is the horizontal kinematic viscosity;

temperature scale $k_{T} \nu / g \alpha d^{3}$, where $g$ is the gravitational acceleration, $\alpha$ the coefficient of volume expansion due to the component analogous to temperature (hereinafter, temperature), $k_{T}$ the horizontal diffusivity coefficient of this component;

solute concentration scale $k_{S} \nu / g \beta d^{3}$, where $\beta$ is the coefficient of the density variation with respect to the variation of the solute concentration, $k_{S}$ the horizontal diffusivity of the solute. 
With these scales, the nondimensionalized governing equations will look as follows:

$$
\begin{gathered}
\frac{\partial \psi}{\partial x} \frac{\partial \omega}{\partial y}-\frac{\partial \psi}{\partial y} \frac{\partial \omega}{\partial x}=\frac{\partial^{2} \omega}{\partial x^{2}}+D_{R} \frac{\partial^{2} \omega}{\partial y^{2}}+\frac{1}{P r} \frac{\partial t}{\partial x}-\frac{1}{S c h} \frac{\partial C}{\partial x} \\
\omega=\frac{\partial^{2} \psi}{\partial x^{2}}+\frac{\partial^{2} \psi}{\partial y^{2}} \\
\frac{\partial \psi}{\partial x}\left(\frac{\partial t}{\partial y}+R a_{T}\right)-\frac{\partial \psi}{\partial y} \frac{\partial t}{\partial x}=\frac{1}{P r}\left(\frac{\partial^{2} t}{\partial x^{2}}+D_{R} \frac{\partial^{2} t}{\partial y^{2}}\right) \\
\frac{\partial \psi}{\partial x} \frac{\partial C}{\partial y}-\frac{\partial \psi}{\partial y} \frac{\partial C}{\partial x}=\frac{1}{S c h}\left(\frac{\partial^{2} C}{\partial x^{2}}+D_{R} \frac{\partial^{2} C}{\partial y^{2}}\right)
\end{gathered}
$$

where horizontal $u$ and vertical $v$ velocities are

$$
u=-\frac{\partial \psi}{\partial y}, \quad v=\frac{\partial \psi}{\partial x}
$$

vorticity

$$
\omega=\frac{\partial v}{\partial x}-\frac{\partial u}{\partial y}
$$

temperature

$$
\Theta=\Theta_{0}+R a_{T} y+t
$$

and $C$ is the solute concentration. Here, $\Theta_{0}$ and $\Theta_{0}+R a_{T}$ are the nondimensional temperature of the lower and upper horizontal walls, respectively, $C$ is the nondimensional solute concentration, $\operatorname{Pr}=\nu / k_{T}=6.7$ is the horizontal Prandtl number, $S c h=\nu / k_{S}=6.7$ is the horizontal Schmidt number, $R a_{T}=g \alpha d^{4}\left(\frac{\Delta T}{d}\right) / k_{T} \nu$ is the temperature Rayleigh number, $D_{R}=\nu^{v} / \nu=k_{T}^{v} / k_{T}=k_{S}^{v} / k_{S}$ is the ratio between the vertical and horizontal diffusion coefficients.

Since the problem was addressed with different possibilities in mind (ranging from a finite box to an infinite horizontal layer), several types of boundary conditions were used: either the stress-free or the no-slip condition at the horizontal and vertical boundaries, as well as either no-flux boundary conditions at the side walls (when combined with the stress-free conditions, these could be regarded as representing an integer of half a horizontal period) or the combination of the no-flux side-wall boundary conditions for the heat with the solute side-wall conditions specifying the same solute gradient at the side walls as imposed on the fluid interior at the horizontal boundaries. After the above transformation of the variable standing for temperature, and nondimensionalization, these boundary conditions could be written as follows: '

$$
\begin{gathered}
\psi=0, \quad \frac{\partial t}{\partial x}=0, \quad \tau \frac{\partial C}{\partial x}+(1-\tau) R a_{S} y=0, \quad(x=0, \quad 0 \leq y \leq 1) \\
\psi=0, \quad \frac{\partial t}{\partial x}=0, \quad \tau \frac{\partial C}{\partial x}+(1-\tau) R a_{S} y=0 \quad(x=\gamma, \quad 0 \leq y \leq 1) \\
\psi=0, \quad t=0, \quad \frac{\partial C}{\partial y}=R a_{S} \quad(0 \leq x \leq \gamma, \quad y=0) \\
\psi=0, \quad t=0, \quad \frac{\partial C}{\partial y}=R a_{S} \quad(0 \leq x \leq \gamma, \quad y=1) .
\end{gathered}
$$

Here, the solute Rayleigh number is defined as $R a_{S}=g \alpha d^{4}\left(\frac{\partial S}{\partial y}\right) / k_{S} \nu$, where $\left(\frac{\partial S}{\partial y}\right)=q$ is the dimensional gradient of the solute reflecting its flux being imposed at the 
horizontal walls, $\gamma=H / d$ is the aspect ratio, $\tau$ is a parameter variation of which from 0 to 1 enables one to switch from the problem with the solute gradient prescribed at the side walls to that with no flux of the solute concentration through these boundaries.

In the latter case, the absolute value of the solute concentration within the domain remains unspecified up to a constant. This could eventually cause unphysical variations in the field of the solute concentration and spontaneous changes in the $\mathrm{Ja}$ cobian sign (based on which symmetry-breaking bifurcation points are detected), due to numerical noise. To avoid these problems, numerical implementation of boundary conditions (5)-(8) was slightly different from what is formulated above. In particular, the difference in solute concentration was specified at the boundary points of one of the vertical cross-sections of the grid (in the middle of the enclosure). Since the prescribed difference was consistent with the flux condition specified at all other points and because of the relative effect of this perturbation tending to zero when the number of grid points is increased to infinity (or when the aspect ratio, to which the number of grid points in horizontal direction is proportional, is large enough), the formulated problem is expected to be qualitatively identical and quantitatively close to the one with the flux condition at the boundary points of all the vertical cross-sections.

Equations (1) - (4) together with boundary conditions (5)-(8) as well as either with the no-slip or with the stress-free condition at the boundaries were discretized by central finite differences on grids $((23+1) \gamma-1) \times 23$. Although some quantitative improvement could be obtained by using a higher resolution, the results on this grid were found to be qualitatively consistent with those in test runs on finer grids, with tolerable quantitative differences in the critical parameters. This grid was also found to give rather close agreement with the results of Cliffe \& Winters (1986) for the Benard problem with Dirichlet boundary conditions. The Euler-Newton and Keller (1977) arclength continuation algorithms were used to trace out the steady branches as $R a_{S}$ was varied and $R a_{T}$ fixed, for different aspect ratios. Stability of the steady solutions computed in this way was studied by examining time response of the linearized time-dependent version of the governing equations to the initial perturbation.

The results will be presented here for $\gamma=3,5$, which was found sufficient to represent the essential qualitative features of the instabilities. Although some computations have been done for $R a_{T}=100000$ and the larger values of this parameter, it is only the results for $R a_{T} \leq 20000$ that will be presented in this report.

\section{Results and interpretation}

\subsection{The equal vertical and horizontal diffusivities}

The principal outcome of this work is that the convective instability was found to set in under a statically stable overall gradient even when the diffusion coefficients are equal, which should be attributed to the effect of the mixed boundary conditions on the diffusion gradients in the perturbed state similar to that described by Welander (1989). The dependence of the solute Rayleigh numbers characterizing the onset of the primary instability in the problem of the aspect ratio $\gamma=5$ with the various boundary conditions is illustrated in fig. 2 . It follows from this figure that the velocity boundary conditions at the side walls of the box with such a large aspect ratio have almost no effect on the critical $R a_{S}$ of the instability, while the onset of the latter depends significantly on the type of the velocity boundary conditions (whether stress-free or no-slip) at the horizontal boundaries. As can be seen from figs. 3,4, representing the aspect ratio $\gamma=3$, the behavior of the bifurcations and the forms of the instabilities are highly consistent with those in the Rayleigh-Benard problem with flux boundary conditions: the primary instability sets in at the most unstable 
wave length that is as long as is allowed by the geometry restrictions and is followed by the instabilities with the decreasing wave length. This is indicative of that zero horizontal wave number, being the critical one for the instability in the above case of Benard convection in an infinite horizontal layer (Hurle, Jakeman \& Pike 1966), is also likely to be the most unstable mode for such a layer with the two-component stratification defined analogously to the configuration considered in this report.

It is interesting to note in this respect that the value of $R a_{S}$ characterizing the primary instability for $\gamma=5, R a_{T}=10000$, and no-flux side-wall boundaries (fig. 2 a) is significantly lower than that for $\gamma=3$ (fig. 3). If one assumes that this trend continues to hold when the aspect ratio is further increased, it appears quite likely that the onset of the primary instability in an infinite horizontal layer would deviate only very little from that for Benard convection, barely depending on the value of the stabilizing temperature gradient at all. The reason why the increase of the rate of temperature stratification may have only relatively small effect on critical $R a_{S}$ is discussed in the end of the Discussion section. The fact that the weak dependence of the critical $R a_{S}$ on $R a_{T}$ seems to become clearly noticeable only for the large aspect ratios could be associated with the relative role of the single vertical crosssection where the values of the solute concentration were specified at the horizontal boundaries being more pronounced for the smaller aspect ratios. In other words, since this cross-section prevents formation of the different diffusion gradients in its vicinity it may have a more significant effect on the two-component problem than on Benard convection (in the latter case, the difference in the critical $R a_{S}$ between $\gamma=3$ and $\gamma=5$ was not found to be as significant as for the double-component stratification). When the same interval of discretization is used, the more significant perturbation caused by the cross-section in the enclosures of the smaller aspect ratio would make the critical parameters in the double-component configuration to be more away from where they would have been without the perturbation (presumably, near the values characteristic of Benard convection).

Conclusions about stability of the steady solutions can be quite reasonably inferred from the results represented in figs. 3,4. In particular, since all the bifurcations are supercritical, the patterns characteristic of the steady solutions not far away from the respective bifurcation points (they are approximately the same as those represented in fig. 4) could be viewed as reflecting the shapes of the respective eigenvectors, which means that all eigenvectors involved are different. Assuming that there is no degeneracy in the eigenvalues, this implies that all eigenvalues becoming zero at the symmetry-breaking bifurcation points are also different. Since at the low values of the solute Rayleigh number all these eigenvalues are stable, each of the sequence of bifurcations is associated with one of the new eigenvalues becoming unstable. Therefore, it is only the solution arising from the first pitchfork bifurcation that is expected to be stable, while all other asymmetric branches (by referring to branches as asymmetric, it is implied that they are asymmetric with respect to at least one type of the two reflectional symmetries characterizing the problem) are characterized by the presence of eigenvalues having become unstable at the preceding bifurcation points. These arguments were directly confirmed by examination of the linear stability of the solutions.

When the destabilizing solute gradient is imposed also at the side-walls, this boundary condition prevents the instability from selecting the zero horizontal mode. For this reason, the observed primary instability is characterized by two counterrotating cells, rather than the single one (figs. 5,6). This was also found similar to the respective events in Benard convection. Although the average cell thickness of the subsequent bifurcations was also found to decrease (like in the formulation with the no-flux solute boundary conditions at the side walls), specification of the solute gradient at the vertical boundaries produces patterns with odd number of cells that are qualitatively different from the case with the no-flux side-wall conditions for the solute: the cells observed in these steady solutions are of the different horizontal sizes. 
Like for the no-flux solute side-wall boundary conditions, it is only the branch arising from the first bifurcation that is stable.

\subsection{Nonequal vertical and horizontal diffusivities}

According to the mechanism following from interpretation of Welander (1989), suggested for the analogue of the diffusive regime, it appears that it is the nonequal gradients in the vertical direction that play the primary role in the instability scenario. Since in the oceanographically relevant situation the diffusion rate is determined by such gradients multiplied by the vertical diffusivities that are much smaller than the horizontal ones, it seemed reasonable to get some insight into what effect the decrease of the vertical/horizontal diffusivity ratio, $D_{R}$, has on the observed instabilities. With these considerations in mind, computations have been performed in the range of $D_{R}=k_{T}^{v} / k_{T}=k_{S}^{v} / k_{S}=\nu^{v} / \nu<1$. Although some results for $D_{R}<0.1$ were also obtained, their relative complexity required more time for processing the data and additional runs for their understanding. Therefore, the attention here is going to be concentrated on the data obtained only for $D_{R}=0.1$.

For understanding the events in the case when the two components are involved, however, it would be useful to have some information about what happens when $D_{R}<$ 1 in Benard convection with the flux boundary conditions (this would be equivalent to $R_{T}=0$ ). As can be seen from figs. 7,8 , the events in this case appear to be qualitatively identical to the corresponding configuration with $D_{R}=1$. The principal quantitative difference of this configuration from Benard convection at $D_{R}=1$ is that the critical Rayleigh number (in our case, it is $R a_{S}$ ) drops from $\sim 120$ (for the stressfree horizontal boundaries) to $\sim 20$. This should probably be attributed to the fact that vertical diffusion provides a mechanism for redistribution of the energy supplied at one of the horizontal boundaries and taken away at the other. Up to a certain point the diffusion along with viscous effects can prevent convection from manifesting itself. When the vertical diffusion is very weak, however, much smaller flux is required to destabilize the system. It is not surprising, therefore, that when $D_{R}$ was decreased below 0.1 , the critical $R a_{S}$ were found to approach zero. It is interesting that this observation appears to substantiate algorithms often incorporated into ocean models according to which denser fluid found above lighter one is interchanged with the latter irrespective of the quantitative parameters of the density difference between the two fluid layers.

Since vertical diffusion is expected to be a destabilizing factor in the two-component system, in contrast to Benard convection, the decrease of $D_{R}$ to 0.1 turns out to have the stabilizing effect: as can be seen from fig. 9 , with the increase of $R a_{T}$, the critical values of the solute Rayleigh number very soon become distinctively higher for $D_{R}=0.1$ than when $D_{R}=1$ (fig. $2 \mathrm{a}, \mathrm{b}$ ). Moreover, the results illustrated in figs. 10,11 demonstrate that the flow patterns in the double-component case with $D_{R}=0.1$ become substantially different from those in Benard convection with the same $D_{R}$.

In particular, two corotating long thin cells (layers) are observed at the onset of the primary instability (fig. $11 \mathrm{a}$ ). The symmetry that they have preserved is reflection between the corner points belonging to a diagonal of the domain. At the second bifurcation, the horizontal symmetry is broken while the vertical one is retained (fig. $11 \mathrm{~b}$ ). Similarly to the situation with the vertical diffusivity being equal to the horizontal one, the subsequent bifurcation is characterized by the doubling of the number of cells in the horizontal direction compared to the convective flow arising from the first bifurcation: the four cells present have the same sense of rotation when compared vertically and the opposite sense when compared horizontally (fig. $11 \mathrm{c}$ ). The symmetry preserved by this flow is the horizontal one. As the solution is continued farther away from bifurcation point $B 3$, the layers at one of the horizontal levels move away from each other (fig. $11 \mathrm{~d}$ ). 
The flow pattern arising at bifurcation point $B 4$, is characterized by the counterrotating cells both in vertical and horizontal comparison (fig. $11 \mathrm{e}$ ). As the solution is continued to the strongly nonlinear regime, however, the weaker cells gradually vanish (fig. $11 \mathrm{f}$ represents the branch oppositely asymmetric to the one in fig. 11 e). Following the same regularity as before, the next bifurcation ( $B 5$ ) is associated with the formation of two layers with four cells in each of them; the cells rotate in the same sense when compared vertically and in the opposite one when compared horizontally (fig. $11 \mathrm{~g}$ ). It appears that the interaction in the interfaces between the respective corotating cells of the two horizontal layers causes suppression of some of the cells from the vertically interacting pairs when the solution is continued to the strongly nonlinear regime (fig. $11 \mathrm{~h}$ ). At bifurcation point $B 6$, all cells arising are counter-rotating with respect to both their vertical and horizontal neighbours (fig. 11 i), which seems to be consistent with the regularity observed for the series of the preceding bifurcations. The net gradient at bifurcation point $B 7$ is already unstable, for which reason the patterns forming are characteristic of Benard convection (fig. 11 j).

Lastly, it is worth mentioning that when some of the branches bifurcating from the no-flow solution were continued, changes of the Jacobian sign were noticed, which indicates that secondary bifurcations should also be expected for the nonequal diffusivities. Although these branches were not computed in the framework of this summer project, their investigation certainly appears to be worthwhile.

\section{Discussion}

Since time limitations did not allow to process some obtained data for the vertical/horizontal diffusivity ratio $D_{R}<0.1$, it is difficult to predict here the details of the behavior of the instabilities when $D_{R}<0.1$. Based on our understanding of the physical mechanisms bringing about these instabilities, however, their nature would have to be crucially related to the different rates of the vertical diffusion. Therefore, it seems reasonable to expect that the further decrease of the vertical diffusivity would make the physical effect associated with the formation of different diffusion gradients due to the mixed boundary conditions less noticeable. This was found consistent with the Jacobian sign change not being observed up to the values of the destabilizing solute gradient being equal to the one specified by the stabilizing temperature difference, during preliminary examination of data for $D_{R} \leq 0.01$. It is only a more detailed analysis, however, that could determine whether this has been a result of all the bifurcation merely moving to the higher values of the solute gradient (until the net gradient becomes neutral and unstable) or there are some other more complex mechanisms responsible for their vanishing.

If the vertical temperature gradient forming in the perturbed state keep increasing with the decrease of the vertical diffusivities, on the other hand, it seems one could expect that the different rates of diffusion might be maintained. This could be possible if the gradient characterizing the background state (this gradient is the origin of the high temperature gradient in the perturbed state) grows with the decrease of $D_{R}$. That is, with the increase of the temperature gradient, the presence of the instabilities for the statically stable net gradient might be restored. That same growing temperature gradient not only increases a stabilizing part of the overall stratification, but also enhances the high gradients in the perturbed state that are responsible for the different rates of diffusion bringing about the instability mechanism. The validity of this conception is planned to be verified at the later stages of this study. 


\section{Conclusions}

It has been demonstrated that, even when the diffusion coefficients of the components are equal, the destabilizing solute gradient maintained by the flux conditions at the horizontal boundaries is capable of generating convective instability when it is just a small fraction of the stabilizing gradient governed by the temperature difference. The instability was found to set in at the long horizontal wave length, and the results suggest that the most unstable wave number for the infinite horizontal layer of fluid could be equal to zero, like in Benard convection with flux boundary conditions. Similarly to the Benard convection configuration, the long wave length primary instability was found to be followed by a series of the bifurcations with a decreasing horizontal wave length. This resemblance should probably be attributed to the fact that in both cases (i.e., with and without the temperature gradient), the instability derives its potential energy from the component maintained by the same, flux-type, boundary condition.

It appears that even a large increase of the stable rate of temperature stratification in a box that is long enough (i.e., where the conditions are sufficiently close to those in an infinite horizontal layer) would produce only a relatively small quantitative change in the critical values of the destabilizing solute Rayleigh number with respect to those with no temperature stratification at all (Benard convection). This would have to be attributed to the two-fold role of the temperature gradient: its increase not only enhances the stabilizing part of the overall stratification but also causes the higher difference in the diffusion gradients.

The velocity boundary conditions at the horizontal walls were also found to have an effect similar to that observed for Benard convection (i.e., when the temperature gradient is absent): for the no-slip boundaries, the instability was found to manifest itself at the larger values of the solute Rayleigh number than for the stress-free ones. The effect of the presence of the consistent value of the solute gradient at the side walls, apart from replacing the most unstable long wave length (presumably, zero wave number) by the one corresponding to the width of the domain, was found to lead to the formation of flow patterns with different cell sizes.

The decrease of the vertical diffusivities with respect to the horizontal ones was found to make the results essentially different from those characterizing the respective case of Benard convection. Unlike Benard convection, in particular, the patterns arising from the series of bifurcation points turned out to have a two-layer structure, and indications of the presence of secondary bifurcations were observed. In addition, the onset of the primary instability for large enough $R a_{T}$, even though observed when the overall gradient is still far from being unstable, is characterized by distinctively higher values of the solute gradient than for the respective formulations with the equal diffusivities, whereas when the temperature gradient is absent, the decrease of the vertical/horizontal diffusivity ratio has a clearly destabilizing effect. This latter observations should probably be attributed to the instability mechanism in the double-component case being essentially due to vertical diffusion, as opposed to vertical diffusion being a stabilizing factor in Benard convection.

\section{Intended developments}

The first issue planned to be addressed is the linear stability analysis of an infinite horizontal layer stratified by two components as considered above, which was begun, but not completed, during this summer program. It is hoped that through this analysis it will be possible to demonstrate analogy of the considered problem with Benard convection with flux boundary conditions (the analogy between the classical finger regime and the conductive Benard problem, for example, was demonstrated by Stern 1960). Both linear stability study and the nonlinear 2-D computations in a box are expected to address the formulation with the nonequal vertical and horizon- 
tal diffusivities in more detail, including the issues of the secondary bifurcations and behavior of the instabilities at the values of $D_{R}$ smaller than those used above. Examination of the effects of rotation on the instabilities also appears to be a reasonable objective.

Towards the end of the Program, it was pointed out by E. Spiegel that the long wave length instability made the non-Boussinesq effects important in Benard convection (Depassier \& Spiegel 1982), which indicates that they may have significance also in the present problem. For this reason, introduction of such effects into the model equations and extension of the steps described above to the non-Boussinesq fluid is also expected to be an interesting development of this work.

\section{Acknowledgements}

I would like to thank the organizers of the 1996 GFD Program for inviting me to participate in it and maintaining creative atmosphere during the course of study. Throughout this Program, conversations on the subject of this work or related issues were held with (the order is approximately chronological) B. Ruddick, M. Stern, S. Meacham, E. Chassignet, E. Knobloch, E. Spiegel, L. Howard, O. Kerr,W. Young, P. Cessi, J. Keller, G. Veronis, K. Julien, A. Woods, N. Balmforth, M. Shelley, usefulness of which is also gratefully acknowledged.

\section{References}

[1] Cliffe K. A. and Winters K. H. (1986) "The use of symmetry in bifurcation calculations and its application to the Benard problem." J. Comp. Phys. $67,310-326$.

[2] Depassier, M. C. \& Spiegel, E. A. (1982) "Convection with heat flux prescribed on the boundaries of the system. I. The effect of temperature dependence of material properties." Geophys. Astrophys. Fluid Dynamics 21, 167-188.

[3] Gargett, A. E. \& Holloway, G. (1991) "Sensitivity of the GFDL ocean model to different diffusivities for heat and salt." J. Phys. Ocean. 22, 1158-1177.

[4] Hurle, D. T. J., Jakeman, E. \& Pike, E. R. (1967) "On the solution of the Benard problem with boundaries of finite conductivity." Proc. Roy. Soc. A $296,469-475$.

[5] Zhang, J., Schmitt, R. W. \& Huang, R. X. (1996) "Sensitivity of GFDL modular ocean model to the parameterization of double-diffusive processes." $J$. Phys. Ocean. submitted.

[6] Schmitt, R. W. (1994) "Double diffusion in oceanography." Annu. Rev. Fluid Mech. 26, 255-285.

[7] Stern, M. E. (1960) "The salt fountain and thermohaline convection." Tellus, $12,172-175$.

[8] H. Stommel (1961) "Thermohaline convection with two stable regimes of flow." Tellus XIII, 224-230.

[9] Stommel, H., Arons, A. B. \& Blanchard, D. (1956) "An oceanographical curiosity: the perpetual salt fountain." Deep-Sea Res. 3, 152-153. 
[10] N. Tsitverblit (1996) "Multiplicity of the equilibrium states in laterally heated thermosolutal systems with the equal diffusivity coefficients." Submitted to Phys Fluids. See also summary of this work in Proceedings of the 1996 GFD program.

[11] Welander, P. (1989) "A new type of double-diffusive instability?" Tellus $41 \mathrm{~A}, 66-72$.

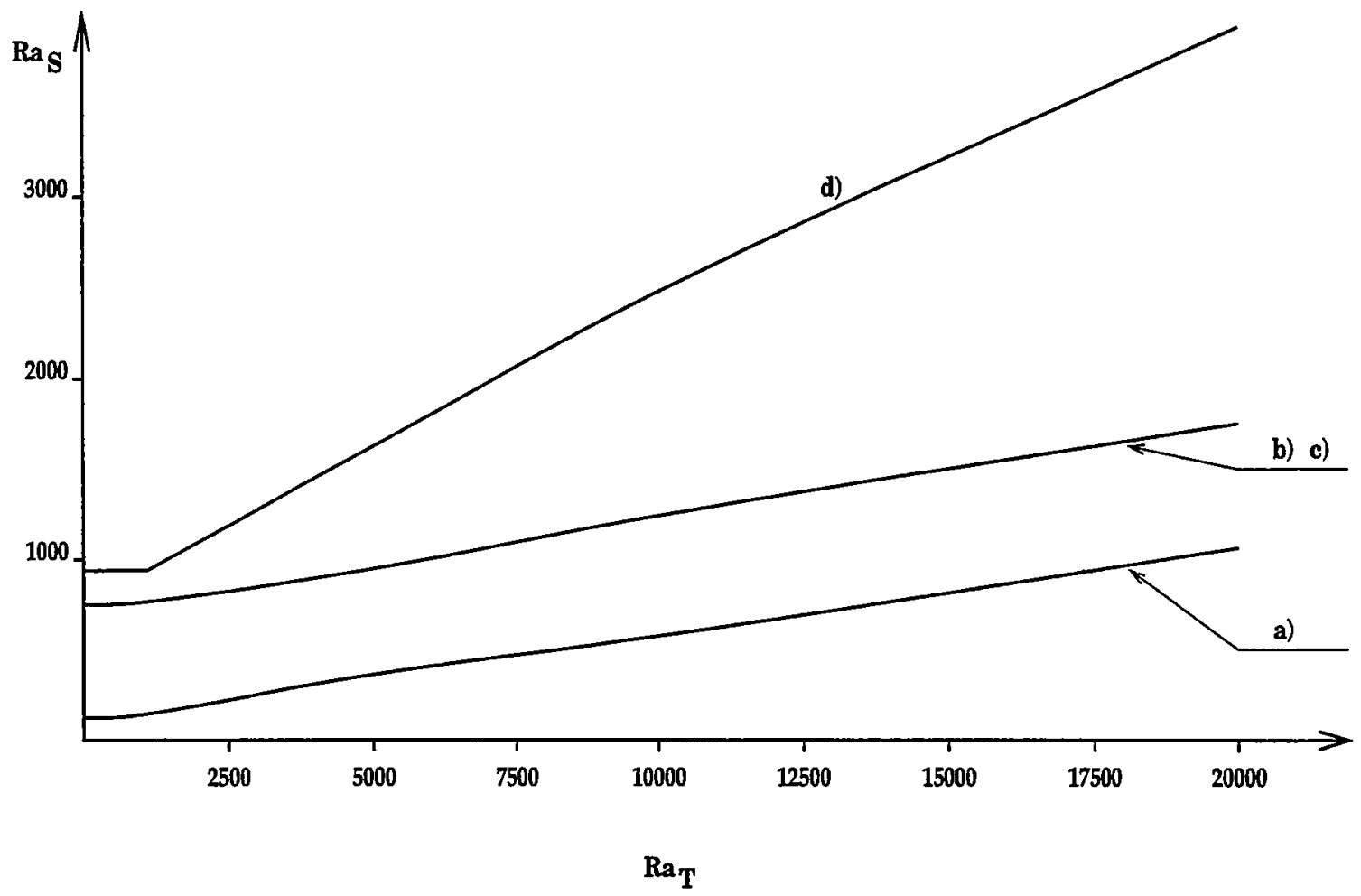

Figure 2: Marginal stability boundaries for the aspect ratio $\gamma=5$; (a) all boundaries being stressfree with no-flux conditions prescribed for both components at the side walls; (b) no-slip horizontal boundaries with stress-free and no-flux (for both components) side walls; (c) all boundaries being no-slip with the no-flux condition prescribed for both components at the side walls; (d) all boundaries being no-slip with the solute gradient and no temperature flux prescribed at the side walls. 


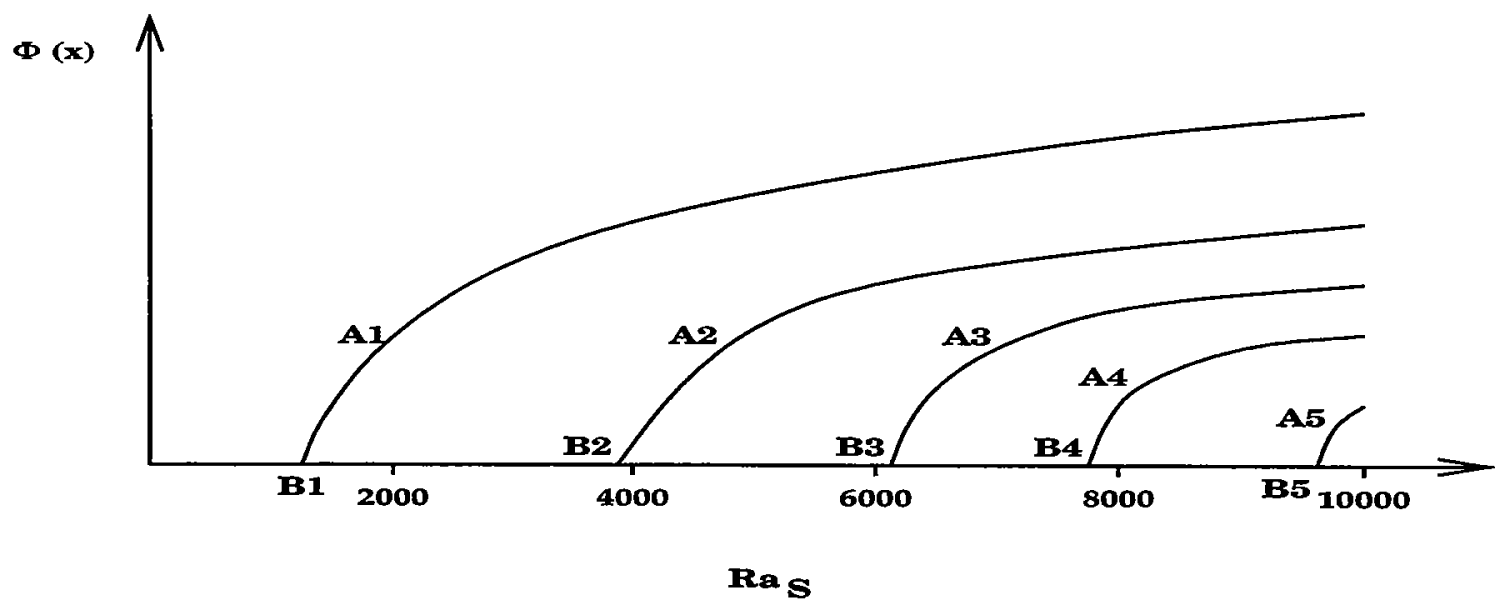

Figure 3: The schematic diagram of the variation of a measure $\phi(\mathbf{x})$ of solution vector $\mathbf{x}$ with solute Rayleigh number $R a_{S} ; \gamma=3, R a_{T}=10000$, the horizontal and side-wall boundaries are stress-free, and no-flux conditions are prescribed for both components at the side walls. $A 1, \ldots, A 5$ are the bifurcating branches, $B 1, \ldots, B 5$ are the symmetry-breaking bifurcation points (only one asymmetric branch for each of the pitchfork bifurcations is depicted).
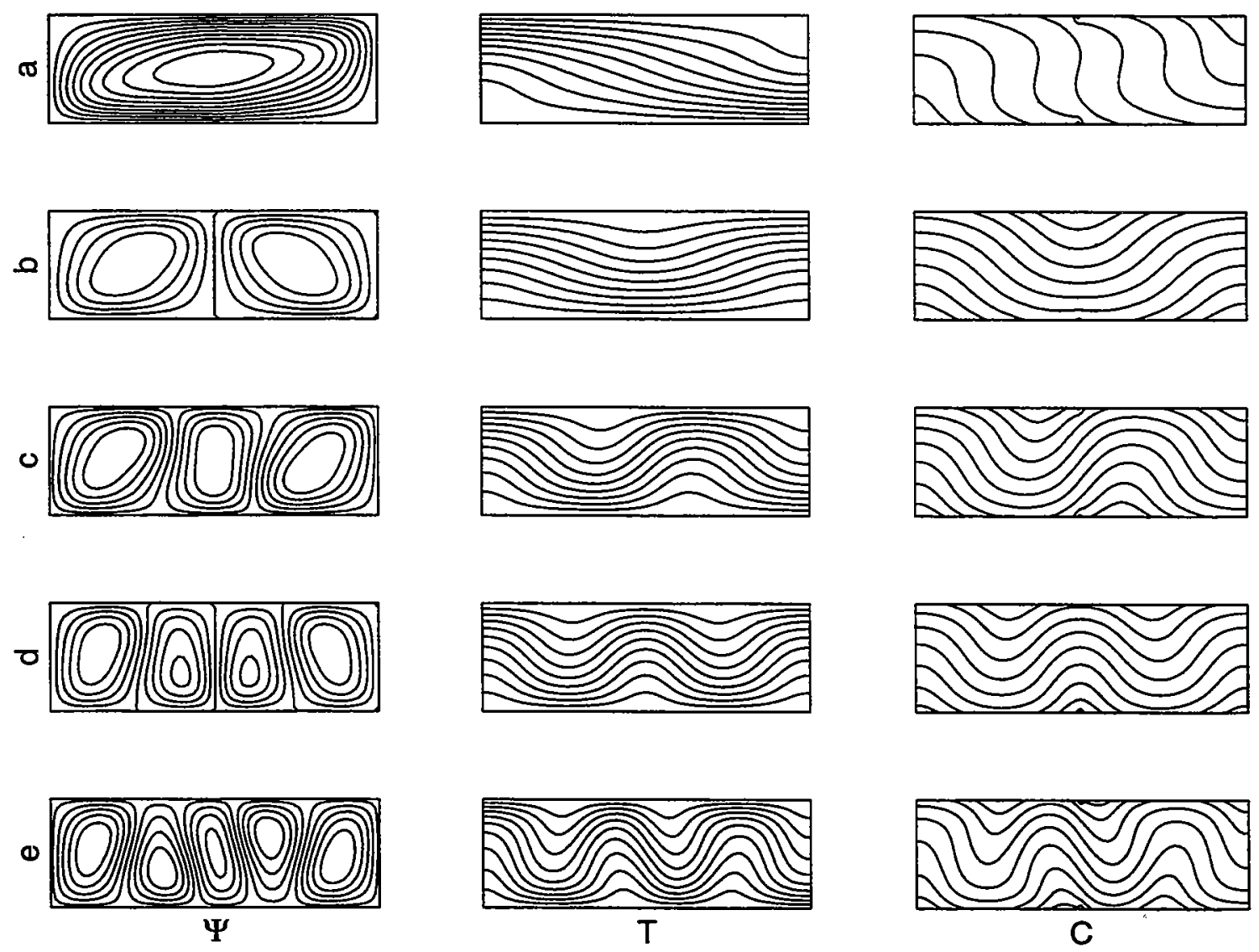

Figure 4: Flow patterns arising from the series of bifurcations as $R a_{S}$ is increased; $\gamma=3$, $R a_{T}=10000$, with all the boundaries being stress-free and no-flux conditions being prescribed for both components at the side-walls; $\Psi$ : streamlines; $T$ : isotherms; $C$ : the lines of the constant solute concentration; (a) branch $A 1, R a_{S}=5220$; (b) branch $A 2, R a_{S}=5139$; (c) branch $A 3$, $R a_{S}=9068$; (d) branch $A 4, R a_{S}=10697$; (e) branch $A 5, R a_{S}=17836$. 


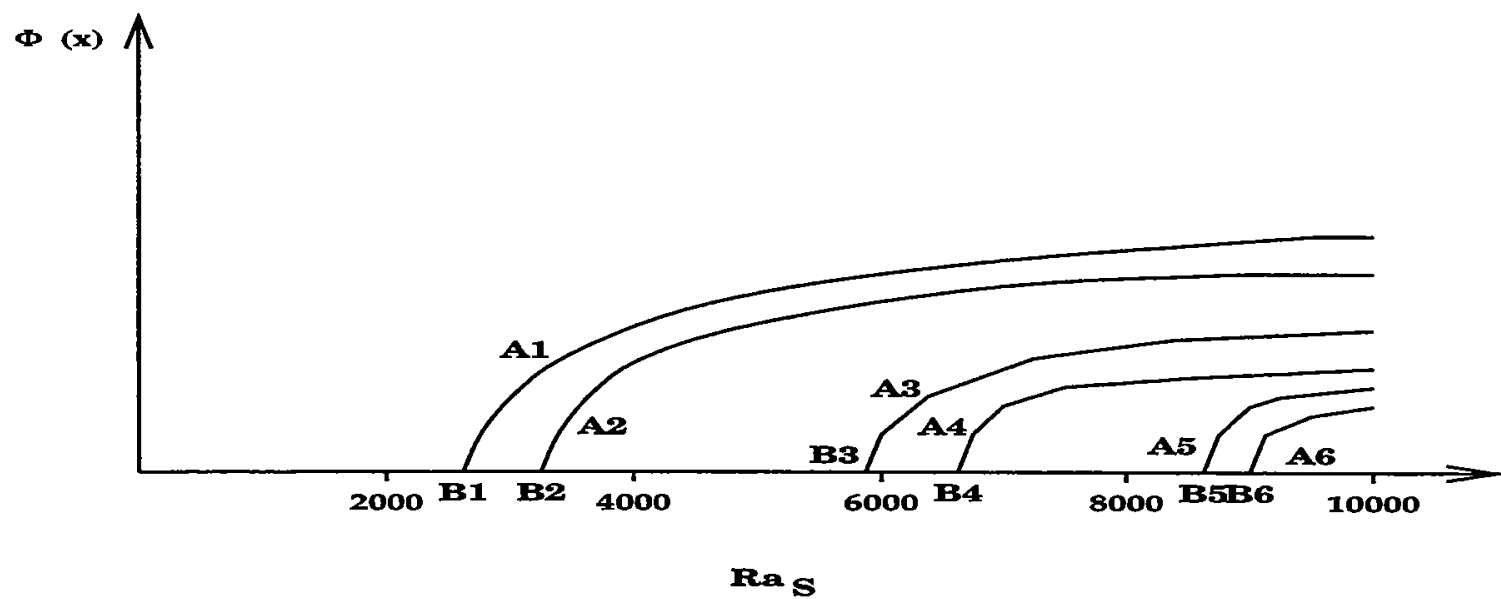

Figure 5: The schematic diagram of the variation of a measure $\phi(\mathbf{x})$ of solution vector $\mathbf{x}$ with solute Rayleigh number $R a_{S} ; \gamma=5, R a_{T}=10000$, the horizontal and side-wall boundaries are no-slip, with the solute gradient and no temperature flux prescribed at the side walls. $A 1, \ldots, A 6$ are the bifurcating branches, $B 1, \ldots, B 6$ are the symmetry-breaking bifurcation points (only one asymmetric branch for each of the pitchfork bifurcations is depicted).

$\omega$

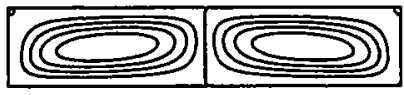

o

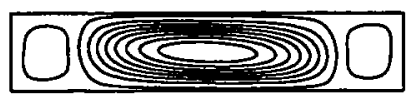

$\circlearrowleft$
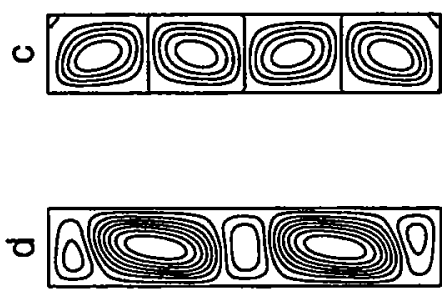

(1)
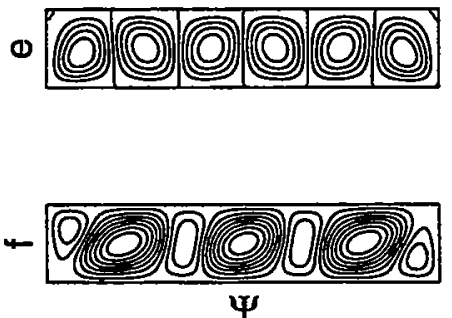
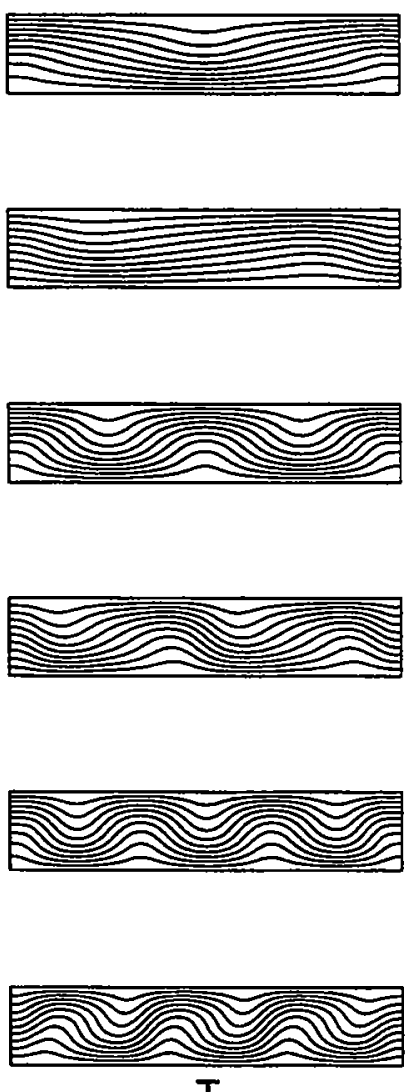

$T$
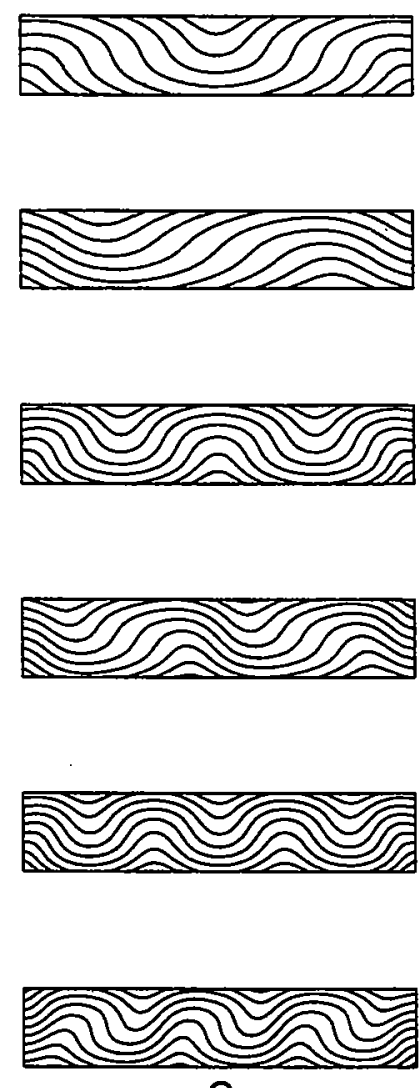

C

Figure 6: Flow patterns arising from the sequence of the bifurcations as $R a_{S}$ is increased; $\gamma=5$, $R a_{T}=10000$, all the boundaries are no-slip, the solute gradient and no temperature flux are prescribed at the side walls; $\Psi$ : streamlines; $T$ : isotherms; $C$ : the lines of the constant solute concentration; (a) branch $A 1, R a_{S}=5128$; (b) branch $A 2, R a_{S}=5099$; (c) branch $A 3, R a_{S}=9644$; (d) branch $A 4, R a_{S}=10370$; (e) branch $A 5, R a_{S}=12177$. 
$\Phi(\mathbf{x})$

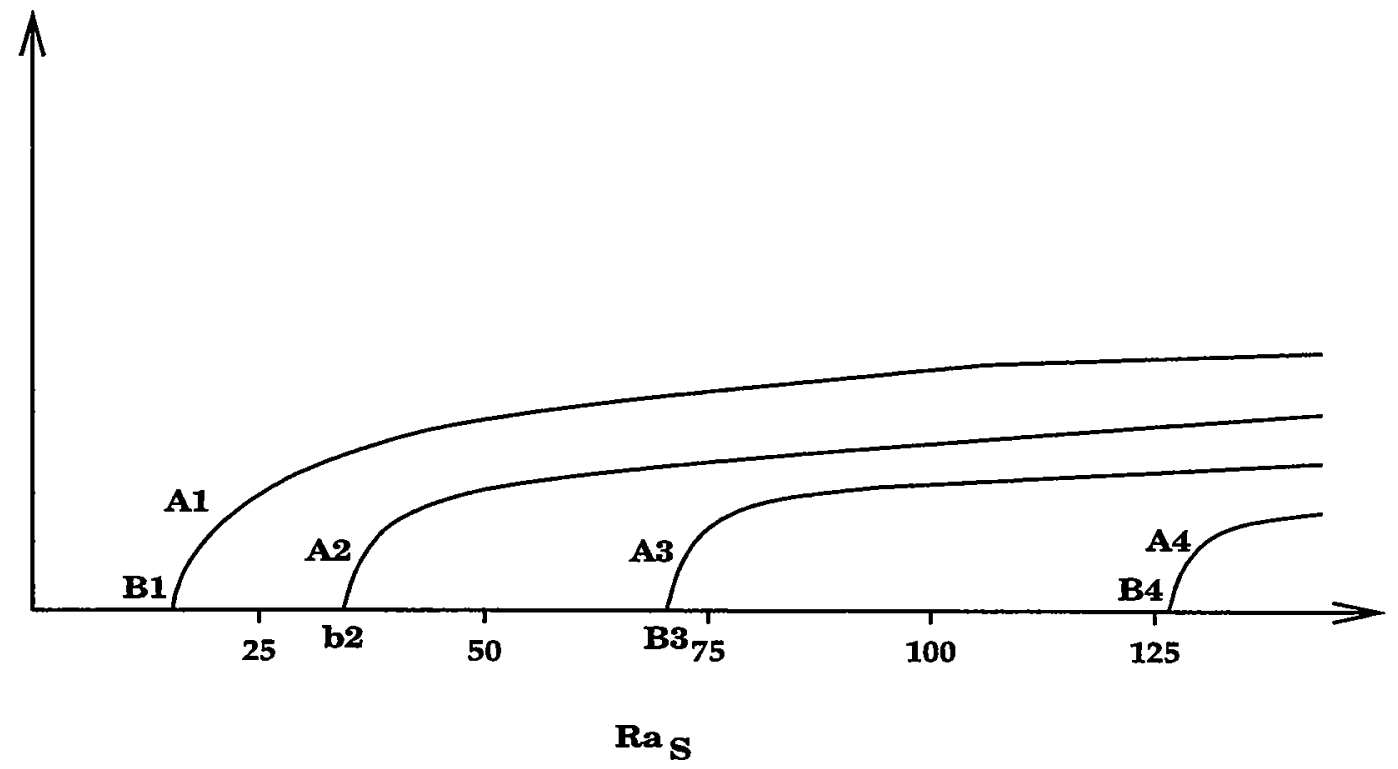

Figure 7: The schematic diagram of the variation of a measure $\phi(x)$ of solution vector $\mathbf{x}$ with solute Rayleigh number $R a_{S} ; \gamma=5, R a_{T}=0$ (Benard convection), $D_{R}=0.1$. The horizontal and side-wall boundaries are stress-free, with no flux of solute prescribed at the side walls. $A 1, \ldots, A 4$ are the bifurcating branches, $B 1, \ldots, B 4$ are the symmetry-breaking bifurcation points (only one asymmetric branch for each of the pitchfork bifurcations is depicted).

๘
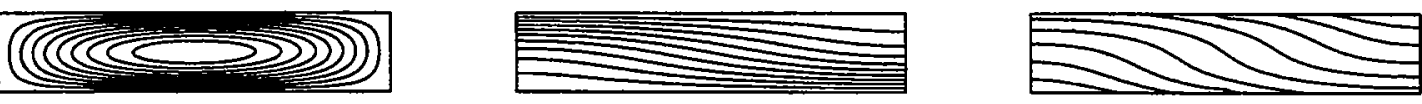

요
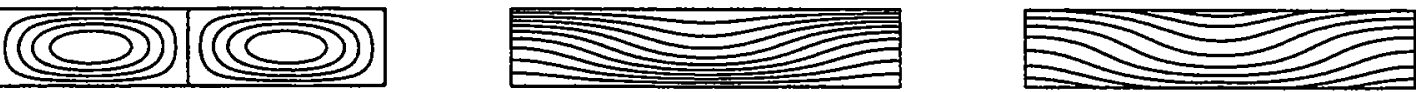

0
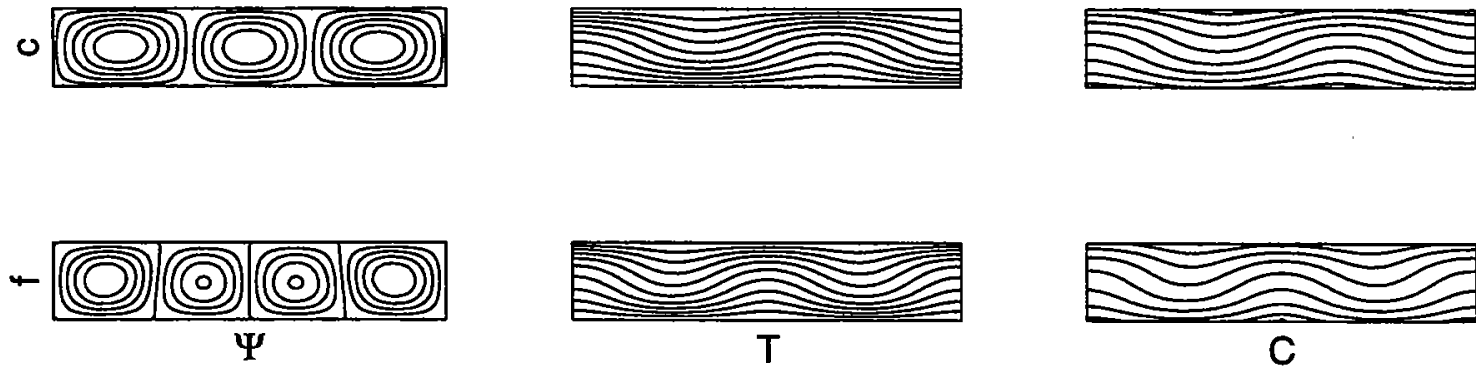

C

Figure 8: Flow patterns arising from the series of bifurcations as $R a_{S}$ is increased; $\gamma=5$, $R a_{T}=0$ (Benard convection), $D_{R}=0.1$. All the boundaries are stress-free, with no flux of solute being prescribed at the side walls. $\Psi$ : streamlines; $T$ : isotherms; $C$ : the lines of the constant solute concentration; (a) branch $A 1, R a_{S}=31$; (b) branch $A 2, R a_{S}=57$; (c) branch $A 3, R a_{S}=111$; (d) branch $A 4, R a_{S}=239$. 


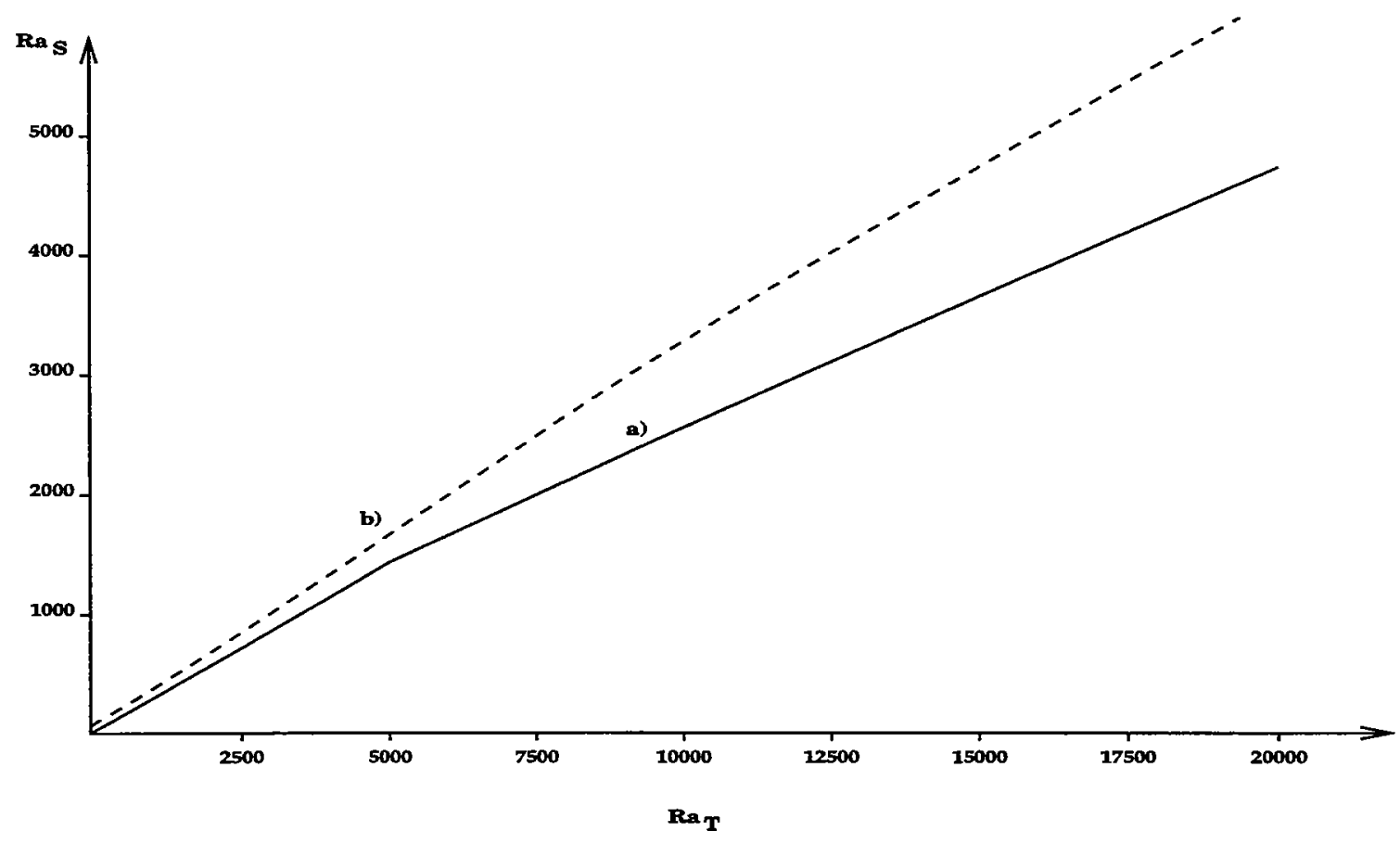

Figure 9: Marginal stability boundaries for the aspect ratio $\gamma=5$ and $D_{R}=0.1$; (a) all the boundaries are stress-free and no-flux conditions are prescribed at the side walls for both components (the solid line); (b) all boundaries are no-slip, with no-flux (for both components) side walls (the dotted line).

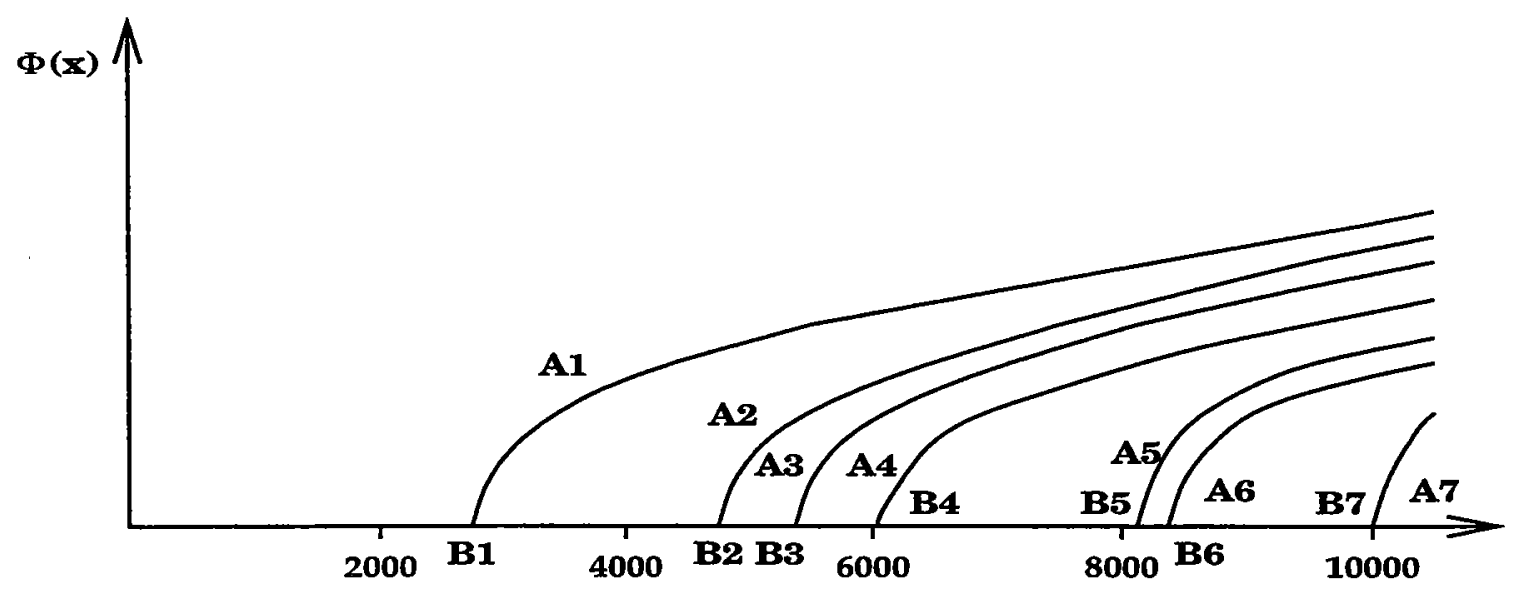

$\mathbf{R a}_{\mathbf{S}}$

Figure 10: The schematic diagram of the variation of a measure $\phi(x)$ of solution vector $\mathbf{x}$ with solute Rayleigh number $R a_{S} ; \gamma=5, R a_{T}=10000, D_{R}=0.1$. The horizontal and side-wall boundaries are stress-free with no flux of temperature and solute prescribed at the side walls. $A 1, \ldots, A 7$ are the bifurcating branches, $B 1, \ldots, B 7$ are the symmetry-breaking bifurcation points (only one asymmetric branch for each of the pitchfork bifurcations is depicted). 
$\boldsymbol{\sigma}$
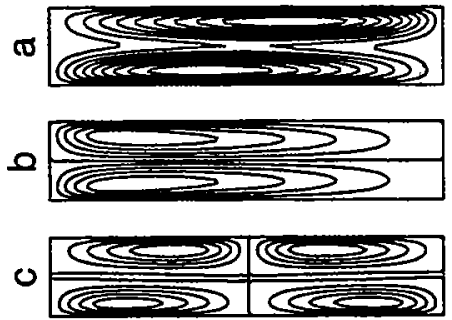

$\boldsymbol{O}$

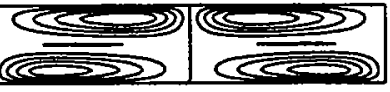

$\boldsymbol{\Phi}$

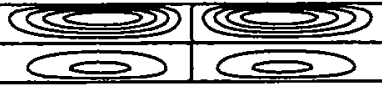

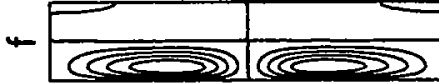
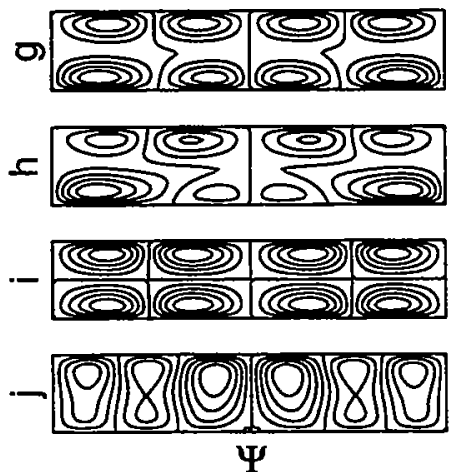
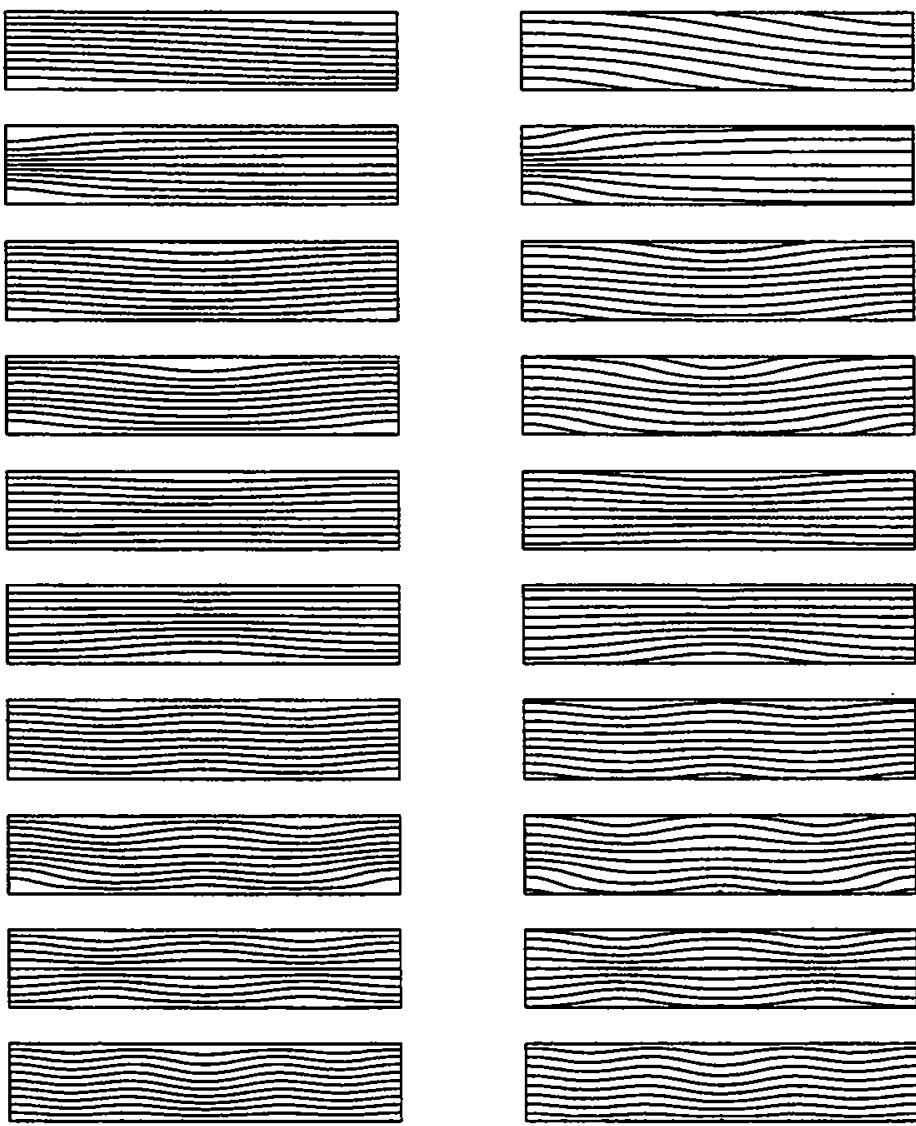

$T$
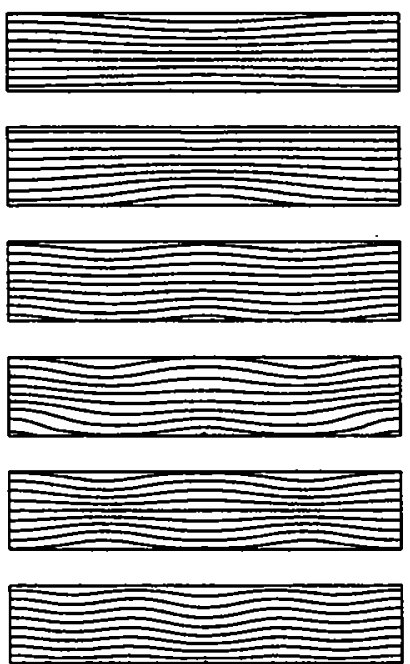

C

Figure 11: Flow patterns arising from the series of bifurcations as $R a_{S}$ is increased; $\gamma=5$, $R a_{T}=10000, D_{R}=0.1$. All the boundaries are stress-free, with no flux of temperature and solute being prescribed at the side walls. $\Psi$ : streamlines; $T$ : isotherms; $C$ : the lines of the constant solute concentration; (a) branch $A 1, R a_{S}=3001$; (b) branch $A 2, R a_{S}=6056$; (c) branch $A 3, R a_{S}=5897$; (d) branch $A 3, R a_{S}=6452$; (e) branch $A 4, R a_{S}=6100$; (f) branch $A 4$ (the branch opposite to the previous one), $R a_{S}=6420$; (g) branch $A 5, R a_{S}=8430$; (h) branch $A 5, R a_{S}=9048$; (h) branch $A 6, R a_{S}=8780$; (i) branch $A 7, R a_{S}=10753$. 


\title{
An Analog of Double-Diffusive Instabilities in Magma Chambers
}

\author{
Tonushree Kundu
}

August 20, 1996

\section{The General Problem}

Flows in magma chambers can be double-diffusive and form "fingers" just as ocean water does. In this instance, however, the compostion of the magma's constituents plays the role of "salt" because of its low diffusivity. A schematic of a typical magma chamber is displayed in Fig 1 . This situation arises when warmer, newer magma $B$ is suddenly injected into the magma chamber containing magma A, which has equilibrated with its surroundings. The properties of the two magmas are highly dependent on the crystals and bubbles in suspension. As magmas heat and cool, different components crystalize or melt in the magma and remain suspended due to the high viscosity. Also, because these crystals are anhydrous, they leave behind excess water. The magma can become saturated in water and then form bubbles. Typical density and viscosity profiles are shown in Fig. 2. For a cooling magma, the density increases when dense crystals form, and the density decreases when light crystals or water bubbles form. Also, viscosity decreases with temperature.

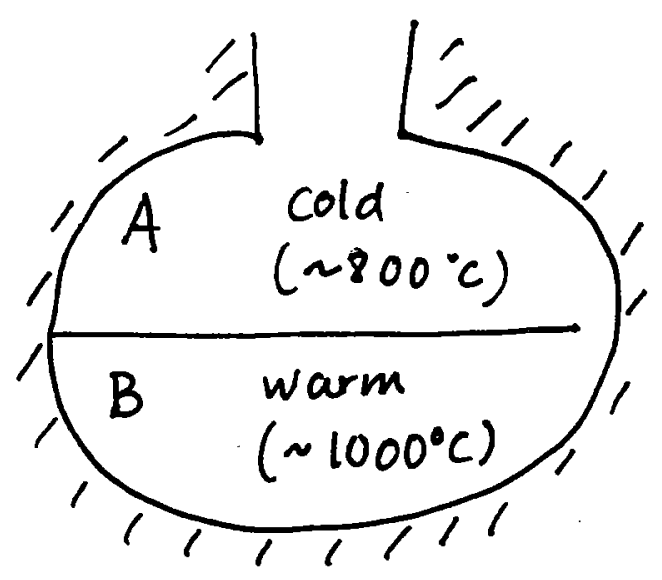

Fig. 1: A schematic of a typical magma chamber. 
Fingering will happen on the portion of the curve where $\rho$ increases with $T$, and the following analysis refers to this situation.
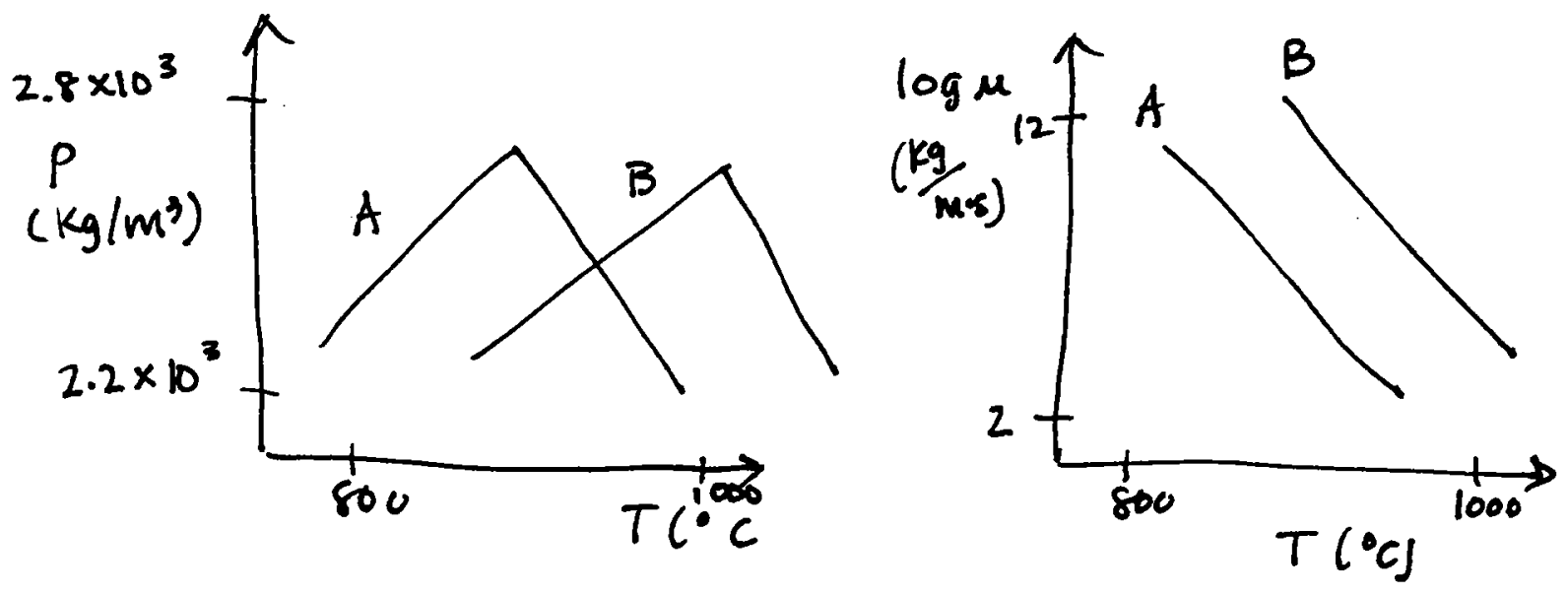

Fig. 2: Typical profiles of $\rho$ v8. $T$ and $\mu$ v8 $T$ for magmas A and B.

The profiles of temperature, concentration, density, and viscosity in the initial state and at time $t$ are shown in Fig. 3. The temperature diffuses on a scale $\delta_{T} \sim \sqrt{\kappa_{\mathrm{r}} t}$ while the concentration diffuses on a scale $\delta_{C} \sim \sqrt{\kappa_{\sigma} t}$ where, $\kappa_{T}\left(\sim 10^{-6} \mathrm{~m}^{2} / \mathrm{s}\right)$ is the diffusivity coefficient of heat and $\kappa_{c}\left(\sim 10^{-9} \mathrm{~m}^{2} / \mathrm{s}\right)$ is the diffusivity of concentration. Since $\kappa_{c} \ll \kappa T$, the concentration profile can be assumed constant over time. The upper magma will then increase in density as it is warmed, and the lower magma will decrease in density. Since the concentration remains constant, the fluid right above this interface can become more dense than fluid right below. It may even become more dense than the whole underlying fluid. In the latter case, the fluid above the interface will want to punch through into the lower layer and form fingers. This case is referred to as "giobally unstable", while the case where the fluid just above the interface is less dense will be referred to as "globally stable". "Neutral" refers to the intermediate case where the fluid right at the interface is as dense as the underlying fluid. For the evolving profile of viscosity, a similar reversal can occur.

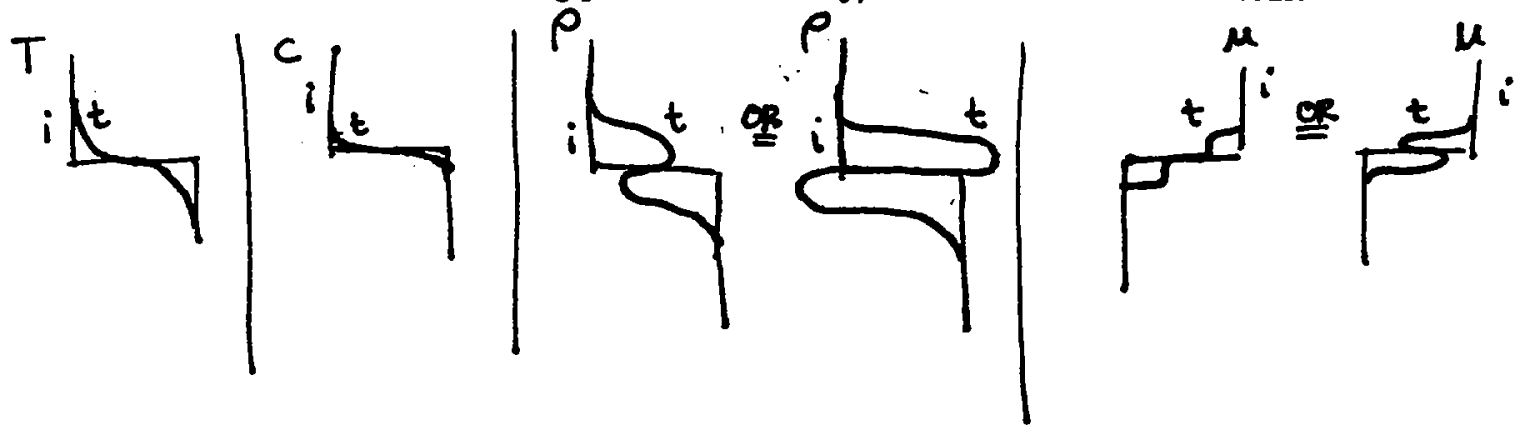

Fig. 3: Typical profiles of $T, C, \rho$, and $\mu$ through the magma chamber in the initial state and at time $t$. 


\section{A Simplified Picture}

In order to simplify the analysis, the real density profile is characterixed by with density jumps and no surface tension (Fig. 4). Since the viscosity is also a function of temperature, each of the four layers may have a different viscosity. The Rayleigh-Taylor instability of these 3 interfaces can be analyzed and then extended to include deepening of the layers as a model of thermal diffusion.

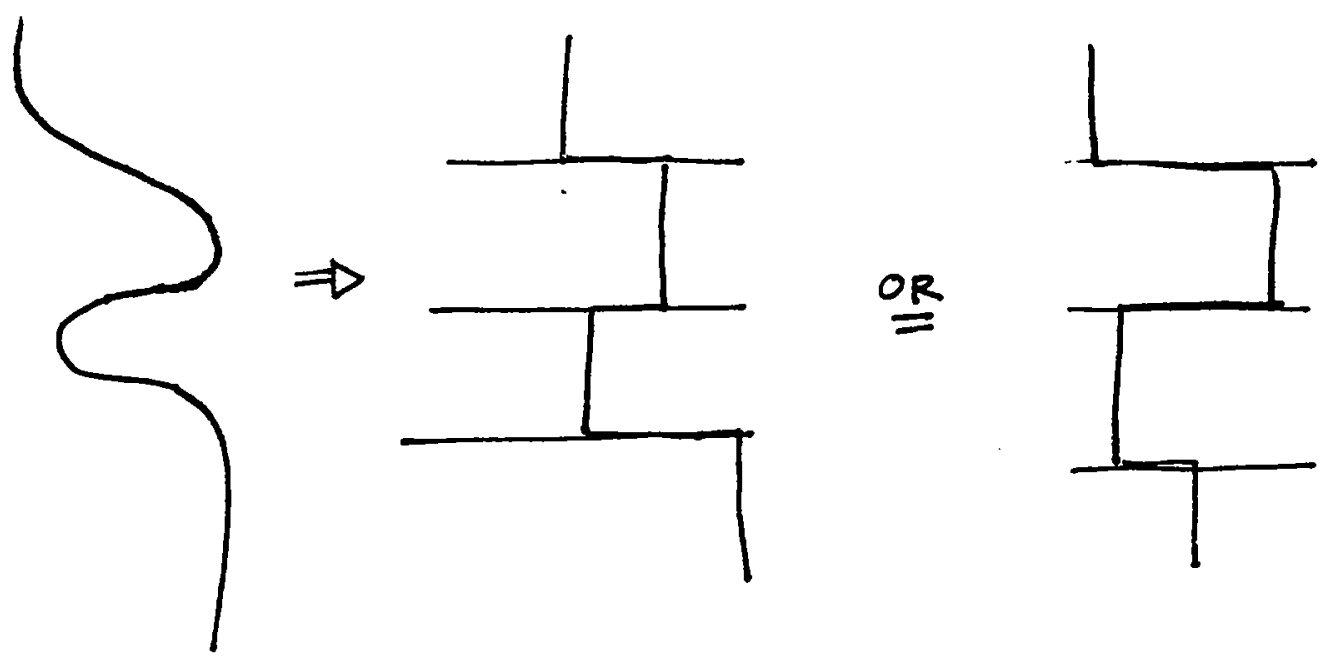

Fig. 4: Simplified density profile.

In a magma chamber, the viscosities are very large and Stokes flow $\left(\nabla^{4} \psi\right)$ can be assumed. In layer $j$, the stream function will have the form

$$
\psi_{j}=\left[\left(A_{j}+B_{j} z\right) e^{-k z}+\left(C_{j}+D_{j} z\right) e^{k x}\right] e^{i k x+\sigma t}
$$

and at the interface the following matching conditions are imposed:

1) Tangential velocity

$$
D \psi_{j-1}=D \psi_{j}
$$

2) Normal velocity

$$
\boldsymbol{\psi}_{j-1}=D \psi_{j}
$$

3) Tangential stress

$$
\mu_{j-1}\left(D^{2}+k^{2}\right) \psi_{j-1}=\mu_{j}\left(D^{2}+k^{2}\right) \psi_{j}
$$


4) Normal stress

$$
\frac{-\mu_{j-1}}{\sigma}\left(D^{2}-3 k^{2}\right) D \psi_{j-1}+\frac{\mu_{j}}{\sigma}\left(D^{2}-3 k^{2}\right) D \psi_{j}=-\frac{k^{2}}{\sigma^{2}} g\left(\rho_{j-1}-\rho_{j}\right) \psi_{j-1}
$$

where $D=\frac{d}{d z}$. In general, this problem involves 12 equations in 12 unknowns.

\section{The Symmetric Problem}

In order to make the problem more tractable and gain some understanding of how the stable upper and lower interfaces react to a perturbation of the unstable middle interface, the symmetric problem shown in Fig. 5 is considered. Note that this situation is not representative of magma. The density jumps across the first and third interfaces are the same, the inner viscosities are equal, and the outer viscosities are equal. The only physically realizable streamfunctions will have the same form at the first and third interfaces. interfaces are identical. This reduces the problem to 6 equations in 6 unknowns. Lengths are non-dimensionalized with respect to $h$ and times are non-dimensionalized with repect to $\frac{\mu_{3}}{\lambda \Delta \rho g h}$. Define the density ratio $Q=\frac{2 \lambda-1}{\lambda}$ and viscosity ratio $R=\frac{\mu_{2}}{\mu_{1}}$. The dispersion relation (with $Q=1.0$ and $R=0.1$ ) is plotted in Fig 6. Asymptotics of the dispersion relation show that as $k \rightarrow 0, \sigma \sim k^{2}$, and as $k \rightarrow \infty, \sigma \sim \frac{1}{k}$. The stable "global mode", in which the stable first and third interfaces deform much more than the unstable middle interface, will decay. The unstable "interfacial mode" where the middle interface deforms and the other two react is considered.

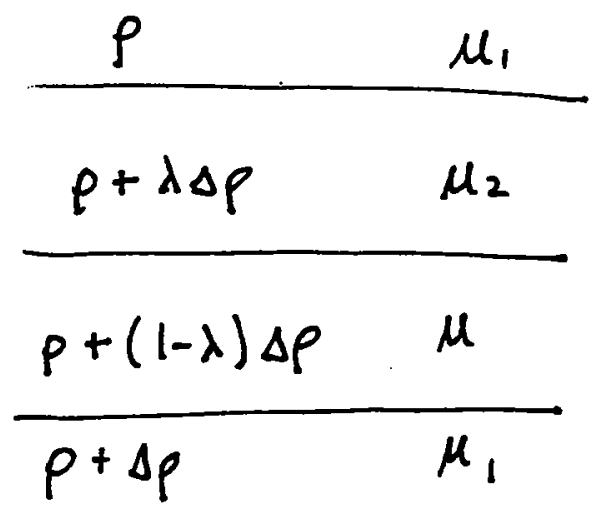

Fig. 5: Schematic of the symmetric problem.

Four limiting cases are displayed in Fig. 7. For $R \rightarrow 0$, the outer fluid is much more viscous than the inner fluid, and, in fact, seems rigid. In this case, the outer interface will not deform at all. For $R \rightarrow \infty$, the cases $Q \rightarrow 0$ and $Q \rightarrow 2$ must be considered separately. For $Q \rightarrow 0$, the unstable density jump in the middle is much smaller than the stable density jumps outside, so 
there will not be enough forcing to deform the outer layers. For $Q \rightarrow 2$, the middle density jump is larger than the outer jumps and there will be enough forcing to deform the outer interfaces. Since $\boldsymbol{R} \rightarrow \infty$, the outer fluid seems inviscid compared to the inner fluid and will just deform like the inside layers.

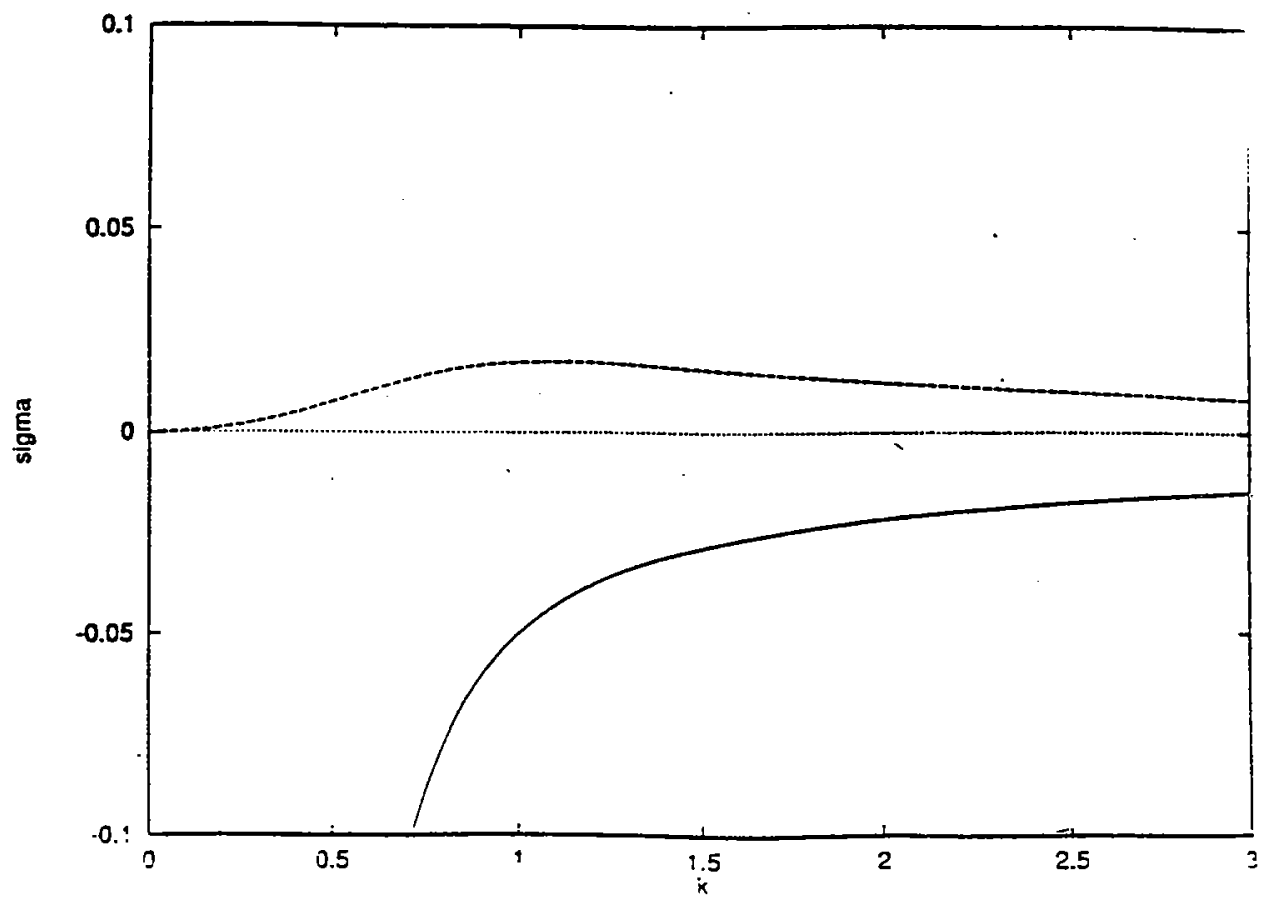

Fig. 6: Growth rate $\sigma$ a function of wavenumber $k$ for $Q=1.0$ and $R=0.1$

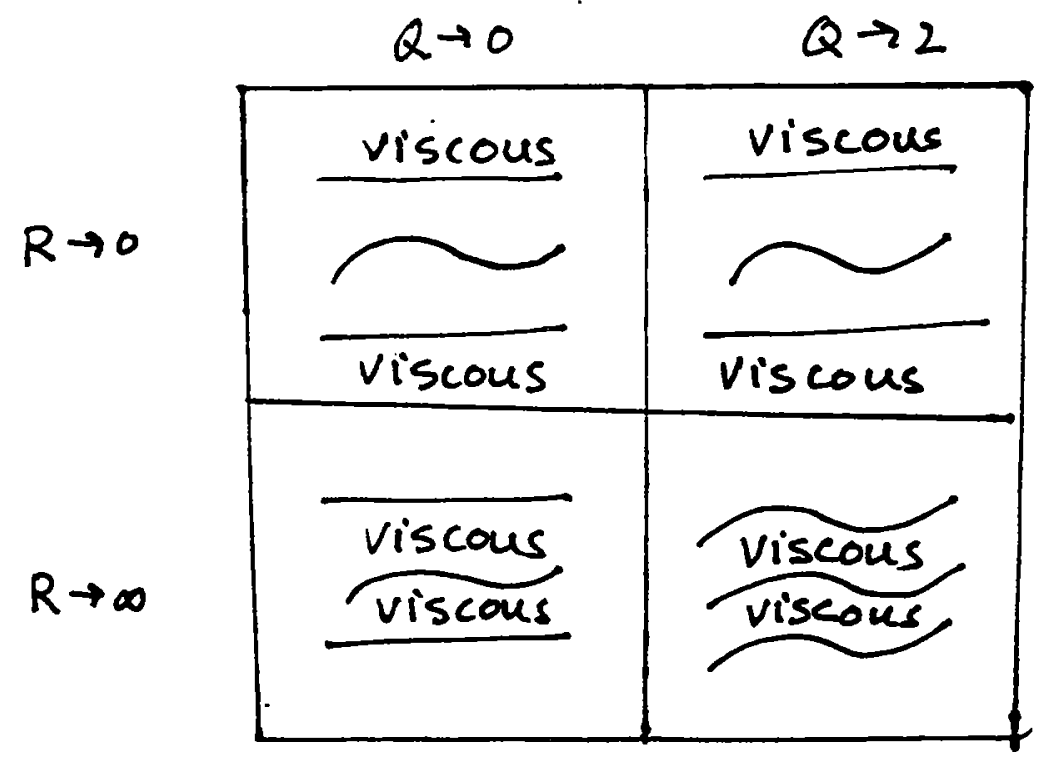

Fig. 7: Limiting cases: $R \rightarrow 0, \infty ; Q \rightarrow 0,2$. 
A plot of the ratio of deformation of the outer interface to the inner interface is given in Fig. 8 and confirms the expected limiting conditions. The asymptotic values of the maximum growth rate, $\sigma^{*}$, and wavenumber of maximum growth, $k^{*}$, are determined. For $R \rightarrow 0$ and all $Q, \sigma^{*}=\frac{Q}{R} f n\left(k^{*}\right)$ and $k^{*}$ is a constant independent of $Q$ and $R$. Note that $\sigma^{*} \sim \frac{1}{R}$ implies that the dimensional growth rate $\sigma_{D} \sim \frac{1}{\mu_{D}}$, i.e. $\sigma_{D}$ is only dependent on the inner viscosity. For $R \rightarrow \infty$ and $Q \rightarrow 0, \sigma^{*}=\frac{Q}{R} f n\left(k^{*}\right), k^{*}$ is independent of $Q$ and $R$, and $\sigma_{D} \sim \frac{1}{\mu_{2}}$. For $R \rightarrow \infty$ and $Q \rightarrow 2, \sigma^{*} \sim \frac{1}{R(2-Q)} f n\left(k^{*}, Q\right), k^{*}=f n(Q)$, and $\sigma_{D} \sim \frac{1}{\mu_{2}}$. In all the asymptotic regimes, the dimensional wavenumber depends only on the inner viscosity. The relation between $\sigma^{*} R$ and $R$ is shown in Fig. 9. For both $R \rightarrow 0$ and $R \rightarrow \infty, \sigma^{*} R$ asymptotes to a constant, confirming the fact that $\sigma^{*} \sim \frac{1}{R}$. For fixed values of $R$, the growth rate increases with increasing $Q$, i.e. more forcing produces faster growth rates.

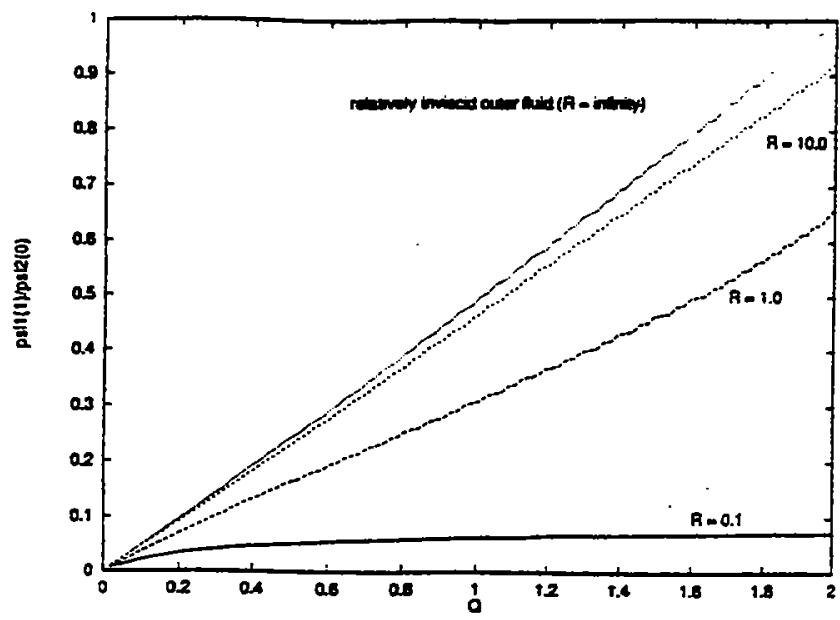

Fig 8: $\psi_{1}(1) / \psi_{2}(0)$ as a function of $Q$ for various $R$.

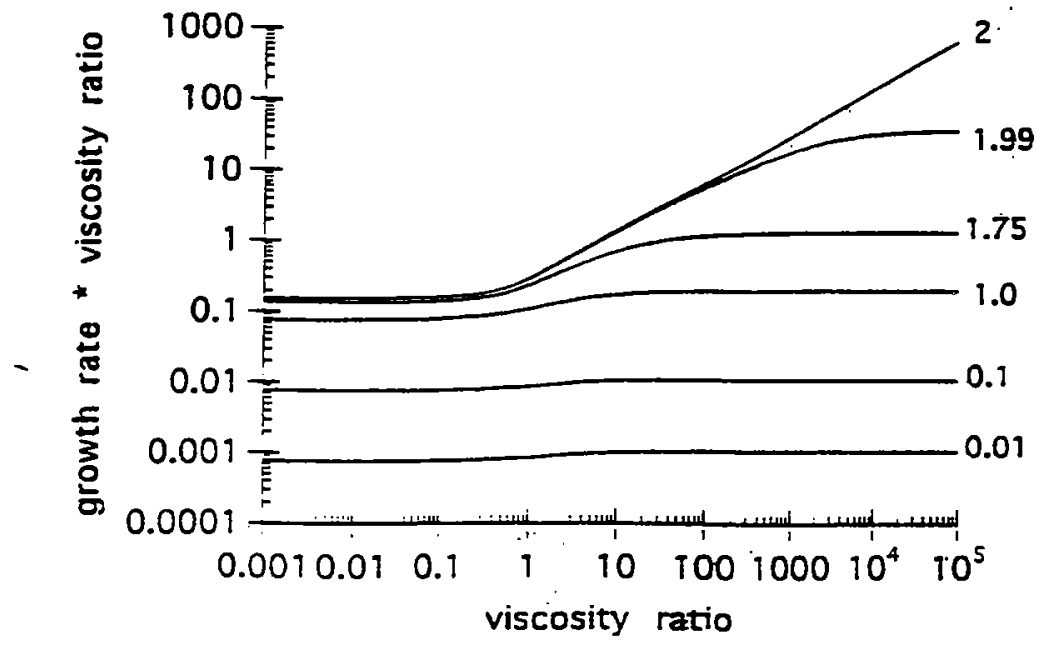

Fig. 9: $\sigma^{*} R$ as a function of $R$. 
The wavenumber $k^{*}$ is plotted against $R$ in Fig. 10. As $R \rightarrow 0, k^{*}$ approaches a constant value independent of $Q$, confirming the asymptotics. In general, there are two competing factors involved in determining the wavelength of the perturbation. Increasing the wavelength causes fluid from further away to be pulled in. For stable density profiles, this would require a large forcing. However, decreasing the wavelength results in higher viscous forces. Thus, at fixed $R$, higher $Q$ would cause more forcing and allow the wavelength to increase (and $k^{*}$ to decrease). At fixed $Q$, increasing $\boldsymbol{R}$ would cause more viscous forces and make the wavelength of perturbation increase. Both of these properties are exhibited in Fig. 10.

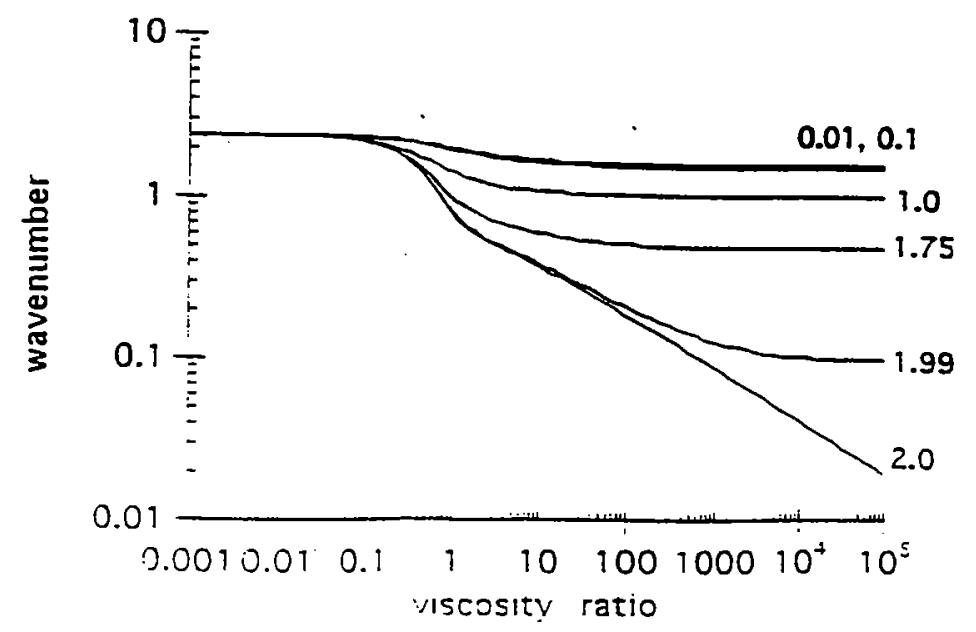

Fig. 10: $k^{*}$ as a function of $R$ for various $Q$.

\section{The Magma Case}

In the case of magma, the convenient symmetries of viscosities as above are not present, but the same density profile can be considered. A schematic of the four magma layers is presented in Fig. 11. Since the upper layer is colder, it is much more viscous than the bottom layer $\left(\mu_{1} \gg \mu_{4}\right)$. The viscosities in the middle layers can take on a large range of values, and both situations $\mu_{3} \gg \mu_{2}$ and $\mu_{2} \gg \mu_{3}$ can be realized. This is still a rather complicated problem, and some simplifications can be made, in particular, $\mu_{1} \gg \mu_{2}, \mu_{3} \gg \mu_{4}$. The top layer now is essentially rigid with respect to the underlying layers, while the bottom layer is relatively inviscid (but still satisfies Stokes flow). The simplified magma case is shown in Fig. 12.

Times are non-dimensionalized with respect to $\frac{\mu_{2}}{\lambda \Delta \rho g h}$ and lengths are non-dimensionalized with respect to $h$. The density and viscosity ratios are defined as $Q=\frac{2 \lambda-1}{\lambda}$ and $R=\frac{\mu_{3}}{\mu_{3}}$. The four limiting cases of $R \rightarrow 0 ; Q \rightarrow 0, Q \rightarrow 2$ and $R \rightarrow \infty ; Q \rightarrow 0, Q \rightarrow 2$ are considered and represented in Fig. 13. As $R \rightarrow 0$, the third layer becomes relatively inviscid. The third layer no longer provides any resistance to motion, and no information about the deforming interface can propagate through to the lower interface. Therefore, regardless of the value of $Q$, the lower interface will not deform and only interfacial mixing will occur. Since the third layer no longer 
affects the motion, $\sigma_{D} \sim f n\left(\mu_{2}\right)$ and becomes independent of $\mu_{2}$.

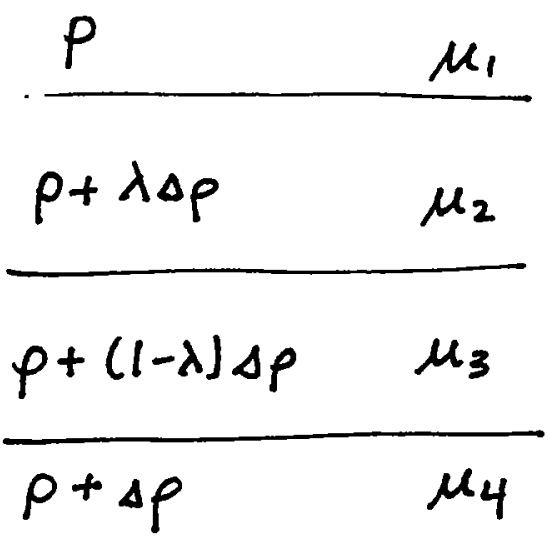

Fig. 11: A model for real magma.

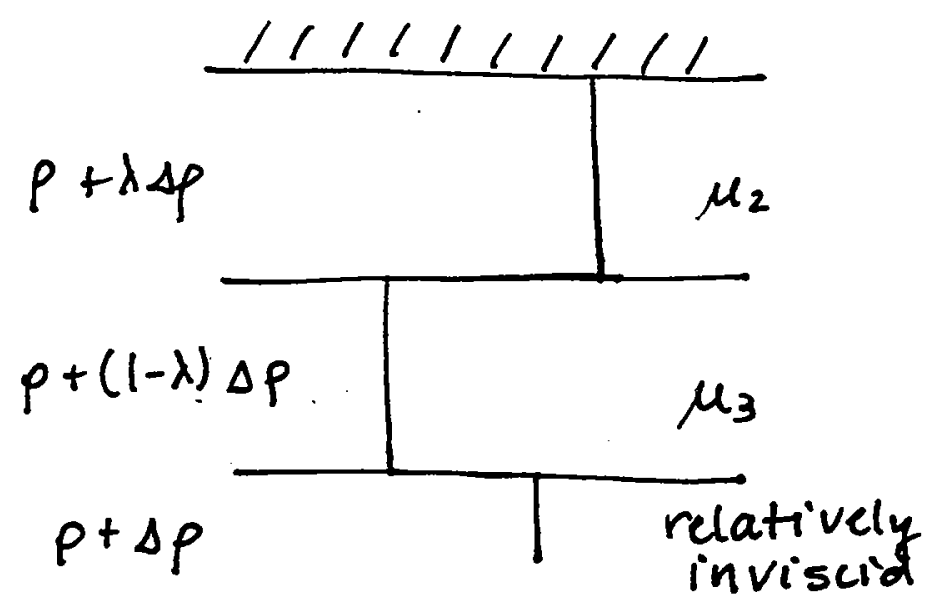

Fig. 12: A simplified model for magma.

As $R \rightarrow \infty$ the third layer becomes much more viscous than the top interface. However, there will be a difference in the behavior of the third interface depending on whether $Q \rightarrow 0$ or $Q \rightarrow 2$. When $Q \rightarrow 0$, the density destabilization is much weaker than the density stabilization at the lower interface. The forcing will not be strong enough to cause the lower interface to move. Since the 
second layer no longer affects the motion, $\sigma_{D} \sim f n\left(\mu_{3}\right)$ and becomes independent of $\mu_{2}$. For the case $Q \rightarrow 2$, the density destabilization is twice as large as the density stabilization at the lower interface, 80 the forcing will be capable of perturbing the bottom interface. Since the bottom layer is so much less viscous, it provides virtually no resistance to motion and will deform along with the middle interface. In fact, the third layer will just be buckling and the shear will be small. In the second layer, however, the fluid is much less viscous, but since it is moving with the third layer at the second interface while maintaining no-slip at the top, there will be high shear. Thus, the second layer is characterised by high shear and low viscosity while the third layer is characterized by low shear and high viscosity. Forces in both layers will be significant, and $\sigma_{D}$ should scale with both $\mu_{2}$ and $\mu_{3}$, i.e. $\sigma_{D} \sim f n\left(\mu_{2}, \mu_{3}\right)$.

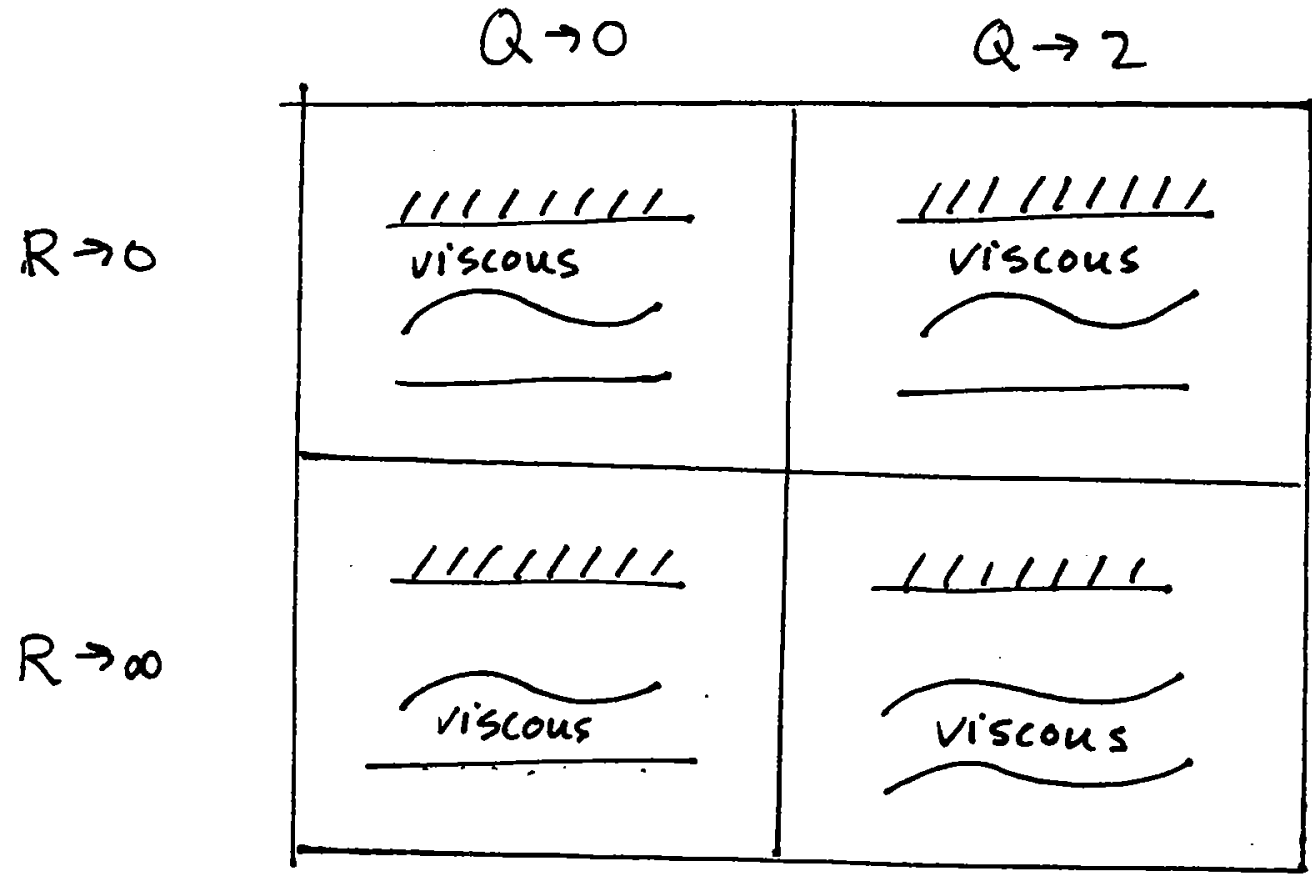

Fig. 13: Limiting cases: $R \rightarrow 0, \infty ; Q \rightarrow 0,2$

A plot of $\psi_{2}(-1) / \psi_{2}(0)$, i.e. the ratio of the deformation of the bottom to the middle interface, is given in Fig. 14. As $R \rightarrow 0$, the deformation of the bottom interface does indeed go to zero regardless of the value of $Q$. At large $R$, the behavior changes as $Q$ changes. For small $Q$, the lower interface barely moves, but at large $Q$, the ratio of streamfunctions approaces unity. This agrees with the limiting cases. 


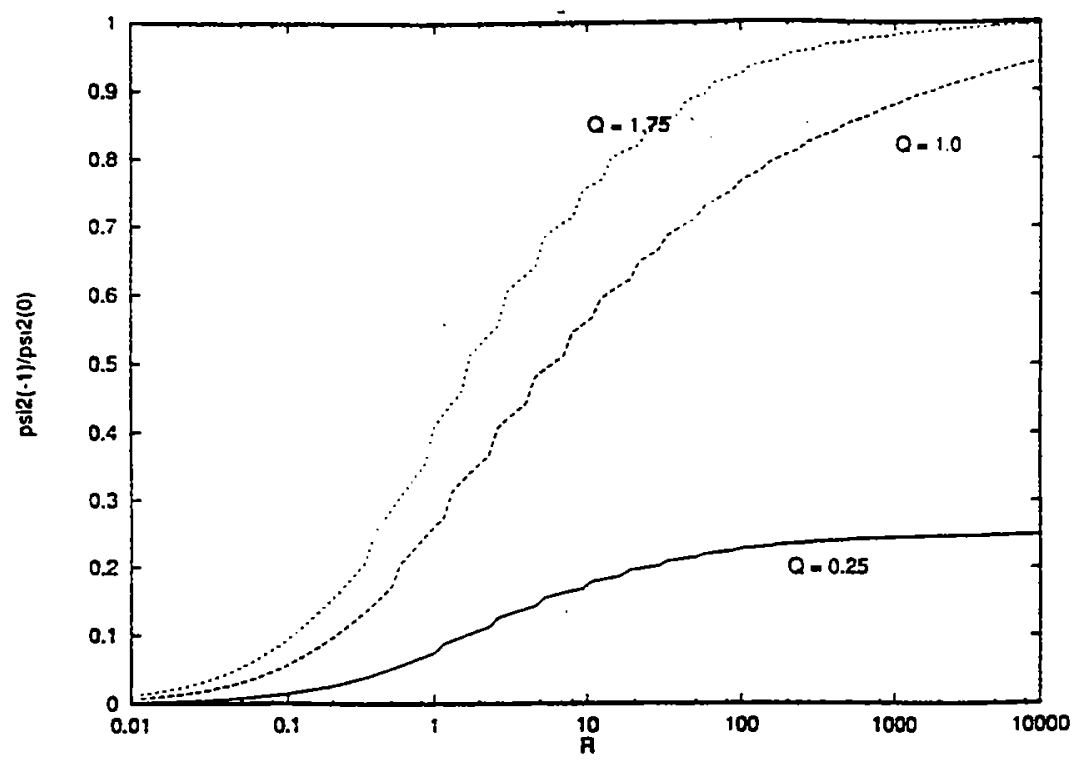

Fig. 14: $\psi_{2}(-1) / \psi_{2}(0)$ as a function of $R$ for various $Q$.

Wavenumber $k^{*}$ is given as a function of $R$ for various $Q$ in Fig. 15. When $R$ gets very large, $\boldsymbol{k}^{*} \sim \boldsymbol{R}^{-\frac{3}{3}}$. stope $-1 / 6$

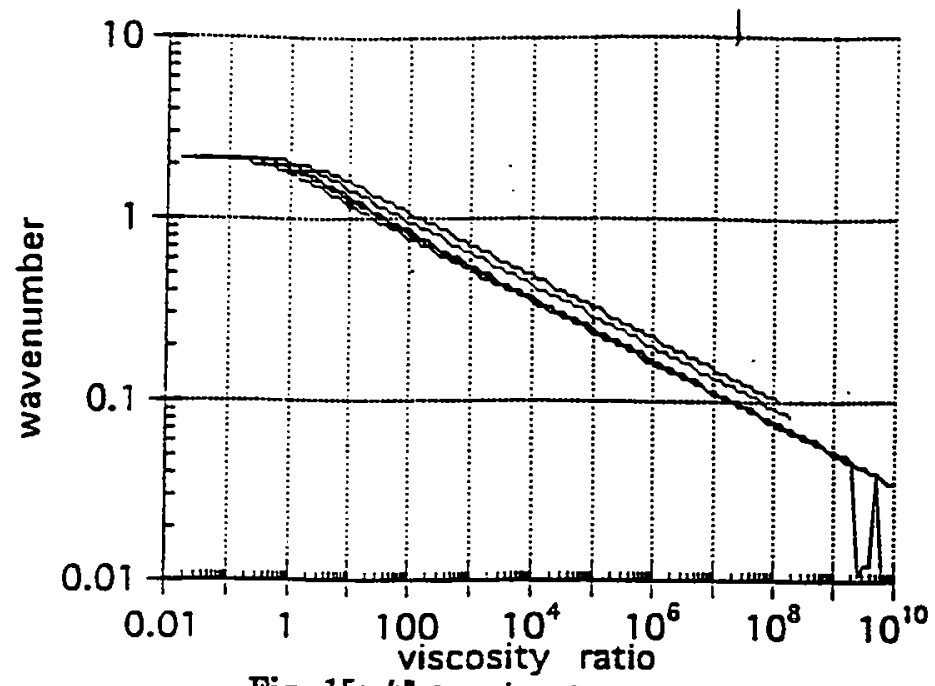

Fig. 15: $k^{*}$ as a tunction of $k$

Fig. 16 shows the growth rate $\sigma^{*}$ as a function of the viscosity ratio $R$. For each of the three regimes $(Q<1, Q=1, Q>1), \sigma^{*} \sim R^{n}$ for some $n$ particular to each of the three regions. For $Q<1$ (globally stable), $\sigma^{*} \sim R$ for large $R$. Note that in this case, $\sigma_{D} \sim \frac{1}{\mu_{s}}$, which was expected from the limiting case $R \rightarrow \infty, Q \rightarrow 0$. For $Q=1$ (neutral), $\sigma^{*} \sim R^{-\frac{2}{3}}$ and for $Q>1.0$ (globally unstable), $\sigma^{*} \sim R^{-\frac{1}{3}}$. This suggests a more compicated scaling for $\sigma_{D}$ when $Q$ has magnitude unity or larger, which is consistent with the physical arguments given above for the limiting case $R \rightarrow \infty, Q \rightarrow 2$. It is interesting to note that in Fig. 16, for $Q$ just slightly less than or greater 
than one, the graph of $\sigma^{*}$ vs $R$ follows the graph for $Q=1$ very closely until $R \rightarrow \infty$. Therefore, for values of $Q$ near 1.0, deviations from the neutral condition are not noticed until $R \rightarrow \infty$.

slopes $-1 ;-2 / 3 ;-1 / 3$

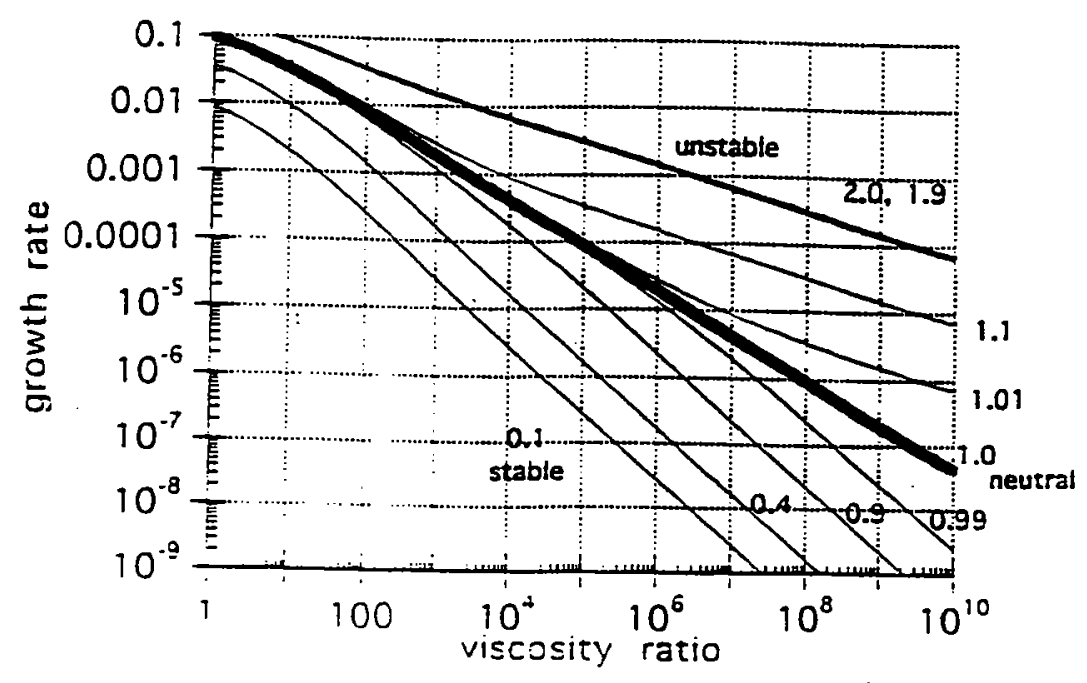

Fig. 16: $\sigma^{*}$ vs. $R$ for various values of $Q$.

\section{The Time-Dependent Problem}

Real magma has thickening layers due to the diffusion of heat, i.e. $d_{z} \sim \sqrt{\kappa_{\tau} t}$ where $d_{\Sigma}$ is the depth of each of the middle layers. The above constant layer thicknesses are modified to accomodate growing layers, and compositional diffusion is neglected. The streamfunction will now have the form

$$
\psi(z, z, t)=A(k, t) e^{i k z} f n(z)
$$

At each time,

$$
\frac{\dot{A}(k)}{A(k)}=\sigma_{D}\left(k_{D}, d_{L}(t)\right)
$$

Note that $\sigma_{D}$ and $k_{D}$ correspond to dimensional quantities. Integrating gives

$$
A\left(k, t_{1}\right)=A(k, 0) e^{\int_{0}^{t_{1}} \sigma_{D}\left(k_{D}, d_{L}(t)\right) d t} .
$$

From the non-dimensionalization,

$$
\sigma_{D}\left(k, d_{L}\right)=\left(\frac{\lambda \Delta \rho g d_{L}}{\mu_{2}}\right) \sigma^{*}(k)
$$




$$
\begin{aligned}
& =\left(\frac{\lambda \Delta \rho g d_{L}}{\mu_{2}}\right) f\left(k_{D} d_{L}\right) \\
& =\left(\frac{\lambda \Delta \rho g \sqrt{\kappa_{T}}}{\mu_{2}}\right) \sqrt{t} f\left(k_{D} \sqrt{\kappa_{T} t}\right)
\end{aligned}
$$

A typical dispersion relation, $f$, is given in Fig. 17 As $t$ increases, the argument of $f$ increases as $\sqrt{t}$, which is motion along the curve $f(x)$ as shown in Fig. 17. Also, the actual magnitude of $\sigma_{D}$ goes as $\sqrt{t}$, so $\sigma_{D}$ increases with time. Fig. 18 shows roughly how the $\sigma_{D}$ vs. $k_{D}$ curve varies with time.

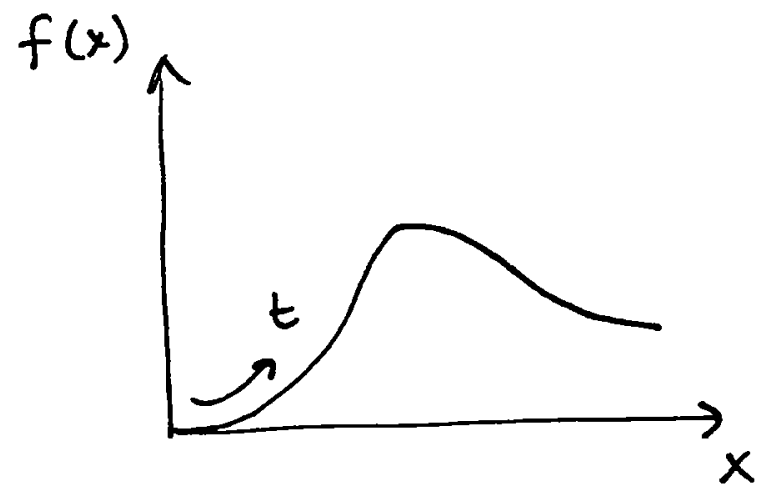

Fig. 17: A typical dispersion relation: $f(x)=\sigma_{D}, x=k_{D}$.

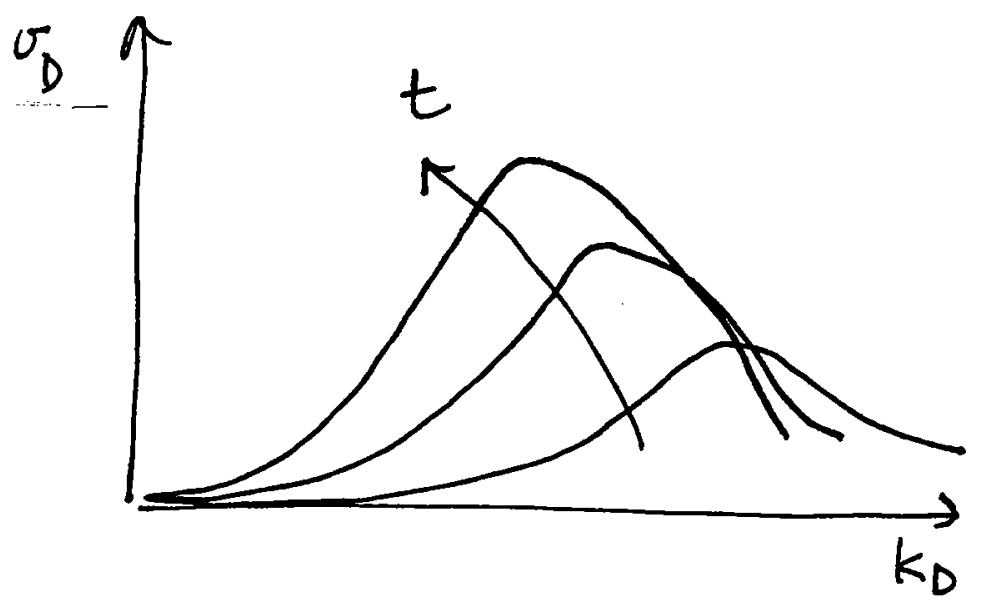

Fig. 18: $\sigma_{D}$ v8. $k_{D}$ with increasing time.

With growing magma layers, the thickness of the fingers will depend on the wavenumber of maximum amplitude rather than the wavenumber of maximum growth rate $\sigma^{*}$. Just as $\sigma^{*}$ changes 
with time, $A_{\max }$ changes with time. Previous results showed that $k\left(\sigma_{\text {mex }}\right)=\frac{k^{\circ}}{d_{L}}$, and it can be shown mathematically that $k\left(A_{\max }\right)=\frac{\hat{k}}{d}$, where $\hat{k}=f n(Q, R)$, i.e. $\hat{k}$ is independent of time. This implies that, for each time $t$, the offset between $k\left(\sigma_{\text {mas }}\right)$ and $k\left(A_{\text {mas }}\right)$ is constant (Fig. 19).

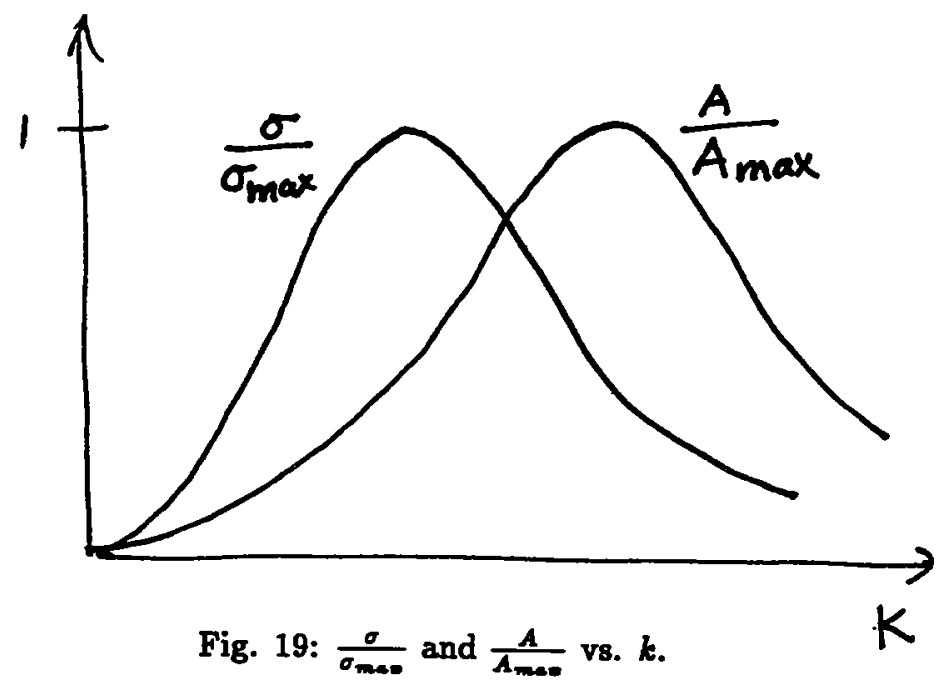

The wavenumbers of maximum growth rate $k^{*}$ and maximum amplitude $\hat{k}$ are shown in Figs. 20-22 for various $Q^{\prime}$ s. For all three regimes of $Q$ (globally stable, neutral, globally unstable), $\hat{k}$ and $k^{*}$ are very similar. Hence, $k^{*}$ is a good approximation for the scale of fingers.

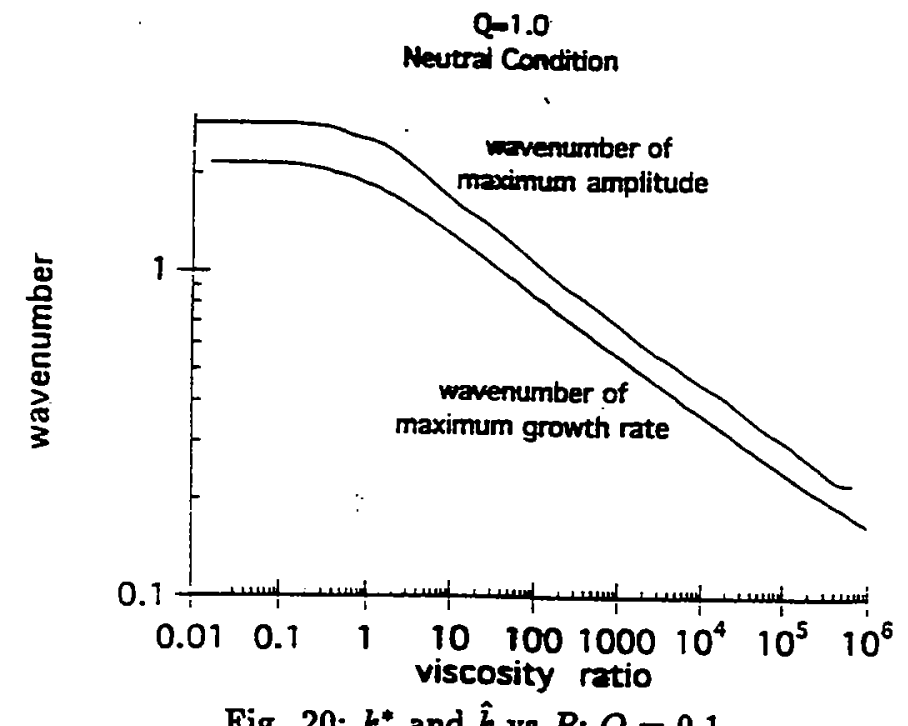

Fig. 20: $k^{*}$ and $\hat{k}$ vs $R ; Q=0.1$ 


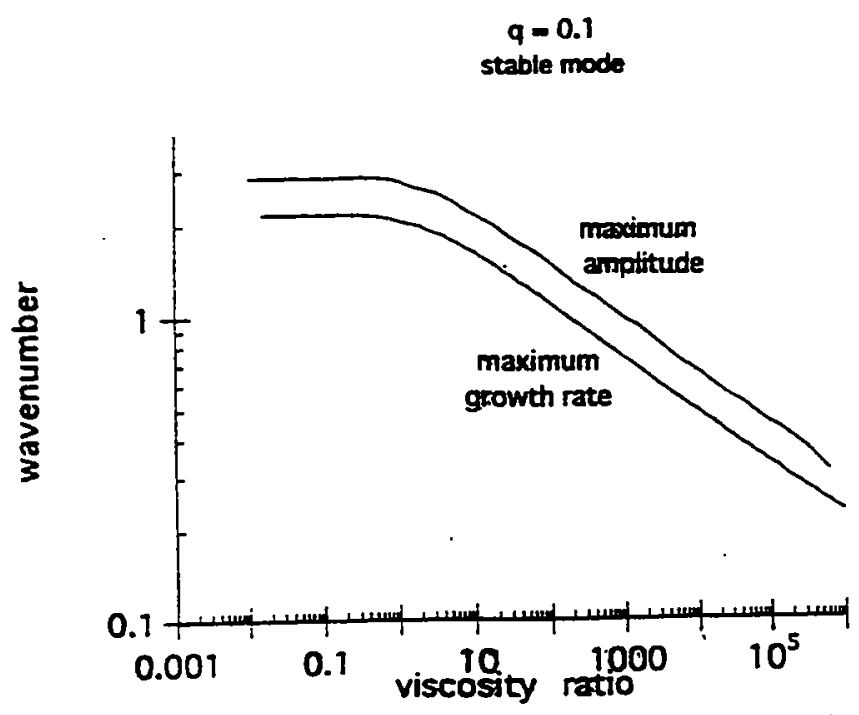

Fig. 21: $k^{*}$ and $\hat{k}$ vs $R ; Q=1.0$

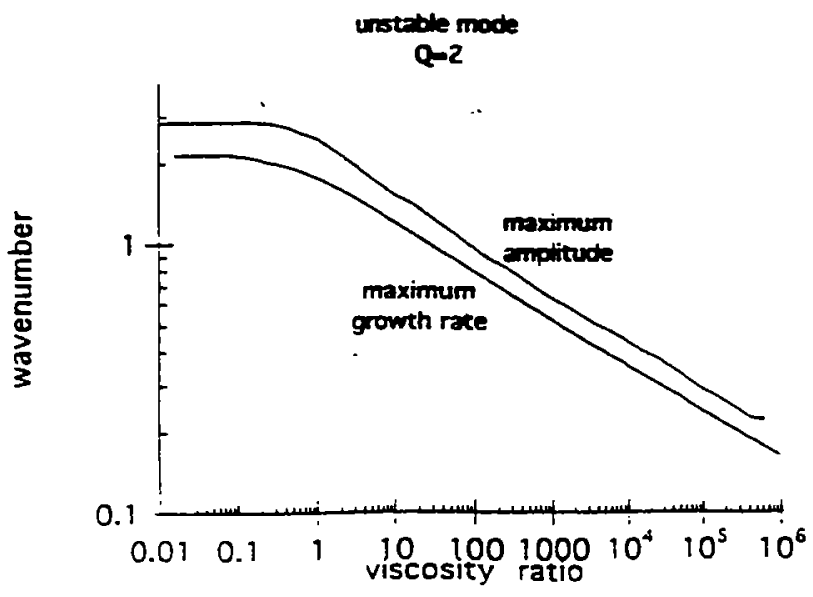

Fig. 22: $k^{*}$ and $\hat{k}$ vs $R ; Q=2.0$

\section{Scaling}

As a rough estimate, nonlinear fingers are expected to develop when

$$
\frac{\dot{A}}{A} \sim \frac{\dot{d_{L}}}{d_{\Sigma}},
$$


i.e. the rate of growth of the perturbation is of the same order as the rate of growth of the thickening layers. The layer depth $d_{L} \sim \sqrt{\kappa_{T} t}$ and $\dot{d}_{L} \sim \sqrt{\frac{k_{T}}{t}}$, so $\frac{d_{L}}{d_{L}} \sim \frac{1}{t}$. Furthermore,

$$
\frac{\dot{A}}{A} \sim \sigma_{D}=\frac{\lambda \Delta \rho g d_{L}}{\mu_{2}} \sigma^{*},
$$

so the critical time when the layers go unstable is

$$
t_{\text {ins }} \sim\left[\frac{\mu_{2}}{\lambda \Delta \rho g \sigma^{*} \sqrt{\kappa_{T}}}\right]^{\frac{2}{3}}
$$

Since $k_{D} \sim \frac{k^{*}}{d_{L}}$, the wavelength of perturbation is

$$
\lambda_{\text {ind }}=\frac{d_{\text {ine }}}{k^{*}}
$$

and $d_{\text {ins }}=\sqrt{\kappa_{\mathrm{r}} t_{\text {crit }}}$.

For a typical magma, rough estimates of viscosity are $\mu_{1} \approx 10^{12} \mathrm{~kg} / \mathrm{m} \cdot \mathrm{s}, \mu_{2} \approx 10^{3}-10^{9} \mathrm{~kg} / \mathrm{m} \cdot \mathrm{s}$, $\mu_{3} \approx 10^{3}-10^{9} \mathrm{~kg} / \mathrm{m} \cdot \mathrm{s}, \mu_{4} \approx 10^{2} \mathrm{~kg} / \mathrm{m} \cdot \mathrm{s}$, also $\Delta \rho \approx 100 \mathrm{~kg} / \mathrm{m}^{3}$ and $\kappa_{\mathrm{x}} \approx 10^{-6} \mathrm{~m}^{2} / \mathrm{s}$. To obtain the scaling for fingers, the asymptotic scalings determined numerically are applied. The extreme cases, $\mu_{2}=10^{3} \mathrm{~kg} / \mathrm{m} \cdot \mathrm{s}$ and $\mu_{2}=10^{9} \mathrm{~kg} / \mathrm{m} \cdot \mathrm{s}$ give a range for $t_{i n s}$ and $d_{i n s}$ (Fig. 23).

\begin{tabular}{|c|c|c|}
\hline $\begin{array}{c}\mu_{2} \\
(\mathrm{~kg} / \mathrm{m} \cdot \mathrm{s})\end{array}$ & $10^{3}$ & $10^{9}$ \\
\hline$R$ & $1-10^{6}$ & $10^{-6}-1$ \\
\hline $\begin{array}{c}t_{\text {ins }} \\
(\mathrm{s})\end{array}$ & $10^{2}-10^{3}$ & $10^{6}-10^{7}$ \\
\hline $\begin{array}{c}\lambda_{\text {ins }} \\
\text { (m) }\end{array}$ & $10^{-2}-10^{-1}$ & $10^{-3}-10^{-4}$ \\
\hline
\end{tabular}

Fig. 23: Time scale and length scale of fingers determined by asymtotics

The timescale of instability is much less than the time the magma resides in the chamber ( 10-10 $0^{3}$ years), so there is certainly enough time for the instabilities to occur. A photo of mafic 
inclusions (Fig. 24) shows "blobs" that have formed in the magme. The diameters of these blobs typically range from 0.1 to $20 \mathrm{~cm}$, which is consistent with the results obtained from scaling. Blobs are formed rather than fingers, and Fig. 25 illustrates a possible mechanism for blob formation. At first, the middle layer is (a) perturbed a bit. The perturbation then (b) grows until it (c) starts being suppressed by the upper, highly viscous magma. Blobs start (d) pinching off and separating at which time the third layer in the original system has risen to meet the highly viscous layer. Diffusion causes (e) the formation of new middle layers and the process repeats itself.

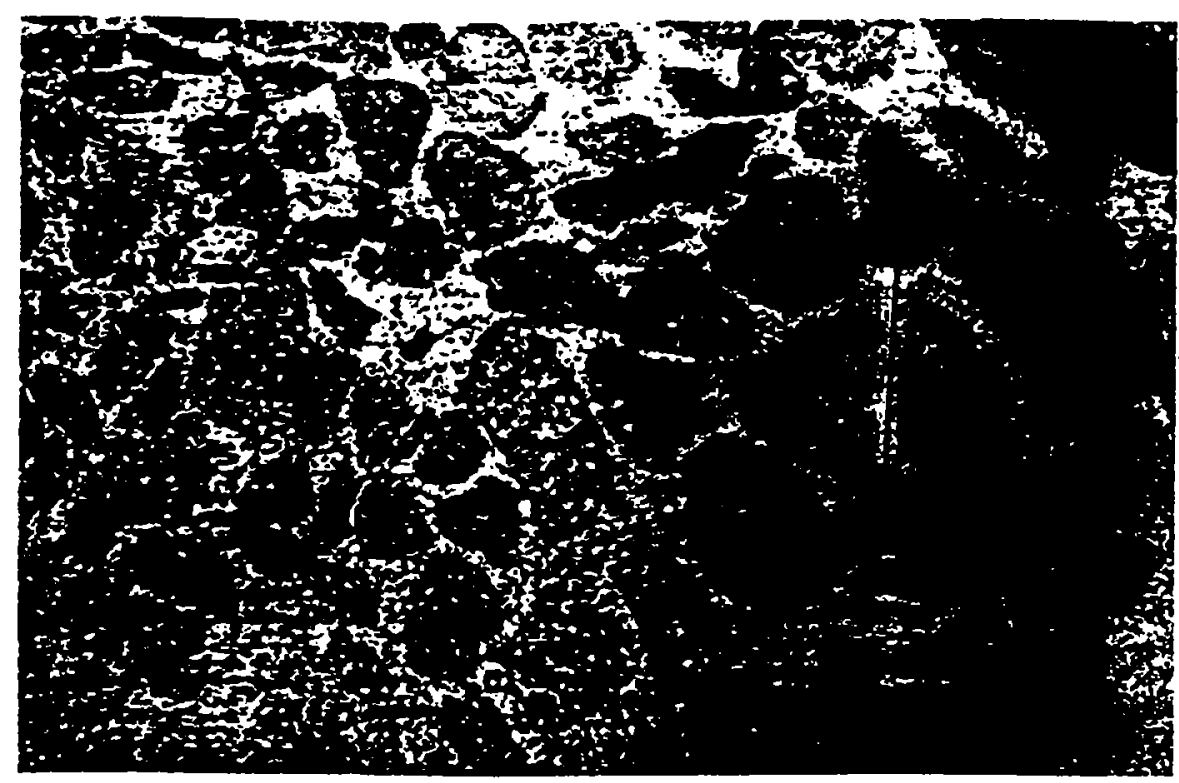

Fig. 24: Mafic inclusions ("blobs")

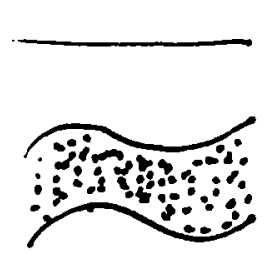

(a)

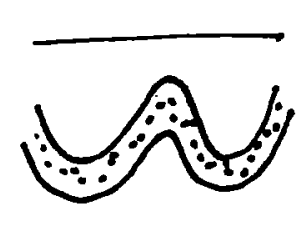

(b)

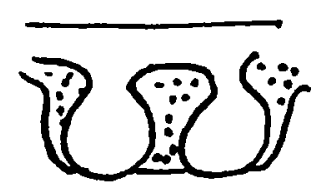

(c)

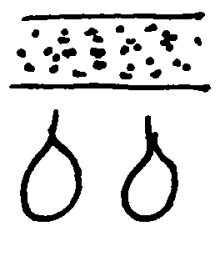

(d)

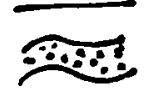

(e)

Fig. 25: A possible mechanism for blob formation. 


\section{Conclusions}

Analysis fo the Rayleigh-Taylor instability for a typical volcanic magma suggests two regimes. In one regime, (realized when the density destabilization at the middle interface becomes small or when the second layer becomes highly viscous) the bottom interface hardly moves and only local mixing occurs at the middle interface. In the other regime (realised when the third layer is highly viscous and the density destabilisation becomes very strong) the bottom interface moves as the middle interface and fingers can form. The scaling determined by this analysis agress quite well with geological data and suggests a possible mechanism for the formation of mafic inclusions ("blobs").

The above analysis involves only linear stability theory, but some nonlinear analysis would be helpful. The linear stability should predict the scaling of the fingers within a reasonable degree of accuracy, but the nonlinear theory (perhaps using Boundary Integral Methods) could be used to determine the evolution of the fingers over time. 


\title{
Semi-convection in stars
}

\author{
Suzanne Talon
}

August 23, 1996

\section{The general problem}

Stellar models may be built using simple considerations (see e.g. Kippenhahn \& Weigert 1994). We use the equations for the conservation of mass, energy and momentum, the last one being further simplified by considering hydrostatic balance, which is suitable for most cases encountered in stellar evolution. We then need to describe how heat is transported, and thus find if radiative conductivity can transport all the heat produced by nuclear burning alone, or if the temperature gradient required to carry all the energy to the surface is too large, thus leading to convection.

In most cases, the answer to that question is rather straightforward and numerical models can be built. We will here concentrate our attention on a more complex situation called "semi-convection" in the astrophysical litterature.

\subsection{Thermal conductivity and chemical composition}

In the core of massive stars, the major source of opacity is electron scattering. The stellar material is completely ionized there and, assuming that the star is made only of helium $(Y)$ and hydrogen $(X)$ (which represent about $98 \%$ of the matter in a normal main sequence star), we can derive a formula that relates the thermal conductivity to the chemical composition:

$$
\kappa_{T} \sim \frac{1}{2-Y} .
$$


That formula expresses the fact that, since helium contributes only 2 electrons for 4 mass units whereas hydrogen contributes 1 for 1 mass unit, helium is less opaque than hydrogen, and the thermal conductivity is higher when the helium mass fraction is higher.

This gives rise to a very interesting process called semi-convection. If we neglect the dependance of the thermal conductivity on any parameter other than chemical composition, in the presence of a (linear) helium gradient, the temperature profile will be parabolic. However, if the helium is homogenized, the temperature profile will become linear, and the maximum value of the temperature gradient will be lowered.

By adding a sufficient amount of helium, it is thus possible to stabilize an unstable temperature profile.

\subsection{Stellar models}

The first people to encounter that situation were Schwarzschild \& Härm (1958), when they built a model for a $30 M_{\odot}$ star, with a growing convective core. The limit of the convective core is given by $\nabla_{\text {rad }}=\nabla_{\text {add }}$, where $\nabla=\frac{d \ln T}{d \ln P}$. Since the pressure, temperature, luminosity and $\nabla_{\text {ad }}$ are all continuous accross the boundary, the chemical discontinuity leads to the condition $\nabla_{\text {rad }}>\nabla_{\text {ad. }}$. However, as soon as convection would start in that region, the mixing of helium would stabilize it, thus impeding the convection. Their conclusion was thus that some process redistributes material outside the convective core until a state where

$$
\nabla_{\mathrm{rad}}=\nabla_{\mathrm{ad}}
$$

throughout the chemical gradient region is reached. They named that process "semi-convection".

One year later, Sakashita \& Hayashi (1959) published a paper where they proposed a similar solution to the problem, but argued that the proper criterion for the onset of overturning convection in the presence of a mean molecular weight gradient was not (2) but rather

$$
\nabla_{\mathrm{rad}}=\nabla_{\mathrm{ad}}+\frac{\beta}{4-3 \beta} \nabla_{\mu} \equiv \nabla_{L}
$$

as showed by Ledoux (1947), where $\nabla_{\mu}=(\mathrm{d} \ln \mu / \mathrm{d} \ln P), \mu$ is the mean molecular weight per particule (for a completely ionized mixture of helium and hydrogen, $\mu=\left(2-\frac{5}{4} Y\right)^{-1}$ ), and $\beta$ is the ratio of gaz pressure to total pressure. That lead to quite different models.

The other important contribution to the field has been made by Kato, in his 1966 paper 


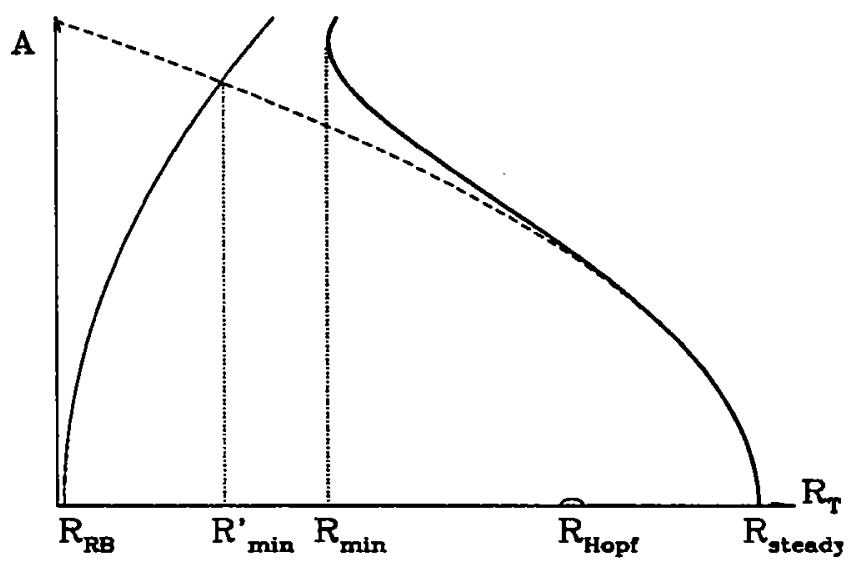

Figure 1: Schematic representation of the "neutrality" condition.

where he describes the possibilty of double-diffusive overstability in the case where

$$
\nabla_{\text {ad }}<\nabla_{\text {rad }}<\nabla_{L}
$$

That lead a few people to use overstability as a source of mixing. However, since in experiments overstability quickly turns into overturning convection, it is not clear whether or not that process is relevent.

\subsection{A new look at an old problem}

In other situations where the "diffusive" stratification of double-diffusion has been studied (see e.g. Veronis 1965), it has been found that the onset of steady convection is actually subcritical, leading to a finite amplitude instability. That is related to the fact that as the instability sets in, it destroys the stabilizing effect of the slowly diffusing component.

If the sub-criticality is strong enough, the minimum Rayleigh number reached by the curve amplitude of the motions vs $R_{T}$ can be lower than that related to the onset of overstability, and the "neutrality" condition of the material should be given by that $R_{\min }$ instead of $R_{\mathrm{RB}}$ (or equivalently 2 ), $R_{\text {steady }}$ (or equivalently 3 ) or $R_{\text {Hopf }}$ (see Fig. 1).

The goal of this project is thus to evaluate $R_{\min }$, which will provide an improved "neutrality" condition. One way to do so is via a weakly non-linear expansion, but this does not give a good approximation for highly nonlinear conditions. Instead, we will use an approximation that will allow us to not evaluate the high order terms. 
As in the interesting approach of Proctor (1981), we need recognize that the semiconvective zone cannot be more unstable than a normal Rayleigh-Bénard (RB) convective zone. In the diagrams amplitude vs $R_{T}$ (where $R_{T}$ is the thermal Rayleigh number that will be formally introduced later), this means that the curve describing steady convection in the presence of helium cannot cross that of RB convection. We will thus use the intersection of our curve, calculated only with the first term to appear in the expansion analysis with the RB curve, defining the point $R_{\min }^{\prime}$ as an approximation of $R_{\min }$ (see Fig. 1).

The turning point of the real curve for steady convection $\left(R_{\min }\right)$ could be evaluated more precisely by using a boundary layer method, as was done by Proctor for the case of thermohaline convection at very low Prandlt number. However, we feel that such an improvement is not essential to stellar structure theory, since it will only marginally affect the value of $R_{\min }$, and not change the physics of the problem.

Note further that for this calculation to remain valid, turbulence must not be important in the semi-convective region. That is consitent with considering a permanent slow rearrangement of the chemical composition profile as the star evolves.

\section{The equations}

As a first step, we will model the semi-convective zone using a weakly non-Boussinesq approximation, where only the dependance of the thermal conductivity on chemical composition will be considered.

\subsection{Basic state}

In the basic state, the fluid is at rest, with a linear gradient of helium. The thermal conductivity $\kappa_{T}$ is function of the chemical composition. Since the major source of opacity is electron scattering, the dependance on the helium abundance (if we neglect elements other than hydrogen and helium) is

$$
\kappa_{T}=\bar{\kappa}(2-Y)^{-1}
$$

where $Y$ is the concentration of helium. We may write it

$$
\kappa_{T}=\bar{\kappa}\left(2-Y_{b}-\eta z^{\prime}\right)^{-1}
$$




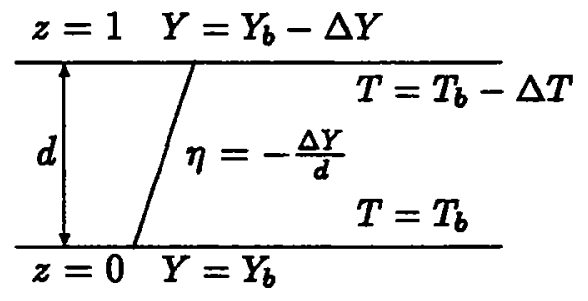

Figure 2: Basic state and boundary conditions

$$
\begin{aligned}
& =\kappa_{0}\left(1-\frac{\eta z^{\prime}}{2-Y_{b}}\right)^{-1} \\
& \simeq \kappa_{0}\left(1+\frac{\eta z^{\prime}}{2-Y_{b}}\right)
\end{aligned}
$$

where $\eta$ is the basic gradient of helium, $0<z^{\prime}<d$ is the vertical distance from the bottom, $Y_{b}$ is the concentration of helium at the base of the considered region, and $\kappa_{0}$ is the thermal conductivity at the same location.

Neglecting any nuclear energy generation and/or stellar contraction/expansion, the static temperature profile is given by

$$
\nabla \cdot(\kappa \nabla \bar{T})=0 \text { or } \kappa \frac{\mathrm{d} \bar{T}}{\mathrm{~d} z}=\mathrm{C}^{\mathrm{st}}
$$

The background temperature gradient is then simply by

$$
\frac{\mathrm{d} \bar{T}}{\mathrm{~d} z}=\frac{\mathrm{d} \bar{T}_{0}}{\mathrm{~d} z}(1+B z) \quad \text { where } \quad B=\frac{\Delta Y}{\left(2-Y_{b}\right)},
$$

$\frac{d \bar{T}_{0}}{d z}$ is the gradient at the base of the region and $z$ is a normalized vertical coordinate. The temperature profile is thus parabolic.

\subsection{General equations}

As mentioned before, the equations are given in a weakly non-Boussinesq approximation. The equations for the conservation of momentum

$$
\frac{1}{\sigma}\left[\partial_{t} \nabla^{2} \psi+J\left(\psi, \nabla^{2} \psi\right)\right]=-R_{T} \theta_{x}-\frac{R_{Y}}{\tau} y_{x}+\nabla^{4} \psi
$$

and for the conservation of helium

$$
\frac{1}{\tau}\left[\partial_{t} y+J(\psi, y)\right]=-\psi_{x}+\nabla^{2} y
$$


are just the double-diffusive equations encountered in the Boussinesq approximation. Here, $J$ is the Jacobian operator defined by $J(a, b)=\partial_{x} a \partial_{z} b-\partial_{x} a \partial_{x} b$ and $\psi$ is the streamfunction and is related to the fluid velocity $\vec{u}=\left(-\psi_{x}, 0, \psi_{x}\right) . \sigma=\nu / \kappa_{0}$ is the Prandtl number, $\tau=\kappa_{Y} / \kappa_{0}$ is the Lewis number, $R_{T}=g \alpha \Delta T d^{3} / \nu \kappa_{0}$ is the thermal Rayleigh number, $R_{Y}=g \beta \Delta Y d^{3} / \nu \kappa_{0}$ is the helium Rayleigh number, the units are $\kappa_{0} / d$ for velocity, $d^{2} / \kappa_{0}$ for time, $\Delta T$ for the temperature fluctuations $\theta$ and $\tau \Delta Y$ for the helium fluctuations $y$. Note that in the context of stellar structure $\sigma$ and $\tau$ have values of about $10^{-8}$. However, we will begin by considering the problem with $\sigma, \tau \sim \mathcal{O}(1)$, and only after will we study the $\sigma$, $\tau \rightarrow 0$ limit.

The equation of heat is slightly modified and becomes

$$
\partial_{t} \theta+J(\psi, \theta)=-\psi_{x}(1+B z)+\nabla^{2} \theta-B \theta_{z}-\frac{1}{\tau} B y_{z}
$$

The boundary conditions are stress-free (for the velocity field) and fixed temperature and helium concentration $\left(\psi=\psi_{z z}=\theta=y=0\right.$ at $\left.z=0,1\right)$. It is important to note that these boundary conditions are uncertain. It is not clear how the semi-convective zone will be affected by the adjacent convective/radiative zones. The influence of the boundary conditions on the final result (neutrality criterion) would be interesting to pursue.

One of the new features of this set of equations is that thay are coupled even for the case $R_{Y}=0$. Note however that if $\Delta Y=0$ we must redefine $B$.

Another important characteristic of the heat equation is that it involves a "Dufour-like" effect, since adding some helium perturbation will help carry heat. However, the form of the equation is somewhat different than for the normal so-called Dufour effect.

\section{The linear problem}

As mentionned in the introduction, we will perform a weakly non-linear analysis on this set of equations. The first step is to solve the linear problem. We expand the function in terms of the small parameter $\varepsilon$.

$$
\Psi=\left(\begin{array}{l}
\psi \\
\theta \\
y
\end{array}\right)=\varepsilon\left(\begin{array}{l}
\psi^{(1)} \\
\theta^{(1)} \\
y^{(1)}
\end{array}\right)+\varepsilon^{2}\left(\begin{array}{c}
\psi^{(2)} \\
\theta^{(2)} \\
y^{(2)}
\end{array}\right)+\varepsilon^{3}\left(\begin{array}{c}
\psi^{(3)} \\
\theta^{(3)} \\
y^{(3)}
\end{array}\right)+\ldots
$$


We will also expand the thermal Rayleigh number

$$
R_{T}=R_{T 0}+\varepsilon R_{T 1}+\varepsilon^{2} R_{T 2}+\ldots
$$

At $\mathcal{O}(\varepsilon)$, we have simply

$$
\left(\begin{array}{ccc}
\nabla^{4}-\frac{1}{\sigma} \partial_{t} \nabla^{2} & -R_{T 0} \partial_{x} & -\frac{1}{\tau} R_{Y 0} \partial_{x} \\
-(1+B z) \partial_{x} & \nabla^{2}-B \partial_{z}-\partial_{t} & -\frac{1}{\tau} B \partial_{z} \\
-\partial_{x} & 0 & \nabla^{2}-\frac{1}{\tau} \partial_{t}
\end{array}\right)\left(\begin{array}{l}
\psi^{(1)} \\
\theta^{(1)} \\
y^{(1)}
\end{array}\right)=0
$$

which is the linear expansion problem. This equation is non-linear and must be solved numerically, or via a perturbation method.

\subsection{Perturbation of the linear problem}

We expand the linear equations with respect to the small parameter $B$.

$$
\begin{aligned}
\psi^{(1)} & =\psi_{0}+B \psi_{1}+\ldots \\
\theta^{(1)} & =\theta_{0}+B \theta_{1}+\ldots \\
y^{(1)} & =y_{0}+B y_{1}+\ldots \\
R_{T} & =R_{T 0}+B R_{T 1}+\ldots \\
R_{Y} & =R_{Y 0}+B R_{Y 1}+\ldots \\
s & =s_{0}+B s_{1}+\ldots \\
k & =k_{0}+B k_{1}+\ldots
\end{aligned}
$$

The temporal dependance is assumed of the form $e^{s t}$. The Laplacian and biharmonic operators become

$$
\begin{aligned}
& \nabla^{2} \rightarrow\left(k_{0}^{2}+\partial_{z}^{2}\right)+2 B\left(k_{0} k_{1}\right)+\ldots \\
& \nabla^{4} \rightarrow\left(k_{0}^{2}+\partial_{z}^{2}\right)^{2}+4 B\left(k_{0}^{3}+k_{0} \partial_{z}\right) k_{1}+\ldots
\end{aligned}
$$

\subsection{1 $\mathcal{O}\left(B^{0}\right)$}

At this order, we recover the same equations as in the well-known linear thermohaline problem.

$$
\frac{1}{\sigma} s_{0} \nabla^{2} \psi_{0}=-R_{T 0} \theta_{0 x}-\frac{1}{\tau} R_{Y 0} y_{0 x}+\nabla^{4} \psi_{0}
$$




$$
\begin{gathered}
s_{0} \theta_{0}=-\psi_{0 x}+\nabla^{2} \theta_{0} \\
\frac{1}{\tau} s_{0} y_{0}=-\psi_{0 x}+\nabla^{2} y_{0}
\end{gathered}
$$

The $z$-dependance can be expressed as $\sin (n \pi z)$. Expanding the $x$-dependance in $e^{i k x}$ leads to

$$
\left(\begin{array}{ccc}
p^{4}+\frac{s_{0}}{\sigma} p^{2} & -i k R_{T 0} & -i k \frac{1}{\tau} R_{Y 0} \\
-i k & -\left(p^{2}+s_{0}\right) & 0 \\
-i k & 0 & -\left(p^{2}+\frac{1}{\tau} s_{0}\right)
\end{array}\right)\left(\begin{array}{l}
\psi_{0} \\
\theta_{0} \\
y_{0}
\end{array}\right)=0
$$

where $p^{2}=n^{2} \pi^{2}+k^{2}$ and which we may write in condensed form $\mathcal{L} \Psi_{0}=0$. The dispersion relation is

$$
\left(p^{4}+\frac{s_{0}}{\sigma} p^{2}\right)\left(p^{2}+s_{0}\right)\left(p^{2}+\frac{1}{\tau} s_{0}\right)-k^{2} R_{T 0}\left(p^{2}+\frac{1}{\tau} s_{0}\right)-\frac{k^{2}}{\tau} R_{Y 0}\left(p^{2}+s_{0}\right)=0 .
$$

The onset of direct convection $(s=0)$ is governed by

$$
p^{6}=k^{2}\left(R_{T 0}+\frac{R_{Y 0}}{\tau}\right)
$$

and the wave number of the most unstable mode is $k_{0}=\pi / \sqrt{2}$ which becomes unstable when $R=\left(R_{T 0}+R_{Y 0} / \tau\right)=R_{c}=27 \pi^{4} / 4$. The solution is then just

$$
\begin{gathered}
\psi_{0}=A e^{i k_{0} x} \sin (\pi z)+\text { c.c. } \\
\theta_{0}=-\frac{i k_{0}}{p_{0}^{2}} A e^{i k_{0} x}(\sin \pi z)+\text { c.c. } \\
y_{0}=-\frac{i k_{0}}{p_{0}^{2}} A e^{i k_{0} x}(\sin \pi z)+\text { c.c. }
\end{gathered}
$$

\subsection{2 $\mathcal{O}\left(B^{1}\right)$}

At $\mathcal{O}\left(B^{1}\right)$, the equations become

$$
\begin{aligned}
& \frac{1}{\sigma} s_{0} p_{0}^{2} \psi_{1}-R_{T 0} i k_{0} \theta_{1}-\frac{1}{\tau} R_{Y 0} i k_{0} y_{1}+p_{0}^{4} \psi_{1}= \\
& -\frac{1}{\sigma} s_{1} p_{0}^{2} \psi_{0}-\frac{2}{\sigma} s_{0} k_{1} k_{0} \psi_{0}+R_{T 1} i k_{0} \theta_{0}+R_{T 0} i k_{1} \theta_{0}+\frac{1}{\tau} R_{Y 1} i k_{0} y_{0}+\frac{1}{\tau} R_{Y 0} i k_{1} y_{0}-4 p_{0}^{2} k_{0} k_{1} \psi_{0} \\
& \quad-s_{0} \theta_{1}-i k_{0} \psi_{1}-p_{0}^{2} \theta_{1}=s_{1} \theta_{0}+z i k_{0} \psi_{0}+i k_{1} \psi_{0}+2 k_{0} k_{1} \theta_{0}+\theta_{0 z}+\frac{1}{\tau} y_{0 z}
\end{aligned}
$$




$$
-\frac{1}{\tau} s_{0} y_{1}-i k_{0} \psi_{1}-p_{0}^{2} y_{1}=\frac{1}{\tau} s_{1} y_{0}+i k_{1} \psi_{0}+2 k_{0} k_{1} y_{0}
$$

We will look for modifications at the onset of convection $\left(s_{0}=s_{1}=0\right)$. In matrix form, the problem is then

$$
\mathcal{L} \Psi_{1}=\left(\begin{array}{c}
R_{T 1} i k_{0} \theta_{0}+R_{T 0} i k_{1} \theta_{0}+\frac{1}{\tau} R_{Y 1} i k_{0} y_{0}+\frac{1}{\tau} R_{Y 0} i k_{1} y_{0}-4 p_{0}^{2} k_{0} k_{1} \psi_{0} \\
z i k_{0} \psi_{0}+i k_{1} \psi_{0}+2 k_{0} k_{1} \theta_{0}+\theta_{0 z}+\frac{1}{\tau} y_{0 z} \\
i k_{1} \psi_{0}+2 k_{0} k_{1} y_{0}
\end{array}\right)
$$

We must now eleminate resonant terms on the rhs of eq. (29). We will thus define the adjoint eigenvector $\Psi^{\dagger}$ and adjoint operator $\mathcal{L}^{\dagger}$ such that $\left\langle\Psi^{\dagger}, \mathcal{L} \Psi\right\rangle=\left\langle\Psi, \mathcal{L}^{\dagger} \Psi^{\dagger}\right\rangle$, where the brackets are used for the inner product $\langle a, b\rangle=\int_{0}^{1} \mathrm{~d} z \int_{0}^{\frac{2 \pi}{k}} \mathrm{~d} x a b$. The adjoint problem is

$$
\mathcal{L}^{\dagger} \Psi^{\dagger}=\left(\begin{array}{ccc}
\nabla^{4} & \partial_{x} & \partial_{x} \\
R_{T 0} \partial_{x} & \nabla^{2} & 0 \\
\frac{1}{\tau} R_{Y 0} \partial_{x} & 0 & \nabla^{2}
\end{array}\right)\left(\begin{array}{c}
\psi^{\dagger} \\
\theta^{\dagger} \\
y^{\dagger}
\end{array}\right)=0
$$

with the boundary conditions $\psi^{\dagger}=\psi_{z z}^{\dagger}=\theta^{\dagger}=y^{\dagger}=0$ at $z=0,1$. We have the same dispersion relation as before and the solution is just

$$
\begin{gathered}
\psi^{\dagger}=e^{i k_{0} x} \sin (\pi z)+\text { c.c. } \\
\theta^{\dagger}=\frac{i k_{0} R_{T 0}}{p_{0}^{2}} e^{i k_{0} x} \sin (\pi z)+\text { c.c. } \\
y^{\dagger}=\frac{i k_{0} R_{Y 0}}{\tau p_{0}^{2}} e^{i k_{0} x} \sin (\pi z)+\text { c.c. }
\end{gathered}
$$

We can now compute the solvability condition $\left\langle\Psi^{\dagger}\right.$, rhs $\rangle=0$.

$$
\begin{array}{r}
\int_{0}^{1} \mathrm{~d} z \int_{0}^{\frac{2 \pi}{h}} \mathrm{e}^{2 i k_{0} x} \mathrm{~d} x \sin ^{2}(\pi z)\left[R_{T 1} \frac{k_{0}^{2}}{p_{0}^{2}}+\frac{R_{Y 1}}{\tau} \frac{k_{0}^{2}}{p_{0}^{2}}-4 p_{0}^{2} k_{0} k_{1}+2 R_{T 0} \frac{k_{0}^{3} k_{1}}{p_{0}^{4}}+2 \frac{R_{Y 0}}{\tau} \frac{k_{0}^{3} k_{1}}{p_{0}^{4}}\right] \\
-z \sin ^{2}(\pi z)\left[R_{T 0} \frac{k_{0}^{2}}{p_{0}^{2}}\right]+\pi \sin (\pi z) \cos (\pi z)\left[R_{T 0} \frac{k_{0}^{2}}{p_{0}^{4}}+\frac{R_{Y 0}}{\tau} \frac{k_{0}^{2}}{p_{0}^{4}}\right]=0
\end{array}
$$

Using $R=R_{T}+\frac{R_{Y}}{\tau}$, remembering that $R_{0}=\frac{p_{0}^{0}}{k_{0}^{2}}$ and calculating the integrals

$$
\int_{0}^{1} \sin (\pi z) \cos (\pi z) \mathrm{d} z=0 ; \int_{0}^{1} \sin ^{2}(\pi z) \mathrm{d} z=\frac{1}{2} ; \int_{0}^{1} z \sin ^{2}(\pi z) \mathrm{d} z=\frac{1}{4}
$$



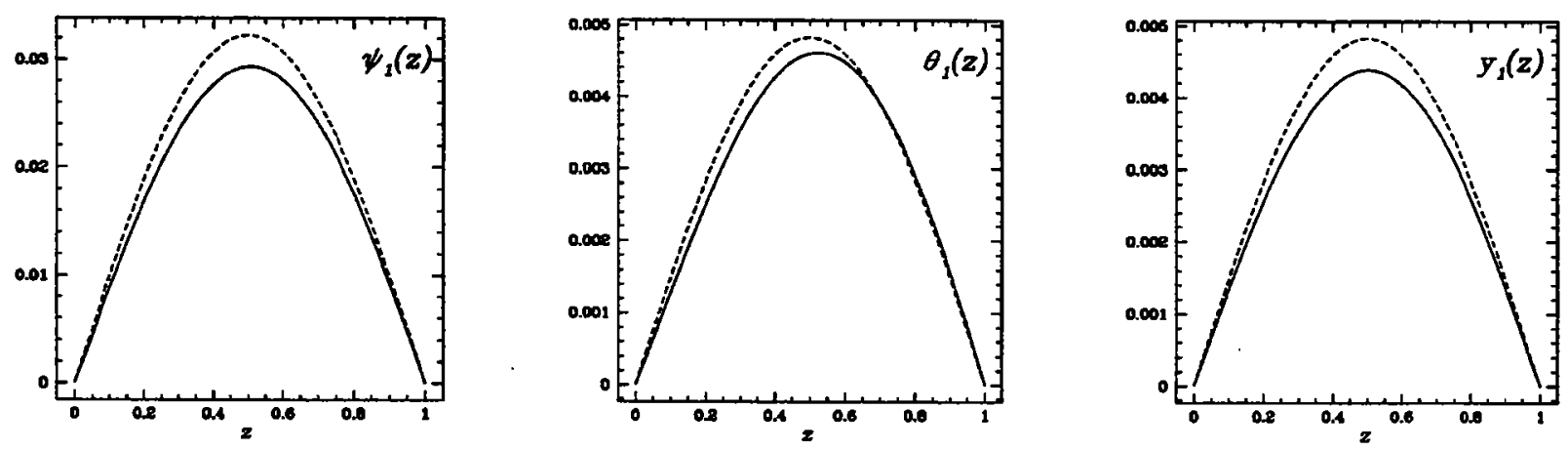

Figure 3: Vertical first order eigenfunctions for the case where $B=0$ (full line) and $B=0.1$ (ticked line), calculated with $R_{Y}=-200, \sigma=0.2, \tau=0.2, k=k_{0}$. Note that in the case $B=0$, these eigenfunctions are $\operatorname{simply} \sin (\pi z)$.

we find that the modification of the wave and Rayleigh numbers at onset are related by

$$
k_{1}=\frac{R_{1}-\frac{1}{2} R_{T 0}}{2 R_{0} k_{0} / p_{0}^{2}} .
$$

Note that this calculations is limited by the condition $B \ll \tau, \sigma, 1$. This tells us that the wave number of the most unstable mode will change. However, that analysis does not tell us what that wave number becomes, since we have only a linear relationship between $R_{T 1}$ and $k_{1}$. Eq. (36) may also be used to find the change of the critical Rayleigh number for a fixed wave number.

\subsection{Numerical results}

As already mentioned, the differences between the standard thermohaline equations and equations (11), (12) and (13) are all located in the heat equation and preceeded by the parameter $B$. It is also worth noticing that the most important term in that equation is the one in $y_{z}$ since it is preceeded by $1 / \tau$, which is small in our case. We expect that the symmetry breaking introduced in the eigenfunctions will be most important for the heat equation, as is illustrated in fig. 3.

Numerically, we can also calculate the marginality curve (that is the Rayleigh number for zero-growth rate $v s k$ ), and see how it is modified by including the $B$ terms. That is illustrated in fig. 4. The comparison between that figure and the perturbation analysis is not straightforward since in the numerical calculation $B \sim \mathcal{O}(\tau, \sigma)$. However, it shows that 


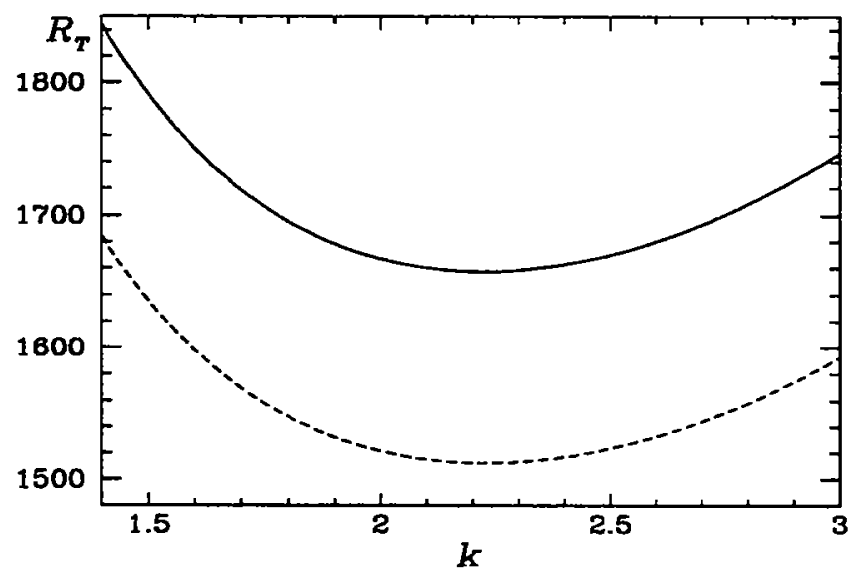

Figure 4: Marginality curve for $B=0$ (solid line) and $B=0.1$ (ticked kine).

even in that regime, the wavenumber of the most unstable mode doesn't change much. We will thus keep $k=k_{0}$ for future calculations.

\section{Weakly non-linear analysis}

We will concentrate our attention on the onset of direct convection and thus eliminate all "fast" time derivatives, present in the first and second order equations. We solve the linear problem

$$
\mathcal{M} \Psi^{(1)}=\left(\begin{array}{ccc}
\nabla^{4} & -R_{T 0} \partial_{x} & -\frac{1}{\tau} R_{Y 0} \partial_{x} \\
-(1+B z) \partial_{x} & \nabla^{2}-B \partial_{z} & -\frac{1}{\tau} B \partial_{z} \\
-\partial_{x} & 0 & \nabla^{2}
\end{array}\right)\left(\begin{array}{c}
\psi^{(1)} \\
\theta^{(1)} \\
y^{(1)}
\end{array}\right)
$$

and then adjoint problem

$$
\mathcal{M}^{\dagger} \Psi^{\dagger}=\left(\begin{array}{ccc}
\nabla^{4} & (1+B z) \partial_{x} & \partial_{x} \\
R_{T 0} \partial_{x} & \nabla^{2}+B \partial_{z} & 0 \\
\frac{1}{\tau} R_{Y 0} \partial_{x} & \frac{1}{\tau} B \partial_{z} & \nabla^{2}
\end{array}\right)\left(\begin{array}{c}
\psi^{\dagger} \\
\theta^{\dagger} \\
y^{\dagger}
\end{array}\right)=0
$$

numerically, using the same boundary conditions as before (stress-free, fixed temperature and helium concentration). For the solutions, we will use the notation

$$
\begin{gathered}
\psi^{(1)}=A\left(T_{2}\right) \cos (k x) \psi_{1}(z) ; \theta^{(1)}=A\left(T_{2}\right) \sin (k x) \theta_{1}(z) ; y^{(1)}=A\left(T_{2}\right) \sin (k x) y_{1}(z) \\
\psi^{\dagger}=\cos (k x) \psi^{\dagger}(z) ; \theta^{\dagger}=\sin (k x) \theta^{\dagger}(z) ; y^{\dagger}=\sin (k x) y^{\dagger}(z)
\end{gathered}
$$


$A\left(T_{2}\right)$ is used here to denote the fact that we allow the amplitude to change on a slow time $T_{2}$ of order $\varepsilon^{2}$. The eigenfunctions in $z$ come from the linear problem (see fig. 3 ).

\section{$4.1 \mathcal{O}\left(\varepsilon^{2}\right)$}

The second order problem is

$$
\mathcal{M} \Psi^{(2)}=\left(\begin{array}{c}
\frac{1}{\sigma} J\left(\psi^{(1)}, \nabla^{2} \psi^{(1)}\right)+R_{T 1} \partial_{x} \theta^{(1)} \\
J\left(\psi^{(1)}, \theta^{(1)}\right) \\
\frac{1}{\tau} J\left(\psi^{(1)}, y^{(1)}\right)
\end{array}\right)
$$

which may be written also

$$
\mathcal{M} \Psi^{(2)}=\left(\begin{array}{c}
\frac{k}{2 \sigma} A^{2} \sin (2 k x)\left[\psi_{1}^{\prime \prime}(z) \psi_{1}^{\prime}(z)-\psi_{1}^{\prime \prime \prime}(z) \psi_{1}(z)\right]+R_{T 1} A k \cos (k x) \theta_{1}(z) \\
-\frac{k}{2} A^{2}\left[\psi_{1}(z) \theta_{1}^{\prime}(z)+\psi_{1}^{\prime}(z) \theta_{1}(z)\right]+\frac{k}{2} A^{2} \cos (2 k x)\left[\psi_{1}(z) \theta_{1}^{\prime}(z)-\psi_{1}^{\prime}(z) \theta_{1}(z)\right] \\
-\frac{k}{2 \tau} A^{2}\left[\psi_{1}(z) y_{1}^{\prime}(z)+\psi_{1}^{\prime}(z) y_{1}(z)\right]+\frac{k}{2 \tau} A^{2} \cos (2 k x)\left[\psi_{1}(z) y_{1}^{\prime}(z)-\psi_{1}^{\prime}(z) y_{1}(z)\right]
\end{array}\right)
$$

using the known $x$ dependance and where the ' denotes a derivative with respect to $z$. In the standard thermohaline case, $J\left(\psi^{(1)}, \nabla^{2} \psi^{(1)}\right)=0$. The symetry breaking introduced in the equations destroys that simple relation. It also introduces the term in $\cos (2 k x)$ in the heat and helium equations. Writing the solvability condition $\left\langle\Psi^{\dagger}\right.$, rhs $\rangle=0$ and integrating over $x$, we are left with

$$
\int_{0}^{1} \mathrm{~d} z R_{T 1} \psi^{\dagger}(z) \theta(z)=0 .
$$

This implies simply $R_{T 1}=0$. The second order solution will have the form

$$
\Psi^{(2)}=\left(\begin{array}{c}
A^{2}\left(T_{2}\right) \psi_{2}(z) \sin (2 k x) \\
A^{2}\left(T_{2}\right) \theta_{2 a}(z)+A^{2}\left(T_{2}\right) \theta_{2 b}(z) \cos (2 k x) \\
A^{2}\left(T_{2}\right) y_{2 a}(z)+A^{2}\left(T_{2}\right) y_{2 b}(z) \cos (2 k x)
\end{array}\right)
$$

The system of equations at this order is then

$$
\begin{gathered}
\psi_{2}^{I V} \sin (2 k x)-8 k^{2} \psi_{2}^{\prime \prime} \sin (2 k x)+16 k^{4} \psi_{2} \sin (2 k x)+2 k R_{T 0} \theta_{2 b} \sin (2 k x) \\
+\frac{2 k}{\tau} R_{Y 0} y_{2 b} \sin (2 k x)=\frac{k}{2 \sigma} \sin (2 k x)\left[\psi^{\prime \prime} \psi^{\prime}-\psi^{\prime \prime \prime} \psi\right] \\
-2 k(1+B z) \psi_{2} \cos (2 k x)+\theta_{2 a}^{\prime \prime}+\theta_{2 b}^{\prime \prime} \cos (2 k x)-4 k^{2} \theta_{2 b} \cos (2 k x)-B \theta_{2 a}^{\prime}-B \theta_{2 b}^{\prime} \cos (2 k x) \\
-\frac{1}{\tau} B y_{2 a}^{\prime}-\frac{1}{\tau} B y_{2 b}^{\prime} \cos (2 k x)=-\frac{k}{2}\left[\psi \theta^{\prime}+\psi^{\prime} \theta\right]+\frac{k}{2} \cos (2 k x)\left[\psi \theta^{\prime}-\psi^{\prime} \theta\right] \\
-2 k \psi_{2} \cos (2 k x)+y_{2 a}^{\prime \prime}+y_{2 b}^{\prime \prime} \cos (2 k x)-4 k^{2} y_{2 b} \cos (2 k x)= \\
-\frac{k}{2 \tau}\left[\psi y^{\prime}+\psi^{\prime} y\right]+\frac{k}{2 \tau} \cos (2 k x)\left[\psi y^{\prime}-\psi^{\prime} y\right]
\end{gathered}
$$



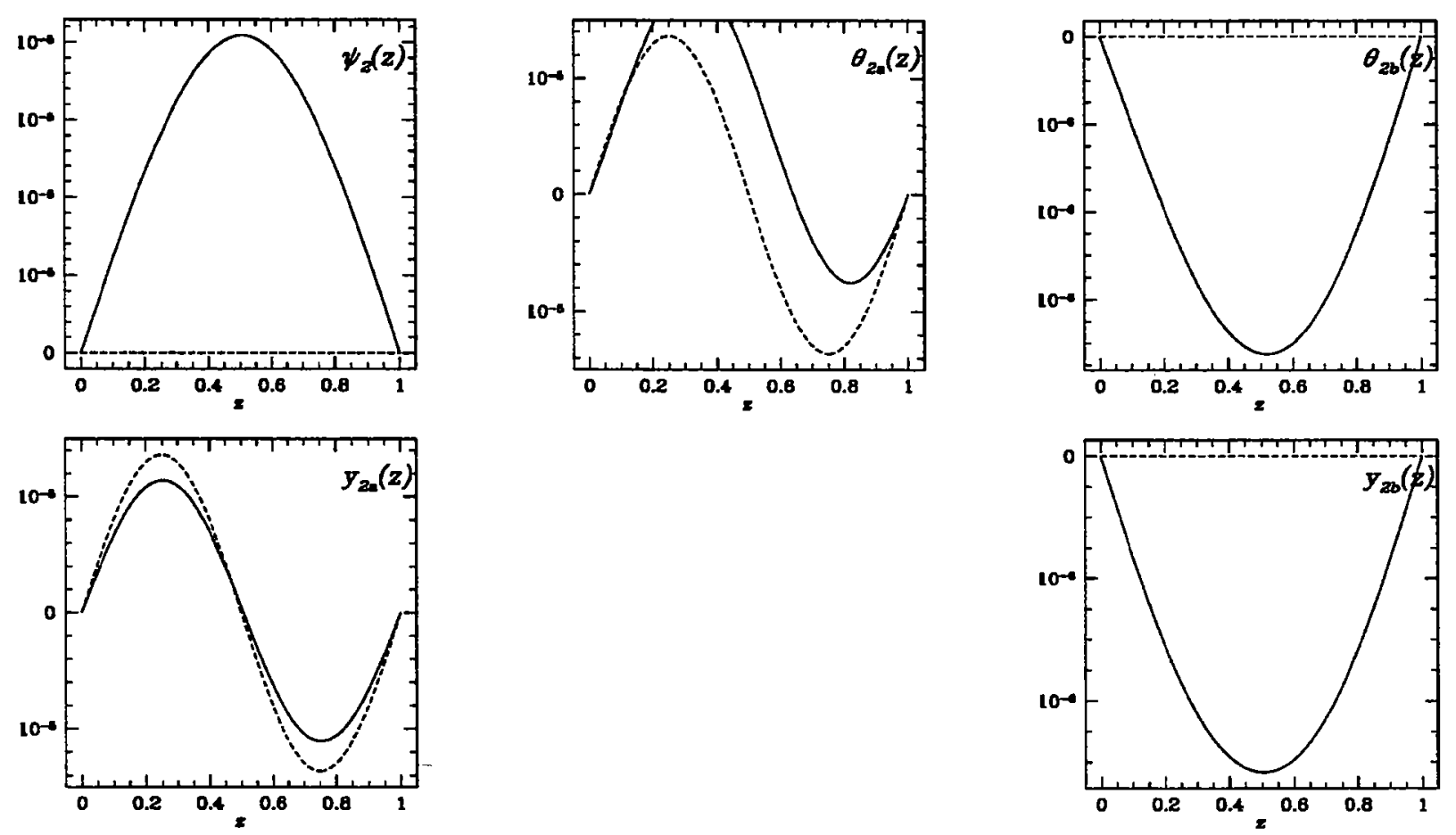

Figure 5: Vertical second order eigenfunctions for the case where $B=0$ (full line) and $B=0.1$ (ticked line), calculated with $R_{Y}=-200, \sigma=0.2, \tau=0.2$, for $k=k_{0}$. The asymmetry is again stronger in the temperature field. Furthermore, some Jacobians which were null in the standard case now have a finite value, giving rise to a more complex second order solution when $B \neq 0$.

That system is solved numerically for $\psi_{2}(z), \theta_{2 a}(z), \theta_{2 b}(z), y_{2 a}(z)$ and $y_{2 b}(z)$.

\section{$4.2 \mathcal{O}\left(\varepsilon^{3}\right)$}

We may now write the third order problem.

$$
\mathcal{M} \Psi^{(3)}=\left(\begin{array}{c}
\frac{1}{\sigma}\left[J\left(\psi^{(1)}, \nabla^{2} \psi^{(2)}\right)+J\left(\psi^{(2)}, \nabla^{2} \psi^{(1)}\right)\right]+R_{T_{2}} \partial_{x} \theta^{(1)}+\frac{1}{\sigma} \partial_{T_{2}} \nabla^{2} \psi^{(1)} \\
J\left(\psi^{(1)}, \theta^{(2)}\right)+J\left(\psi^{(2)}, \theta^{(1)}\right)+\partial_{T_{2}} \theta^{(1)} \\
\frac{1}{\tau}\left[J\left(\psi^{(1)}, y^{(2)}\right)+J\left(\psi^{(2)}, y^{(1)}\right)\right]+\frac{1}{\tau} \partial_{T_{2}} y^{(1)}
\end{array}\right)
$$




$$
\begin{aligned}
& \text { or } \\
& \mathcal{M} \Psi^{(3)}=\left(\begin{array}{l}
\frac{2 k}{\sigma} A^{3} \sin ^{2}(k x) \cos (k x)\left[\psi_{2}^{\prime} \psi_{1}^{\prime \prime}-\psi_{2} \psi_{1}^{\prime \prime \prime}-\psi_{2}^{\prime \prime \prime} \psi_{1}+\psi_{1}^{\prime} \psi_{2}^{\prime \prime}+3 k^{2}\left(\psi_{2}^{\prime} \psi_{1}-\psi_{1}^{\prime} \psi_{2}\right)\right] \\
+\frac{2 k}{\sigma} A^{3} \cos ^{3}(k x)\left[\psi_{2} \psi_{1}^{\prime \prime \prime}-\psi_{1}^{\prime} \psi_{2}^{\prime \prime}+3 k^{2} \psi_{2} \psi_{1}^{\prime}\right]+A R_{T 2} k \cos (k x) \theta_{1}+\frac{1}{\sigma} A_{T_{2}} \cos (k x)\left(\psi_{1}^{\prime \prime}-k^{2} \psi_{1}\right) \\
-k A^{3} \sin (k x)\left[\psi_{1} \theta_{2 a}^{\prime}+\psi_{1} \theta_{2 b}^{\prime}-2 \theta_{1}^{\prime} \psi_{2}\right] \\
+k A^{3} \sin (k x) \cos ^{2}(k x)\left[-2 \psi_{1} \theta_{2 b}^{\prime}+4 \psi_{1}^{\prime} \theta_{2 b}+4 \psi_{2} \theta_{1}^{\prime}-2 \psi_{2}^{\prime} \theta_{1}\right]+A_{T_{2}} \theta_{1} \sin (k x) \\
-\frac{k}{\tau} A^{3} \sin (k x)\left[\psi_{1} y_{2 a}^{\prime}+\psi_{1} y_{2 b}^{\prime}-2 y_{1}^{\prime} \psi_{2}\right] \\
+\frac{k}{\tau} A^{3} \sin (k x) \cos ^{2}(k x)\left[-2 \psi_{1} y_{2 b}^{\prime}+4 \psi_{1}^{\prime} \theta_{2 b}+4 \psi_{2} y_{1}^{\prime}-2 \psi_{2}^{\prime} y_{1}\right]+\frac{1}{\tau} A_{T_{2}} y_{1} \sin (k x)
\end{array}\right.
\end{aligned}
$$

The solvability condition at this order will tell us is the bifurcation is sub- or supercritical.

Integrating over $x$, we get

$$
\begin{gathered}
\int_{0}^{1} \mathrm{~d} z \psi^{\dagger} \frac{1}{\sigma}\left[2\left(\psi_{2}^{\prime} \psi_{1}^{\prime \prime}-\psi_{2} \psi_{1}^{\prime \prime \prime}-\psi_{2}^{\prime \prime \prime} \psi_{1}+\psi_{1}^{\prime} \psi_{2}^{\prime \prime}+3 k^{2}\left(\psi_{2}^{\prime} \psi_{1}-\psi_{1}^{\prime} \psi_{2}\right)\right)+6\left(\psi_{2} \psi_{1}^{\prime \prime \prime}-\psi_{1}^{\prime} \psi_{2}^{\prime \prime}+3 k^{2} \psi_{1}^{\prime}\right)\right] \\
+\theta^{\dagger}\left[-2 \psi_{1} \theta_{2 b}^{\prime}+4 \psi_{1}^{\prime} \theta_{2 b}+4 \psi_{2} \theta_{1}^{\prime}-2 \psi_{2}^{\prime} \theta_{1}-4\left(\psi_{1} \theta_{2 a}^{\prime}+\psi_{1} \theta_{2 b}^{\prime}-2 \theta_{1}^{\prime} \psi_{2}\right)\right] \\
+\frac{1}{\tau} y^{\dagger}\left[-2 \psi_{1} y_{2 b}^{\prime}+4 \psi_{1}^{\prime} \theta_{2 b}+4 \psi_{2} y_{1}^{\prime}-2 \psi_{2}^{\prime} y_{1}-4\left(\psi y_{2 a}^{\prime}+\psi_{1} y_{2 b}^{\prime}-2 y_{1}^{\prime} \psi_{2}\right)\right] \\
+\frac{4}{A^{2}} \psi^{\dagger} \theta_{1} R_{T 2}+\frac{A_{T_{2}}}{A^{3}}\left[\frac{1}{\sigma} \psi^{\dagger}\left(\psi_{1}^{\prime \prime}-k^{2} \psi_{1}\right)+\theta^{\dagger} \theta_{1}+\frac{1}{\tau} y^{\dagger} y_{1}\right]=0
\end{gathered}
$$

Peforming the integration we will be able to write an amplitude equation of the form

$$
\alpha A_{T_{2}}=\beta R_{T_{2}} A+\gamma A|A|^{2} \text {. }
$$

In all computed cases, $\boldsymbol{\gamma}$ was found to be positive, indicating a subcritical bifurcation. In fig. 6, we have the amplitude vs $R_{T}$ diagram for Rayleigh-Bénard convection, the standard thermohaline case $(B=0)$ and two values of $B(0.01$ and 0.1$)$. For these parameters, we see that $R_{R B}$ is a very good approximation of $R_{\min }$.

\section{$5 \quad$ The $\nu \rightarrow 0, \kappa_{Y} \rightarrow 0$ limit}

In stars, we are in the limit where both $\nu$ and $\kappa_{Y}$ are very small. To study that limit properly, we will use a new scaling for the variables $\left(u \sim \nu / d, t \sim d^{2} / \nu, T \sim \kappa_{T} \nu / g \alpha d^{3}\right.$, $\left.Y \sim \kappa_{T} \nu / g \beta d^{3}\right)$. We will also introduce a new non-dimensionnal number, the Schmidt number $\sigma=\nu / \kappa_{Y}$. There is some uncertainty as to the real value of that parameter in stars, then however, it is reasonable to assume $\sigma \sim \mathcal{O}(1)$. We will have to change the notation for the Prandtl number, now $\mathcal{P}$.

$$
\partial_{t} \nabla^{2} \psi+J\left(\psi, \nabla^{2} \psi\right)=-\theta_{x}-y_{x}+\nabla^{4} \psi
$$




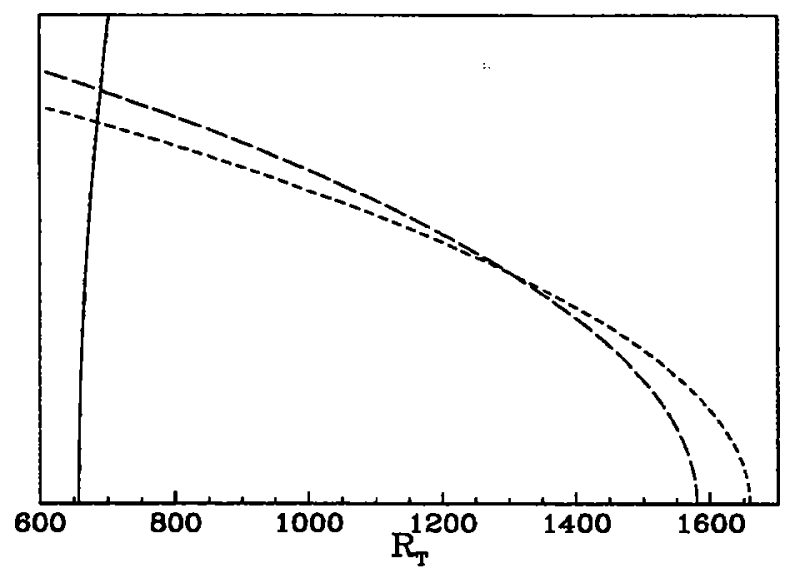

Figure 6: Amplitude vs $R_{T}$ diagram showing the neutrality condition for the case with $B=0$ (short dashed line) and $B=0.1$ (long dashed line). For that calculation, we used $\tau=0.2$, $\sigma=0.2, R_{Y}=-200$ and $k=k_{0}$. The full line is the classical Rayleigh-Bénard problem.

$$
\begin{gathered}
\partial_{t} \theta+J(\psi, \theta)=-R_{T}(1+B z) \psi_{x}+\frac{1}{\mathcal{P}}\left[\nabla^{2} \theta-B \theta_{z}-\frac{R_{T}}{R_{Y}} B y_{z}\right] \\
\partial_{t} y+J(\psi, y)=-R_{Y} \psi_{x}+\frac{1}{\sigma} \nabla^{2} y
\end{gathered}
$$

This limit corresponds effectively to study the case of infinite thermal conductivity. From equation (49), we see that we can drop the time derivative, as well as the advection term in the heat equation. The heat profile is thus slaved to the behavior of the velocity and helium fields.

In the case with $B=0$, we can use $\sin (n \pi z)$ for the $z$-dependance of the eigenfunctions, and in particular, we can thus calculate the dispersion relation

$$
s^{2}\left(p^{4} \sigma\right)+s\left(p^{6}(1+\sigma)-k^{2} \mathcal{P} \sigma R_{T}\right)+p^{8}-k^{2} p^{2}\left(\mathcal{P} R_{T}+\sigma R_{Y}\right)=0
$$

We can thus show that overstability still exists provided that

$$
\sigma R_{Y}<\frac{-p^{6}}{k^{2}}
$$

The value of the thermal Rayleigh number corresponding to zero-growth rate is then

$$
\mathcal{P} R_{T}=\frac{p^{6}(1+\sigma)}{k^{2} \sigma} .
$$

Note that the relevant parameters of the problem are now $\mathcal{P} R_{T}$ and $\sigma R_{Y}$. 

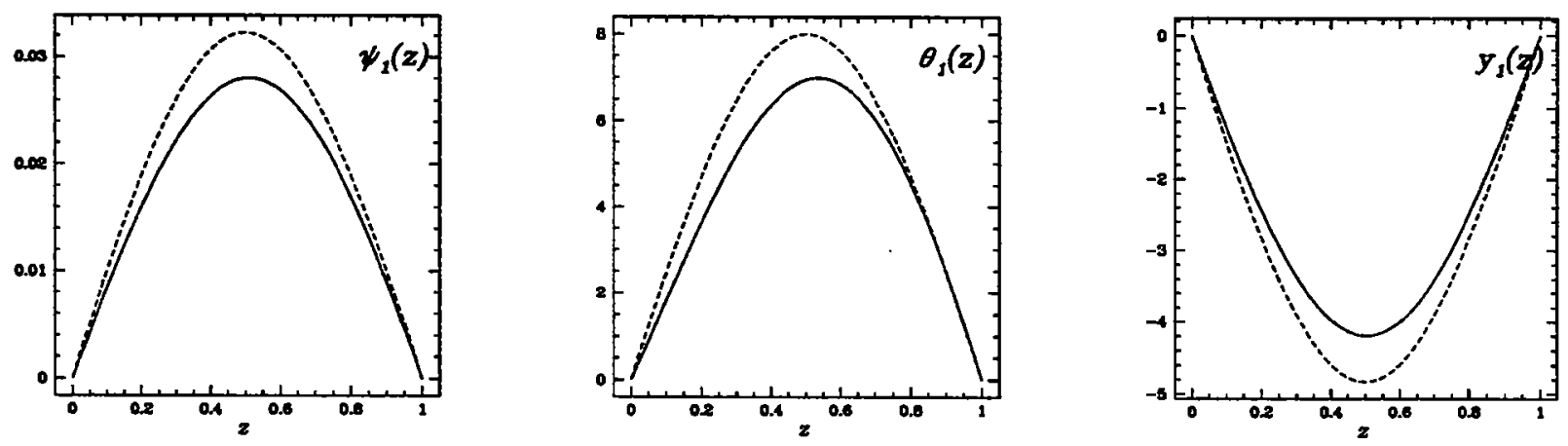

Figure 7: Vertical first order eigenfunctions for the case where $B=0$ (full line) and $B=0.1$ (ticked line), calculated with $R_{Y}=-1000, \sigma=1, \mathcal{P}=10^{-6}, k=k_{0}$.

\subsection{Weakly non-linear analysis}

As previously, we will concentrate ourselves on the onset of direct convection. The linear problem is very similar to what it was before

$$
\mathcal{N} \Psi^{(1)}=\left(\begin{array}{ccc}
\nabla^{4} & -\partial_{x} & -\partial_{x} \\
-\mathcal{P} R_{T}(1+B z) \partial_{x} & \nabla^{2}-B \partial_{z} & -\frac{R_{x}}{R_{Y}} B \partial_{z} \\
-\sigma R_{Y} \partial_{x} & 0 & \nabla^{2}
\end{array}\right)
$$

whose adjoint problem is just

$$
\mathcal{N}^{\dagger} \Psi^{\dagger}=\left(\begin{array}{ccc}
\nabla^{4} & \mathcal{P} R_{T}(1+B z) \partial_{x} & \sigma R_{Y} \partial_{x} \\
\partial_{x} & \nabla^{2}+B \partial_{z} & 0 \\
\partial_{x} & \frac{R_{x}}{R_{Y}} B \partial_{z} & \nabla^{2}
\end{array}\right)\left(\begin{array}{c}
\psi^{\dagger} \\
\theta^{\dagger} \\
y^{\dagger}
\end{array}\right)=0
$$

These problems are solved numerically, using the same boundary conditions (stress free, fixed temperature and helium concentration), and notations as previously.

\subsection{1 $\mathcal{O}\left(\epsilon^{2}\right)$}

The second order problem is

$$
\mathcal{N} \Psi^{(2)}=\left(\begin{array}{c}
J\left(\psi^{(1)}, \nabla^{2} \psi^{(1)}\right) \\
0 \\
\sigma J\left(\psi^{(1)}, y^{(1)}\right)
\end{array}\right)
$$


which may be written also

$$
\mathcal{N} \Psi^{(2)}=\left(\begin{array}{c}
\frac{k}{2} A^{2} \sin (2 k x)\left[\psi_{1}^{\prime \prime}(z) \psi_{1}^{\prime}(z)-\psi_{1}^{\prime \prime \prime}(z) \psi_{1}(z)\right] \\
0 \\
-\frac{k}{2} A^{2}\left[\psi_{1}(z) y_{1}^{\prime}(z)+\psi_{1}^{\prime}(z) y_{1}(z)\right]
\end{array}\right),
$$

using the known $x$ dependance. Once more, the solvability condition leaves us with $R_{T 1}=0$. The second order solution will have the same form as before

$$
\Psi^{(2)}=\left(\begin{array}{c}
A^{2}\left(T_{2}\right) \psi_{2}(z) \sin (2 k x) \\
A^{2}\left(T_{2}\right) \theta_{2 a}(z)+A^{2}\left(T_{2}\right) \theta_{2 b}(z) \cos (2 k x) \\
A^{2}\left(T_{2}\right) y_{2 a}(z)+A^{2}\left(T_{2}\right) y_{2 b}(z) \cos (2 k x)
\end{array}\right)
$$

The system of equations is then

$$
\begin{aligned}
& \psi_{2}^{I V} \sin (2 k x)-8 k^{2} \psi_{2}^{\prime \prime} \sin (2 k x)+ 16 k^{4} \psi_{2} \sin (2 k x)+2 k \theta_{2 b} \sin (2 k x) \\
&+2 k y_{2 b} \sin (2 k x)=\frac{k}{2} \sin (2 k x)\left[\psi_{1}^{\prime \prime} \psi_{1}^{\prime}-\psi_{1}^{\prime \prime \prime} \psi_{1}\right] \\
&-2 k \mathcal{P} R_{T 0}(1+B z) \psi_{2} \cos (2 k x)+ \theta_{2 a}^{\prime \prime}+\theta_{2 b}^{\prime \prime} \cos (2 k x)-4 k^{2} \theta_{2 b} \cos (2 k x)-B \theta_{2 a}^{\prime}-B \theta_{2 b}^{\prime} \cos (2 k x) \\
&-\frac{R_{T 0}}{R_{Y 0}} B y_{2 a}^{\prime}-\frac{R_{T 0}}{R_{Y 0}} B y_{2 b}^{\prime} \cos (2 k x)=0 \\
&-2 k \sigma R_{Y 0} \psi_{2} \cos (2 k x)+y_{2 a}^{\prime \prime}+y_{2 b}^{\prime \prime} \cos (2 k x)-4 k^{2} y_{2 b} \cos (2 k x)= \\
&-\frac{k \sigma}{2}\left[\psi_{1} y_{1}^{\prime}+\psi_{1}^{\prime} y_{1}\right]+\frac{k \sigma}{2} \cos (2 k x)\left[\psi_{1} y_{1}^{\prime}-\psi_{1}^{\prime} y_{1}\right]
\end{aligned}
$$

\subsection{2 $\mathcal{O}\left(\varepsilon^{3}\right)$}

The third order problem is now

$$
\mathcal{N} \Psi^{(3)}=\left(\begin{array}{c}
J\left(\psi^{(1)}, \nabla^{2} \psi^{(2)}\right)+J\left(\psi^{(2)}, \nabla^{2} \psi^{(1)}\right)+\partial_{T_{2}} \nabla^{2} \psi^{(1)} \\
J\left(\psi^{(1)}, \theta^{(1)}\right)+\partial_{T_{2}} \theta^{(1)}+R_{T_{2}}\left[\mathcal{P}(1+B z) \partial_{x} \psi^{(1)}+\frac{B}{R_{Y_{0}}} \partial_{z} y^{(1)}\right] \\
\sigma J\left(\psi^{(1)}, y^{(2)}\right)+\sigma J\left(\psi^{(2)}, y^{(1)}\right)+\sigma \partial_{T_{2}} y^{(1)}
\end{array}\right)
$$

or

$$
\mathcal{N} \Psi^{(3)}=\left(\begin{array}{l}
2 k A^{3} \sin ^{2}(k x) \cos (k x)\left[\psi_{2}^{\prime} \psi_{1}^{\prime \prime}-\psi_{2} \psi_{1}^{\prime \prime \prime}-\psi_{2}^{\prime \prime \prime} \psi_{1}+\psi_{1}^{\prime} \psi_{2}^{\prime \prime}+3 k^{2}\left(\psi_{2}^{\prime} \psi_{1}-\psi_{1}^{\prime} \psi_{2}\right)\right] \\
+2 k A^{3} \cos ^{3}(k x)\left[\psi_{2} \psi_{1}^{\prime \prime \prime}-\psi_{1}^{\prime} \psi_{2}^{\prime \prime}+3 k^{2} \psi_{2} \psi_{1}^{\prime}\right]+A_{T_{2}}\left(\psi_{1}^{\prime \prime}-k^{2} \psi_{1}\right) \cos (k x) \\
-\frac{k}{2} A^{3}\left(\psi_{1} \theta_{1}^{\prime}+\psi_{1}^{\prime} \theta_{1}\right)+\frac{k}{2} A^{3} \cos (2 k x)\left(\psi_{1} \theta_{1}^{\prime}-\psi_{1}^{\prime} \theta_{1}\right)+A_{T_{2}} \theta_{1} \sin (k x) \\
-\left[\mathcal{P}(1-B z) A k \sin (k x) \psi_{1}+\frac{B}{R_{Y 0}} A \sin (k x) y_{1}^{\prime}\right] R_{T_{2}} \\
-\sigma k A^{3} \sin (k x)\left[\psi_{1} y_{2 a}^{\prime}+\psi_{1} y_{2 b}^{\prime}-2 y_{1}^{\prime} \psi_{2}\right] \\
+\sigma k A^{3} \sin (k x) \cos ^{2}(k x)\left[-2 \psi_{1} y_{2 b}^{\prime}+4 \psi_{1}^{\prime} \theta_{2 b}+4 \psi_{2} y_{1}^{\prime}-2 \psi_{2}^{\prime} y_{1}\right]+\sigma A_{T_{2}} y_{1} \sin (k x)
\end{array}\right)
$$




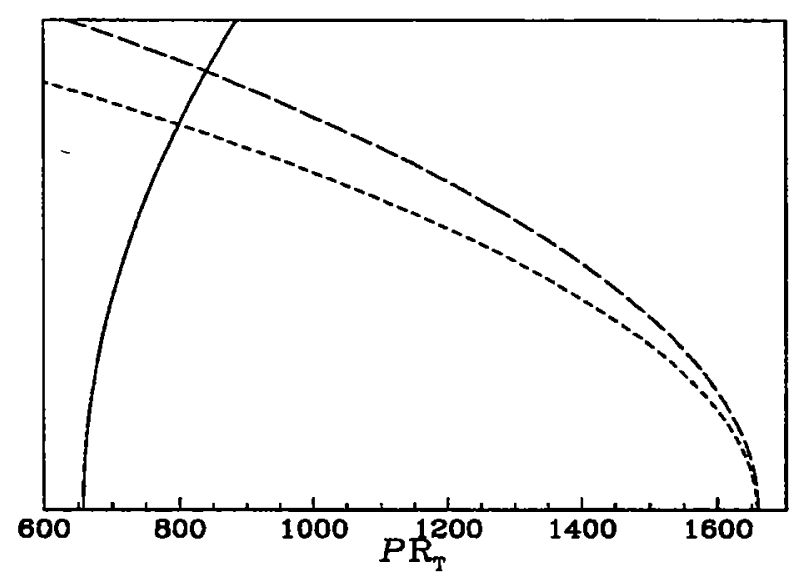

Figure 8: Amplitude vs $R_{T}$ diagram showing the neutrality condition for the case with $B=0$ (short dashed line) and $B=\mathcal{P}$ (long dashed line). For that calculation, we used $\mathcal{P}=10^{-6}$, $\sigma=1, R_{Y}=-1000$ and $k=k_{0}$.

We now just have to compute the solvability condition to get an amplitude equation similar to eq. (47).

This new scaling relevant to the stellar case also leads to a subcritical bifurcation. Figure 8 shows the neutrality condition obtained using a new set of parameters, more appropriate for a stellar case.

\section{Perspectives}

This project demonstrated that in the regime relevant to stellar structure, the onset of steady convection is sub-critical. Furthermore, it is quite likely that the minimum Rayleigh number for the onset of a finite amplitude instability is smaller than what is required for the onset of overstability.

The last step would now be to complete that analysis for compressible stellar matter and realistic values of $B, R_{Y}$ and $R_{T}$. We also need to "translate" the approximate $R_{\min }$ into temperature and helium gradients that will result in the stellar model. In particular, a new scaling might be required in the case where $B \gg \mathcal{P}$. 


\section{Acknowledgements}

I would like to thank first Jean-Paul Zahn, my thesis supervisor who sent me here, and Ed Spiegel who worked with me on this project. Many thanks also to Neil Balmforth who help me deal with all these equations, even though I am doing astrophysics. Thanks to Keith Julian, for his help with the numerics, and to the GFD fellows who promotted me to the status of "Princess Suzanna" this summer. Last but not least, thanks to Jakot who suffered a lot during the last two weeks of this program.

\section{References}

[1] Kato, S. 1966, PASP, 18, 374

[2] Kippenhahn, R. \& Weigert, A. 1994, Stellar structure and evolution, Berlin : New York : Springer-Verlag

[3] Ledoux, P. 1947, ApJ, 105, 305

[4] Proctor, M. 1981, JFM, 105, 507

[5] Sakashita, S. \& Hayashi, C. 1959, Prog. Theor. Phys., 22, 830

[6] Schwarzschild, M. \& Härm, R. 1958, ApJ, 128, 348

[7] Veronis, G. 1965, J. Marine Res., 23, 1 


\title{
Aspects of Double Diffusion in a Thin Slot
}

\author{
Joseph A. Biello
}

August 24, 1996

\section{Introduction}

A fluid with stable salt stratification, when heated from below forms convective layers from the instability of a vertically advancing thermal boundary layer (Huppert \& Linden [1]) Veronis [3] found analytically that such a system goes unstable through overstable oscillations. The primary instability of such a system is difficult to observe experimentally (see Shirtcliffe [2]) but is important in determining the subsequent evolution of the fluid. This paper considers five aspects of the instability of such a stably stratified system in the presence of a background constant temperature gradient.

If one could have an actual constant background temperature gradient in the fluid and slowly increase this gradient it may by possible to see the onset of overstability. We describe, in Section 2, an experiment which was conceived for this purpose. The outcome does not seem to show this overstability, but rather a primary instability to convection driven by horizontal temperature differences. A brief description of . the subsequent non-linear evolution of the system is provided.

In section 3 we discuss the linear theory for onset of instability in a tall, thin geometry. We show that the instability can set in at zero vertical wavenumber in parameter regimes where the density is stably stratified.

Sections 4,5 and 6 extend the linear theory to the weakly non-linear regime. In each section we derive amplitude equations for the zero wavenumber oscillatory eigenfunctions which arise for different sets of boundary conditions. The equation 
of section 4 has a subcritical bifurcation while the modified system of section 5 can be supercritical. The secondary instability of this latter system is touched upon. We also derive the dispersion relation that arises for the secondary instability of the amplitude equation of section 6 and show that is displays an Eckhaus instability.

We conclude with a description of our future plans for this research.

\section{The Experiment}

\subsection{Set Up}

The apparatus consists of a tank $80 \mathrm{~cm}$ high $(\mathrm{z}), 80 \mathrm{~cm}$ across $(\mathrm{y})$ and $1.2 \mathrm{~cm}$ back to front $(x)$. The front wall is $1 \mathrm{~cm}$ of perspex while the back wall is a $1 \mathrm{~cm}$ aluminum plate. Behind the top of the plate, running the breadth of the tank is attached a cooling bath; similarly there is a heating bath at the bottom. We use the standard double bucket technique to fill the tank with a constant salt-water concentration gradient (saltier on the bottom).

The thermal diffusion time across the slot is of order 1 minute and changes to the background temperature profile were made on times considered slow with respect to this. $(\Delta \rho / \rho)_{\text {Salt }}=1.5 \times 10^{-3}$ across the height of the tank so $\Delta T_{\max }=6.2^{\circ} \mathrm{C}$ would destabilize the density. We increased the temperature gradient about room temperature at the center of the plate. The rate was less than $\mp 0.5^{\circ} \mathrm{C}$ per hour at the top and bottom bath, respectively. Thermal diffusion in the z-direction for aluminum is comparable to the 1 minute diffusion time given above. The velocity field was visualized by using dissolved fish flakes and the salt water was dyed. The salt Rayleigh number started at 1900 while the thermal Rayleigh number was turned up to 1750 .

\subsection{Early Behavior}

An early time snapshot is shown in Figure 1. Note primarily the long straight rolls in the top part of the tank which form from the top and bottom boundaries and continue forming towards the midline as the temperature is turned up. The velocity 


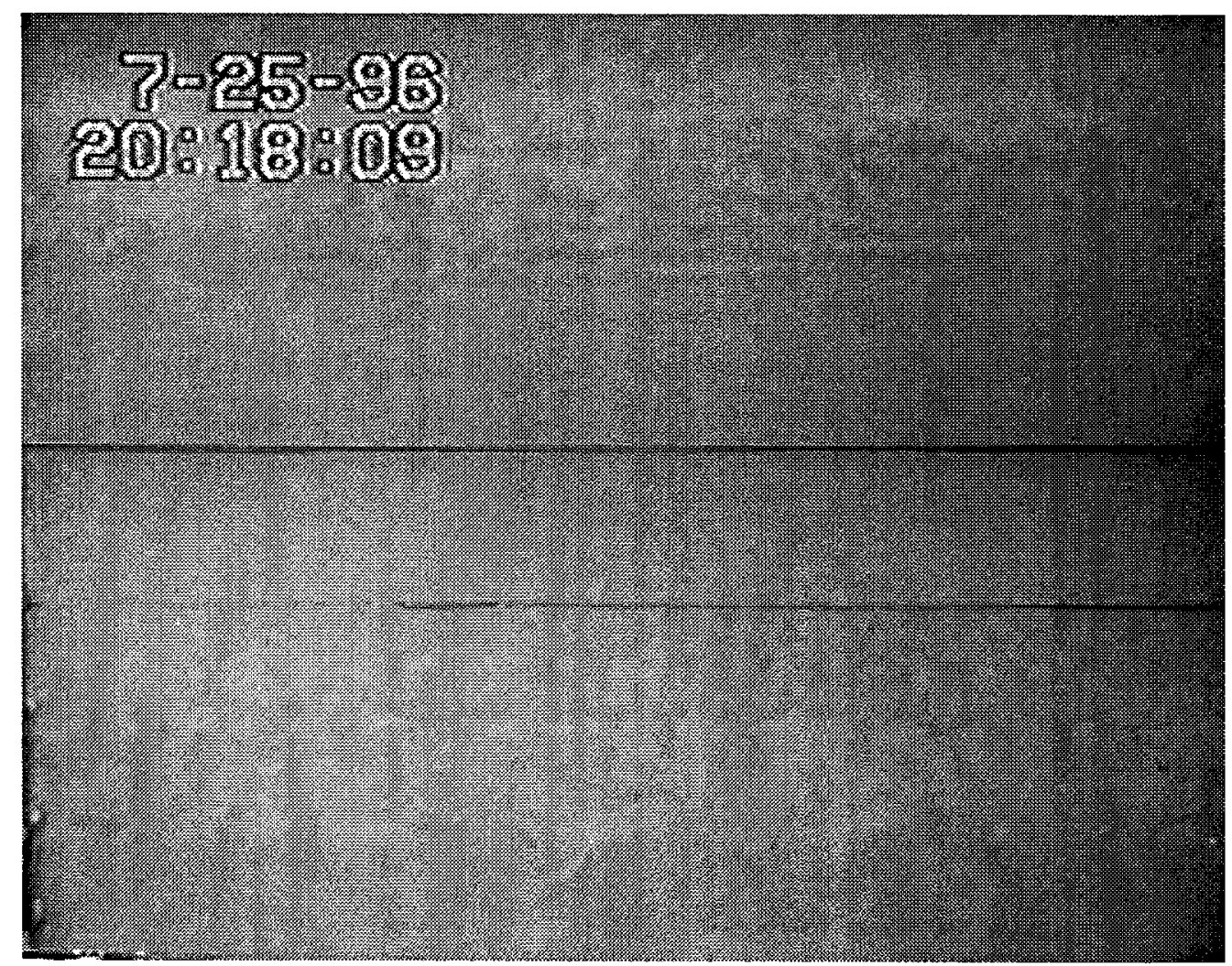

Figure 1: Early Time Photo of the experiment

field of the rolls is entirely in the $x-z$ plane and shows no $y$ dependence save for some boundary effects.

It seems that, as the temperature gradient is turned up, the primary instability sets in due to a temperature difference between the front and back walls. The hypothesis is that in the top half of the apparatus, the rear wall temperature is colder than the front (which is apparently at room temperature) and the fluid begins to fall. This falling is halted by the density contrast in the salt field and gives rise to the rolls. The same process takes place in the bottom half of the tank, but with fluid rising along the back wall.

At the time of this snapshot (Figure 1), the rolls in the top half rolls are becoming unstable to a vertical zig-zag along their axis. After a longer time this zig-zag undergoes a transition to slow rolls in the $y-z$ plane as is seen at the bottom of the tank, at which point the velocity field shows no $x$-dependence. 


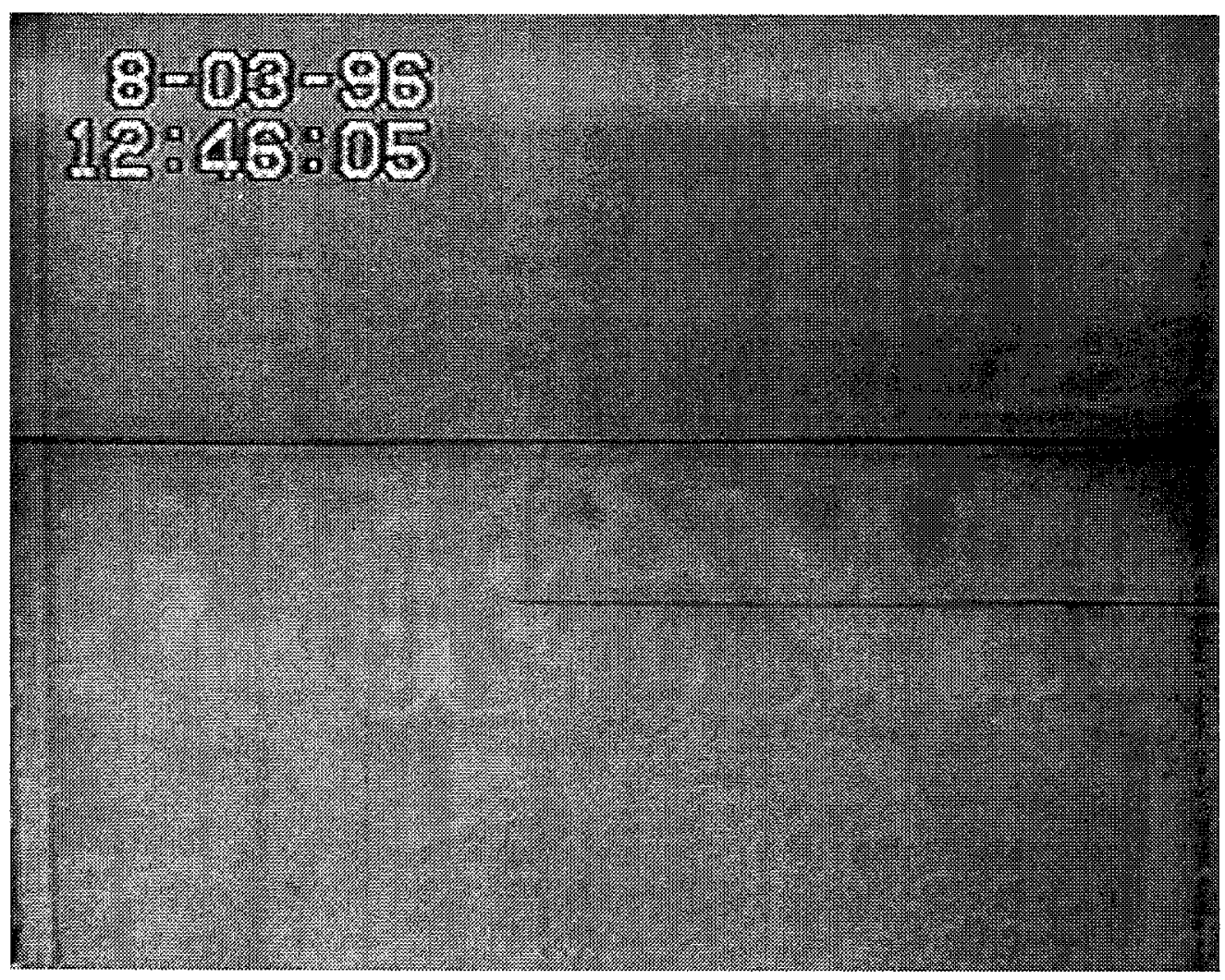

Figure 2: Late Time Photo of the Experiment

\subsection{Late Time Behavior}

In Figure 2, the fluid velocity is entirely $y-z$ Hele-Shaw-like. What began as regular rolls has now become turbulent thermal convection in layers. The salt concentration of these layers is relatively constant, since the convection keeps them mixed, and increases toward the bottom of the tank. The shear at the layer interfaces slowly decreases the salt contrast between layers. Convective blobs impinging upon the interfaces from above and below excite waves of ever-increasing amplitude as the salt proves a less effective barrier. After these interfacial waves start breaking, it then takes a short time for two layers to merge. Layer merging proceeds until there are just two remaining, dividing the tank in half. At this point the interfacial waves are quite large and dramatic and the last interface quickly breaks up. The salt becomes well mixed and highly turbulent thermal convection proceeds unimpeded throughout the tank. 


\section{Linear Theory}

\subsection{The Equations}

Consider the Boussinesq approximation for a fluid with a background linear profile of salt and temperature: in this paper we will be concerned with the diffusive stratification, that is to say, hotter and saltier below. The non-dimensional form of the momentum, heat and salt equations are, respectively,

$$
\begin{gathered}
{\left[\frac{1}{\sigma} \partial_{t}-\nabla^{2}\right] \nabla^{2} \psi+R_{T} \theta_{x}-R_{S} \Sigma_{x}=\frac{J\left(\psi, \nabla^{2} \psi\right)}{\sigma}} \\
{\left[\partial_{t}-\nabla^{2}\right] \theta+\psi_{x}=J(\psi, \theta)} \\
{\left[\partial_{t}-\tau \nabla^{2}\right] \Sigma+\psi_{x}=J(\psi, \Sigma)}
\end{gathered}
$$

Where:

$$
\begin{gathered}
\vec{u}=\left(\psi_{z}, 0,-\psi_{x}\right) \\
R_{T}=\frac{g \alpha \Gamma d^{4}}{\nu \kappa_{T}} \\
R_{S}=\frac{g \beta \Delta d^{4}}{\nu \kappa_{T}}
\end{gathered}
$$

$\Delta$ and $\Gamma$, defined as positive, are the background vertical gradients of salt and heat, $-\beta$ and $\alpha$ are their respective coefficients of expansion. $\sigma=\nu / \kappa_{T}$ is the Prandtl number, $\tau=\kappa_{S} / \kappa_{T}$ is the Lewis number and $d$ is the thickness of the slot in the $x$ direction. The temperature and salt equations are written for the perturbations of these fields from their linear background.

In an effort to explain the onset of instability seen in the experiment, the configuration we consider is one in which the vertical dimension is much greater than the horizontal. However, we emphasize that our analysis is not directly applicable to the experiment since we completely disregard effects of a temperature gradient in the thin direction as the back plate temperature is turned up. 


\subsection{Boundary Conditions}

In this and the following section we will consider boundary conditions most appropriate to the experiment. On the front and back walls no slip on the velocity field, $\psi=\psi_{x}=0$ and no flux of salt $\Sigma_{x}=0$. The temperature is strongly forced by the aluminum plate on the back wall so $\theta=0$ on $x=0$, and no heat flux through the front, so $\theta_{x}=0$ on $x=1$. These boundary conditions are used for the linear problem and will be changed for the derivation of the amplitude equations in subsequent sections.

\subsection{The problem}

Assuming all fields proportional to $e^{i k z}$ and no dependance on $y$, the linear problem becomes

$$
L_{k} \Psi=0
$$

where

$$
\boldsymbol{L}_{k} \equiv\left[\begin{array}{ccc}
\left(\frac{1}{\sigma} \partial_{t}+k^{2}-\partial_{x x}^{2}\right)\left(\partial_{x x}^{2}-k^{2}\right) & R_{T} \partial_{x} & -R_{S} \partial_{x} \\
\theta \\
\partial_{x} & \left(\partial_{t}+k^{2}-\partial_{x x}^{2}\right) & 0 \\
\partial_{x} & 0 & \left(\partial_{t}+\tau k^{2}-\tau \partial_{x x}^{2}\right)
\end{array}\right] .
$$

We will be considering fixed $\tau=10^{-2}$ and $\sigma=7$ in all that follows.

\subsubsection{Stationary Bifurcation}

Setting $\partial_{t}=0$ in equation (9) and fixing all other parameters gives an eigenvalue problem for $R_{T}$. At $k=0$ it is straightforward to solve analytically and yields

$$
R_{T}=R_{T_{C}} \equiv \frac{R_{S}}{\tau}+\alpha^{4}
$$

where

$$
\cos (\alpha) \cosh (\alpha)=1
$$

gives $\alpha$ implicitly. The first root of this equation corresponds to the lowest mode eigenfunction. 

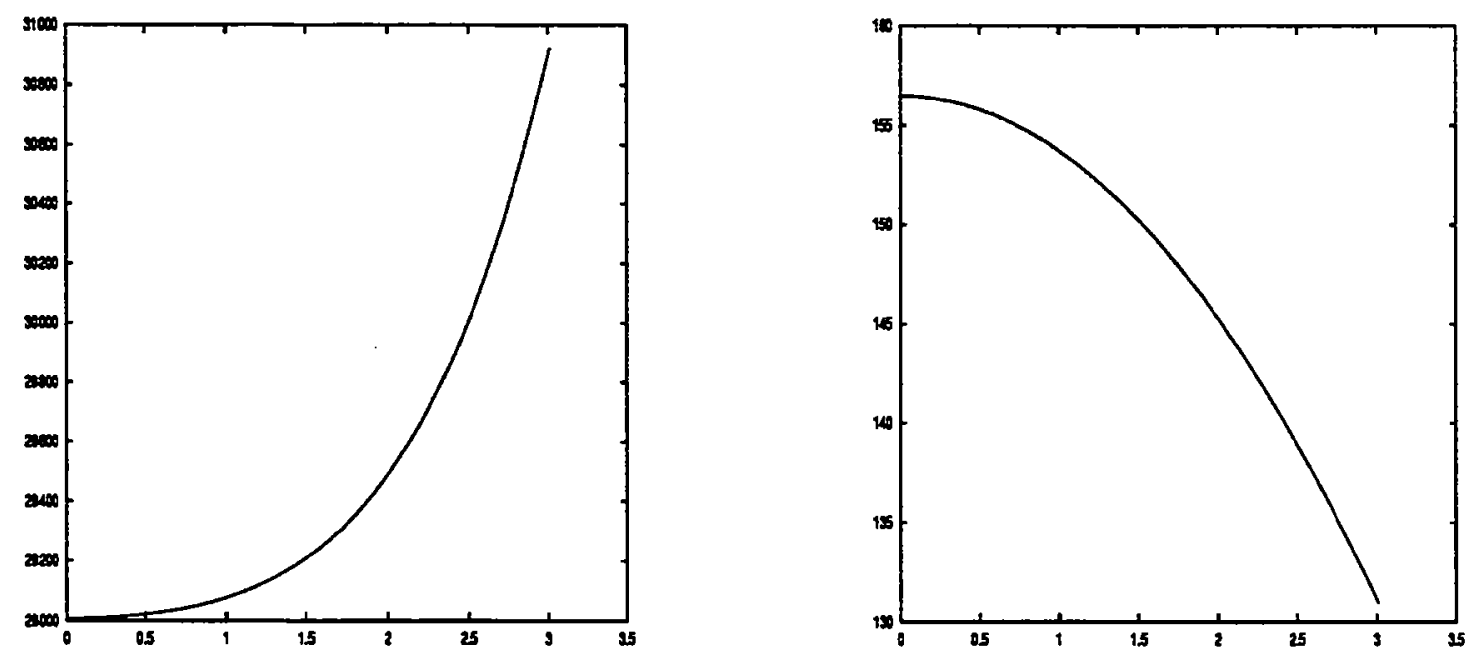

Figure 3: Critical $R_{T}$ and $\omega$ versus vertical wavenumber for $R_{S}=30000$.

For non-zero vertical wavenumber, solutions are obtained numerically using a Newton-Raphson routine (NRK). The zero-wavenumber mode has the lowest critical thermal Rayleigh number. It is important to note that the stationary bifurcation occurs when density is very unstably stratified for small $\tau\left(\tau=10^{-2}\right.$ for heat/salt).

\subsubsection{Oscillatory Bifurcation}

Now search for purely oscillatory modes in (9) $\partial_{t}=i \omega$ : we once again resort to the NRK routine to get the marginal curves and eigenfunctions. Figure 3 shows that the instability sets in at $k=0$ for a stably stratified density distribution when $R_{S}=30000$. In particular, the frequency of the oscillatory instability remains finite as the vertical wavenumber goes to zero. The real part of the marginal eigenfunction is shown in Figure 4.

For comparison, marginal curves are plotted for $R_{S}$ of 8000 and 80000 in figure 5. In the case of the weaker salt stratification instability sets in at zero wavenumber but at unstable densities. For the greater salt stratification instability sets in at stable densities but at a finite wavenumber. 

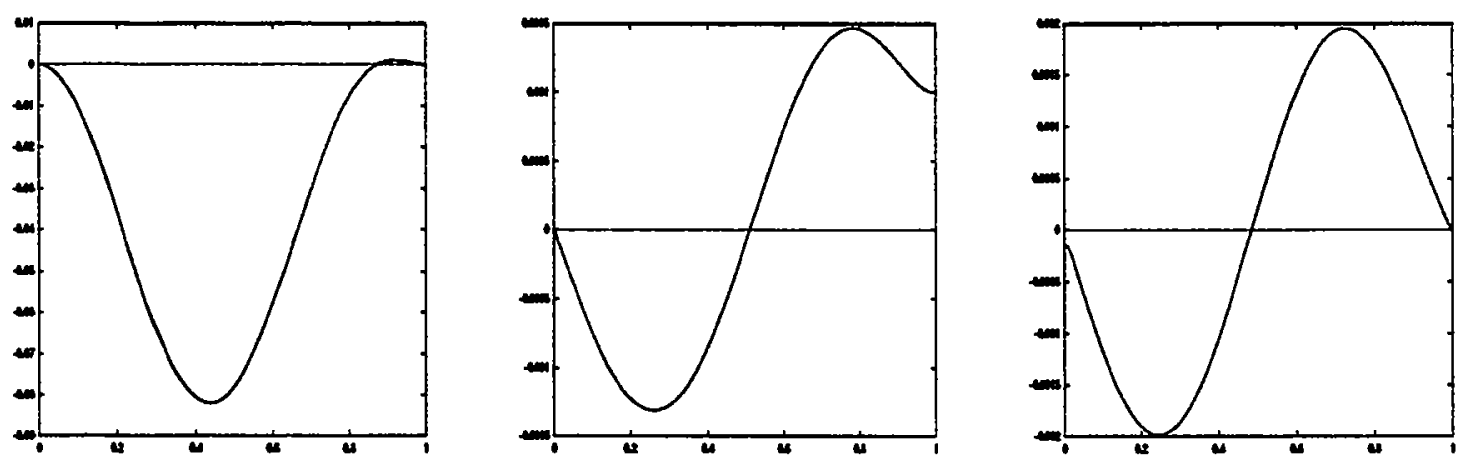

Figure 4: Stream, temperature and salt eigenfunctions at $k=0$ for $R_{S}=30000$
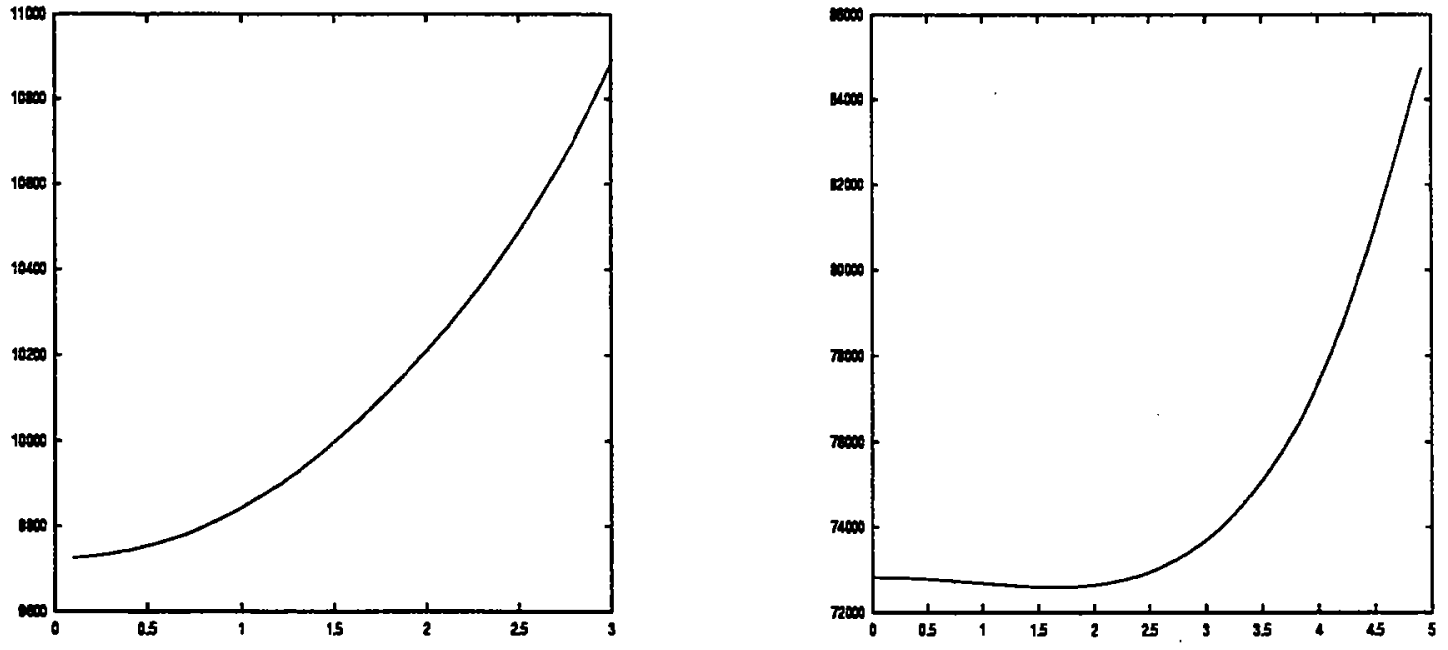

Figure 5: Marginal Curves for $R_{S}=8000$ and 80000 


\section{Amplitude Equations for Mixed Temperature and Fixed Salt Flux Boundary Conditions}

\subsection{Derivation}

\subsubsection{Scalings}

The zero wavenumber instability with non-zero frequency seen in the linear problem motivates a long wave expansion for the thermohaline equations (1) - (3). If we scale

$$
\begin{gathered}
z \longrightarrow \epsilon z \\
\partial_{t} \longrightarrow \partial_{t}+\epsilon^{2} \partial_{T}
\end{gathered}
$$

and recognize that the quadratic form of the marginality curve allows

$$
R_{T}=R_{0}+\epsilon^{2} R_{2}+\epsilon^{4} R_{4}
$$

our system can be written formally as

$$
L_{\circ} \Psi+\epsilon^{2} L_{2} \Psi+\epsilon^{4} L_{4} \Psi=\epsilon N_{1}(\psi, \Psi)+\epsilon^{3} N_{3}(\psi, \Psi)
$$

Where,

$$
\begin{gathered}
L_{2} \equiv\left[\begin{array}{ccc}
\left(\frac{1}{\sigma} \partial_{t} \partial_{z z}^{2}-2 \partial_{x x z z}^{4}+\frac{1}{\sigma} \partial_{T} \partial_{x x}^{2}\right) & R_{2} \partial_{x} & 0 \\
0 & \left(\partial_{T}-\partial_{z z}^{2}\right) & 0 \\
0 & 0 & \left(\partial_{T}-\tau \partial_{z z}^{2}\right)
\end{array}\right] \\
L_{4} \equiv\left[\begin{array}{ccc}
\left(\frac{1}{\sigma} \partial_{T}-\partial_{z z}^{2}\right) \partial_{z z}^{2} & R_{4} \partial_{x} & 0 \\
0 & 0 & 0 \\
0 & 0 & 0
\end{array}\right] \\
N_{1}(\psi, \Psi) \equiv J\left(\psi,\left[\begin{array}{c}
\frac{1}{\sigma} \psi_{x x} \\
\theta \\
\Sigma
\end{array}\right]\right)
\end{gathered}
$$

$L_{0} \Psi$ is defined as in equation (9) with $k=0$ and $N_{3}$ is defined similar to $N_{1}$ with $\psi_{x x}$ replaced by $\psi_{z z}$.

For the mixed temperature, fixed salt flux and the fixed heat and salt flux amplitude equations (considered in the following section) it is natural to begin the 
expansion of our fields at $O(\epsilon)$ in order to allow the second order linear operator to enter at the same order as the first order non-linear operator. The expansion of our variables now allows the odd powers to decouple from the even ones so we can take:

$$
\Psi=\epsilon \Psi_{1}+\epsilon^{3} \Psi_{3}+\cdots
$$

\subsection{2 $O(\epsilon)$}

At first order equation (11) becomes

$$
L_{0} \Psi_{1}=0
$$

which is just the linear problem of the previous section evaluated at $k=0$. There are two marginal eigenvectors in this problem, $\hat{\Psi}_{1}^{a}$ which denotes the eigenvectors of the previous section with eigenvalues $\omega$ and $\hat{\Psi}_{1}^{b}$ which has zero eigenvalue,

$$
\hat{\mathbf{\Psi}}_{1}^{b} \equiv\left[\begin{array}{l}
0 \\
0 \\
1
\end{array}\right] .
$$

This latter solution arises due to the no flux boundary conditions of salt on the side walls and it represents the redistribution of the mean salt field by the flow [4]. So, the general solution at this order is

$$
\mathbf{\Psi}_{1}=A(z, T) \hat{\mathbf{\Psi}}_{1}^{a} e^{i \omega t}+A^{*}(z, T) \hat{\mathbf{\Psi}}_{1}^{a *} e^{-i \omega t}+B(z, T) \hat{\Psi}_{1}^{b}
$$

where an asterisk denotes complex conjugate.

The adjoint problem is obtained by multiplying by the adjoint eigenvector and integrating over $x$ by parts. Let a dagger denote the adjoint vector, then it is a straightforward exercise to show that $\hat{\mathbf{\Psi}}_{1}^{b \dagger}=\hat{\mathbf{\Psi}}_{1}^{b}$ and

$$
\hat{\mathbf{\Psi}}_{1}^{\alpha \dagger}=\left[\begin{array}{c}
\hat{\psi}_{1}^{*}(x) \\
-R_{0} \hat{\theta}_{1}^{*}(x) \\
R_{S} \hat{\Sigma}_{1}^{*}(x)
\end{array}\right]
$$

\subsection{3 $O\left(\epsilon^{3}\right)$ - The Amplitude Equations}

$$
L_{0} \Psi_{3}=N_{2}\left(\psi_{1}, \Psi_{1}\right)-L_{2} \Psi_{1}
$$


In this equation the inhomogeneous terms can resonate with the linear operator. To solve the equation at this order we must therefore impose the solvability conditions:

$$
\left\langle\hat{\mathbf{\Psi}}_{1}^{a \dagger}, R H S\right\rangle=\left\langle\hat{\mathbf{\Psi}}_{1}^{b \dagger}, R H S\right\rangle=0
$$

the inner product denoting integration over $x=0,1$. The solvability conditions translate into equations for the amplitudes, $A$ and $B$, we find

$$
\begin{gathered}
A_{T}=\eta A_{z z}+\beta A B_{z}+\gamma A \\
B_{T}=\tau\left[B_{z z}-\alpha|A|_{z}^{2}\right] .
\end{gathered}
$$

$\mathrm{B}$ and $\alpha$ are real while $\mathrm{A}, \eta$ and $\beta$ are, in general, complex since at onset of instability for $k=0$ there is a non-zero oscillation frequency. $\tau$ is still the Lewis number. The coefficients are defined as follows,

$$
\begin{aligned}
& \eta=\frac{1}{r} \int_{0}^{1}\left[2\left|\hat{\psi}_{1 x}\right|^{2}+R_{0}\left|\hat{\theta}_{1}\right|^{2}-\tau R_{S}\left|\hat{\Sigma}_{1}\right|^{2}\right] d x+\frac{i \omega}{r \sigma} \int_{0}^{1}\left|\hat{\psi}_{1}\right|^{2} d x \\
& \beta=\frac{R_{S}}{r} \int_{0}^{1}\left[\tau\left|\hat{\Sigma}_{1 x}\right|^{2}+i \omega\left|\hat{\Sigma}_{1}\right|^{2}\right] d x \\
& \alpha=2 \int_{0}^{1}\left|\hat{\Sigma}_{1 x}\right|^{2} d x \\
& \gamma=R_{2} \\
& r=\int_{0}^{1}\left|\hat{\theta}_{1 x}\right|^{2} d x
\end{aligned}
$$

\subsection{A Steady Solution}

Steady solutions of (21) and (22) are obtained by setting time derivatives to zero. So

$$
B_{z}=\alpha|A|^{2}
$$

and

$$
0=\eta A_{z z}+\alpha \beta A|A|^{2}+\gamma A
$$

If, for a moment, we take $\eta$ and $\beta$ to be real and consider periodic solutions, $A=$ $R e^{i k z}$, we can disregard boundary conditions for (24). $R$ is given implicitly by

$$
\gamma=\eta_{r} k^{2}-\alpha \beta_{r} R^{2}
$$

Since the real parts of $\eta$ and $\beta$ are positive, curves of constant $R$ in (25) are parabolas intersecting the negative $\gamma$-axis in the $\gamma v s . k$ plane. Therefore solutions of this form 
exist only for negative $\gamma$ and we have a subcritical bifurcation. In physical terms, the oscillatory mode is advecting so much of the background salt field as to destroy its ability to stabilize the system. This oscillation is then unstable; the well mixed salt will now have $R_{S}=0$ and thermal convection will ensue.

\section{Amplitude Equations for Fixed Heat and Salt Fluxes at the Boundaries}

\subsection{Derivation}

The redistribution of the mean salt field by the flow is the mechanism for subcriticality in eq. (25) so, in order to saturate the instability, the mean temperature field must be allowed to redistribute itself at the same order. Changing the temperature boundary conditions to no flux on both walls $\left(\theta_{x}=0\right)$ allows another marginally stable eigenfunction at first order, the temperature mode,

$$
\hat{\mathbf{\Psi}}_{1}^{c} \equiv\left[\begin{array}{l}
0 \\
1 \\
0
\end{array}\right]
$$

It was analytically verified that the zero wavenumber instability still occurs for these boundary conditions in a parameter range where the density is stably stratified $\left(R_{T_{C}}<R_{S}\right)$, allowing us to use the same asymptotic expansion as before.

Now the general first order solution becomes

$$
\Psi_{1}=A(z, T) \hat{\Psi}_{1}^{a} e^{i \omega t}+A^{*}(z, T) \hat{\mathbf{\Psi}}_{1}^{a *} e^{-i \omega t}+B(z, T) \hat{\Psi}_{1}^{b}+C(z, T) \hat{\Psi}_{1}^{c}
$$

Once again we proceed to third order and project the inhomogeneous terms onto the, now three, first order marginal eigenfunctions. This yields evolution equations for the A, B and C modes

$$
\begin{gathered}
A_{T}=\eta A_{z z}+\beta A B_{z}-\delta A C_{z}+\gamma A \\
B_{T}=\tau\left[B_{z z}-\alpha|A|_{z}^{2}\right] \\
C_{T}=C_{z z}-\xi|A|_{z}^{2}
\end{gathered}
$$


where,

$$
\begin{aligned}
& \delta=R_{0}+\frac{i \omega}{r} \int_{0}^{1}\left|\hat{\theta}_{1}\right|^{2} d x \\
& \xi=2 \int_{0}^{1}\left|\hat{\theta}_{1 x}\right|^{2} d x
\end{aligned}
$$

All coefficients are defined such that their real parts are manifestly positive. They are defined in the same manner as before but are now solutions to a different linear problem since the boundary conditions have been changed.

Only the derivatives of $B$ and $C$ enter in (28) so we can rewrite our system, defining $\tilde{B}=B_{z}$ and $\tilde{C}=C_{z}$, as

$$
\begin{gathered}
A_{T}=\eta A_{z z}+\beta A \tilde{B}-\delta A \tilde{C}+\gamma A \\
\tilde{B}_{T}=\tau\left[\tilde{B}-\alpha|A|^{2}\right]_{z z} \\
\tilde{C}_{T}=\left[\tilde{C}-\xi|A|^{2}\right]_{z z}
\end{gathered}
$$

\subsection{Steady Solutions}

Seek solutions of equations (32)-(34) of the form

$$
A=R e^{i(k z+n T)}, \quad \tilde{B}=\tilde{B}_{0}, \quad \tilde{C}=\tilde{C}_{0}
$$

such that $\partial_{T} \tilde{B}=\partial_{T} \tilde{C}=0$ and the time dependence of $\mathrm{A}$ is given explicitly through $\Omega$. Therefore

$$
\begin{aligned}
\gamma & =\eta_{r} k^{2}+\Lambda_{r} R^{2} \\
\Omega & =\eta_{i} k^{2}-\Lambda_{i} R^{2} \\
\Lambda & \equiv \xi \delta-\alpha \beta
\end{aligned}
$$

and

$$
\tilde{B}_{0}=\alpha R^{2}, \quad \tilde{C}_{0}=\xi R^{2}
$$

In order for the bifurcation to be supercritical $\Lambda_{r}$ must be greater than zero. It is evident from the definition that this can be easily achieved for small $\tau$ if the salt and temperature fields are of comparable magnitude. Curves of constant roll amplitude are plotted in figure 6. 


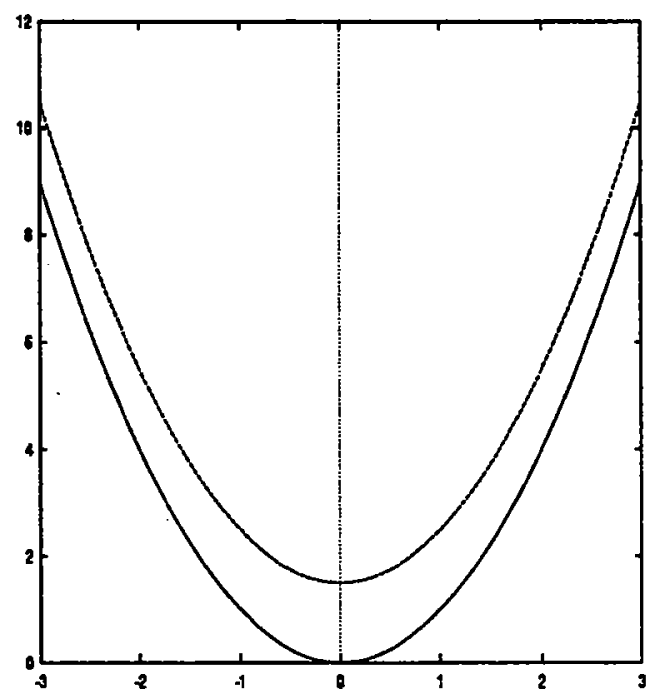

Figure 6: Schematic curves of Constant $\mathrm{R}$ in the $\gamma$ vs. $k$ plane for steady solutions of the fixed flux amplitude equations

\subsection{Secondary Instability}

On perturbing the solutions (38) such that

$$
\begin{aligned}
& A=[R+r(z, T)] e^{i(k z+\Omega T+\phi(z, T))} \\
& \tilde{B}=\tilde{B}_{0}+b(z, T) \\
& \tilde{C}=\tilde{C}_{0}+c(z, T)
\end{aligned}
$$

keeping only linear terms in $r, \phi, b$ and $c$ and assuming that all perturbation fields are proportional to $e^{\lambda T+i q z}$ yields a quartic dispersion relation

$$
\begin{aligned}
0= & \left(\lambda+q^{2}\right)\left\{\left(\lambda+\tau q^{2}\right)\left[\left(\eta_{r} q^{2}+2 i \eta_{i} k q+\lambda\right)^{2}+\left(\eta_{i} q^{2}-2 i k q \eta_{r}\right)^{2}\right]\right. \\
& \left.-2 \alpha \tau R^{2} q^{2}\left[q^{2}\left(\beta_{i} \eta_{i}+\beta_{r} \eta_{r}\right)+2 i k q\left(\beta_{r} \eta_{i}-\beta_{i} \eta_{r}\right)+\lambda \beta_{r}\right]\right\} \\
& +2 \xi R^{2} q^{2}\left(\lambda+\tau q^{2}\right)\left[q^{2}\left(\eta_{i} \delta_{i}+\eta_{r} \delta_{r}\right)+2 i k q\left(\delta_{r} \eta_{i}-\delta_{i} \eta_{r}\right)+\lambda \delta_{r}\right] .
\end{aligned}
$$

We have not yet explored the possible solutions to this dispersion relation but point out that even with all imaginary coefficients set to zero (i.e. if the initial Hopf instability occurs with zero frequency) there is the possibility for very rich dynamics in this equation. One or more modes may go unstable simultaneously; they can go unstable singly, through a stationary bifurcation, or in pairs through a Hopf. Such an investigation will be carried out in future work. 


\section{The Amplitude Equation for Fixed Tempera- ture and Salt Boundaries}

\subsection{Derivation}

The fields which redistribute the background temperature and salt stratification can be suppressed by changing the boundary conditions to $\theta=\Sigma=0$ on both $x=0,1$. As such, we will be left with a lower order set of equations for the amplitudes. It turns out that allowing the fields to come in at lowest order gives a different structure to the $A$ equation than in the previous analyses, so let

$$
\Psi=\Psi_{0}+\epsilon \Psi_{1}+\epsilon^{2} \Psi_{2}+\cdots
$$

So our formal system, equation (11), becomes

$$
\begin{array}{r}
L_{0} \Psi_{0}+\epsilon L_{0} \Psi_{2}+\epsilon^{2} L_{0} \Psi_{2}+\epsilon^{2} L_{2} \Psi_{0}+O\left(\epsilon^{3}\right)= \\
\epsilon N_{1}\left(\psi_{0}, \Psi_{0}\right)+\epsilon^{2}\left[N_{1}\left(\psi_{1}, \Psi_{0}\right) N_{1}\left(\psi_{0}, \Psi_{1}\right)\right]
\end{array}
$$

A complete solution of this problem involves solving the linear inhomogeneous problem for the new boundary conditions. In an effort to elucidate the possible dynamics of this system, we will restrict ourselves to the formal solution of the equation, rather than the full problem. The numerical solution of the linear homogeneous problem confirms that a zero wavenumber Hopf bifurcation occurs in this case for $R_{S} \sim 8000$ so we can proceed with a long wave expansion for this case.

\subsection{1 $O\left(\epsilon^{0}\right)$}

At this order the problem is similar to the previous linear problem (only the boundary conditions have been modified) so

$$
\Psi_{\mathrm{o}}=A(z, T) \hat{\Psi}_{0} e^{i \omega t}+A^{*}(z, T) \hat{\Psi}_{0}^{*} e^{-i \omega t}
$$

and the adjoint eigenfunction is defined as in (19).

\subsection{2 $O\left(\epsilon^{1}\right)$}

$$
L_{\mathrm{o}} \Psi_{1}=N_{1}\left(\psi_{0}, \Psi_{\mathrm{o}}\right)
$$


Since only terms in $e^{i \omega t}$ and constant in time appear on the right hand side of (44) there is no resonance and the inhomogeneous problem can be solved at this order. Formally, we write

$$
\Psi_{1}=A A_{z} \hat{\mathbf{\Psi}}_{1}^{a} e^{2 i \omega t}+A A_{z}^{*} \hat{\mathbf{\Psi}}_{1}^{b}+\text { c.c. }
$$

where c.c. denotes complex conjugation and $\hat{\mathbf{\Psi}}_{1}^{a}, \hat{\mathbf{\Psi}}_{1}^{b}$ are particular solutions of (44).

\subsection{3 $O\left(\epsilon^{2}\right)$ - The Amplitude Equation}

$$
L_{\circ} \Psi_{2}=N_{1}\left(\psi_{1}, \Psi_{0}\right)+N_{1}\left(\psi_{0}, \Psi_{1}\right)-L_{2} \Psi_{\circ}
$$

Once again there are resonances that must be eliminated by projecting onto $\hat{\Psi}_{0}^{\dagger}$. The resulting amplitude equation

$$
A_{T}=\eta A_{z z}+\gamma A+\alpha A\left|A_{z}\right|^{2}+\beta|A|^{2} A_{z z}+\zeta A^{*} A_{z}^{2}+\delta A^{2} A_{z z}^{*}
$$

has, in general, all coefficients except $\gamma$ complex.

Since the coefficients remain undetermined, this turns out to be the most general equation up to cubic order in $A$ that respects all of the symmetries of the problem and, as such, could have been determined entirely on symmetry arguments. That is to say, $A$ is equivariant under each of

$$
\begin{array}{rl}
z \longrightarrow z+c & z \longrightarrow-z \\
T \longrightarrow T+T_{0} & A \longrightarrow A^{*}
\end{array}
$$

the last of these expressing the symmetry under $x \rightarrow-x$. As in the previous cases, a complete solution is necessary to determine both the magnitudes and signs of the coefficients.

\subsection{Steady Solution}

Again, consider solutions of (47) of the form $A=R e^{i(k z+\Omega T)}$. Therefore

$$
\begin{array}{r}
\gamma=k^{2}\left\{\eta_{r}+R^{2} \Xi_{r}\right\} \\
\Omega=-k^{2}\left\{\eta_{i}+R^{2} \Xi_{i}\right\} \\
\Xi \equiv \beta+\zeta+\delta-\alpha
\end{array}
$$




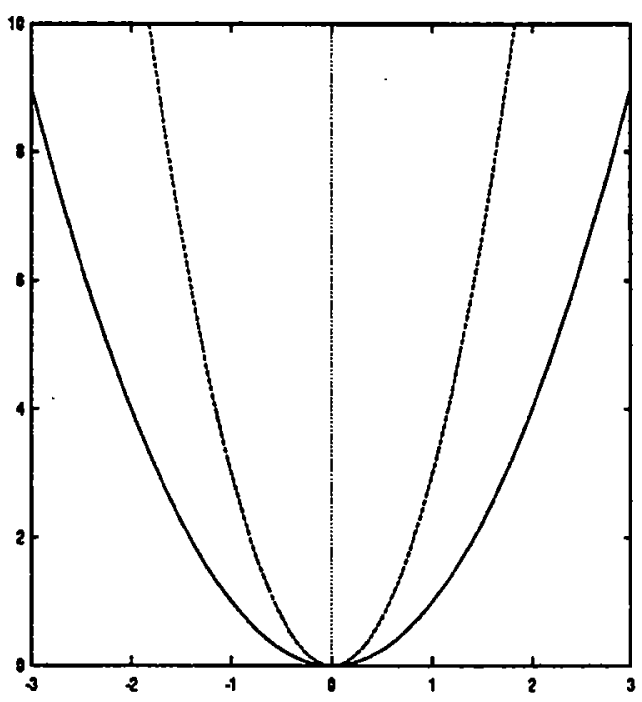

Figure 7: Schematic curves of constant $R$ in the $\gamma$ vs. $k$ plane for steady solutions of the fixed temperature and salt amplitude equation. No solutions exist below the lowest curve, stable solutions above the uppermost and unstable ones in between.

In order to have solutions for positive $\gamma$ (supercritical) the coefficient of $R^{2}$ in (50) must be positive, and we restrict our attention to this situation. Contrasting with the fixed temperature and salt flux case we see that curves of constant $R$ all pass through the origin of Figure 7 whereas in the $A-B-C$ case they were parametrized by their y-intercept.

\subsection{Secondary Instability}

Focusing on the case of real coefficients we perturb (50) and linearize as in (41), the dispersion relation is

$$
\begin{aligned}
0= & \hat{\lambda}^{2}+\hat{\lambda}\left[\hat{q}^{2}\left(2+(\mu+\nu) R^{2}\right)+2 \Xi R^{2}\right] \\
& +\left(1+\mu R^{2}\right)\left(1+\nu R^{2}\right) \hat{q}^{4}+2 R^{2} \Xi\left(1+\nu R^{2}\right) \hat{q}^{2}- \\
& 4 \hat{q}^{2}\left(1+\Xi R^{2}\right)\left(1+(\nu+\zeta) R^{2}\right)
\end{aligned}
$$

where the rescaled wavenumber and growth rate are $\hat{q}=q / k$ and $\hat{\lambda}=\lambda / k^{2}$ and the parameters $\mu=\beta+\delta$ and $\nu=\beta-\delta$. Dispersion relations are plotted in Figure 8. For real coefficients it can be shown that, at leading order in $q$, the unstable 


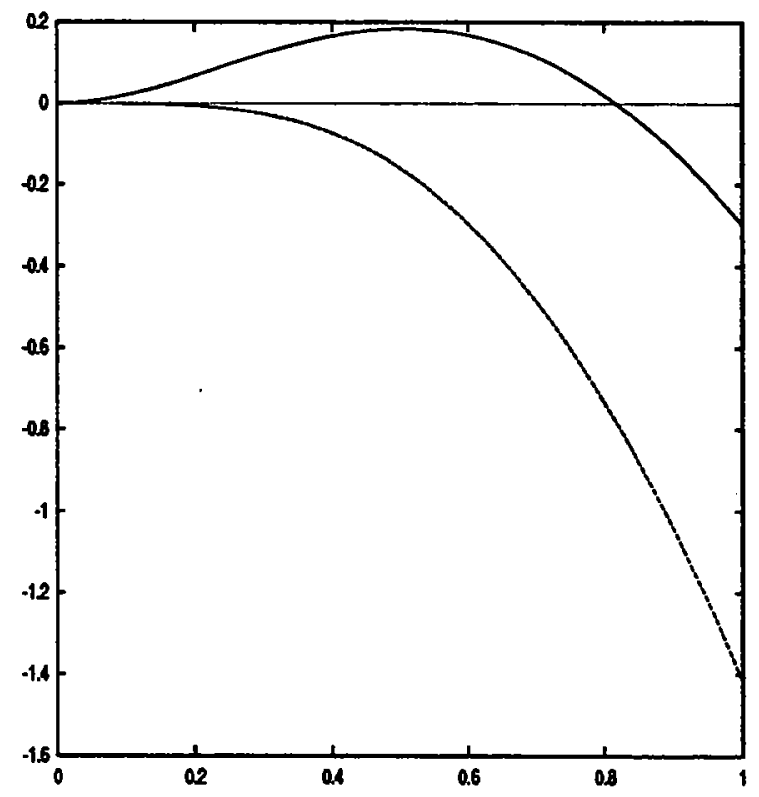

Figure 8: Scaled growth rate versus scaled wavenumber for $R=1$ and $R=R^{*}=$ $\sqrt{ } 2, \mu=2, \nu=1, \Xi=1, \zeta=-1$.

eigenvector of (52) lies entirely along $\phi$, the phase. Requiring $\hat{\lambda}(\hat{q})$ to have a nonzero root defines $R=R$, such that all rolls with amplitude $0<R<R$, have a band of $\hat{q}$ over which they are unstable. This Eckhaus-unstable band, as discussed in Fauve [5], is shown in Figure 7.

\section{Discussion}

The experiment was intended to be purely qualitative and to motivate theoretical investigations; to that end it was successful. There are some fascinating dynamics in the breakdown of the original rolls, the slow regular convection in layers and the later layer breakdown through interfacial wave breaking. The experimental investigation was not exhaustive and there is potential for some quantitative work here. In particular, the temperature distribution on the aluminum plate is not known and not necessarily linear. The front boundary should also be insulated better in order to minimize losses there, but this would make flow visualization much more difficult. In order to understand this experiment fully, one must include thermal diffusion effects the back plate and, at later stages, understand how the 
heat advected by the fluid affects the temperature of the aluminum.

The unique feature of the linear theory is the zero wavenumber instability with non-zero frequency which we have shown to be due to the geometry of the problem - not to the details of the boundary conditions.

In the weakly non-linear theory the fixed salt/temperature case showed an Eckhaus instability when we restricted attention to parameters with zero imaginary part. Including some of the imaginary parts will be the next step in studying this amplitude equation.

We will be carrying out an investigation of secondary instabilities in the mixed temperature case and the fixed temperature and salt flux case with coefficients derived directly from the linear problem. We hope to study the bifurcation structure of the three amplitude system of equations in all parameter regimes as there is the possibility for rich new dynamics here.

Other avenues that could be explored include an investigation of the 3-D vorticity mode (the zig-zag instability which was seen in the experiment). A Hele-Shaw description of the flow at large $R_{T}$ and a study of the layer dynamics could ultimately lead to a mixing length theory for turbulent layers.

\section{Acknowledgments}

An NSF graduate fellowship funded my existence. To Neil Balmforth, I am eternally indebted for hours upon hours of conversation and for pressing me on when I otherwise would not have; to Paola Cessi and Bill Young, for many ideas leading to the salt mode, tomatoes and basil; George Veronis for patience, in conversation and baseball; Barry Ruddick, who told me how to properly conduct an experiment; Oliver Kerr, who helped interpret it. Jack Whitehead gave me strong encouragement to proceed with the experiment and John Salzig built the apparatus in a few days. Finally, I thank the other GFD Wretches and Summer Fellows and Alice who made everyone fall in love with her this summer. For Stephanie. 


\section{References}

[1] Huppert, H. E., and P. F. Linden, 1979. On heating a stable salinity gradient from below. JFM, 95, pp 431-464.

[2] Shirtcliffe, T.G.L, 1967. Thermosolutal convection: observation of an overstable mode. Nature, 213, 489-490.

[3] Veronis, G., 1968. Effect of a stabilizing gradient of solute on thermal convection. JFM, 34, pp 315-336.

[4] Cessi, P. \& W. R. Young, 1992. Fixed-Flux convection in a tilted slot. JFM, 237, pp 57-71.

[5] Fauve, S. 1991. Patterns in Fluid Flow, Principal Lectures GFD 1991. 


\title{
Convection with radiation : the fixed flux case
}

\author{
S. Aumaître
}

\section{Introduction}

There are three means to transfer heat : conduction, which involves a molecular energetic exchange; convection, which involves a mass transfer, and radiation which involves light. In atmospheric dynamics these three processes are strongly connected because temperature drives the radiative emission, the gas expansion and the conducting flux. But often the light-matter interactions are neglected and all the radiative effects are included in the conduction. We only consider the case where radiation increases the thermal conductivity. But in some media light-matter interactions cannot be negleted. In this case it is interesting to know the radiative effect on gas motion. This provides the motivation for studies which consider the well known problem of Rayleigh-Bénard convection where some radiative component is added.

Since Goody [1] the stabilising effect of radiation was well understood for grey media. In the static state, radiation involves a non-linear profile of temperature which is more stable than the linear one. This work has been confirmed by experimental results obtained by Gille and Goody [2]. Continuing from this work, other works have tried to describe more precisely the light interaction with the convective gas and the wall boundary. But the case with a fixed total flux (of temperature and radiation) are curiously less studied. We would like to try to understand better the onset of instability in this case.

Before considering the fixed flux case, we will describe a set of equations for temperature and fluid velocity, the boundary condition added by radiation and the approximation introduced. In our conclusion we will give some possible extensions in this subject. 


\section{Equations for convection with radiative trans- fer}

\subsection{Equations for the fluid motion}

The motivation for studying convection with radiation comes from the stellar atmosphere. In this case the Boussinesq approximation is not strickly valid, but we adopt it for simplicity. The fluid is assumed to be incompressible except in the gravitational term where the thermal expansion is present. We derive these equations from the Navier-Stokes equation [3].

We can assume that radiation only affects the heat equation. Hence we obtain the most simple set of equations which describe the fluid motion with radiative transfer:

$$
\begin{gathered}
\rho_{o} D_{t} \vec{u}=-\vec{\nabla} p+\rho_{o} g\left(1-\alpha\left(T-T_{o}\right)\right) \overrightarrow{e_{z}}+\eta \vec{\nabla}^{2} \vec{u} \\
\rho_{o} C_{v} D_{t} T=k \vec{\nabla}^{2} T-\vec{\nabla} \overrightarrow{q_{r}} \quad(2.1 . b) \\
\vec{\nabla} \vec{u}=0 \quad(2.1 . c) .
\end{gathered}
$$

We denote $D_{t}=\partial_{t}+\vec{u} \cdot \vec{\nabla}$, the velocity as $\vec{u}$, the pressure $p$, the temperature as $T$ the mean density as $\rho_{0}$ and is the gravity field as $-g \vec{e}_{z}$. the thermoconductivity as $k$, the specific heat coefficient as $C_{v}$ the viscosity as $\eta$ and the density expansion as $\alpha$. We denote the radiative flux as $\overrightarrow{q_{r}}$, a new variable introduced by radiation. We need a new set of equations to describe this term.

\subsection{Equation for the radiative flux.}

Radiative flux is described by the light intensity equation, but we need some approximation to derive this equation. A rigorous description of interaction between light and gas in motion is actually very complex. It involves a relativistic treatment of photon and particle motion [4]. Except for several extreme astrophysical cases this is not necessary. But even without a relativistic treatment the light intensity 
equation is still an integro-differential equation [5].

So let us just consider only grey media. We can describe them in terms of opacity which is characterised by an absorption coefficient. It seems reasonable to suppose a local thermodynamic equilibrium (L.T.E.) when the temperature difference between the two walls is much smaller than the mean temperature. In this case the gas light emission can be described by the black body law. The light intensity equation can be approximated by :

$$
\frac{1}{c} \partial_{t} I(\Omega, \nu)+l_{i} \partial_{i} I(\Omega, \nu)=\kappa_{\nu} \rho_{o}(B(\nu)-I(\Omega, \nu))
$$

where $I(\Omega, \nu)$ is the light intensity, $\kappa_{\nu}$ is an absorption coefficient and $B(\nu)$ is the Planck law. $\nu$ is the light frequency, $\vec{l}$ gives the light beam's direction and $\Omega$ is a solid angle definied by $l_{i} . c$ is the light velocity which is much bigger than the other hydrodynamical velocity. We can reasonably neglect the time dependence in the above equation.

We must now introduce some global optical momentum. We define the radiative energy density $u_{r}$ by :

$$
u_{r} c=\frac{j}{4 \pi} \int_{\nu, \Omega} I(\Omega, \nu) d \nu d \Omega,
$$

where $j$ is introduced to simplify the equations below.

The radiative flux $\vec{q}_{\boldsymbol{r}}$ is

$$
\overrightarrow{q_{r}}=\int_{\nu, \Omega} I(\Omega, \nu) \cdot \vec{l} d \nu d \Omega
$$

The radiative pressure tensor $\Pi_{i k}$ is given :

$$
\Pi_{i k}=\int_{\nu, \Omega} I(\Omega, \nu) l_{i} l_{k} d \nu d \Omega
$$

We need a infinite set of moments as is the case when solving the light intensity equation (2.2). We relate these moments by successive integration of equation (2.2). 
A first integration gives :

$$
\vec{\nabla} \overrightarrow{q_{r}}=4 \pi \kappa_{o p t} \rho_{o}\left(\frac{\sigma}{\pi} T^{4}-j\right)
$$

If we multiply (2.2) by $l_{k}$ we obtain

$$
\partial_{i} \Pi_{i k}=\kappa_{\text {opt }} \rho_{\circ} q_{r k}
$$

We must now close our problem. The L.T.E. assumption suggests we take an isotropic tensor pressure proportional to the radiative energy density. As is the case for the black body law we have :

$$
\Pi_{i k}=4 \pi j / 3 \delta_{i k}
$$

If we introduce this relation in (2.3.b) and combine it with (2.3.a) we get an equation in $\mathrm{j}$ which closes our problem :

$$
\frac{1}{\tilde{\tau}} \vec{\nabla}^{2} j=\tau \kappa_{\text {opt }} \rho_{o}\left(j-\frac{\sigma}{\pi} T^{4}\right)
$$

where $\tilde{\tau}=\sqrt{3} \kappa_{\text {opt }} \rho_{\mathrm{o}}$

We also need to specify the boundary condition for the velocity, the temperature, and our new optical variable $j$.

\subsection{The boundary condition.}

We consider the classical Rayleigh-Benard problem of an infinite layer of fluid between two horizontal parallel walls at $z=-d / 2$ and $d / 2$. We fix the wall temperature or the total flux. We have

$$
\left(-k \vec{\nabla} T+\vec{\nabla} \overrightarrow{q_{r}}\right)=F
$$

where $F$ is a control parameter in the latter case. 
We often use the stress free boundary for the velocity at both walls, we could consider also the no slip condition or different boundary conditions at the top and at the bottom. We recall that free slip means $w=0$ and $\partial_{x} u=0$ on the wall, where $u$ and $w$ are the horizontal and the vertical coordinates of the velocity respectively. For no slip we have $\vec{u}=\overrightarrow{0}$ on the wall. We also need boundary conditions for our optical parameter $j$. It is characterised by the 'color' of the boundary. We give below some idea of how we understand the two extremes cases.

In the mirror case the wall reflects all the incident radiation. The radiative flux must vanish on the wall, thus we have $\left.\overrightarrow{q_{r}} \cdot \overrightarrow{e_{z}}\right|_{\omega}=0$. The equation (2.3.b) and our closure relation involve a condition on $j$ :

$$
\left.\partial_{z} j\right|_{w}=0 \quad(2.6 . a) .
$$

On the other hand we must consider the black wall case where all the incident flux is absorbed. In the equilibrium black body case where all the walls are at the same temperature the walls absorb all the incident radiation and emit following the black body law. By analogy let us assume : $\left.\vec{q}_{\tau} \cdot \vec{e}_{z}\right|_{w}=c \Delta u_{r}$ where $\Delta u_{r}$ is the energy density difference between the top and the bottom. The heuristic argument above is not a proof but I hoped that it could help to understand the origin of the following boundary condition on $j$ for black wall :

$$
\partial_{z} j \pm\left.\tilde{\tau} j\right|_{w^{ \pm}}=0 \quad(2.6 . b) .
$$

We can introduce a coefficient $\zeta_{\boldsymbol{w}}$ which measures the color of the wall to describe the intermediate case $: \partial_{z} j \pm\left.\zeta_{w \pm} \tilde{\tau} j\right|_{w^{ \pm}}=0 \quad$ (2.6.c).

The previous arguments amont to the Milne-Eddington Approximation for radiative transfer. For more information there are several papers and books on this subject, ref [6] and [7] for instance.

\subsection{The stabilizing effect of radiation.}

Goody showed clearly the stabilizing effect of radiative transfer on the onset of convective instability. In the static state, radiation involves a non-linear gradient of 
temperature more stable than the linear one. As we have assumed $\left(T_{0} \gg \Delta T_{0}\right.$ the temperature difference between the wall) we can linearize this set of equation. The gradient is given by [1], [2], [8] :

$$
D \Delta T(z)=M+H \cdot \cosh (\alpha z)
$$

where $D=\frac{d}{d z}, M$ and $H$ are some function of $\tilde{\tau}$ and $T_{0}$ and $\alpha^{2}=\tilde{\tau}^{2}+\frac{16 \sigma T_{0}^{3}}{k}$.

The profile of temperature gradient is shown in Fig(1) with $\alpha=10$. We can understand the stabilising effect of this profile if we remember that in the RayleighBenard problem the onset of instablity is given by a critical value of the dimensionless Rayleigh number proportional to $\beta \cdot d^{4}$, where $\beta$ is the initial temperature gradient (linear in the Rayleigh-Benard problem) and $d$ is the distance between the horizontal walls. We can see from Fig(1) that radiation decreases the thickness where the temperature gradiant is strong. It is depth the $\delta \sim \alpha^{-1}$ which is important for the onset of instability. We can expect that the critical Rayleigh number grows as $d^{3} / \delta^{3}$ with this approximate reasoning.

This simple dimensional argument must be confirmed by a more complete study of the linear stability. Goody used a variatonal principle and showed how the critical Rayleigh number increases with the optical parameter. There are some alternative methods [8] but at this level all solutions need some numerical computation.

This effect-was studied by several authors with different radiative boundary conditions, and for different approximations for the gas and light interaction. But the fixed total flux regime seems less often studied. Moreover the long wave expansion developed by Chapman and Proctor [9] could permit us to get some analytical results. 


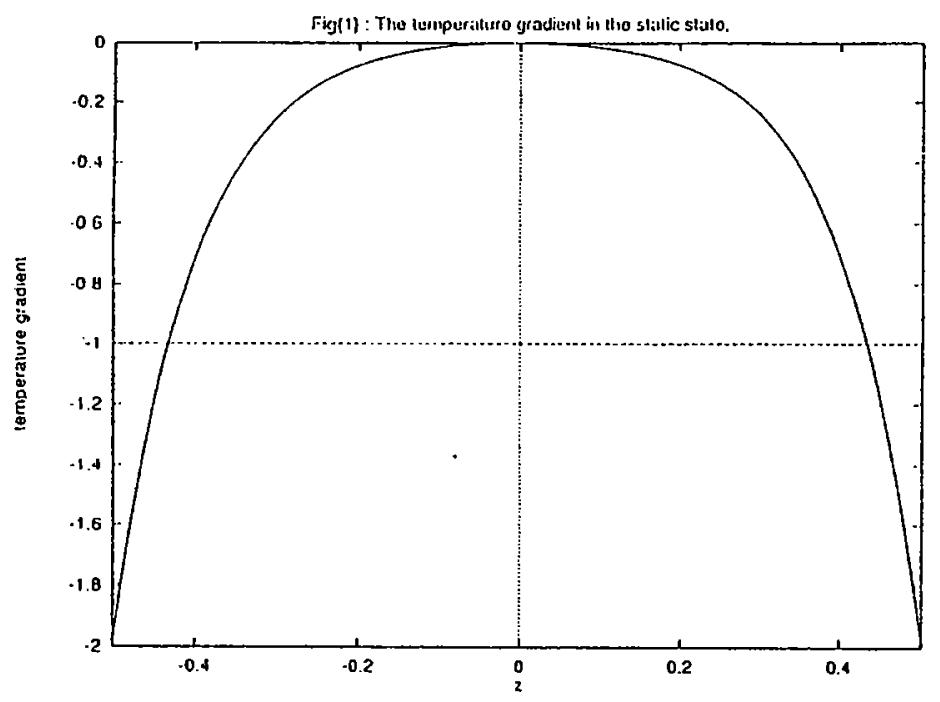

Figure 1: Static Temperature Gradient between two isothermal wall with arbitrary vertical unit.

\section{The total fixed flux case.}

\subsection{The dimensionless equations.}

As before we assume that the temperature difference is small enough to linearise our equations. We use the classical length scale $d$ and time scale $d^{2} / \kappa$, where $\kappa=k / \rho_{o} C_{v}$ is the thermal conductivity. The fixed flux boundary condition is

$$
k \partial_{z} T+\left.\frac{4 \pi}{\sqrt{3} \tilde{\tau}} \partial_{z} j\right|_{w}=-F
$$

which suggests the following scaling for temperature : $T_{d} \longleftarrow F d T_{a} / k$ and $j$ : $j_{d} \longleftarrow\left(\sqrt{3} \tilde{\tau} / 4 \pi 0 F d j_{a}\right.$ where the subscriptes $\{\mathrm{d}, \mathrm{a}\}$ means represents the dimensional and dimensionless variables respectively.

Under this scaling the equations become :

$$
\begin{gathered}
\operatorname{Pr}^{-1} D_{t} \vec{u}=\vec{\nabla} P+R a \Delta T+\vec{\nabla}^{2} \vec{u}(3.2 . a) \\
D_{t} \Delta T=\vec{\nabla}^{2}(j+\Delta T)(3.2 . b) \\
\vec{\nabla}^{2} j=\tau^{2}(j-\lambda \Delta T+\chi)(3.2 . c) \\
\vec{\nabla} \cdot \vec{u}=0(3.2 . d)
\end{gathered}
$$

where all our variables are now dimensionless. The dimensionless parameters are $R a=\left(\alpha g F d^{4}\right) /(\kappa \nu k)$, the rescaled Rayleigh number, $\operatorname{Pr}=\nu / \kappa$, the Prandtl number, $\tau=\tilde{\tau} \cdot d$ the optical length and $\lambda=\left(8 \sigma T_{o}^{3} d / \sqrt{3} \tau k\right)$ which could be interpreted 
as the rate of the heat transfer by radiation over the normal heat conductivity. The coefficient $\chi=\left(4 \pi \sigma T_{0}^{4}\right) /(\sqrt{3} \tau F)$ depends on the external parameter $F$ but is neither present in the static temperature gradient nor in the perturbation equations. Therefore this dependance is unimportant.

We must specify the boundary condition on $j$. The simplest case is the mirror. Let us first consider the case where $\left.\partial_{z} j\right|_{w}=0$. We can note that this set of equation with this boundary condition does not break the Boussinesq symmetry. Radiation has no effect in this sense but we can easly introduce some asymmetry in our boundary condition later with different $\zeta_{w^{+}}$and $\zeta_{w^{-}}$.

\subsection{The static state.}

Without fluid motion, $T$ and $j$ are a function of $z$ only. The static state is given by the two following equations :

$$
\begin{gathered}
D^{2}\left(\Delta T_{\circ}(z)+j_{\circ}(z)\right)=0(3.3 . a) \\
D^{2} j_{\circ}(z)-\tau j_{\circ}(z)=-\tau \lambda \Delta T_{\circ}(z)-\chi(3.3 . b) .
\end{gathered}
$$

With the above boundary condition we can easily obtain the temperature gradient:

$$
D \Delta T_{\circ}(z)=-\left[\frac{\tau^{2}}{\alpha^{2}}\left(\frac{\lambda \cosh (\alpha z)}{\cosh (\alpha / 2)}+1\right)\right]
$$

where $\alpha^{-2}=\tau^{-2}(1+\lambda)^{-1}$ is an important length scale of our problem as in the constant temperature case. We note that the mirror boundary conditition involves $\left.D \Delta T_{o}(z)\right|_{w}=-1$ or $\left.D \Delta T_{o}(z)\right|_{w}=-F$ with the dimensional variables. Having obtained the static backgroundstate, we can now investigate its linear stability.

\subsection{The pertubation equations.}

Squires theorem tells us that we need consider only the two dimensional case. Let us add a small pertubation to our static state. We call $\theta$ the temperature perturbation, $j$ the radiative perturbation and we introduce the two dimensional stream-function defined by $\partial_{x} \psi=w$ and $\partial_{z} \psi=-u$, where $\psi$ is the stream function and $u$ and $w$ are the two components of the velocity (horizontal and vertical respectively). We 
derive equation (3.2.a) in such a manner that we can eliminate the pressure term. We obtain

$$
\begin{gathered}
\operatorname{Pr}^{-1} D_{t} \vec{\nabla}^{2} \psi=R a \theta_{x}+\vec{\nabla}^{4} \psi \\
D_{t} \theta+D \delta T_{o}(z) \psi_{x}=\vec{\nabla}^{2}(\theta+j) \\
\vec{\nabla}^{2} j-\tau^{2} j=-\tau^{2} \lambda \theta
\end{gathered}
$$

where $D_{t}=\partial_{t}+\psi_{x} \partial_{z} \cdot-\psi_{z} \partial_{x}$. includes the non linear term.

First we neglect the non-linear term and we take :

$$
\left(\begin{array}{c}
\psi \\
\theta \\
j
\end{array}\right)=\exp (\omega t-i a x)\left(\begin{array}{c}
\Psi(z) \\
\Theta(z) \\
J(z)
\end{array}\right)
$$

where $\Psi(z), \Theta(z), J(z)$ are functions of $z$ only. $\Re(\omega)$ gives us the growth rate of $\Psi(z), \Theta(z), J(z)$ at the horizontal wave number $a$. The above equation gives :

$$
\begin{gathered}
\left(D^{2}-a^{2}\right)\left(D^{2}-a^{2}-\frac{\omega}{P r}\right) \Psi(z)-i a \Theta(z)=0 \\
\left(D^{2}-a^{2}-\omega\right) \Theta(z)+i a \Psi(z) D \Delta T_{o}(z)-\left(D^{2}-a^{2}\right) J(z)=0 \\
\left(D^{2}-a^{2}-\tau^{2}\right) J(z)+\tau^{2} \lambda \Theta(z)=0
\end{gathered}
$$

We assume that we have no overstability as in the Rayleigh-Benard case, hence $\Im(\omega)=0$. The first instable mode is given by $\Re(\omega)=0$. We must solve the above equation with $\omega=0$ to get the marginal stability curve, which gives the critical Rayleigh number for any wave number. The minimum of this curve gives us the wave number of the first unstable mode and the critical Rayleigh number. We cannot make analytical progress because of the non-linear gradient of temperature, but we can solve it numerically as a eigenvalue problem.

The results of numerical computation are given fig(2). There are many difference with the non radiative case. When the radiative transfer is zero we know from Hurle and al. [10] that for a constant flux, the most unstable wavenumber is equal to 0 because it is a minimum of the marginal stability curve. We can see from Fig(3) that 


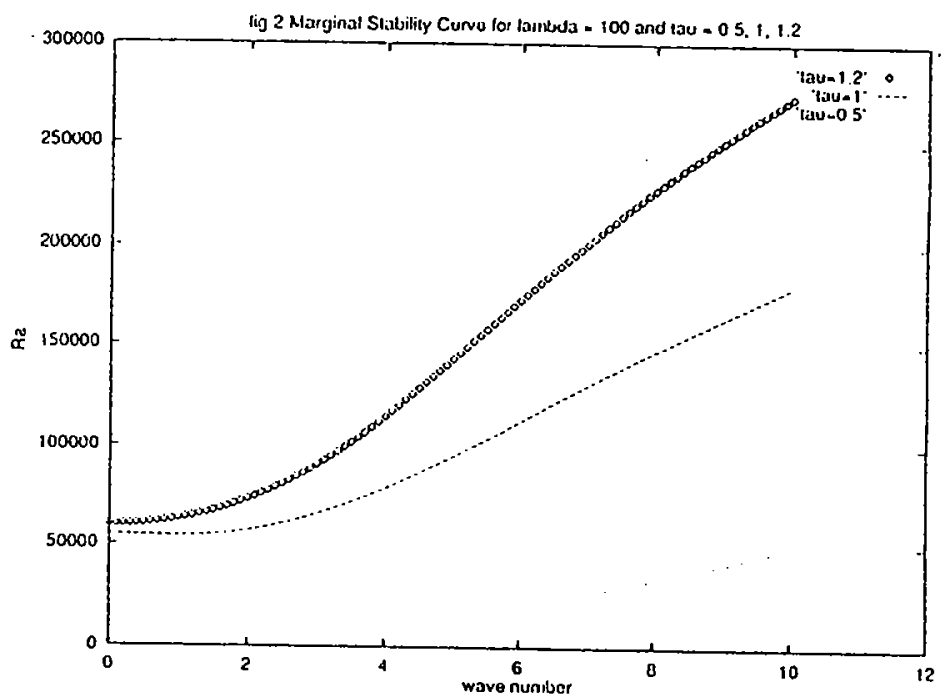

Figure 2: Marginal Stability curves for $\lambda=100$ and $\tau=1.2,1,0.5$

is not always the case with radiative transfer. There is a range of optical parameters $(\tau<1.1)$ where the marginal stability is a maximum for a zero wave number. It means that the first unstable mode has a finite wave length. This phenomenon seems to have some similarity with the case of penetrative convection with fixed flux [11]. We must do a long-wave expansion to understand those phenomena better.

\subsection{A long wave expansion.}

Let us assume that the marginal stability curve has a minimum at zero wavenumber. We must study our equation around this point in the weakly non-linear regim. We take $X=\epsilon^{-1} x$ to be the adopted length scale and following Chapman and Proctor [9] we take $T=\epsilon^{-4} t$ to be the adopted timescale where $\epsilon \sim d / l \ll 1$ is small. $L$ is the horizontal length of the cell. In the new variable (3.5) becomes :

$$
\begin{gathered}
\epsilon^{4} \partial_{T} \vec{\nabla}^{2} \psi+\epsilon \psi_{X} \vec{\nabla}^{2} \psi_{z}-\epsilon \psi_{z} \vec{\nabla}^{2} \psi_{X}=\epsilon R a \theta_{X}+\vec{\nabla}^{4} \psi \\
\epsilon^{4} \partial_{T} \theta+\epsilon \psi_{X}\left[D \Delta T(z)+\theta_{z}\right]-\epsilon \psi_{z} \theta_{X}=\vec{\nabla}^{2}(\theta+j) \\
\vec{\nabla}^{2} j-\tau^{2} j=-\tau^{2} \lambda \theta
\end{gathered}
$$

where $\vec{\nabla}^{2}=\left(\partial_{z z}^{2}+\epsilon^{2} \partial_{X X}^{2}\right)$. For the distinguished scaling which keeps a connection between $\theta$ and $\psi$ we must rescale $\psi$ by $\epsilon \phi$. We also get :

$$
\begin{gathered}
\epsilon^{4} \partial_{T} \vec{\nabla}^{2} \phi+\epsilon \phi_{X} \vec{\nabla}^{2} \phi_{z}-\epsilon^{2} \phi_{z} \vec{\nabla}^{2} \phi_{X}=R a \theta_{X}+\vec{\nabla}^{4} \phi \\
\epsilon^{4} \partial_{T} \theta+\epsilon^{2} \phi_{X}\left[D \Delta T(z)+\theta_{z}\right]-\epsilon^{2} \phi_{z} \theta_{X}=\vec{\nabla}^{2}(\theta+j) \\
\vec{\nabla}^{2} j-\tau^{2} j=-\tau^{2} \lambda \theta \quad(3.7 . c)
\end{gathered}
$$


The boundary condition for the perturbation quantity is $\left.\partial_{z}(\theta+j)\right|_{w}=0$. An integration of (3.7.b) over $z$ with this boundary condition gives the solvability condition:

$$
\epsilon^{4} \partial_{T}<\theta>+\epsilon^{2}<\phi_{X} D \Delta T(z)>+\epsilon^{2}<\phi \theta_{z}>_{X}=\epsilon^{2}<\theta+j>_{X X}
$$

where $\left\langle>=\int_{-1 / 2}^{1 / 2} \cdot d z\right.$. We expand our variables as :

$$
\left(\begin{array}{l}
\phi \\
\theta \\
j
\end{array}\right)=\left(\begin{array}{c}
\phi_{0} \\
\theta_{0} \\
j_{0}
\end{array}\right)+\epsilon^{2}\left(\begin{array}{c}
\phi_{2} \\
\theta_{2} \\
j_{2}
\end{array}\right)+\cdots
$$

Similarly we write our control parameter :

$$
R a=R a_{\circ}+\epsilon^{2} R a_{2}+\epsilon^{4} R a_{4}+\cdots
$$

where $R a_{0}, R a_{2}, \ldots$ are the first coefficients of the Taylor expansion of the marginal stability curve.

We must solve our set of equations at each order, the solvability condition at the next order gives us the required information to construct the marginal stability curve, since the sign of $R a_{2}$ show us whether $R a(0)$ is a minimum or a maximum.

\subsubsection{The first order.}

We introduce the previous expansion in (3.7)and at the leading order we obtain

$$
\begin{gathered}
\phi_{o z z z z}+R a_{o} \theta_{0 X}=0 \\
\theta_{o z z}+j_{o z z}=0 \\
j_{o z z}-\tau^{2} j_{0}=-\tau^{2} \lambda \theta_{0}
\end{gathered}
$$

We can solve the above equation with the boundary condition :

$$
\left.\phi_{o z z}\right|_{w}=\left.\phi_{o}\right|_{w}=0
$$




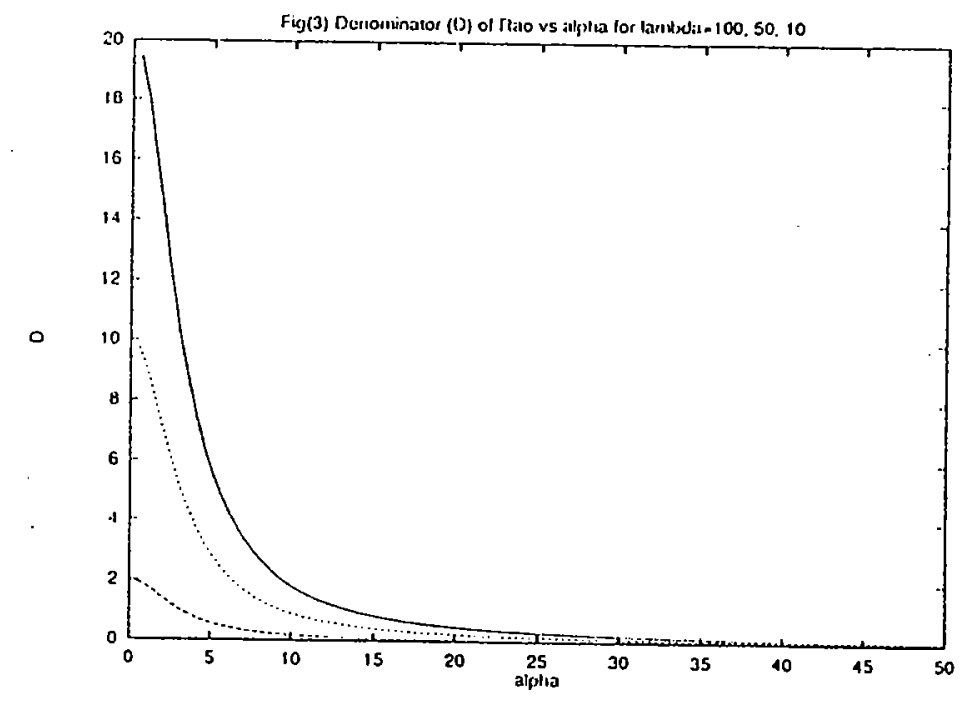

Figure 3: the denominator of $R a_{\circ}$ vs $\alpha$ for $\lambda=100,50,10$

$$
\begin{gathered}
\theta_{o z}+\left.j_{o z}\right|_{w}=0 \\
\left.j_{o z}\right|_{w}=0
\end{gathered}
$$

If we integrate equation (3.9.b) twice we obtain :

$$
\theta_{0}+j_{0}=f(X, T)
$$

where we call $f(X, T)$ the form function,

$$
j_{0}=\frac{\tau^{2} \lambda}{\alpha^{2}} f(X, T), \theta_{0}=\frac{\tau^{2}}{\alpha^{2}} f(X, T) \text { and } \phi_{0}=-\frac{R_{0} \tau^{2}}{4 ! \alpha^{2}}\left(z^{4}-\frac{3}{2} z^{2}+\frac{5}{16}\right) f_{X}(X, T) .
$$

The critical value of $R a_{0}$ is given by the solvability condition

$$
<\phi_{0 X} D \Delta T(z)>=<\theta_{0}+j_{0}>_{X X} .
$$

If we take $f_{X X}(X, T) \neq 0$ we must impose

$$
R a_{o}=\frac{\alpha^{4} 4 !}{\tau^{4}\left[\left(\frac{48}{\alpha^{5}} \tanh (\alpha / 2)-\frac{24}{\alpha^{4}}+\frac{2}{\alpha^{2}}\right) \lambda+\frac{1}{5}\right]}(3
$$

The above denominator seems always positive ( $\mathrm{cf}$ Fig(3)).

\subsubsection{The second order $O\left(\epsilon^{2}\right)$}

If we want to know the sign of $R a_{2}$ we must consider the second order where our set of equations becomes : 


$$
\begin{gathered}
\phi_{2 z z z z}+R a_{o} \theta_{2 X}=\phi_{o X} \phi_{o z z z}-\phi_{o z} \phi_{o X z z}-2 \phi_{o X X z z}-R a_{2} \theta_{o X} \\
\theta_{2 z z}+j_{2 z z}=-\phi_{o z} \theta_{o X}+\phi_{o X} D \Delta T_{o}(z)-f_{X X}(X, T) \\
j_{2 z z}-\tau^{2} j_{2}+\tau^{2} \lambda \theta_{2}=j_{o X X}
\end{gathered}
$$

and $\phi_{2}, \theta_{2}, j_{2}$ must satisfy

$$
\left.\left.\partial_{T}<\theta_{0}>+<\phi_{2 X} D \Delta T(z)>+<\phi_{0} \theta_{2 z}\right\rangle_{X}=<\theta_{2}+j_{2}\right\rangle_{X X}
$$

We must solve a differential equation involving a sixth order polynomial multiplied by some hyperbolic function. We must then integrate the given expression four times. Even if all the above functions have an analytical expression, we are unable to resolve it during this summer. However we can deduce the form of the equation given by the above solvability condition by symmetry argument. With our boundary condition we do not break any Boussinesq symmetry and the form function has the same form as the equation without radiation [9].

$$
f_{T}=-\frac{R a_{2}}{R a_{o}} f_{X X}-A(\tau, \lambda) f_{X X X X}-B(\tau, \lambda)\left(f_{X}^{3}\right)_{X}
$$

We must find the $f(X, T)=f_{0} \exp (i K X)$ on the marginality curve. In this case

$$
R a_{2}=R a_{\circ} A(\tau, \lambda) K^{2}
$$

therefore $A(\tau, \lambda)$ gives the sign of $R a_{2}$. If we want to know the growth rate of the most unstable mode we must take $f(X, T)=f_{0} \exp (\Omega T+i K X)$ and the equation (3.13) when linearized gives

$$
\Omega=\frac{R a_{2}}{R a_{o}} K^{2}-A(\tau, \lambda) K^{4}
$$

The maximum of this curve $K_{1 \max }=\left(R a_{2} / 2 A R a_{0}\right)^{1 / 2}$ gives us the most unstable mode in the linear regime. However, this maximum does not give us the preferred mode of the horizontal structure in the nonlinear regime. Indeed equation (3.13) defines a Lyapunov functional. If we multiply it by $f_{T}$ and integrate we get

$$
-\int\left(f_{T}\right)^{2} d X=\frac{d V[f]}{d t}
$$




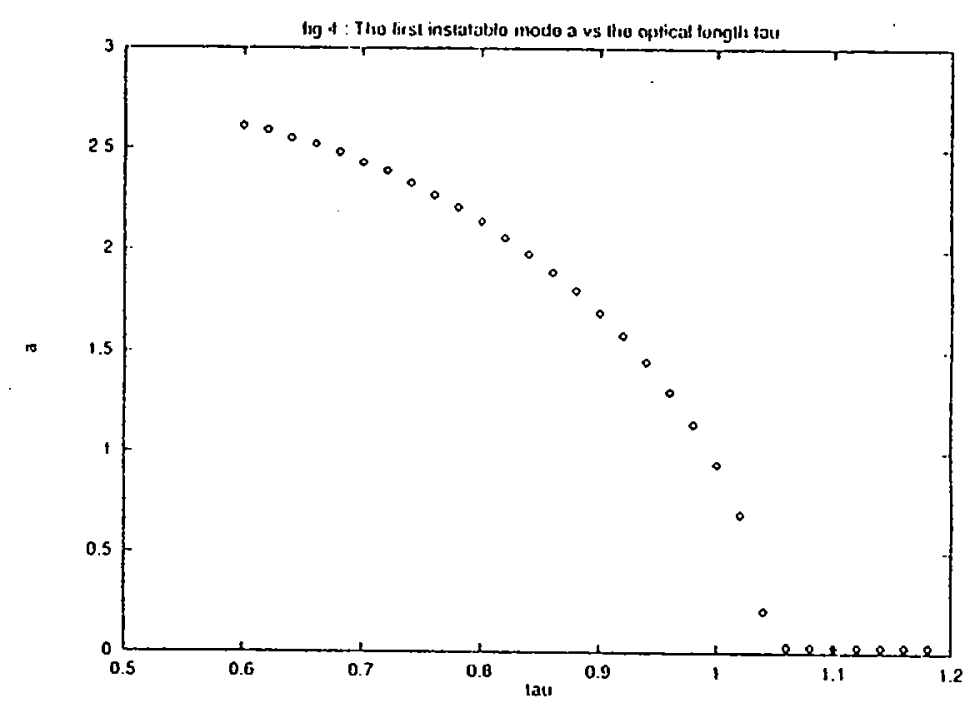

Figure 4: the first unstable mode of the marginality curve vs $\tau$ for $\lambda=100$

where $V[f]$ is the Lyapunov functional which is a decreasing function of time. Chapman and Proctor [9] used this functional to prove that all growing modes are unstable when competing with a mode of larger wavenumber. The only stable mode is the one with the largest wavenumber which can fit the container.

There is some value of $\left(\tau^{\dagger}, \lambda^{\dagger}\right)$ where $R a_{2}=0$. We must rescale $\theta$ and $j$ by $\epsilon$ and $\psi$ by $\epsilon^{2}$. The form equation becomes

$$
f_{T}=-\frac{R a_{4}}{R a_{o}} f_{X X}+C(\tau, \lambda) f_{X X X X X X}-D(\tau, \lambda)\left(f_{X}^{3}\right)_{X}
$$

where $C(\tau, \lambda)$ gives the sign of $R a_{4}$. We expect $C(\tau, \lambda)>0$ for $A(\tau, \lambda) \leq 0$. The maximum growth rate is at wavenumber $K_{2 \max }=\left(R a_{2} / 3 A R a_{o}\right)^{1 / 4}$. However we can also derive a Lyapunov functional from (3.16) and the most unstable mode cannot be deduced from a simple linear analysis.

When $R a_{2}<0$ we cannot do a long wave expansion except when the minimum is at a distance $\epsilon$ from zero. Fig(4) shows that the wavenumber which gives the minimum of the marginal stability curve grows quickly for $\tau<1.1$. The long wave expansion becomes inappropriate very fast.

It could be very interesting to know the value of the coefficients. However, in the limited time available I was only able to calculate one of them. We anticipate that the computation is more tractable if we assume that

$$
D \Delta T_{\mathrm{o}}(z) \sim\left[-\frac{\tau^{2}}{\alpha^{2}}\left(\frac{\lambda\left(1+\alpha^{2} z^{2}\right)}{1+\alpha^{2} / 4}+1\right)\right] .
$$

However, even with this simplification this computation is still difficult. 
To summarize our result we have : For $\alpha>\alpha^{\dagger}$ we have $R a_{2}>0$ and the marginal stability curve grows continuously. We can make a long-wave expansion and we find the same form equation (3.13) than for the non-radiative case. Radiation only increases the instability onset. For $\alpha=\alpha^{\dagger}$ we have $R a_{2}=0$. In this case we need to rescale our variables to find an equation for the form function. For $0<\alpha<\alpha^{\dagger}$ $R_{2}$ is negative and the long wave expansion becomes inappropriate. For $\alpha=0$ we find the classical Rayleigh -Bénard problem where $R a(0)=5$ ! .

\section{Conclusions and Perspectives.}

The work which I completed during the summer showed that convection with radiative transfer has some extremely interesting unexplored behaviour. I have shown that Rayleigh-Bénard problem is a singular limit of the above model. This will be explored further in subsequent work.

During this study I was examining the limit of very small interaction and very hot temperature. The radiation increases only the thermal conductivity in this case. We can derive a set of equation for the Boussinesq approximation for a zero Prandtl number limit. But as noted by Malkus [3] the convection motion needs two dimensinless parameters to describe it. A better approach may be to derive a new set of equations from the Navier-Stokes equations.

If we have asymmetric boundary conditions we must add a term proportional to $\left(f_{X}^{2}\right)_{X X}$ in equation (3.13). If consider the more realistic case where the optical thickness depends on temperature we can expect a term proportional to $\left(f^{2}\right)_{X X}$ as shown by Depassier and Spiegel [12]. The form equation becomes :

$f_{T}=-\frac{R a_{2}}{R a_{o}} f_{X X}-A(\tau, \lambda) f_{X X X X}-B(\tau, \lambda)\left(f_{X}^{3}\right)_{X}+H(\tau, \lambda)\left(f_{X}^{2}\right)_{X X}+K(\tau, \lambda)\left(f^{2}\right)_{X X}$.

It could be interesting to compute the solutions as function of our optical paramaters.

Another interesting problem is to consider the radiative influence on the spatial distribution of different sorts of gases. We could study the convection with radiation and two gases with very different absorption coefficients. Coupled with Soret or Du- 
four effects, this problem will become very complex but certainly very interesting. Also of interest is the reintroduction of the time dependence in the light intensity equation (2.2), relevant to very hot astrophysical objects.

\section{Acknowledgements}

I would like to thank Neil Balmforth for all his help during this summer. Thanks to Steve Meacham, Keith Julien and Ed Spiegel for their good advice. Thank to all the G.F.D fellows especially Jim Stephens, Jean-Luc Thiffeault and Jon Wylie who helped me with my report. This report has taken advantage of all the questions raised during my talk. Lastly, I would thank Stephan Fauve who sent me here.

\section{References}

[1] Goody, R.M 1956, JFM, vol 1

[2] Gille, J. \& Goody, R.M 1964, JFM, vol 20, part 1

[3] Malkus, W.V.R 1964, G.F.D summer school Lecture notes

[4] Simon, R. Journal Quant. Spectrosc. Radiat. Trans, vol 3

[5] Christophorudis, S. \& Davis, S.H 1970, Phys. of Fluids, 13

[6] Chandrasekhar, S. 1960, radiative transfer, Dover

[7] Krook, M. 1965, M. ApJ, 122

[8] Apaci, V.S \& Gozüm, D. 1974, Physic of fluids, 16

[9] Chapman, C.J and Proctor, M.R.E 1980, JFM, vol 1, part 4

[10] Hurle, D.T.J Jackeman, E. \& Pike E.R. 1967, Proc. Royl. Soc. A, vol 296

[11] Robert, A.J. 1981, G.F.D summer school Fellow Report

[12] Depassier, M.C \& Spiegel, E.A. 1982, Geophys. astrophys. Fluid Dynamics, vol 21 


\title{
A Few Steps Toward Staircases
}

\author{
Francesco Paparella
}

\section{The staircase problem}

One of the most fascinating aspects of double diffusion is the formation of layered structures. In such sructures the vertical profiles of the two diffusing quantities have shapes that look like staircases (figures 1,2 in the first Ray Schmitt lecture, this volume). What is even more interesting is that staircases are observed not only in the laboratories, but also in the sea. Furthermore, the staircases influence the rates of vertical mixing of heat and salt, so that those elusive structures may play a role in the overall thermohaline dynamics of the seas.

Although there is an abundance of experimental and observational data, at this time no theoretical model seems to be able to reproduce (or even to mimic) the process of the formation of a multi-layered structure from initial constant gradient conditions.

One of the big problems that one must face is the extreme complexity of the flow along the horizontal direction. In fact, convection (with high Rayleigh number) develops inside the layers and, when the system is driven by the fall of the less diffusive substance, the interface itself is characterized by salt fingers.

The idea that underlies this work is that to model the observed vertical profiles of temperature and salt it should be possible to get rid of the complications of the horizontal direction and cast a set of equations that depend only on the height and the time. I have been comforted by the fact that such an approach has already been attempted in the study of the Rayleigh-Bénard convection [5]. Single-mode equations similar to those presented here have been studied also by Gough and Toomre [6], but they didn't take in to account the possibility of a horizontal shear.

\section{The equations}

My primitive equations are the Navier-Stokes equations in the Boussinesq approximations, coupled with advection-diffusion equations for the temperature and the salinity field. I decided to work in two spatial dimensions because this permits a description of the flow velocities through a single scalar field (namely the streamfunction $\psi$ ) and because this simplifies the algebraic process of averaging out the horizontal dependency. The non-dimensional equations read: 


$$
\begin{aligned}
\partial_{t} \nabla^{2} \psi-J\left(\psi, \nabla^{2} \psi\right) & =-\sigma R_{T} \partial_{x} T+\sigma R_{S} \partial_{x} S+\sigma \nabla^{4} \psi \\
\partial_{t} T-J(\psi, T) & =\nabla^{2} T \\
\partial_{t} S-J(\psi, S) & =\tau \nabla^{2} S
\end{aligned}
$$

where $R_{T}$ is the temperature Rayleigh number, $R_{S}$ is the salinity Rayleigh number, $J($, is the Jacobian operator, $T$ is temperature, and $S$ is salinity (here I used the same nondimensionalization as in [3]). The velocities can be recovered from the streamfunction: $u=\partial_{z} \psi ; \boldsymbol{w}=-\partial_{x} \psi$, hence $\nabla \cdot \mathbf{u}=0$.

In order to write horizontally averaged equations I split the three variables $\psi, T$ and $S$ as:

$$
\begin{array}{r}
\psi=\bar{\psi}(z, t)+\psi^{\prime}(x, z, t) \\
T=\bar{T}(z, t)+T^{\prime}(x, z, t) \\
S=\bar{S}(z, t)+S^{\prime}(x, z, t)
\end{array}
$$

where the overbar denotes horizontal average and the variables with a prime are the fluctuations around this average. Plugging this definition into the primitive equations and averaging over $x$ one gets the equations for the averaged variables $\bar{\psi}, \bar{T}$ and $\bar{S}$ :

$$
\begin{aligned}
\partial_{t} \partial_{z z} \bar{\psi} & =\partial_{z}\left(\overline{\partial_{x} \psi^{\prime} \nabla^{2} \psi^{\prime}}+\sigma \partial_{z z z} \bar{\psi}\right) \\
\partial_{t} \bar{T} & =\partial_{z}\left(\overline{T^{\prime} \partial_{x} \psi^{\prime}}+\partial_{z} \bar{T}\right) \\
\partial_{t} \bar{S} & =\partial_{z}\left(\overline{S^{\prime} \partial_{x} \psi^{\prime}}+\tau \partial_{z} \bar{S}\right)
\end{aligned}
$$

Those equations still depend on the variables with a prime. To close the problem one has to take into account the equations for the fluctuations:

$$
\begin{aligned}
\partial_{\varepsilon} \nabla^{2} \psi^{\prime}-J\left(\psi^{\prime}, \nabla^{2} \psi^{\prime}\right)+\overline{J\left(\psi^{\prime}, \nabla^{2} \psi^{\prime}\right)}-\partial_{x} \psi^{\prime} \partial_{z z z} \bar{\psi}+\partial_{z} \bar{\psi} \partial_{x} \nabla^{2} \psi^{\prime} & = \\
-\sigma R_{T} \partial_{x} T^{\prime}+\sigma R_{S} \partial S^{\prime}+\sigma \nabla^{4} \psi^{\prime} & \\
\partial_{t} T^{\prime}-J\left(\psi^{\prime}, T^{\prime}\right)+\partial_{z} \overline{\left(T \partial_{x} \psi^{\prime}\right)}-\partial_{x} \psi^{\prime} \partial_{z} \bar{T}+\partial_{z} \bar{\psi} \partial_{x} T^{\prime} & =\nabla^{2} T^{\prime} \\
\partial_{t} S^{\prime}-J\left(\psi^{\prime}, S^{\prime}\right)+\partial_{z} \overline{\left(S \partial_{x} \psi^{\prime}\right)}-\partial_{x} \psi^{\prime} \partial_{z} \bar{S}+\partial_{z} \bar{\psi} \partial_{x} S^{\prime} & =\tau \nabla^{2} S^{\prime}
\end{aligned}
$$

Note that the quantity $U(z, t)=\partial_{z} \bar{\psi}$ represents a horizontal velocity field. In most past works $([5],[6],[8])$ it has been put to zero. Then more recently there has been experimentally observed the spontaneous generation of a horizontal shearing velocity in Rayleigh-Bénard convection $([2],[9])$. Furthermore, it is commonly believed that a horizontal shear is the main factor limiting the growth of salt fingers in the sea (see the second of the Eric Kunze's lectures, this volume). Hence, $U$ will be a significant element in my calculations.

The next step is to expand the horizontal structure of those equations and to drastically truncate the expansion after an arbitrarily chosen number of terms. This is the very idea of a Galerkin truncation, with the only difference that the dependence of one of the spatial coordinates (the height $z$ ) will be retained here. 
tion:

It is convenient to expand the vertical velocity fluctuations, rather than the streamfunc-

$$
w^{\prime}=\sum_{k} f_{k} W_{k}
$$

From continuity it follows that:

$$
\begin{gathered}
u^{\prime}=\sum_{k} a_{k}^{-2} \partial_{x} W_{k} \partial_{x} f_{k} \\
\psi^{\prime}=\sum_{k} a_{k}^{-2} W_{k} \partial_{x} f_{k}
\end{gathered}
$$

Temperature and salinity fluctuations are expanded in a analogous way:

$$
\begin{aligned}
T^{\prime} & =\sum_{k} f_{k} \Theta_{k} \\
S^{\prime} & =\sum_{k} f_{k} \Phi_{k}
\end{aligned}
$$

The functions $f_{k}$ used in the expansion are the planform functions of the linear theory, that is, the solutions of the eigenvalue problem for the Laplacian operator. Because there is only one horizontal coordinate, the eigenvalue problem is simply:

$$
\partial_{z z} f_{k}=-a_{k}^{2} f_{k}
$$

whose solutions are sines and cosines. So, in two dimensions this expansion turns out to be a Fourier transform. $W_{k}(z, t), \Theta_{k}(z, t), \Phi_{k}(z, t)$ are, respectively, the vertical velocity, the temperature and the salinity fluctuations associated with the horizontal scale $1 / a_{k}$.

Plugging those expansions into the equations (3) for the averaged quantities, and into the equations (4) for the fluctuations, after a lot of algebra and some care in multiplying the (4) by $f_{k}$ and averaging over $x$, one gets a close a set of (an infinite number of) equations that involve $\bar{\psi}, \bar{T}, \bar{S}, W_{k}, \Theta_{k}$, and $\Phi_{k}$.

\section{Single mode equations for double diffusion}

All this equation crunching was needed for two pourposes: hide the horizontal dependence into the $f_{k}$, and split the equations in modes associated with a spatial scale. The simplification consists in assuming that the dynamics of the flow will be dominated by the contribution of just a few of those modes. For example, in the Rayleigh-Bénard convection, even at very high Rayleigh numbers, the overall flow circulates with patterns having roughly the same spatial scale of the convection cells that appears just after the first instability.

For the fingering double-diffusive convection it seems that at least two modes should be retained: one associated with the big scale of the convection inside the layers, the other associated with the smaller scale of the salt fingers that develops between the layers. 
However, the simplest thing to do is to retain just one spatial scale. Although this won't be a complete model with fingering, such a crude truncation is still expected to show at least the early stages of the layering process, what could be called a layering bifurcation.

The single mode equations are:

$$
\begin{aligned}
\partial_{t} U & =\partial_{z}\left(\sigma \partial_{z} U+\frac{1}{\alpha} W^{+} \partial_{z} W^{-}-\frac{1}{\alpha} W^{-} \partial_{z} W^{+}\right) \\
\partial_{t} \bar{T} & =\partial_{z}\left(\partial_{z} \bar{T}-W^{+} \Theta^{+}-W^{-} \Theta^{-}\right) \\
\partial_{t} \bar{S} & =\partial_{z}\left(\tau \partial_{z} \bar{S}-W^{+} \Phi^{+}-W^{-} \Phi^{-}\right) \\
\partial_{t} \Theta^{+} & =\mathcal{D} \Theta^{+}+\alpha U \Theta^{-}-W^{+} \partial_{z} \bar{T} \\
\partial_{t} \Theta^{-} & =\mathcal{D} \Theta^{-}-\alpha U \Theta^{+}-W^{-} \partial_{z} \bar{T} \\
\partial_{t} \Phi^{+} & =\tau \mathcal{D} \Phi^{+}+\alpha U \Phi^{-}-W^{+} \partial_{z} \bar{S} \\
\partial_{t} \Phi^{-} & =\tau \mathcal{D} \Phi^{-}-\alpha U \Phi^{+}-W^{-} \partial_{z} \bar{S} \\
\partial_{t} \mathcal{D} W^{+} & =\sigma \mathcal{D}^{2} W^{+}-\sigma \alpha^{2}\left(R_{T} \Theta^{+}-R_{S} \Phi^{+}\right)-\alpha W^{-} \partial_{z z} U+\alpha U \mathcal{D} W^{-} \\
\partial_{t} \mathcal{D} W^{-} & =\sigma \mathcal{D}^{2} W^{-}-\sigma \alpha^{2}\left(R_{T} \Theta^{-}-R_{S} \Phi^{-}\right)+\alpha W^{+} \partial_{z z} U-\alpha U \mathcal{D} W^{+}
\end{aligned}
$$

Here $W^{+}, \Theta^{+}, \Phi^{+}$are the coefficients of $\sin (\alpha x)$, while $W^{-}, \Theta^{-}, \Phi^{-}$are the coefficients of $\cos (\alpha x)$. The operator $\mathcal{D}$ that appears in the diffusion terms is defined as $\mathcal{D}=\left(\partial_{z z}-\alpha^{2}\right)$ and $1 / \alpha$ is the only horizontal scale retained in the truncation. It is convenient to split the average temperature and salinity into a linear part, that is the static, conductive solution, plus a term that becomes non zero when the convection sets in:

$$
\begin{aligned}
& \bar{T}=z+\tilde{T} \\
& \bar{S}=z+\tilde{S}
\end{aligned}
$$

The choice of the sign of the linear part determines the type of stratification that one is imposing on the problem. The stratification $(+z,+z)$ has a stabilizing temperature and a destabilizing salinity (fingering regime). The stratification $(-z,-z)$ has a destabilizing temperature and a stabilizing salinity (diffusive regime). The two other possibilities, namely $(+z,-z)$ and $(-z,+z)$ correspond to a completely stable and a completely unstable stratification.

In this work I assume that the fluid is confined between two horizontal slabs having fixed temperature and salinity, and I will use stress free boundary conditions. This is very convenient in writing a spectral code to solve numerically the single-mode equations (9) because those boundary conditions are fullfilled by a discrete sine transform along the $z$ coordinate for the variables $\tilde{T}, \tilde{S}, W^{ \pm}, \Theta^{ \pm}, \Phi^{ \pm}$, and a discrete cosine transform for $U$.

Following [1] I will also assume that the non-dimensional slab height and the nondimensional temperature and salinity differences are all equal to $\pi$, instead of the usual value of 1 . This allows me to get rid of a lot of annoying $\pi s$ in the code, but it also means that all the Rayleigh numbers that I will mention must be multiplied by $\pi^{4}$ in order to get the usual values. 
As the linear theory shows that the first unstable mode has a horizontal scale comparable to the layer thickness, I will choose to set $\alpha=1 / \sqrt{2}$, that is the value of the most unstable wavelength in the Rayleigh-Bénard convection.

\section{First results}

As a way to test the calculations, I checked that it was possible to recover from the singlemode equations (9) two previous results. I do a two-mode Galerkin truncation along the vertical direction assuming that:

$$
\begin{aligned}
& \psi=A \sin (\alpha x) \sin (z)+B \sin (z)+C \cos (\alpha x) \sin (2 z) \\
& T=D \cos (\alpha x) \sin (z)+E \sin (2 z)+F \sin (\alpha x) \sin (2 z)-z \\
& S=G \cos (\alpha x) \sin (z)+H \sin (2 z)+I \sin (\alpha x) \sin (2 z)-z
\end{aligned}
$$

where $A$ through $I$ are amplitudes that depend only on time. In particular, the terms $B \sin (z), E \sin (2 z)-z$ and $H \sin (2 z)-z$ represent, respectively, the horizontal velocity $U$, the horizontally averaged temperature $\bar{T}$ and salinity $\bar{S}$. The stratification is that of the diffusive case. Plugging the expansion (11) into the Boussinesq equations (1) or into the single-mode equations (9), one gets the same set of 9 ordinary differential equations (O.D.E.s):

$$
\begin{aligned}
\dot{A} & =-\sigma\left(1+\alpha^{2}\right) A-\frac{\sigma \alpha}{1+\alpha^{2}}\left(R_{T} D-R_{S} G\right)+\frac{\alpha}{2}\left(\frac{3+\alpha^{2}}{1+\alpha^{2}}\right) B C \\
\dot{B} & =-\sigma B-\frac{3}{4} \alpha A C \\
\dot{C} & =-\sigma\left(4+\alpha^{2}\right) C+\frac{\sigma \alpha}{4+\alpha^{2}}\left(R_{T} F-R_{S} I\right)-\frac{\alpha^{3}}{2\left(4+\alpha^{2}\right)} A B \\
\dot{D} & =-\left(1+\alpha^{2}\right) D-\alpha A-\alpha A E-\frac{\alpha}{2} B F \\
\dot{E} & =-4 E+\frac{\alpha}{2} A D \\
\dot{F} & =-\left(4+\alpha^{2}\right) F+\alpha C+\frac{\alpha}{2} B D \\
\dot{G} & =-\tau\left(1+\alpha^{2}\right) G-\alpha A-\alpha A H-\frac{\alpha}{2} B I \\
\dot{H} & =-4 \tau H+\frac{\alpha}{2} A G \\
\dot{I} & =-\tau\left(4+\alpha^{2}\right) I+\alpha C+\frac{\alpha}{2} B G
\end{aligned}
$$

Switching off the salinity variables $G, H$ and $I$ one recovers the 6 O.D.E.s that Howard and Krishnamurti used to mimic the onset of the shear instability in Rayleigh-Bénard convection [1]. 
Switching off the shear and the possibility of having tilted convective cells (that means the variables $B, C, F, I)$ one recovers the 5 O.D.E.s used by Veronis [3] to study the overstability in double diffusive convection.

Finally, on leaving only the variables $A, D, E$, one finds the three equations in which Lorenz [4] first found deterministic chaos.

An illustrative example of the behaviour of these 9 O.D.E.s is figure 1 , showing the average temperature amplitude and the shear amplitude of the whole system against the same variables of the Howard and Krishnamurti model. Here the thermal Rayleigh number is $R_{T}=200$, the salinity Rayleigh number is $R_{S}=100$, the Prandtl number is $\sigma=10$ and the Lewis number is $\tau=1 / 3$. Because of the highly stabilizing effect of the salt, the temperature amplitude falls on a steady value and the shear is absent (the small initial perturbation is damped out very soon), while in the Howard and Krishnamurti model there is a fast time dependence.

Lowering the salinity Rayleigh number to $R_{S}=50$ (figure 2) the temperature gets a time dependence in the 9 O.D.E.s too, but the shear is still absent.

Finally, when the salinity Rayleigh number is as low as $R_{S}=15$ one observes a strong, time dependent shear also in the 9 O.D.E.s (figure 3).

\section{Rayleigh-Bénard convection}

I want now to explore the capabilities of a single-mode truncation with $z$ dependence to describe the Rayleigh-Bénard convection after the onset of a large scale shearing velocity. One expects that with the $z$ dependence retained, the description of the vertical heat transport should be qualitatively improved.

Early works [7],[8] shows that the Nusselt number $N u$ (which is a non-dimensional measure of the vertical heat flow) is related to the Rayleigh number by the following power law:

$$
N u \propto R_{T}^{1 / 3}
$$

A recent experimental work [9] shows that for very high Rayleigh numbers the scaling of the Nusselt number is:

$$
N u \propto R_{T}^{2 / 7}
$$

This change of the exponent value has been attributed to the effect of the shearing velocity $\mathrm{U}$ [9].

I numerically integrated the equations 9 for the case of Rayleigh-Bénard convection (that means without the salinity variables and with the stratification $\bar{T}=-z+\tilde{T}$ ). The result is presented in figure 4, where is plotted the Nusselt number versus the Rayleigh number. The monotonically ascending branch corresponds to an integration in which the variables $U, W^{-}$ and $\Theta^{-}$have been switched off. This is the case of the single-mode equations presented in [5] who are known as to have steady solutions that show the $1 / 3$ scaling (unpublished result by L. Howard). 
As the shear sets in the Nusselt number drops. The descending branch in figure 4 corresponds to steady state solutions with a nonzero $U$. With further increase of the Rayleigh number, the solutions become time-dependent and the Nusselt number rises with $R_{T}$. At approximately $R_{T}=225$ the direction of $U$ begins to reverse with time. This marks a steep ascending branch in the graph that ends at approximately $R_{T}=700$. After this value the direction of the shearing velocity doesn't reverse anymore and the rise of the Nusselt number with the Rayleigh number is milder.

I will not state anything about the asimptotic limit of $N u$ for large values of $R_{T}$ because I haven't been able to explore a range of Rayleigh numbers high enough. In fact, as $R_{T}$ increases, even smaller time steps and higher resolutions are needed in order to carry out a meaningful numerical integration, because of faster time dependence of the solutions and of sharper gradients that develops inside the boundary layers.

However, it is reasonable that, to recover the $2 / 7$ scaling law, it will be necessary to include smaller spatial scales in the truncation. These scales could play an important role describing the interaction between the large scale shear and the convective cells.

\section{The overturning case}

I obtained the first numerical result for the full single-mode system (9) with the following parameters: $\sigma=10, \alpha=1 / \sqrt{2}, R_{T}=10, R_{S}=333, \tau=1 / 50$. The imposed stratification is that of the fingering case.

With that choice of parameters the system is in a statically unstable situation: the water near the top boundary is so salty that its density is greater than that of the fresher, colder water near the bottom layer. So, the gravity doesn't need any help from the thermal diffusion to set the beginning of the convection.

Even so, the system is still a double-component one, and an inspection at the behaviour of the salinity $\bar{S}$ reveals interesting features (figure 5). In the middle of the slab an instability develops that leads to the formation of a sharp gradient that splits in two wave-like disturbancies, one travelling upwards, the other donwards, until they are absorbed by the boundary layers. This process repeats over and over, but with these parameters its time dependence is irregular.

With continous lowering of the salinity Rayleigh number and the Lewis number along the line $R_{S} \cdot \tau=6 . \overline{6}$ the system become regular, showing a sharp gradient that forms and splits periodically in time.

With a still lower Rayleigh and Lewis number the solutions become steady, without showing any layering, at approximately $R_{S}=107$ and $\tau=1 / 16$.

I found numerical evidence that this is a subcritical bifurcation by raising again the two numbers, and finding steady solutions up to $R_{S}=127$ and $\tau=1 / 19$. 


\section{Layering!}

Adjusting the two Rayleigh numbers so as to produce a statically stable situation (that is in a true fingering regime) and keeping the same Lewis number, I didn't find any layering. However, for $R_{T}=1000, R_{S}=666, \tau=1 / 3 \mathrm{I}$ find the results of figure $6 \mathrm{a}$, where two layers separated by a high gradient interface are clearly visible in the salinity profile. In a few nondimensional time units, the numerical solution evolves from a small sinusoidal perturbation of the initially linear stratification to a steady solution corresponding to the shown profiles. The convection that develops in that layer also is able to excite a shear. In figure $6 \mathrm{~b}$ the profile of the horizontal velocity $U$ is shown.

A second run, with $R_{T}=5000, R_{S}=3333, \tau=1 / 3$, showed three layers separated by two interfaces. The temperature, salinity and horizontal velocity profiles are shown in figure 7. Note the much stronger shear than in the previous case.

\section{Future work}

A modal expansion with amplitudes that depend on both $z$ and $t$ seems to be a useful tool in the study of convection. Even with the severest truncation (only one horizontal scale) it reproduces the behaviour of the Rayleigh-Bénard convection at low Rayleigh numbers and

gives a realistic scenario for higher Rayleigh numbers, provided that the horizontal shearing velocity is not neglected.

Future work is needed to asses the asymptotic relationship of the Nusselt number with the Rayleigh number for the single-mode truncation.

More puzzling are the results for the double-diffusive convection. A single-mode truncation is quite likely not enough to reproduce laboratory or in-situ observations. Nevertheless, a set of equations for a single horizontal scale should be considered as a basic building block for a physical decription of the flow, until the nonlinear interaction between scales is mild and the consequent creation of new ones is negligible. For this reason, future work will focus on the study of the equations (9) varying the horizontal scale $\alpha$ and the Lewis number $\tau$. Also, the role of the shear needs to be further investigated. A run in which the variable $U$ was switched off showed a milder interface gradient than the corresponding run with the full set of variables.

Finally, I am working on a two-scale version of the spectral code that I hope will be able to simulate a much more realistic fingering convection.

\section{Acknowledgements}

I wish to thank Mike Shelley, who gave to me an initiation to the art of programming spectral codes; Lou Howard, George Veronis, Paola Cessi, Barry Ruddick, Bill Young, Nell Balmforth, Steve Meacham, Melvin Stern, Raffaele Ferrari for many enlightening discussions; 
Steve Meacham and Jean-Luc Thiffeault who opened to me accounts on Milou and Fido; and all the fellows for the wonderful Summer.

But most of all I have to thank Ed Spiegel that has been to me as good a guide through the unknowns of the Physics as Virgilius was for Dante through the dangers of the Inferno:

Lo duca e io per quel cammino ascoso

intrammo a ritornar nel chiaro mondo;

e sanza cura aver d'alcun riposo,

salimmo sù, el primo e io secondo, tanto ch' $i$ ' vidi de le cose belle

che porta l ciel, per un pertugio tondo.

\section{References}

[1] Howard, L. N. and R. Krishnamurti, 1986. Large-scale flow in turbulent convection: a matematical model. J. Fluid Mech. 70385

[2] Krishnamurti, R. and L. N. Howard, 1981. Large-scale flow in turbulent convection. Proc. Natl. Acad. Sci. USA 781981

[3] Veronis, G., 1965. On finite amplitude instability in thermohaline convection. J. Mar. Res. 231

[4] Lorenz, E. N., 1963. Deterministic nonperiodic flow. J. Atmos. Sci. 20130

[5] Gough, D. O., E. A. Spiegel and J. Toomre, 1975. Modal equations for cellular convection. J. Fluid Mech. 68695

[6] Gough, D. O. and J. Toomre, 1982. Single-mode theory of diffusive layers in thermohaline convection. J. Fluid Mech. 12575

[7] Malkus, W. V. R. 1954. Discrete transitions in turbulent convection. Proc. R. Soc. Lond. A225 185

[8] Kraichnan, R. H., 1962. Turbulent thermal convection at arbitrary Prandtl number. Phys. Fluids 51374

[9] Castaing, C. et al., 1989. Scaling of hard thermal turbulence in Rayleigh-Bénard convection. J. Fluid Mech. 2041 

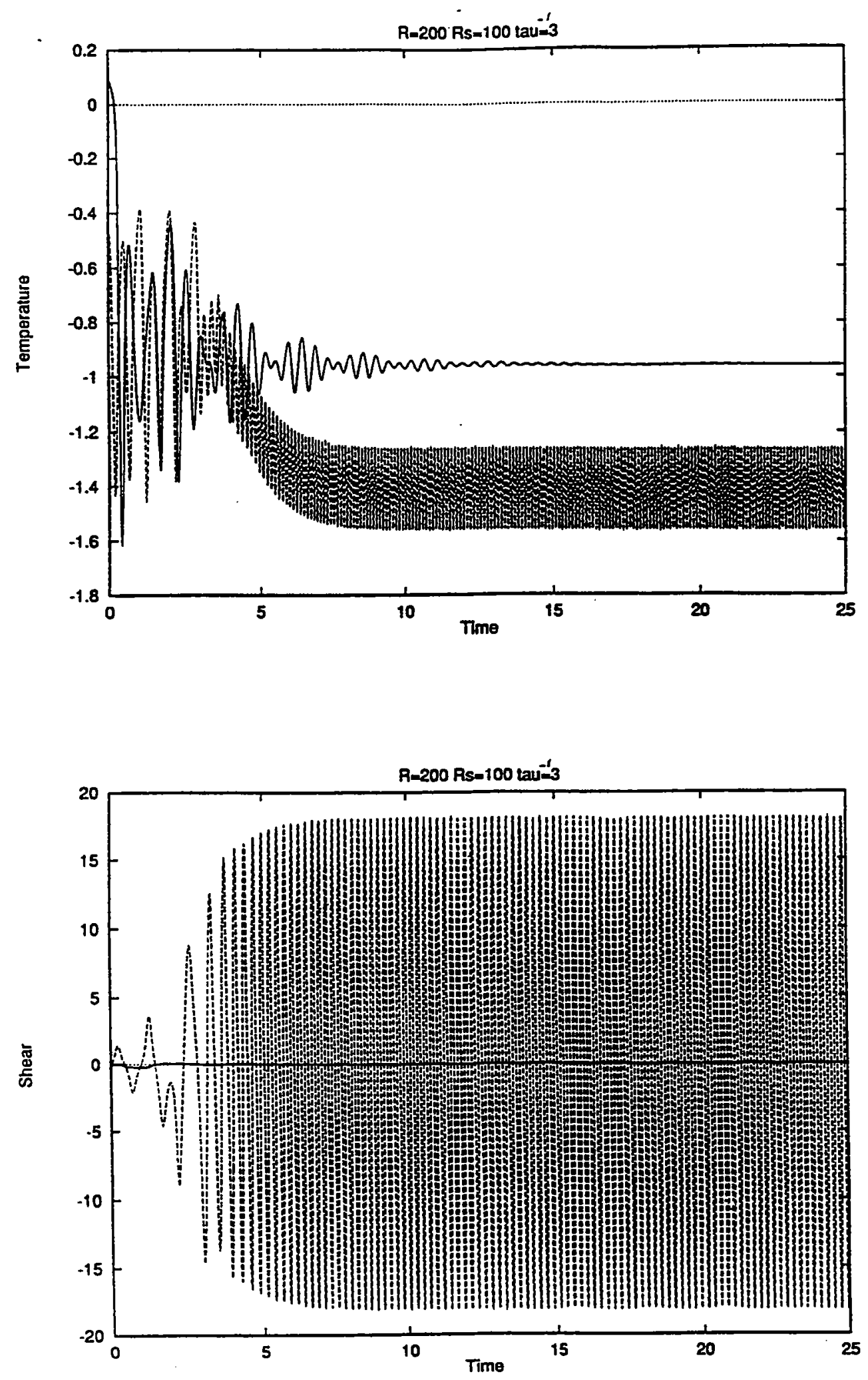

Figure 1. Average temperature and horizontal velocity amplitudes (variables $E$ and $B$ ) of the 9 O.D.E.s for double-diffusive convection (solid line) versus the same variables of the 6 O.D.E.s by Howard and Krishnamurty for Rayleigh-Bénard convection (dashed lines). 

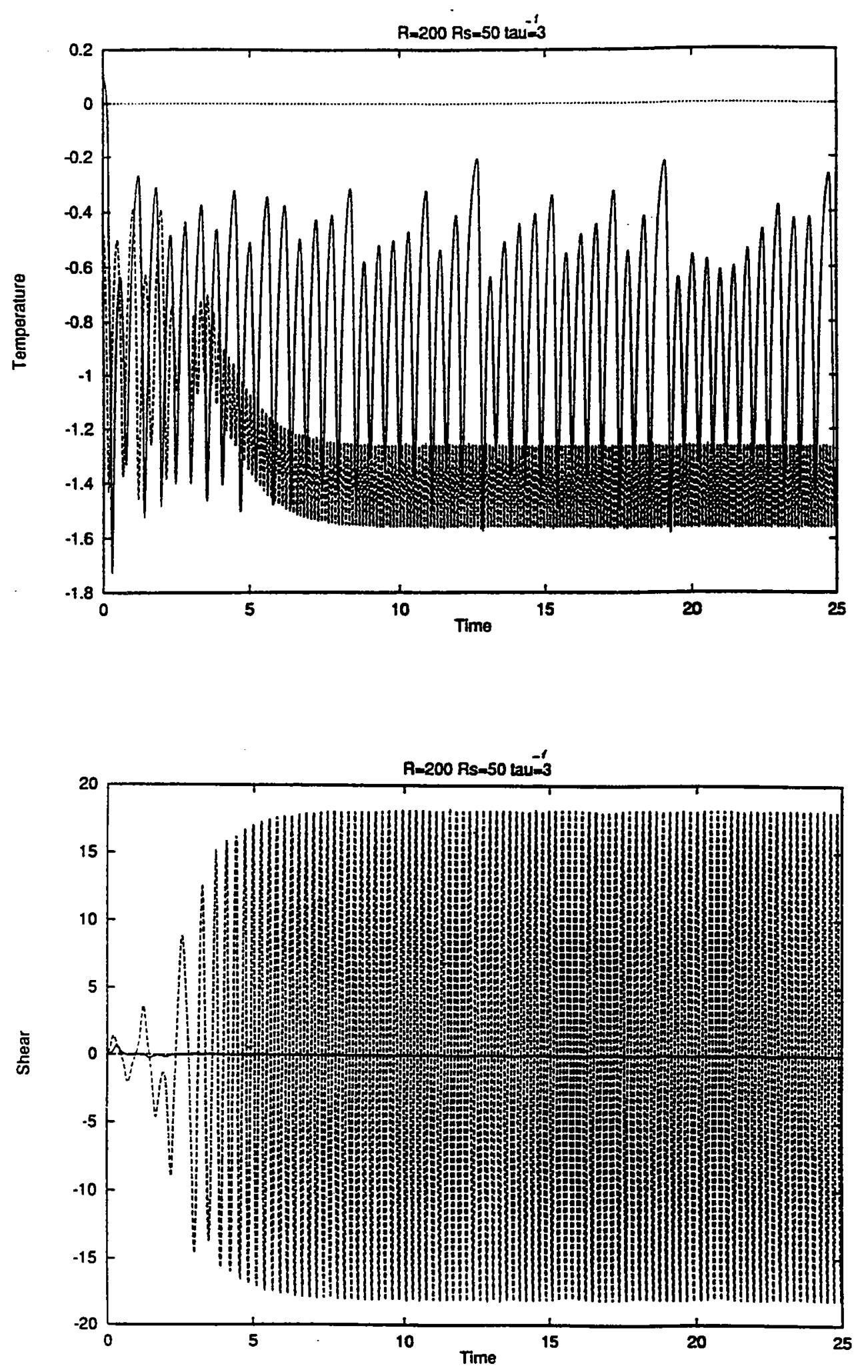

Figure 2. Same as in fig. 1, but with $R_{S}=50$. 

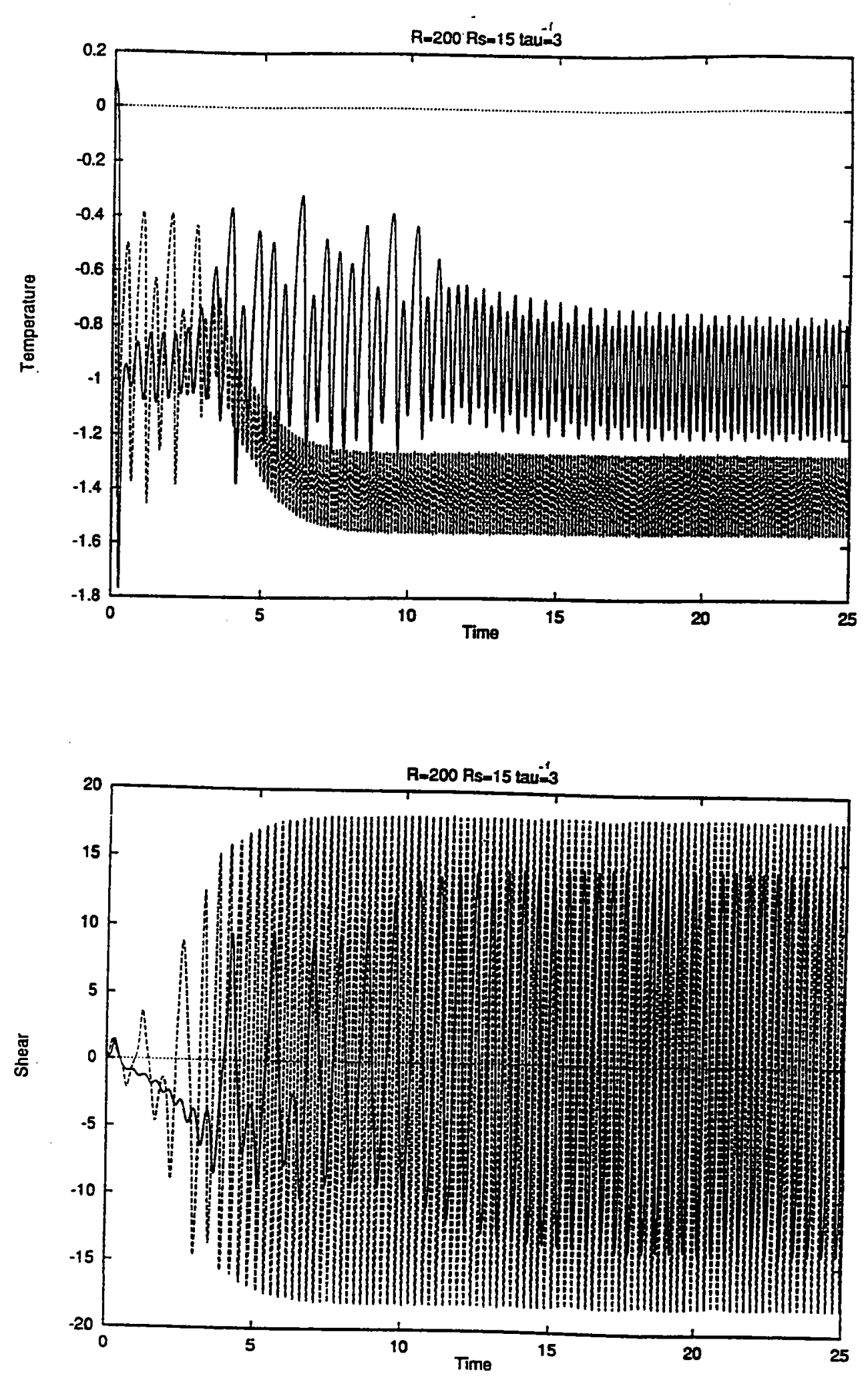

Figure 3. Same as in fig. 1, but with $R_{S}=15$. 


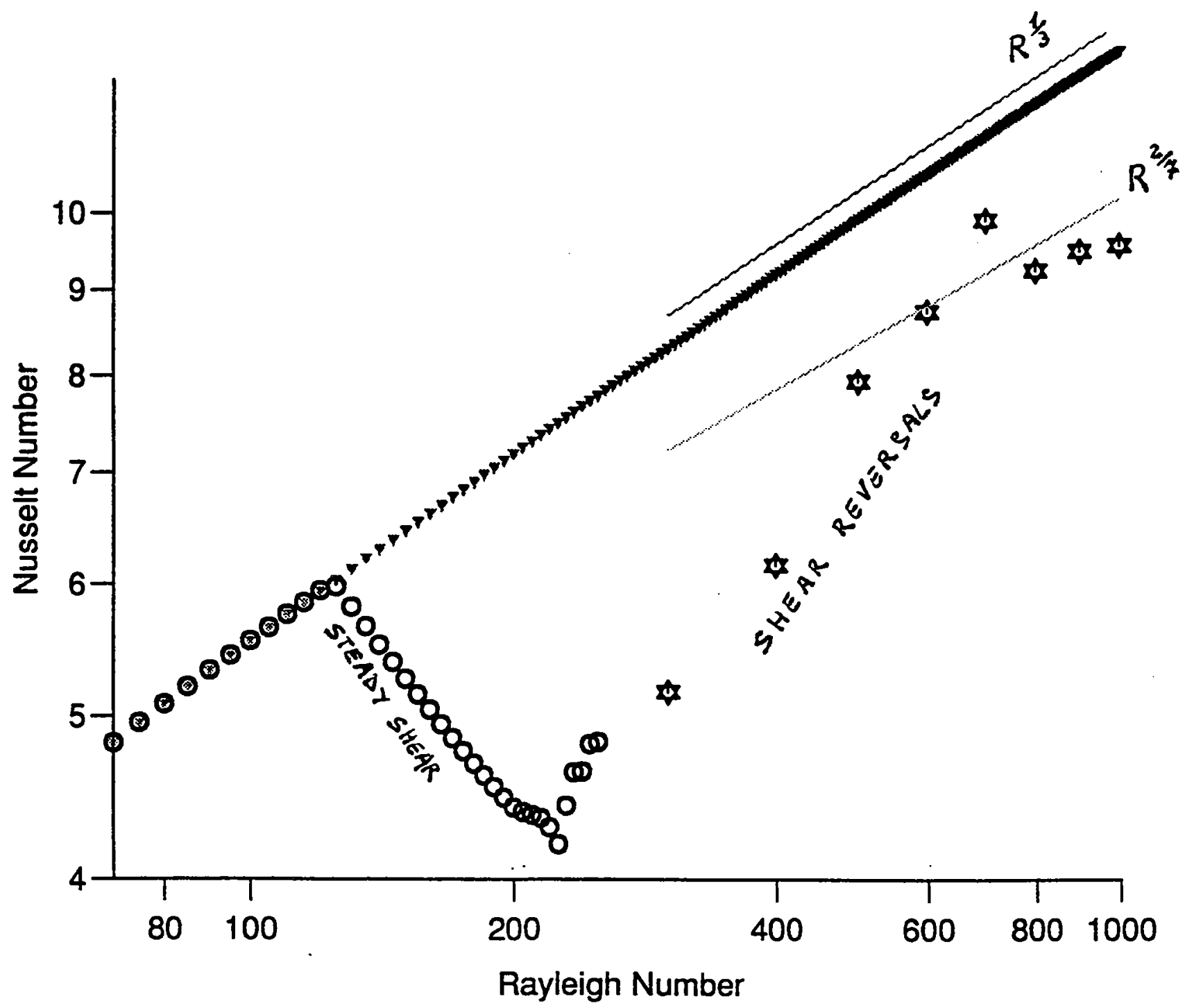

Figure 4. Nusselt number vs. Rayleigh number for a single-mode system. The Prandtl number is $\sigma=10$, and the horizontal wavelength is $\alpha=1 / \sqrt{2}$. Triangles are results obtained putting to zero the variables $U, W^{-}$and $\Theta^{-}$. The spatial resolution is 64 grid points. The timestep is $\Delta t=10^{-4}$ non-dimensional time units. The solutions found are steady. Circles are results for the full system. Timestep and resolution as for triangles. Stars are results obtained with a resolution of 256 grid points and a timestep $\Delta t=10^{-5}$. The Nusselt number is averaged over 5 non-dimensional time units after a 50 time-units-long run needed to damp out the any transient. 

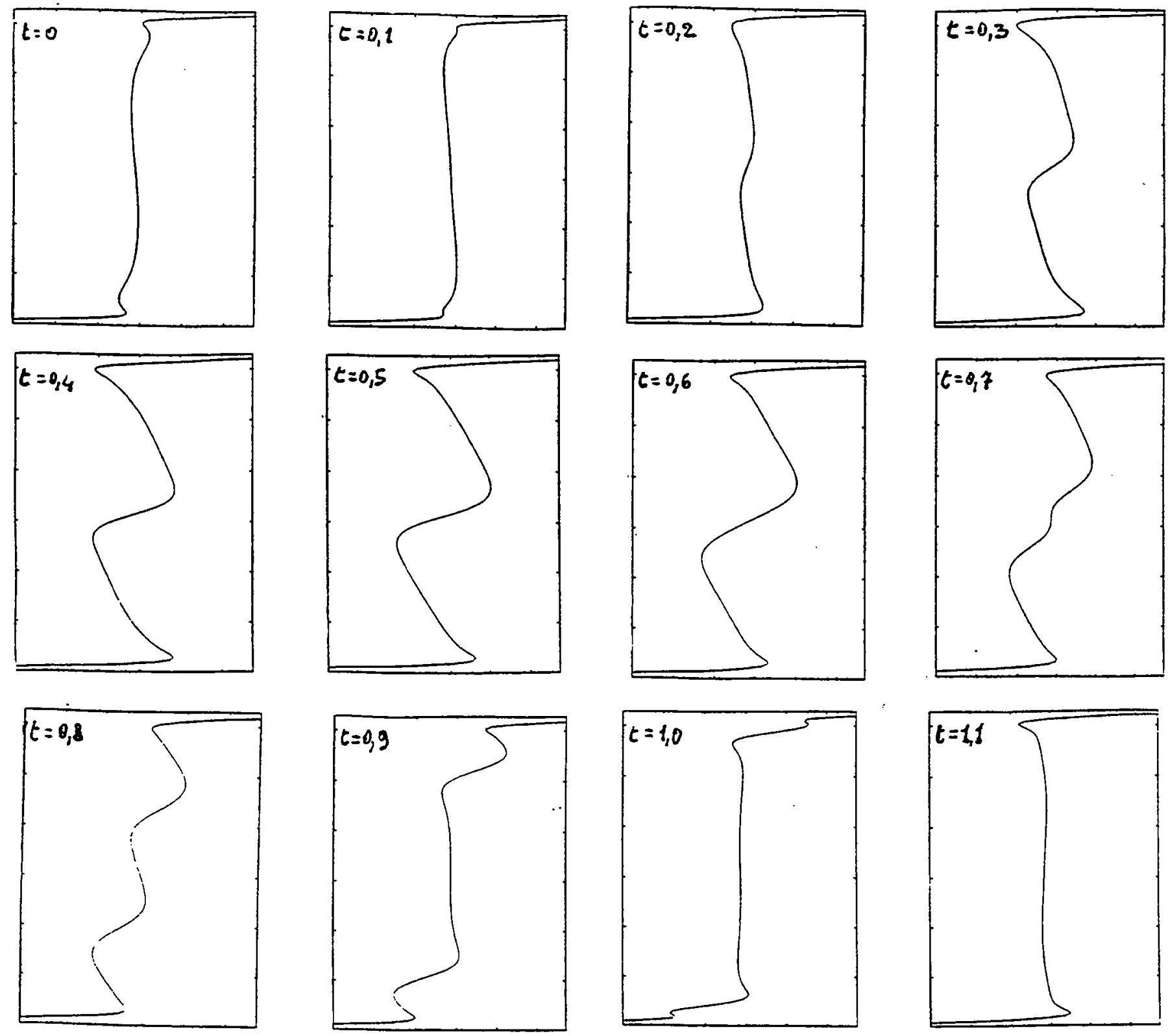

Figure 5. Time sequence of the horizontally averaged salinity $\bar{S}$ for $\sigma=10, \alpha=1 / \sqrt{2}$, $R_{T}=10, R_{S}=333, \tau=1 / 50$. The spatial resolution is 128 grid points, the time step is $\Delta t=2 \cdot 10^{-5}$. 

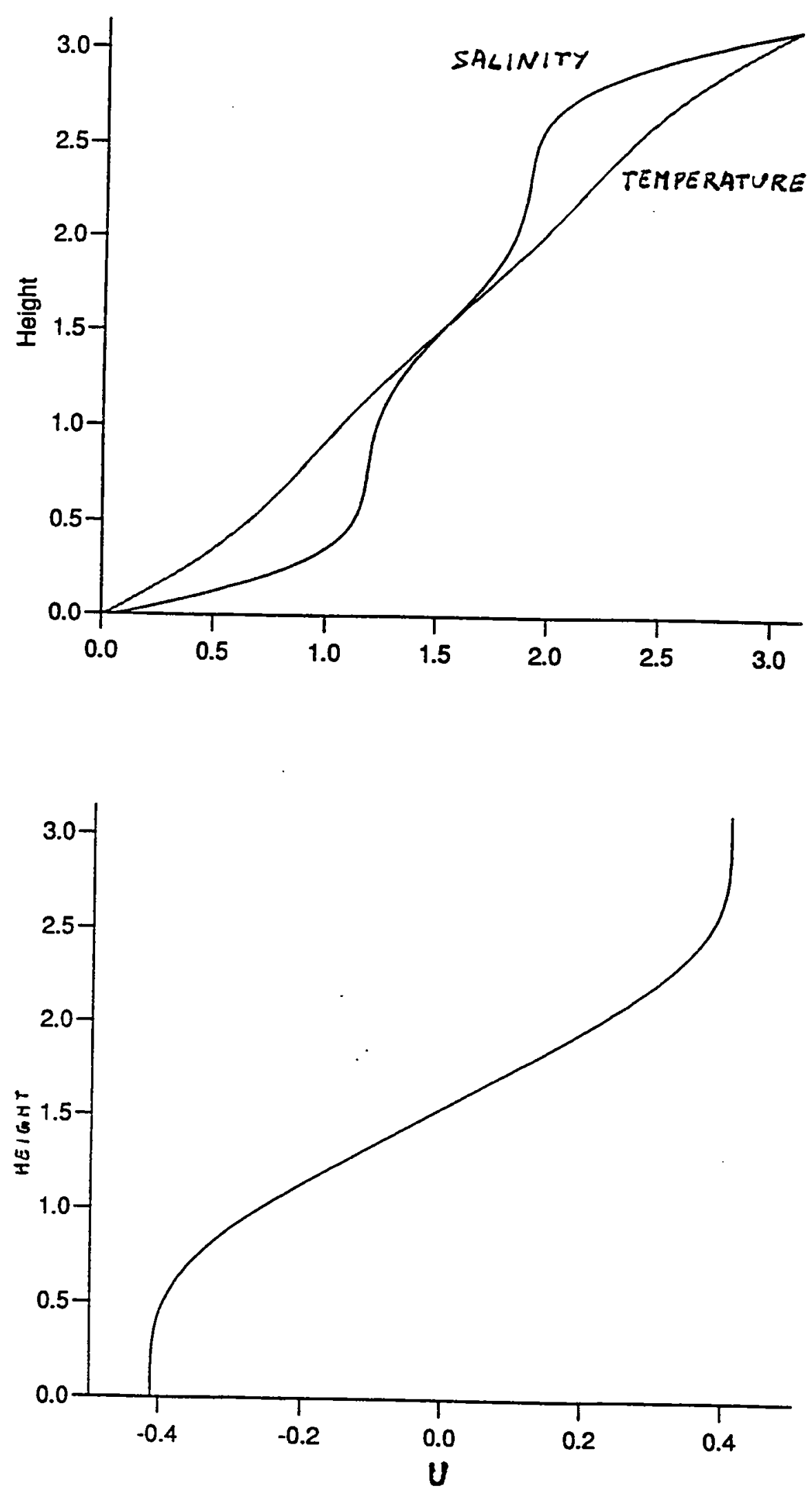

Figure 6. The upper panel shows the horizontally averaged salinity and temperature profiles of the steady solution of the equations (9). The parameters are $R_{T}=1000, R_{S}=666$, $\tau=1 / 3$. The vertical resolution is 128 grid points and the time step is $\Delta t=2 \cdot 10^{-5}$.

The lower panel shows the horizontal shearing velocity $U$ for the same run. 

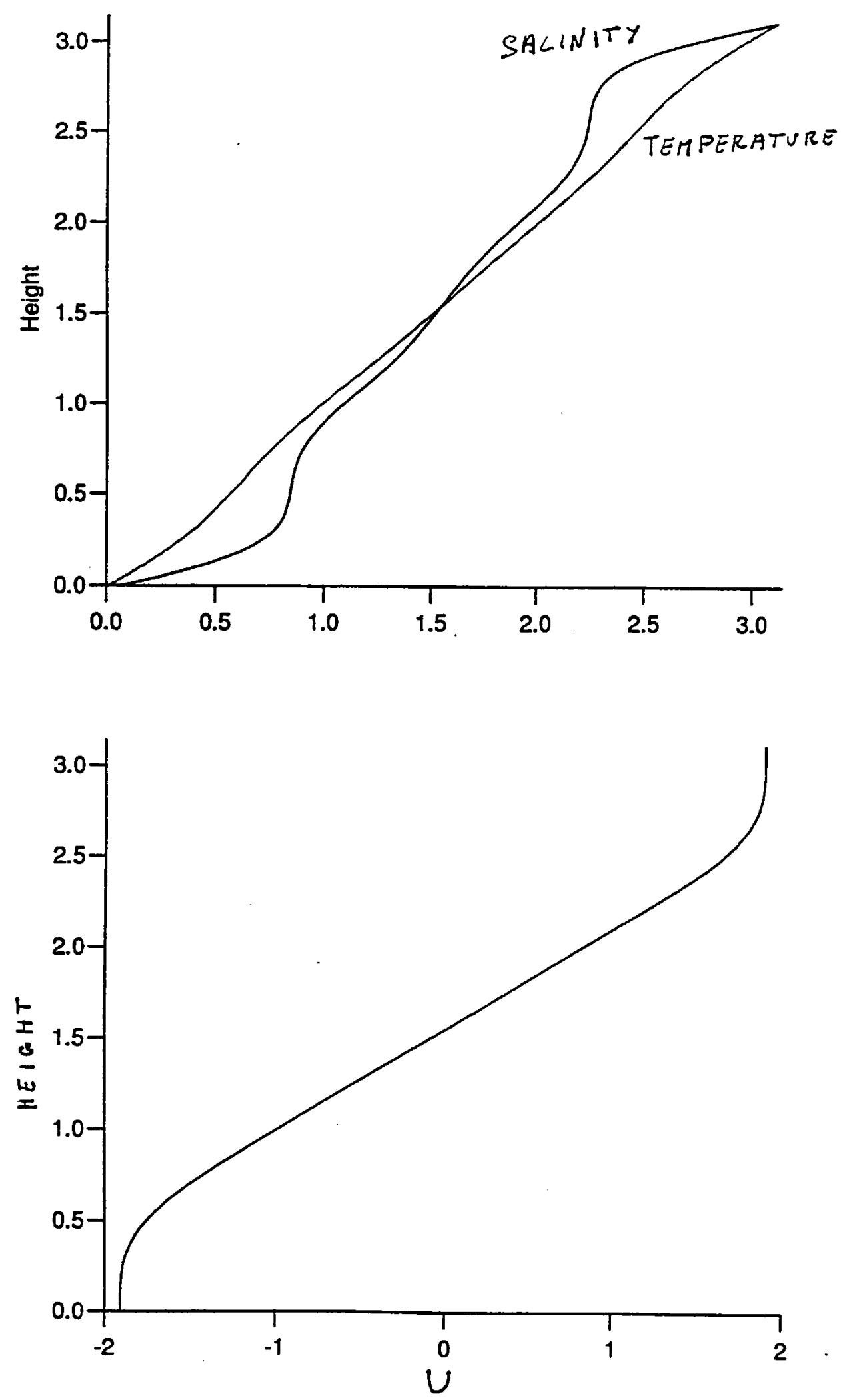

Figure 7. As in figure 6 but here is $R_{T}=5000, R_{S}=3333$. 


\title{
Double Diffusion With a Density Interface
}

\author{
By Alexander Casti
}

\section{Introduction}

When a fluid has two constituent components diffusing at different rates, the dynamics can be remarkably different than those of fluids where only a single element is diffusing. In the classic Rayleigh-Bénard problem of single component (heat) diffusion [1], an initially quiescent fluid heated from below eventually becomes buoyantly unstable, resulting in a bifurcation to steady convection if the boundary conditions are sufficiently ideal. A doubly diffusing fluid with heat and salt can involve more complicated dynamics at onset. If the basic state is in the diffusive sense, for which heat is the unstably stratified component and salt the stably stratified one, the onset of convection can be oscillatory [2]. This happens even when the fluid's density decreases upward through the layer. The essential mechanism behind this lies in the differing rates of diffusion for heat and salt. When the fluid at the lower boundary gets too hot, it expands and starts to rise. After a fluid parcel has travelled upwards a while, it eventually diffuses away its excess heat to the surrounding, cooler fluid. Owing to the slower diffusion of salt, the parcel finds itself saltier and heavier than its neighbors and begins to sink. Eventually, it begins to feel the heat again and will expand just as before, and the cycle continues until nonlinear effects take control. However, the temperature field lags the displacement field, so the parcel in fact overshoots, a situation referred to as overstable [3]. In what follows, the slowly diffusing component will be referred to as "salt," and the quickly diffusing component as "heat," even though the only qualifications for a doubly diffusive fluid is that there be two or more components with differing diffusivities.

For the purposes of this report, diffusively stratified phenomena will be classified according to two categories - weakly and strongly nonlinear. When a critical temperature gradient throughout the layer is slightly exceeded, the fluid is weakly nonlinear and begins to convect. The initial oscillatory bifurcation has been verified experimentally [4]. In contrast, a highly supercritical, strongly nonlinear thermohaline system will develop vigorously convecting, well-mixed layers separated by thin interfaces across which there are sharp gradients of heat and salt [5]. They are dubbed "diffusive interfaces" because the primary mechanism for heat and salt transport across them is diffusion. In the initial stages of layering, the linear, background profiles are mixed up and homogenized near the heated boundary, and eventually another layer develops on top of the thin interface, and then another layer atop of this one, and so on. This 
phenomena is not limited to the laboratory; diffusive interfaces are observed in Antarctic lakes, the Red Sea, and in many other bodies of water [6]. One should be aware, however, that oceanic interfaces are a result of lateral intrusions of water, not from a vertically destabilizing temperature gradient.

The primary purpose of this report is to explore the dynamics behind the breakup of an interface in a diffusively stratified fluid. The point of view adopted will be weakly nonlinear theory, so any connection to interface breakup in an actual experiment is tenuous. However, it will be seen that many of the rough features of interface destruction can be captured by a simple model, so in that sense it is well worth exploring. For instance, in an experiment conducted this summer at WHOI [7], a 2-Layer fluid of cool, fresh water above hot, salty water was seen to evolve in the following manner: Initially, both layers were quiescent. As the temperature gradient was increased from below, convection ensued in both layers, the interface remaining fairly sharp. After a while, the interface began to oscillate, until eventually fluid from both layers entrained across the interface. After a longer period, the interface began to break up, until a point was reached where the salty fluid below finally punched through and obliterated the interface, leaving a one-layer, turbulently convecting fluid with a uniform salt distribution. An attempt is made here to capture some aspects of this phenomena by considering an arbitrary background salinity profile, a sharp hyberbolic tangent function say, and exploring the effects of a sharp gradient region in the middle of a two-dimensional, Boussinesq fluid. It will be assumed that the dynamics of interest occur on a much shorter timescale than the interface can diffuse. That is, the Lewis number, as defined below, will be very small. Furthermore, no consideration will be given as to how the interface got there in the first place, or as to what is keeping it there (i.e. some sort of salinity source function).

\section{The Equations of Motion}

The following momentum, temperature, salinity, state, and mass continuity equations for a Boussinesq fluid will be used throughout:

$$
\begin{aligned}
\partial_{t} \mathbf{u}+\mathbf{u} \cdot \nabla \mathbf{u} & =-\frac{1}{\rho_{0}} \nabla p-\frac{g \rho}{\rho_{0}} \hat{\mathbf{z}}+\nu \nabla^{2} \mathbf{u} \\
\partial_{t} T+\mathbf{u} \cdot \nabla T & =\kappa_{T} \nabla^{2} T \\
\partial_{t} S+\mathbf{u} \cdot \nabla S & =\kappa_{S} \nabla^{2} S \\
\rho & =\rho_{0}\left(1-\alpha\left(T-T_{0}\right)+\beta\left(S-S_{0}\right)\right) \\
\nabla \cdot \mathbf{u} & =0
\end{aligned}
$$

where $\mathbf{u}=(u, w)$ is the velocity, $\mathrm{g}$ the gravitational acceleration, $\mathrm{p}$ the pressure, $\nu$ the molecular viscosity, $\mathrm{T}$ the temperature, $\mathrm{S}$ the salinity, $\alpha>0$ the coefficient of thermal expansion, $\beta>0$ the coefficient of salt expansion, and $\rho_{0}, T_{0}$, and $S_{0}$ reference density, temperature, and salinity values. The thickness of the layer will be denoted by $d$. 
Employ the following non-dimensionalizations, where tilde denotes a non-dimensional variable:

$$
\tilde{\mathbf{x}}=\frac{1}{d} \mathbf{x}, \tilde{\mathbf{u}}=\frac{d}{\kappa_{T}} \mathbf{u}, \tilde{T}=\frac{T-T_{0}}{\Delta T}, \tilde{S}=\frac{S-S_{0}}{\Delta S}, \tilde{t}=\frac{\kappa_{T}}{d^{2}} t,
$$

Upon dropping the tilde notation, taking the curl of (1), and defining the streamfunction $\mathbf{u} \equiv\left(-\partial_{z} \psi, \partial_{x} \psi\right)$, we are left with the following non-dimensionalized vorticity, heat, and salt equations:

$$
\begin{aligned}
\sigma^{-1} \partial_{t} \nabla^{2} \psi-\nabla^{4} \psi-R_{T} \partial_{x} \theta+R_{s} \partial_{x} S & =-J\left(\psi, \nabla^{2} \psi\right) \\
\partial_{t} \theta-\nabla^{2} \theta-\partial_{x} \psi & =-J(\psi, \theta) \\
\partial_{t} S-\tau \nabla^{2} S & =-J(\psi, S),
\end{aligned}
$$

where $R_{T}=\frac{g \alpha \Delta T d^{3}}{\nu \kappa_{T}}$ is the thermal Rayleigh number, $R_{S}=\frac{g \beta \Delta S d^{3}}{\nu \kappa_{T}}$ is the salt Rayleigh number, $\sigma=\frac{\nu}{\kappa_{T}}$ the Prandtl number, $\tau=\frac{\kappa_{S}}{\kappa_{T}}$ the Lewis number, and $J(f, g) \equiv$ $\partial_{x} f \partial_{z} g-\partial_{z} f \partial_{x} g$ is the Jacobian derivative. In many situations of interest $\tau$ is very small; for instance, $\tau=\frac{1}{80}$ for water. Note that in equation (8), the temperature has been written $T=T_{b}+\theta$, where $\frac{d T_{b}}{d z}=-1$, and that $\Delta T$ and $\Delta S$ measure the temperature and salinity deviations from their reference values. For a sharp hyberbolic tangent profile $S_{b}=-\tanh (\mathrm{bz}), \Delta S$ measures the salinity jump through the middle of the layer.

\section{First Model: A Salinity Step Function}

\subsection{Forumlation of the Problem}

As a first attempt to form a simple model of doubly diffusive interface dynamics, consider a step-function salinity profile

$$
\begin{aligned}
& S_{b}(x, z, t)=1-H(z-\eta(x, t)) \\
& H(z-\eta(x, t))= \begin{cases}1, & z>\eta \\
0, & z<\eta\end{cases}
\end{aligned}
$$

Since the notion of an interface that is diffusing is somewhat dubious, set $\tau=0$ and feed equation(10) into (7), and ignore the salt equation (9) since the salt is a passive tracer. The variable $\eta$ represents the height of the interface above its equilibrium position at $z=0$. The general scenario is sketched in figure (1). In order to deal with an interface with a density jump, we need to define jump conditions normal to the interface. The normal and tangential vectors to the interface are defined by

$$
\begin{aligned}
& \hat{\mathbf{n}}=\frac{1}{\sqrt{1+\left(\partial_{x} \eta\right)^{2}}}\left(-\partial_{x} \eta, 1\right)^{\dagger} \\
& \hat{\mathrm{t}}=\frac{1}{\sqrt{1+\left(\partial_{x} \eta\right)^{2}}}\left(1, \partial_{x} \eta\right)^{\dagger} .
\end{aligned}
$$




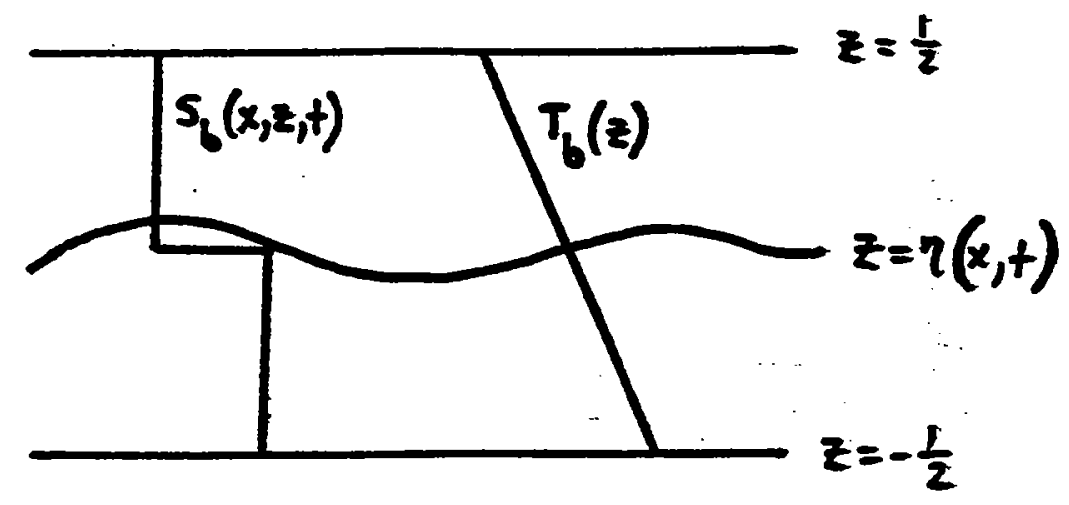

Figure 1: Discontinuous Salinity Profile With a Jump At $z=0$

The appropriate jump conditions for a fluid of identical material properties with no surface tension at the interface are [8]

$$
\begin{array}{rlrl}
\partial_{t} \eta-\partial_{z} \psi \partial_{x} \eta+\partial_{x} \psi \partial_{z} \eta & =\partial_{x} \dot{\psi} & & \text { (kinematic condition) } \\
{\left[-\partial_{z} \psi\right]} & =0 & & \text { (horizontal velocity) } \\
{\left[\partial_{x} \psi\right]} & =0 & & \text { (vertical velocity) } \\
{[\hat{\mathbf{t}} \cdot \mathbf{T} \cdot \hat{\mathbf{n}}]} & =0 & & \text { (lateral stress) } \\
{\left[\partial_{n}^{3} \psi\right]} & =\frac{1}{\sqrt{1+\left(\partial_{x} \eta\right)^{2}}} R_{S} \partial_{x} \eta \quad \text { (vorticity jump) } \\
{[\theta]} & =0 & & \text { (temperature) } \\
{[\hat{\mathbf{n}} \cdot \nabla \theta]} & =0 & & \text { (heat flux), }
\end{array}
$$

where [.] denotes the normal jump of a quantity, $\mathbf{T}$ is the viscous stress tensor for an incompressible fluid $T_{i j}=-p \delta_{i j}+\mu\left(\frac{\partial u_{i}}{\partial x_{j}}+\frac{\partial u_{j}}{\partial x_{i}}\right), \mu$ the dynamic viscosity, $\partial_{n}$ the normal derivative to the interface, and $\delta_{i j}$ the Kronecker delta function.

\subsection{The Linear Problem}

The equations of motion and the interface conditions linearized about the $z=0$ reference state are as follows:

$$
\begin{aligned}
\sigma^{-1} \partial_{t} \nabla^{2} \psi_{j}-\nabla^{4} \psi_{j}-R_{T} \partial_{x} \theta_{j} & =0 \\
\partial_{t} \theta_{j}-\nabla^{2} \theta_{j}-\partial_{x} \psi_{j} & =0 \\
\partial_{t} \eta & =\partial_{x} \psi_{j} \\
{\left[-\partial_{z} \psi\right] } & =0 \\
{\left[\partial_{x} \psi\right] } & =0 \\
{\left[\partial_{z}^{2} \psi\right] } & =0 \\
{\left[\partial_{z}^{3} \psi\right] } & =R_{s} \partial_{x} \eta \\
{[\theta] } & =0 \\
{\left[\partial_{z} \theta\right] } & =0,
\end{aligned}
$$


where $j=a, b$ denotes the "above" layer and the "below" layer. Equations. (21)(29) must be solved in each layer for some set of boundary conditions at $z= \pm \frac{1}{2}$. Note that the only boundary condition in the linear problem that makes the situation non-trivial is the jump condition (27). If $R_{s}=0$, then we simply have two RayleighBénard problems stacked upon one another, and the eigenvalue problem should recover a critical thermal Rayleigh number of 16 times the usual critical Rayleigh number in this limit (take $d \rightarrow \frac{d}{2}, \Delta T \rightarrow \frac{\Delta T}{2}$ ). Now, decompose the field variables into the usual normal modes

$$
\left(\begin{array}{c}
\psi_{j} \\
\theta_{j} \\
\eta
\end{array}\right)=\left(\begin{array}{c}
\psi_{j}(z) \\
\theta_{j}(z) \\
\eta_{0}
\end{array}\right) e^{i k x+s t}+\text { c.c. }
$$

One could plow through the algebra and solve the eigenvalue problem, but the easiest way to proceed is simply to hunt through parameter space and search for marginal eigenvalues $s$, with $\Re(s)=0$, on a computer using a shooting method. For both rigid and stress free boundary conditions at the top and bottom, and fixed temperature, it turns out that there are no steady bifurcations other than 2-layer convection. It is easy to see why when one writes out (27) and substitutes for $\eta$ using (23). Interface condition (27) then becomes

$$
s \partial_{z a}^{3} \psi=s \partial_{z b}^{3} \psi-k^{2} R_{s} \psi_{b}
$$

Thus, if $s=0$ is to be an eigenvalue, (31) demands that $\psi_{b}=0$ at the boundary, i.e. the vertical velocity must vanish at the interface! This means that no matter how small $R_{S}$ is, we will always have 2-Layer convection at marginality. This is contrary to physical intuition, which would suggest that if $R_{s}$ is extremely small, the fluid would not feel the salinity jump at $z=0$, and normal, full layer Rayleigh-Bénard convection would ensue. Clearly then, too much has been stripped out of the model. The fluid feels an essentially impenetrable barrier! One might understand this better if the salt Rayleigh number, which scales proportional to the $\Delta S$ jump across the interface, is written as

$$
R_{S}=\frac{g \beta \frac{d S_{b}}{d z} d^{4}}{\nu \kappa_{T}}
$$

where $\frac{d S_{b}}{d z}$ is the salt gradient through the interface. Since $\frac{d S_{b}}{d z}$ is infinite in this problem, both layers see an infinite gradient at the interface. In addition to this disappointing result, Hopf bifurcations could not be found. In light of a desire to explore competitive mechansisms between 2-Layer and 1-Layer convection, this model is unsatisfactory since the linear problem does not allow a rich variety of marginal solutions about which to expand a weakly nonlinear theory. Perhaps something interesting might still come of it, but at this point it seems that more progress might be made by considering a continuous salt profile, especially since a computer is so repulsed by discontinuities. 


\section{Some Comments About The Linear Salt Profile Problem}

The diffusive problem for which the background salt profile is linear has been examined in great detail [9]. Returning to the full Boussinesq equations (7)-(9) and writing

$$
\begin{array}{r}
S=S_{b}+\Sigma, \quad \frac{\mathrm{dS}_{\mathrm{b}}}{\mathrm{d} z}=-1 \\
\left(\begin{array}{c}
\psi \\
\theta \\
\Sigma
\end{array}\right)=\left(\begin{array}{c}
i \psi_{e} \\
\theta_{e} \\
\Sigma_{e}
\end{array}\right) e^{i k x+s t} \sin \pi z+\text { c.c. }
\end{array}
$$

one can solve the linear problem and obtain the dispersion relation

$$
\begin{aligned}
s^{3}+(\sigma+\tau+1) q^{2} s^{2}+((\sigma & \left.+\tau+\sigma \tau) q^{4}-\frac{\sigma k^{2}}{q^{2}}\left(R_{T}-R_{S}\right)\right) s \\
& +\left(\sigma \tau q^{6}+\sigma k^{2}\left(R_{S}-\tau R_{T}\right)\right)=0,
\end{aligned}
$$

where $q^{2} \equiv\left(\pi^{2}+k^{2}\right)$. Free slip, fixed temperature boundary conditions have been assumed. Among the myriad of interesting results, one can deduce from (35) that oscillatory convection corresponds to the lowest critical $R_{T}$ provided $R_{S}$ is large enough (or, equivalently, $\tau$ is small enough). That is, the stabilizing salt field must be strong enough to sustain oscillations. It is interesting that no matter how small $R_{s}$ may be, one can always choose a small enough Lewis number $\tau$ such that the Hopf bifurcation will be the first. From (35), one can also derive the following marginality relations:

$$
\begin{array}{r}
R_{T}^{\text {Steady }}=\frac{R_{S}}{\tau}+\frac{q^{6}}{k^{2}} \\
R_{T}^{O s s}=\frac{\sigma+\tau}{\sigma+1} R_{s}+(1+\tau)\left(1+\sigma^{-1} \tau\right) \frac{q^{6}}{k^{2}} \\
\omega_{i}^{2}=(\sigma \tau+\sigma+\tau) q^{4}-\sigma\left(R_{T}-R_{S}\right) \frac{k^{2}}{q^{2}}
\end{array}
$$

where $\omega_{i}$ is the frequency of the Hopf mode should it occur, and $R_{T}^{\text {Steady }}$ and $R_{T}^{O s c}$ are the critical Rayleigh numbers for a given mode with wavenumber $\mathrm{k}$ to become marginal to steady convection and oscillatory convection, respectively. From a mathematical viewpoint, a curious feature of the marginality curves is that when Hopf modes are present, the Hopf branch always intersects the steady branch at two points for fixed $R_{s}, \tau$, and $\sigma$. These points of intersection, commonly referred to as Takens-Bogdanov codimension-2 points [10], correspond to the simultaneous onset of steady and oscillatory convection. They are of special interest since they provide a natural location in parameter space at which to investigate the competing effects of steady, overturning convection and any oscillations that might occur in the system. A noteworthy feature 


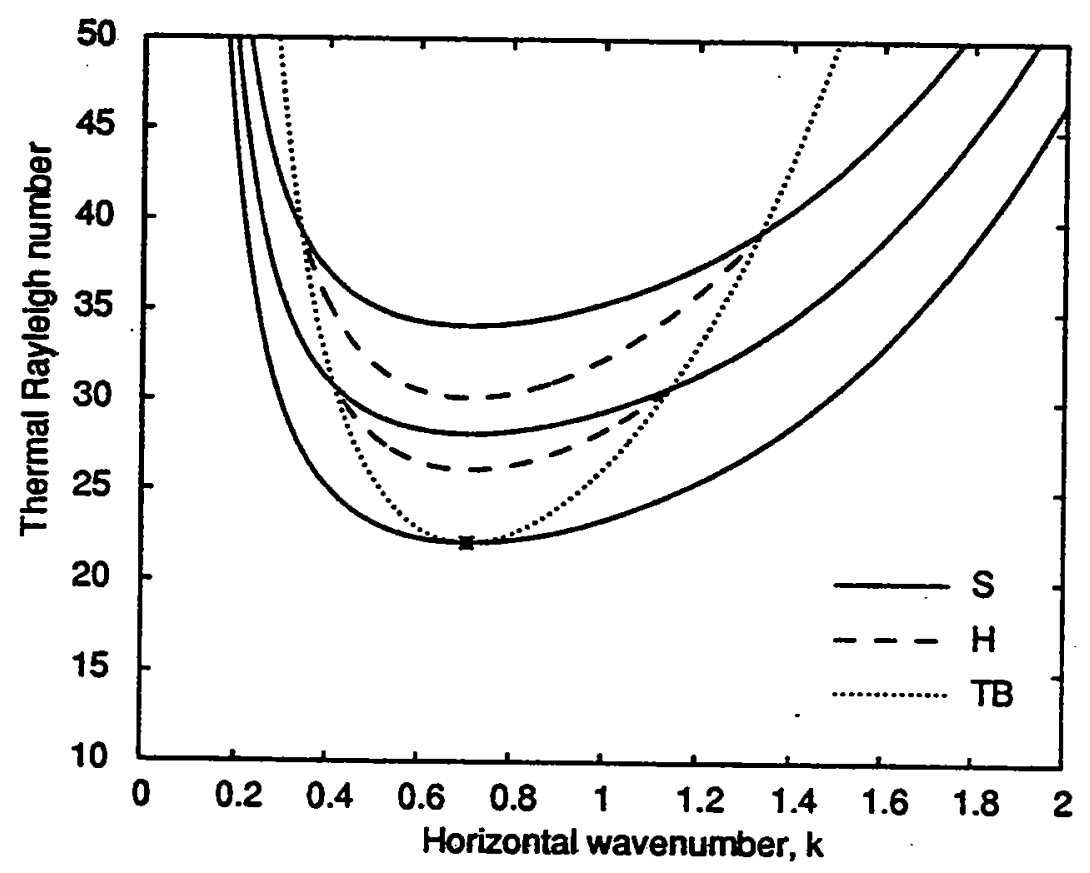

Figure 2: Coalescence of Marginal Curves, $\tau=0.666, \mathrm{H}=$ Hopf Branch, $\mathrm{S}=$ Steady Branch

is that for fixed $R_{s}$, the oscillatory branch can be made to disappear. In fact, for $R_{S}<\mathrm{R}_{\mathrm{S}}^{\text {crit }}=\frac{q^{6} \tau^{2}(\sigma+1)}{\sigma k^{2}(1-\tau)}$, the Hopf branch does not exist. As $\tau$ is varied appropriately, the two Takens-Bogdanov points merge at the minimum of the steady branch. This is a degenerate, codimension-2 point explored elsewhere in this volume [11]. A plot of how these curves coalesce as $\tau$ is varied is shown in figure (2). It will be of interest later to see how much of these results for a linear salt profile hold true for an arbitrary background profile $S_{b}$, with $\frac{d S_{b}}{d z}<0$.

\section{$5 \quad$ Arbitrary $S_{b}(z)$, Small $R_{S}$ limit}

We now turn to a model with an arbitrary, monotonically decreasing background salt field. Since some analytical progress can be made in a small $R_{s}$ limit, we explore this first. Suppose the following expansions [12]:

$$
\begin{aligned}
\psi=\epsilon^{2} \psi_{2}+\epsilon^{4} \psi_{4}+\cdots, & \theta=\epsilon^{2} \theta_{2}+\epsilon^{4} \theta_{4}+\cdots \\
S=S_{b}+\Sigma_{0}+\epsilon^{2} \Sigma_{2}+\cdots & \\
R_{S}=\epsilon^{4} r_{4}+\cdots, & R_{T}=R_{0}\left(1+\epsilon^{2} \rho+\cdots\right) \\
\tau=\epsilon^{2} \tau_{2}+\cdots, & T=\epsilon^{2} t \Rightarrow \partial_{t} \rightarrow \epsilon^{2} \partial_{T} \\
R_{0}=\frac{q^{6}}{k^{2}}=\frac{\left(\pi^{2}+k^{2}\right)^{3}}{k^{2}} &
\end{aligned}
$$


The idea of this expansion is to couple the salt field weakly to the usual Rayleigh-Bénard convection equations, such that the lowest order problem does not involve the salinity. The question of interest pertains to the effect of the $S_{b}(z)$ profile on convection rolls. For instance, can the salt field halt the overturning convection and cause the rolls to oscillate on a slow time scale? Note that the small amplitude expansion does not employ the usual Malkus-Veronis [13] scalings, since the perturbation to the thermal Rayleigh number scales as the stream function and temperature amplitudes. Malkus and Veronis used the scaling $\psi=\epsilon \psi_{1}+\cdots$, with an analagous scaling for the temperature. Thus, the convection is weaker in this small $R_{s}$ limit. Upon equating $\epsilon$ powers in equations (7)-(9) we find

$$
\begin{aligned}
& \mathcal{O}\left(\epsilon^{2}\right): \quad \mathcal{L}_{2} \equiv\left(\begin{array}{cc}
-\nabla^{4} & -R_{0} \partial_{x} \\
-\partial_{x} & -\nabla^{2}
\end{array}\right)\left(\begin{array}{c}
\psi_{2} \\
\theta_{2}
\end{array}\right)=0 \\
& \psi_{2}=-\mathrm{iA}(\mathrm{T}) \mathrm{e}^{\mathrm{ikx}} \sin \pi z+\text { c.c. } \\
& \theta_{2}=\theta_{\mathrm{e}} \mathrm{A}(\mathrm{T}) \mathrm{e}^{\mathrm{ikx}} \sin \pi z+\text { c.c. } \\
& \partial_{T} \Sigma_{0}-\tau_{2} \nabla^{2} \Sigma_{0}+S_{b}{ }^{\prime} \partial_{x} \psi_{2}+J\left(\psi_{2}, \Sigma_{0}\right)=0,
\end{aligned}
$$

where $\theta_{e}=\frac{k}{q^{2}}$ and $q^{2}=\pi^{2}+k^{2}$. The task is now to derive an evolution equation for the slow-time amplitude $A(T)$, and to couple this with (47). This is accomplished by applying a solvability condition at the next order.

$$
\mathcal{O}\left(\epsilon^{4}\right): \quad \mathcal{L} \mathbf{v}_{\mathbf{4}}=\mathbf{I}_{4} \equiv\left(\begin{array}{c}
-\sigma^{-1} \partial_{T} \nabla^{2} \psi_{2}+\rho R_{0} \partial_{x} \theta_{2}-r_{4} \partial_{x} \Sigma_{0}-J\left(\psi_{2}, \nabla^{2} \psi_{2}\right) \\
-\partial_{T} \theta_{2}-J\left(\psi_{2}, \theta_{2}\right)
\end{array}\right)
$$

We now enforce a solvability constraint on (48) to remove the resonant terms. To do this, we need to define the matrix equation adjoint to $\mathcal{L}$ :

$$
\text { Adjoint Equation : } \quad \begin{gathered}
\mathcal{L}^{\dagger} \mathbf{v}_{\mathbf{a d}} \equiv\left(\begin{array}{cc}
-\nabla^{4} & \partial_{x} \\
R_{0} \partial_{x} & -\nabla^{2}
\end{array}\right)\left(\begin{array}{c}
\psi_{a d} \\
\theta_{a d}
\end{array}\right)=0 \\
\psi_{a d}=\mathrm{i} \psi_{\mathrm{a}} \mathrm{e}^{\mathrm{ikx}} \sin \pi z+\text { c.c. } \\
\theta_{a d}=\theta_{\mathrm{a}} \mathrm{e}^{\mathrm{ikx}} \sin \pi \mathrm{z}+\text { c.c. }
\end{gathered}
$$

where $\psi_{a}=k$ and $\theta_{a}=\frac{k^{2} R_{0}}{q^{2}}$. The solvability conditon at this order is defined by

$$
\left\langle\mathrm{v}_{\mathrm{ad}}, \mathbf{I}_{4}\right\rangle \equiv \frac{k}{2 \pi} \int_{0}^{\frac{2 \pi}{k}} \int_{0}^{1} d z d x \mathrm{v}_{a d}^{H} \mathbf{I}_{4}=0
$$

where the superscript $\mathrm{H}$ denotes the Hermitian conjugate transpose. Application of the solvability constraint (52) yields the evolution equation for $\mathrm{A}(\mathrm{T})$

$$
\begin{gathered}
\partial_{T} A-\mu A+r\left(k^{-1} \psi_{a d}, \partial_{x} \Sigma_{0}\right\rangle=0 \\
\mu=\frac{\sigma q^{2}}{\sigma+1} \rho, r=\frac{2 r_{4}}{q^{2}(\sigma+1)} .
\end{gathered}
$$


The task at hand is to analyze the following, coupled equations:

$$
\begin{aligned}
\partial_{T} A-\mu A+r\left\langle k^{-1} \psi_{a d}, \partial_{x} \Sigma_{0}\right\rangle & =0 \\
\partial_{T} \Sigma_{0}-\tau_{2} \nabla^{2} \Sigma_{0}-\gamma(z) \partial_{x} \psi_{2}+J\left(\psi_{2}, \Sigma_{0}\right) & =0,
\end{aligned}
$$

where $\gamma(z) \equiv-S_{b}{ }^{\prime}$. Note that $\gamma=1$ recovers the case of the linear profile, and that $\psi_{a d}$ is given by (50) and $\psi_{2}$ by (45).

\subsection{Linearized Amplitude Equation}

Consider the linearized version of (56) by ignoring the Jacobian term. If we write

$$
\Sigma_{0}=B(T) e^{i k x} \hat{S}_{0}(z)+\text { c.c. }
$$

we have

$$
\begin{aligned}
A_{T}-\mu A+r\left\langle-i e^{-i k x} \sin \pi z+\text { c.c. }, i k B(T) e^{i k x} \hat{S}_{0}(z)+\text { c.c. }\right\rangle & =0 \\
B_{T} \hat{S}_{0}(z)-k A \gamma \sin \pi z-\tau_{2} \mathrm{~B}\left(\partial_{z}^{2} \hat{S}_{0}-\mathrm{k}^{2} \hat{S}_{0}\right) & =0 .
\end{aligned}
$$

There is a nice trick that allows one to solve these equations. Take (59) and apply the operator $\langle\sin \pi z, \cdot\rangle$, and integrate the diffusion term by parts, making use of fixed salt boundary conditions and the equation

$$
\hat{S}_{0}=0, \quad z=0,1
$$

Applying this operation reduces (58) and (59) to

$$
\begin{aligned}
A_{T}-\mu A+k r B & =0 \\
B_{T}-k J A+q^{2} \tau_{2} B & =0
\end{aligned}
$$

where we have chosen the normalization and defined $\mathrm{J}$ by

$$
\begin{array}{r}
I_{0} \equiv \int_{0}^{1} d z \sin \pi z \hat{\mathrm{S}}_{0}=1 \\
J \equiv \int_{0}^{1} d z \gamma \sin ^{2} \pi z
\end{array}
$$

Note that all of the information about the basic state salinity profile is buried within the parameter $J$.

\subsection{Takens-Bogdanov Point}

Just as in the linear salt profile case, equations (61) and (62) have Takens-Bogdanov bifurcation points. To see this, write

$$
\left(\begin{array}{c}
A \\
B
\end{array}\right)=\left(\begin{array}{c}
A_{e} \\
B_{e}
\end{array}\right) e^{s T}+\text { c.c. }
$$


to derive the dispersion relation

$$
s^{2}+\left(\tau_{2} q^{2}-\mu\right) s+\left(r k^{2} J-\mu q^{2} \tau_{2}\right)=0
$$

There are some important things to take notice of in equation (66). First, it is a second order equation, which means that our center manifold projection has resulted in a dispersion relation one order lower than the usual third order equation. This is essentially a reflection of the lowest order decoupling of the salt field. Also, we will see later that higher order terms in the expansion, particularly the terms that generate a mean temperature field by the perturbations, are crucial to the stability of the system. Second, there would be no non-trivial, steady solutions to (66) without the diffusion term, which means that a model that neglected the diffusion in this limit would almost certainly be unstable. Third, there are no marginal, oscillatory solutions. Despite the latter, unpleasant feature, there are still Takens-Bogdanov points defined by the parameter relations

$$
\mu=\tau_{2} q^{2} \quad, \quad r k^{2} J=\mu q^{2} \tau_{2} .
$$

One of the more interesting features of this analysis is that the dynamics seem relatively insensitive to the basic state salinity profile used! All that the parameter $J$ contributes is a multiplicative factor to the displacement of the marginal stability curve for steady modes. The steady branch is now defined by

$$
R_{T}^{S t e a d y}=R_{0}+\epsilon^{2}\left(\frac{2 r_{4} J}{\tau_{2}}\right),
$$

and since $J>0$ the effect of the background salt profile is fairly trivial. The bifurcation is still supercritical, so long as $S_{b}$ is a monotonically decreasing function and $r_{4}>0$. We will now proceed to build upon the linear analysis by unfolding the Takens-Bogdanov bifurcation. Will the nonlinear terms quash the linear instability?

\subsection{Unfolding The Takens-Bogdanov Bifurcation}

Let us now investigate the stability of the steady solutions defined by $r k^{2} J=\mu q^{2} \tau_{2}$. To do so, expand (55) and (56) about the Takens-Bogdanov point using the following perturbation scheme:

$$
\begin{array}{r}
A=\epsilon A_{1}+\epsilon^{2} A_{2}+\cdots, \Sigma_{0}=\epsilon S_{1}+\epsilon^{2} S_{2}+\cdots \\
r=r_{0}+\epsilon r_{1}+\epsilon^{2} r_{2}+\cdots ; \tau_{2}= \\
\tau_{20}+\epsilon \tau_{21}+\epsilon^{2} \tau_{22}+\cdots \\
T=\epsilon T_{1} \Rightarrow \partial_{T} \rightarrow \epsilon \partial_{T_{1}} .
\end{array}
$$

\subsubsection{Order $\epsilon$}

At $\mathcal{O}(\epsilon)$, we recover the linear problem

$$
\begin{aligned}
-\mu A_{1}+r_{0}\left\langle k^{-1} \psi_{a d}, \partial_{x} S_{1}\right\rangle & =0 \\
-\gamma k\left(A_{1} e^{i k x} \sin \pi z+\text { c.c. }\right)-\tau_{00} \nabla^{2} S_{1} & =0 .
\end{aligned}
$$


Now employ the same solvability trick as before and write

$$
S_{1}=B_{1}(T) e^{i k x} \hat{S}_{1}(z)+\text { c.c. }
$$

Upon projection of (73) onto $\sin \pi z$, the resulting equations are

$$
\begin{aligned}
-\mu A_{1}+k r_{0} I_{1} & =0 \\
-k J A_{1}+\tau_{00} q^{2} I_{1} & =0 \\
\hat{S}_{1}{ }^{\prime}-k^{2} \hat{S}_{1} & =-\frac{k}{\tau_{00}} \gamma \sin \pi z \\
I_{1}(T) & \equiv B_{1}(T) \int_{0}^{1} d z \sin \pi z \hat{\mathrm{S}}_{1} \equiv B_{1}(T) I_{0}
\end{aligned}
$$

where ' $=\frac{d}{d z}$. We will identify $B_{1}=A_{1}$, and though the introduction of the $I_{1}(T)$ term is somewhat pedantic, it helps to flesh out the nature of the codimension-2 expansion. Also note that $I_{0}$ will not be normalized to unity, and by making use of $(77)$, one can show that $I_{0}=\frac{k J}{\mu}$. A solvability constraint for non-trivial solutions of (75), (76) yields the parameter relations that put us at the Takens-Bogdanov(TB) point:

$$
\begin{aligned}
\mu & =\tau_{00} q^{2} \\
\mu \tau_{00} q^{2} & =k^{2} J r_{0} .
\end{aligned}
$$

\subsubsection{Order $\epsilon^{2}$}

Here we have

$$
\begin{array}{cc}
-\mu A_{2}+r_{0}\left\langle k^{-1} \psi_{a d}, \partial_{x} S_{2}\right\rangle= & -r_{1}\left\langle k^{-1} \psi_{a d}, \partial_{x} S_{1}\right\rangle-\partial_{T_{1}} A_{1} \\
-\gamma k\left(A_{2} e^{i k x} \sin \pi z+\text { c.c. }\right)-\tau_{00} \nabla^{2} S_{2}= & -\partial_{T_{1}} S_{1}+\tau_{11} \nabla^{2} S_{1} \\
\cdot & -J\left(-A_{1} e^{i k x} \sin \pi z+\text { c.c. }, S_{1}\right) .
\end{array}
$$

Upon projecting (82) onto $e^{i k x} \sin \pi z$ we have

$$
\begin{aligned}
-\mu A_{2}+k r_{0} I_{2} & =-r_{1} k I_{0} A_{1}-\partial_{T_{1}} A_{1} \\
-k J A_{2}+\tau_{00} q^{2} I_{2} & =-\tau_{11} q^{2} A_{1}-I_{0} \partial_{T_{1}} A_{1} .
\end{aligned}
$$

A more illuminating expression of these equations is

$$
\begin{gathered}
\mathcal{M} \mathrm{v}_{2} \equiv\left(\begin{array}{cc}
-\mu & k r_{0} \\
-k J & \tau_{00} q^{2}
\end{array}\right)\left(\begin{array}{c}
A_{2} \\
I_{2}
\end{array}\right)=\mathbf{R}_{2} \\
\mathbf{R}_{2}=-\left(\begin{array}{c}
\frac{\mu r_{1}}{\tau_{0}} \\
\tau_{11} q^{2}
\end{array}\right) A_{1}-\frac{\mu}{k r_{0}}\left(\begin{array}{c}
k r_{0} \\
\mu
\end{array}\right) \partial_{T_{1}} A_{1} .
\end{gathered}
$$

The relations $\operatorname{det}(\mathcal{M})=0$ and $\operatorname{Tr}(\mathcal{M})=0$ are the TB conditions. At this stage one needs to define the matrix adjoint problem to (85). It is good enough to define 
the adjoint eigenvectors, since at each epsilon power the solvability condition will be $\mathbf{e}_{\mathbf{1}}^{\dagger} \cdot \mathbf{R}_{\mathbf{j}}=0$, where $\mathbf{e}_{\mathbf{1}}^{\dagger}$ is an adjoint nullvector, and $\mathbf{R}_{\mathbf{j}}$ is the right hand side column vector, after projection, at each order $j$. Choose the following set of eigenvectors and their adjoints:

$$
\begin{aligned}
& \mathcal{M} \mathrm{e}_{1}=0, \mathrm{e}_{1}=\left(\begin{array}{c}
k r_{0} \\
\mu
\end{array}\right) \\
& \mathcal{M} \mathrm{e}_{2}=-\frac{\mu}{k r_{0}} \mathbf{e}_{1} \quad, \quad \mathbf{e}_{2}=\left(\begin{array}{c}
1 \\
0
\end{array}\right) \\
& \mathbf{e}_{1}^{\dagger} \mathcal{M}=0, \quad \mathbf{e}_{1}^{\dagger}=\left(\begin{array}{c}
-\mu \\
k r_{0}
\end{array}\right)^{\dagger} .
\end{aligned}
$$

Applying solvability to (86), which ensures that the right side of (86) has no component in the space of the generalized eigenvector $\mathbf{e}_{2}$, yields the following constraint between the unfolding parameters $\tau_{11}$ and $r_{1}$ :

$$
\tau_{11}=\frac{\mu^{2} r_{1}}{k r_{0}^{2} q^{2}}
$$

We will need a particular solution to equations (81),(82) before proceeding. Homogeneous solutions are ignored since they will be non-resonant at the next order. After a bit of effort, this solution can be written

$$
\begin{aligned}
S_{2} & =S_{2}^{(0)}+S_{2}^{(1)}+S_{2}^{(2)} \\
S_{2}^{(0)} & =\left|A_{1}\right|^{2} \Delta_{0}(z) \\
S_{2}^{(1)} & =\left(A_{2}-\frac{\tau_{11}}{\tau_{00}} A_{1}\right) \hat{S}_{1} e^{i k x}+\frac{1}{\tau_{00}} \partial_{T_{1}} A_{1} \Delta_{1}(z) e^{i k x}+\text { c.c. } \\
S_{2}^{(2)} & =A_{1}{ }^{2} e^{2 i k x} \Delta_{2}(z)+\text { c.c. } \\
-A_{2} & =\frac{r_{1}}{r_{0}} A_{1}+\frac{1}{\mu} \partial_{T_{1}} A_{1},
\end{aligned}
$$

where the set of $\Delta_{j}$ 's $(j=0,1,2)$ satisfy the following differential equations:

$$
\begin{aligned}
\Delta_{0}{ }^{\prime \prime} & =\frac{2 k}{\tau_{00}}\left(\sin \pi \mathrm{z} \hat{\mathrm{S}}_{1}\right)^{\prime} \\
\Delta_{1}{ }^{\prime \prime}-k^{2} \Delta_{1} & =\hat{S}_{1} \\
\Delta_{2}{ }^{\prime \prime}-4 k^{2} \Delta_{2} & =\frac{k}{\tau_{00}}\left(\sin \pi \mathrm{z} \hat{\mathrm{S}}_{1}^{\prime}-\pi \cos \pi z \hat{\mathrm{S}}_{1}^{\circ}\right) \\
\Delta_{j} & =0, \quad \mathrm{z}=0,1 .
\end{aligned}
$$

\subsubsection{Order $\epsilon^{3}$, Amplitude Equation}

The equations at this order are 


$$
\begin{aligned}
-\mu A_{3}+r_{0}\left\langle k^{-1} \psi_{a d}, \partial_{x} S_{3}\right\rangle= & -\partial_{T_{1}} A_{2}-r_{1}\left\langle k^{-1} \psi_{a d}, \partial_{x} S_{2}\right\rangle \\
& -r_{2}\left(k^{-1} \psi_{a d}, \partial_{x} S_{1}\right\rangle \\
-\gamma k\left(A_{3} e^{i k x} \sin \pi z+\text { c.c. }\right)-\tau_{00} \nabla^{2} S_{3}= & -\partial_{T_{1}} S_{1}+\tau_{11} \nabla^{2} S_{2}+\tau_{22} \nabla^{2} S_{1} \\
& -J\left(-i A_{1} e^{i k x} \sin \pi z+\text { c.c., } S_{2}\right) \\
& -J\left(-i A_{2} e^{i k x} \sin \pi z+\text { c.c., } S_{1}\right)
\end{aligned}
$$

Applying solvability and the maxim that patience is a virtue, one finds the amplitude equation

$$
\partial_{T_{1}}^{2} A_{1}+a \partial_{T_{1}} A_{1}+b A_{1}=c\left|A_{1}\right|^{2} A_{1}
$$

where the real coefficients are defined by

$$
\begin{aligned}
a & =\frac{r_{1} I_{0}}{r_{0}}(1-\mu)+\frac{r_{1}}{r_{0}}\left(\mu+k r_{0} I_{0}-\mu r_{1} I_{0}\right) \\
b & =\left(\frac{k J r_{1}}{r_{0}}\right)^{2}(\mu-1)-\mu q^{2} \tau_{22}+I_{0} k r_{1}\left(\frac{\mu^{2} r_{1}}{k r_{0}^{2}}-\frac{\mu r_{2}}{r_{1}}-1\right) \\
c & =k r_{0}\left(I_{a}+I_{b}+2 \pi I_{c}\right) \\
I_{a} & =\int_{0}^{1} d z \sin ^{2} \pi z \Delta_{0}^{\prime}>0 \\
I_{b} & =\int_{0}^{1} d z \sin ^{2} \pi z \Delta_{2}^{\prime}>0 \\
I_{c} & =\frac{1}{2} \int_{0}^{1} d z \sin 2 \pi z \Delta_{2}>0 .
\end{aligned}
$$

\subsection{Shortcomings of the Amplitude Equation}

Equation (102) is unsatisfactory for a variety of reasons. First, there are no oscillatory solutions, so the rolls cannot oscillate, whatever the background salt profile. Second, all steady solutions are unstable. In this limit, the salt is impotent and cannot stifle the runaway growth of the convective cells; the salt field just goes along for the ride until it is completely mixed up by the convection. In retrospect, one can trace the runaway growth to the absence of a mean temperature field that is generated at higher order by the perturbations. This mean temperature field is the culprit that typically saturates the growth of the convection [13]. In the language of amplitude equations, the mean temperature field manifests itself as a cubic nonlinearity with a negative coefficient. But do not be misled - this term is contained within the small $R_{s}$ expansion used here, but we have not gone to high enough order to see it. If we reconstitute the equations, or do a Galerkin projection, we could bring it back in. However, in this scaling a damping, nonlinear term would be asymptotically small compared to the lower order terms, so one cannot say that these terms would squelch the growth of the rolls and at the same time claim that the results are asymptotic. Some other perturbative scheme 
would have to be employed to achieve this. One possibility is to treat the equations as a boundary layer problem [15], and assume that all of the salt is confined to a thin layer, similar to the approach taken by Proctor [14] in his study of steady, subcritical thermohaline convection. A scheme that brings in the damping nonlinearities and retains an arbitrary salt profile in a thin boundary remains as future work.

Another way to understand what happened in this expansion is to consider the normal form equations for a Takens-Bogdanov bifurcation in a system with translational and reflectional symmetry, i.e. a system with $O(2)$ symmetry. The doubly-diffusive Boussinesq equations have the $O(2)$ symmetry, so we can consult the work of Dangelmayr and Knobloch [16] and write down the normal form equations immediately:

$$
\begin{aligned}
& \partial_{t} B=A \\
& \partial_{t} A=\mu B+\nu A+\left(e|B|^{2}+f|A|^{2}+g(B \bar{A}+\text { c.c. })\right) B+h|B|^{2} A .
\end{aligned}
$$

In the context of the small $R_{s}$ amplitude equation (102), the normal form tells us that our coefficients $f, g, h$ are all zero. Thus, the small $R_{s}$ expansion has picked out a highly degenerate Takens-Bogdanov point, a point at which no oscillatory solutions exist. The degeneracy of the TB expansion here is a direct result of all the physics we have thrown out by looking at such a weak salt coupling to the Rayleigh-Bénard convection. If we had launched our analysis from the Takens-Bogdanov point on the original, microscopic equations, we would have picked up the missing terms from the normal form (109) and obtained oscillatory solutions. In addition, we would obtain a cubic nonlinearity with a negative coefficient, which is necessary for the saturation of the steady solutions. As is outlined in [11], however, it is not clear how to bring in the presently missing normal form terms in an asymptotically valid way. They will still be of higher order than the terms in equation (102). Still, it is noteworthy that an arbitrary, monotonically decreasing salt profile has such a negligible effect, even if this limit of small $R_{s}$ is restrictive.

\section{Numerics for the Linear Problem and Larger $R_{S}$ Regimes}

We now turn to numerical results for some insight into what happens in a regime of larger $R_{s}$. All numerical work on the linearized equations (7)-(9) was done with a basic state $S_{b}=-\tanh (b z)$, where $b$ was chosen so that the $\tanh$ profile reached its extremal values (to within double precision) outside a thin region near the center that encompassed roughly twenty percent of the layer. Special care was taken to ensure that the sharp salinity gradient was adequately resolved by clustering points near the center of the layer. The background salinity profile is shown in figure (3).

It is expected that if $R_{s}$ is too large, the convection will have a difficult time punching through the layer. Thus, the first bifurcation (lowest $R_{T}$ ) to a marginal eigenvalue should look roughly like 2-Layer convection if the Lewis number is not 


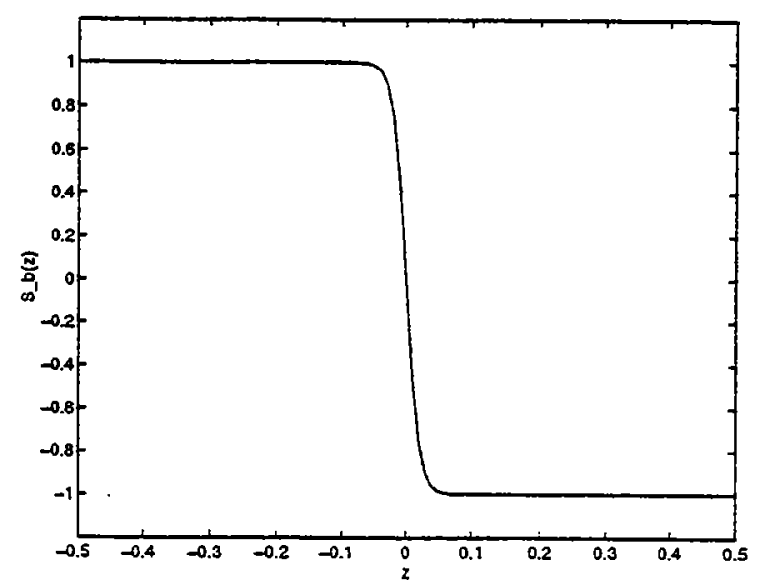

Figure 3: Background Salt Profile Used In The Numerical Runs

compensatingly small. It is important to be aware that for such large $R_{\varepsilon}$ regimes, the eigenfunctions are greatly distorted, so when one speaks of "2-Layer convection," it should be interpreted as an approximate description of the dynamical structure.

It is observed that no matter how large $R_{s}$ is, one can always tune the Lewis number to be so small that the first bifurcation is oscillatory, and for certain tunings a variety of marginal curves can collide. This is analagous to what was seen in the linear profile, but we shall see below that in a larger $R_{s}$ regime the dynamics of modal competition are fundamentally different. Physically, the rough picture is that modes wanting 1Layer convection and modes wanting 2-Layer convection are competing; each having an equal say at parameter values corresponding to a resonance. Since the latter type of dynamics is suggestive of an interface on the verge of destruction, let us concentrate on this.

\subsection{1:1 Resonance}

When a system is said to have a 1:1 Resonance [17], it means that two modes of the same wavelength are competing at marginality. In terms of the linear dynamics, it manifests itself as a collision of the neutral curves, whereby they get closer and closer as certain parameters are tuned. In this case, the NRK (Newton-Raphson-Kantorovich) solver used here [18] shows that for a fixed $R_{s}$ (10000 for the figures below), one can tweak the Lewis number so that two steady branches collide and pinch off, ultimately connected by a Hopf branch. This is the paradigm of 1:1 resonance, as is sketched in figure (4).

Some numerical runs are presented in figure (5). This figure shows two steady branches, the higher one corresponding to 1-Layer tendencies and the lower one to 2-Layer tendencies, each connected by a dotted Hopf branch. As $\tau$ is increased from 0.3 to 0.6 , the curves collide and pinch off. The left branch shot off to infinity and could not be latched onto in the numerical runs performed. This run is presented in 


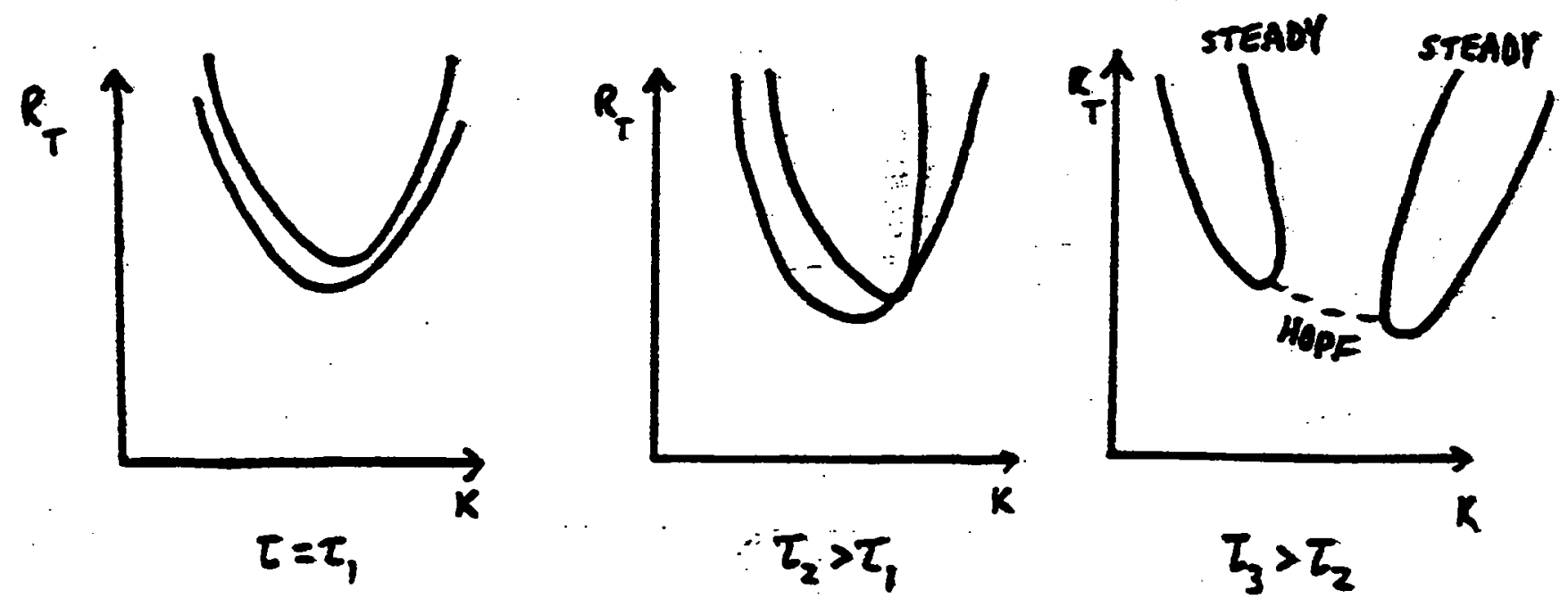

Figure 4: 1:1 Resonance and the pinching off of marginal curves.

figure (6). The eigenfunctions of the veritical velocity, temperature, and salinity for the upper and lower steady branches are plotted in figures (7) and (8).

\subsection{Normal Forms For a 1:1 Resonance}

Given a 1:1 resonance in a system with $O(2)$ symmetry, we can write down the normal form amplitude equations immediately [1i]. With $A$ and $B$ corresponding to the two competing modes of nearly equal wavelength we have

$$
\begin{aligned}
\partial_{t} A= & \mu A+\chi B-\left(a_{1}|A|^{2}+b_{1}|B|^{2}+c_{1} A \bar{B}\right) A \\
& -\left(b_{2}|A|^{2}+a_{2}|B|^{2}+c_{2} \bar{A} B\right) B \\
\partial_{t} B= & \nu B+\lambda A-\left(a_{4}|A|^{2}+b_{4}|B|^{2}+c_{4} A \bar{B}\right) A \\
& -\left(b_{3}|A|^{2}+a_{3}|B|^{2}+c_{3} \bar{A} B\right) B,
\end{aligned}
$$

where the set of coefficients $\left\{a_{i}, b_{i}, c_{i}\right\}$ are parameter dependent functions to be determined according to the particular problem. To obtain the coefficients, one must perform a center manifold reduction on the system. However, many things can be said from knowing the normal form. Among the most interesting is that resonant phase interactions manifest themselves in the linear terms, so it is possible for the basic state solution to switch from a steady bifurcation to a Hopf bifurcation at the point of resonance. If the resonance was weaker [17], a 1:2 resonance for instance, there is no phase coupling at lowest order, and the phase of either mode remains undetermined in the weakly nonlinear limit. To ascertain what types of solutions are dynamically preferable at any point in parameter space, the specific coefficients of (110) must be worked out, which is a matter left for future work. 


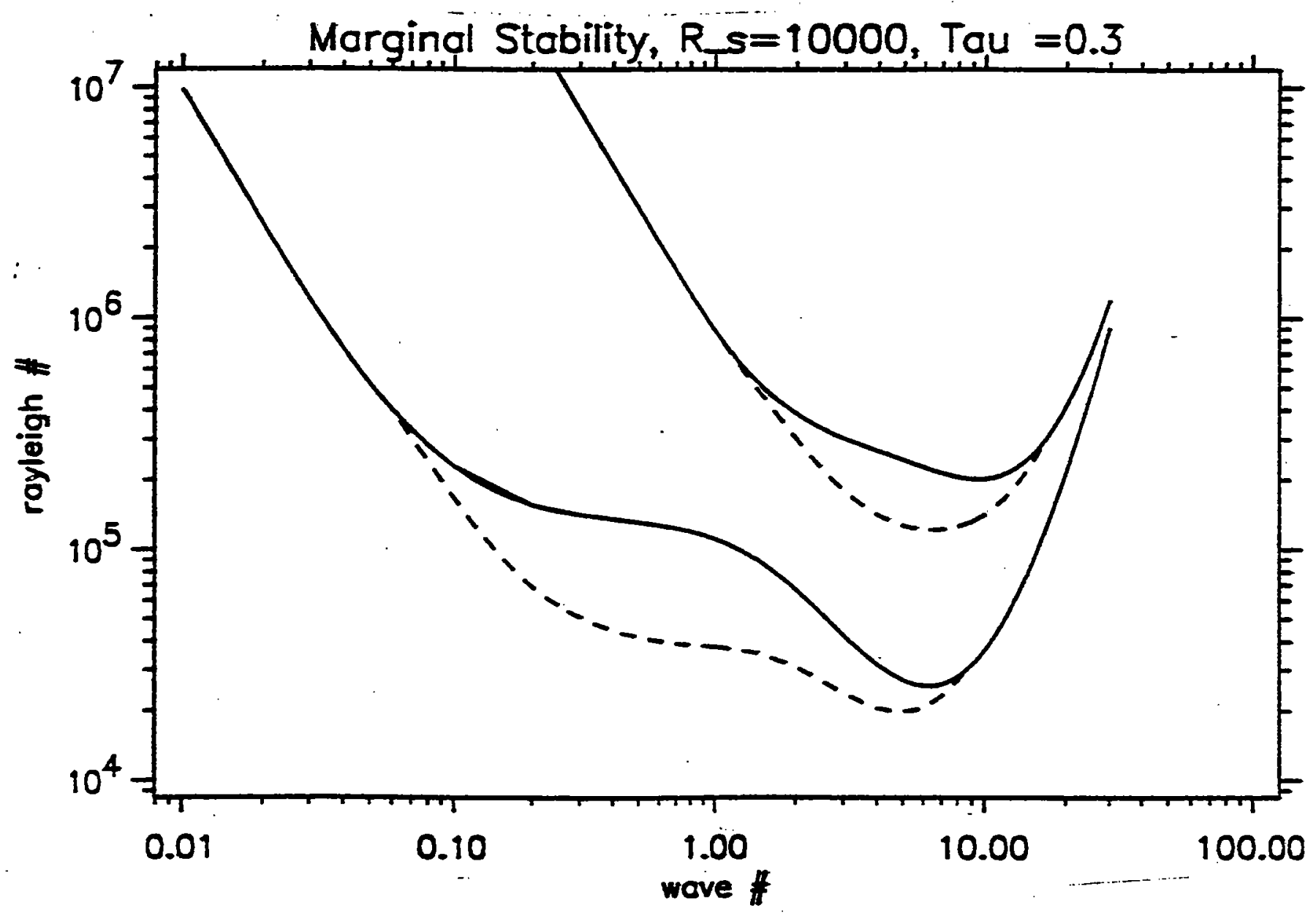

Figure 5: Marginal Curves Close To Pinching. Solid lines are steady branches, Dotted lines are Hopf. 

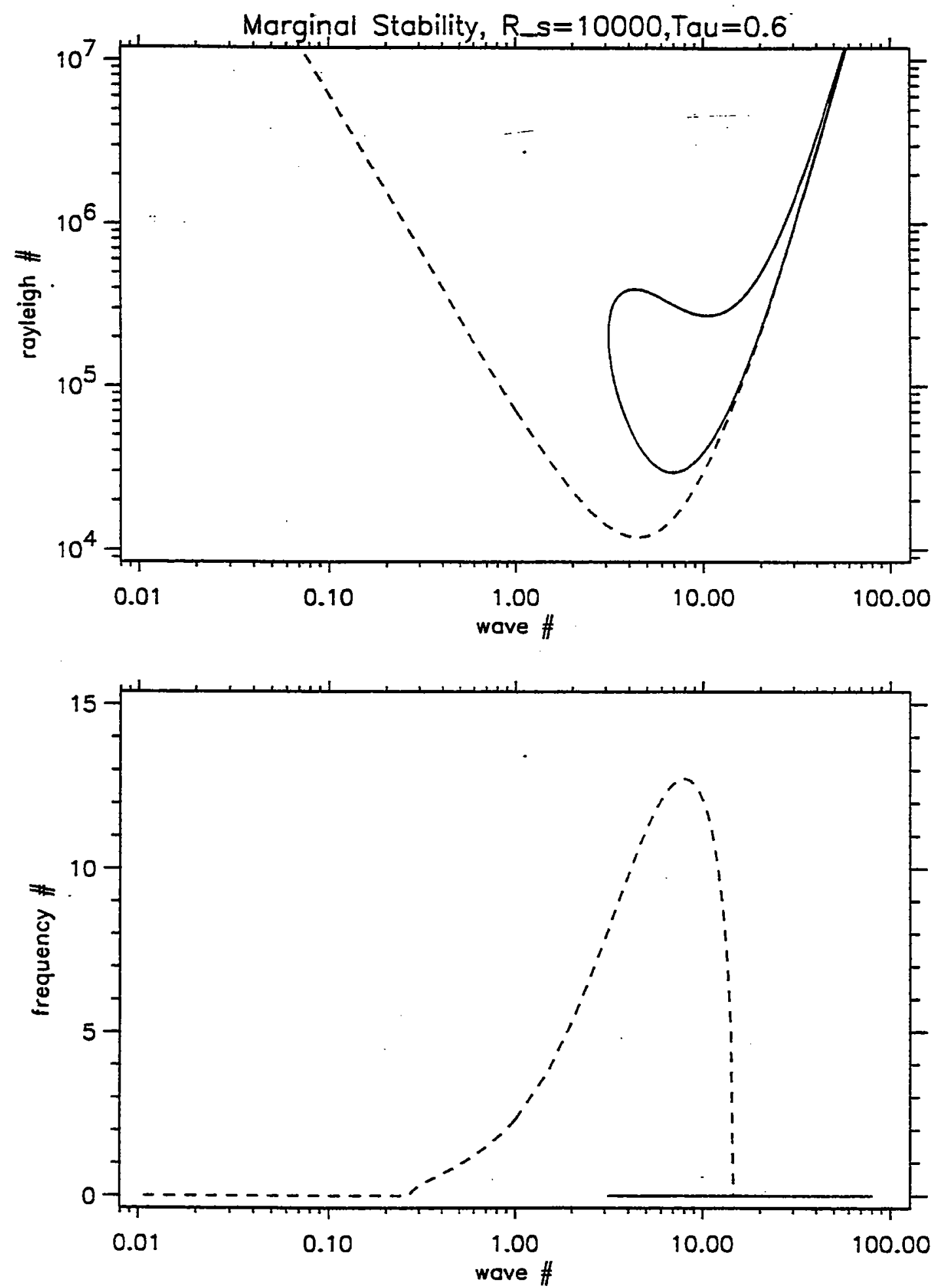

Figure 6: Pinching of the marginal curves, $\tau=0.6$, Dotted branch=Hopf , Solid branch $=$ Steady 

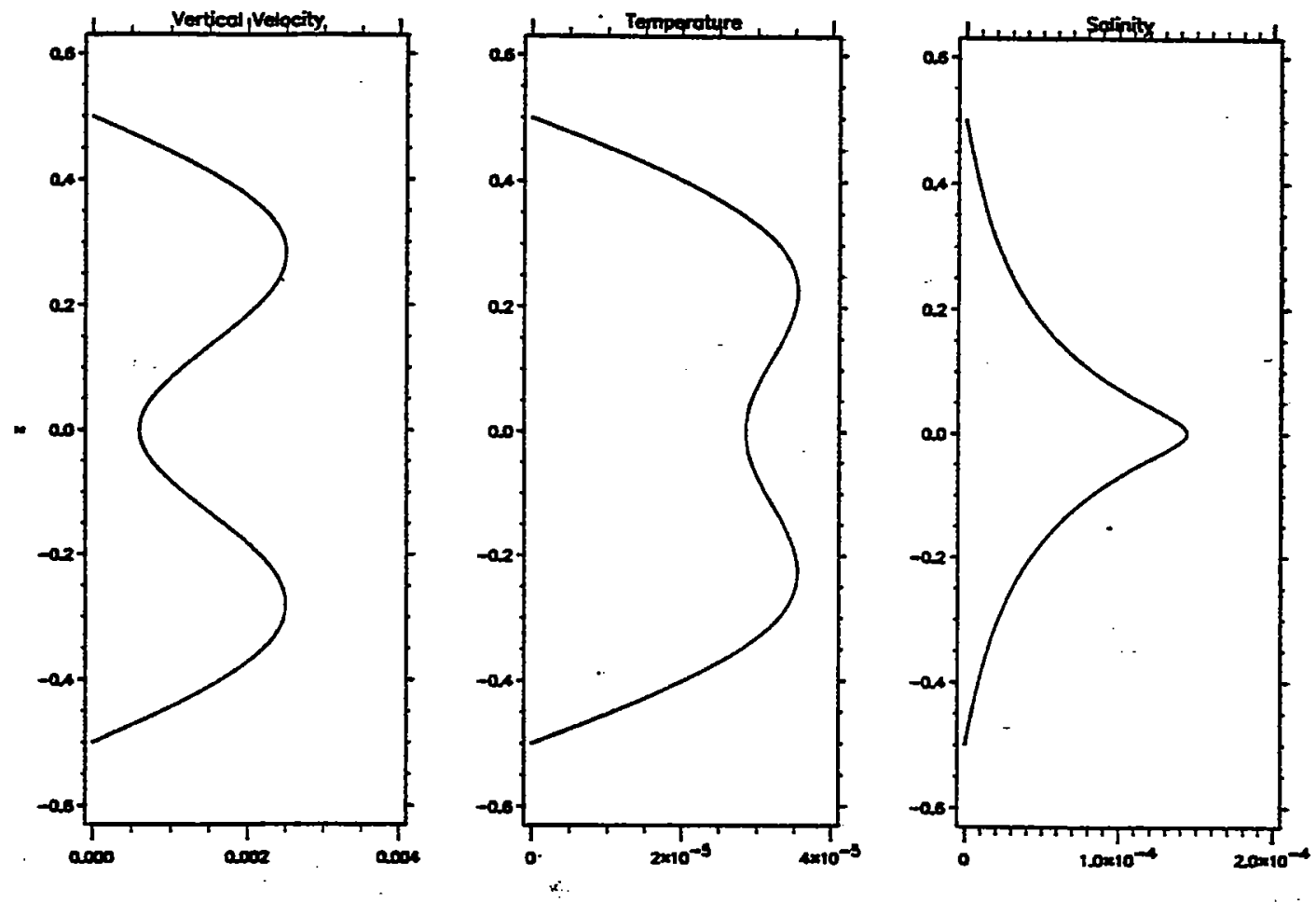

Figure 7: Eigenfunctions for the lower, steady branch, $\tau=0.6$
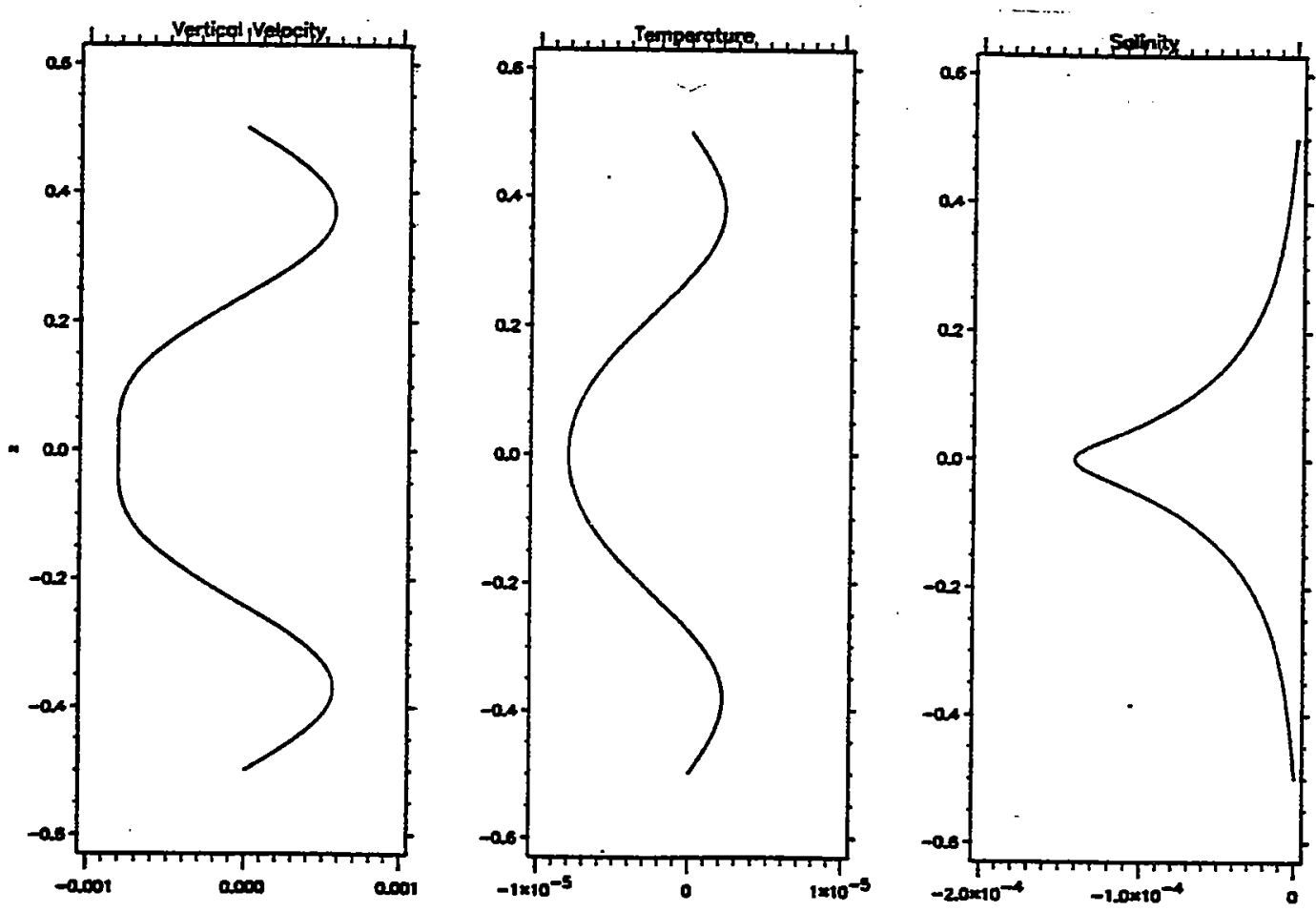

Figure 8: Eigenfunctions for the upper, steady branch, $\tau=0.6$ 


\section{Discussion}

We have seen that a doubly-diffusive fluid with a sharp gradient region in the more slowly diffusing component can exhibit fundamentally different behaivor than the well studied linear salt profile scenario. Though the actual laboratory experiments to date involve highly nonlinear processes, 1:1 resonant interaction of modes favoring 1-Layer convection and 2-Layer convection appears worthy of further study as a paradigm for interfacial obliteration in fluids amenable to weakly nonlinear analysis. Whether this situation is realizable in a geophysical or laboratory context is unknown to the author at this time. In a small $R_{s}$ limit with an arbitrary, monotonically decreasing background salt profile, it was seen that unless mean temperature field effects generated by the perturbations are accounted for, the salt is unable to influence the runaway growth of full layer convection. Work currently in progress involves bringing in these saturating effects in such a way that an arbitrary background salt field can be retained, so that the effects of a sharp gradient region can be more thoroughly investigated. One possibility is to return to the classic scalings of Malkus and Veronis, while confining the salt to a thin boundary layer so that a similar analysis to that of section 5 is applicable. In this case, however, it is hoped that parameter dependences can be introduced into the cubic term of the amplitude equation (102) so that a Takens-Bogdanov analysis captures a richer spectrum of solutions than allowed in the present work.

\section{Acknowledgements}

The author would like to thank everybody present at the 1996 Geophysical Fluid Dynamics Summer Program for providing the most exciting work environment I have ever encountered. Special thanks goes to the following people: Keith Julien, for providing me a first dose of a cure for numerical incompetency, for his invaluable advice, for his great humor, and for his cannon arm at third base (Nobody strikes fear into the hearts of distant softball spectators like Dr.Julien.) Bill Young, for his brilliant suggestions. Neil Balmforth, for his tireless effort in keeping me and the other fellows on track, and also for his brilliant suggestions. Andy Woods, for suggesting the research problem (you too Keith). Michael Shelley, for his...jokes. My advisor, Edward Spiegel, for finally letting me win at pool. Steve Meacham, our esteemed director, for helping me through some rough times. Joe Keller, Lou Howard, Gorgeous George Veronis, Barry Ruddick, Willem Malkus, Stewart Turner, and the rest of the sages for their inspiration. And finally the other fellows, who kept me sane. "ok..."

\section{References}

[1] Chandrasekhar, S., (1961) Hydrodynamic and Hydromagnetic Stability, Dover Publications Inc., New York. 
[2] Veronis, G., (1965), "On Finite Amplitude Instability In Thermohaline Convection," J.Mar.Res. 23, pp.1-17.

[3] Moore, D.W. and Spiegel, E.A., (1966) "A Thermally Excited Non-linear Oscillator," Astrophys.J., 143, pp.871-887.

[4] Shirtcliffe, T.G.L., (1967) "Thermosolutal Convection: Observation of an Overstable Mode," Nature, Lond. 213, pp.489-490.

[5] Turner, J.S., (1968) "The Behaivor of a Stable Salinity Gradient Heated From Below," J.Fluid.Mech. 33, pp.183-200.

[6] Huppert, H.E., and Turner, J.S., (1981) "Double-diffusive Convection," J.Fluid Mech. 106, pp.555-556.

[7] Biello, J., (1996) These proceedings.

[8] Joseph, D.D., and Renardy, Y.Y., (1993) Fundamentals of Two-Fluid Dynamics, Part 1: Mathematical Theory and Applications, Springer-Verlag.

[9] Huppert,H.E. and Moore,D.R., (1976) "Nonlinear Double-Diffusive Convection," J.Fluid.Mech. 78, pp.821-854.

[10] Coullet,P. and Spiegel,E.A., (1983) "Amplitude Equations For Systems With Competing Instabilities," Siam J.Appl.Math, 43, pp.776-821.

[11] Balmforth, N., and Julien, K., (1996) These proceedings.

[12] Private Communication with Bill Young at WHOI, (1996)

[13] Malkus, W.V.R., and Veronis, G., (1958) "Finite Amplitude Cellular Convection," J.Fluid Mech. 4, pp.225-260.

[14] Proctor, M.R.E., (1981) "Steady Subcritical Thermohaline Convection," J.Fluid Mech. 105, pp.507-521.

[15] Private communication with Bill Young and Neil Balmforth at WHOI, (1996)

[16] Dangelmayr, G. and Knobloch, E., (1987) "The Takens-Bogdanov Bifurcation With O(2) Symmetry," Phil.Trans.R.Soc.Lond.A 322, pp.243-279.

[17] Julien, K.A., (1991) "Strong Spatial Interactions With 1:1 Resonance: A ThreeLayer Convection Problem," Nonlinearity 7, pp.1655-1693.

[18] The author is indebted to Keith Julien for supplying the NRK code used in this work. 


\title{
Long-wave Instability in Double-diffusive Marangoni Convection
}

\author{
Jean-Luc Thiffeault
}

\section{Introduction}

In fluid convection with a free surface at the top it is sometimes the case that surface tension effects become important. For instance in conditions of microgravity (e.g. crystal growth in a space shuttle when a free surface is present) the gravitational forces are negligible [1, 2]; Or, as in Bénard's original experiments, the layer of fluid is very thin and the convection is surface-tension dominated [3, 4]. We consider here the case of two diffusing quantities (for convenience referred to as heat and salt) with those surface tension effects taken into account. We shall be particularly interested in the diffusive regime where overstability occurs. We will derive a small amplitude long-wave planform equation for the case where fixed fluxes of heat and salt are imposed at the top and bottom boundaries, and find a long-wave equation capturing the bifurcation structure of a codimension two Takens-Bogdanov point. We discuss the possibility of capturing a larger portion of the bifurcation by adding surface deformation effects to tune out resonant nonlinear terms.

\section{Governing Equations of the System}

The equations describing the temporal evolution of the system are the usual ones for two-dimensional thermohaline convection in the Boussinesq approximation:

$$
\begin{aligned}
\frac{1}{\sigma}\left(\partial_{t} \nabla^{2} \Psi+\left\{\Psi, \nabla^{2} \Psi\right\}\right) & =R_{T} \partial_{x} \Psi-R_{S} \partial_{x} \Psi+\nabla^{4} \Psi \\
\partial_{t} T+\{\Psi, T\} & =\partial_{x} \Psi+\nabla^{2} T \\
\partial_{t} S+\{\Psi, S\} & =\partial_{x} \Psi+\tau \nabla^{2} S .
\end{aligned}
$$

Here $\Psi$ is the stream function, $T$ is the temperature, and $S$ is the salinity. Both $T$ and $S$ are deviations from linear profiles. The dimensionless constants used are the temperature and salt Rayleigh numbers

$$
R_{T}=\frac{g \alpha \bar{T}_{z} d^{4}}{\nu \kappa_{T}}, \quad R_{S}=\frac{g \beta \bar{S}_{z} d^{4}}{\nu \kappa_{T}}
$$


and the Prandtl and Schmidt numbers

$$
\sigma=\frac{\nu}{\kappa_{T}}, \quad \tau=\frac{\kappa_{S}}{\kappa_{T}}
$$

where $x$ is the horizontal direction, $z$ the vertical, $g$ is the acceleration due to gravity, $\alpha$ and $\beta$ are the coefficients of thermal expansions for the heat and salt, $\bar{T}_{z}$ and $\bar{S}_{z}$ are the mean gradients of heat and salt, $d$ is the vertical thickness of the fluid layer, $\nu$ is the viscosity of the fluid, $\kappa_{T}$ and $\kappa_{S}$ are the coefficients of heat and salt diffusion. The Jacobian is

$$
\{F, G\}=\partial_{x} F \partial_{z} G-\partial_{x} G \partial_{z} F .
$$

The problem was nondimensionalised so that $t \sim d^{2} / \kappa_{T}, x, z \sim d, T \sim \bar{T}_{z} d, S \sim \bar{S}_{z} d$. When surface tension effects are not present, for $R_{T}<0$ and $R_{S}<0$ the system is in the fingering regime, whereas for $R_{T}>0$ and $R_{S}>0$ the system is in the diffusive regime. The onset of instability for the fingering regime is direct, and for the diffusive case it is oscillatory (overstability) [5].

The difference between normal thermohaline convection and its Marangoni counterpart comes from the boundary conditions. We consider an infinite layer of fluid bounded above and below. At the bottom of the fluid there is a rigid plate with fixed flux boundary conditions on the heat and salt:

$$
\Psi=\partial_{z} \Psi=\partial_{z} T=\partial_{z} S=0, \quad z=0,
$$

whereas at the top there is a free surface having a surface tension with a linear temperature and salinity dependence

$$
\sigma=\sigma_{0}-\gamma_{T}\left(T-T_{0}\right)-\gamma_{S}\left(S-S_{0}\right),
$$

so that there is a stress exerted at the top of the fluid $[6,7]$. The boundary conditions at the top surface are thus

$$
\begin{aligned}
\Psi & =\partial_{z} T=\partial_{z} S=0, \\
\nabla^{2} \Psi & =M_{T} \partial_{x} T+M_{S} \partial_{x} S, \quad z=1,
\end{aligned}
$$

where the temperature and salinity Marangoni numbers are defined as

$$
M_{T}=\frac{\gamma_{T} \bar{T}_{z} d^{2}}{\rho \nu \kappa_{T}}, \quad M_{S}=\frac{\gamma_{S} \bar{S}_{z} d^{2}}{\rho \nu \kappa_{T}},
$$

and $\rho$ is the density of the fluid. The quantities $\gamma_{T, S}$ can have either sign depending on the diffusing components, and in particular for the heat-salt system

$$
\gamma_{T}=0.157 \mathrm{dyn} \mathrm{cm}^{-1} \mathrm{~K}^{-1}, \quad \gamma_{S}=-0.367 \mathrm{dyn} \mathrm{cm}^{-1} \mathrm{wt}^{-1} \text {. }
$$

We neglect here possible deformation of the surface (for inclusion of this effect see [8, $9,10,11])$. At lowest order there it is possible to use the crispation number to take surface deformation effects into account, which leads to a long wave instability. We also find such instabilities here, but as a result of the fixed heat and salt flux condition in Eqs. 7 and 9, which distinguishes this work from previous ones. $[6,12]$ as 


\section{Linear Stability}

The system of equations 1-3 can be written with a linear and a nonlinear part

$$
\mathcal{L} \Psi=\mathcal{N}(\Psi, \Psi),
$$

where the state vector is

$$
\Psi=\left(\begin{array}{c}
\Psi \\
T \\
S
\end{array}\right)
$$

the linear operator is given by

$$
\mathcal{L}=\left(\begin{array}{ccc}
\nabla^{4}-\frac{1}{\sigma} \partial_{t} \nabla^{2} & R_{T} \partial_{x} & -R_{S} \partial_{x} \\
\partial_{x} & \nabla^{2}-\partial_{t} & 0 \\
\partial_{x} & 0 & \tau \nabla^{2}-\partial_{t}
\end{array}\right)
$$

and the nonlinear terms by

$$
\mathcal{N}(\Psi, \Psi)=\left(\begin{array}{c}
\left\{\Psi, \nabla^{2} \Psi\right\} \\
\{\Psi, T\} \\
\{\Psi, S\}
\end{array}\right)
$$

We now look at the linear problem. The nonlinear terms $\mathcal{N}$ drop out and we can focus our attention on only one Fourier mode in the $x$ direction with wavenumber $a$, so we write

$$
\Psi=A e^{\mathrm{i}(\alpha x \pm \omega t)}\left(\begin{array}{c}
\psi_{ \pm}(z) \\
-\mathrm{i} \Theta_{ \pm}(z) \\
-\mathrm{i} \Sigma_{ \pm}(z)
\end{array}\right)+\text { c.c. }
$$

where we separate the $x$ and $z$ dependence and in doing so define the complex functions $\psi, \Theta$, and $\Sigma$. We have set the real part of the time dependence to zero to look for marginal modes. We then have to solve the linear set of equations

$$
\begin{aligned}
\left(\left(D^{2}-a^{2}\right) \mp \mathrm{i} \frac{\omega}{\sigma}\right)\left(D^{2}-a^{2}\right) \psi_{ \pm} & =-a R_{T} \Theta_{ \pm}+a R_{S} \Sigma_{ \pm} \\
\left(\left(D^{2}-a^{2}\right) \mp \mathrm{i} \omega\right) \Theta_{ \pm} & =a \psi_{ \pm} \\
\left(\tau\left(D^{2}-a^{2}\right) \mp \mathrm{i} \omega\right) \Sigma_{ \pm} & =a \psi_{ \pm} .
\end{aligned}
$$

We vary $a$ and solve numerically for $R_{T}$ and $\omega$ using a code written by N. Baker and D. Moore implementing the Newton-Raphson-Kantorovich method. The marginal stability curve and Hopf frequency plotted against $a$ are shown in Figure 1 for the diffusive case $\left(R_{T}>0, R_{S}>0\right)$. There is an instability at $a=0$, corresponding to the diffusive instability. There is also another instability at nonzero $a$, a mode driven by surface tension. It can be seen from the figure that by varying parameters it is possible for these two instabilities to come in at the same critical Rayleigh number $R_{T c}$. 


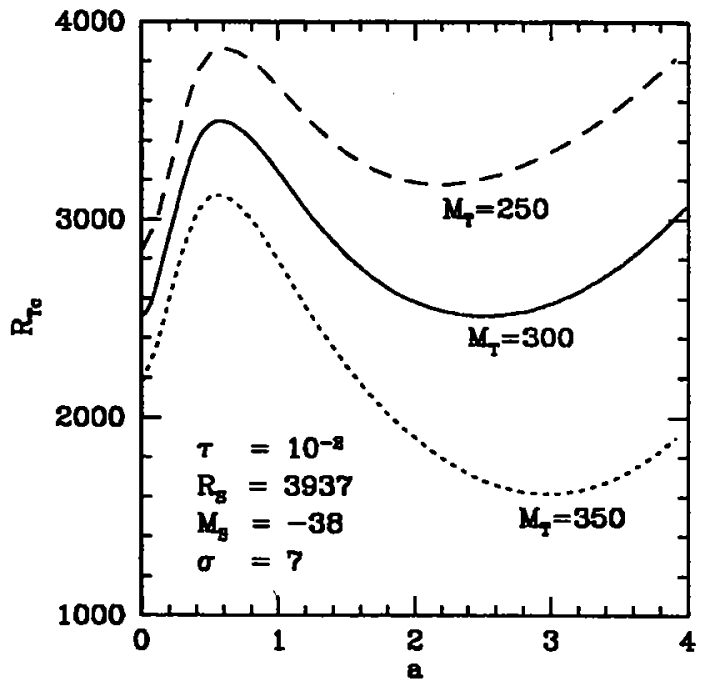

(a)

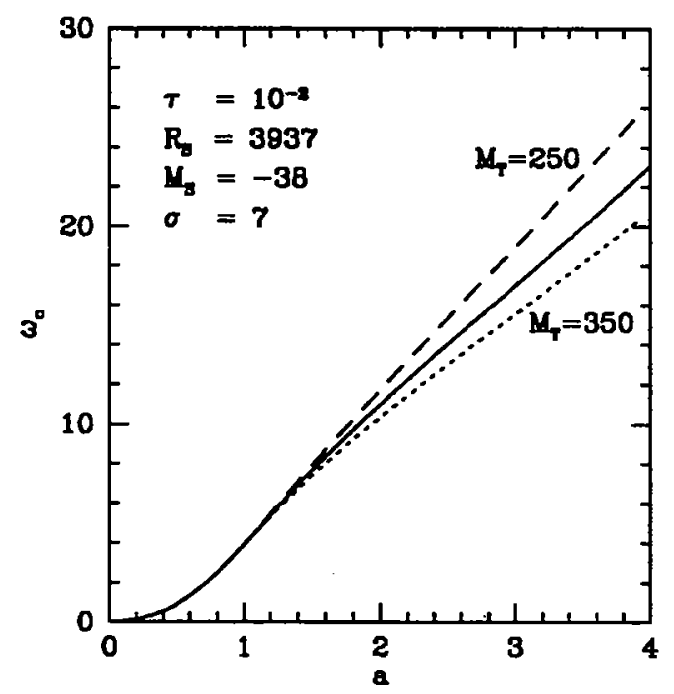

(b)

Figure 1: Marginal stability curve (a) and Hopf frequency (b) for the diffusive case. $M_{T}=300$ represents the case for which both the $a=0$ (double-diffusive) and nonzero $a$ (surface tension) instabilities linearly come in together.

The density ratio $R_{\rho}$ is defined to be the density gradient of the stabilizing component over the destabilizing one, which for the diffusive case is

$$
R_{\rho}=\frac{\beta \bar{S}_{z}}{\alpha \bar{T}_{z}}=\frac{R_{S}}{R_{T}} .
$$

All the curves in Figure 1 have $R_{\rho}>1$ and correspond therefore to statically stable situations in the absence of surface tension.

\section{Finite Wavenumber Instability}

Figure 2 shows the eigenfunctions of the system for the solid line in Figure 1. Note the real part of the salinity eigenfunction has an extremum below $z=1$. This may be due to the much lower diffusivity of salt.

Some idea of the development of the instability at finite wavenumber may be gained by the usual kind of weakly nonlinear theory. This must be done numerically, at least partly, for this instability and requires us to evaluate the adjoint to the linear system Eqs. 17-19. We mention here that the equations for the adjoint problem for this system are straightforward to obtain. Specifically, one just takes the transpose of the matrix Eq. 14 and let $\partial_{x} \rightarrow-\partial_{x}$ and $\partial_{t} \rightarrow-\partial_{t}$. However, a complication arises because of the surface terms when one writes

$$
\int_{0}^{1} \Psi^{\dagger} \mathcal{L} \Psi d z=\text { surface terms }+\int_{0}^{1} \mathcal{L}^{\dagger} \Psi^{\dagger} \Psi d z
$$



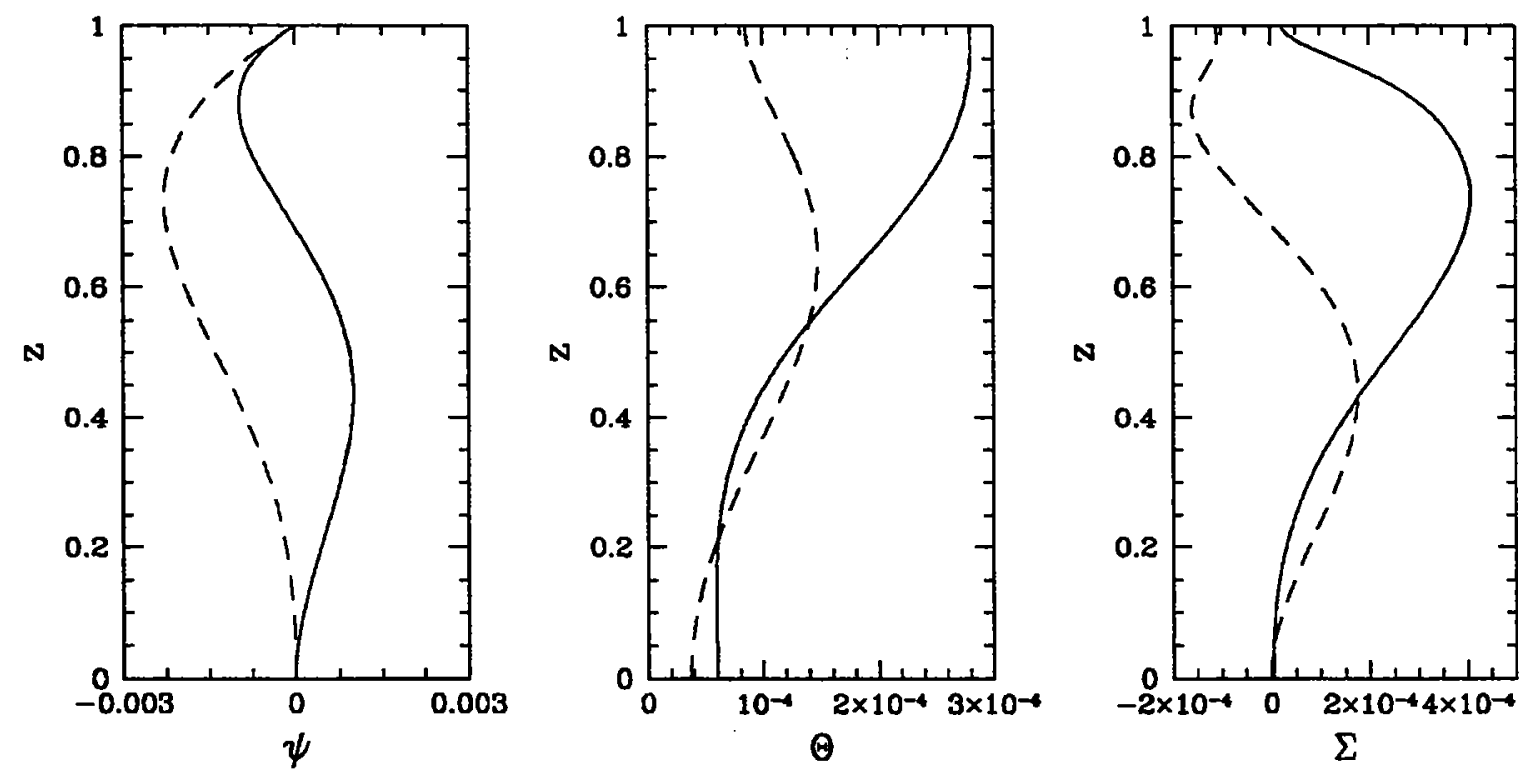

Figure 2: Linear eigenfunctions of $\psi, \Theta$, and $\Sigma$ at the $a=2.5$ minimum of the solid curve $\left(M_{T}=300\right)$ in Figure 1. The solid line is the real part and the dashed line the imaginary part. The Hopf frequency is $\omega=14.1$.

We will not show this in detail here, but in order for the surface terms to vanish, one must take for the boundary conditions on the adjoint

$$
\Psi^{\dagger}=D \Psi^{\dagger}=D T^{\dagger}=D S^{\dagger}=0, \quad z=0,
$$

at the bottom surface and

$$
\begin{aligned}
\Psi^{\dagger} & =0 \\
D T^{\dagger} & =M_{T} \partial_{x} D \Psi^{\dagger}, \\
D S^{\dagger} & =M_{S} \partial_{x} D \Psi^{\dagger}, \quad z=1
\end{aligned}
$$

at the top.

We can then use the solution to the adjoint problem to derive an amplitude equation for the nonzero $a$ instability, calculating the coefficients numerically. This equation is valid for cases like the dotted curve $\left(M_{T}=350\right)$ in Figure 1 , where the nonzero wavenumber instability occurs at a smaller thermal Rayleigh number. The amplitude equation obtained is, as expected, a complex Ginzburg-Landau equation:

$$
\partial_{T} A=a_{1} A+a_{2} A_{X X}-a_{3}|A|^{2} A .
$$

The analysis of this equation, and its coupling with an equation for the long-wave instability, will be the topic of later work. 


\section{Long-Wave Expansion}

We now focus our attention on the instability at $a=0$ of the kind seen in Figure 1 . We shall assume we are in a parameter range such that the marginal stability curve looks like the dashed line $\left(M_{T}=250\right)$ in Figure 1 , so that the long wave mode goes unstable before the surface-tension driven instability at nonzero $a$. In the usual manner we assume that there is an expansion in a small parameter $\varepsilon$ for the spatial and temporal dependence [13]

$$
\begin{aligned}
& \partial_{x}=\varepsilon^{1 / 2} \partial_{X} \\
& \partial_{t}=\varepsilon \partial_{T_{1}}+\varepsilon^{2} \partial_{T_{2}}
\end{aligned}
$$

The absence of a zeroth order $x$ derivative reflects the fact that this instability occurs at $a=0$. We define $\phi$ by

$$
\psi(z)=\varepsilon^{1 / 2} \phi_{X}(z),
$$

so that all the equations contain only integer powers of $\varepsilon$. The remaining variables are also expanded in powers of $\varepsilon$, assuming their amplitudes are small:

$$
\begin{array}{r}
\phi\left(X, z, T_{1}, T_{2}\right)=\varepsilon \phi_{1}+\varepsilon^{2} \phi_{2}+\ldots \\
T\left(X, z, T_{1}, T_{2}\right)=\varepsilon T_{1}+\varepsilon^{2} T_{2}+\ldots \\
S\left(X, z, T_{1}, T_{2}\right)=\varepsilon S_{1}+\varepsilon^{2} S_{2}+\ldots,
\end{array}
$$

and the control parameters

$$
\begin{aligned}
R_{T} & =R_{T 0}+\varepsilon R_{T 1}+\varepsilon^{2} R_{T 2}+\ldots \\
R_{S} & =R_{S 0}+\varepsilon R_{S 1}+\varepsilon^{2} R_{S 2}+\ldots \\
M_{T} & =M_{T 0}+\varepsilon M_{T 1}+\varepsilon^{2} M_{T 2}+\ldots \\
M_{S} & =M_{S 0}+\varepsilon M_{S 1}+\varepsilon^{2} M_{S 2}+\ldots
\end{aligned}
$$

We choose not to expand $\tau$ and $\sigma$. The operators $\mathcal{L}$ and the nonlinear terms $\mathcal{N}$ also have an expansion

$$
\begin{aligned}
\mathcal{L} & =\mathcal{L}_{0}+\varepsilon \mathcal{L}_{1}+\varepsilon^{2} \mathcal{L}_{2}+\ldots \\
\mathcal{N} & =\varepsilon^{2} \mathcal{N}_{2}+\varepsilon^{3} \mathcal{N}_{3}+\ldots
\end{aligned}
$$

where

$$
\begin{aligned}
\mathcal{L}_{0} & =\left(\begin{array}{ccc}
D^{4} \partial_{X} & R_{T 0} \partial_{X} & -R_{S 0} \partial_{X} \\
0 & D^{2} & 0 \\
0 & 0 & D^{2}
\end{array}\right), \\
\mathcal{L}_{1} & =\left(\begin{array}{cccc}
2 D^{2} \partial_{X}^{3}-\sigma^{-1} \partial_{T_{1}} D^{2} \partial_{X} & R_{T 1} \partial_{X} & -R_{S 1} \partial_{X} \\
& \partial_{X}^{2} & \partial_{X}^{2}-\partial_{T_{1}} & 0 \\
\partial_{X}^{2} & 0 & \tau \partial_{X}^{2}-\partial_{T_{1}}
\end{array}\right),
\end{aligned}
$$




$$
\begin{aligned}
& \mathcal{L}_{2}=\left(\begin{array}{ccc}
\partial_{X}^{5}-\sigma^{-1}\left(\partial_{T_{2}} D^{2} \partial_{X}+\partial_{T_{1}} \partial_{X}^{3}\right) & R_{T_{2}} \partial_{X} & -R_{S_{2}} \partial_{X} \\
0 & -\partial_{T_{2}} & 0 \\
0 & 0 & -\partial_{T_{2}}
\end{array}\right), \\
& \mathcal{N}_{2}=\left(\begin{array}{c}
\sigma^{-1}\left(\phi_{0 X X} D^{3} \phi_{0 X}-D \phi_{0 X} D^{2} \phi_{0 X X}\right) \\
\phi_{0 X X} D \Theta_{0}-D \phi_{0 X} \Theta_{0 X} \\
\phi_{0 X X} D \Sigma_{0}-D \phi_{0 X} \Sigma_{0 X}
\end{array}\right), \\
& \mathcal{N}_{3}=\left(\begin{array}{c}
\sigma^{-1}\left(\phi_{0 X X} D^{3} \phi_{1 X}-D \phi_{0 X} D^{2} \phi_{1 X X}+\phi_{1 X X} D^{3} \phi_{0 X}-D \phi_{1 X} D^{2} \phi_{0 X X}\right) \\
\phi_{0 X X} D \Theta_{1}-D \phi_{0 X} \Theta_{1 X}+\phi_{1 X X} D \Theta_{0}-D \phi_{1 X} \Theta_{0 X} \\
\phi_{0 X X} D \Sigma_{1}-D \phi_{0 X} \Sigma_{1 X}+\phi_{1 X X} D \Sigma_{0}-D \phi_{1 X} \Sigma_{0 X}
\end{array}\right),
\end{aligned}
$$

where we are using a slightly modified state vector with $\phi$ in the first slot instead of $\psi$ :

$$
\Phi\left(X, z, T_{1}, T_{2}\right)=\left(\begin{array}{c}
\phi \\
\Theta \\
\Sigma
\end{array}\right) .
$$

The boundary conditions at the bottom rigid surface are

$$
\phi_{X}=D \phi_{X}=D \Theta=D \Sigma=0, \quad z=0 ;
$$

and at the top surface

$$
\begin{aligned}
& \phi_{X}=D \Theta=D \Sigma=0, \\
& D^{2} \phi_{X}=M_{T} \Theta_{X}+M_{S} \Sigma_{X}, \quad z=1 ;
\end{aligned}
$$

where the last boundary condition is different at each order since there is an expansion for $M_{T}$ and $M_{S}$.

For the case of fixed flux boundary conditions the solvability condition at each order is simply

$$
\int_{0}^{1} D^{2} \Theta d z=\int_{0}^{1} \tau D^{2} \Sigma d z=0 .
$$

We are now ready to proceed with the expansion.

\subsection{Order $\varepsilon^{0}$}

The zeroth order equations are simply $\mathcal{L}_{0} \Phi_{0}=0$ :

$$
\begin{aligned}
D^{4} \phi_{0 X} & =-R_{T 0} \Theta_{0 X}+R_{S_{0}} \Sigma_{0 X}, \\
D^{2} \Theta_{0} & =0, \\
\tau D^{2} \Sigma_{0} & =0 .
\end{aligned}
$$

The $\Theta_{0}$ and $\Sigma_{0}$ equations together with the boundary conditions tell us that these two quantities are independent of $z$. Hence we can integrate the first equation four times and solve for $\phi_{0 X}$, which after applying the boundary conditions becomes

$$
\begin{aligned}
\phi_{0 X} & =\frac{z^{2}\left(z^{2}-1\right)}{48}\left[\left((3-2 z) R_{T 0}+12 M_{T 0}\right) \Theta_{0 X}-\left((3-2 z) R_{S 0}-12 M_{S 0}\right) \Sigma_{0 X}\right] \\
& =-b_{T 0}(z) \Theta_{0 X}+b_{S 0}(z) \Sigma_{0 X},
\end{aligned}
$$


which defines $b_{T 0}(z)$ and $b_{S 0}(z)$. The solvability condition is automatically satisfied at this order, since the right-hand-side of the temperature and salinity equations vanishes.

\subsection{Order $\varepsilon^{1}$}

The first order system is $\mathcal{L}_{0} \Phi_{1}+\mathcal{L}_{1} \Phi_{0}=0$ :

$$
\begin{aligned}
D^{4} \phi_{1 X}= & -R_{T 1} \Theta_{0 X}+R_{S_{1}} \Sigma_{0 X}-R_{T 0} \Theta_{1 X}+R_{S 0} \Sigma_{1 X} \\
& -2 D^{2} \phi_{0 X X X}+\sigma^{-1} \partial_{T_{1}} D^{2} \phi_{0 X} \\
D^{2} \Theta_{1}= & -\phi_{0 X X}-\Theta_{0 X X}+\partial_{T_{1}} \Theta_{0} \\
\tau D^{2} \Sigma_{1}= & -\phi_{0 X X}-\tau \Sigma_{0 X X}+\partial_{T_{1}} \Sigma_{0}
\end{aligned}
$$

where we have used the fact that $D \Theta_{0}=D \Sigma_{0}=0$. We integrate the $\Theta_{1}$ and $\Sigma_{1}$ equations to get the solvability condition

$$
\begin{aligned}
\left\langle D^{2} \Theta_{1}\right\rangle & =-\left\langle\phi_{0 X X}\right\rangle-\Theta_{0 X X}+\partial_{T_{1}} \Theta_{0}=0 \\
\left\langle\tau D^{2} \Sigma_{1}\right\rangle & =-\left\langle\phi_{0 X X}\right\rangle-\tau \Sigma_{0 X X}+\partial_{T_{1}} \Sigma_{0}=0
\end{aligned}
$$

where

$$
\langle f\rangle \equiv \int_{0}^{1} f d z .
$$

Using the previous order result, we have

$$
\begin{aligned}
\left\langle\phi_{0 X X}\right\rangle & =\left\langle b_{S 0}\right\rangle \Sigma_{0 X X}-\left\langle b_{T 0}\right\rangle \Theta_{0 X X}, \\
& =\left(\frac{R_{S 0}}{320}-\frac{M_{S 0}}{48}\right) \Sigma_{0 X X}-\left(\frac{R_{T 0}}{320}+\frac{M_{T 0}}{48}\right) \Theta_{0 X X} .
\end{aligned}
$$

Then we can rewrite Eq. 34 as

$$
\partial_{T_{1}}\left(\begin{array}{c}
\Theta_{0} \\
\Sigma_{0}
\end{array}\right)=M\left(\begin{array}{c}
\Theta_{0 X X} \\
\Sigma_{0 X X}
\end{array}\right)
$$

where

$$
M=\left(\begin{array}{cc}
1-\left\langle b_{T 0}\right\rangle & \left\langle b_{S 0}\right\rangle \\
-\left\langle b_{T 0}\right\rangle & \tau+\left\langle b_{S 0}\right\rangle
\end{array}\right) .
$$

Now assume $M$ has a complex eigenvalues $\lambda_{ \pm}$. Then the solution to Eq. 37 is that the linear combinations of $\Theta_{0}$ and $\Sigma_{0}$ along the eigenvectors of $M$ (call them $\widetilde{\Theta}_{ \pm}$) evolve as

$$
\tilde{\Theta}_{ \pm}\left(X, T_{1}, T_{2}\right)=e^{\alpha X+\lambda_{ \pm} \alpha^{2} T_{1}} \widehat{\Theta}_{ \pm}\left(T_{2}\right),
$$

where $\alpha$ is a constant. We require that the real part of $\lambda_{ \pm}$vanish to avoid exponentially growing or decaying solutions at this order. If we assume that the $\lambda_{ \pm}$are real and that only $\lambda_{+}$vanishes, then we have a steady bifurcation; continuing with the long-wave expansion then leads to an equation as in [17]. If the imaginary part of $\lambda_{ \pm}$does 
not vanish, we get a diffusive-type linear O.D.E., thereby fixing the $X$ dependence of $\Theta_{0}$ and $\Sigma_{0}$. Thus we need to introduce another, longer length scale in order to get a partial differential equation and in the end we get a complex Ginzburg-Landau equation. This calculation simply amounts to rescaling the problem and doing an expansion like that in Section 4. Both the oscillatory and steady instabilities can be captured in a single long-wave equation if we can tune the system such that both the eigenvalues of $M$ vanish in real and imaginary part. This specially tuned system corresponds to the Takens-Bogdanov bifurcation point, where branches of oscillatory and steady instabilities meet [14].

The tuning is achieved by imposing that $M$ have zero eigenvalues, i.e. that its determinant and trace vanish:

$$
\tau\left(1-\left\langle b_{T 0}\right\rangle\right)+\left\langle b_{S 0}\right\rangle=0, \quad\left\langle b_{S 0}\right\rangle-\left\langle b_{T 0}\right\rangle+\tau+1=0 .
$$

The values of $\left\langle b_{T 0}\right\rangle$ and $\left\langle b_{S 0}\right\rangle$ at this point are

$$
\left\langle b_{T 0}\right\rangle=\frac{1}{1-\tau}, \quad\left\langle b_{S 0}\right\rangle=\frac{\tau^{2}}{1-\tau},
$$

and the matrix $M$ simplifies to

$$
M=\frac{\tau}{\tau-1}\left(\begin{array}{cc}
1 & -\tau \\
\tau^{-1} & -1
\end{array}\right) .
$$

In terms of the physical parameters, we have

$$
\begin{aligned}
& R_{T 0}=\frac{320}{1-\tau}-\frac{20}{3} M_{T 0} \\
& R_{S 0}=\frac{320 \tau^{2}}{1-\tau}+\frac{20}{3} M_{S 0}
\end{aligned}
$$

that is, the co-dimension two tuning of $R_{T}$ and $R_{S}$ at the Takens-Bogdanov point. The matrix $M$ has only one eigenvector, $\mathbf{e}_{1}=(\tau 1)$. Any other vector is projected onto $\mathbf{e}_{1}$ by $M$. We could make a coordinate transformation to put $M$ in Jordan form

$$
\widetilde{M}=\left(\begin{array}{ll}
0 & 1 \\
0 & 0
\end{array}\right),
$$

but we will not find it necessary to do so here.

Thus we have

$$
\Theta_{0}=\tau \Sigma_{0}
$$

and the solvability condition, Eq. 37, is now just

$$
\partial_{T_{1}}\left(\begin{array}{c}
\Theta_{0} \\
\Sigma_{0}
\end{array}\right)=0
$$


It now remains for us to solve the system of equations given by 33 . We can rewrite the solution for $\phi_{0 X}$ as

$$
\phi_{0 X}=P_{1}(z) \theta_{0 X}
$$

where

$$
P_{1}(z)=\frac{z^{2}(z-1)}{48}\left[(3-2 z)\left(R_{T 0}-\frac{R_{S 0}}{\tau}\right)+12\left(M_{T 0}+\frac{M_{S 0}}{\tau}\right)\right] .
$$

Integrating the temperature and salinity equations twice and applying the fixed flux boundary conditions we find

$$
\begin{aligned}
\Theta_{1} & =\left(\frac{z^{2}}{2}-P_{1}^{(-2)}(z)\right) \Theta_{0 X X}+\theta_{1,0} \\
\tau \Sigma_{1} & =\left(\frac{z^{2}}{2}-P_{1}^{(-2)}(z)\right) \Theta_{0 X X}+\tau \Sigma_{1,0} \\
& =\Theta_{1}-\Theta_{1,0}+\tau \Sigma_{1,0}
\end{aligned}
$$

where the notation $P_{1}^{(n)}(z)$ means that $P_{1}$ is differentiated $n$ times, or integrated for $n<0$. The $z$-independent quantities $\Theta_{1,0}$ and $\Sigma_{1,0}$ are integration constants that can depend on $X$ and $T_{2}$. The equation for $\phi_{1 X}$ is

$$
\begin{aligned}
D^{4} \phi_{1 X}= & -R_{T 0} \Theta_{1,0 X}+R_{S 0} \Sigma_{1,0 X}+\left(\frac{R_{S 1}}{\tau}-R_{T 1}\right) \Theta_{0 X} \\
& +\left[\left(\frac{R_{S 0}}{\tau}-R_{T 0}\right)\left(\frac{z^{2}}{2}-P_{1}^{(-2)}(z)\right)-2 P_{1}^{(2)}(z)\right] \Theta_{0 X X X} .
\end{aligned}
$$

We wish to integrate this four times and apply the boundary conditions. The calculation will not be shown in detail here. Instead, we write

$$
\phi_{1 X}=-b_{T 0}(z) \Theta_{1,0 X}+b_{S 0}(z) \Sigma_{1,0 X}+P_{2}(z) \Theta_{0 X}+P_{3}(z) \Theta_{0 X X X} .
$$

\subsection{Order $\varepsilon^{2}$}

At this order we have $\mathcal{L}_{0} \Phi_{2}+\mathcal{L}_{1} \Phi_{1}+\mathcal{L}_{2} \Phi_{0}=\mathcal{N}_{2}$ :

$$
\begin{aligned}
D^{4} \phi_{2 X}= & -R_{T 0} \Theta_{2 X}+R_{S 0} \Sigma_{2 X}-R_{T_{1}} \Theta_{1 X}+R_{S 1} \Sigma_{1 X} \\
& -R_{T 2} \Theta_{0 X}+R_{S 2} \Sigma_{0 X}-2 D^{2} \phi_{1 X X X}-\phi_{0 X X X X X}+\sigma^{-1} \partial_{T_{2}} D^{2} \phi_{0 X} \\
& +\sigma^{-1}\left(\phi_{0 X X} D^{3} \phi_{0 X}-D \phi_{0 X} D^{2} \phi_{0 X X}\right), \\
D^{2} \Theta_{2}= & -\phi_{1 X X}-\Theta_{1 X X}+\partial_{T_{2}} \Theta_{0}-D \phi_{0 X} \Theta_{0 X}, \\
\tau D^{2} \Sigma_{2}= & -\phi_{1 X X}-\tau \Sigma_{1 X X}+\partial_{T_{2}} \Sigma_{0}-D \phi_{0 X} \Sigma_{0 X} .
\end{aligned}
$$

We integrate the heat and salt equations in the usual manner to get the solvability conditions

$$
\begin{aligned}
& \Theta_{0 T_{2}}=\left\langle\phi_{1 X X}\right\rangle+\left\langle\Theta_{1 X X}\right\rangle \\
& \Sigma_{0 T_{2}}=\left\langle\phi_{1 X X}\right\rangle+\left\langle\tau \Sigma_{1 X X}\right\rangle
\end{aligned}
$$


where the nonlinear terms dropped out because of the boundary conditions. We can rewrite this as

$$
\left(\begin{array}{c}
\Theta_{0 T_{2}} \\
\Sigma_{0 T_{2}}
\end{array}\right)=M\left(\begin{array}{c}
\Theta_{1,0 X X} \\
\Sigma_{1,0 X X}
\end{array}\right)+\left\langle P_{2}\right\rangle\left(\begin{array}{c}
\Theta_{0 X X} \\
\Sigma_{0 X X}
\end{array}\right)+\left\langle P_{3}-P_{1}^{(-2)}+\frac{z^{2}}{2}\right\rangle\left(\begin{array}{c}
\Theta_{0 X X X X} \\
\Sigma_{0 X X X X}
\end{array}\right) .
$$

We define $\chi$ by

$$
\begin{aligned}
\left(\begin{array}{c}
\Theta_{1,0} \\
\Sigma_{1,0}
\end{array}\right) & =\Sigma_{1,0}\left(\begin{array}{l}
\tau \\
1
\end{array}\right)+\frac{\tau-1}{\tau}\left(\begin{array}{c}
\chi \\
0
\end{array}\right), \\
& =\Sigma_{1,0} \mathbf{e}_{1}+\frac{\tau-1}{\tau} \chi \mathbf{e}_{2},
\end{aligned}
$$

or

$$
\chi=\frac{\tau}{\tau-1}\left(\Theta_{1,0}-\tau \Sigma_{1,0}\right) .
$$

These conditions on the parameters amount to tuning the Takens-Bogdanov point at order $\varepsilon^{2}$. The solvability condition Eq. 53 can be written

$$
\left(\begin{array}{c}
\Theta_{0 T_{2}} \\
\Theta_{0 T_{2}} / \tau
\end{array}\right)=\left(\begin{array}{c}
\chi_{X X} \\
\chi_{X X} / \tau
\end{array}\right)+\left\langle P_{2}\right\rangle\left(\begin{array}{c}
\Theta_{0 X X} \\
\Sigma_{0 X X}
\end{array}\right)+\left\langle P_{3}-P_{1}^{(-2)}+\frac{z^{2}}{2}\right\rangle\left(\begin{array}{c}
\Theta_{0 X X X X} \\
\Sigma_{0 X X X X}
\end{array}\right) .
$$

These two equations can only be consistent for $\tau \neq 1$ if

$$
\left\langle P_{2}\right\rangle=\left\langle P_{3}-P_{1}^{(-2)}+\frac{z^{2}}{2}\right\rangle=0,
$$

which means, after integrating the polynomials,

$$
\begin{aligned}
\frac{R_{S 2}}{\tau}-R_{T 2} & =\frac{20}{3}\left(M_{T 2}+\frac{M_{S 2}}{\tau}\right) \\
M_{T 0}+\frac{M_{S 0}}{\tau} & =48 \frac{(32 \pm \sqrt{210194})}{241} .
\end{aligned}
$$

Then the solvability condition at this order is

$$
\Theta_{0 T_{2}}=\chi_{X X}
$$

\subsection{Order $\varepsilon^{3}$}

Avoiding detailed calculations, we simply quote the result for the solvability condition at this order ${ }^{1}$

$$
\chi_{T_{2}}=-\nu \chi_{X X}+\mu \Theta_{0 X X X X}+\lambda \Theta_{0 X X}-\rho \chi_{X X X X}-\gamma \Theta_{0 X X X X X X}+\zeta\left(\Theta_{0 X}^{2}\right)_{X X},
$$

\footnotetext{
${ }^{1}$ This calculation was done on Wolfram Research's Mathematica software package.
} 
where

$$
\begin{aligned}
\nu= & \frac{(1-\tau)}{960}\left(3 R_{T 2}+20 M_{T_{2}}\right) \\
\mu= & \sqrt{\frac{9577}{22}} \frac{\left(R_{T_{2}} \tau-R_{S_{2}}\right)}{75600}, \\
\lambda= & \frac{M_{T_{4}} \tau+M_{S 4}}{48}, \\
\rho= & \frac{142}{1687}+\frac{5 \sqrt{\frac{9577}{22}}}{15183}-\frac{M_{T 0}}{90720}-\frac{\sqrt{\frac{9577}{22}} M_{T_{0}}}{22680}+\frac{M_{T_{0}} \tau}{90720} \\
\gamma= & \frac{(144945779034529+64636181572 \sqrt{210694})}{10129277026793925} \tau, \\
\zeta= & \frac{-1}{439092360 \sigma}\left(523104000 \sigma+3766800 \sqrt{210694} \sigma-18603995 M_{T 0} \sigma\right. \\
& -9640 \sqrt{210694} M_{T_{0}} \sigma+293982984 \tau+887832 \sqrt{210694} \tau \\
& \left.+18603995 M_{T 0} \sigma \tau+9640 \sqrt{210694} M_{T 0} \sigma \tau\right) .
\end{aligned}
$$

We have eliminated $M_{S_{0}}$ from these coefficients by taking the positive solution of Eq. 57 for definiteness.

\section{Analysis of the System}

From now on we write $T$ for $T_{2}$ and drop the subscript 0 on $\Theta_{0}$. The system of long wave equations describing the small amplitude behaviour is thus

$$
\begin{aligned}
& \Theta_{T}=\chi_{X X}, \\
& \chi_{T}=-\nu \chi_{X X}+\mu \Theta_{X X X X}+\lambda \Theta_{X X}-\rho \chi_{X X X X}-\gamma \Theta_{X X X X X X}+\zeta\left(\Theta_{X}^{2}\right)_{X X}
\end{aligned}
$$

This can be written as a partial differential equation, second order in time:

$$
\begin{aligned}
\Theta_{T T}= & -\nu \Theta_{X X T}-\rho \Theta_{X X X X T}+\mu \Theta_{(6 X)}+\lambda \Theta_{X X X X} \\
& -\gamma \Theta_{(8 X)}+\zeta\left(\Theta_{X}^{2}\right)_{X X X X} .
\end{aligned}
$$

We now make a spectral expansion of the system.

\subsection{Galerkin Truncation}

We use a truncated expansion for $\theta$ :

$$
\Theta=A e^{\mathrm{i} K X}+B e^{2 \mathrm{i} K X}+\text { c.c. }
$$


neglecting higher harmonics. Then by using Eq. 60 we obtain a set of coupled O.D.E.'s for $A$ and $B$ :

$$
\begin{aligned}
& \ddot{A}=K^{2}\left(\nu-\rho K^{2}\right) \dot{A}+K^{4}\left(\lambda-\mu K^{2}-\gamma K^{4}\right) A+4 \zeta K^{6} A^{*} B \\
& \ddot{B}=4 K^{2}\left(\nu-4 \rho K^{2}\right) \dot{B}+16 K^{4}\left(\lambda-4 \mu K^{2}-16 \gamma K^{4}\right) B-16 \zeta K^{6} A^{2} .
\end{aligned}
$$

Let

$$
\begin{aligned}
& \alpha(K)=\lambda-\mu K^{2}-\gamma K^{4}, \\
& \beta(K)=\nu-\rho K^{2},
\end{aligned}
$$

and abbreviate $\alpha(K)$ by $\alpha_{1}$ and $\alpha(2 K)$ by $\alpha_{2}$ (similarly for $\beta_{1}$ and $\beta_{2}$ ). The linear dispersion relation for mode $j$ is

$$
\Gamma_{j}^{2}-K_{j}^{2} \beta_{j} \Gamma_{j}-K_{j}^{4} \alpha_{j}=0 .
$$

For a direct mode slightly above criticality we have $\Gamma_{j}=\epsilon \ll 1$, so that

$$
\Gamma_{j}=\epsilon=-K_{j}^{2} \frac{\alpha_{j}}{\beta_{j}} .
$$

For a Hopf mode slightly above criticality, we have $\Gamma_{j}=\epsilon+i \omega$ and

$$
\epsilon=K_{j}^{2} \frac{\beta_{j}}{2}, \quad \omega^{2}=-K_{j}^{4}\left(\frac{\beta_{j}^{2}}{4}+\alpha_{j}\right) .
$$

\subsection{Low-order Bifurcation Structure}

We first look for steady nonlinear solutions of Eqs. 62. Assume $\dot{A}=\dot{B}=0$. Then we can solve for the amplitude of $A$ :

$$
|A|^{2}=-\frac{\alpha_{1} \alpha_{2}}{4 K^{4} \zeta^{2}} .
$$

We see that the mode will be supercritical if $\alpha_{1}$ and $\alpha_{2}$ have opposite signs, and subcritical if they have the same sign. The sign of $\zeta$ is immaterial. This first case is illustrated in Figure 3. The solid line corresponds to the $K=1$ mode and the dashed line is the $K=2$ mode, which is unstable. The second case is seen in Figure 4(a). The $B$ mode (solid line) now becomes unstable before the subcritical $A$ mode (dashed line). Figure 4(b) shows a plot of $\Theta$ as a function of $X$ for different values of $\lambda$ along the subcritical dashed line of (a). It can be seen from this figure that this is a mixed mode, and the mode develops a higher harmonic as the unsteady branch nears the steady $B$ branch.

For a Hopf mode the amplitude and frequency are

$$
\begin{aligned}
|A|^{2} & =\frac{4 K^{4} \omega^{2} \beta_{1} \beta_{2}-\left(\omega^{2}+K^{4} \alpha_{1}\right)\left(\omega^{2}+16 K^{4} \alpha_{2}\right)}{64 K^{12} \zeta^{2}}, \\
\omega^{2} & =-4 K^{4} \frac{\alpha_{1} \beta_{2}+4 \alpha_{2} \beta_{1}}{\beta_{1}+4 \beta_{2}}
\end{aligned}
$$




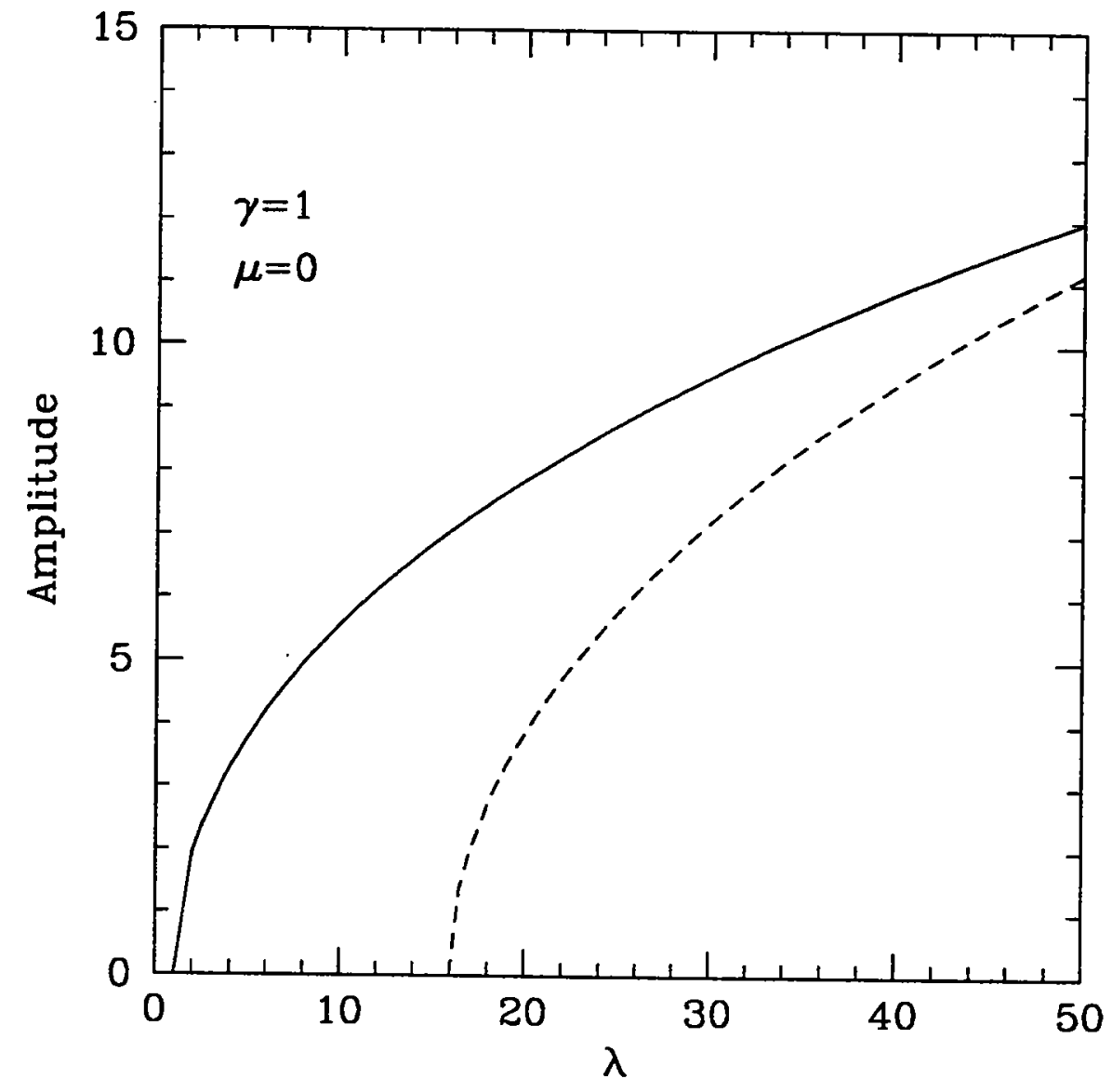

Figure 3: Summed amplitude of Fourier modes of a Galerkin truncation containing 20. modes, for the case in which $A$ is supercritical and $B$ is unstable. The dynamics of the system are very well approximated by the two-mode truncation. 

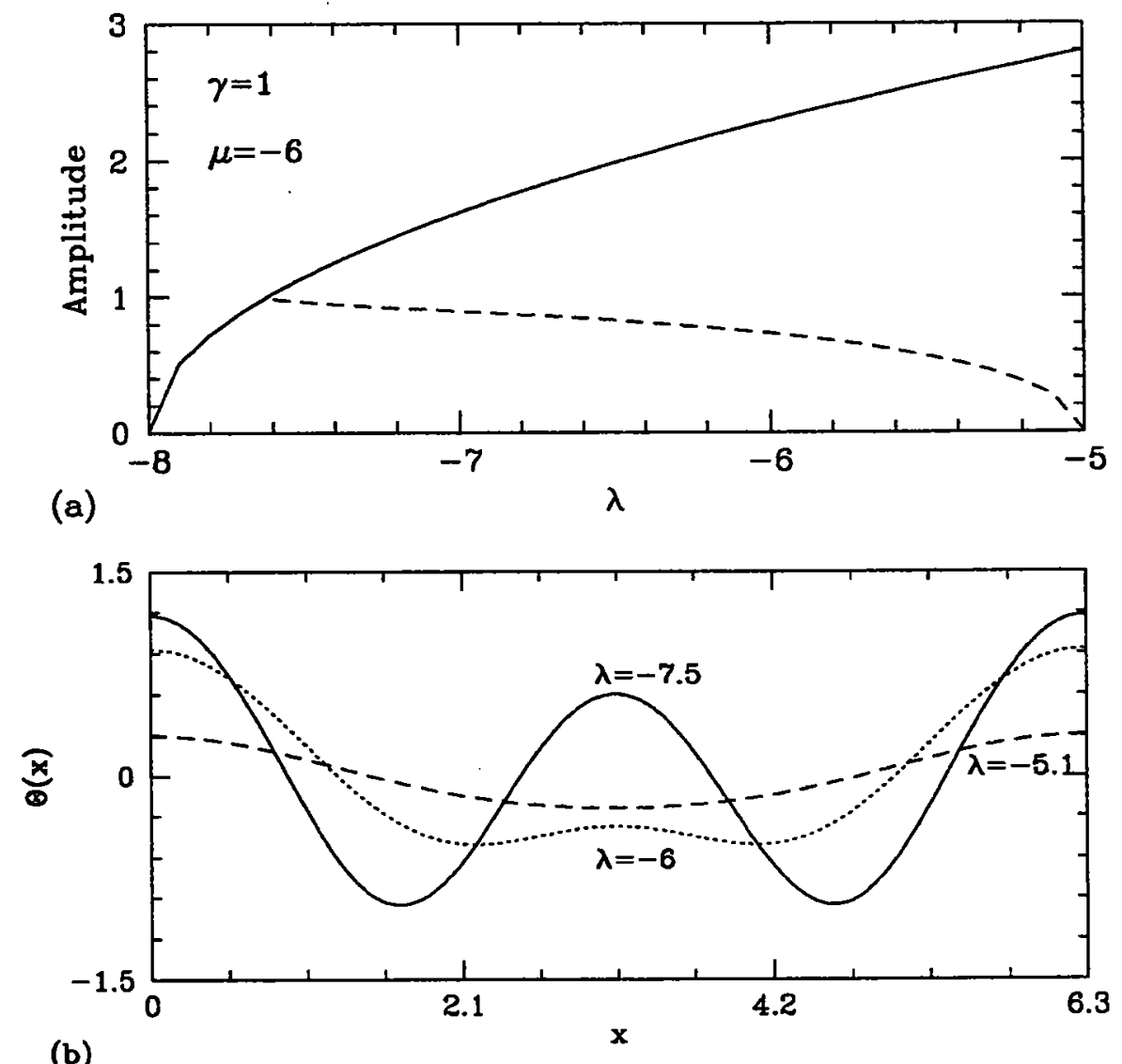

(b)

Figure 4: (a) Summed amplitude of Fourier modes of a Galerkin truncation containing 30 modes, for the case in which the $B$ mode (solid line) becomes unstable before the subcritical $A$ mode (dashed line). The two mode truncation approximates the dynamics very well. (b) Plot of $\Theta$ as a function of $X$ for different values of $\lambda$ along the subcritical dashed line of (a). It can be seen from this that the mode develops a higher harmonic as the unsteady branch nears the steady $B$ branch, and is a pure $2 K$ mode when they meet. 
The Hopf branch is supercritical if

$$
\frac{\left(\alpha_{1}-16 K^{4} \alpha_{2}\right)^{2}}{4 K^{2} \beta_{2}}>K^{2} \alpha_{1} \beta_{2} .
$$

Now if the $B$ mode is damped when the Hopf bifurcation for the $A$ mode occurs $\left(\alpha_{1}<0\right.$ for Hopf), we will have a subcritical bifurcation. The question is now whether this Hopf branch turns around at the next order $\left(|A|^{4} A\right)$. We have not yet worked this out, though the author and Neil J. Balmforth have a wager on the matter. If the branch does not turn around it will point to the fact that the expansion only captures the lowest order Takens-Bogdanov bifurcation structure, a result which is expected. [15]

After including a $C \exp (3 \mathrm{i} K X)$ term in the Galerkin expansion we find the amplitude of the Hopf mode to order $|A|^{4} A$ satisfies (letting $K=1$ )

$$
\begin{aligned}
A= & -\frac{64 \zeta^{2}|A|^{2} A}{\left(\alpha_{1}+\mathrm{i} \beta_{1} \omega+\omega^{2}\right)\left(\alpha_{2}+4 \mathrm{i} \beta_{2} \omega+16 \omega^{2}\right)} \\
& +\frac{48 \cdot 16^{2} \cdot 81 \zeta^{4}|A|^{4} A}{\left(\alpha_{1}+\mathrm{i} \beta_{1} \omega+\omega^{2}\right)\left(\alpha_{2}+4 \mathrm{i} \beta_{2} \omega+16 \omega^{2}\right)^{2}\left(\alpha_{3}+9 \mathrm{i} \beta_{3} \omega+81 \omega^{2}\right)} .
\end{aligned}
$$

The problem is that in this system there is no way to tune out the $|A|^{2} A$ term without also getting rid of the quintic nonlinearity. Hence the above equation is quite difficult to solve for $A$ and $\omega$.

\section{Conclusions}

We have a derived a small amplitude long wave equation for the double-diffusive Marangoni convection system. The expansion contains only a quadratic nonlinearity, of a different nature than that derived by Depassier and Spiegel [16] because of the additional $X$ derivative: they had a nonlinearity of the form $\left(f^{2}\right)_{x x}$, whereas we have $\left(f_{x}^{2}\right)_{x x}$. The nonlinear term thus has the opposite effect (stabilizing for steady bifurcations) on the criticality of the system, as in [17].

We chose a small amplitude expansion because the physical system we studied does not contain enough parameters to tune out the resonant nonlinearities that arise in the order $\varepsilon^{2}$ solvability condition, Eq. 55, when the physical variables are of order one. However, including surface displacement effects (to lowest order, crispation $[6,12]$ ) should allow us to do so and capture the full bifurcation structure of the TakensBogdanov point. We expect a system of the form

$$
\begin{aligned}
\Theta_{T}= & \chi_{X X}, \\
\chi_{T}= & -\nu \chi_{X X}+\mu \Theta_{X X X X}+\lambda \Theta_{X X}-\rho \chi_{X X X X}-\gamma \Theta_{X X X X X X}+q_{1}\left(\Theta_{X}^{2}\right)_{X X X X} \\
& +q_{2}\left(\Theta_{X X}^{2}\right)_{X X}+q_{3}\left(\Theta_{X}^{2}\right)_{X X}+q_{4}\left(\Theta_{X X}^{3}\right)_{X X X}+q_{5}\left(\Theta_{X}^{4}\right)_{X X} \\
& +q_{6}\left(\chi \Theta_{X X}\right)_{X X}+q_{7}\left(\chi_{X} \Theta_{X}\right)_{X X}+q_{8}\left(\chi_{X} \Theta_{X X}\right)_{X}
\end{aligned}
$$


The equation has all the terms allowed by symmetry. If the system had an additional up-down symmetry, only one nonlinearity with coefficient $\left(q_{4}\right)$ would survive. Hence the equations above are potentially much richer than the Boussinesq up-down symmetric case. We can also use planform equations like the above to look for steady nonlinear solutions of the system [17].

Another direction to explore is the interaction of the $a=0$ mode with the nonzero $a$ mode when they occur together, as shown by the solid line in Figure 1 . There will be a complex Ginzburg-Landau equation like Eq. 23 for the nonzero $a$ mode and an equation of the type derived here for the $a=0$ mode, with a coupling between them which should lead to interesting dynamics (see $[8,11]$ for theory and $[9,10]$ for experiments concerning the steady case, Marangoni single-diffusion). Another possible system for the study of this sort of long and finite length scale interaction is the case of compressible convection [13].

\section{Acknowledgments}

I am especially thankful to Neil Balmforth for being a great and available project supervisor, and for teaching me weakly nonlinear theory together with Keith Julien. I'm also indebted to nearly all the staff of the G.F.D. program, but I'd like to mention conversations with Lou Howard, Joe Keller, Oliver Kerr, Edgar Knobloch, Melvin Stern, Barry Ruddick, Edward Spiegel, Stewart Turner, and George Veronis. If I haven't learned double-diffusion properly from these people, I never will. Finally, I am grateful to all the other Fellows, for being a good laugh.

\section{References}

[1] C. F. Chen and T. F. Su, "Effect of surface tension on the onset of convection in a double-diffusive layer," Physics of Fluids A 4, 2360 (1992).

[2] J. Tanny, C. C. Chen, and C. F. Chen, "Effects of interaction between Marangoni and double-diffusive instabilities," Journal of Fluid Mechanics 393, 1 (1995).

[3] H. Bénard, "Les tourbillons cellulaires dans une nappe liquide," Revue générale des Sciences pures et appliquées 11, 1261 (1900).

[4] J. R. A. Pearson, "On convection cells induced by surface tension," Journal of Fluid Mechanics 4, 489 (1958).

[5] J. S. Turner, Buoyancy Effects in Fluids (Cambridge University Press, Cambridge, 1973).

[6] R. W. Zeren and W. C. Reynolds, "Thermal instabilities in two-fluid horizontal layers," Journal of Fluid Mechanics 53, 305 (1972). 
[7] D. A. Nield, "Onset of convection in a fluid layer overlying a layer of a porous medium," Journal of Fluid Mechanics 81, 513 (1977).

[8] A. A. Golovin, A. A. Nepomnyashchy, and L. M. Pismen, "Interaction bewteen short-scale Marangoni convection and long-scale deformational instability," Physics of Fluids 6, 34 (1994).

[9] M. F. Schatz, S. J. VanHook, W. D. McCormick, J. B. Swift, and H. L. Swinney, "Onset of surface-tension-driven Bénard convection," Physical Review Letters 75, 1938 (1995).

[10] S. J. VanHook, M. F. Schatz, W. D. McCormick, J. B. Swift, and H. L. Swinney, "Long-wavelength instability in surface-tension-driven Bénard convection," Physical Review Letters 75, 4397 (1995).

[11] A. A. Golovin, A. A. Nepomnyashchy, L. M. Pismen, and H. Riecke, "Steady and oscillatory side-band instabilities in Marangoni convection with deformable interface," Preprint (1996).

[12] C. Pérez-García, "Linear stability analysis of Bénard-Marangoni convection in fluids with a deformable free surface," Physics of Fluids A 3, 292 (1991).

[13] M. C. Depassier and E. A. Spiegel, "The large-scale structure of compressible convection," Astronomical Journal 86, 496 (1981).

[14] P. H. Coullet and E. A. Spiegel, "Amplitude equations for systems with competing instabilities," SIAM Journal of Applied Mathematics 43, 776 (1983).

[15] E. A. Spiegel, "Double convection," Proceedings of the 1981 Summer School in Geophysical Fluid Dynamics, p. 43, Woods Hole Oceanographic Institution (1981).

[16] M. C. Depassier and E. A. Spiegel, "Convection with heat flux prescribed on the boundaries of the system. I. The effect of temperature dependence of material properties," Geophysical and Astrophysical Fluid Dynamics 21, 167 (1982).

[17] C. J. Chapman and M. R. E. Proctor, "Nonlinear Rayleigh-Bénard convection between poorly conducting boundaries," Journal of Fluid Mechanics 101, 759 (1980). 


\title{
Linear Stability Analysis Of A Gravity Current Over Topography
}

\author{
J. Stephens
}

\section{Introduction}

Here we consider gravity currents under the influence of the Earth's rotation vector, where they may adjust geostrophically against boundaries to form a perimeter current, with either one or two fronts. A comprehensive review has been conducted by Griffiths(1986).

Gravity currents are important for transporting heat, salt and momentum, in oceanic, atmospheric, and other environments. The Denmark Straits overflow for example, in the North Atlantic, transports significant quantities of cold water equatorward, whilst the Mediterranean Undercurrent is an important source of heat and salt in the Eastern North Atlantic. On a smaller scale, gravity currents may be observed in lakes if the deformation radius is small enough that they may feel the earths rotation.

A study of a 2-fronted current on a slope in a 1-layer reduced gravity system was considered by Griffiths, Killworth \& Stern(1982), hereafter denoted GKS(1982). They discovered that a zero potential vorticity current was unstable to linearised perturbations over a finite range of wavenumbers. This range was bounded by a short wave cut-off at wavelengths comparable to the jet width, and extended to infinite wavelength. As the wavelength approached infinity the growth rate asymptoted to zero in proportion to the square of the wavenumber. 
The instabilities take the form of normal modes that couple the free streamlines of the current and extract both kinetic and potential energy from the flow. In the long wavelength limit these become sinuous modes.

Laboratory studies that GKS(1982) conducted demonstrated the unstable nature of a gravity current with two free streamlines.

Here we extend the study conducted by GKS(1982) to a 2-layer system. It will be seen that the presence of the upper layer in combination with the slope serves to modify the shear instability in GKS(1982) and stabilises the flow to long wavelength perturbations.

\section{Basic State}

Our basic state consists of a 2-fronted gravity current on a slope of gradient $\alpha$ in a 2-layer fluid with small density difference between the upper $\left(\rho_{1}\right)$ and the lower $\left(p_{2}\right)$ layers. To simplify the analysis, we choose layer 1 to be at rest in the unperturbed state, and for the current in layer 2 to possess a uniform zero potential vorticity (PV) distribution. The domain is divided into 4 regions as shown in fig(1) below. We use a shallow-water description of the dynamics.

\section{FIG 1 BASIC STATE}

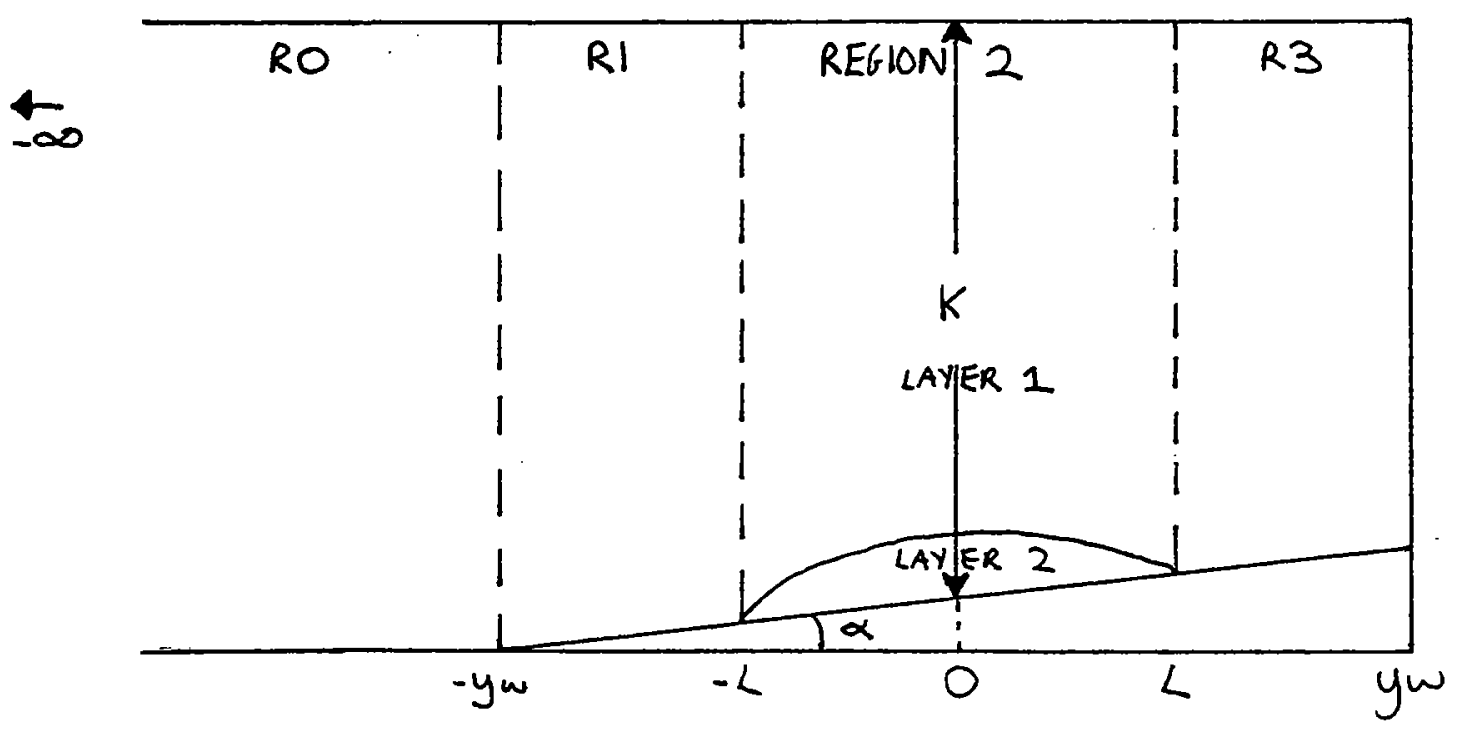




\subsection{Region 2}

For the unperturbed current we have

$$
\bar{u}_{2}-U=-\frac{g^{\prime}}{f} \partial_{y} \bar{h}_{2} \quad \text { where } U=-\frac{g^{\prime} \alpha}{f}
$$

$U$ is the slope velocity associated with the geostrophic balance of the current against the slope. $g^{\prime}$ is the reduced gravity effect $g^{\prime}=g^{\rho_{2}-\rho_{1}} \frac{\text { The additional term involving }}{\rho}$. The $\partial_{y} \bar{h}_{2}$ represents the geostrophic velocity induced by the slope of the interface.

To simplify the basic state we define layer 1 to be at rest and allow no cross-stream basic state velocity.

$$
\bar{u}_{1}=\bar{v}_{1}=\bar{v}_{2}=0
$$

In addition we choose a current with zero potential vorticity. This restriction implies that

$$
\bar{h}_{2}=\frac{f^{2}}{2 g^{\prime}}\left(L^{2}-y^{2}\right)
$$

\subsection{Regions 1 \& 3}

We define

$$
\begin{gathered}
\bar{u}_{1}=\bar{v}_{1}=0 \\
\bar{h}_{1}=K-\alpha y
\end{gathered}
$$

$K$ is the total depth of layers $1 \& 2$ at $y=0$.

\subsection{Region 0}

$$
\begin{gathered}
\bar{u}_{1}=\bar{v}_{1}=0 \\
\bar{h}_{1}=K-\alpha y_{w}
\end{gathered}
$$




\section{Perturbation Equations}

Using primes to denote perturbation quantities and $\eta^{\prime}$ for the displacement of the free surface, the linearised momentum and continuity equations take the following Boussinesq form:

\subsection{Region 2, Layer 1}

$$
\begin{aligned}
& \partial_{t} u_{1}^{\prime}-f v_{1}^{\prime}=-g \partial_{x} \eta^{\prime} \quad \text { where } \eta^{\prime}=h_{1}^{\prime}+h_{2}^{\prime} \\
& \partial_{t} v_{1}^{\prime}+f u_{1}^{\prime}=-g \partial_{y} \eta^{\prime} \\
& \partial_{t} h_{1}^{\prime}+\bar{h}_{1} \partial_{x} u_{1}^{\prime}+\partial_{y}\left(v_{1}^{\prime} \bar{h}_{1}\right)=0
\end{aligned}
$$

\subsection{Region 2, Layer 2}

$$
\begin{aligned}
& \partial_{t} u_{2}^{\prime}+\bar{u}_{2} \partial_{x} u_{2}^{\prime}+v_{2}^{\prime} \partial_{y} \bar{u}_{2}-f v_{2}^{\prime}=-g^{\prime} \partial_{x} h_{2}^{\prime}-g \partial_{x} \eta^{\prime} \\
& \partial_{t} v_{2}^{\prime}+\bar{u}_{2} \partial_{x} v_{2}^{\prime}+f u_{2}^{\prime}=-g^{\prime} \partial_{y} h_{2}^{\prime}-g \partial_{y} \eta^{\prime} \\
& \partial_{t} h_{2}^{\prime}+\bar{u}_{2} \partial_{x} h_{2}^{\prime}+\bar{h}_{2} \partial_{x} u_{2}^{\prime}+\partial_{y}\left(\bar{h}_{2} v_{2}^{\prime}\right)=0
\end{aligned}
$$

\subsection{Regions 0,1,3}

$$
\begin{aligned}
& \partial_{t} u_{1}^{\prime}-f v_{1}^{\prime}=-g \partial_{x} \eta^{\prime} \\
& \partial_{t} v_{1}^{\prime}+f u_{1}^{\prime}=-g \partial_{y} \eta^{\prime} \\
& \partial_{t} h_{1}^{\prime}+\bar{h}_{1} \partial_{x} u_{1}^{\prime}+\bar{h}_{1} \partial_{y} v_{1}^{\prime}=0
\end{aligned}
$$

\section{Boundary Conditions}

The boundary conditions we use are simply $v=0$ at $y=-\infty$ and $y=y_{w}$. 


\section{Non-dimensional Perturbation Equations}

Two dynamical length scales of interest will be used in the non-dimensionalisation. The first is an internal deformation radius based on the reduced gravity felt by the current and the height of the current at $y=0$. It is defined

$$
L_{d}=\frac{\sqrt{g^{\prime} H}}{f} \quad \text { where } \quad H=\bar{h}_{2}(0)
$$

We also define a non-dimensional wavenumber $\varepsilon$ defined

$$
\varepsilon=\frac{2 \pi}{\lambda} L_{d}
$$

where $\lambda$ is the non-dimensional wavelength.

For the most part following GKS(1982) we obtain the following non-dimensional variables:

$$
\begin{aligned}
& x=\frac{x^{*} \sqrt{g^{\prime} H}}{\varepsilon f} \quad y=\frac{y^{*} \sqrt{g^{\prime} H}}{f} \\
& (u, U)=\left(u^{*}, U^{*}\right) \sqrt{g^{\prime} H} \quad v=v^{*} \varepsilon \sqrt{g^{\prime} H} \\
& t=\frac{t^{*}}{\varepsilon f} \quad h=h^{*} H \\
& \eta=\eta^{*} \frac{g^{\prime}}{g} H \quad \alpha=\alpha^{*} \frac{L_{d}}{H} \quad K=K^{*} H
\end{aligned}
$$

We apply a normal mode perturbation to the non-dimensional momentum equations of the following form (dropping ${ }^{*}$ 's):

$$
\left(u^{\prime}, v^{\prime}, h^{\prime}, \eta^{\prime}\right)=(u,-i v, h, \eta) e^{i(x-c t)}
$$

The resulting equations become

\subsection{Region 2, Layer 1}

$$
\begin{aligned}
& -c u_{1}+v_{1}=-\eta \\
& -c \epsilon^{2} v_{1}+u_{1}=-\partial_{y} \eta \\
& \bar{h}_{1} u_{1}-\partial_{y}\left(v_{1} \bar{h}_{1}\right)-c h_{1}=0
\end{aligned}
$$




\subsection{Region 2, Layer 2}

$$
\begin{aligned}
& u_{2}\left(\bar{u}_{2}-c\right)=-\left(\eta+h_{2}\right) \\
& \epsilon^{2} v_{2}\left(\bar{u}_{2}-c\right)+u=-\partial_{y}\left(\eta+h_{2}\right) \\
& \bar{h}_{2} u_{2}-\partial_{y}\left(v_{2} \bar{h}_{2}\right)+\left(\bar{u}_{2}-c\right) h_{2}=0
\end{aligned}
$$

\subsection{Regions 1,3}

$$
\begin{aligned}
& -c u_{1}+v_{1}=-\eta \\
& -\epsilon^{2} c v_{1}+u_{1}=-\partial_{y} \eta \\
& \bar{h}_{1} u_{1}-\partial_{y}\left(v_{1} \bar{h}_{1}\right)-c \frac{g^{\prime}}{g} \eta=0
\end{aligned}
$$

\subsection{Region 0}

$$
\begin{aligned}
& -c u_{1}+v_{1}=-\eta \\
& -\epsilon^{2} c v_{1}+u_{1}=-\partial_{y} \eta \\
& \bar{h}_{1} u_{1}-\bar{h}_{1} \partial_{y} v_{1}-c \frac{g^{\prime}}{g} \eta=0
\end{aligned}
$$

\section{Non-dimensional Basic State}

The non-dimensional form of the basic state in Region 2 is

$$
\begin{aligned}
& \bar{u}_{2}=U+y \\
& \bar{h}_{2}=1-\frac{1}{2} y^{2} \\
& \bar{h}_{1}=K-\bar{h}_{2}-\alpha y \quad \text { where } \quad K=1+\bar{h}_{1}(0)
\end{aligned}
$$

We can see that since $\bar{h}_{2}(0)=1$, that $\bar{h}_{1}(0)$ is just the ratio of the layer depths at $y=0$. The edges of the unperturbed jet lie at $y= \pm L= \pm \sqrt{2}$. 


\section{Solutions Of The Equations}

We now make an approximation to simplify the equations, namely that $\bar{h}_{1}=K$ everywhere, except where it is differentiated in the continuity equations. This enables simple analytic solutions to be found in Regions $1 \& 3$. Where $\bar{h}_{1}$ is differentiated with respect to $y$, it is taken to be $-\alpha$ or $-\left(\alpha+\partial_{y} \bar{h}_{2}\right)$ as appropriate. By manipulating the non-dimensional perturbation equations we obtain single equations for $\eta^{\prime}$ in Regions 0, 1 \& 3:

\subsection{Region 0}

$$
\partial_{y y} \eta-b \eta=0 \quad \text { where } \quad b=\left(\frac{1}{\bar{h}_{1}}+\varepsilon^{2}-\frac{\varepsilon^{2} c^{2}}{\bar{h}_{1}}\right)
$$

The solution is simply

$$
\eta=A_{0} e^{\sqrt{b} y}+A_{1} e^{-\sqrt{b} y}
$$

The boundary condition at $y=-\infty$ requires $A_{1}=0$.

\subsection{Regions 1/3}

$$
\partial_{y y} \eta-\tilde{a} \partial_{y} \eta-\tilde{b} \eta=0 \quad \text { where } \quad \tilde{a}=\frac{\alpha}{\bar{h}_{1}} \quad \text { and } \quad \tilde{b}=\left(b+\frac{\tilde{a}}{c}\right)
$$

The solution is

$$
\eta=A_{2} e^{P_{1} y}+A_{3} e^{P_{2} y} \quad \text { where } \quad P_{1,2}=\frac{\tilde{a} \pm \sqrt{\tilde{a}^{2}+4 \tilde{b}}}{2}
$$

\subsection{Region 2}

In this 2-layer region the equations are more complicated due to coupling between the layers. We can obtain

$$
\partial_{y y} \eta=-\eta \frac{y-\alpha}{\bar{h}_{1}}-\eta\left(\frac{y-\alpha}{\bar{h}_{1} c}-b\right)+\frac{u_{2}}{\bar{h}_{1}}\left(\bar{u}_{2}-c\right)\left(1-c^{2} \varepsilon^{2}\right)
$$




$$
\partial_{y}\left(\bar{h}_{2} \partial_{y} u_{2}\right)=-\varepsilon^{2}\left(-\bar{h}_{2} u_{2}+u_{2}\left(\bar{u}_{2}-c\right)^{2}+\eta\left(\bar{u}_{2}-c\right)\right.
$$

Because of the appearance of $u_{2}$ in (38) and $\eta$ in (39) the problem is 4th order. We note that it is homogeneous, with $y$-dependent coefficients. The solution was found numerically using standard Runge-Kutta methods and so the method will not be expounded upon in detail.

\section{Dynamical Modes}

It is both instructive and interesting to recognise the various wave modes present in this, and the GKS('82) study:

\begin{tabular}{llr}
\multicolumn{1}{c}{ Mode } & GKS('82) & Present Study \\
Barotropic Topographic Waves (slow) & No & Yes \\
Barotropic Poincare Waves (fast) & No & Yes * \\
Barotropic Kelvin Waves (fast) & No & Yes * \\
Baroclinic Internal Gravity Waves & Yes & Yes \\
Baroclinic Kelvin Waves & Yes & Yes \\
Shear waves & Yes & Yes
\end{tabular}

We do not expect these fast modes to be dynamically important, since they will not be able to couple resonantly with the much slower current.

\subsection{Barotropic Topographic Mode}

The barotropic topographic mode is the most significant new addition to the problem in the 2-layer case, however this study did not explore a resonant-type coupling between the shear flow and the topographic mode: The phase speed of topographic waves is given by

$$
c=\frac{\alpha f / K}{k^{2}+l^{2}+L_{e}^{-2}}
$$


$L_{e}$ is the external Rossby radius of deformation, and $k$ and $l$ are wavenumbers in the $x$ and $y$ directions respectively.

Taking the long wave limit corresponding to the fastest modes and therefore those which are most likely to couple, and neglecting the $L_{e}^{-2}$ term which will be small, we obtain

$$
c=\frac{\alpha f}{K l^{2}}
$$

Scaling $L \sim L_{d} \sim \frac{e}{k} \sim \frac{1}{l}$ and substituting, we obtain

$$
c \sim-\frac{\alpha g^{\prime}}{f} \frac{h_{2}}{K} \sim U \frac{h_{2}}{K}
$$

This is a factor $\frac{h_{2}}{K}$ smaller than $U$ (which is the average current velocity). In the present study we restricted our attention to currents that were unidirectional. Moreover, the minimum velocities in the basic current were usually greater than $\frac{U h_{2}}{K}$, thus we do not expect to see resonant coupling.

\section{Shear Instability}

The shear instability which dominated the GKS(1982) study was caused by an interaction between disturbances to the two free streamlines at the edges of the current. This produced modes which grew exponentially, by releasing kinetic and potential energy from the basic state. In the $k=0$ limit corresponding to perturbations of infinite downstream length scale, the imaginary part of the phase speed of the eigenmode asymptoted to zero and the structure of the mode became a sinuous meandering disturbance.

The shear instability only occurs at wavelengths with large enough cross-stream length scale to cause an interaction across the current. We can scale the cross-stream wavenumber $l=k / \varepsilon$, and thus waves with a larger downstream wavenumber have larger $l$, ie smaller cross-stream wavelength, and are stable to this mode of instability. The mechanism for the instability will become apparent in the next section.

The shear instability found by GKS('82) was found to be present in this study as expected, and fig(3) shows the $\eta$ eigenfunction structure for one of the shear modes, corresponding to a non-dimensional wavelength of $21 L_{d}$, and $\bar{h}_{1}=10$. The figure shows large displacements in $\eta$ (the free surface displacement) at the edges of the 
FIG 3 SHEAR INSTABIUTY

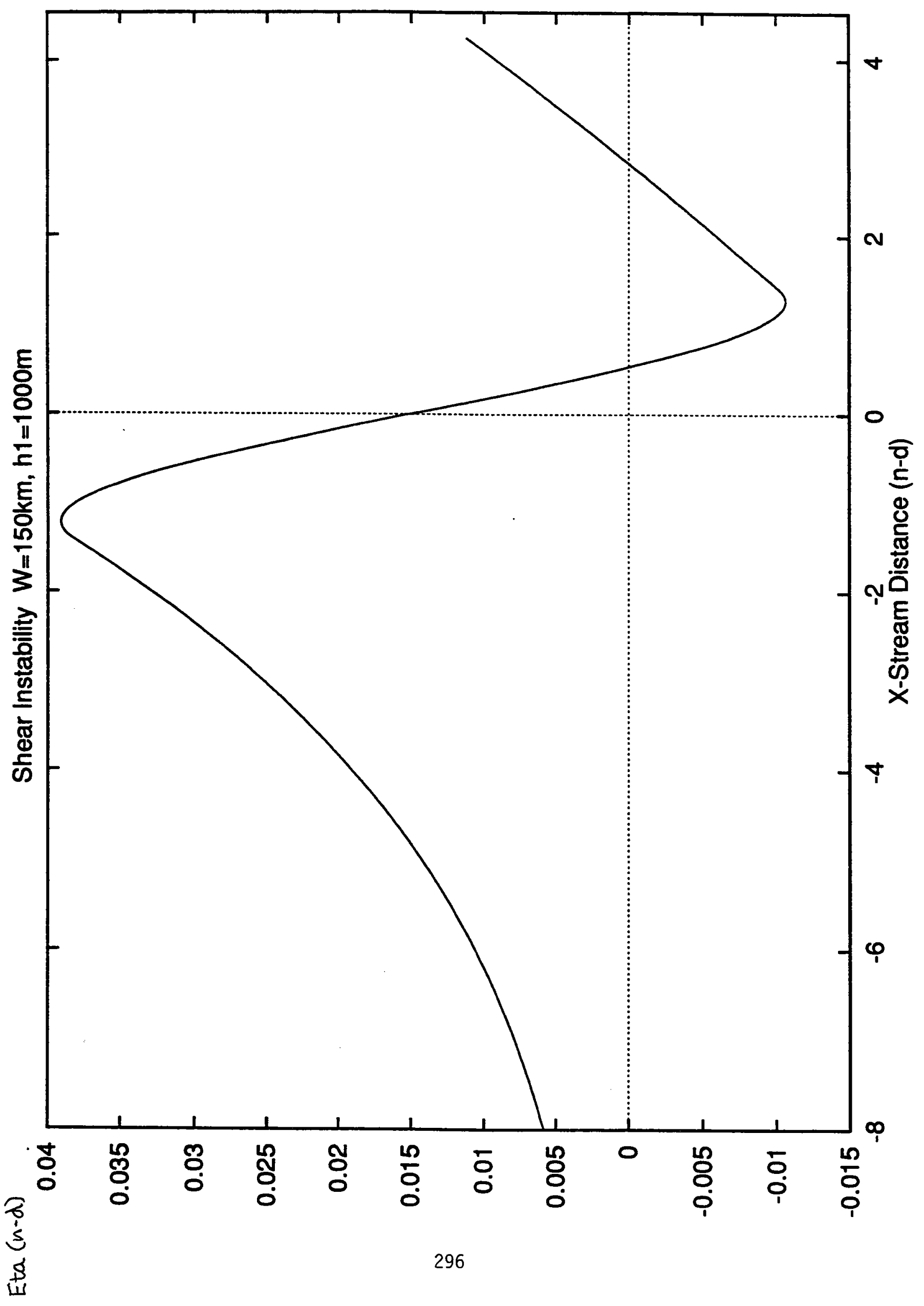


current associated with the instability.

Fig(4) shows the displacement in the cross-stream direction of the free streamlines associated with the instability. We can see that there is a small phase shift between the disturbance on the two fronts. This is a necessary characteristic of barotropic (shear) instability as we will see shortly.

\subsection{Changing Slope And Upper Layer Depth}

The band of unstable shear waves was tracked through wavenumber space for varying slope and depth, $\bar{h}_{1}$.

Fig(5) shows a plot of growth rate $\left(\varepsilon c_{i m}\right)$ vs wavenumber for varying slopes in the case where $\bar{h}_{1}=10$. We can see that as the slope increases the band of unstable waves narrows and moves to longer wavelengths, and the maximum growth rate decreases. It is evident that at some slope slightly greater than .033 , the flow is stabilised to long waves.

Fig(6) shows a similar plot for $\bar{h}_{1}=20$. We see that now a steeper slope is necessary to stabilise the flow, and the band of instability for a given slope has shifted to longer wavelengths.

Fig(7) displays the $\bar{h}_{1}=40$ case. Now even a slope of .05 is not enough to stabilise the flow and the band of unstable waves is still quite wide. The instability extends further towards $k=0$, in particular, for steeper slopes where we see that waves for $k=0.08$, corresponding to $\lambda=75 L_{d}$ are unstable.

Note that for values of slope greater than .008 in the $\bar{h}_{1}=10$ case, .016 in the $\bar{h}_{1}=20$ case, and .024 in the $\bar{h}_{1}=40$ case, the $\bar{h}_{1}=K$ approximation is no longer satisfied at $y_{w}$. We do not expect this to introduce a significant qualitative change to the results discussed. 
FIG 4 STREAMLINE PERTURBATIONS

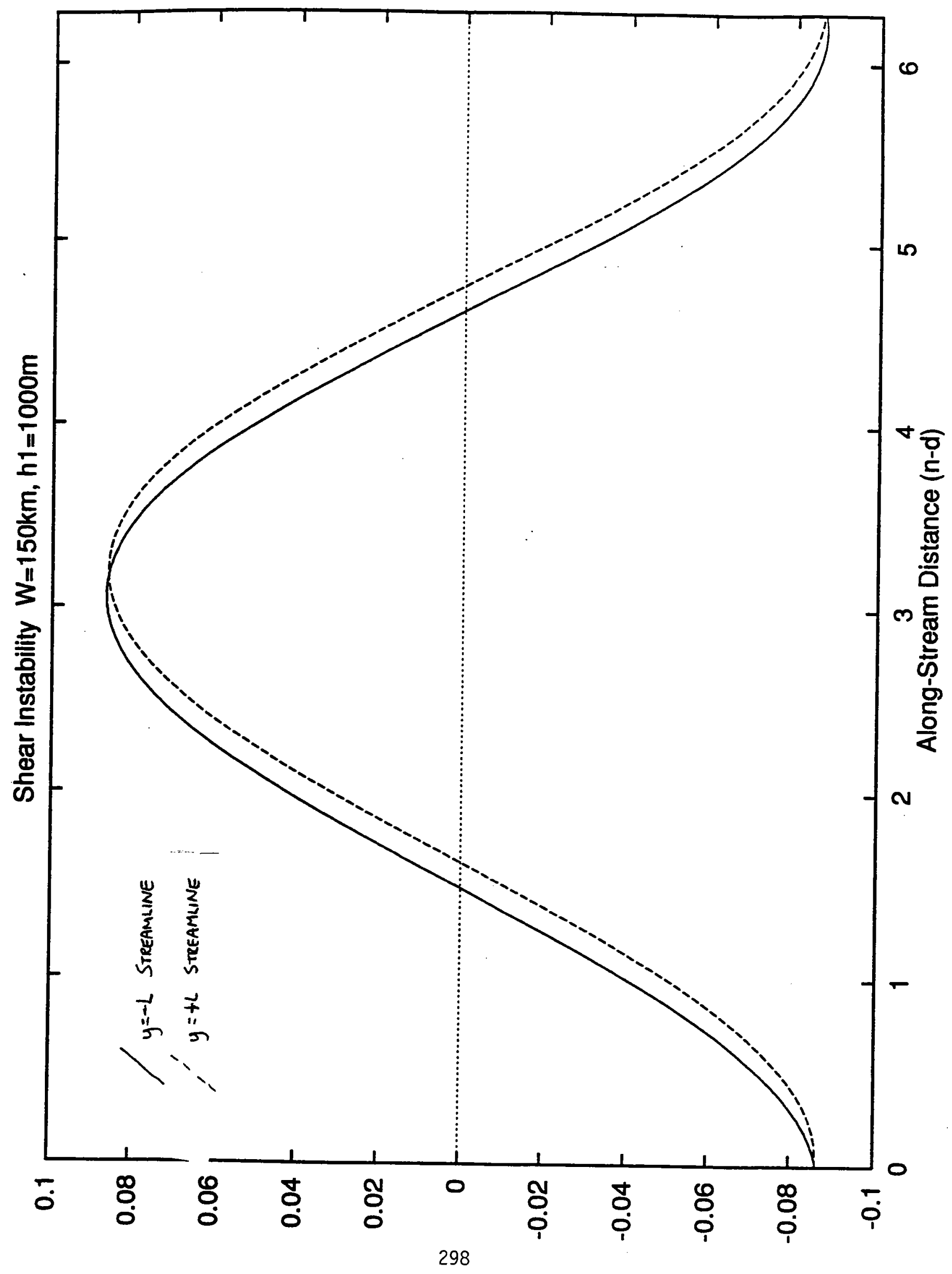


FIG $5 \quad \bar{h}_{1}=10$

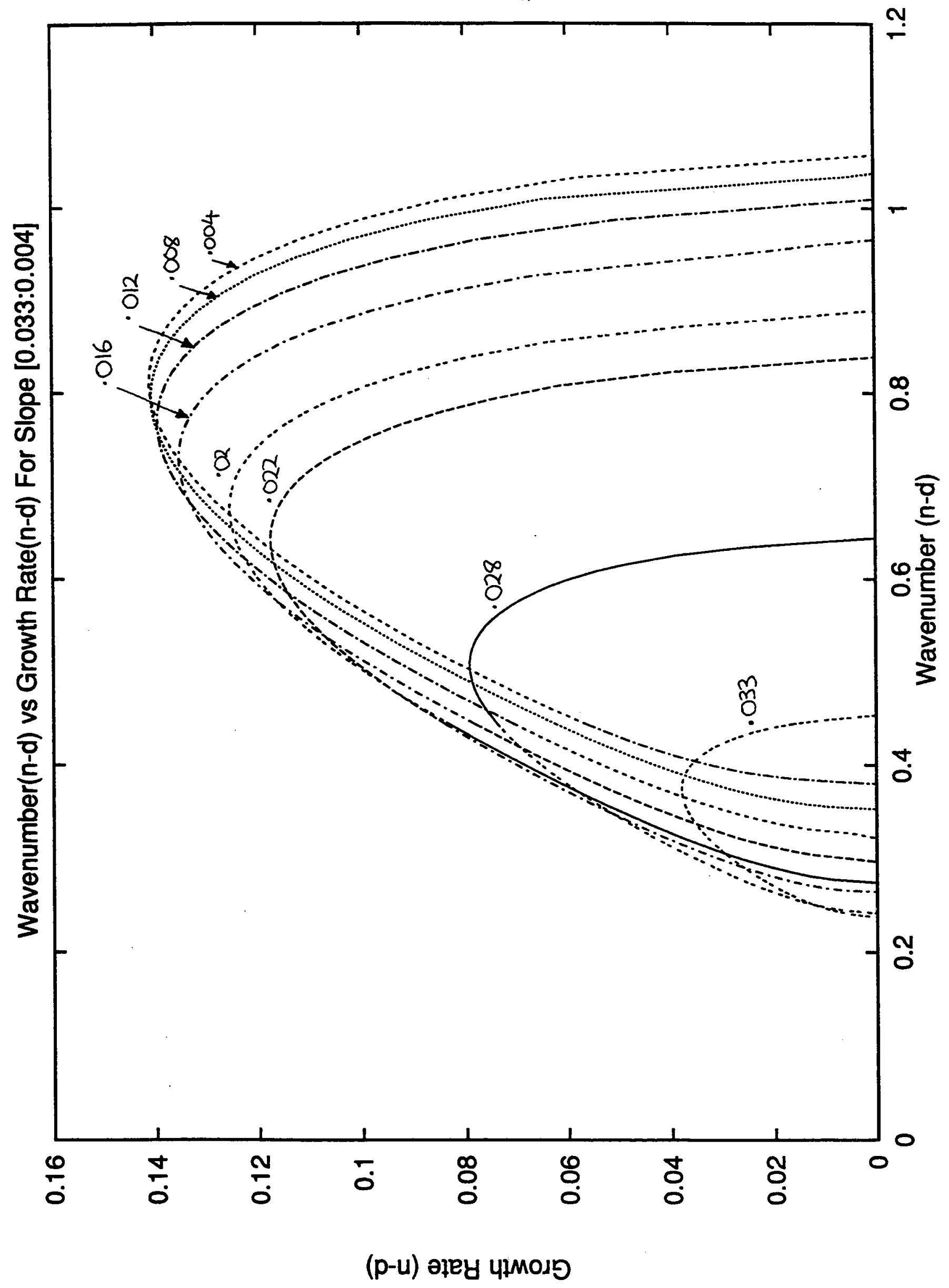


FIG $6 \quad \bar{h}_{1}=20$

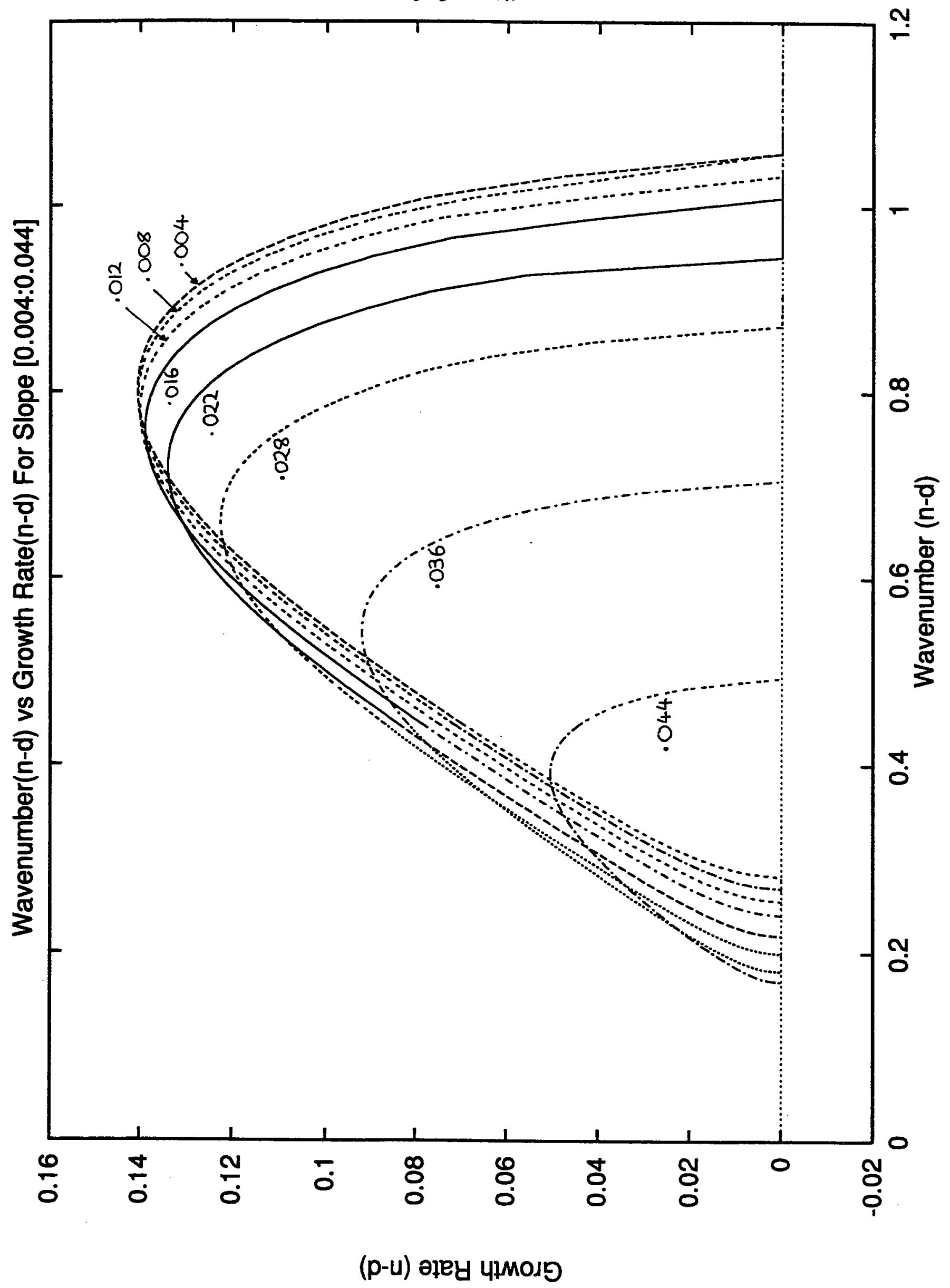




\section{$F i g 7 \quad \bar{h}_{1}=40$}

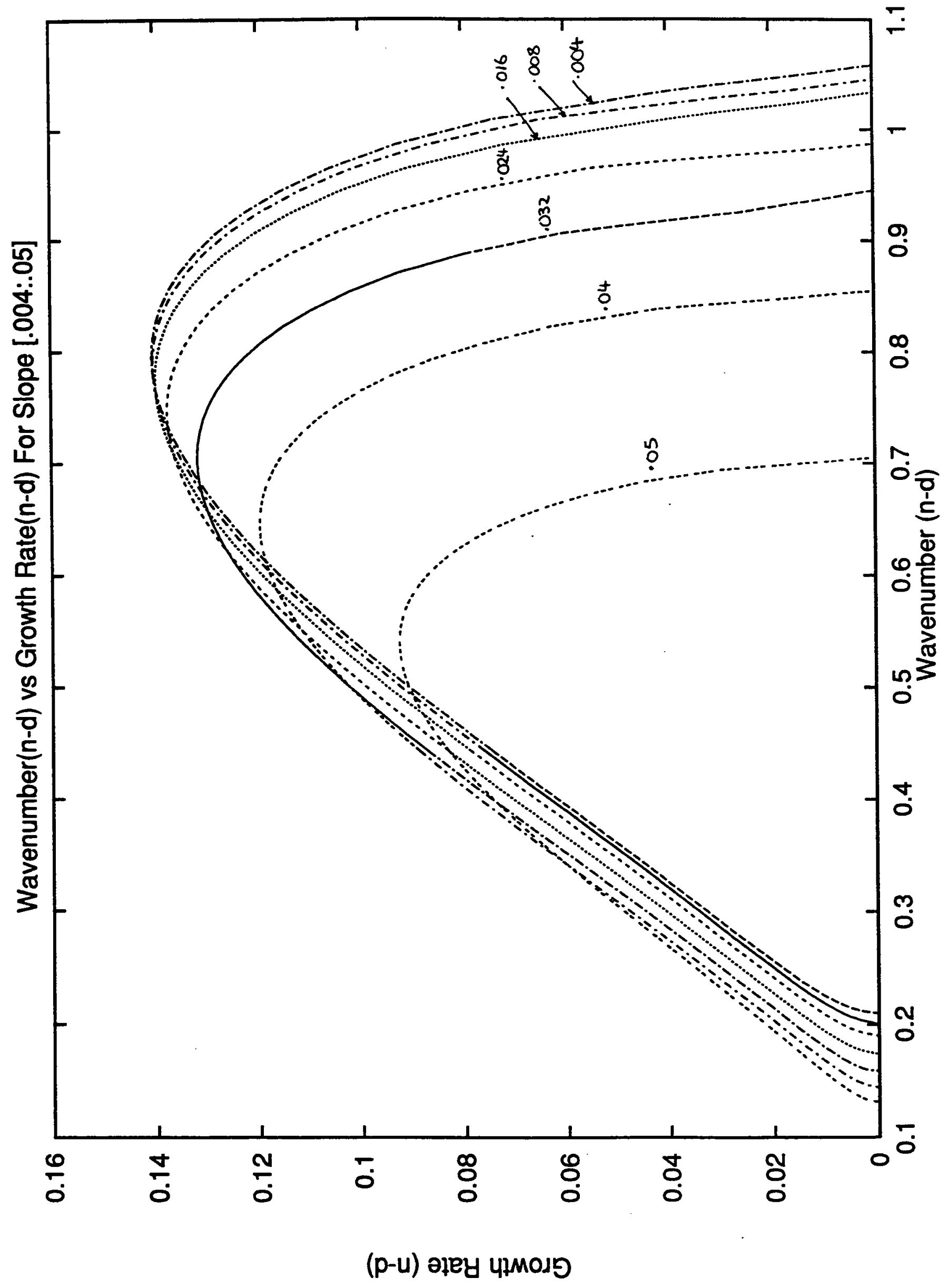




\subsection{Mechanism Of Shear Instability}

We have seen that smaller upper layer depths and steeper slopes are more stable to long wavelength perturbations. In the next section we will see why, but let us first consider the mechanism for the instability itself:

NO PHASE SHIFT

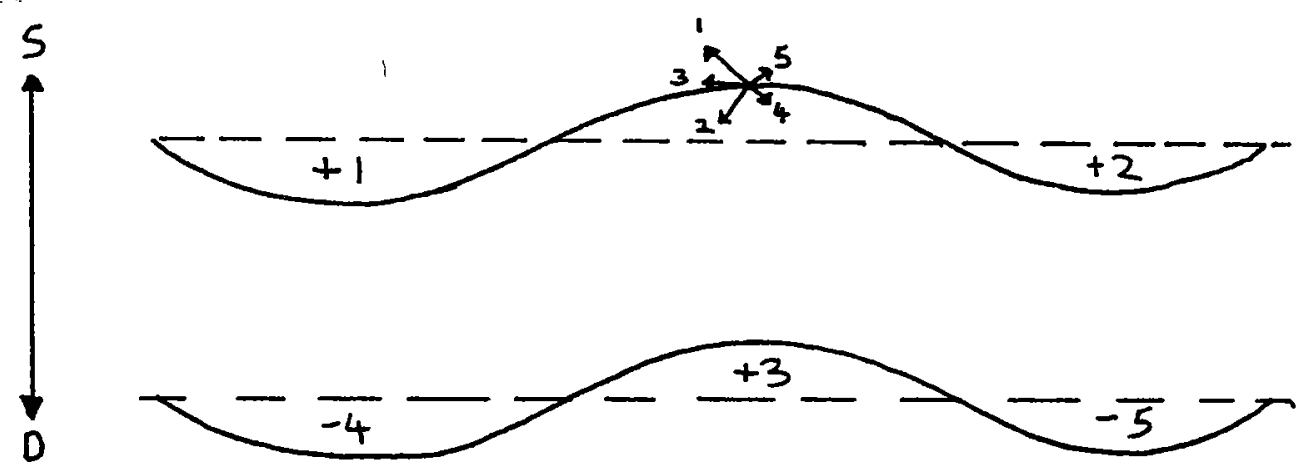

PHASE SHIFT

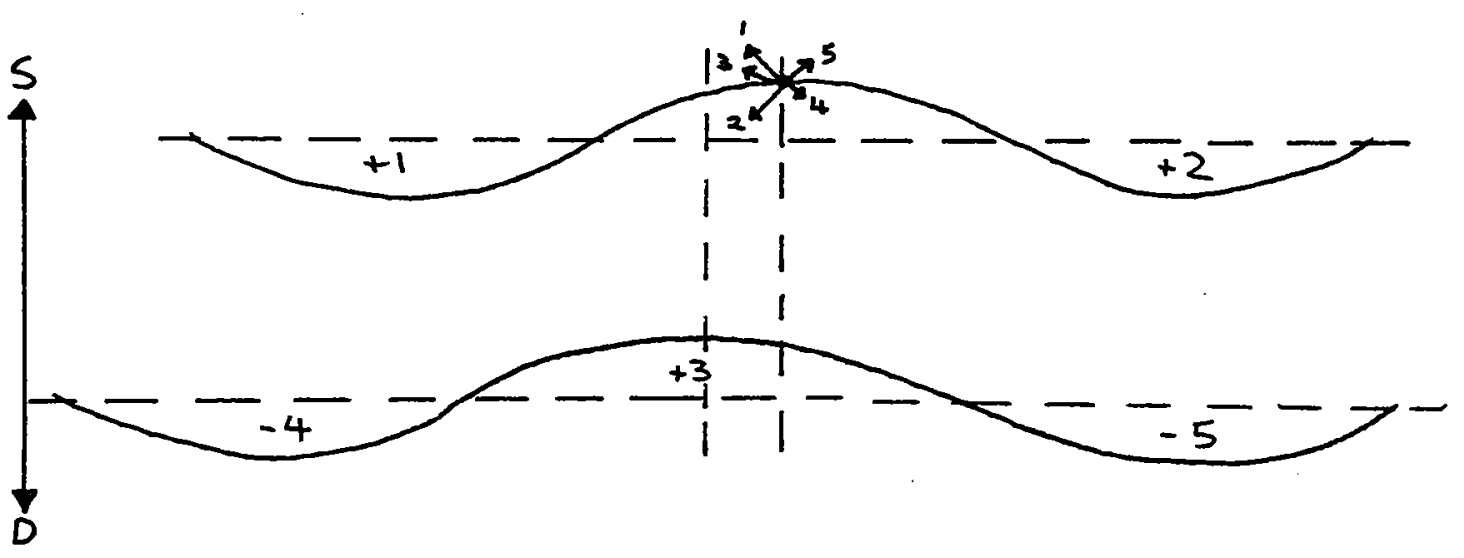

FI 62

The +'s and -'s in the above diagram denote relative vorticity anomalies associated with the displacement of the current by the normal mode perturbations. The ambient fluid surrounding the current (our layer 1 fluid) has zero basic state relative vorticity as there is no flow. Since the current has - ve relative vorticity associated with its linear shear (to obtain zero potential vorticity), then perturbations of the 
current to regions where there was previously no relative vorticity generate - ve relative vorticity anomalies. Similarily, areas to where the ambient fluid has moved to replace space left by the perturbed current have +ve relative vorticity anomalies.

The subscripts on the vorticity anomalies correlate with arrows on the meander in the topmost streamline pictured in both cases. These arrows represent the vectors of velocity induction by the surrounding vorticity anomalies on the point from which the arrows emanate.

In the first case, where there is no phase shift in the disturbance to the two sides of the current, we see that the resultant of the vorticity induction vectors is purely a downstream propagation of the disturbance at that point. This situation corresponds to the $\operatorname{GKS}(1982) k=0$ limit.

In the second case, where there is a phase shift in the disturbance, the magnitude and direction of the contributions from the vorticity anomalies is changed. We can see that there is now a cross-stream component to the resultant of the vorticity induction vectors, due to vorticity anomaly (3), and also from the resultant of (4) and (5). The sense of this component is such as to make the meander grow, producing instability. We note that if the phase shift were reversed then the meander would decay.

Note: This mechanism is analagous to the two-dimensional instability of a shear layer. It does not directly require the presence of topography. The topography induces an average along-slope velocity which simply contributes to the along-slope propagation of the unstable wave. The topographic slope has been marked on the diagram to assist in the explanation of the stabilisation of the current.

\subsection{Stabilisation Of Shear Instability}

When the upper layer has a finite depth, the role of topography is enhanced. Relative vorticity generation in the upper layer by vortex stretching and compression due to the perturbed current serves to modify the instability described.

Consider vorticity anomaly (3) in Fig(2). Upper layer fluid that moves upslope (to replace the space left by the perturbed current), will acquire - ve relative vorticity from a vortex compression effect as it ascends the slope. This counteracts the +ve vortex anomaly associated with the absence of the current and thus decreases the 
cross-stream component of the vorticity induction vector due to (3), helping to stabilise the current.

At points (1) \& (2), there is an enhancement of the positive vorticity anomaly by a downslope vortex stretching effect, however this only serves to enhance the downstream propagation of the disturbance to the free streamline.

The relative vorticity generation in the upper layer is enhanced if the upper layer is thinner (since the volume of fluid is decreased) and/or the slope is steeper, and thus for some slope and upper layer depth we would expect that it might inhibit the instability. At some point (taking into account the cross-stream component due to (4) \& (5)), there will be no cross-stream component to the resultant of the vorticity induction vectors at our point of consideration in Fig(2). For a steeper slope and/or thinner upper layer depth, the resultant of the vorticity induction vectors would be such as to provide a restoring effect to any perturbations.

In GKS(1982), where the upper layer is effectively infinitely deep, there can be no counteracting upper layer relative vorticity generation and thus no stabilising effect on the current.

Interestingly, this mechanism is not the whole story. Fig(8) shows the plot of growth rate ( $\left.\varepsilon c_{i m}\right)$ vs wavenumber for $\alpha=0$ (the case of no slope), when $\bar{h}_{1}=10$. For $\alpha=0$ we know that the stabilising mechanism discussed above cannot operate, and in the absence of any other effects would expect the longwave instability to extend to $k=0$. The above discussion omits the stretching and compression of upper layer fluid columns associated with the movement of the interface separating the two layers. We beleive that this gives rise to an additional stabilizing mechanism.

\section{Second Instability}

Another type of instability was found at higher wavenumbers and Fig(9) shows a plot of growth rate vs wavenumber for this band, where $\alpha=.05$, and $\bar{h}_{1}=20$. The instability was not found to be present for lower slopes or larger upper layer depths. In fact the instability was enhanced for smaller upper layer depths, although it is correct to say that the $\bar{h}=K$ approximation is greatly violated for such a large slope when the upper layer depth is small. 
FIG $8 \quad \bar{h}_{1}=20$

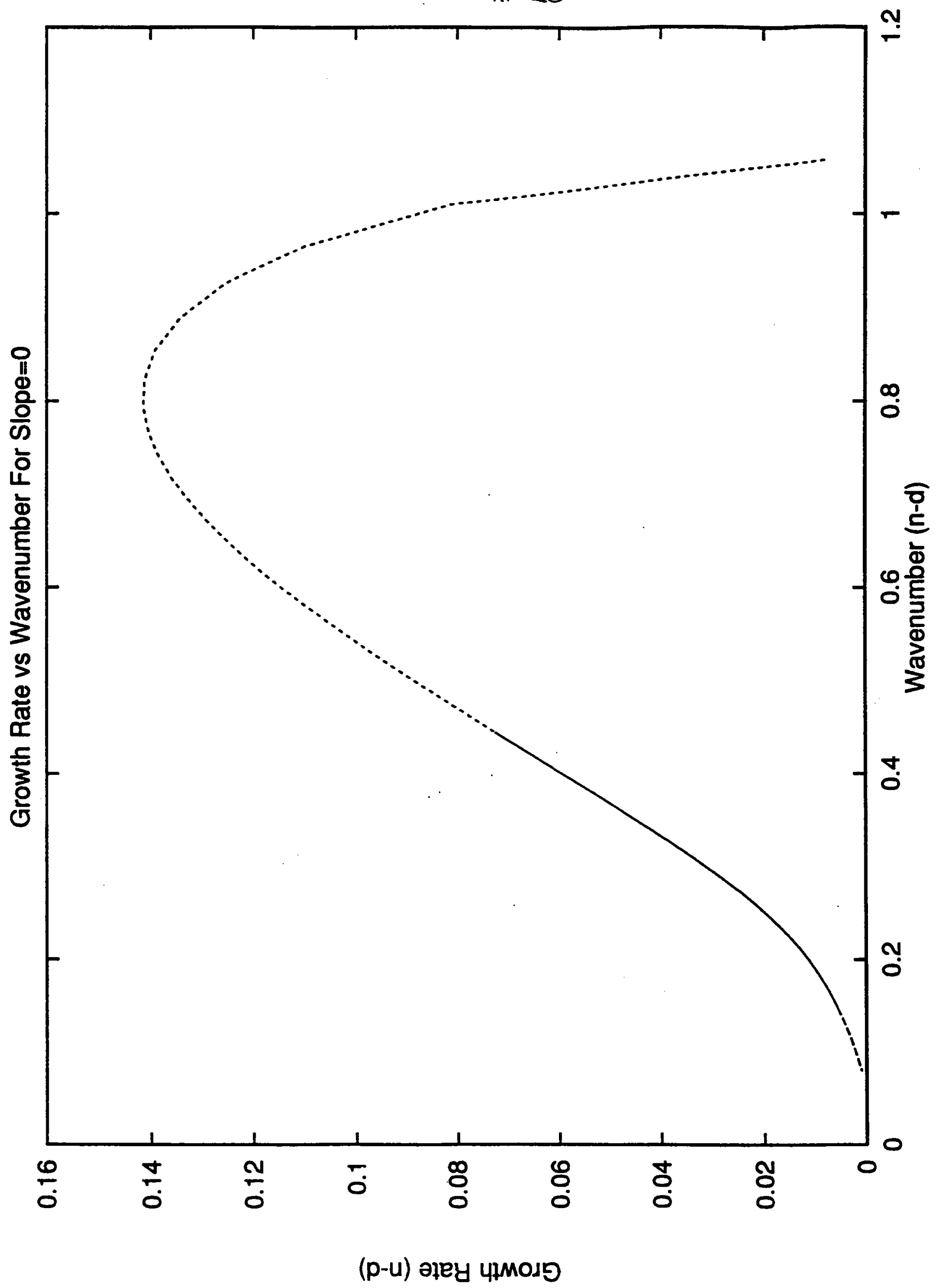




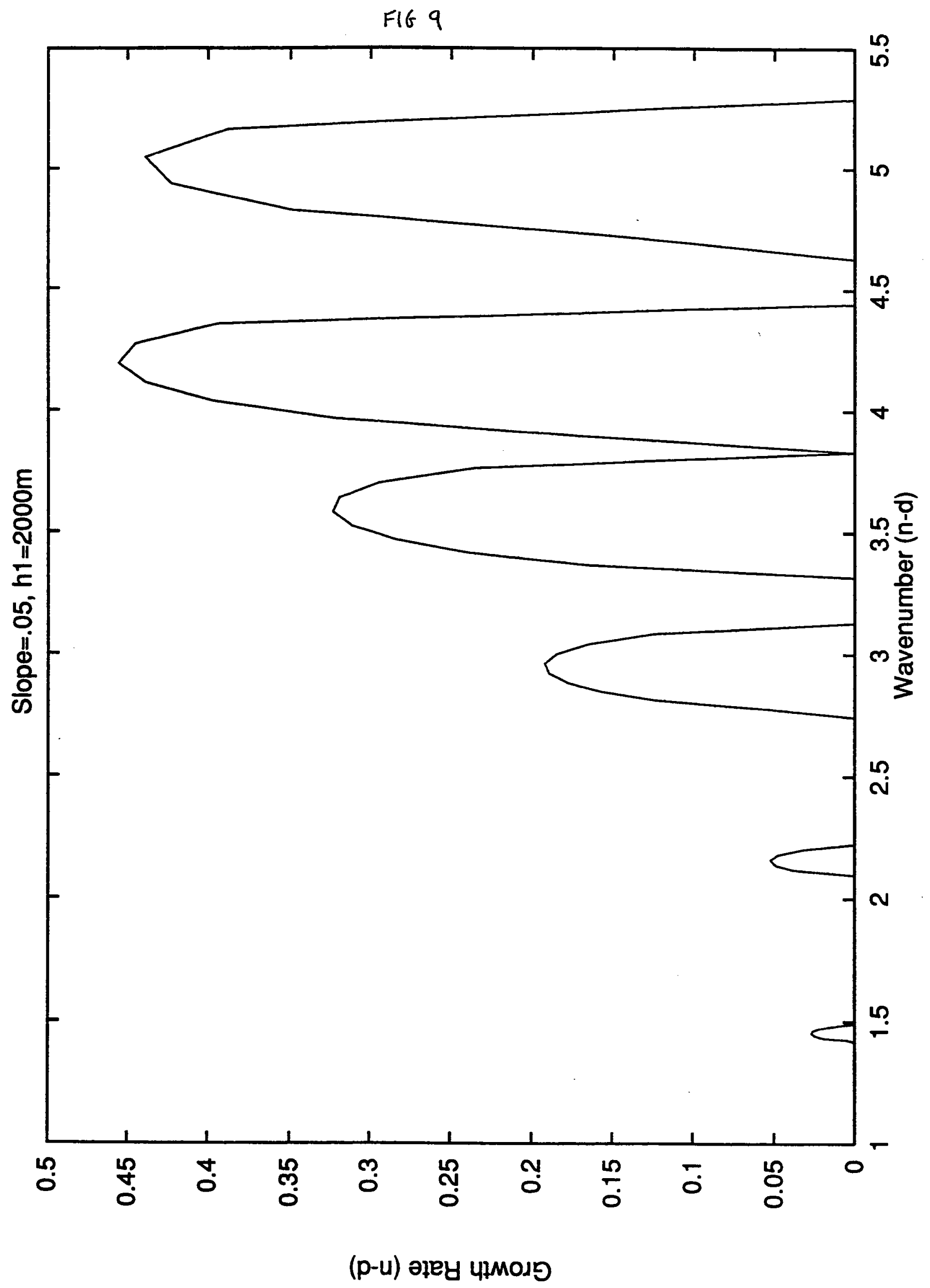


Fig(10) shows a plot of $\eta$ for the unstable mode $k=2.96$ which corresponds to a wavelength of approximately $3 L_{d}$.

Fig(11) shows the free streamline perturbations, which exhibit both a meandering and varicose. nature.

Further work needs to be conducted to determine the nature of the instability via energetics, to determine the energy conversion terms. The instability could be baroclinic, in which case we would expect to see cross-stream heat fluxes associated with potential energy release from the basic state. If the instability were barotropic, we would expect to observe kinetic energy release form the basic state, though of course it could be a mixed mode.

\section{Conclusions}

(1) Two-layer effects exert a stabilising influence on the flow for long wavelengths. The band of unstable waves decreases in width and the growth rate decreases in magnitude with increasing slope and decreasing $\bar{h}_{1}$.

The stabilising mechanism proposed depends on the relative vorticity generation by vortex stretching and compression in the upper layer due to cross-stream perturbations of the current. This vorticity can cancel vorticity anomalies due to the moving current (which has -ve relative vorticity); anomalies which would otherwise serve to destabilise the flow.

(2) Further work needs to be conducted to determine the nature of the second instability, and it is not possible at the present time to make comment.

\section{Acknowledgements}

The author wishes to express his thanks to Steve Meacham, for his many hours of patient supervision, Barry Ruddick and Melvin Stern for their many helpful ideas \& comments, and everyone in the program for helping to make it a wonderful summer. 
FIG 10

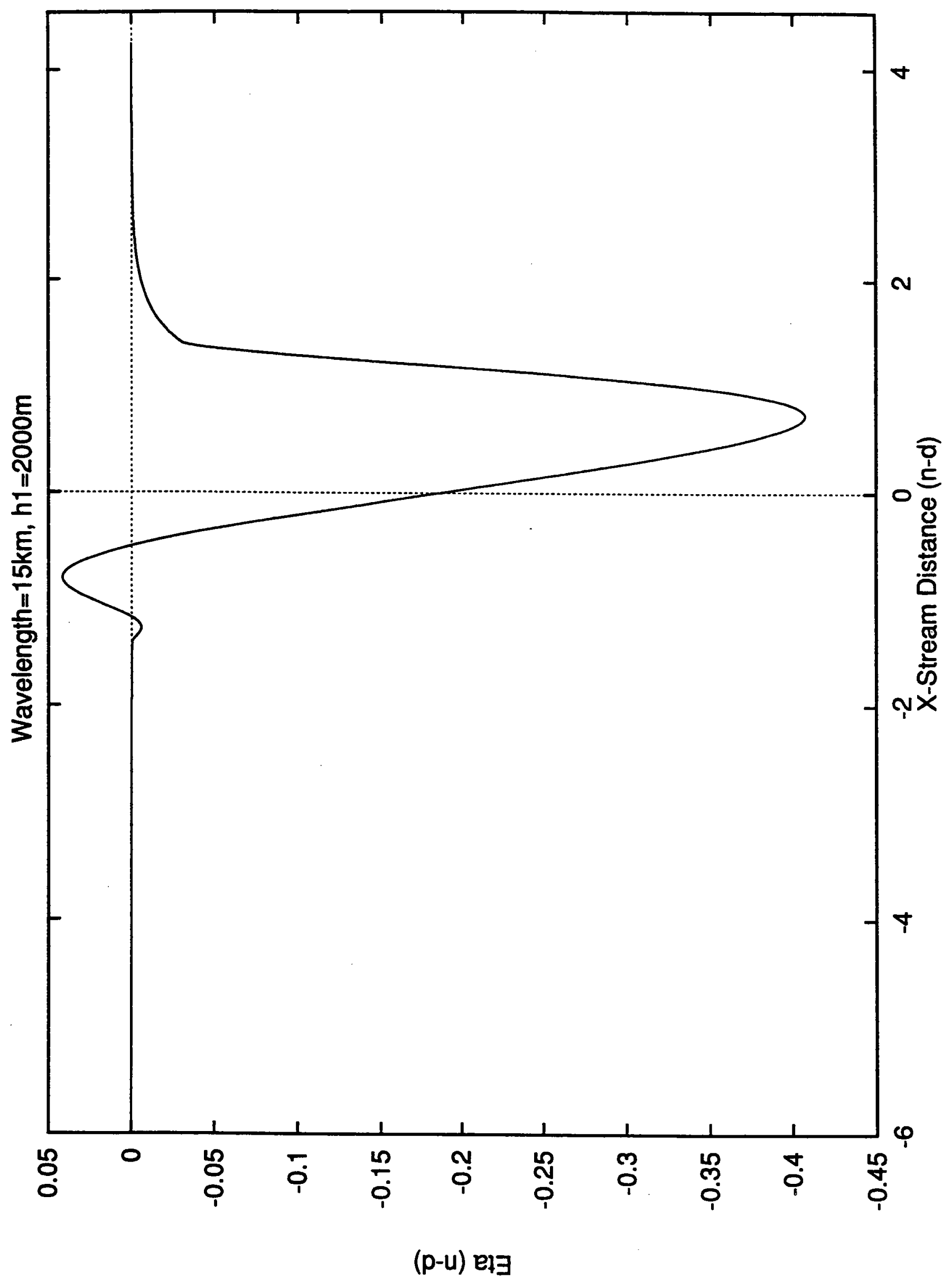


FIG 11 streamline perturbattons

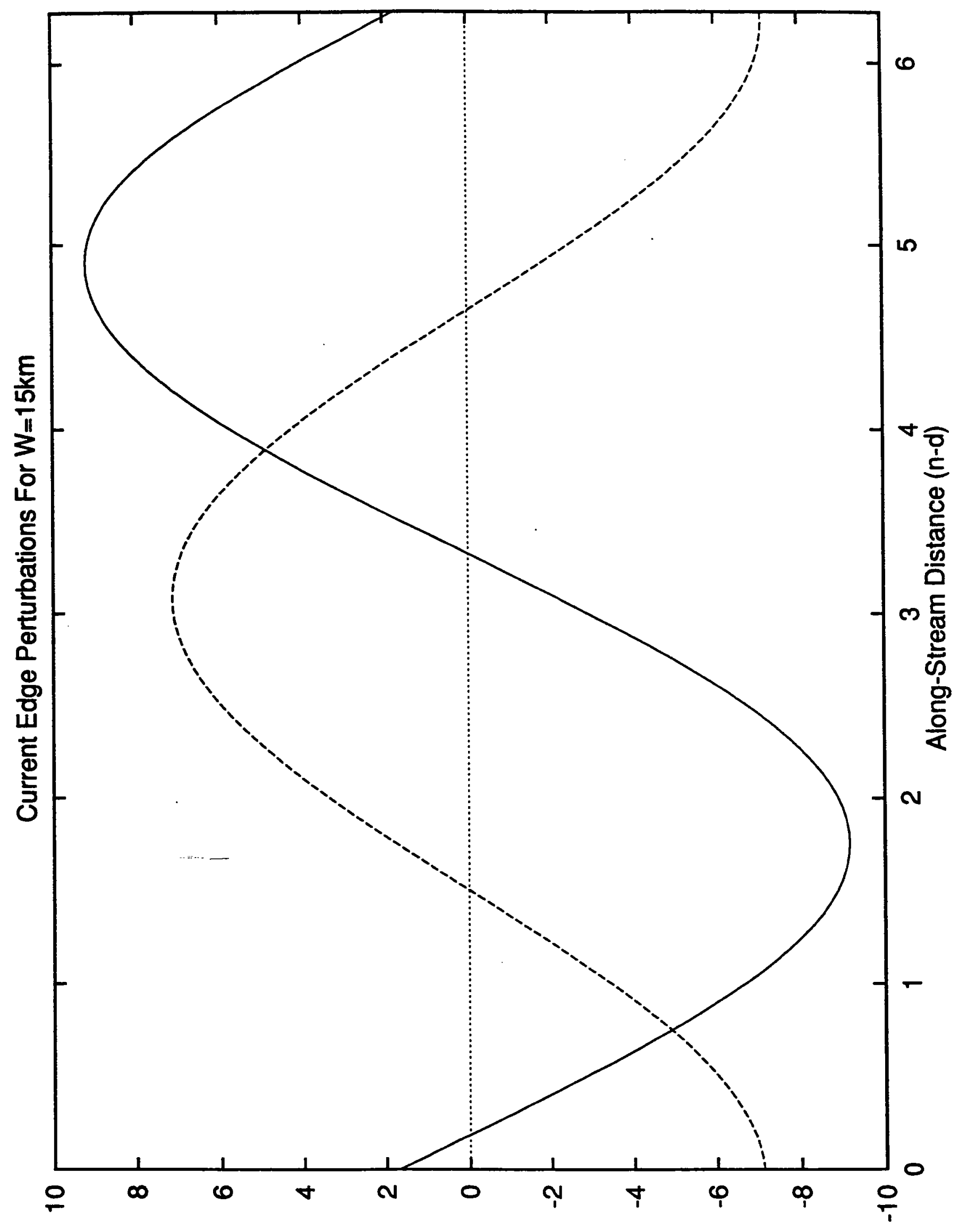




\section{References}

(1) Griffiths R.W, Killworth P.D, Stern M.E, Ageostrophic Instability Of Ocean Currents. JFM (1982) vol 117, pp. 343-377.

(2) Griffiths R.W, Gravity Currents In Rotating Systems. Ann Rev Fluid Mech (1986) vol 18, pp. 59-89. 


\title{
The Takens-Bogdanov bifurcation in an extended system
}

\author{
N. J. Balmforth and K. A. Julien
}

In thermohaline convection the onset of instability can be either through an overstable oscillation (a Hopf bifurcation), or via a steady mode of overturning (typically a subcritical pitchfork bifurcation when overstable oscillations exist). Which of these pathways is actually taken is dependent upon the particular fluid system under consideration. Here we will be concerned with the situation in which the two possible bifurcations occur simultaneously. This "degenerate" situation is often referred to as the Takens-Bogdanov bifurcation.

The unfolding of the Takens-Bogdanov bifurcation for thermohaline convection was previously carried out by Knobloch and Proctor (1981), and Coullet and Spiegel (1983). They concentrated on two-dimensional fluid systems bounded above and below by rigid, stress-free plates, at fixed temperature and salinity and which were assumed strictly periodic in the horizontal. Here we take their analysis further by considering a fluid with the same vertical boundary conditions, but which is extensive in the horizontal. Rather than deriving an amplitude equation of the form of an ordinary differential equation, this leads us to find a partial differential system governing the development of the instability. An alternative approach to this problem was taken by Bretherton (1981), who used a Galerkin truncation to find amplitude equations.

\section{Formulation}

The equations governing a Boussinesq, two-dimensional fluid can be cast in the form,

$$
\begin{gathered}
\nabla^{4} \psi+R_{T} \Theta_{x}-R_{S} \Sigma_{x}=\frac{1}{\sigma} \partial_{t} \nabla^{2} \psi+J\left(\psi, \nabla^{2} \psi\right) \\
\nabla^{2} \Theta+\psi_{x}=\partial_{t} \Theta+J(\psi, \Theta)
\end{gathered}
$$

and

$$
\tau \nabla^{2} \Sigma+\psi_{x}=\partial_{t} \Sigma+J(\psi, \Sigma)
$$

for the fluctuating parts of the streamfunction, $\psi(x, z, t)$, temperature, $\Theta(x, z, t)$, and salinity, $\Sigma(x, z, t)$. These equation are free of dimensions, which has lead to the inclusion of the thermal and saline Rayleigh numbers, $R_{T}$ and $R_{S}$, the Prandtl number, $\sigma$, and the inverse of the Lewis number, $\tau$. The symbol $J(X, Y)$ represents the usual Jacobian.

Equations (1) - (3) are to be solved subject to the boundary conditions,

$$
\psi=\psi_{z z}=\Theta=\Sigma=0
$$

on the plates, which we locate at $z=0$ and $\pi$. Note that the system (1)-(4) possesses $O(2)$-symmetry with translation, $x \rightarrow x+d$, and reflection, $x \rightarrow-x$, invariance. 


\section{Linear theory}

On neglecting the nonlinear terms in (1)-(3), introducing the dependences, $\exp (i k x+$ $\lambda t) \sin z$ (so we consider only the gravest mode in the vertical), and solving for the eigenvalue, $\lambda$, we arrive at the cubic dispersion relation,

$\lambda^{3}+p^{2}(1+\sigma+\tau) \lambda^{2}+p^{4}\left[(\sigma+\tau+\sigma \tau)-\frac{\sigma k^{2}}{p^{6}}\left(R_{T}-R_{S}\right)\right] \lambda+\sigma \tau\left[p^{6}-k^{2}\left(R_{T}-\frac{R_{S}}{\tau}\right)\right]=0$

(cf. Veronis, 1965), where $p^{2}=1+k^{2}$.

When the final term on the left-hand side of (5) vanishes, the system is marginally unstable to a direct instability. That is, for

$$
R_{T}^{s t e a d y}=\frac{R_{S}}{\tau}+\frac{p^{6}}{k^{2}}
$$

For $R_{T}$ greater than this critical value, the mode with wavenumber $k$ is unstable. If $\lambda=i \omega$, with $\omega$ real, then

$$
\begin{gathered}
\omega^{2}=p^{4}\left[(\sigma+\tau+\sigma \tau)-\frac{\sigma k^{2}}{p^{6}}\left(R_{T}-R_{S}\right)\right] \\
=\frac{\sigma \tau}{p^{2}(1+\sigma+\tau)}\left[p^{6}-k^{2}\left(R_{T}-\frac{R_{S}}{\tau}\right)\right]
\end{gathered}
$$

and the system undergoes a Hopf bifurcation. By combining (7) and (8) we arrive at another critical condition, $=R_{T}^{h o p f}$. However, we will be dealing with the Takens-Bogdanov point for which the two bifurcation occur simultaneously. This occurs when the frequency of the Hopf mode vanishes which reveals both (6) and the relation,

$$
R_{T}=R_{S}+\frac{p^{6}}{\sigma k^{2}}(\sigma+\tau+\sigma \tau)
$$

The curves of marginal stability and the loci of the Takens-Bogdanov points for $\sigma=7$ and $\tau=0.9$ are shown in figure 1 . The important features of this figure that the curves of marginal oscillatory instability are bounded by the locus of the Takens-Bogdanov points and disappear at the minimum of this locus where two Takens-Bogdanov points collide.

It is clear from (6) and (9) that the Takens-Bogdanov point is the co-dimension two bifurcation located on tuning the two parameters, $R_{T}$ and $R_{S}$. These parameters are then defined as functions of the remaining parameters, $\sigma$ and $\tau$, and the wavenumber, $k$. The wavenumber appears in these formulae through the quantity, $p^{6} / k^{2}=\left(1+k^{2}\right)^{3} / k^{2}$. This quantity has its minimum at the value, $k=k_{c}=1 / \sqrt{2}$. Hence, if $R_{T}$ and $R_{S}$ take the values given by (6) and (9) with $k=k_{c}$, then the state is marginally stable with respect to this critical wavenumber, and all other wavenumbers are stable. The critical values satisfying (6) and (9) with $k=k_{c}$ we denote by

$$
R_{T}=R=\frac{p^{6}}{k^{2}} \frac{(\sigma+\tau)}{\sigma(1-\tau)}, \quad R_{S}=S=\frac{p^{6}}{k^{2}} \frac{\tau^{2}(\sigma+1)}{\sigma(1-\tau)} .
$$


Our goal is to now perturb the system around this special point, and derive a weakly nonlinear amplitude equation. The important point is that for $R_{T}>R$ and $R_{S}>S$, there are bands of unstable modes. These bands may be associated with either direct instabilities or overstability, or mixtures of the two (see figure 2). As $R_{T} \rightarrow R$ and $R_{S} \rightarrow S$, these bands shrink to a point around the marginal value, $k=k_{c}$ (see figure 1). In other words, the parameter values $R_{T}=R, R_{S}=S$ and $k=k_{c}$ reflect a state in which not only do we have a Takens-Bogdanov bifurcation, but it is at marginal stability. Furthermore, this state actually corresponds to the collision and disappearance of two Takens-Bogdanov points approaching from different wavenumbers. Thus, in the perturbation expansion, we tune both $R_{T}$ and $R_{S}$ and introduce a long length scale $X=\left(k-k_{c}\right)^{-1}$ to unfold this doubly degenerate bifurcation.

\section{Asymptotic expansion}

We now pose the asymptotic expansions,

$$
\psi=\epsilon \psi_{1}+\epsilon^{2} \psi_{2}+\ldots, \quad \Theta=\epsilon \Theta_{1}+\epsilon^{2} \Theta_{2}+\ldots \quad \text { and } \quad \Sigma=\epsilon \Sigma_{1}+\epsilon^{2} \Sigma_{2}+\ldots
$$

rescale time by $T=\epsilon t$, and introduce the multiple length scales, $x$ and $X=\epsilon x$. As part of the unfolding of the Takens-Bogdanov degeneracy, we further pose

$$
R_{T}=R+\epsilon r_{1}+\epsilon^{2} r_{2} \quad \text { and } \quad R_{S}=S+\epsilon s_{1}+\epsilon^{2} s_{2} .
$$

To leading order, the equations (1)-(3) become,

$$
\begin{gathered}
\nabla^{4} \psi_{1 x}+R \Theta_{1 x x}-S \Sigma_{1 x x}=0 \\
\nabla^{2} \Theta_{1}+\psi_{1 x}=0
\end{gathered}
$$

and

$$
\tau \nabla^{2} \Sigma_{1}+\psi_{1 x}=0 .
$$

By virtue of our choices, $R_{T}=R$ and $R_{S}=S$, these equations have a unique solution,

$$
\left(\begin{array}{c}
\psi_{1 x} \\
\Theta_{1} \\
\Sigma_{1}
\end{array}\right)=\left[A(X, T) e^{i k_{c} x}+\text { c.c. }\right] \sin z\left(\begin{array}{c}
1 \\
2 / 3 \\
2 / 3 \tau
\end{array}\right) .
$$

At next order, we have the system,

$$
\begin{gathered}
\nabla^{4} \psi_{2}+R \Theta_{2 x}-S \Sigma_{2 x}=-\frac{3}{2 \sigma} \psi_{1 T}-R \Theta_{1 X}+S \Sigma_{1 X}+6 \psi_{1 X x}-r_{1} \Theta_{1 x}+s_{1} \Sigma_{1 x} \\
\nabla^{2} \Theta_{2}+\psi_{2 x}=\Theta_{1 T}+\frac{4}{3}|A|^{2} \sin 2 z-2 \Theta_{1 X x}-\psi_{1 X}
\end{gathered}
$$

and

$$
\tau \nabla^{2} \Sigma_{2}+\psi_{2 x}=\Sigma_{1 T}+\frac{4}{3 \tau}|A|^{2} \sin 2 z-2 \tau \Sigma_{1 X x}-\psi_{1 X}
$$


The solution for $\left(\psi_{2 x}, \Theta_{2}, \Sigma_{2}\right)$ can be divided into two pieces (a further, homogeneous solution can be ignored). The first is the harmonic of the original mode:

$$
\left(\begin{array}{c}
\psi_{2 x}^{(2)} \\
\Theta_{2}^{(2)} \\
\Sigma_{2}^{(2)}
\end{array}\right)=-\frac{1}{3}|A|^{2} \sin 2 z\left(\begin{array}{c}
0 \\
1 \\
1 / \tau^{2}
\end{array}\right)
$$

whereas the second has dependence $e^{i k_{c} x} \sin z$. This second piece requires some consideration since we need to avoid it being unbounded. The equation for second component can be written in the form, $\left(\psi_{2 x}^{(1)}, \Theta_{2}^{(1)}, \Sigma_{2}^{(1)}\right)^{T} e^{i k_{c} x} \sin z$, where

$$
M\left(\begin{array}{c}
\psi_{2 x}^{(1)} \\
\Theta_{2}^{(1)} \\
\Sigma_{2}^{(1)}
\end{array}\right)=A_{T}\left(\begin{array}{c}
1 \\
2 / 3 \\
2 / 3 \tau
\end{array}\right)+i k_{c} A_{X}\left(\begin{array}{c}
-\sigma \\
2 / 3 \\
2 / 3
\end{array}\right)+A\left(\begin{array}{c}
-\sigma\left(r_{1}-s_{1} / \tau\right) / 3 \\
0 \\
0
\end{array}\right)
$$

and

$$
M=\left(\begin{array}{ccc}
-3 \sigma / 2 & \sigma R / 3 & -\sigma S / 3 \\
1 & -3 / 2 & 0 \\
1 & 0 & -3 \tau / 2
\end{array}\right)
$$

The matrix, $M$, has both a null vector, $\mathbf{e}_{1}$, and a generalized eigenvector, $\mathbf{e}_{2}$, defined by

$$
\mathbf{e}_{1}=\left(\begin{array}{c}
1 \\
2 / 3 \\
2 / 3 \tau
\end{array}\right) \quad \text { and } \quad \mathbf{e}_{2}=\left(\begin{array}{c}
0 \\
-4 / 9 \\
-4 / 9 \tau^{2}
\end{array}\right)
$$

that is, $M \mathrm{e}_{1}=\mathbf{0}$ and $M \mathrm{e}_{2}=\mathrm{e}_{1}$. Moreover, there is an adjoint vector to $\mathrm{e}_{1}$,

$$
\mathbf{e}_{1}^{\dagger}=(1,2 \sigma R / 9,-2 \sigma S / 9 \tau),
$$

with property, $\mathrm{e}_{1}^{\dagger} M=0$. Provided the vector on the right-hand side of (21) is therefore orthogonal to $\mathbf{e}_{1}^{\dagger}$, we find a bounded solution. In fact, on taking the product of $\mathrm{e}_{1}^{\dagger}$ with (21), we discover that we must insist that $r_{1}=s_{1} / \tau$. Then the solution can be written in the form,

$$
\left(\begin{array}{c}
\psi_{2 x} \\
\Theta_{2} \\
\Sigma_{2}
\end{array}\right)=A_{T} e^{i k_{c} x} \sin z \mathbf{e}_{2}+i k_{c} A_{X} e^{i k_{c} x} \sin z\left(\begin{array}{c}
0 \\
-4 / 9 \\
-4 / 9 \tau
\end{array}\right)+c . c .-\frac{1}{3}|A|^{2} \sin 2 z\left(\begin{array}{c}
0 \\
1 \\
1 / \tau^{2}
\end{array}\right) .
$$


We now proceed to order $\epsilon^{3}$. The system that needs to be solved is

$$
\begin{gathered}
\nabla^{4} \psi_{3}+R \Theta_{3 x}-S \Sigma_{3 x}= \\
3 \psi_{1 X X}-R \Theta_{2 X}+S \Sigma_{2 X}-r_{1} \Theta_{2 x}+s_{1} \Sigma_{2 x}-r_{2} \Theta_{1 x}+s_{2} \Sigma_{1 x}+O . T . \\
\nabla^{2} \Theta_{3}+\psi_{3 x}=\Theta_{2 T}+\frac{1}{3}|A|^{2} A e^{i k_{c} x} \sin z-2 \Theta_{2 X x}-\Theta_{1 X X}+\text { O.T. }
\end{gathered}
$$

and

$$
\tau \nabla^{2} \Sigma_{3}+\psi_{3 x}=\Sigma_{2 T}+\frac{1}{3 \tau^{2}}|A|^{2} A e^{i k_{c} x} \sin z-2 \tau \Sigma_{2 X x}-\tau \Sigma_{1 X X}+\text { O.T. }
$$

where O.T. indicates other, non-resonant terms. We can solve (26)-(28) provided we take the Fredholm Alternative,

$$
A_{T T}+\mu A_{T}+\gamma A-\delta A_{X X}-2|A|^{2} A=0,
$$

where

$$
\begin{aligned}
& \mu=-\frac{2 \sigma(1-\tau) r_{1}}{9(1+\sigma+\tau)} \\
& \gamma=-\frac{\sigma \tau\left(r_{2}-s_{2} / \tau\right)}{3(1+\sigma+\tau)}
\end{aligned}
$$

and

$$
\delta=\frac{6 \sigma \tau}{(1+\sigma+\tau)} .
$$

Equation (29) is the amplitude equation governing the instability.

\section{Regularization}

Although the asymptotic calculation outlined above leads to what at first sight appears to be a perfectly sensible amplitude equation, it is degenerate and the formulation is fundamentally flawed. This can be seen immediately on looking for travelling wave solutions to (29); if one introduces the travelling wave solution, $A(\xi)=A(X-c T)$, then separates real and imaginary parts, the only solution is for $c=0$. In other words, the travelling wave solutions that emerge at the Hopf bifurcations which unfold from the degenerate Takens-Bogdanov point are not captured by the asymptotic amplitude equation (29). The equation does however capture the steady, overturning solutions, but it remains an incomplete description.

This problem is deep-rooted in the asymptotic unfolding of the Takens-Bogdanov bifurcation. For periodic boxes, there is a similar problem in the ordinary amplitude equation (Spiegel, 1981). In fact, (29) reduces to the form of that equation if one simply omits the $X$-derivatives.

To recover the missing pieces in the unfolding, one must resort to the more sophisticated techniques of normal-form theory in the context of periodic systems. Such a theory does not yet exist for extended systems. Instead we need to follow the somewhat inelegant, alternative route of reconstitution (Spiegel, 1981). In the purely periodic case, this 
amounts to proceeding to next order, then adding certain of the new terms back into the original equation for the amplitude, $a(t)$. This leads one to add a term of the form, $a^{2} \dot{a}$, into the amplitude equation. This term models a nonlinear dissipation and also emerges from the normal-form analysis (Coullet and Spiegel, 1983).

If we follow a similar procedure here to obtain the corresponding term, we arrive at the regularized amplitude equation,

$$
A_{T T}+\mu A_{T}+i k \nu A_{X T}+\gamma A-\delta A_{X X}-2|A|^{2} A+\varpi\left(|A|^{2} A_{T}+\frac{11}{8} A|A|_{T}^{2}\right)=0,
$$

where

$$
\varpi=\epsilon(2 / 3)^{5} \frac{(\tau+\sigma)(\tau+1)+1}{\tau(1+\sigma+\tau)} .
$$

In principle, one should also reconstitute terms of higher order in the spatial derivatives, and higher-order nonlinear terms. However, for now, we omit these. Equation (33) is the regularized amplitude equation, some of whose solutions we will describe next. Note that, on omitting the spatial derivatives, (33) reduces to the normal form presented by Dangelmayr and Knobloch (1984) with the exception of a term $\left|A_{T}\right|^{2} A$ which formally occurs at higher order.

\section{Linear theory again}

If we now retain only the linear terms of (33), we can again consider the stability of the hydrostatic background. If we set $A=\exp \lambda T+i K X$, then the eigenvalue satisfies

$$
\lambda=-\frac{1}{2} \mu \pm \sqrt{\frac{1}{4} \mu^{2}-\gamma-\delta K^{2}}
$$

At $K=0$, this is just

$$
\lambda_{0}=-\frac{1}{2} \mu \pm \sqrt{\frac{1}{4} \mu-\gamma} .
$$

For $\mu>0$, there is therefore a real eigenvalue (namely that with the + sign) that passes through zero as $\gamma$ decreases through zero. That is, the bifurcation of direct modes occurs for $K=0$ at $\gamma=0$. In addition, for $\gamma<0$, there is a Hopf bifurcation at $\mu=K=0$, and for $\mu<0$ there is overstability. 


\section{Finite amplitude solutions}

Equation (33) admits a class of steady solutions, $A=R e^{i K(X-c T)}$, with

$$
\left(\delta-c^{2}\right) K^{2}-i c \mu K+\gamma=(2+i c \varpi K) R^{2} .
$$

The real parts of (37) imply

$$
R^{2}=\frac{1}{2}\left[\gamma+\left(\delta-c^{2}\right) K^{2}\right],
$$

whereas the imaginary terms give

$$
c\left(\varpi R^{2}+\mu\right)=0 .
$$

We may solve these two equations for either stationary solutions, or for travelling waves of finite amplitude. For the former, $c=0$ and so

$$
R^{2}=\frac{1}{2}\left(\gamma+\delta K^{2}\right),
$$

Since $\gamma<0$ indicates the degree of direct instability, equation (40) evidently corresponds to a steady branch of subcritical solutions. These solutions are therefore unstable.

The travelling waves have $c \neq 0$, and rearrangement of (38) and (39) gives

$$
R^{2}=-\mu / \varpi
$$

and

$$
c^{2}=\delta+\frac{\left(\gamma-2 R^{2}\right)}{K^{2}}=\delta+\frac{\varpi \gamma+2 \mu}{\varpi K^{2}} .
$$

From linear theory we know that overstability sets in when $K=0$ and $\mu \leq 0$. Thus the travelling wave solutions emerge supercritically. Note that the amplitude and wavespeed of these solutions diverge as $\varpi \rightarrow 0$.

\section{Discussion}

This note has described the derivation of an amplitude equation at the marginally stable, Takens-Bogdanov point. For thermohaline convection with boundaries that are stress-free, and of fixed temperature and salinity, the asymptotic theory does not uncover a complete description of the dynamics. Rather, additional nonlinear dissipative terms need to be recovered from higher order as in the standard Takens-Bogdanov problem.

One method around the difficulty with the amplitude equation would be to try different physical systems for which the coefficient of the cubic term could also be made to vanish at, for example, some values of $\sigma$ and $\tau$. The analysis could then be taken to higher order with a different scaling for the time derivative (in fact, one like the Malkus-Veronis scaling, $\left.T=\epsilon^{2} t\right)$. However, that is not possible for the current system since the relevant coefficient in (29) is negative definite and independent of the parameters. Such problems highlight the need for a normal-form theory for extended systems. 
For the thermohaline system considered here, the points in parameter space for which the cubic coefficient vanishes are also co-dimension two bifurcations. This point is actually given by $R_{T}=R_{T}^{s t e a d y}$ and $R_{S}=R_{S}^{c u b i c} p^{6} \tau^{3} / k^{2}\left(1-\tau^{2}\right)$. Thus as for Taken-Bogdanov, such points also lie on parabolic loci on the $k-R_{T}$ plane. Moreover, when $k=k_{c}$, the cubic coefficient vanishes at the point of marginality. Furthermore, since $R_{S}^{c u b i c}<S$, the steady bifurcation must always be subcritical in the neighbourhood of the Taken-Bogdanov points. At $R_{S}^{c u b i c}$, an asymptotic reduction results in an amplitude equation of the form,

$$
A_{T}+\gamma A-A_{X X}+\delta|A|^{2} A-|A|^{4} A=0 .
$$

A combination of this equation and (33) is what one would derive if one could force the Takens-Boganov bifurcation to occur simultaneously with the vanishing of the cubic coefficient. Presumably, that amalgamation would contain a wealth of dynamics.

For the regularized system (33), we have provided some steady and travelling solutions. Of these, the steady rolls are subcritical and therefore unstable. The travelling solutions are supercritical, but they may be unstable to secondary instabilities of the Eckhaus variety (see Fauve, 1991). This must be uncovered by looking at the linear stability of the finiteamplitude solutions, which leads into a version of phase dynamics for the thermohaline system. As pointed out by Bretherton (1981), the system may also lead to solitary wave solutions in the limit that (34) approaches a Klein-Gordon equation. In that circumstance, soliton perturbation theory can be used to elucidate the solitary wave dynamics. Such directions are worthy of future work.

\section{References}

- Bretherton, C., 1981. Double diffusion in a long box. GFD summer project.

- Coullet, P., and E. A. Spiegel, 1983. Amplitude equations for systems with competing instabilities. SIAM J. App. Math., 43, 776-821.

- Dangelmayr, G., and E. Knobloch, 1987. Takens-Bogdanov bifurcation with O(2)symmetry. Phil. Trans. of Royal Soc. Lond., 322, 243-279.

- Fauve, S., 1991. Patterns in fluid Hlow. GFD Lecture Notes.

- Knobloch, E., and M.R.E. Proctor 1981. Nonlinear periodic convection in doublediffusive systems. J. Fluid Mech., 108, 291-316.

- Spiegel, E. A., 1981. Physics of convection. GFD Lecture Notes.

- Veronis, G., 1965. On finite amplitude instability in thermohaline convection. J. Mar. Res., 23, 1-17. 


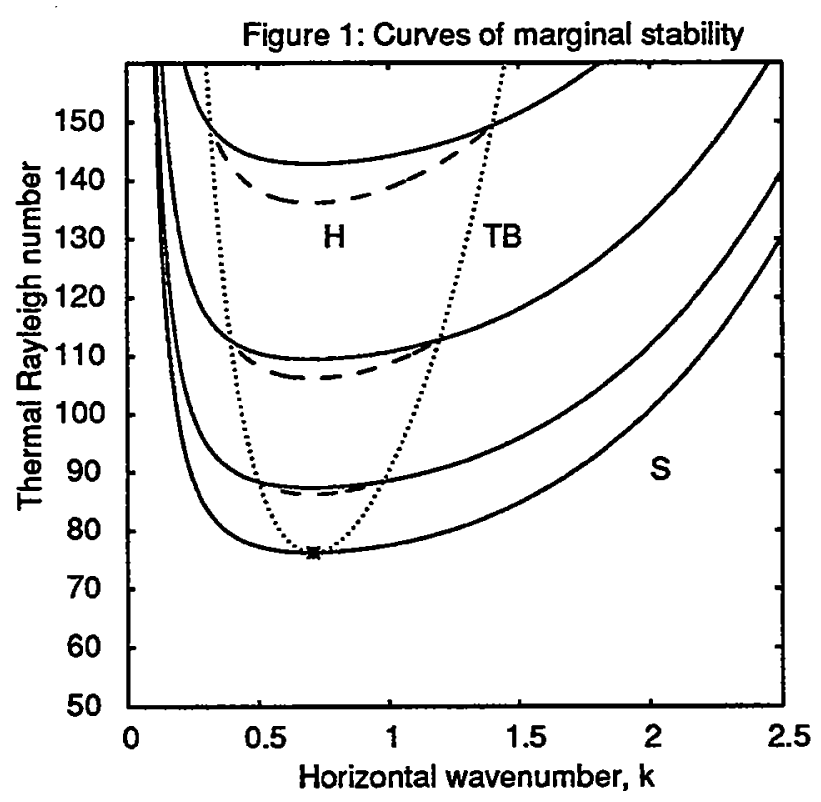

Figure 1: Curves of marginal stability for $\sigma=7$ and $\tau=0.1$. The solid curves (marked $S$ ) show the onset of steady convection, and the dashed curves (marked $H$ ) indicate the onset of overstability, for $R_{S}=S+10 j$ with $j=0,1,2$ and 3 . The dotted curve shows the locus of Takens-Bogdanov points $(\operatorname{marked} T B)$.

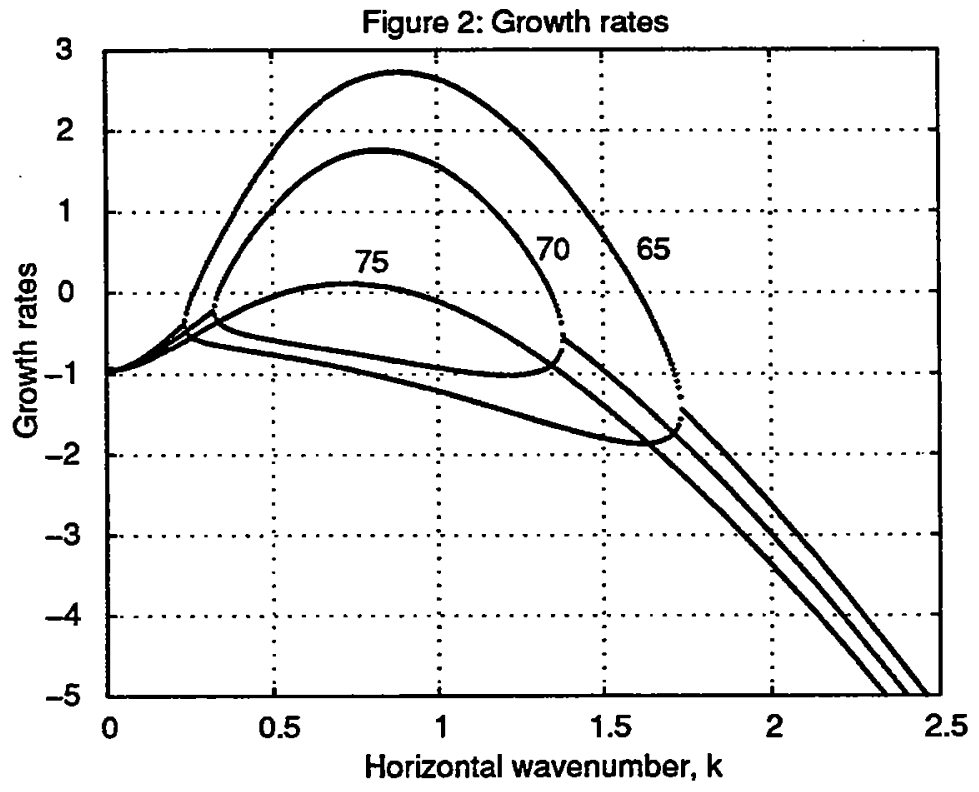

Figure 2: Growth rates against $k$ for $R_{T}=90$ and $R_{S}=65,70$ and 75 . For $R_{S}=65$ and 70 , there is a band of direct instability. When $R_{S}=75$, the system possesses a band of overstabilities. 


\title{
Woods Hole Oceanographic Institution \\ Program of Summer Study \\ Geophysical Fluid Dynamics \\ - 1996 -
}

\section{Double-Diffusive Intrusions Across the Arctic Ocean}

\author{
E. C. Carmack \\ Institute of Ocean Sciences (IOS), Sidney, B.C., V8L 4B2, CANADA
}

\begin{abstract}
Ddata obtained during the Canada/U.S. 1994 Arctic Ocean Section shows that a major warming and ventilation of the mid-depth layers of the Arctic Ocean (200-1000 m) occured due to influx of anomalously warm waters from the Atlantic. This transition from one stationary thermohaline structure to another appears to have begun in the early 1990s; now, anomalously warm waters are found in the Nansen, Amundsen, and Makarov basins, with the largest temperature difference, as much as $1{ }^{\circ} \mathrm{C}$, in the core of the Atlantic layer. The transition is occuring via multiple intrusions, $40-60 \mathrm{~m}$ thick, extending laterally through the Atlantic and upper deep waters; these features appear to be laterally coherent across the Arctic Ocean $(>2000 \mathrm{~km})$ and to persist over time-scales of several years. Once formed, the layers can support both diffusive and salt finger convection. Potential temperature $(\theta)$ versus salinity (S) correlation curves for each successive diffusive and salt finger regime are parallel, with higher values of the stability frequencey $(N)$ in the diffusive regime than in the salt finger regime. However, the process of thermohaline transition via self-organized and self-propelled layers has not been simulated, parameterized, or accounted for in GCMs.
\end{abstract}

\section{Introduction}

Thermohaline transitions are changes, either temporal or spatial, from one stationary thermohaline structure to another. The phenomenology generally involves one water mass either displacing or lying in juxaposition to another, slightly different, water mass, with lateral exchanges occuring via the formation of intrusions along near-isopycnal surfaces. Such intrusions give rise to alternating layers of diffusive and salt finger convection (Ruddick, 1992). Spatial transitions are well known to exist in ocean settings such as frontal regimes or across mesoscale features (cf. Federov, 1970). Similar intrusions can also occur in the deep waters of lakes; for example, where distinct water masses occur in basins separated by sills (cf. Wuest et al., 1988).

Observations of recent changes in the thermohaline structure of the Arctic Ocean obtained aboard the CCGS Louis S. St-Laurent during the Canada/U.S 1994 Arctic Ocean Section 
(Arctic-94; Figure 1) are presented. Data were collected using a Neil Brown conductivitytemperature-depth (CTD) system (with accuracies estimated at $\mathrm{T}= \pm 0.001{ }^{\circ} \mathrm{C} ; \mathrm{S}= \pm 0.002$; and $\mathrm{D}= \pm 2 \mathrm{~m}$ ). Potential temperature $(\theta)$ and density $(\sigma)$ were calculated from algorithms in Unesco (1983).

\section{Thermohaline Changes in the Arctic Ocean}

Warm, relatively salty water of Atlantic origin enters the Arctic Ocean through Fram Strait and the Barents Sea, subducts beneath the cold and relatively fresh surface water, and flows counterclockwise along the continental slope and submarine ridges (Figure 1; Carmack et al., 1990). Fresher and less dense water of Pacific origin enters through Bering Strait and likewise flows in a counterclockwise direction (Aagaard, 1989). Both waters exit the Arctic into the North Atlantic via Fram Strait and the complex Canadian Archipelago. Earlier views held that this flow maintains quasi-stationary water properties in the interior Arctic Ocean, and that significant variability occured only on the adjacent marginal seas and shelves; for example, data obtained in the Canada and Makarov basins prior to the 1990's show the Atlantic layer temperature maximum $\left(\Theta_{\max }\right)$ between 0.4 to $0.6^{\circ} \mathrm{C}$ (cf. Carmack et al., 1995). Above this layer cold, nutrient-rich Pacific water was believed to form a stationary structure which extended across the Canada and Makarov basins to the Lomonosov Ridge (Kinney, et al., 1970; Pounder, 1985). During the past five years, however, evidence has appeared of a major thermohaline transition event within the core of the Atlantic layer, first in 1990 in the Nansen Basin north of Svalbard (Quadfasel et al., 1991) and then in 1993 in the Makarov Basin north of the East Siberian Sea (Carmack et al., 1995; McLaughlin et al., 1996). Data from Arctic-94 show that this trend now extends across the Nansen, Amundsen, and Makarov basins (Carmack et al., 1996). For example, the vertical section of $\Theta$ from the Chukchi Sea to the North Pole (Figure 2) shows the three main thermal layers of the Arctic Ocean: cold $\left(\Theta<0^{\circ} \mathrm{C}\right)$ surface water in the upper $150-200 \mathrm{~m}$, warm Atlantic water $\left(\Theta>0{ }^{\circ} \mathrm{C}\right)$ at mid-depths, and cooler deep water $\left(\Theta<0^{\circ} \mathrm{C}\right)$ below about $800 \mathrm{~m}$. The multiple cores of water warmer than $0.8^{\circ} \mathrm{C}$ between $200-350 \mathrm{~m}$ are associated with topographically-steered currents following the continental slope and submarine ridges crossed by the ship. Comparison with earlier data North American ice camps prior to the 1990s shows that the largest temperature change, over $2{ }^{\circ} \mathrm{C}$, occurs near $\overline{200} \mathrm{~m}$ since the Atlantic core is now both warmer and shallower. However, near-surface water $(50-100 \mathrm{~m})$ is now actually colder due to the replacement of waters of Pacific origin by surface waters from the Eurasian Basin. The missing Pacific water likely exited into the North Atlantic or the Labrador Sea via Fram Strait or the Canadian Archipelago.

\section{Thermohaline Structure}

Selected profiles of $\Theta$ and $S$ versus depth (Figure 3) show the basic thermohaline structure of the Arctic Ocean. In the Atlantic layer above $\Theta_{\max }$ both temperature and salinity increase with depth (cf. Padman and Dillon, 1987), while below $\Theta_{\max }$ the water column is stabilized by both $\Theta$ and $\mathbf{S}$ gradients. Superimposed on the large scale distributions are multiple, finescale horizontal intrusions in which $\Theta$ inversions are stabilized by a density compensating salinity profile. The upper portion of each finescale intrusion may support the diffusive instability, 
while the lower portion may support the salt finger instability (cf. Schmitt (1994). Profiles of the density ratio, $R_{p}$, show relative constant values within each regime for each successive layer; e.g. about 0.2 to 0.4 for the diffusive regimes and 1.5 to 1.8 for the salt finger regimes.

Expanded scale correlation $\Theta / S$ diagrams from all Arctic-94 stations (Figure 4) show that the intrusion of new water occurs in coherent, well-defined layers. Further, the intrusions are aligned in $\Theta / S$ space, and appear immune to mixing and disruption by shear flow and internal waves. Surprisingly, the intrusions occur in exactly the same place on $\Theta / S$ space as observed in other parts of the Arctic in 1991 (Rudels et al., 1994) and 1993 (Carmack et al., 1995a). Hence, the layers are both laterally coherent across the entire Arctic Basin and quasistationary in time. The aspect ratio (length/height) is near $2 \times 10^{-6}$, much smaller than scales typically reported for ocean finestructure (cf. Federov, 1979).

Figure 4 also shows that the slopes of $\Theta / S$ for each successive layer in the vertical form a set of near-parallel lines for both the diffusive and salt finger regimes. The salt finger regimes cross isopycnals at a small angle $\left(\mathrm{R}_{\mathrm{p}}\right.$ about 1.2$)$ suggesting that the transformation of layers as they spread laterally is not a purely isopycnal mixing process, but rather is such as to increase the salinity (and density) more rapidly that than can be compensated for by the heat flux. The $\Theta / S$ slopes associated with the diffusive regimes (about 4 ) are close to those giving maximum contraction on mixing, while the $\Theta / S$ slopes associated with the salt finger regimes (about 20) are close that those yielding maximum stability to mixing (minimum potential energy) when the effects of contraction on mixing and differential compressibility are considered (cf. Bennett, 1996).

Because the salt finger regimes of each intrusion have constant $\Theta / S$ slopes, it is useful to "rotate" the salinity axis by defining $S_{\text {mix }}=S-(\Delta S / \Delta \Theta) \Theta$, where $\Delta S / \Delta \Theta=-0.056$, so as to vertically align the salt finger regimes, as is done in the $\Theta / S_{\text {mix }}$ scatter diagram shown in Figure 5. Here, each $\Theta / \mathrm{S}_{\operatorname{mix}}$ data point represents a layer of water $4 \mathrm{~m}$ thick, so that the density of points represents the volumetric distribution of water properties. Also shown in Figure 5 is the distribution of stability frequency, $N=((g / \rho)(\delta \rho / \delta z))^{1 / 2}$. Here, several features are evident. First, the water is volumetrically "banded" or quantum-like in $\Theta / S_{\operatorname{mix}}$ space; that is, there is a greater volume of water occurring along narrow $S_{\operatorname{mix}}$ lines aligned with the salt finger regime than elsewhere. Second, while $\mathbf{N}$ shows a general decrease with depth from values of about $4 \mathrm{hr}^{-1}$ in the thermocline region to about $0.5 \mathrm{hr}^{-1}$ in the deep water, the pattern is dominated by a series of alternating values associated with each intrusion: within each diffusive regime $\mathbf{N}$ is maximum; within each salt finger regime $\mathbf{N}$ is near-zero. Within each individual intrusion $\mathbf{N}$ (and potential vorticity) is constant across the full width of the Arctic Ocean.

The thermohaline transitions span not only the Atlantic layer, but in places extend well into the deep water. For example, Figure 6 is a $\Theta / S_{\operatorname{mix}}$ plot expanded in scale to show detail in the deep water. Here, layer structure is clearly evident to depths exceeding about $2000 \mathrm{~m}$, the approximate sill depth of the Lomonosov Ridge which separates waters of the Makarov Basin from the Atlantic. Inspection of individual station data suggest that the intrusions eminate from new water entering the Makarov Basin along the flank of the Alpha-Mendeleyev Ridge. 


\section{Conclusions}

Data from the first oceanographic crossing of the Arctic Ocean document what appears to be a recent influx of anomalously warm water from the Atlantic into the Arctic Ocean at middepth. These data to signal a major change in the thermohaline structure of the Arctic Ocean driven by changes in the transport of Atlantic waters through Fram Strait and the Barents Sea, and an internal re-arrangement of water mass boundaries. However, the process of theromhaline transition via self-organized and self-propelled double-diffusive intrusions has not been simulated, parameterized, or accounted for in GCMs.

\section{Acknowledgements}

I must acknowledge the talents and efforts of the Arctic-94 hydrography team: Knut Aagaard, Jim Swift, Ron Perkin, Fiona McLaughlin, Peter Jones, and Robie Macdonald. I deeply appreciate the efforts of the officers and crew of the CCGS Louis S. St-Laurent, in making Arctic-94 a successful mission. We are indebted to the extraordinarily competent technical support of all science personnel on Arctic-94; in particular: J. Elliott, D. Muus, R. Nelson, R. Pearson, D. Sieberg, and R. Williams.

\section{References}

Aagaard, K., 1989: A synthesis of the Arctic circulation, Rapp. P.-v Reun. Cons. int Explor. Mer. 188, 11-22.

Aagaard, K., and E. C. Carmack, 1989: On the role of sea-ice and other freshwater, $J$. Geophys. Res. 94, 14485-14498.

Aagaard, K., and E. C. Carmack, 1994: The Arctic and climate: A perspective, In: The Polar Oceans and Their Role in Shaping the Global Environment, O. M. Johannessen, R. D. Muench, and J. E. Overland, Eds., pp. 4-20, Geophys. Monogr. 85, Am. Geophys. Un.

Anderson, L. G., G. Bjork, O. Holby, E. P. Jones, G. Kattner, K. P. Koltermann, B. Liljeblad, R. Lindegren, B. Rudels, and J. Swift, 1994: Water masses and circulation in the Eurasian Basin: Results from the Oden-91 expedition, J. Geophys. Res., 99, 32733283.

ACSYS, Arctic Climate Systems Study, Initial Implementation Plan, WCRP-85, 66 p., 1994.

Bennett, E. B., 1996: Stationary Ocean Structures. Prog. Oceanogr., Submitted.

Carmack, E. C., 1990: Large-scale oceanography. In: Polar Oceanography, Part A, W. O. Smith Ed. pp. 171-222, Academic, San Diego.

Carmack, E. C., R. W. Macdonald, R. G. Perkin, and F. A. McLaughlin., 1995: Evidence for warming of Atlantic Water in the southern Canadian Basin. Geophys Res. Lett.

Carmack, E. C., K. Aagaard, J. H. Swift, R. G. Perkin, F. A. McLaughlin, R. W. Macdonald, E. P. Jones, J. Smith, K. Ellis, and L. Kilius, 1996: Large thermohaline changes in the Arctic Ocean. Nature, submitted.

Gorshkov, S. G., 1983: World Ocean Atlas, Vol. 3, Arctic Ocean (in Russian) 189 p. Pergamon, New York.

Kinney , P., M. E. Arhelger, and D. C. Burrell, 1970: Chemical characteristics of water masses in the Amerasian Basin of the Arctic Ocean, J. Geophys. Res., 75, 4097-4104. 
Padman, L., and T. M. Dillon, 1987: Vertical fluxes through the Beaufort Sea thermohaline staircase. J. Geophys. Res., 93, 10,799-10,806.

Perkin, R. G., and E. L. Lewis, 1984: Mixing in the West Spitsbergen Current, J. Phys. Ocean., 14, 1315-1325.

Pounder, E. R. 1986: Physical oceanography near the North Pole, J. Geophys. Res., 91, 11,763-11, 773, 1986.

Quadfasel, D., A. Sy, D. Wells, and A. Tunik, 1991: Warming in the Arctic, Nature, 350, 385.

Ruddick, B., Intrusive mixing in a mediterannean salt lens - Intrusion slopes and dynamical mechanisms, J. Phys. Oceanogr., 22, 1274-1285.

Rudels, B., E. P. Jones, L. G. Anderson, and G. Kattner, 1994: On the intermediate depth waters of the Arctic Ocean, In: The Polar Oceans and Their Role in Shaping the Global Environment, O. M. Johannessen, R. D. Muench, and J. E. Overland, Eds., Geophys. Monogr. 85, Am. Geophys. Un.

Schmitt, R. W., 1994: Double Diffusion in Oceanography. Annu. Rev. Fluid Mech., 26, 255285.

Unesco, 1983: Algorithms for computation of fundamental properties of seawater. Tech. Pap. Mar. Sci., 44, 53 pp..

Wuest, A., D. M. Imboden, and M. Schurter, 1988: Origin and size of hypolimnic mixing in Umersee, the southern basin of Vierwaldstattersee (Lake Lucerne), Schweiz, $Z$. Hydrol., 50, 40-70.

\section{Figure Captions}

Figure 1. Map showing the Arctic Ocean and Arctic-94 station locations.

Figure 2. Sections across the Canadian Basin of (a) $\Theta$ and (b) $\Theta$ anomaly.

Figure 3. Vertical profiles of $\Theta, S$, and density ratio $\left(R_{p}\right)$ at station 17 from Arctic-94.

Figure 4. Correlation $\Theta / S$ plots for Arctic-94 stations; also shown are lines of constant $\sigma_{0.5}$.

Figure 5 Correlation plots for Arctic-94 stations showing $\Theta / S_{\operatorname{mix}}$ and $N / S_{\operatorname{mix}}$, where $S_{\operatorname{mix}}$ is a parameter obtained by rotating the salinity axis to be parallel with the mixing bands, and $\mathrm{N}$ is the stability frequency.

Figure 6. Plots from Station 23 showing (a) vertical profile $s(100$ to $500 \mathrm{~m})$ of $\Theta$, $S$, and $R_{p}$, and (b) correlations of $\Theta / S$ and $\Theta / N$ in the range $\Theta=0.6$ to $-0.8{ }^{\circ} \mathrm{C}$ and $S_{\operatorname{mix}}=34.8$ to 35.0 . 


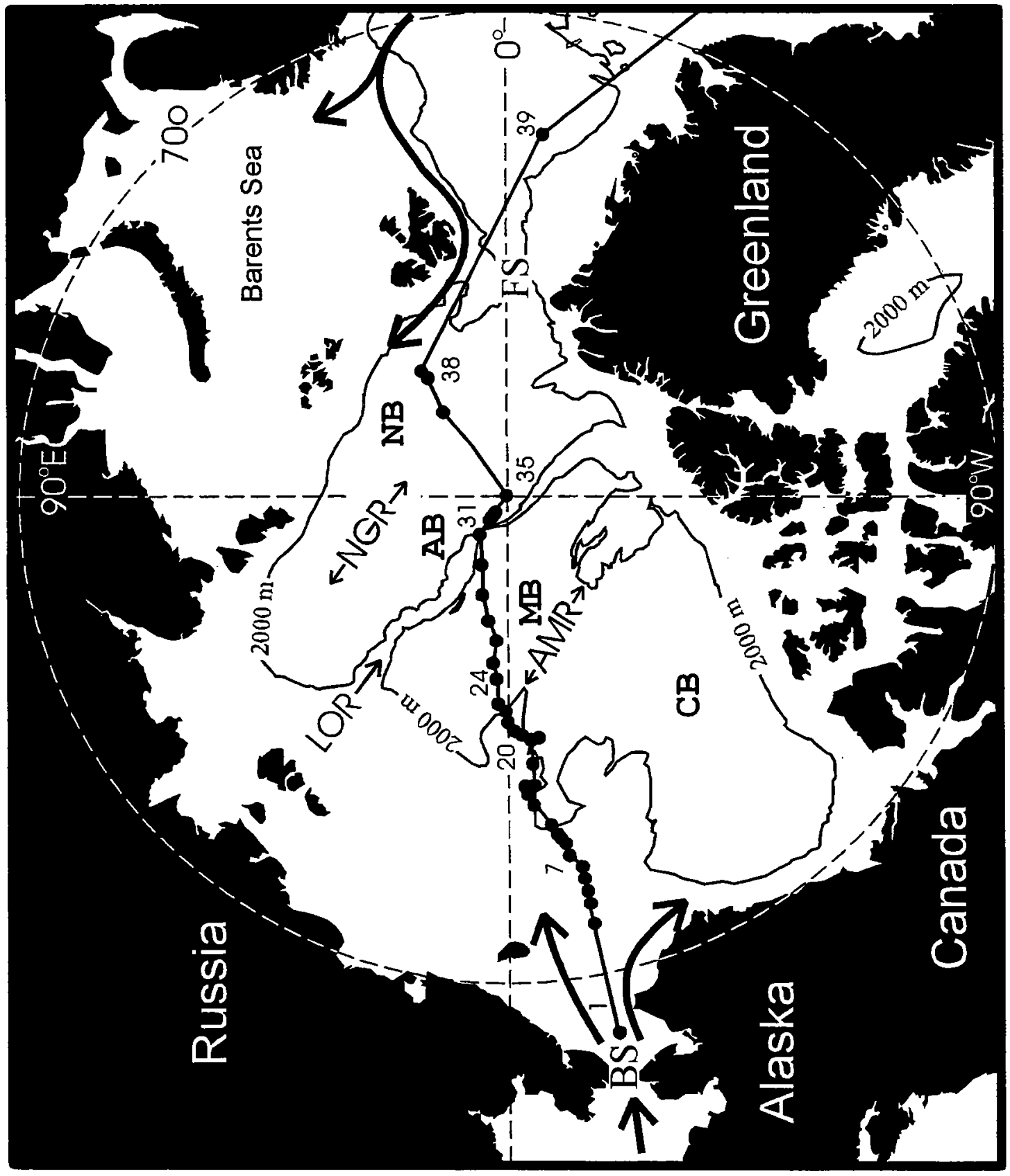

D్ 
Stations

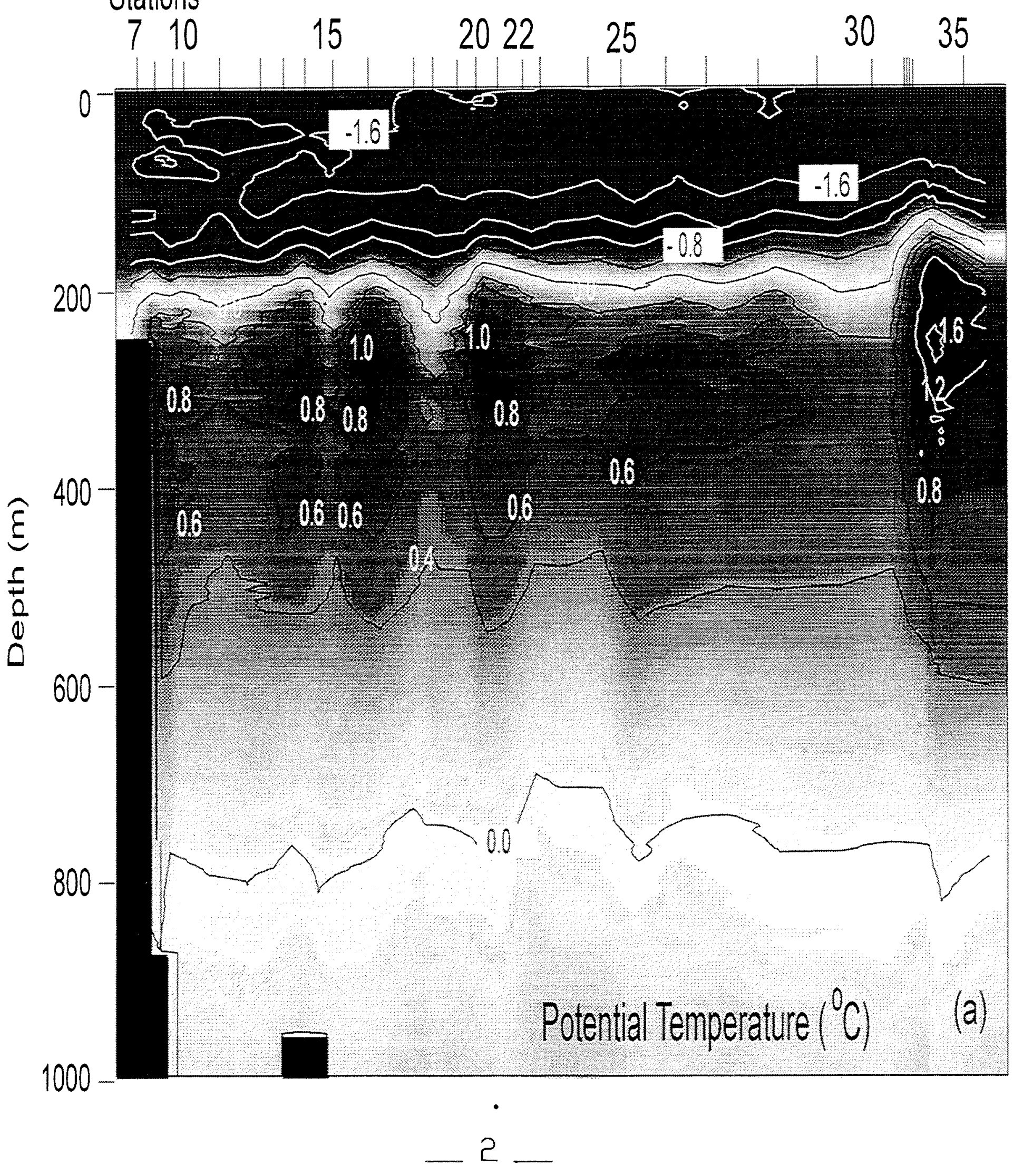




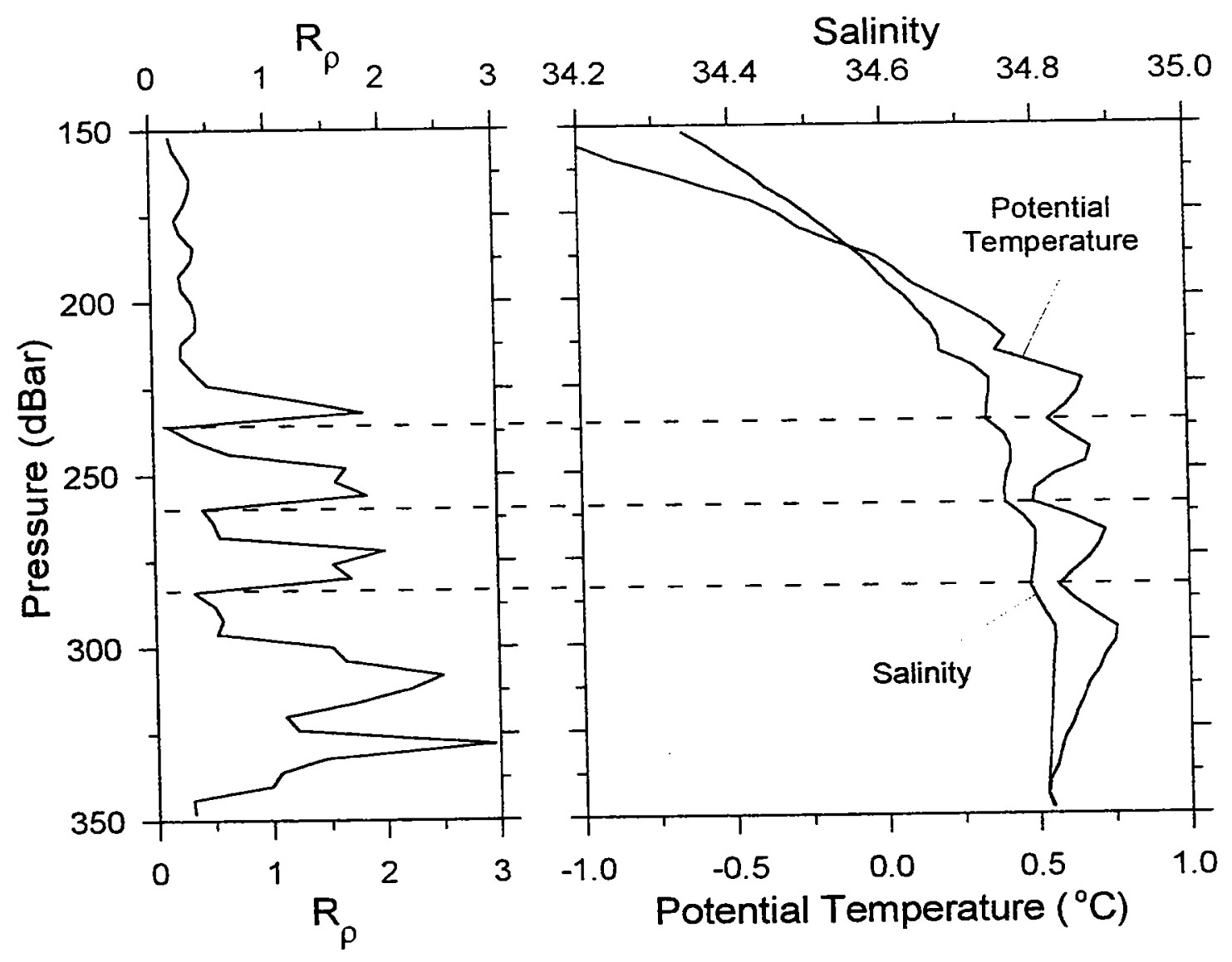

3 


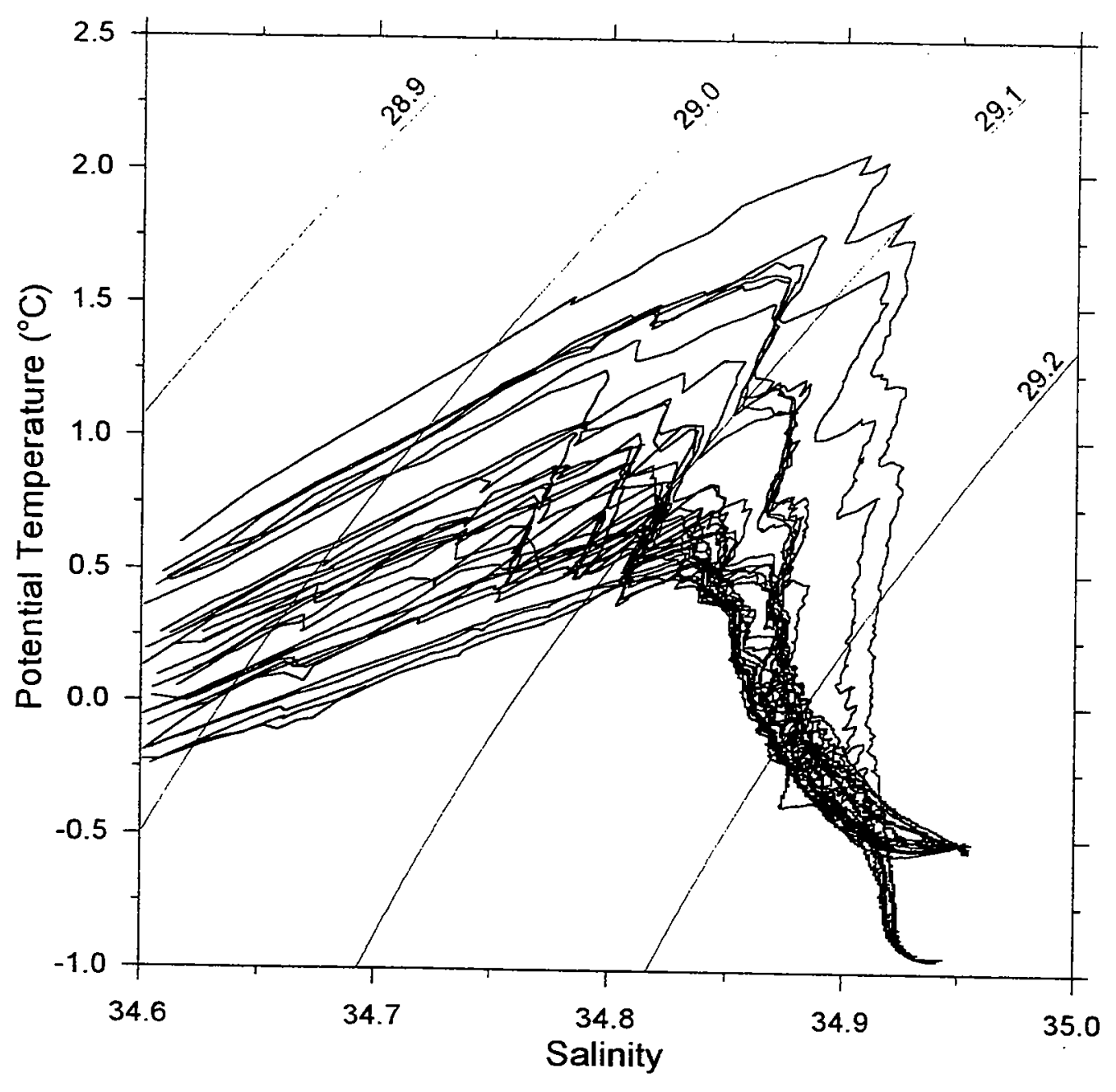

$-4$ 


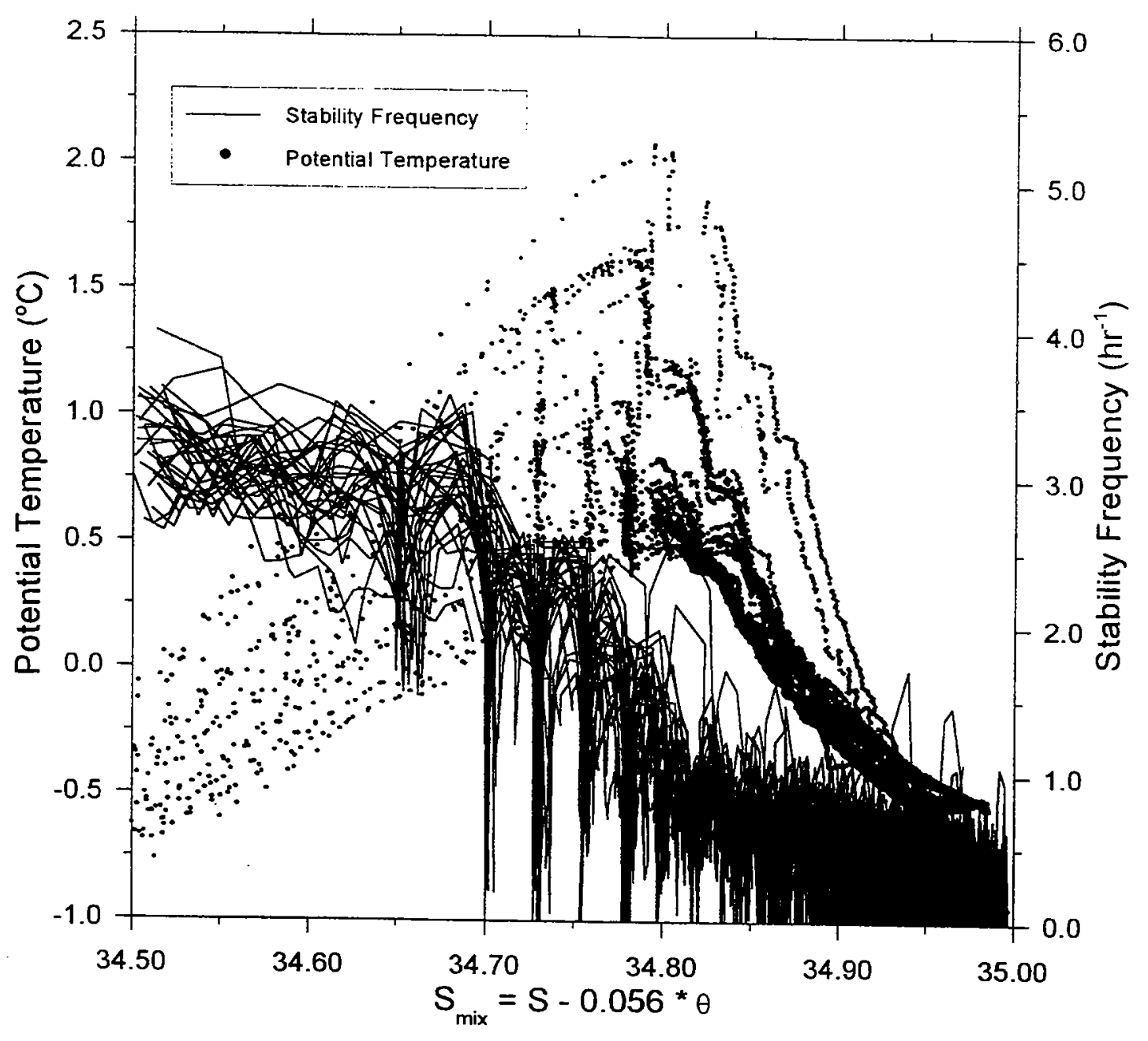

$-5$ 


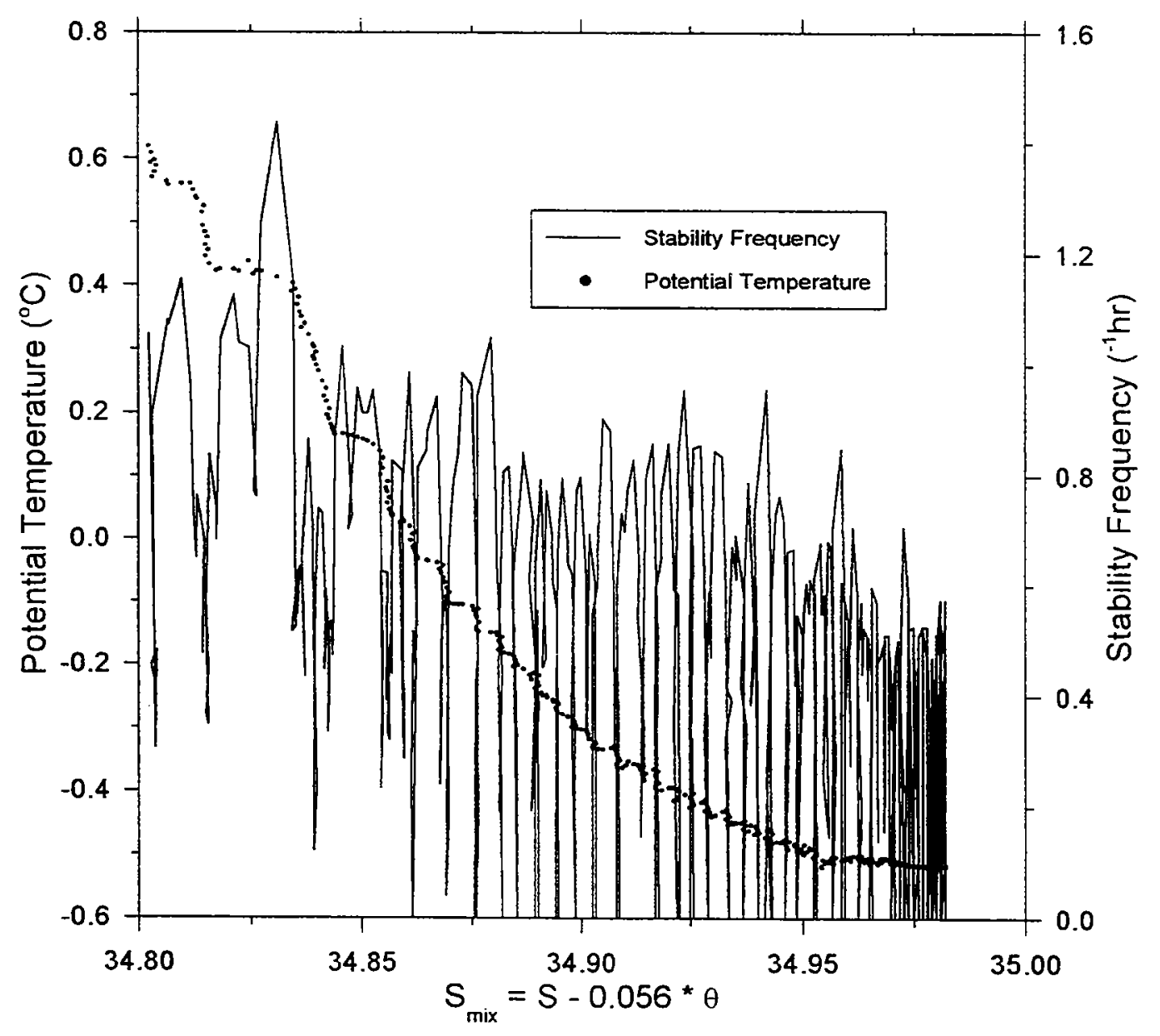

$-6$ 


\title{
Horizontal variability of thermohaline staircases in the Western Mediterranean
}

\author{
Gerd Krahmann \\ Institut für Meereskunde \\ Düsternbrooker Weg 20 \\ 24105 Kiel, Germany
}

Thermohaline staircases have been observed in large parts of the central Western Mediterranean Sea, the Algero-Provençal-Basin, during two hydrographic surveys in January and October 1994. The cruises were carried out in the framework of the THETIS-2 project [18].

The general circulation of the Mediterranean is dominated by the concentrating character of this basin: the integrated evaporation is larger than the input by precipitation and by river run-offs. This causes an inflow-outflow regime in the strait of Gibraltar with a low-salinity water mass flowing into the Mediterranean above an outward flowing high-salinity water mass. The temperature difference between the inand outflow (the inflow is warmer than the outflow) shows that the net surface heat fluxes in the Mediterranean must be directed from the sea to the atmosphere. Both net fluxes (of freshwater and of heat) cause the outflowing water mass to be denser than the inflowing and thus to be located below the inflow. A similar picture can be drawn for the eastern Mediterranean and the strait of Sicily.

In the western Mediterranean three major water masses (see fig. 1) can be found at different depth intervals (e.g. Millot [12]): Modified Atlantic Water (MAW) in the uppermost level $(0-150 \mathrm{~m})$, Levantine Intermediate Water (LIW) in the middle $(250-500 \mathrm{~m}$ ) and Western Mediterranean Deep Water (WMDW) in the deepest level ( $1400 \mathrm{~m}$ to the bottom). The depths between these levels are filled with mixed types of the three water masses.

The observed staircases occur in the depth range from 500 to $1300 \mathrm{~m}$, right below the temperature and salinity maximum of the Levantine Intermediate Water. The vertical density ratio $R_{\rho}$ ranges from 1.05 to 1.3 with a mean of 1.15 and decreasing values with depth. The vertical extension of the well-mixed layers and the size of the temperature and salinity steps is variable. However, the $\theta$-S diagram (potential temperature-salinity diagram) reveals that the well-mixed layers in different profiles are not independent from each other. The $\theta-S$ values form characteristic bands which suggest that the layers are strongly connected with each other but that divergences or convergences of the thermohaline fluxes lead to horizontal variations of the charac- 


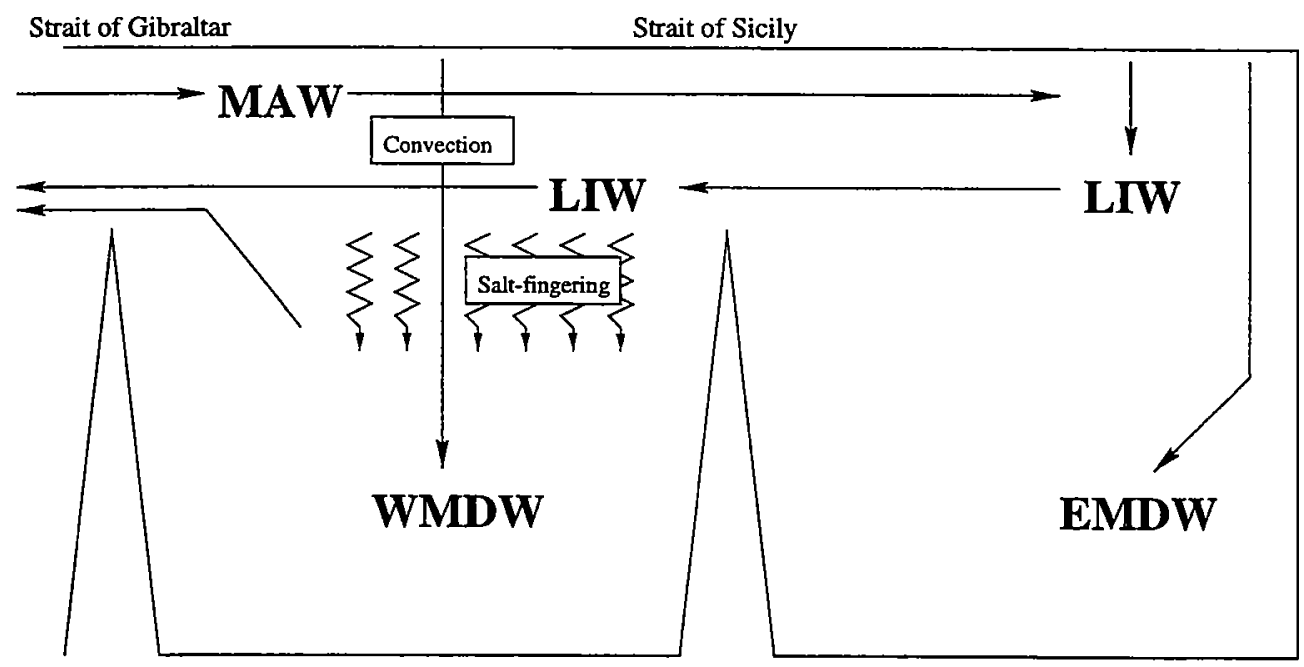

Figure 1: Watermasses of the Mediterranean Sea.

teristics. Since the temperature and salinity characteristics remain the same between the two cruises, a quasistationary equilibrium must exist between the along-layer and across-step thermohaline fluxes.

The step structures show a high variability in their pattern: some have inverted layers, a sign of active horizontal exchanges; others show very regular layers; and some with only weak-gradient interfaces between well-mixed layers of decreased thickness. This variability is used to distinguish regions with active layer-forming, with fully developed step structures, with reduced layering and without steps.

\section{Watermasses and Circulation in the Western Mediter- ranean}

Atlantic water enters the basin through the Strait of Gibraltar, is modified by mixing and thermohaline fluxes, and feeds the Algerian Current which follows the Algerian coast eastward. Part of it passes the Straits of Sardinia and Sicily (see fig. 2 for straits and basins) and takes part in the circulation in the Eastern Mediterranean, whereas other parts follow the coastlines of Sardinia and Corsica and Italy northward and merge together in the Northern Current (e.g. Sammari et al. [15] or Astraldi et al. [1]). This current turns westward, passes the southern coast of France and parts 
of it recirculate north of the Balearic islands, other parts pass the Balearic islands southward.

The LIW enters the Western Mediterranean through the Strait of Sicily below the eastward flow of the MAW. It is present throughout the whole basin as an intermediate temperature and salinity maximum. The flow of the LIW is generally cyclonic. Following approximately the $300 \mathrm{~m}$ depth contour it forms an intermediate boundary current around the eastern and northern coastlines. The central basin is entered via two passages: the Strait of Corsica and the Strait of Sardinia. Along the west coast of Sardinia and Corsica a current of LIW flows northward and merges at the northern tip of Corsica with the flow through the Strait of Corsica and feeds the Northern Current (Astraldi and Gasparini [1]) at the southern coast of France. Because of mixing with the surrounding water masses the temperature and salinity maxima become less pronounced during the spreading of the LIW so that the vertical gradients between the LIW and the homogeneous Western Mediterranean Deep Water (WMDW) become smaller.

The WMDW is formed by deep convection in the Gulf of Lions south of France (e.g. MEDOC Group [11] or Leaman and Schott [7]). Because of its high density and the blocked passage at Gibraltar (sill depth $300 \mathrm{~m}$ ) it fills the deep parts of the Western Mediterranean. The WMDW has a very uniform potential temperature and salinity (e.g. Lacombe et al. [6]). Parts of it participate in the outflow at Gibraltar together with the LIW.

\section{Measurements}

During the ocean acoustic tomography experiment THETIS-2 [18] two hydrographic surveys were carried out in the central basin of the Western Mediterranean (see fig. 3 for station maps). The first of which on R/V Poseidon (cruise 201, leg 1) lasted from 9 th of January 1994 to 4 th of February 1994. The second was on board the french R/V Suroit from 11th of October 1994 to 3rd November 1994. A total of 84 CTD station were occupied during the two cruises. The CTD measurements were obtained with a Neil Brown Mark III sonde. Bottle samples were taken together with the CTD to calibrate the conductivity sensor. The accuracy reached \pm 0.003 for salinity and $\pm 0.003^{\circ} \mathrm{C}$ for temperature. The location of the profiles was chosen to sample the connections between a large-scale mooring network within single cruises (THETIS-2 final report [18]). Thus the spacing between the stations is not optimal for the examination of thermohaline staircases. The profiles used in the analysis were averaged onto $1 \mathrm{dbar}$ steps, so that single salt-finger interfaces, which have vertical extensions of only 20 to $30 \mathrm{~cm}$, are not resolved. Nevertheless this resolution seems to be sufficient since no 


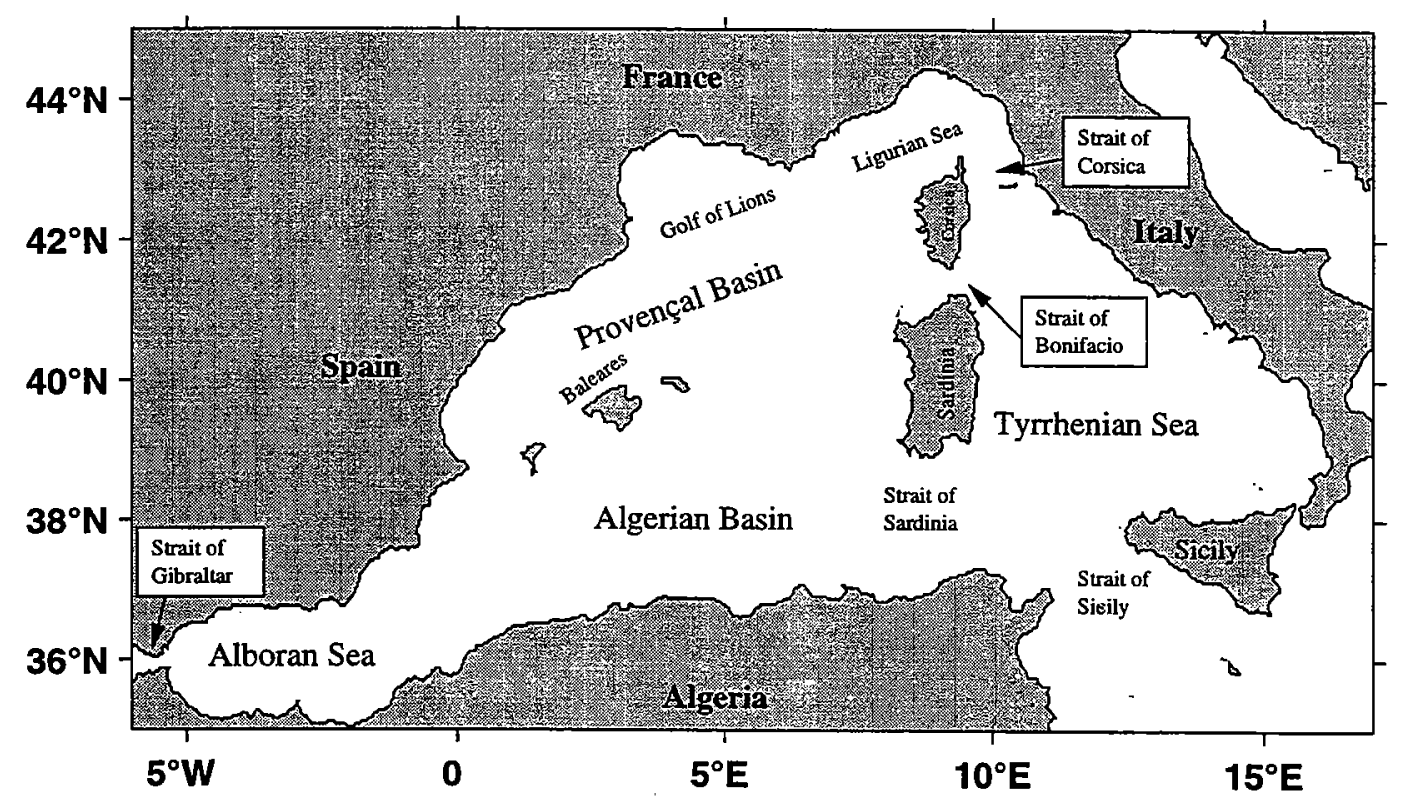

Figure 2: Names of Straits and Basins of the Western Mediterranean Sea.

observed thermohaline high-gradient interface showed thicknesses of less than $5 \mathrm{~m}$.

\section{The vertical step structure}

The thermohaline step structures in the CTD profiles in the Algero-Provençal-Basin are highly variable in difference to those found in the Tyrrhenian Sea by Molcard and Tait [14] and Johannessen and Lee [5] (see fig. 8). The temperatures, salinities and depths of steps and layers differ between the profiles. However a look into the $\theta$-S diagram shows that well-mixed layers in different profiles must have a strong connection with each other (see fig. 4) since the $\theta$-S values of the layers are located in seperate bands in the diagram.

Such a pattern with bands of values has also been found in the C-SALT area by Fleury and Lueck [4] and Schmitt et al. [16]. The band-like distribution of the $\theta$-S values allows identification of the layers, which is impossible from a simple overlaying of the profiles. Moreover most of the step structures are very similar even though the first look does not show that (see fig. 5). After application of similarity transformations (linear transformations of the form $\theta^{\prime}=c_{1} \theta+c_{2}$ and $z^{\prime}=c_{3} z+c_{4}$ ) both to the potential temperature and the depth of the profile the similarity is much more obvious 
Stations Poseidon 201 Jan 1994

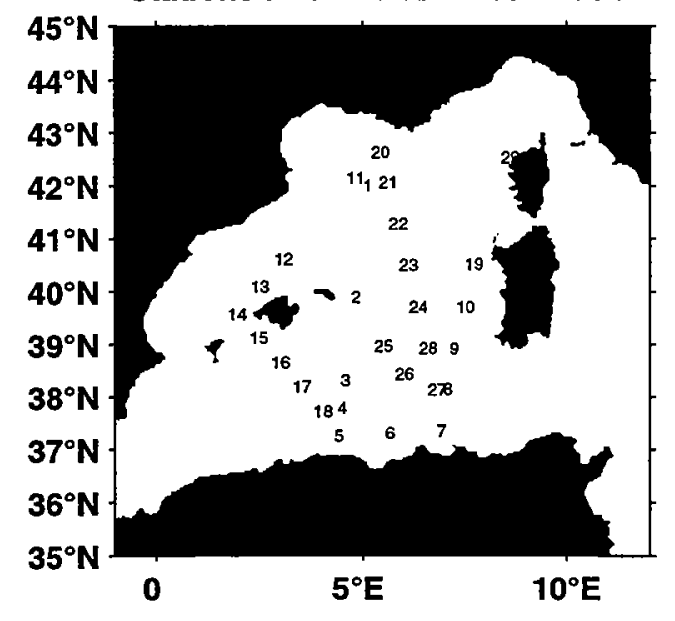

Stations Suroit Oct 1994

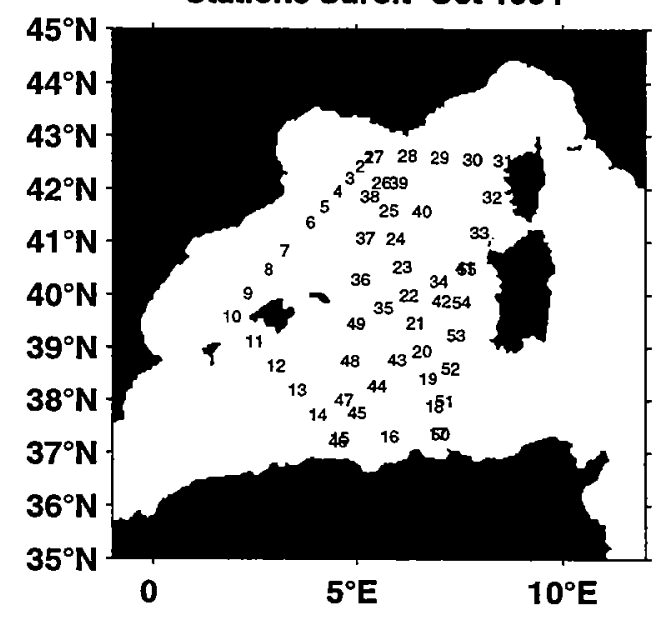

Figure 3: CTD-station maps of the two hydrographic surveys in central Western Mediterranean. The upper was made on R/V Poseidon in January 1994, the lower on R/V Le Suroit in October 1994.

(see fig. 5). The transformation preserves the ratios between temperature and depth gradients. Thus the typical pattern of each profile with a series of varying thick or thin layers and large or small steps is unchanged by the transformation. For the calculation of the transformation coefficients the profile 50 of the Suroit cruise has been taken as a reference because of its most regular pattern. The coefficients $c_{1}, c_{2}, c_{3}, c_{4}$ have been determined by a non-linear fitting routine in the way that the transformed profile matched best with the reference profile. The result of the transformation can be seen in figure 6 , where a waterfall plot of all step containing $\theta$-profiles is plotted with and without transformation. Here it can be seen that the pattern of the thermohaline staircase is similar for all profiles. After applying the transformation the identification of the well-mixed layers is much easier.

For those profiles which have the most regular step structures (Suroit profiles 13, $20,44,50$ ) we find thicknesses of the layers from 25 to $85 \mathrm{~m}$ and of the interfaces of 10 to $20 \mathrm{~m}$. The temperature and salinity changes across the interfaces range from 0.02 to $0.06^{\circ} \mathrm{C}$ and from 0.004 to $0.013 \mathrm{psu}$, respectively. The properties of the seven layers which we analysed are summarized in table 1. 

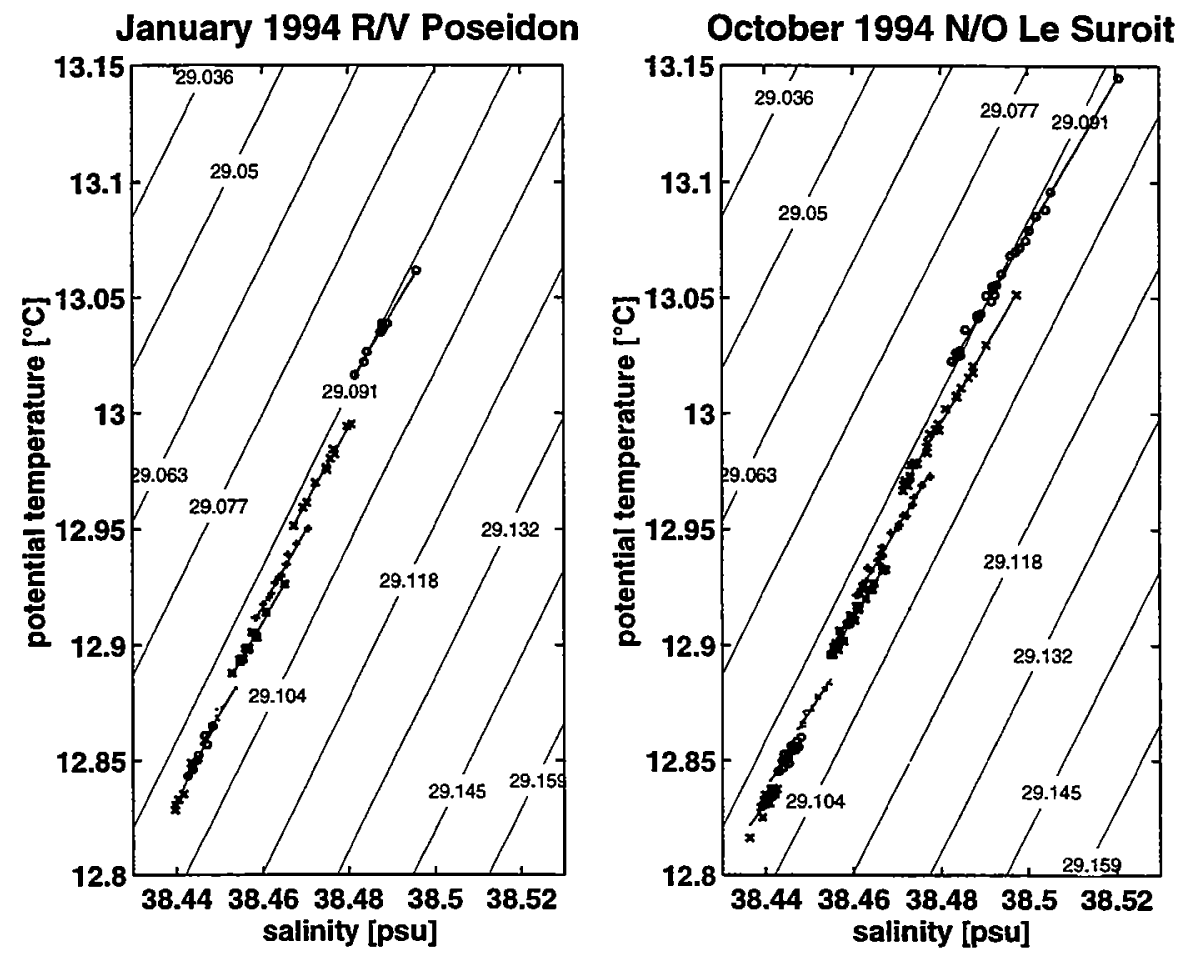

Figure 4: $\theta$-S diagrams for the two cruises containing the potential temperaturesalinity characteristics of the well mixed layers of the thermohaline staircases.

\begin{tabular}{|c|c|c|c|c|c|c|c|c|}
\hline $\begin{array}{c}\text { layer } \\
\text { no. }\end{array}$ & $\begin{array}{c}\langle\theta\rangle \\
{\left[{ }^{\circ} \mathrm{C}\right]}\end{array}$ & $\begin{array}{c}\langle\mathrm{psu}] \\
{[\mathrm{psu}]}\end{array}$ & $\begin{array}{c}\langle\sigma\rangle \\
{\left[\mathrm{kg} / \mathrm{m}^{3}\right]}\end{array}$ & $\begin{array}{c}\langle\Delta Z\rangle \\
{[\mathrm{m}]}\end{array}$ & $\begin{array}{c}\langle\Delta \theta\rangle \\
{\left[{ }^{\circ} \mathrm{C}\right]}\end{array}$ & $\begin{array}{c}\langle\Delta S\rangle \\
{[\mathrm{psu}]}\end{array}$ & $\begin{array}{c}\langle\Delta \sigma\rangle \\
{\left[\mathrm{kg} / \mathrm{m}^{3}\right]}\end{array}$ & $\left\langle R_{\rho}\right\rangle$ \\
\hline \hline 1 & 13.046 & 38.490 & 29.091 & 48 & 0.059 & 0.013 & 0.0024 & 1.26 \\
2 & 12.987 & 38.478 & 29.094 & 61 & 0.050 & 0.012 & 0.0018 & 1.20 \\
3 & 12.937 & 38.466 & 29.095 & 65 & 0.029 & 0.007 & 0.0008 & 1.11 \\
4 & 12.908 & 38.459 & 29.095 & 43 & 0.037 & 0.009 & 0.0010 & 1.17 \\
5 & 12.871 & 38.450 & 29.096 & 67 & 0.021 & 0.005 & 0.0003 & 1.12 \\
6 & 12.851 & 38.446 & 29.097 & 28 & 0.018 & 0.005 & 0.0002 & 1.07 \\
7 & 12.833 & 38.440 & 29.097 & 36 & & & \\
\hline
\end{tabular}

Table 1: Average properties of the layer and step parameters. 

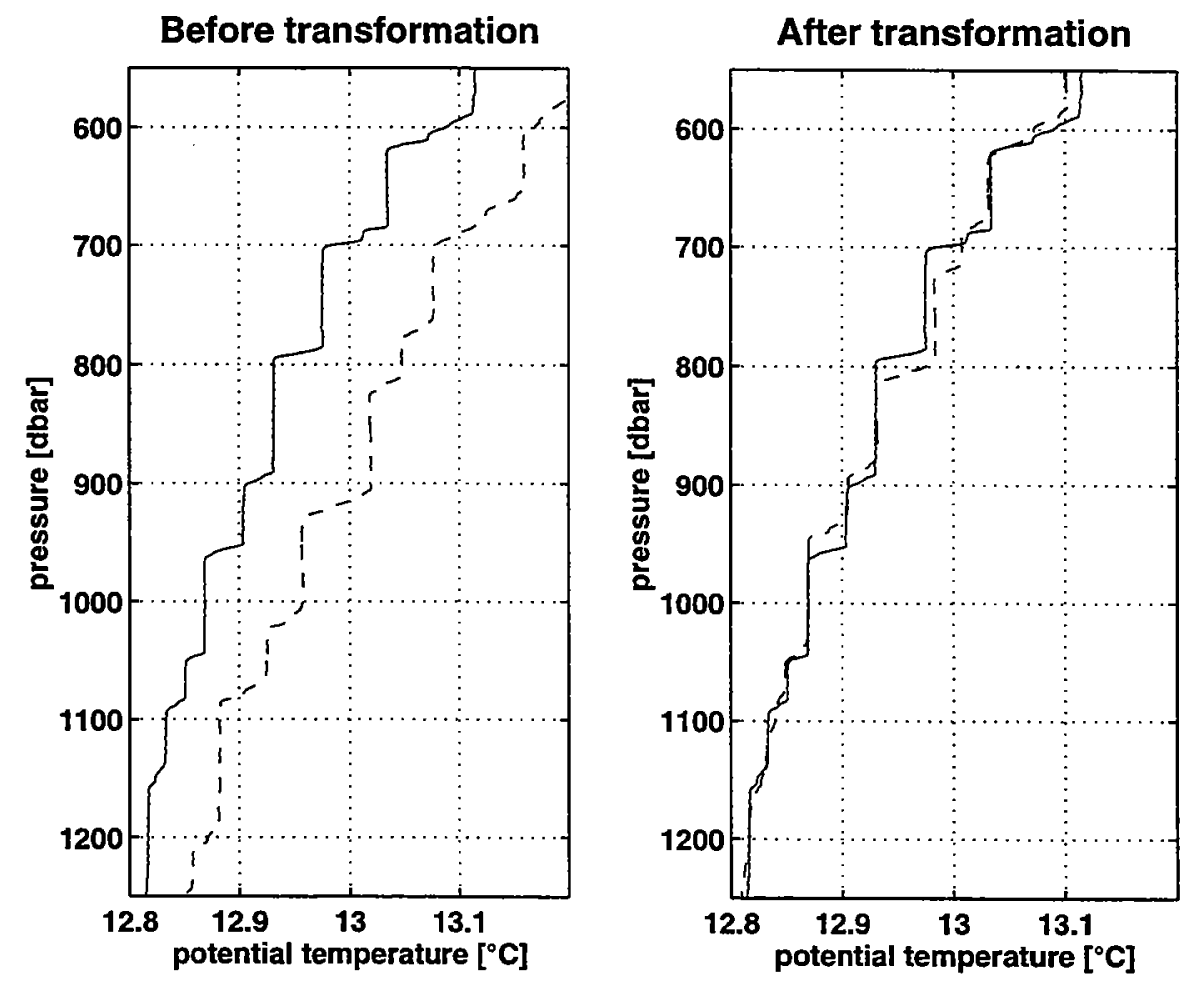

Figure 5: Two potential temperature profiles from the R/V Le Suroit cruise in October 1994. The left plot shows the profiles 50 (solid) and 33 (dashed). In the right plot profile 33 is transformed with a similarity transformation to match profile 50 . After application of the transformation the similarity of the thermohaline staircase pattern is obvious. 

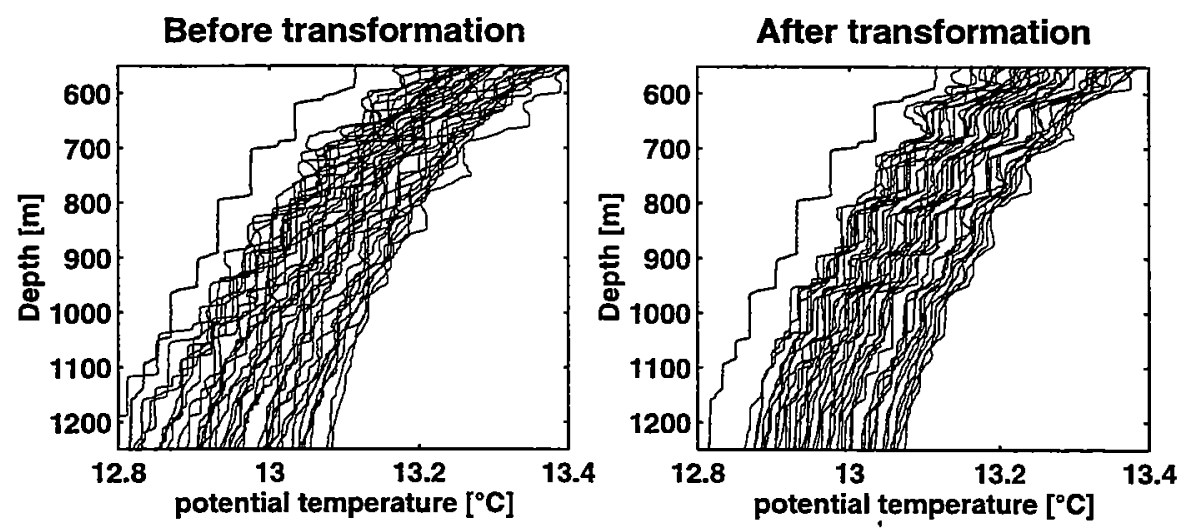

Figure 6: Waterfall plot of all profiles containing thermohaline staircases. The left plot shows the profiles without transformation, while the right shows the transformed profiles. The transformed profiles show the high similarity of the typical pattern of all profiles.

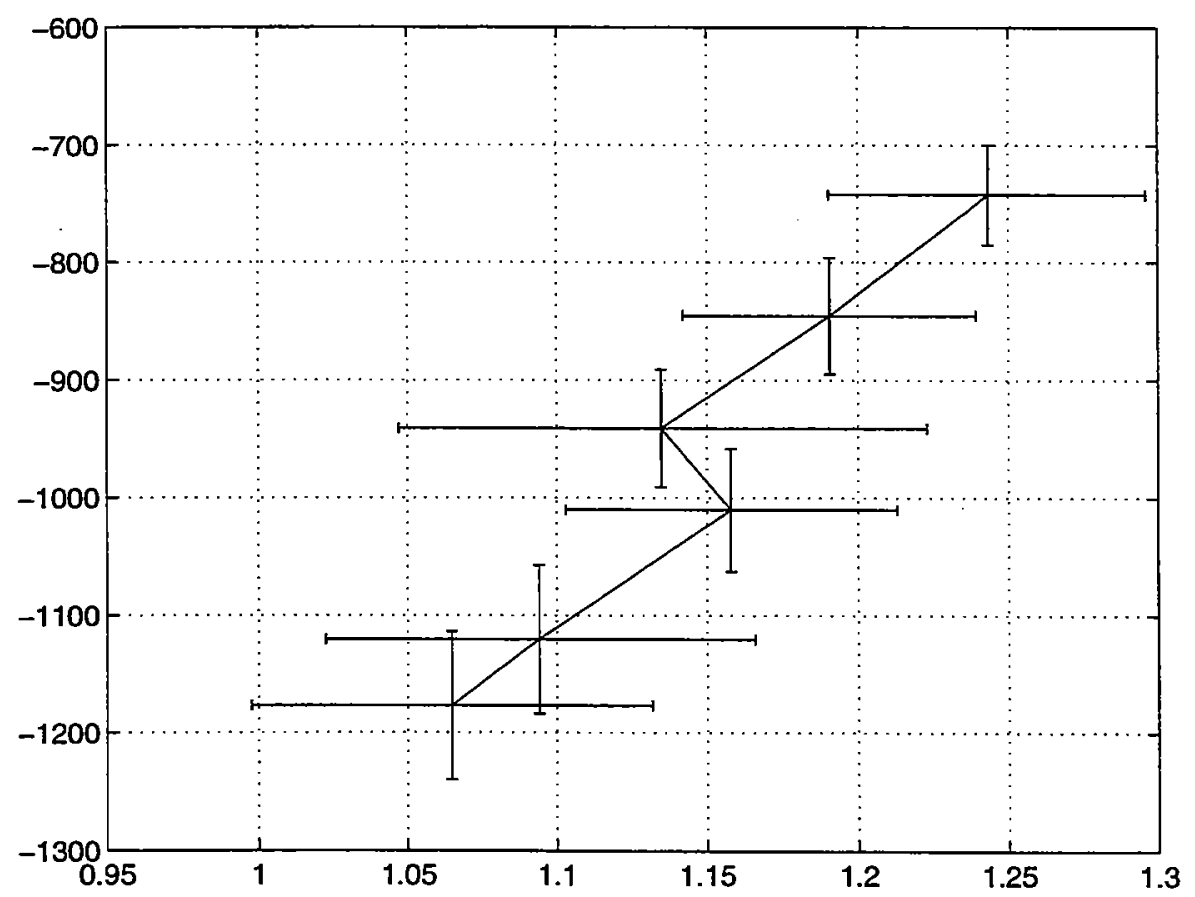

Figure 7: Average vertical density ratios and depths for the 6 examined thermohaline steps. Bars denote the standard deviation of the respective density ratios and depths. 


\section{Variability of the step structure}

The potential temperature and salinity profiles show some different characteristics, which can be used to classify them in order to distinguish between certain regimes :

- The sharpness of the step structures becomes stronger from the west to the east.

- The temperature/salinity amplitude of the steps follows the amplitude of the LIW temperature/salinity maximum.

- The easternmost profiles show layers with inverted vertical temperature and salinity gradients.

\section{The sharpness of the steps}

The step structures of profiles in the eastern part of the experiment area are sharper than of those in the central part of the Algero-Provençal-Basin while the profiles near the Balearic islands do not show any steps. Profiles in the center of the area suggest that diffusion slowly removes the step structure when the step generating process is not any longer active. The diffusion leads to profiles which have reduced well-mixed layers and larger interfaces with nearly constant $\theta$ - and S-gradients (see figure 8 ). In these cases the thickness of the interfaces reached up to $50 \mathrm{~m}$. Interestingly one-dimensional diffusion is not able to generate similar profiles. A simple one-dimensional model with diffusion with a constant diffusion coefficient changes the shape of our most regular profile (profile 50 of the October cruise, figure 12) much more to rounded edges, whereas the measured profiles have a linear interfacial part with sharp edges between interface and well-mixed part (see figure 8).

\section{Amplitudes of steps}

The temperature and salinity jump of the interfaces between the layers varies with the location of the profile. The closer the profile is located to the source of the LIW (southern tip of Sardinia) the larger the steps are. Coincident with the decrease of the amplitude of the steps we find an increase of the size of the well-mixed layers. This can be seen best in figure 10, where the first order transformation coefficients of the depth and temperature similarity transformations are plotted against each other. A strong relation (correlation coefficient 0.91) exists between the thermohaline step size and the thickness of the well-mixed layers. The larger the steps are (integrated over whole profiles), the thinner the well-mixed layers will be. The regression coefficient between the two first order transformation coefficients is $0.96 \pm 0.17$. This relation 


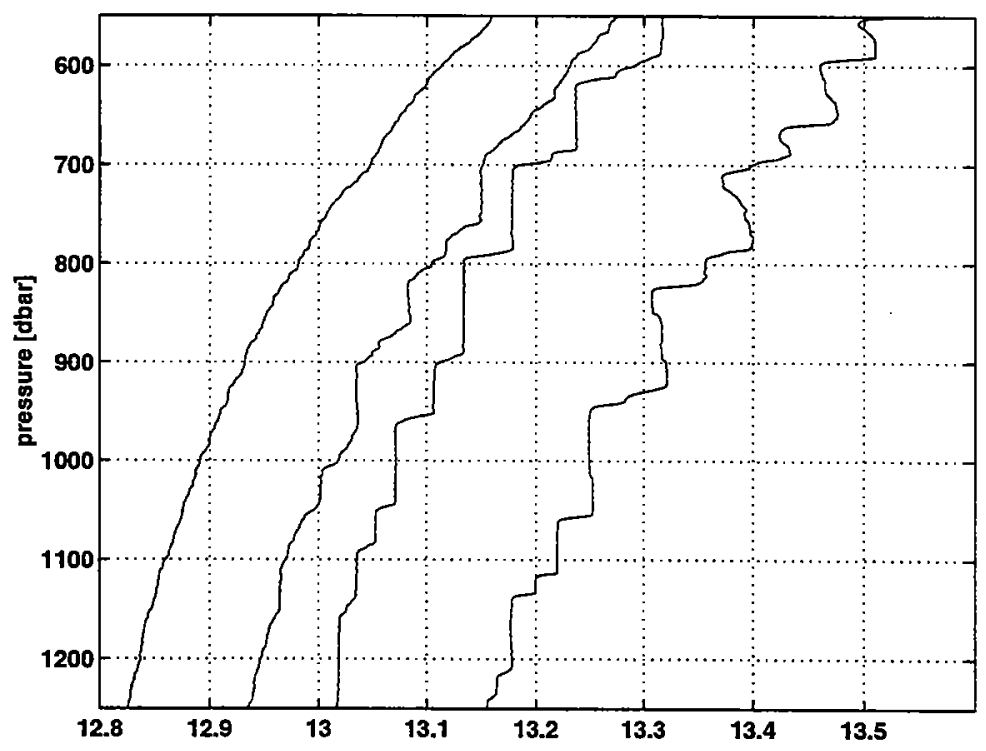

Figure 8: Four typical profiles for the different regions. From left to right profiles without, with diffused, with regular and with inverted steps are plotted. The profiles are offset by $0.1^{\circ} \mathrm{C}$.
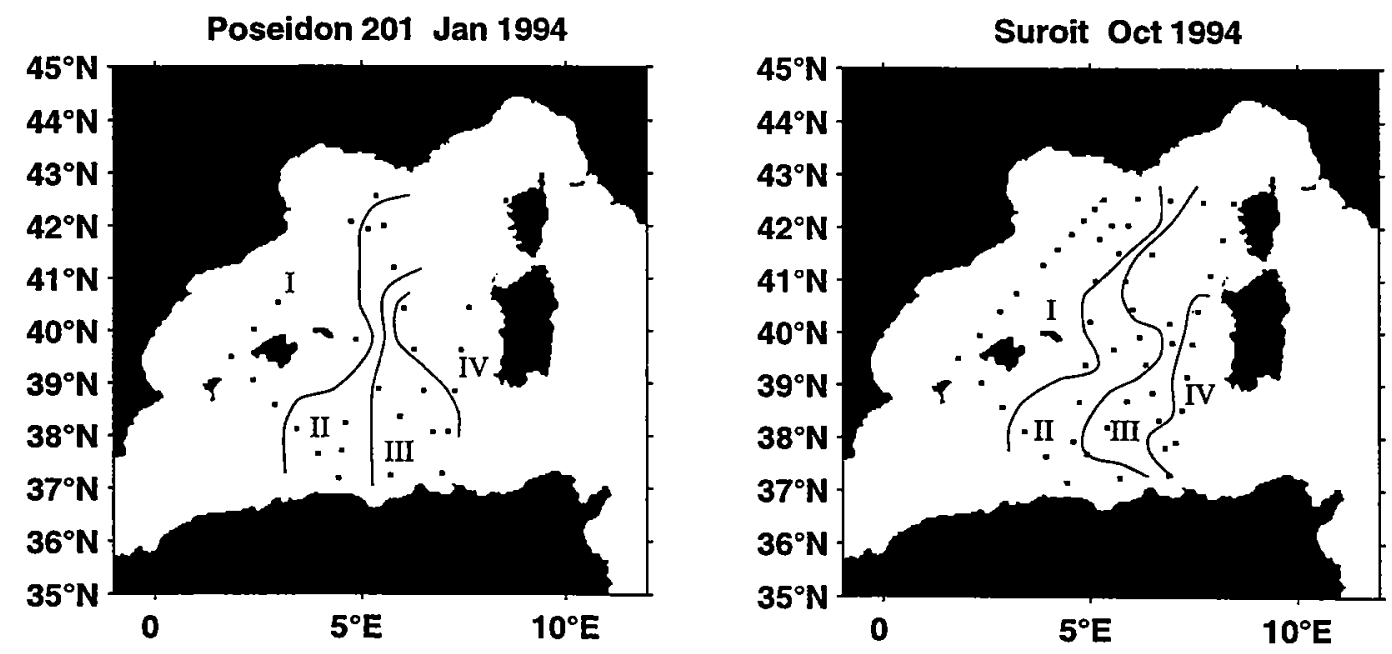

Figure 9: Map of the classification of the observed thermohaline staircases. The numbering of the regions corresponds to the sequence of the four profiles in figure 8 . 


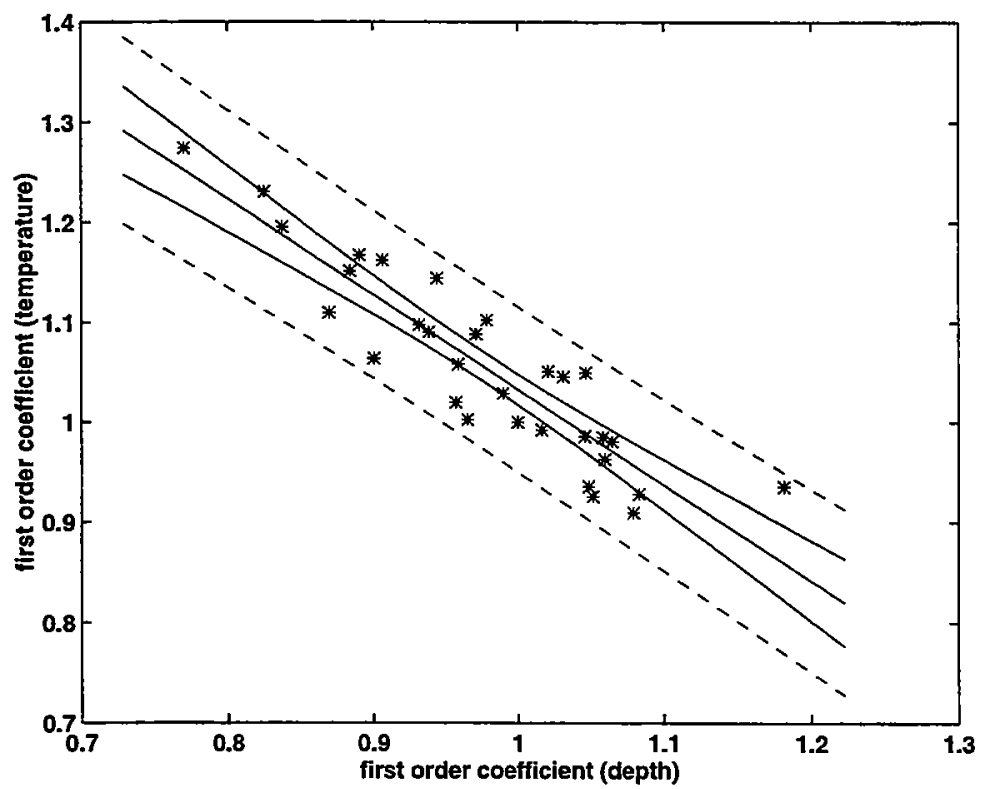

Figure 10: Scatterplot of the first order transformation coefficients of the similarity transformation $\left(c_{1}, c_{3}\right)$. The obvious linear relation between the coefficient connects decreasing thermohaline step sizes with increasing mixed layer sizes.

close to one suggests a one-dimensional diffusive downward transport of heat and salt under conservation of the heat and salt content.

\section{Inversions in the step structures}

Profiles close to the straits of Sardinia and Bonifacio show an interesting characteristic which may be related to the forming and maintainance of the thermohaline steps in the Algero-Provençal-Basin. The usual gradient of temperature and salinity with higher above lower values is inverted in certain otherwise well-mixed layers. McDougall [10] predicted such patterns for salt-fingering conditions in frontal regions. The region where we found inversions is close to the entering area of the LIW into the Algero-Provençal-basin. Calculations of the absolute value of the horizontal gradients from climatological temperature and salinity data show that the regions with inverted layers coincide with regions with highest horizontal gradients. A map of the average temperatures in $700 \mathrm{~m}$ depth is plotted in figure 11 .

For the inverted layers in the experiment area we find diffusive density ratios of $R_{\rho}^{d}=1.5 \pm 0.3\left(1 / R_{\rho}^{d}=R_{\rho}\right)$. This is close to the results of Marmarino [8] in the C- 


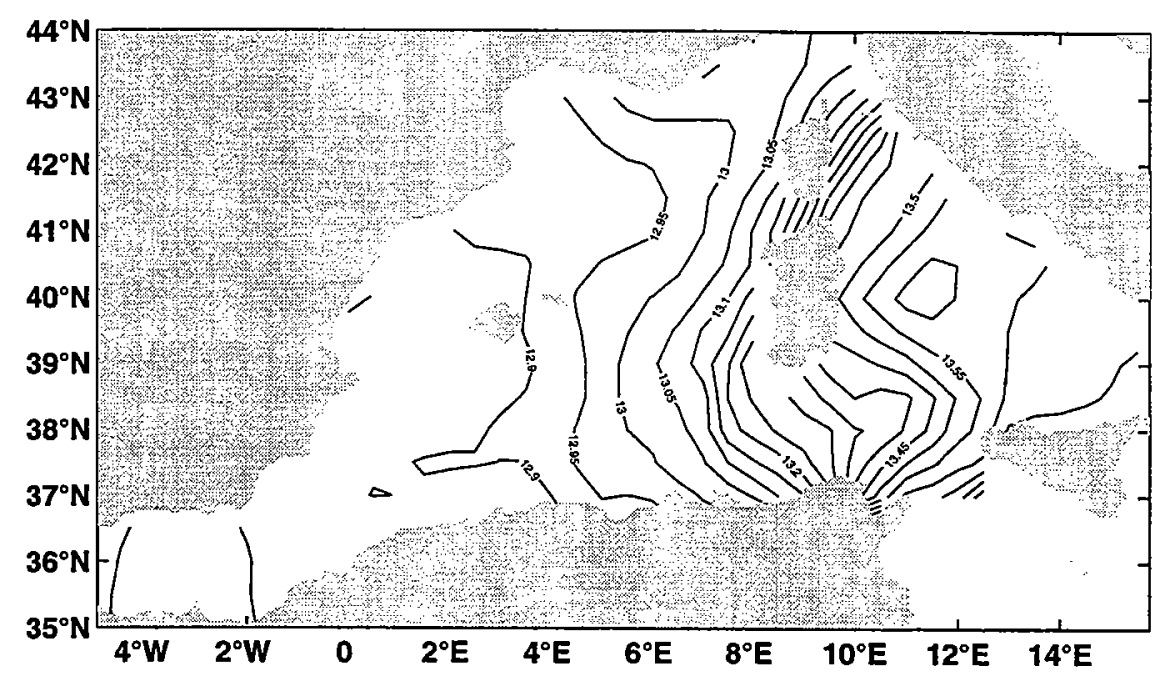

Figure 11: Contour plot of the potential temperatures in $700 \mathrm{~m}$ depth from the THETIS-2 climatology.

SALT area. He found diffusive density ratios of 1.2-2.6. Diffusive density ratios close to 1 are a sign for double diffusive unstable conditions in which temperature is layered unstable (higher temperatures below lower) and the stable layered salinity (higher salinity below lower) counteracts the temperature effect on density. The measurements of Marmarino [8] showed that inversions in well-mixed layers may be the vertical signature of horizontal intrusions into the layered system which are be driven by horizontal $\theta-\mathrm{S}$ gradients (Turner [19], McDougall [10]).

\section{Temporal variability of the step structures}

The temporal variability in the experiment area may be examined by comparing the step structures of the two cruises in January 1994 and in October 1994. The comparison shows that the structures during both cruises are strikingly similar (see figure 12). Moreover not only single profiles at certain locations remain the same, but also the banded structure in the $\theta$-S diagram is the same within the accuracy of our measurements (see figure 4). This means that the step-layer pattern and the horizontal variability of the thermohaline steps have not changed between January and October. This is a parallel to the the results of Molcard and Tait [14] who found that the structures in the neighbouring Tyrrhenian basin remained constant for at 

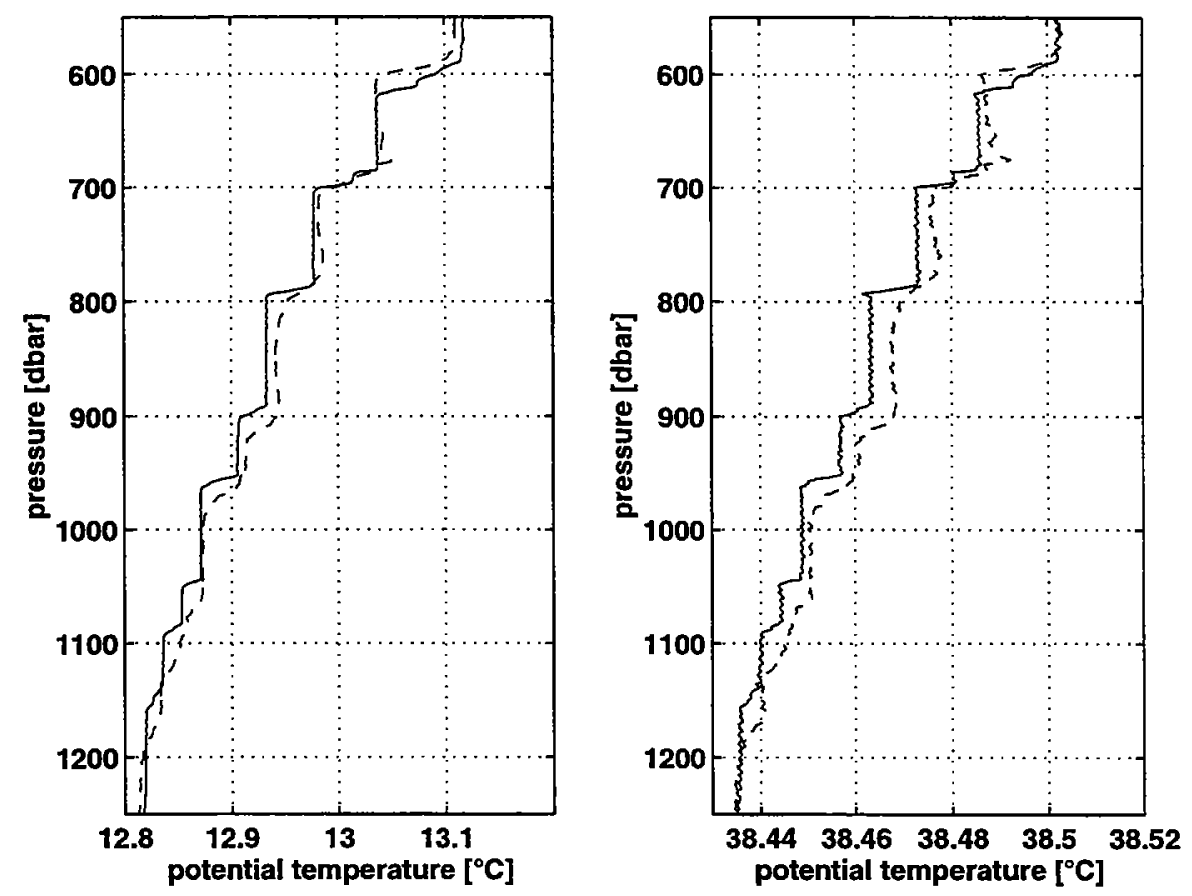

Figure 12: Profiles 25 of the January cruise (dashed) and 50 of the October cruise (solid) show the temporal constancy of the thermohaline staircase pattern. Profile 50 of the October cruise has been used as reference for the similarity transformation. 
least 3 years and with Schmitt et al. [16] who found a system of layers in the C-SALT area being the same for at least 8 month.

\section{$5 \quad$ Four different regimes}

In figure 8 we have plotted four different types of profiles which we found in the Algero-Provençal-Basin. These profiles can be used to divide the measurement area into four regions in which the different types of profiles are preferably found. Figure 9 shows the resulting regions for the two cruises. The regions deduced of the two cruises coincide within the achievable accuracy. The region of inverted layers is located close to the Strait of Sardinia, where the LIW enters the Algero-Provençal-Basin, it follows the further path of the LIW as a boundary current along the western coast of Sardinia. The second region with well developed step structures and no inverted layers is found as a band around the first. The diffusively reduced layers of the third region are located further to the north and to the west next to the second region.

The different regimes are colocated with the amplitude of the horizontal gradient of temperature or salinity. Climatological values (THETIS-group [18]) have been used to calculate horizontal gradients of temperature and salinity in the depth layers in which steps have been found. The region with highest gradients falls together with the first region, where inverted layers have been found. The areas with diffused step structures or no steps at all fall together with regions of weak horizontal gradients. This supports the idea that horizontal gradients are an ingredient for developing step structures. This has also been found by Washburn and Käse [20] in the Mediterranean outflow region in the North Atlantic and supports the calculations of McDougall [10]. In the outflow region step structures were preferably found around meddies but not directly below them, where vertical but not horizontal gradients were present.

\section{The lateral density ratio}

In section 3 we argued that there must be a connection between related layers of different profiles even though they do not have uniform $\theta$-S characteristics because of $\theta-\mathrm{S}$ values of each layer forming characteristic bands in the $\theta$-S diagram in figure 4 . The local inclination of these lines relative to the isopycnals gives the density ratio between water masses lying on the lines. Since the different points in the diagram represent different profiles at different locations, these density ratios are given by the mainly horizontal (lateral) along-layer temperature and salinity variations. The lateral density ratios which we find in our measurements are summarized in table

2. The average lateral density ratio is $R_{\rho}^{h}=0.92 \pm 0.06\left(R_{\rho}^{h}=\alpha \theta_{h} / \beta S_{h}\right.$, where 


\begin{tabular}{|c|c|c|c|c|c|}
\hline $\begin{array}{c}\text { layer } \\
\text { no. }\end{array}$ & $R_{p}^{h}$ & $\begin{array}{c}\text { Suroit } \\
\text { order 1 }\end{array}$ & $\begin{array}{c}\text { Suroit } \\
\text { order 0 }\end{array}$ & $\begin{array}{c}\text { Poseidon } \\
\text { order 1 }\end{array}$ & $\begin{array}{c}\text { Poseidon } \\
\text { order 0 }\end{array}$ \\
\hline \hline 1 & $0.94 \pm 0.04$ & $3.17 \pm 0.22$ & $-109.0 \pm 8.5$ & $3.24 \pm 0.14$ & $-111.8 \pm 5.3$ \\
\hline 2 & $0.95 \pm 0.04$ & $3.29 \pm 0.21$ & $-113.5 \pm 8.0$ & $3.21 \pm 0.16$ & $-110.7 \pm 6.2$ \\
\hline 3 & $0.93 \pm 0.05$ & $3.26 \pm 0.28$ & $-112.3 \pm 10.6$ & $3.17 \pm 0.21$ & $-109.2 \pm 7.9$ \\
\hline 4 & $0.92 \pm 0.06$ & $3.21 \pm 0.32$ & $-110.5 \pm 12.4$ & $3.10 \pm 0.26$ & $-106.4 \pm 9.9$ \\
\hline 5 & $0.87 \pm 0.09$ & $3.08 \pm 0.50$ & $-105.7 \pm 19.3$ & $2.92 \pm 0.42$ & $-99.5 \pm 16.1$ \\
\hline 6 & $0.92 \pm 0.17$ & $3.72 \pm 0.91$ & $-130.0 \pm 35.2$ & $2.64 \pm 0.87$ & $-88.6 \pm 33.5$ \\
\hline 7 & $1.15 \pm 0.24$ & $4.77 \pm 1.28$ & $-170.5 \pm 49.4$ & $2.70 \pm 1.35$ & $-91.0 \pm 52.0$ \\
\hline
\end{tabular}

Table 2: Results of the linear regressions applied to the mixed-layer characteristics in figure $4 . R_{p}^{h}$ is the along layer (lateral) density ratio calculated from the linear regression of both cruises. The other values give the regression coefficients of the two cruises.

$h$ denotes the along layer direction). The values for the two deepest layers, which have large uncertainties due to the very low horizontal gradients in these depths, are neglected. This value is in good agreement with the value of 0.93 expected by McDougall [10] for the case of a steady state interleaving process with a high turbulent Prandtl number. The regression coefficients in table 2 of both cruises agree within the given uncertainties (with a $95 \%$ confidence). The lateral density ratio can be obtained from the regression coefficients by multiplying the first order coeffcient with the appropriate $\alpha / \beta$. The work of McDougall [10] based on a steady state interleaving process. With our measurements we can not decide wether a steady state (of the small scale intrusions) was reached or not. We can only say that the overall system of steps and layers is in a steady state. 


\section{References}

[1] Astraldi, M., G. P. Gasparini and S. Sparnocchia : The Seasonal and Interannual Variability in the Ligurian-Provençal Basin. in Seasonal and Interannual Variability of the Western Mediterranean Sea, P. E. La Violette (Ed.), AGU 1995.

[2] Boyd, J.D. and H.Perkins, 1987 : Characteristics of thermohaline steps off the northeast coast of South America, July 1983. Deep-Sea Res., 34, 337-364.

[3] Bryden, H. L. and T. H. Kinder, 1991 : Steady two-layer exchange through the Strait of Gibraltar. Deep-Sea Research, 38, Suppl. 1. 445-463.

[4] Fleury M. and R. G. Lueck, 1991 : Fluxes across a thermohaline interface. DeepSea Res., 38, 745-769.

[5] Johanneesen, O.M. and O.S. Lee, 1974 : Thermohaline staircase structure in the Tyrrhenian Sea. Deep-Sea Res., 21, 629-639.

[6] Lacombe, H., J.C. Gascard, J. Gonella and J.P. Bethoux, 1981 : Response of the Mediterranean to the water and energy fluxes across its surface, on seasonal and interannual scales. Oceanol. Acta, 4, 2, 247-255.

[7] Leaman, K.D. and F.A. Schott, 1991 : Hydrographic structure of the convection regime in the Gulf of Lions: Winter 1987. J. Phys. Ocean., 21(4), 575-598.

[8] Marmorino, G.O., 1991 : Deep-Sea Res., 38, 11, 1431-1454.

[9] McDougall, T. J., 1985 : Double-Diffusive Interleaving. Part I: Linear Stability Analysis. J. Phys. Ocean., 15, 1532-1541.

[10] McDougall, T. J., 1985 : Double-Diffusive Interleaving. Part II: finite amplitude, steady state interleaving. J. Phys. Ocean., 15, 1542-1556.

[11] MEDOC Group, 1970 : Observation of formation of deep water in the Mediterranean Sea, 1969. Nature, 227, 1037-1040.

[12] Millot, C., 1987 : The Circulation in the western Mediterranean Sea. Oceanol. Acta, 10(2), 143-149.

[13] Molcard, R., and A. J. Williams : Deep stepped structure in the Tyrrhenian Sea. Mem. Soc. Roy. Sci. Liege Collect. 6, 7, 191-201, 1975. 
[14] Molcard, R. and Tait, 1977 : The steady state of the step structure in the Tyrrhenian Sea. In: $A$ voyage of discovery: George Deacon Seventieth Anniversary Volume, M. Angel, editor, Pergamon Press, 221-233.

[15] Sammari, C., C. Millot and L. Prieur, 1995 : Aspects of the seasonal and mesoscale variablilities of the Northern Current in the western Mediterranean Sea inferred from the PROLIG-2 and PROS-6 experiments. Deep-Sea Res., 42, No $6,893-917$.

[16] Schmitt, R.W., H. Perkins, J.D. Boyd and M.C. Stalcup , 1987 : C-SALT: an investigation of the thermohaline staircase in the western tropical North Atlantic. Deep-Sea Res., 34, 1655-1666.

[17] Schmitt, R. W., 1994 : Double Diffusion in Oceanography. Annu. Rev. Fluid Mech., 26, 255-285.

[18] THETIS group, 1995 : THETIS-2 final report.

[19] Turner, J.S., 1978 : Double-Diffusive Intrusions Into a Density Gradient. J. Geophys. Res., 83, 2887-2901.

[20] Washburn, L. and R.H. Käse, 1987 : Double Diffusion and the Distribution of the Density Ratio in the Mediterranean Waterfront Southeast of the Azores. J. Phys. Ocean., 17, 12-25. 


\title{
The statistical stability of convection dynamos
}

\author{
Willem V. R. Malkus
}

Dept. of Mathematics, Mass. Inst. of Technology, Cambridge, MA 02139.

In a reversal of the usual kinematic dynamo approach, here the velocity field is deduced, $a$ posteriori, from the results for an optimum magnetic field. In a forthcoming paper (Malkus, 1996), and in this lecture, a form of the magnetohydrodynamic equations is derived which reduces to Navier-Stokes-like equations for a single vector field, for that limit in which the magnetic energy density is arbitrarily larger than the kinetic energy density. Also in that paper, relative stability arguments determine a parameter range in which that solution of maximum magnetic momentum transport will be statistically stable.

Here, quantitative implications of those solutions are explored to establish the relation between heat flux from the core and (the recently observed) differential rotation of inner and outer core. For this lecture, the formal discussion was simplified by using the geometry of a cylindrical annulus and choosing the ratio of the kinematic viscosity and magnetic diffusivity to be one. Hirsching and Busse (1994) chose the ratio to be four and Glatzmaier and Roberts (1995) chose the ratio to be five hundred in their numerical studies of dynamo processes. However, the estimate of this ratio in the Earth's core is one-millionth. Although it is yet to be established, dynamo dependence on this ratio appears small indeed.

Soward's (1980) solutions for a (multi wave number) upper bound on magnetic transport may clarify the weak dependence on these dissipation coefficients. Although his study was in parallel plane geometry, with no large scale mean velocity, the reinterpretation here establishes that exact magnetogeostrophic balance can be achieved in the body of the fluid with this solution translated to annulus geometry and with a derived weak mean toroidal flow. First estimates of this upper bound relation between heat flux and core rotation are independent of Rayleigh numbers and the Prandtl numbers, yet well within an order of magnitude of current measurements.

\section{References}

Glatzmaier, G.A. and P.H. Roberts (1995) Phys. Earth Planet. Inter. 91, 63.

Hirsching, W. and F.H. Busse (1994) Phys. Earth Planet. Inter. 90, 24.

Malkus, W.V.R. (1996) Physica D.

Soward, A.M. (1980) Geo. Astro. Fluid Dynamics, 15, 317. 


\title{
Anomalous effects of distant boundaries on traveling-wave instabilities
}

\author{
Michael Proctor \\ University of Cambridge, U.K.
}

Many immportant instabilities of pattern-forming type lead, in an infinite region, to growing waves that propagate in only one direction. In these circumstances, the onset of instability in a finite region, even one very long compared with the basic wavelength, takes place via a confined "wall mode" at a value of the instability parameter that is related to the absolute instability of the travelling wave modes. The non-linear solution takes the form of a nearuniform wave train in part of the domain, whose wavelength and frequency are controlled by the boundaries. Secondary instabilities can lead to curious solutions with two regions of different frequency and wave number, separated by a chaotic front. 


\title{
What determines salt finger amplitude in un- bounded T-S gradient layers.
}

\author{
Melvin E. Stern \\ Dept. of Oceanography, Florida State Univ., Tallahassee, FL.
}

The theoretical and experimental studies that have contributed to our understanding of salt finger convection in the ocean have mostly dealt with a fully developed (asymptotic) regime consisting of two deep, well-mixed reservoirs with specified temperatures and salinities (or solute concentrations in the sugar-salt case), between which, a well-defined gradient (equilibrium) layer containing vertically coherent fingers forms; their amplitude and flux are determined by the vertical salinity difference, the density ratio, the viscosity $\nu$, and the thermal diffusivity, $\kappa_{T}$; especially when the salt diffusivity, $\kappa_{S}$, or $\tau,=\kappa_{S} / \kappa_{T}$, is small. Although such thin finger layers are sometimes observed in ocean microstructure measurements, more often [in the C-SALT measurements ${ }^{1}$ ] the vertical gradient region between mixed layers is much too thick for the asymptotic regime, and any fingers therein could only be vertically coherent over a relatively small fraction of the layer. We will determine the amplitude of the weak fingers in this regime, a question which is of theoretical interest because there is a well-known exact nonlinear solution for a vertically unbounded gradient region in which the depth independent vertical velocity continues to increase exponentially with time. Nevertheless, this model has been extensively used, along with various suggestions for other effects which limit the growth, such as the ambient shear in the ocean ${ }^{2}$. There are, however, laboratory experiments ${ }^{3,4}$ with a thick gradient region and no externally applied shear in which thin wiggly fingers with quite small amplitude are realized, and which are vertically coherent over only a fraction of the depth. A secondary (Floquet) instability ${ }^{5}$ has been proposed to limit the growth of long fingers in this case, but this theory still furnishes no quantitative measure of a statistically steady amplitude. To obtain a start on the problem, a very weak, nonlinear theory has been sought ${ }^{6}$, similar to the one developed by Malkus and Veronis ${ }^{7}$ for pure thermal convection at slightly supercritical Rayleigh number. In order to obtain thin salt fingers, Proctor and Holyer ${ }^{6}$ expanded in inverse powers of a very large but finite depth (i.e. thermohaline Rayleigh numbers). They were, however, unable to focus on the most unstable wavelength of interest, and were unable to obtain definitive amplitude information. We will be able to obtain this by considering a somewhat different parameter regime.

Let $\bar{T}_{z}, \bar{S}_{z}$ be the uniform vertical gradients in a completely unbounded spatial region, where the expansion and contraction coefficients are absorbed in all the following $T-S$ quantities so that these have densimetric units. Analytical tractability will be achieved for a large Prandtl number $\left(\nu / \kappa_{T}\right)$ fluid with

$$
\epsilon=\tau^{-1} R^{-1}-1 \rightarrow 0^{+}, \quad \tau=\kappa_{S} / \kappa_{T}=O(1), \quad R=\bar{T}_{z} / \bar{S}_{z}=O(1) .
$$


Although this restricts the quantitative result to the sugar-salt experiment, we expect qualitative relevance for heat-salt $(\tau \ll 1)$. This has proven to be true for the thin finger experiments in both cases where the " $4 / 3$ " flux law holds; and the lateral intrusions observed in sugar-salt experiments have also proven to be relevant to oceanic observations of heat-salt intrusions. We shall obtain a prediction of the amplitude of the horizontal $T$-variation in (1), and a qualitative explanation of the way equilibration occurs; in addition, we suggest the feasibilty of extending the calculation numerically to larger $\epsilon$ (small $\tau$ or $R$ ), where breakdown of fingers to convective layers is expected.

The equations of motion are first non-dimensionalised using $d=\left(\kappa_{T} \nu / g \bar{T}_{z}\right)^{1 / 4}$ as the length sca;e, $\bar{T}_{z} d$ as the scale for the total temperature deviation $\left(T^{\prime}(x, y, z, t)+\theta(z, t)\right)$ and salinity deviation $\left(S^{\prime}+\sigma(z, t)\right)$ from the undisturbed $\left(\bar{T}_{z}, \bar{S}_{z}\right), \kappa_{T} / d$ as the velocity scale, $d^{2} / \kappa_{T}$ as the time scale, and $\nu \kappa_{T} / d^{2}$ as the pressure scale. When $\nu / \kappa_{T} \rightarrow \infty$, with (1), the asymptotic Boussinesq equations become

$$
\begin{gathered}
0=-\nabla p+\nabla^{2} \boldsymbol{v}+\left(T^{\prime}-S^{\prime}\right) \hat{k}, \quad \nabla \cdot v=0, \\
\frac{d}{d t}\left[T^{\prime}(x, y, z, t)+\theta(z, t)\right]+w=\nabla^{2}\left(T^{\prime}+\theta\right), \\
\frac{1}{\tau} \frac{d}{d t}\left[S^{\prime}(x, y, z, t)+\sigma(z, t)\right]+\frac{w}{\tau R}=\nabla^{2}\left(S^{\prime}+\sigma\right),
\end{gathered}
$$

Let us look for solutions which are spatially periodic in the sense

$$
\bar{T}^{\prime}=0=\bar{S}^{\prime}=\bar{w}, \quad\langle w>=0=\langle\theta\rangle=\langle\sigma\rangle
$$

where $\left(^{-}\right)$denotes a horizontal average and $\langle>$, a vertical average. In these equations, the undisturbed temperature gradient is unity, and the undisturbed salinity gradient is $R^{-1}<1$.

The horizontal averages of $(3 a, b)$ are the mean field equations

$$
\frac{\partial \theta}{\partial t}+\frac{\partial}{\partial z} \overline{w T^{\prime}}=\frac{\partial^{2} \theta}{\partial z^{2}}, \quad \tau^{-1}\left(\frac{\partial \sigma}{\partial t}+\frac{\partial}{\partial z} \overline{w S^{\prime}}\right)=\frac{\partial^{2} \sigma}{\partial z^{2}},
$$

and when these are subtracted from $(3 a, b)$, the result is

$$
\begin{gathered}
D T^{\prime}+w=-w \frac{\partial \theta}{\partial z}-\left(\nabla \cdot\left(v T^{\prime}\right)-\frac{\partial}{\partial z} \overline{w T^{\prime}}\right) \\
\boldsymbol{D}_{\tau} S^{\prime}+R^{-1} \tau^{-1} w=-\tau^{-1} w \frac{\partial \sigma}{\partial z}-\tau^{-1}\left(\nabla \cdot\left(\boldsymbol{v} S^{\prime}\right)-\frac{\partial}{\partial z} \overline{w S^{\prime}}\right) \\
\boldsymbol{D} \equiv \frac{\partial}{\partial t}-\nabla^{2}, \quad \boldsymbol{D}_{\tau} \equiv \frac{1}{\tau} \frac{\partial}{\partial t}-\nabla^{2}, \quad \nabla_{2}^{2} \equiv \nabla^{2}-\frac{\partial^{2}}{\partial z^{2}}
\end{gathered}
$$


By eliminating the horizontal velocity components $(u, v)$ from $(2)$, we obtain the linear equation

$$
\nabla^{4} w=\nabla_{2}^{2}\left(S^{\prime}-T^{\prime}\right)
$$

and when $\left(S^{\prime}-T^{\prime}\right)$ is eliminated using (5) and (6), we get

$$
\boldsymbol{L}\left(\frac{\partial}{\partial t}, \frac{\partial}{\partial x}, \frac{\partial}{\partial y}, \frac{\partial}{\partial x}\right) w=-\frac{1}{\tau} \boldsymbol{D} \nabla_{2}^{2} w \frac{\partial \sigma}{\partial z}+D_{\tau} \nabla_{2}^{2} w \frac{\partial \theta}{\partial z}+N
$$

where the linear operator is

$$
L\left(\frac{\partial}{\partial t}, \frac{\partial}{\partial x}, \frac{\partial}{\partial y}, \frac{\partial}{\partial x}\right) \equiv D_{\tau} D \nabla^{4}-\left[\left(R^{-1} \tau^{-1}-1\right) \nabla^{2}+\tau^{-1}\left(1-R^{-1} \frac{\partial}{\partial t}\right)\right] \nabla_{2}^{2} .
$$

Since the last terms in (5) and (6) are independent of $(x, y)$, the value of $N$ is

$$
N=-\tau^{-1} D \nabla_{2}^{2} \nabla \cdot\left(v S^{\prime}\right)+D_{\tau} \nabla_{2}^{2} \nabla \cdot\left(v T^{\prime}\right)
$$

The solution of the linear problem, $L(w)=0$ for $w, T^{\prime}, S^{\prime}$ gives the eigenfunctions in the form $\exp \left(\lambda_{m} t\right)$ multiplied by

$$
\left(W_{m}, T_{m}, S_{m}\right) \propto \sin k x \cos l y \cos m z
$$

and for subsequent rescaling of the wavenumbers, we need

$$
(k, l, m) \equiv \epsilon^{1 / 4}(\hat{k}, \hat{l}, \hat{m}), \quad k_{0}^{2}=k^{2}+l^{2}, \quad \hat{k}_{0}^{2}=\hat{k}^{2}+\hat{l}^{2} .
$$

The eigenvalue equation $L(w)=0$ for the growth rate $\lambda_{m}$ is .

$$
\left(\lambda_{m}+k_{0}^{2}+m^{2}\right)\left[\lambda_{m}+\tau\left(k_{0}^{2}+m^{2}\right)\right]\left(k_{0}^{2}+m^{2}\right)^{2}+\lambda_{m} k_{0}^{2}\left(1-R^{-1}\right)-k_{0}^{2}\left(k_{0}^{2}+m^{2}\right) \tau \epsilon=0,
$$

and in the $\epsilon \rightarrow 0$ limit, the simplified $\lambda_{m} \rightarrow 0$ solution is

$$
\begin{aligned}
\lambda_{m} & =\frac{\epsilon \tau k_{0}^{2}\left(k_{0}^{2}+m^{2}\right)-\tau\left(k_{0}^{2}+m^{2}\right)^{4}}{\left(k_{0}^{2}+m^{2}\right)^{3}(1+\tau)+k_{0}^{2}\left(1-R^{-1}\right)}+\ldots, \\
& =\tau \epsilon^{3 / 2}\left(\hat{k}_{0}^{2}+\hat{m}^{2}\right) \frac{\left[\hat{k}_{0}^{2}-\left(\hat{k}_{0}^{2}+\hat{m}^{2}\right)^{3}\right]}{(1-\tau) \hat{k}_{0}^{2}}+\ldots,
\end{aligned}
$$

where (10a) has been used to rescale the wavenumbers. In this $\epsilon \rightarrow 0$ limit (with $R^{-1} \tau^{-1} \approx 1$ ), the $T_{m}, S_{m}$ eigenfunctions obtained from the linearization of (5), (6) simplify to

$$
T_{m}=S_{m}=-\frac{w_{m}}{k_{0}^{2}+m^{2}}=-\frac{w_{m}}{\left(\hat{k}_{0}^{2}+\hat{m}^{2}\right) \epsilon^{1 / 2}}
$$

For "thin" fingers with finite $m^{2} / k_{0}^{2} \ll 1$, the maximum of (11) is

$$
\max \lambda_{m}=\sqrt{ } \frac{4}{27} \frac{\tau}{1-\tau} \epsilon^{3 / 2}, \hat{k}_{0}^{2}=\frac{1}{\sqrt{ } 3}
$$

The steady solutions of (4) for vertically periodic $\theta, \sigma$ are

$$
\frac{\partial \theta}{\partial z}=\overline{w T^{\prime}}-<\overline{w T^{\prime}}>, \quad \frac{\partial \sigma}{\partial z}=\tau^{-1} \overline{w S^{\prime}}-\tau^{-1}<\overline{w S^{\prime}}>,
$$

and these will be substituted into (8) along with $D=D_{\tau}=\nabla^{2}$, and also $L=L\left(0, \frac{\partial}{\partial x}, \frac{\partial}{\partial y}, \frac{\partial}{\partial z}\right)$. For small $\epsilon$ and $\lambda$, the latter operator may be expanded as 
$\boldsymbol{L}(0, \ldots)=\boldsymbol{L}\left(\lambda_{m}, \ldots\right)-\lambda_{m} \partial \boldsymbol{L}(0, \ldots) / \partial \lambda_{m}+\ldots$, where

$$
\frac{\partial \boldsymbol{L}(0, \ldots)}{\partial \lambda_{m}}=-\left(1+\tau^{-1}\right) \nabla^{6}-\left(\tau^{-1}-1\right) \nabla_{2}^{2},
$$

so that the expansion of $(8)$ may be written as

$$
\begin{aligned}
& L\left(\lambda_{m}, \frac{\partial}{\partial x}, \frac{\partial}{\partial y}, \frac{\partial}{\partial z}\right) w= \\
& \left.\quad \lambda_{m} \frac{\partial L}{\partial \lambda_{m}}(w)+\tau^{-2} \nabla_{2}^{2} \nabla^{2} w\left[\overline{w S^{\prime}}-<\overline{w S^{\prime}}>\right]-\nabla_{2}^{2} \nabla^{2} w \overline{w T^{\prime}}-<\overline{w T^{\prime}}>\right]+N+\ldots .
\end{aligned}
$$

The small finite amplitude $A$ in the leading term of

$$
w=A w_{m}+O\left(A^{2}\right)
$$

is now obtained from the solvability condition of the inhomogeneous differential equation (16).

As in the classical Rayleigh convection problem ${ }^{7}$, it is convenient to first consider the $l \equiv 0$ (2D, "sheets") case because $N$ vanishes identically [c.f. $\nabla_{2}^{2}\left(w \partial T^{\prime} / \partial z+u \partial T^{\prime} / \partial x\right)$ in (9b)]. We then multiply (16) by the eigenfunction $w_{m}$ of the $L\left(\lambda_{m}, \ldots\right)$ operator and integrate over all $(x, z)$, using (12) in (14) to get

$$
\begin{aligned}
& 0=A \lambda_{m}\left\langle\overline{w_{m} \frac{\partial L(0, \ldots)}{\partial \lambda_{m}} w_{m}}\right\rangle \\
& -A^{3}\left(\tau^{-2}-1\right)\left(k_{0}^{2}+m^{2}\right)^{-1}\left\langle w_{m} \nabla_{2}^{2} \nabla^{2} w_{m}\left(\overline{w_{m}^{2}}-<\overline{w_{m}^{2}}>\right)\right\rangle \\
& =A \lambda_{m}\left\langle\overline{w_{m} \frac{\partial \bar{L}}{\partial \lambda_{m}} w_{m}}\right\rangle \\
& \left.-A^{3}\left(\tau^{-2}-1\right) k_{0}^{2}\left(k_{0}^{2}+m^{2}\right)^{-1}\left\langle\left(k_{0}^{2} \overline{w_{m}^{2}}+\overline{\left(\frac{\partial w_{m}}{\partial z}\right)^{2}}\right) \overline{\left(w_{m}^{2}\right.}-<\overline{w_{m}^{2}}>\right)\right\rangle
\end{aligned}
$$

The final step is to make use of the asymptotic wave number scaling [eqs (10a)-(11)] in simplifying (15) to $\partial L / \partial \lambda_{m}=\left(\tau^{-1}-1\right) k_{0}^{2}+\ldots$, so that the $\epsilon \rightarrow 0$ limit of $(18 \mathrm{~b})$ is given by

$$
0=\lambda_{m}\left(\tau^{-1}-1\right)-A^{2} \frac{\left.\left\langle\left(k_{0}^{2} \overline{w_{m}^{2}}+\overline{\left(\frac{\partial w_{m}}{\partial z}\right)^{2}}\right) \overline{\left(w_{m}^{2}\right.}-<\overline{w_{m}^{2}}>\right)\right\rangle}{\left(k_{0}^{2}+m^{2}\right)<\overline{w_{m}^{2}}>}
$$

For $l=0,\left\langle\overline{w_{m}^{2}}\right\rangle=1 / 4,\left\langle\left(\overline{w_{m}^{2}}\right)^{2}\right\rangle=(1 / 4)(3 / 8),\left\langle\overline{w_{m}^{2}\left(\partial w_{m} / \partial_{z}\right)^{2}}\right\rangle=(1 / 4)\left(m^{2} / 8\right)$, and therefore (19) with (13) becomes

$$
0=\epsilon^{3 / 2}\left(\hat{k}_{0}\right)^{-2}\left(\hat{k}_{0}^{2}+\hat{m}^{2}\right)\left[\hat{k}_{0}^{2}-\left(\hat{k}_{0}^{2}+\hat{m}^{2}\right)^{3}\right]-\left(A^{2} / 8\right)\left(\tau^{-2}-1\right)\left(\hat{k}_{0}^{2}-\hat{m}^{2}\right)\left(\hat{k}_{0}^{2}+\hat{m}^{2}\right)^{-1}
$$


For long thin sheets with $\hat{m}^{2} / \hat{k}_{0}^{2} \ll 1$ with the fastest growing wavelength $k_{0}^{2}=1 / \sqrt{ } 3$, the squared amplitude

$$
A^{2}=\frac{16}{3 \sqrt{ } 3}\left[\frac{1}{R \tau}-1\right]^{3 / 2} /\left(\tau^{-2}-1\right)
$$

is quite finite and quite different from the previously mentioned $m \equiv 0$ (exponentially increasing) solution, and therefore the latter is a singular limit (of $m$ ).

For rectangular planform $(l \neq 0)$ solutions, the $N$ term no longer vanishes, but it is easy to see that $u \partial T^{\prime} / \partial x+v \partial T^{\prime} / \partial y+w \partial T^{\prime} / \partial z$ and $N$ are directly proportional to $m$. Therefore $N$ will have a negligible contribution to $A$ for long thin fingers $\left(m / k_{0} \ll 1\right)$, and the only modification necessary in our previous results is to introduce a factor $\overline{\cos ^{2} l y}=1 / 2$ in computing the averages in the numerator and denominator of (19). We conclude that for all long thin rectangular planform fingers, the squared amplitude is

$$
A^{2}=\frac{32}{3 \sqrt{ } 3}\left[\frac{1}{R \tau}-1\right]^{3 / 2} /\left(\tau^{-2}-1\right)
$$

$\left(m / k_{0} \ll 1 \hat{k}_{0}^{2}=\hat{k}^{2}+\hat{l}^{2}=1 / \sqrt{ } 3\right)$. The corresponding $T^{\prime}$ fluctuation (which might be measured in double-gradient sugar/salt experiments) has an amplitude $A \epsilon^{-1 / 2} \sqrt{ } 3$, or in dimensional units

$$
\frac{T_{\operatorname{dim}}^{\prime}}{\bar{T}_{z}\left[\kappa_{T} \nu /\left(g \bar{T}_{z}\right)\right]^{1 / 4}}=\left(\frac{32}{\sqrt{ } 3}\right)^{1 / 2}\left[\frac{1}{R \tau}-1\right]^{1 / 4} /\left(\tau^{-2}-1\right)^{1 / 2}
$$

The mechanism of amplitude equilibration is suggested by computing the modified mean density gradient $d \bar{\rho} / d z=d \sigma / d z-d \theta / d z$ from (14) and (12). The result is proportional to $-A^{2} \cos 2 m z$, i.e. $d \bar{\rho} / d z$ has a negative extremum (stabilizing stratification) where the finger velocities are extremal, and it has a positive extremum (destabilizing) where $w=0$. It is expected that higher order (larger $\epsilon$ ) effects on the larger scale (half the vertical finger wavelength) of modified density field will have a major effect on large groups of fingers, possibly leading to convective layers where $d \bar{\rho} / d z$ is destabilizing. This effect seems accessible via numerical solutions of (2)-(3b), primarily because $\tau=O(1)$ implies a single dissipative scale.

\section{References}

1. Schmitt, R.W., H. Perkins, J.D. Boyd and M.C. Stalcup. (1987) C-SALT: an investigation of the thermohaline staircase in the western tropical North Atlantic. Deep-Sea Res. 34, 16551666.

2. Kunze, E. (1994) A proposed flux constraint for salt fingers in shear. J. Mar. Res. 52, 999-1016.

3. Stern, M.E. and J.S. Turner. (1969) Salt fingers and convecting layers. Deep-Sea Res. 16, 497-511. 
4. Taylor, J.R. (1993) Anisotropy of salt fingers. J. Phys. Oceanog. 23, 554-565.

5. Holyer, J.Y. (1984) The stability of long steady, two-dimensional salt fingers. J. Fluid Mech. 147, 169-185.

6. Proctor, M.R.E. and J.Y. Holyer. (1986) Planform selection in salt fingers. J. Fluid Mech. 168, 241-253.

7. Malkus, W.V.R. and G. Veronis. (1958) Finite amplitude cellular convection. J. Fluid Mech. 4, 225-261. 


\title{
Multiplicity of the equilibrium states in laterally heated thermosolutal systems with and without the difference in the diffusivity coefficients
}

\author{
Naftali A. Tsitverblit \\ Oceanography, Lamont-Doherty Earth Observatory of Columbia \\ University P.O. BOX 1000 /RT 9W/ PALISADES, \\ NY 10964-8000, USA
}

July 17,1996

As established by Thorpe et al. (1969), Hart (1971), Thangam et al. (1981), a stably stratified solution subjected to a horizontal temperature gradient is eventually characterized by the formation of convective layers, which has been attributed to the double-diffusive instability. Although the analysis in the works just mentioned has been performed for the steady instability in an infinite vertical slot, there are a number of reasons for one to believe that the corresponding finite steady formulation would be relevant to the various other laterally heated systems, possessing inherently nonsteady features. For example, the results of Huppert \& Turner (1980) accentuate that the behavior of the layers is mainly determined by the horizontal temperature gradient and is independent of the ice-melting process at a vertical boundary: the layer height was found to be described by the Chen scale (Chen, Briggs \& Wirtz 1971) irrespective of whether the lateral heating/cooling is due to the meting-ice surface or to the temperature difference between the vertical side walls of an enclosure.

Another factor emphasizing relevance of the steady formulations is associated with the established validity of quasi static approximations. In particular, when assumptions of such a type were made by Tanny \& Tsinober (1988), for the interpretation of their experimental data, and by Kerr (1989), theoretically, the critical values of the Rayleigh numbers obtained in both these works showed reasonable agreement with the marginal stability curve computed for an infinite vertical slot by Hart (1971) and Thangam et al. (1980). Moreover, a weakly nonlinear analysis by Kerr (1990), where the quasi static assumption was also made, demonstrated that, like in the results obtained by Hart (1973) for the slot, it is the subcritical bifurcation that is responsible for the onset of the layers in the single-side-wall heating problem.

Although the earlier studies have determined some general characteristics of the layer behavior (such as, for example, the Chen scale, "successive" and "simultaneous" modes of layer formation; see Chen et al. 1971), specific features of the behavior of the layers, namely as to under what circumstances they merge and what the thickness of the resulting layer is, turned out to be hardly susceptible of description in terms of any simple mechanism (such as the doubling of the layer height initially suggested by Wirtz \& Reddy 1979, for example), but were found likely to be associated with some rather complex scenarios (Tanny \& Tsinober 1988, Jeevaraj \& Imberger 1991). Moreover, it was noted by Tanny \& Tsinober (1988) that the layer height during the merging process can even be irreproducible despite practically identical conditions 
maintained in the conducted experiments. Among some other issues, it also remained unclear whether the boundary between the "successive" and "simultaneous" modes of layer formation should be associated with the primary instability of the system or with a secondary bifurcation, or else, attributed to a phenomenon other than instability at all.

The purpose of the study the results of which were discussed during the first part of the lecture (Tsitverblit \& Kit 1993, Tsitverblit 1995, hereinafter, TK and $\mathrm{T}$, respectively) was to examine the possibility that the diversity of the previously observed flow characteristics would virtually be associated with the variety of the properties of steady states in the appropriately formulated finite-enclosure heat-salt problems.

The reflectional symmetry of the problem a priory implies that symmetry-breaking bifurcations, in addition to limit points, are also feasible in the system. Consequently, the attention was primarily concentrated on the higher-order mechanisms responsible for the formation of such singularities. In particular, the presented results exhibited the key role of two basic types of the scenarios giving rise to the multiple steady solutions as the value of the salinity Rayleigh number is increased from that designating the commencement of the double-diffusive region (Hart 1971, Thangam et al. 1981). One of them is associated with the progressive bending of a solution branch and forming limit points on it. As for the large and small thermal Rayleigh numbers the solution is unique and symmetric, in application to the symmetric solutions the limit points form only in pairs, each of which constitutes an $S$-like structure arising from a preceding non-degenerate hysteresis point. Although new asymmetric branches can arise also from single limit points, forming at the existing asymmetric branches as a result of the exchange of the criticality of a symmetry-breaking bifurcation point already present, it is the second mechanism, consisting of the emergence of an isola of asymmetric solutions and its subsequent splitting into the pair of symmetry-breaking bifurcations on a symmetric branch, that is basically responsible for the existence of the asymmetric solutions.

It was demonstrated that, owing to the above mechanisms, the structure of steady flows in a vertical rectangular enclosure of the aspect ratio $\gamma=3$ becomes extremely complex even when the salinity Rayleigh number is still relatively small. The growing complexity of the bifurcation diagram is associated with expansion of the region of solution multiplicity, increase of the number of the multiple solutions present over certain intervals of the bifurcation parameter $\left(R a_{T}\right)$, and enrichment of the variety of the types of the steady flows. The latter trend has in particular been represented by the maximal number of cells, present in the set of the steady solutions characterizing a value of the salinity Rayleigh number, being incremented when $R a_{S}$ exceeds certain critical values.

In spite of the appreciably different range of the salinity Rayleigh numbers, the characteristics of the obtained steady solutions have been found to qualitatively reflect the whole diversity of the flow patterns observed in the previous studies both throughout the transitional processes and at the steady, or quasi steady, stages. Among the computed steady solutions, in particular, there were found symmetric and asymmetric flows characterized by one, two, three, four, and five cells. Like in the steady (quasi steady) and transient flows observed in the previous studies, the presence of a stagnant flow region in the steady solutions was found to depend on whether their buoyancy ratio is above or below certain critical interval of its values in the vicinity of one of the computed limit points, which may suggest that this singularity plays the role of the boundary between the "successive" and "simultaneous" regimes of layer formation (Chen et al. 1971).

Within the considered range of parameters, most of the relevant flow patterns, the multicell flows, have been found linearly unstable. Instability of the steady solutions was found susceptible of consistent interpretation as being associated with the mechanisms responsible for the promotion of the solution multiplicity. The results 
presented, however, are indicative of the growth rates of the instabilities being so small that the corresponding steady flows could long be observed in the experiment and perhaps affect essentially the characteristics of such systems. In particular, it is the cell height of unstable multicell flows that turned out to be in good agreement with the physically meaningful length scales of the layers (they were found to be close to the Chen scale) observed in the previous studies. It was also emphasized that the critical values of the parameter $Q=\frac{\left(1-\frac{k_{S}}{k_{T}}\right)^{6} g(\alpha \Delta T)^{6}}{\nu k_{S} d^{2}\left(\beta \frac{\Delta S}{H}\right)^{5}}$, whose construction could also be justified by meaningful physical arguments (Kerr 1989), fell well inside the region of the flow multiplicity. These results give evidence of the important role played by the multiplicity of not only the stable but also the unstable steady flows in the physical mechanisms responsible for the diversity of the earlier documented flow features.

The instabilities found by Thorpe et al. (1969), Hart (1971), Thangam et al. (1981), Kerr (1989), TK and T, as well as the related diversity in the layer characteristics observed in other studies, have been commonly associated with the utterly different molecular diffusivities of heat and salt. Multiplicity of the equilibrium states, however, proved feasible in heat-salt systems described also in terms of the equal diffusivity coefficients, in particular, where the two components are distinguished in terms of the "mixed" boundary conditions (see, among many others, Cessi \& Young 1992, Quon \& Ghil 1992,1995, where highly simplified ocean thermohaline circulation models were addressed). It is important to emphasize that the mechanism bringing about the instabilities in these works is not double-diffusive (this mechanism is discussed, in particular, by Walin 1985, whereas the principal idea behind it dates back to the seminal work by Stommel 1961). As emphasized by Welander (1989), however, the "mixed" boundary conditions can eventually also lead to manifestation of the doublediffusive effects, by virtue of the nonequal gradients of the components, even when the diffusivity coefficients of both density-effecting properties are equal.

In view of the "mixed" boundary conditions at the vertical side walls in all the earlier studies addressing the laterally heated systems (no salt flux is imposed at these boundaries together with the temperature difference), the purpose of the work addressed in the second part of the lecture (Tsitverblit 1996) has been to inquire about the physical nature of the bifurcation phenomena uncovered in TK and T (and believed to be responsible for diversity of the previously observed phenomena) with particular emphasis on the role played by the different diffusivity coefficients versus that of the "mixed" side-wall boundary conditions. This objective was fulfilled by examining the steady solution structure in such systems with the diffusivity of the solute, $k_{S}$, being equal to that of the temperature, $k_{T}$, and comparing the results with those in $\mathrm{T}$.

Without dwelling on a number of other interesting observations arising from this study, the attention during the lecture was concentrated on aspects of the fact that, despite some simplification in the solution structure and quantitative differences in the critical thermal Rayleigh numbers, most of the main qualitative features of the bifurcation phenomena and multiple steady flows in such systems with the equal diffusivity coefficients were found to be basically the same as for the heat-salt diffusivity ratio. In particular, the $R a_{T}$ characterizing the bifurcations obtained exhibit an appreciable drop as the vertical solute stratification exceeds a certain critical value, close to that associated in the previous works with the commencement of the double-diffusive region, and grow with the further increase of this parameter, like in the results of Hart (1971), Thangam et al. (1981), and T, where $L e w=\frac{k_{T}}{k_{S}}=101$. As $R a_{S}$ increases, the multiple steady flows form by the same scenarios as distinguished in $\mathrm{T}$ for $\frac{k_{T}}{k_{S}}=101$ and only within a region of some intermediate values of the buoyancy ratio, with the unique solutions at the very large and very small values of this parameter. Similarly to the results in $T$, the region of multiple steady states was found to expand with the solute Rayleigh number growing. In addition, the sequence of 
the exchanges between the different flow patterns proved to be essentially the same as at $L e w=101$ (i.e., like in T, two-, four-, five-, three-, and one-cell patterns were successively observed along the symmetric branches at the highest $R a_{S}$ considered), and the variety of the types of the multiple steady flows was found to enhance with $R a_{S}$ crossing some its critical values, which was also the case in $\mathrm{T}$.

The qualitative persistence of the bifurcation phenomena even for the equal diffusivity coefficients was interpreted as being due to the mixed boundary conditions for the temperature and the solute imposed at the vertical side walls of the enclosures in many previous studies. It was suggested, in particular, that by virtue of the different ways of specification of the boundary conditions, a perturbation would inevitably cause the higher increase of the horizontal gradient for the temperature than for the solute and, thereby, the different rates of the lateral diffusion of these components even when their diffusivity coefficients are the same. This mechanism was found to be essentially identical to the one the principal possibility of which was suggested by Welander (1989) in application to the oscillatory instability in a configuration with vertical gradients of two components.

Apart from the consistency with the results for the boundary conditions of the mixed type, the suggested interpretation was also found to be more directly supported by the data obtained with the Dirichlet side-wall boundary conditions for the solute. The latter are characterized by the absence of all the instabilities in the problem with the equal diffusivities and by a number of the bifurcations at $L e w=\frac{k_{T}}{k_{S}}=101$ being much smaller than in T. Based on these additional observations, it was concluded that the instabilities observed for $L e w=1$ are indeed associated with the different diffusion gradients of the temperature and the solute that are generated by the perturbation in their respective isolines by virtue of the mixed side-wall boundary conditions, rather than simply with the unequal gradients of these components characterizing the background motion itself. This conclusion accentuates that the nature of the bifurcation phenomena and the related diversity of the flow features observed in laterally heated thermohaline systems should be attributed to the double-diffusive effects that are due not only to the different diffusivity coefficients of heat and salt, but also to the different types of the side-wall boundary conditions often maintained for the involved components.

\section{References}

[1] Chen, C. F. Briggs, D. G. and Wirtz, R. A. (1971) "Stability of thermal convection in a salinity gradient due to lateral heating." Int. J. Heat Mass Transfer 14, 57-65.

[2] Cessi, P. and Young, W. R. (1992) "Multiple equilibria in two-dimensional thermohaline circulation." J. Fluid Mech. 241, 291-309.

[3] Hart, J. E. (1971) "On sideways diffusive instability." J. Fluid Mech. 49, 279-288.

[4] Hart, J. E. (1973) "Finite amplitude sideways diffusive convection." J. Fluid Mech. 59, 47-64.

[5] Huppert, H. E. and Turner, J. S. (1980) "Ice blocks melting into a salinity gradient." J. Fluid Mech 100, 367-384.

[6] C. G. Jeevaraj and J. Imberger (1991) "Experimental study of doublediffusive instability in sidewall heating." J. Fluid Mech. 222, 565-586. 
[7] O. S. Kerr "Heating a salinity gradient from a vertical sidewall: linear theory." J. Fluid Mech. 207, 323-352, (1989).

[8] O. S. Kerr "Heating a salinity gradient from a vertical sidewall: nonlinear theory." J. Fluid Mech. 217, 529-546, (1990).

[9] Quon, C., and Ghil, M. (1992) "Multiple equilibria in thermosolutal convection due to salt-flux boundary conditions." J. Fluid Mech. 245, 449-483.

[10] Schmitt, R. W. (1994) "Double diffusion in oceanography." Annu. Rev. Fluid Mech. 26, 255-285.

[11] H. Stommel (1961) "Thermohaline convection with two stable regimes of flow." Tellus XIII, 224-230.

[12] Thangam, S., Zebib, A., and Chen, C. F. (1981) "Transition from shear to sideways diffusive instability in a vertical slot." J. Fluid Mech. 112, 151-160.

[13] Thorpe, S. A., Hutt, P. K., and Soulsby, R. (1969) "The effect of horizontal gradients on thermohaline convection." J. Fluid Mech. 38, 375-400.

[14] N. Tsitverblit and E. Kit (1993) "The multiplicity of steady flows in confined double-diffusive convection with lateral heating." Phys. Fluid $A$ 5(4), 1062-1064.

[15] N. Tsitverblit (1995) "Bifurcation phenomena in confined thermosolutal convection with lateral heating: Commencement of the double-diffusive region." Phys. Fluid 7(4), 718-736.

[16] N. Tsitverblit (1996) Multiplicity of the equilibrium states in laterally heated thermosolutal systems with the equal diffusivity coefficients. Submitted.

[17] G. Walin (1985) "The thermohaline circulation and the control of ice ages." Palaeogeography, Palaeoclimatology, Palaeocology 50, 323-332.

[18] Welander, P. (1989) "A new type of double-diffusive instability?" Tellus 41A, 66-72.

[19] R. A. Wirtz and C. S. Reddy (1979) "Experiments in convective layer formation and merging in a differentially heated slot." J. Fluid Mech. 91, 451464. 


\section{A Laboratory Study of the Effect of Bottom Topography on Ocean Circulation by George Veronis}

During spring, 1996, Ross Griffths of Australian National University and I carried out a series of experiments to study the effects of a sloping side boundary on the circulation of a homogeneous fluid in a rotating sliced cylinder. Pedlosky and Greenspan (1967) and Beardsley (1969) studied the same problem when the side wall is vertical. In the present experiments the container was made by cutting a circular cone (90 degree apex angle) with a plane that has a slope of 0.1 with respect to the horizontal (see figure 1).

As in the earlier experiments the slope of the inclined plane is meant to simulate the beta-effect on the beta-plane (i.e., the linear variation of the Coriolis parameter, $f$, with latitude). The analogy with ocean circulation enters through the potential vorticity, $(\zeta+f) / H$, the variation of which determines the large scale circulation. Here, $\zeta(=\partial v / \partial x-\partial u / \partial y)$ is the vertical component of relative vorticity and $H$ is the depth of the fluid. It is evident that an increase (decrease) in $f$ can be simulated by a decrease (increase) in $H$. Although the sloping bottom and the sloping sides both involve depth variation, the effect of the sloping bottom is interpreted as a beta-effect while the effect of the 1:1 sloping side is interpreted directly as a depth variation. Thus, minimum depth above the sloping bottom indicates "north" and maximum depth indicates "south".

The tank diameter was $97.3 \mathrm{~cm}$ and the minimum depth of the interior was $8.48 \mathrm{~cm}$; the maximum depth was $15.79 \mathrm{~cm}$. The flow was driven by the top lid which rotated with the angular velocity, $\Omega+\Delta \Omega$.The range of the Rossby number, $R o=\Delta \Omega / \Omega$, was $0.0021 \leq R o \leq 0.175$ The range of the Ekman number, $E=\nu /\left(h^{2} \Omega\right)$, was $2.110^{-5} \leq E \leq 1.2610^{-4}$.

For the linear problem potential vorticity reduces to $f / H$. Hence curves of constant potential vorticity, called geostrophic contours, are circles near the rim and D-shaped curves elsewhere, as seen in figure 2. The curve of intersection of bottom slope and side slope is an ellipse. The innermost circular geostrophic contour (shown by the dashed circle in figure 2) is tangent to the northernmost point on the ellipse. In contrast to the vertical wall case, where all geostrophic contours intersect the rim at east and west, all geostrophic contours in our set-up close upon themselves.

The main results obtained with the sloping boundary are the following: 
1) For weak driving (nearly linear flow) fluid between the rim and the dashed circle travels along a circle with an azimuthal velocity independent of azimuthal angle. The flow inside the dashed circle but still on the slope also is largely azimuthal along a portion of the circles of the D-shaped curves.

2) Fluid in the "interior" (over the gently sioping bottom) travels from or to points on the northwestern quadrant to or from points on the eastern side of the ellipse. Each of these trajectories is neariy independent of position when the forcing is weak. This interior flow has an eastward component for $\Delta \Omega<0$ and a westward component for $\Delta \Omega>0$.

3) In all cases (weak or strong forcing) azimuthal flow on the D-shaped curves cuts sharply across the circular parts of contours closer to the center and joins the interior flow. This cross-contour flow occurs on the eastern half of the ellipse. The flow is from the slope region to the interior for $\Delta \Omega<0$ and vice versa for $\Delta \Omega>0$. This sharply cross-contour flow is associated with strong frictional dissipation. Thus, in strong contrast to the vertical wall case, and indeed to all of the intuition that we have developed since Stommel's (1948) westward intensification paper, in the present case the bulk of the dissipation takes place on the eastern side of the basin. This explicit result is not expected to carry over to ocean basins since they are not enclosed by closed geostrophic contours, but it does indicate that sloping boundaries can allow dissipation to take place at locations that are far from those that one would expect from the vertical wall case.

4) Cyclonic forcing $(\Delta \Omega>0)$ leads to a circulation inside of D-shaped curves consisting of a northwestward interior flow, a counterclockwise azimuthal flow, and a short cross-contour traverse between the azimuthal flow and the northwestward interior flow. The only effect of strong driving is to make the interior flow more zonal. There are negligible kinks and no evidence of instabilities. The flow takes on the form of a Fofonoff gyre although the dynamical balance in the present situation is different from Fofonoff's.

5) Strong anticyclonic forcing $(\Delta \Omega<0)$ generates strong anticyclonic and cyclonic loops where the azimuthal flow enters the interior in the northwest. These loops can pinch off and become eddies. Eddy activity is confined to the northwestern part of the interior except when $\Delta \Omega$ is very large when the eddies may extend even into the southeastern quadrant. Eddies are formed because the azimuthal flow overshoots the latitude to which it must return in order to connect up to the slow southeastward interior flow. In contrast, with cyclonic forcing, flow enters the interior from the south and east so the 
fluid has the same direction as that required by the interior flow, hence there is no overshoot and no eddy activity.

6) The stability of the system with anticyclonic forcing depends on the values of the Rossby and Ekman numbers (figure 3). The sloping wall causes the system to become unstable at values of $R o$ that are smaller than those for a vertical wall.

7) In general, the sloping boundary serves as a conduit which can take fluid from a point where it enters from the interior and carry it without changing the value of potential vorticity to a distant point where it may enter the interior. It is this feature that should be applicable to ocean basins and that may give rise to features that are very different from those in the flat-bottomed case.

\section{References}

Beardsley, R.C., A laboratory model of the wind-driven ocean circulation J. Fluid Mech., 38, 255-271, 1969.

Pedlosky, J. and H.P. Greenspan, A simple laboratory model for the oceanic circulation. J. Fluid Mech., 27, 291-304, 1967.

Stommel, H. The westward intensification of wind-driven ocean circulation. Trans AGU 1948 

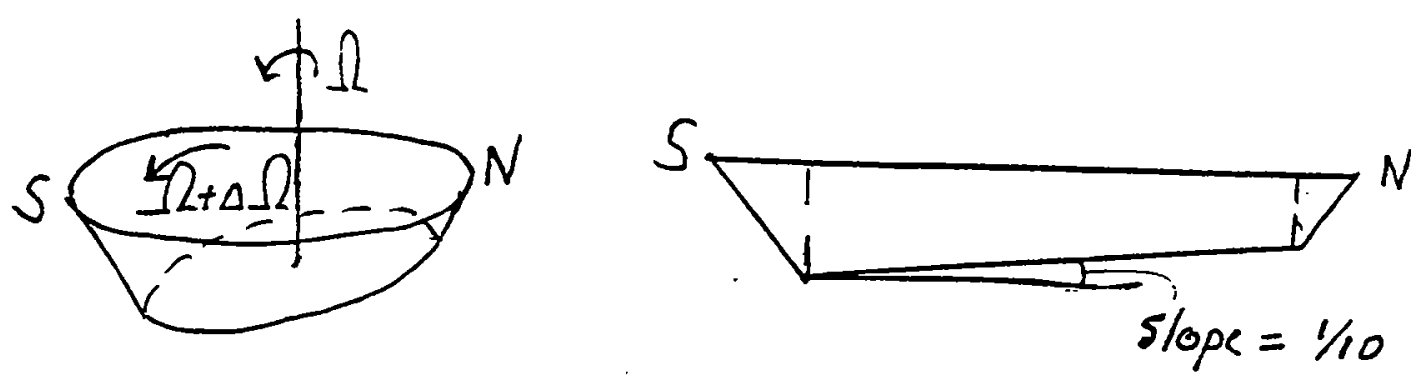

Figure 1. (a) A perspective view of the sliced cylinder tank with sloping sidewalls. (b) The sliced cylinder with sloping walls viewed from the east.

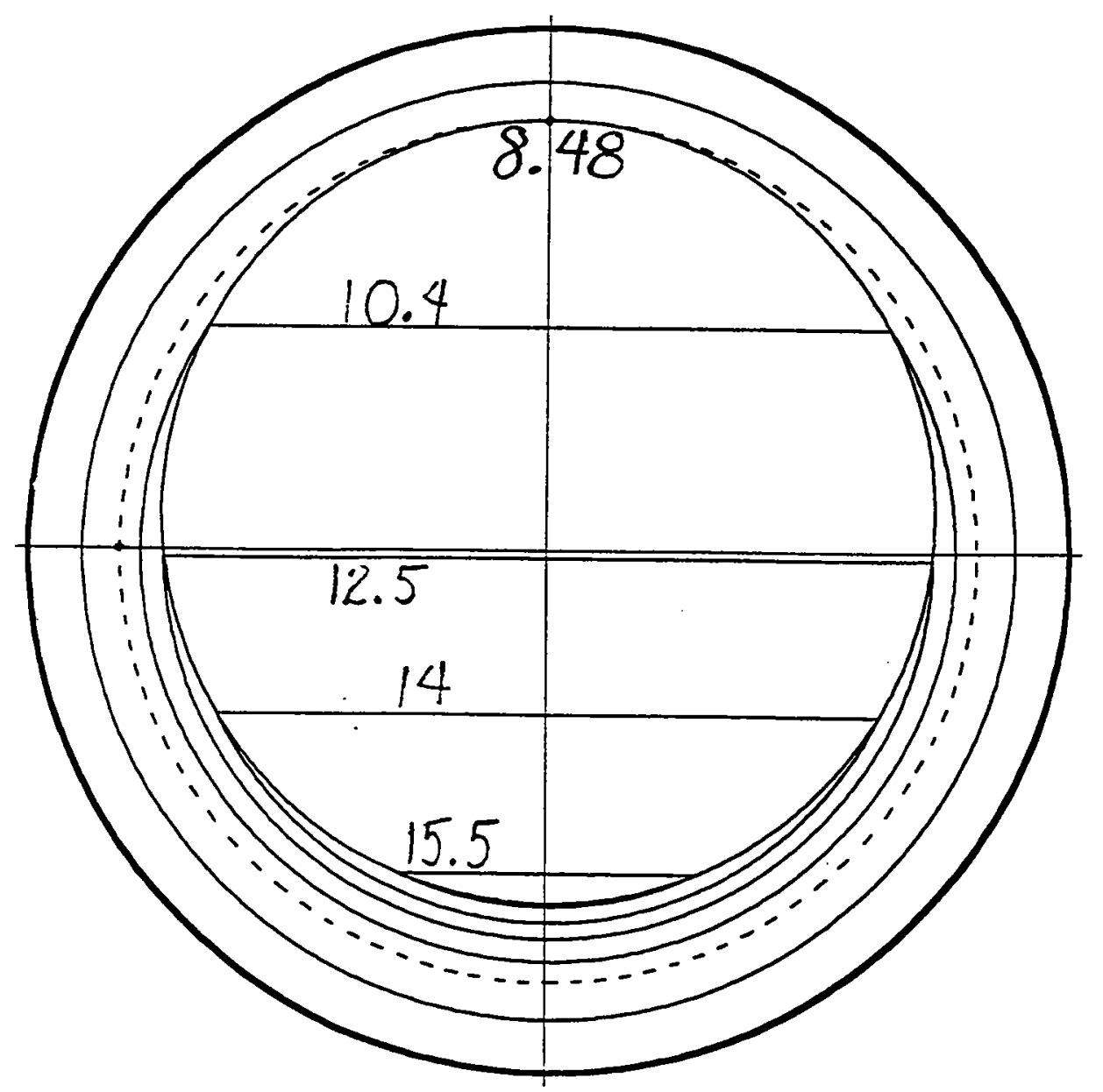

Figure 2. Contours of equal depth (looking down on the tank). The dashed circle is tangent to the northernmost point of the gently sloping bottom. For depths greater than $8.48 \mathrm{~cm}$ the contours are D-shaped curves. 


\section{B-plane with sloping wall \\ Anticyclonic forcing \\ Stability regimes}

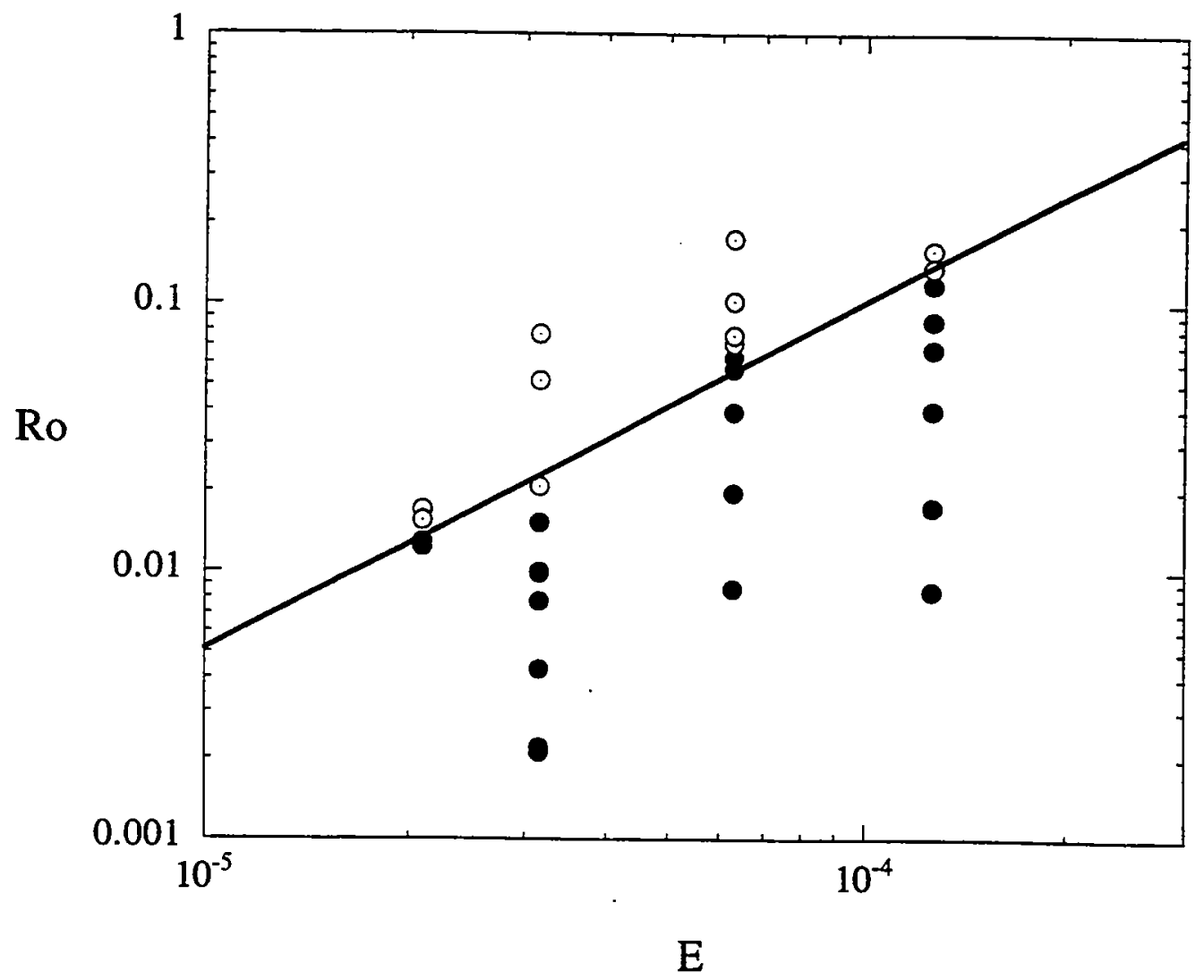

Figure 3. The stability diagram in the Ro vs $E$ plane. Solid circles indicate stable flow, open circles indicate flow with eddies. The transition to instability occurs at lower values of Ro than it does for the case with a vertical wall. 


\title{
Double Diffusive Effects in Magmas
}

\author{
Andrew W Woods, School of Mathematics, \\ University of Bristol, Bristol, England
}

In this lecture we reviewed a number of processes concerning the dynamics of magma chambers, which contain molten rock and are located $5-10 \mathrm{~km}$ below the earth's surface at active volcanoes. Much of the work in the lecture has been described in the papers by Nilson (1985), Chen and Turner (1980), McBirney (1985) and Huppert and Turner (1980). Magma chambers are cooled at their upper surface and side-walls from the surrounding cold crust and this leads to cooling and crystallisation of the melt. As the melt cools, any changes in the density of the melt depend on the degree of cooling and of changes in composition of the melt. The changes in composition result from crystallisation which occurs as the melt cools. The crystals may either remain attached to the boundary of the chamber or they may be suspended in the melt, in which case they also affect the density of the melt. A magma chamber thereby provides an environment in which there is diffusion of both heat and composition and therefore in which double diffusive effects may develop.

The main focus of the lecture was on double-diffusive boundary currents which develop at the cooled side-wall of the chamber. The cooling of the melt may produce either a buoyant or a dense flow along the side-wall of the chamber; in some cases the thermal and compositional effects act in parallel and the flow may be described by similarity solutions, with an inner compositional layer, a central thermal layer and an outer viscous layer. In other cases, the thermal and compositional effects act in opposition. As a result of the different scales for thermal and compositional diffusion, this leads to counterflowing boundary layers. There are no simple similarity solutions to describe such flows, since the thermal layer advects information downwards (upwards) while the compositional layer advects information upwards (downwards). Instead, the motion may calculated numerically (Nilson et al.,1985).

As the boundary layers advance along the boundary, they entrain adjacent fluid by viscous and thermal diffusion, inceasing the volume flux in the current. This boundary current drains back into the interior of the chamber, either at the top or base of the chamber and a net circulation is set up in the interior. The rate of descent of the first front associated with the interior filling box flow was described and the variation of density with position behind the front was also discussed following Worster and Leitch (1985). In this way, the interior fluid becomes stratified as a result of the boundary flow, even if it is initially homogeneous. As a result, double diffusive layering may then develop in the interior of the chamber: as the buoyant boundary layer rises through a stratified interior, lateral intrusions may develop from the boundary flow. This is because the density difference between the boundary flow and the interior progressively decreases until the boundary flow is no longer buoyant. The 
boundary flow then intrudes into the interior fluid. For a stratification $\frac{\partial \rho}{\partial z}$ and a boundary current of buoyancy $\Delta \rho$, the vertical separation of these lateral intrusions scales as $\Delta \rho / \frac{\partial \rho}{\partial z}$.

The main part of the lecture concluded by noting that the process of layering formed by the boundary current in a magma chamber has some similarities with the layers which form as an iceberg melts in a stratified ocean (Huppert and Turner 1980). In that case, a current of fresh melt water runs up the side of the iceberg, and the outer part of this current intrudes into the interior as it mixes with the relatively saline deeper fluid and rises into the less saline environment. The presence of and the circulation set up by the layers tends to localise the melting on the iceface producing a rippled texture on the ice-face.

Chen CF and Turner JS Crystallisation in a double-diffusive system, 1980, J Geophys Res., $85,2573-2593$

Huppert HE and Turner JS Ice blocks melting into a salinity gradient, 1980, J Fluid Mech., $100,367-384$

McBirney, A.H., Mixing and Unmixing of Magmas, J Volc Geoth Res., 7, 357-371

Nilson, R.H., 1985, Countercurrent convection in a double-diffusive boundary layer, J Fluid Mech., 160, 181-210

Worster MG and Leitch A, 1985 Laminar free convection in confined regions, J Fluid Mech, $156,301-319$ 


\section{GFD PROGRAM 1996}

\section{Monday, June 17th}

10:00 A Lawrence Peirson III, Associate Dean, Woods Hole Oceanographic Institution

Welcome and Introduction

10:15 Barry Ruddick, Dalhousie University

Double diffusion: An introduction. Pt. I

Tuesday, June 18th

10:00 Barry Ruddick, D.U. and George Veronis, Yale University

Double diffusion: An introduction. Pt. II

Wednesday, June 19th

10:00 George Veronis, Yale University

Double diffusion: An introduction. Pt. III

19:00 Andy Woods, University of Bristol

Double diffusion in geological systems

\section{Thursday, June 20th}

10:00 Ray Schmitt, W.H.O.I.

Macro-scale signatures of double diffusion in the ocean

14:00 Jack Whitehead, Woods Hole Oceanographic Institution

Doubly-driven catastrophes in my lab.

Friday, June 21th

10:00 Barry Ruddick, Dalhousie University and/or George Veronis, Yale University

A model for salt fingers

\section{Monday, June 24th}

10:00 Barry Ruddick, Dalhousie University

Double diffusion: Laboratory experiments

\section{Tuesday, June 25th}

10:00 George Veronis, Yale University

Thermohaline fronts

13:00 Gerd Krahmann, University of Kiel

Thermohaline steps in the Algero-Provencal-Basin (Western

Mediterranean) - spatial and temporal variability

\section{Wednesday, June 26th}

10:00 Ray Schmitt, W.H.O.I.

Micro-scale signatures of salt fingers in the ocean

14:00 Eddy Carmack, IOS, British Columbia

Double-Diffusive Intrusions in the Arctic Ocean: A Mechanism of Transition

\section{Thursday, June 27th}

10:00 Eric Kunze, University of Washington

Limits on growing, finite-length fingers: A gradient Froude number constraint 
14:00 Eddy Carmack IOS, British Columbia

Three Vignettes: 1) Diffusive Instabilities driven by Geothermal

Heating; 2) Light Quantum Principles in Oceanography; 3) the Thermal

Curtain Hypothesis.

17:00 Staff

Discussion of possible projects.

Friday, June 28th

10:00 Eric Kunze, University of Washington

Effects of vertical shear on salt fingers

14:00 Eric Chassignet, U. Miami

On the choices of eddy diffusivity in ocean general circulation models

Monday, July 1st

10:00 Oliver Kerr, City University, London

Double-diffusive intrusions at boundaries

Tuesday, July 2nd

10:00 Keith Julien, University of Colorado

Weakly nonlinear convection I: Rayleigh-Benard Convection

17:00 The Fellows

Project outlines

Wednesday, July 3rd

10:00 Keith Julien, University of Colorado

Weakly nonlinear convection II: Strong spatial resonances in multi-layer convection

14:00 Phil Morrison, University of Texas at Austin T.B.A.

Thursday, July 4th

10:00 G. Washington, University of Delaware

Friday, July 5th

No lectures

Monday, July 8th

10:00 Paul Kolodner and Hermann Riecke

Binary fluid convection I

Tuesday, July 9th

10:00 Paul Kolodner and Hermann Riecke

Binary fluid convection II

13:00 Isom Herron, RPI

The Two-Dimensional stability of flows with constant vorticity

Wednesday, July 10th

10:00 Paul Kolodner and Hermann Riecke

Binary fluid convection III

14:00 Charles Doering

Convection, Stability and Turbulence 


\section{Thursday, July 11th}

10:00 Paul Kolodner and Hermann Riecke

Binary fluid convection IV

\section{4:00 Paul Kolodner}

Viscoelastic Convection

Friday, July 12th

10:00 Edgar Knobloch, UC Berkeley

Oscillatory convection in large aspect ratio containers

14:00 Paul Dellar, University of Cambridge

Ambipolar diffusion: astrophysical applications of porous media

\section{Monday, July 15th}

10:00 Edgar Knobloch, UC Berkeley

Transition to chaos in standing and travelling wave convection

Wednesday, July 17th

14:00 Naftali Tsitverblit

Multiplicity of the equilibrium states in laterally heated

thermosolutal systems.

\section{Monday, July 22nd}

10:00 Willem Malkus, Massachussetts Institute of Technology

The geodynamo -- Analytic (and numerical) idealizations.

Tuesday, July 23rd

10:00 Mike Proctor, University of Cambridge

Magnetoconvection: fundamentals

\section{Wednesday, July 24th}

10:00 Mike Proctor, University of Cambridge

Magnetoconvection: simulations

\section{Thursday, July 25th}

10:00 Melvin Stern, Florida State University

A prediction of finger fluxes in an unbounded $\mathrm{T}-\mathrm{S}$ gradient region.

14:00 Mike Proctor, University of Cambridge

Anomalous effects of distant boundaries on the onset of travelling-wave instabilities

\section{Friday, July 26th}

NO LECTURES!

\section{Monday, July 29th}

NO LECTURES

\section{Tuesday, July 30th}

10:00 Stewart Turner, Australian National University

Laboratory models of double-diffusive processes

Wednesday, July 31st

10:00 Stewart Turner, Australian National University

Stratification and circulation produced by double-diffusive sources

in closed regions

Thursday, Aug 1st - Thursday, August 8th

NO LECTURES 
Friday, Aug 9th

10:00 George Veronis, Yale University

A laboratory study of topographic effects on ocean circulation

Monday, Aug 12th -- Friday, Aug 16th

NO LECTURES

Monday, Aug 19th

10:00

Fellow's Lecture I: Jon Wylie

Surface wave propagation in granular media

\section{1:00}

Fellow's Lecture II: Naftali Tsitverblit

The finger regime of double-diffusive convection with equal diffusion coefficients and mixed boundary conditions

\section{3:30}

Fellow's Lecture III: Tuni Kundu

Stability of volcanic magma

Tuesday, Aug 20th

10:00

Fellow's Lecture IV: Suzanne Talon

Semi-convection in stars

\section{1:00}

Fellow's Lecture V: Joseph Biello

Double-diffusion in a thin slot

\section{4:00}

Fellow's Lecture VI: Sebastien Aumaitre

Convection in the presence of radiative transfer

\section{Wednesday, Aug 21st}

10:00

Fellow's Lecture VII: Francesco Paparella

Single-mode convection with shear and salt

\section{1:00}

Fellow's Lecture VIII: Alex Casti

Double-diffusion with a sharp density interface

\section{3:30}

Fellow's Lecture IX: Jean-Luc Thiffeault

Long-wave instability in thermohaline-Marangoni convection

\section{4:30}

Fellow's Lecture X: Jim Stephens

Stability analysis of a gravity current over topography 


\section{BIBLIOGRAPHY}

Acheson, D. J., 1979. 'Stable' density stratification as a catalyst for instability. Journal of Fluid Mechanics, 96, 723--733. Ahlers, G., and I. Rehbert, 1986. Convection in a binary fluid mixture heated from below. Physics Review Letters, 56, $1371-1376$.

Ambrosone, L., Costantino, L., Paduano, L., and Vitagliano V, Ecological problems and diffusion in Trends in Ecological Physical Chemistry, Bonati, L., Cosentino,U., Lasagni, M., Moro, G., Pitea, D., Schiraldi, A., Eds., Elsevier, Amsterdam 1993, pp. 65-78.

Anderson, D. L., Accelerated plate tectonics, Science, 187, 1077-1079, 1975.

Anderson, S. A., 1992. Shear, strain and thermohaline vertical fine structure in the upper ocean, Ph.D. thesis, University of California, San Diego.

Anderson, S. P., and R. Pinkel, Double Diffusively Unstable Intrusions Near an Oceanic Front: Observations from R/P FLIP. In Double Diffusive Convection A. Brandt and H.J.S. Fernando (eds), pp. 195-211, AGU, 1995.

Antoranz, J. C., and M. G. Velarde, 1979. Thermal diffusion and convective stability. Physics of Fluids, 22, 1038--1043. Armi, L., 1978. Some evidence for boundary mixing in the deep ocean. Journal of Geophysical Research, 83, 1971--1979. Armi, L., and R. C. Millard, 1976. The bottom boundary layer of the deep ocean. Journal of Geophysical Research, 81, 4983- -4990.

Armi, L., and W. Zenk, 1984. Large lenses of highly saline Mediterranean water. Journal of Physical Oceanography, 14, $1560-1576$.

Armi, L., D. Hebert, N. Oakey, J. Price, P. Richardson, T. Rossby, and B. Ruddick, 1989. Two years in the life of a Mediterranean Salt Lens. J. Phys. Oceanogr. 19, 354-370.

Asaeda, T., N. Tamai, and H. Ozaki, 1984. Study on the heat and mass transfer at the density interface in double-diffusive processes. JSCE, 342, 135--141, (in Japanese).

Auchmuty, J. F. G., and G. Nicolis, 1975. Bifurcation analysis of nonlinear reaction-diffusion equations - I. evolution equations and the steady state solutions, Bull. Math. Bio., 37, 323-365.

Ayers, J. C., Brenan, J. B., Watson, E. B., Wark, D. A., and Minarik, W. G., 1992. A new capsule technique for hydrothermal experiments using the piston-cylinder apparatus, Am. Mineral., 77, 1080-1086,.

Babarutsi, S., and V. 14. Chu, Dye-concentration distribution in shallow recirculating flows, J. Hydr. Eng., 117, 5, 643-659, 1991.

Baines, P. G., and A. E. Gill, 1969. On thermohaline convection with linear gradients. Journal of Fluid Mechanics, 37, 289-306.

Baines, W.D., J.S. Turner, and I.H. Campbell, Turbulent fountains in an open chamber. J. Fluid Mech. 212, 557-592, 1990. Baker, D. J., Jr., 1971. Density gradients in a rotating stratified fluid: Experimental evidence for a new instability. Science, $172,1029--1031$.

Batchelor, G.K. and R.W. Janse van Rensburg, Structure formation in bidisperse sedimentation J. Fluid Mech. Vol 166 pp 379-407, 1986.

Bauer, E., and G. Siedler, 1988. The relative contributions of advection and isopycnal and diapycnal mixing below the subtropical salinity maximum, Deep-Sea Research, 35, 811--837.

Benard, H., 1901. Les tourbillons cellulaires dans un nappe liquide transportant de la chaleur par convection en regime permanent.Ann. Chim. Phys., 23, 62-144.

Benilov, A. Y., and D. W. Collins, A Quasi-Lagrangian Analysis of Particle Motion in Different Kinds of Background Stratification. In Double Diffusive Convection A. Brandt and H.J.S. Fernando (eds), pp. 57-68, AGU, 1995.

Bergantz, G.W., Convection and solidification in tall magma chambers, Ph.D. Thesis, Johns Hopkins Unv. Baltimore, MD, $127 \mathrm{pp}, 1988$.

Bergman, T. L., and A. Ungan, 1988. A note on lateral heating in a double-diffusive system. Journal of Fluid Mechanics, 194, 175--186.

Bergman, T. L., F. P. Incropera, and R. Viskanta, 1982. A multi-layer model for mixing layer development in a double-diffusive thermohaline system heated from below. International Journal of Heat and Mass Transfer, 25, 1411-1418. Berman, T., Salt Finger in a warm core eddy in the Eastern Mediterranean (in Hebrew). M.Sc. Thesis, Hebrew University, Jerusalem, Israel, 1992.

Berman, T., and S. Brenner, and N. Paldor, Salt Fingering in the Cyprus Eddy. In Double-Diffusive Convection A. Brandt and H.J.S. Fernando (eds), pp.251-260, AGU, 1995.

Best, J. L., and A. G. Roy, Mixing-layer distortion at the confluence of channels of different depth, Nature, 350, 411413, 1991.

Black, R. D. C. (ad.), Papers and Correspondence of William Stanley Jevons, Macmillan Press, London, 7 vols., 1972-1981.

Blankenbach, B. et al., A benchmark comparison of mantle convection codes, Geophys. J. Int., 98, 23-38, 1989.

Bormans, M., Effect of Rp on double diffusive interleaving, Deep Sea Res., 39, 871-884, 1992. 
Boudreau, B. P., and P. H. Leblond, A Simple Evolutionary Model for Water and Salt in the Black Sea, Paleoceanography, 4, 157-166, 1989.

Boussinesq, J., 1903. Theorie analytique de la chaleur., Paris, Gathier-Villars.

Boyd, J. D. 1989. Properties of thermal staircase off the northeast coast of South America, Spring and Fall 1985. Journal of Geophysical Research, 94, 8303--8312.

Boyd, J. D., and H. Perkins, 1987. Characteristics of thermohaline steps off the northeast coast of South America, July 1983. Deep-Sea Research, 34, (3), 337--364.

Boyd, J. D., and H. T. Perkins, 1984. Estimates of vertical heat and salt fluxes in a thermohaline staircase. EOS, $65,954$.

Boyd, J. D., H. T. Perkins, and K. D. Saunders, 1983. Characteristics of thermohaline step structures off the northeast coast of South America. EOS, 64, 1058.

Brand, H. and V. Steinberg, Nonlinear effects in the convective instability of a binary mixture near threshold, Phys. Lett. A, 93, 333-336, 1983 b.

Brand, H. R., P. C. Hohenberg and V. Steinberg, 1984. Codimension-2 bifurcations for convection in binary fluid mixtures. Physical Review A., 30, 2548--2561.

Brand, H., P.C. Hohenberg and V. Steinberg, Amplitude equation near a polycritical point for the convective instability of a binary fluid mixture in a porous medium, Phys. Rev. A, 27, 591-594, 1983.

Brand,H. and V. Steinberg, Convective instabilities in binary mixtures in a porous medium, Physica A, 119, 327-338, 1983a. Brenner, S., Long term evolution and dynamics of a persistent warm core eddy in the Eastern Mediterranean Sea Deep Sea Res. 40, 1193-1206, 1993.

Brenner, S., Z. Rozentrub, J. Bishop, M. Krom, The mixed layer/ thermocline cycle of a persistent warm core eddy in the Eastern Mediterranean. Dyn.. Atmos. Oceans., 15, 457- 476,1991.

Brewer, P. G., and D. W. Spencer, Distribution of Some Trace Elements in Black Sea and Their Flux Between Dissolved and Particulate Phases. In: The Black Sea Geology, Chemistry and Biology, E. T. Degens and D. A. Ross (editors), Am. Assoc. Pet. Geol. Memoir 20, Tulsa, Oklahoma, 137-143, 1974.

Broecker, W.S., T.S Peng, Tracers in the sea. 690 pp. A publication of Lamont-Doherty Observatory, Columbia University, Palisades, NY, 1982.

Broughton, J. M., 1972. Experiments on steady layered convection in a doubly diffusive system. Masters Thesis, Colorado State University, Fort Collins, $\mathrm{CO}$.

Bruce, J. G., J. L. Kerling, and W. H. Beatty II, 1984. Temperature steps off Northern Brazil. Tropical Ocean-Atmosphere Newsletter, (23), January, 10--11.

Bruno, M. S., M. Muntisov, and H. B. Fischer, Effect of buoyancy on transverse mixing in streams, J. Hydr.. Eng., I I 6 , 1484-1494, 1990.

Brunt, D., 1927. The period of simple vertical oscillations in the atmosphere. Quart. J. Roy. Meteor. Soc., 53, $30--32$.

Buesseler, K. O., Livingstone, H. D., and S. Casso, Mixing Between Oxic and Anoxic Waters of the Black Sea as Traced by Chernobyl Cesium Isotopes, Deep-Sea Res., 38, S725- S745, 1991.

Buffett, B.A., Huppert, H.E., Lister, J.R. and A.W. Woods, Analytical model for solidfication of the Earth's core, Nature, 356, 329-331, 1992.

Busse, P. G., and J. A. Whitehead, 1971. Instabilities of convection rolls in a high Prandtl number fluid. Journal of Fluid Mechanics, 47, 305--320.

Caldwell, D. R., 1983. Small-scale physics of the ocean. U.S. Nat'1 Report to the Int'l Union of Geodesy and Geophysics 1979-1982. Reviews of Geophysics and Space Physics, 21, 1192--1205.

Caldwell, D.R. 1973 Measurement of negative thermal diffusion coefficients by observing the onset of thenmohaline convection. J. Phys. Chem.77, 2004-2008.

Caldwell, D.R. 1974 Experimental study on the onset of thermohaline convection. J. Fluid Mech. 64, $347-367$.

Caldwell, D.R. 1976 Thermosolutal convection in a solution with large negative Soret coefficient. J. Fluid Mech., 74, 129-142.

Calman, J., 1977. Experiments on high Richardson number instability of a rotating stratified shear flow. Dynamics of Atmospheres and Oceans, 1, 277--297.

Campbell, I. H., T. J. McDougall and J. S. Turner, 1984. A note on Fluid dynamic processes which can influence the deposition of massive sulphides. Economic Geology, 79, 1905--1913.

Campbell, I.H., and J.S. Turner, Turbulent mixing between fluids with different viscosities, Nature, 313, 39-42, 1985.

Carmack, E. C., and P. D. Killworth, 1978. Formation and interleaving of abyssal water masses off Wilkes Land, Antarctica. Deep-Sea Research, 25, 357--369.

Carmack, E. C., and T. D. Foster, 1975. On the flow of water out of the Weddell Sea. Deep-Sea Research, 22, $711--724$.

Carmack, E., and K. Aagaard, 1973. On the deep water of the Greenland Sea. Deep-Sea Research, 20, 687--715.

Castillo, J. L., and M. G. Velarde, 1982. Buoyancy-thermocapillary instability: the role of interfacial deformation in one- and two-component fluid layers heated from below and above. Journal of Fluid Mechanics, 125, 463--474.

Cbarlson, G. S., and Sani, R. L., On thermoconvective instability in a bounded cylindrical fluid layer, Int. J. Heat Mass 
Transfer, 14, 2157-2160, 1971.

Cbarlson, G. S., and Sani, R. L., Thermoconvective instability in a bounded cylindrical fluid layer, Int. J. Heat Mass Transfer, 13, 1479-1496, 1970.

Chandrasekhar, S., Hydrodynamic and Hydromagnetic Stability, Clarendon Press, Oxford, 1961.

Charnock, H., Advances in physical oceanography. Recent Advances in Meteorology and Physical Oceanography, Royal Meterological Society, 67-81, 1983.

Chen F. and J.W. Lu, Influence of viscosity variation on salt-finger instability in a fluid layer, a porous layer and their superposition, J.Appl. Phys. 70, 4121-4131, 1991.

Chen, C. F., 1974. Onset of cellular convection in a salinity gradient due to a later temperature gradients. Journal of Fluid Mechanics, 63, 563--576.

Chen, C. F., 1975. Double-diffusive convection in an inclined slot. Journal of Fluid Mechanics, 72, $721--729$.

Chen, C. F., 1978. Time-dependent double-diffusive instability in a density-stratified fluid along a heated inclined wall. Journal of Heat Transfer, 100, 653--658.

Chen, C. F., and D. H. Johnson 1984. Double-diffusive convection: A report on an Engineering Foundation conference. Journal of Fluid Mechanics, 138, 405--416.

Chen, C. F., and F. J. Millero, Precise thermodynamic properties for natural waters covering only the limnological range, Limnology and Oceanography, 31, 657-662, 1986.

Chen, C. F., and J. S. Turner, 1980. Crystallization in double-diffusive system. Geophysical Research, 85, $2573--2593$.

Chen, C. F., and R. D. Sandford, 1976. Sizes and shapes of salt fingers near the marginal state. Journal of Fluid Mechanics, 78, 601--607.

Chen, C. F., and R. D. Sanford, 1977. Stability of time-dependent double diffusive convection in an inclined slot. Journal of Fluid Mechanics, 83, 83--95.

Chen, C. F., and W. W. Skok, 1974. Cellular convection in a salinity gradient along a heated inclined wall. International Journal of Heat and Mass Transfer, 17, 51--60. Chen, C. F., and S. B. Wong, 1974. Double-diffusive convection along a sloping wall. Bulletin of the American Physical Society, 19, 1153.

Chen, C.F. and Su, T.F. 1992 Effects of surface tension on the onset of convection in a double-diffusive layer. \{lit Phys. Fluids\}, \{lbf A 4 \}, 2360.

Chen, C. F., Briggs, D. G., Wirtz, R. A., Stability of thermal convection in a salinity gradient due to lateral heating, Int. J. Heat Mass Itansfer, 14, 57-65, 1971.

Chen, C. F., D. G. Briggs, and R. A. Wirtz, 1971. Stability of thermal convection in a salinity gradient due to lateral heating. International Journal of Heat and Mass Transport, 14, 57--65.

Chen, C. F., R. C. Paliwal, and S. B. Wong, 1976. Cellular convection in a density stratified fluid: Effect of inclination of the heated wall. Proceedings of the 1976 Heat Transfer and Fluid Mechanics Institute, Stanford University Press, Palo Alto,

California, 18--32.

Chen, F., and C. F. Chen, 1988. Onset of finger convection in a porous layer underlying a fluid layer. Trans. ASMEC C: Journal of Heat Transfer, 110, 403--409.

Chereskin, T. K., and P. F. Linden, 1986. The effect of rotation on intrusions produced by heating a salinity gradient. Deep-Sea Research, 33, 305--322.

Chinake, C.R. and R.H. Simoyi, Fingering patterns and other interesting dynamics in the chemical waves generated by the Chlorite-Thiourea Reaction, J. Phys. Chem., 98, 4012-4019, 1994.

Chossat, P., and G. Iooss, Primary and secondary bifurcations in the Couette-Taylor problem, Japan J. Applied Math., 2 , $37-68,1985$.

Christensen, U.R. and D.A. Yuen, Layered convection induced by phase transitions, J. Geophys. Res., 90, $10291-10$ 300,

1985.

Churchill, J. H., P. C. Cornillon, and P. Hamilton, 1989. Velocity and hydrographic structure of subsurface shelf waters at the Gulf Stream's edge. Journal of Geophysical Research, 94(C8), 10,791--10,800.

Clark, S., Spera, F.J. and D.A. Yuen, Steady-state double-diffusive convection in magma chambers heated from below, in Magmatic Processes: Physicochemical Principles, pp 289-305, ed. by B.O. Mysen, The Geochemical Society, 1987.

Clarke, R. A., S. H. Swift, J. L. Reid, and K. P. Koltermann, 1990. The formation of Greenland Sea Deep Water:

Double-diffusion or deep convection? Deep-Sea Research, 37(9A), 1385--1424.

Clune, T. and Knobloch, E., Pattern selection in three dimensional magnetoconvection, Physica, 74D, 151-176, 1994.

Codispoti, L. A., Friederich, G. E., Murray, J. W., and C. Sakamoto, Chemical Variability in the Black Sea: Implications of Data Obtained with a Continuous Vertical Profiling System that penetrated the Oxic/ Anoxic Interface, Deep-Sea Res., 38, S691-S710, 1991.

Cohen, C.F. and R.D. Sandford, Stability of time-dependent double-diffusive convection in an inclined slot. J. Fluid Mech. 8S, 83-95, 1977.

Cooper, A. R. Jr., Model for multi-component diffusion, Phys. Chem. Glasses, 6, 55-61, 1965.

Cooper, J. W., and H. Stommel, 1968. Regularly spaced steps in the main thermocline near Bermuda. Journal of Geophysical 
Research, 73, 5849--5854.

Cortecci, G., R. Molcard, and P. Noto, 1974. Isotopic analysis of the deep staircase structure in the Tyrrhenian Sea. Nature, 250, 134--136.

Costantino, L., Della Volpe, C., Ortona, O., and Vitagliano, V., Isothermal diffusion in a peculiar ternary system: The microemulsion AOT - Water - Heptane, J. Chem. Soc. Faraday Trans., 88, 61-63 (1992).

Cox, C. S., Y. Nagata, and T. Osborn, 1969. Oceanic fine structure and internal waves. Bull. Japan Soc. Fish. Oceanogr., Prof. Uda's Commemorative Paper, 67--72.

Cox, S.M., and S. Leibovich, Langmuir circulations in a surface layer bounded by a strong thermoline, J. Phys. Oceaneog., 23, 1330-1345, 1993.

Cox, S.M., S. Leibovich, I.M. Moroz and A. Tandon, Nonlinear dynamics in Langmuir circulations with $0(2)$ symmetry, J. Fluid Mech., 241, 669-704, 1992a.

Cox, S.M., S. Leibovich, I.M. Moroz, and A. Tandon, Hopf bifurcations in Langmuir circulations, Physica D, 59, 226-254, $1992 b$.

Craik, A.D.D., and S. Leibovich, A rational model for Langmuir circulations, J. Fluid Mech., 73, 401-426, 1977.

Crapper, P. F., 1975. Measurements across a diffusive interface. Deep-Sea Research, 22, 537--545.

Crapper, P. F., 1976. The transport across a diffusive interface in the presence of externally imposed turbulence. Journal of Physical Oceanography, 6, 982--984.

Cross, M.C. and P.C. Hohenberg, Rev. Mod. Phys. 65851 (1993).

Cussler, E. I., 1976. Multicomponent Diffusion. Elsevier.

Da Costa, L. N., E. Knobloch, and N. O. Weiss, 1981. Oscillations in double-diffusive convection. Journal of Fluid

Mechanics, 109, 25--43.

Davaille, A. and C. Jaupart, Thermal convection in lava lakes, Geophys. Res. Letr., 20, 1827-1830, 1993.

Davis, R.H. and Acrivos, A., Sedimentation of noncollodial particles at low Reynolds number, Ann. Rev. Fluid Mech. Vol 17, pp 91-118, 1985

de Groot, S. R., and Mazur, P., Non-Equilibrium Thermodynamics, Dover, New York, 1962.

Dean, A. E., E. Knobloch, and J. Toomre, 1989. Traveling waves and chaos in thermosolution convection. Physics Review A, 36(6), 2862--2869.

Deane, A. E., E. Knobloch, and J. Toomre, 1989. Traveling waves in large-aspect-ratio thermosolutal convection. Physics

Review A, 37 (5), 1817--1820.

Degens, E. T., and D. A. Ross, 1969. Hot Brines and Recent Heavy Metal Deposits in the Red Sea, Springer-Verlag, New York, 599pp.

Delnore, V. E. 1980. Numerical simulation of thermohaline convection in the upper ocean. Journal of Fluid Mechanics, 96, 803--826.

Demenkow, J. W., 1973. A study of the two layer salt finger system, Ph.D. Dissertation, University of Rhode Island, Kingston, RI.

Dijkstra, H.A., Molemaker, M.J., Van der Ploeg, A., Botta, E.F.F., An efficient code to compute non-parallel flows and their linear stability, submitted to Comp. Fluids, 1994.

Dijkstra, H.A., On the structure of cellular solutions in Rayleigh Benard-Marangoni flows in small-aspect-ratio containers, J. Fluid Mech., 243, 73-102, 1992.

Dijkstra, H.A., Transient Marangoni convection in a square container. Physico Chemical Hydrodynamics 10, $493515,1988$.

Drazin, P. G., and Reid, W. H., Hydrodynamic Stability, Cambridge University Press, 1981.

Drew, D. A., Mathematical Modeling of two-phase flow, Ann. Rev. Fluid Mech., Vol 15, pp 261-291, 1983

Du, Y., Dissipative Structure, 1996. Non-Uniform Strain Fields, and Formation of Seismicity Patterns In Double-Diffusive

Convection A. Brandt and H.J.S. Fernando (eds), pp. 125-133, AGU, 1996.

Dugan, J. P., B. W. Stalcup, and R. L. DiMarco, Statistics of small-scale activity in the upper ocean, J. Geophys. Res., 97(C4), 5665-5675, 1992.

Eckart, C., 1948. An analysis of the stirring and mixing processes in incompressible fluids. Journal of Marine Research, 7 , 265--275.

Ekman, V. W., 1906. On dead water. Scientific Results of the Norwegian North Polar Expedition, 1893--1896, 5(15), 1--152.

Elder, J. W., 1968. The unstable thermal interface, Journal of Fluid Mechanics, 32, 69--96.

Elder, J. W., 1969. Numerical experiments with thermohaline convection. Physics of Fluids, 12, (Suppl. II), $194--197$.

Elliot, A. J., and R. I. Tait, 1977. On the steady-state nature of the Mediterranean Outflow step structure. A Voyage of

Discovery, G.Deacon 70th Anniversary Volume, Suppl. to Deep-Sea Research, 213--220.

Elliott, A. J., M. R. Howe, and R. I. Tait, 1974. The lateral coherence of a system of thermohaline layers in the deep ocean. Deep-Sea Research, 21, 95-- 107.

Elsasser, W. M., Convection and stress propagation in the upper mantle, in The application of modern physics to the Earth and planetary interiors, edited by S. K. Runcorn, Wiley Interscience, New York, 223-246, 1969. 
Emms, P.W. and A.C. Fowler, Compositional convection in the solidification of binary alloys, J. Fluid Mech., 262. I I 1 -139, 1994.

Eriksen, C. C., 1978. Measurements and models of finestructure, internal gravity waves, and wave breaking in the deep ocean. Journal of Geophysical Research, 83, 2989--3009.

Erofeev, A. Yu., and I. D. Lozovatsky, Comparative analysis of lateral fine structure parameters in the Lomonosov current and Canary upwelling, Oceanological Res., vol. 42, SO-61,1990 (in Russian).

Escowitz, E. C., and A. F. Amos, 1971. Steps on the thermocline northeast of Barbados (abstract). Transactions of the American Geophysical Union, 52, 232.

Evans, D. L., 1975. Large and small-scale temperature-salinity correlations in the ocean. Ph.D. Dissertation, University of Rhode Island, Kingston, RI.

Evans, D. L., 1981. Velocity shear in a thermohaline staircase. Deep-Sea Research, 28, 1409--1415.

Evans, D. L., 1982. Observations of small scale shear and density structure in the ocean. Deep-Sea Research, 29, 581--595.

Fader, A., J., A tribute to Henry Stommel. Oceanus, 35, (special issue), 30-33, 1992.

Federov, K. N., 1970. On the step-like structure of temperature inversions in the ocean. Atmospheres and Ocean Physics, 6, 704--709.

Federov, K. N., 1978. The thermohaline fine structure of the ocean. Pergamon, Oxford. 170pp.

Federov, K. N., 1988. Layer thicknesses and effective diffusivities in "diffusive" thermohaline convection in the ocean. In: Small-Scale Turbulence and Mixing in the Ocean, J. Nihoul and B. Jamart, editors, Elsevier Oceanography Series, 46, Elsevier, New York, pp.471--480.

Fedorov, K. N., 1980. Intrusive fine structure in frontal zones and indication of double-diffusion. In: Marine Turbulence, J.Nihoul, editor, New York, Elsevier, pp.57--63.

Fedorov, K. N., Layer Thicknesses and Effective Diffusivities in "Diffusive" Thermohaline Convection in the Ocean, In: Small Scale Turbulence and Mining in the Ocean, J. C. J. Nihoul and B. M. Jamart, (editors), Elsevier, 471-480, 1989. Fedorov, K. N., The Physical Nature and Structure of Oceanic Fronts, C. Garrett (editor), Springer-Verlag, Berlin, 333 pp., 1986.

Fedorov, K. N., Thermohaline ocean structure., 184 pp., Hydrometeorological Publisher, Leningrad,1976 (in Russian)

Feliks, Y., Downwelling along the northeast coast of the Eastem Mediterranean. J. Phys. Oceanogr., 511-526, 1990.

Feliks, Y., S. Itzikowitz, Movement and geographic distribution of anticyclonic eddies in the Eastern Levantine Basine. Deep Sea Res., 34, 1499-1508, 1987.

Fernando, H. J. S., and C. Y. Ching, An Experimental Study on Thermohaline Staircases, In: Double Diffusion in Oceanography: Proceedings of a Meeting, September 26-29, 1989, R. W. Schmitt (editor), Technical Report, Woods Hole Oceanographic Institution, 141-149, 1991.

Fernando, H. J. S., Oceanographic Implications of Laboratory Experiments on Diffusive Interfaces, J. Phys. Oceanogr., 19, 1707-1715, 1989.

Fernando, H.J.S., Buoyancy transfer across a diffusive interface, J. Fluid Mech., 209, 1-34, 1989.

Fernando, H.J.S., The formation of a layered structure when a stable salinity gradient is heated from below. J. Fluid Mech. 182, 525-541, 1987.

Fisher, H. B., 1971. The dilution of an undersea sewage cloud by salt fingers. Water Research, 5, 909--915.

Fleury, M., and R. G. Lueck, 1991. Fluxes across a thermohaline staircase. Deep-Sea Research, 38(7), 745--747.

Fleury, M., and R. G. Lueck, Fluxes across a thermohaline interface. Deep Sea Res., 55, 745-769, 1991.

Foster, T. D., 1972. An analysis of the cabbeling instability in sea water. Journal of Physical Oceanography, 2, 294--301.

Foster, T. D., 1983. The temperature and salinity finestructure of the ocean under Ross Ice Shelf. Geophysical Research, 88 , 2556--2564.

Foster, T. D., and E. C. Carmack, 1976. Temperature and salinity structure in the Weddell Sea. Journal of Physical Oceanography, 6, 36--44.

Foster, T. D., Effect of boundary conditions on the onset of convection, Phys. Fluids, 11, 1257-1262, 1968.

Fradkov, A. S., G. Nauheimer, and H. J. Neugebauer, 1996. Volume Separation in Double Diffusive Convection Systems. In Double-Diffusive Convection, A. Brandt and H. J .S. Fernando (eds), pp. 151-169, AGU, 1996.

Gargett, A. E., 1976. An investigation of the occurrence of oceanic turbulence with respect to fine structure. Journal of Physical Oceanography, 6, 139--159.

Gargett, A. E., 1978. Microstructure and fine structure in an upper ocean frontal regime. Journal of Geophysical Research, 83, 5123--5134.

Gargett, A. E., 1988. The scaling of turbulence in the presence of stable stratification. Journal of Geophysical Research, 93, 5021--5036.

Gargett, A. E., and R. W. Schmitt, 1982. Observations of salt fingers in the central waters of the eastern North Pacific. Journal of Geophysical Research, 87(C10), 8017--8029.

Gargett, A. E., and R. W. Schmitt, Observations of salt fingers in the central waters of the Eastern North Pacific, J. Geophys. Res., 87, 8017-8092, 1982. 
Gargett, A. E., Holloway, G. 1992. Sensitivity of the GFDL ocean model to different diffusivities for heat and salt. J. Phys. Ocean, 22(10), 1158--77.

Garrett, C. and E. P. W. Horne, 1978. Frontal circulation due to cabbeling and double diffusion. Journal of Geophysical Research, 83, 4651--4656.

Garrett, C. J. R., and W. H. Munk, 1972. Oceanic mixing by breaking internal waves. Deep-Sea Research, 19, 823--832.

Garrett, C., 1982. On the parameterization of diapycnal fluxes due to double-diffusive intrusions. Journal of Physical

Oceanography, 12, 952--959.

Garrett, C., Mixing in the Ocean Interior, Dyn. Atmos. Oceans, 3, 239-265, 1979.

Garrett, C., The Role of Secondary Circulation in Boundary Mixing, J. Geophys. Res., 95, 3181-3188, 1990.

Garrett, C.J.R., 1973. The effect of internal wave strain on

Georgi, D. T. and R. W. Schmitt, 1983. Fine and microstructure observations on a hydrographic section from the Azores to the Flemish Cap. Journal Physical Oceanography, 13, 632--647.

Georgi, D. T., 1978. Fine-structure in the Antarctic polar front zone: its characteristics and possible relationship to internal waves. Journal of Geophysical Research, 83, 4579--4588.

Georgi, D., Fine structure in the Antarctic Polar Front Zone: its characteristics and possible relationship to internal waves, J. Geophys. Res., 83, 45:9-3671, 1978.

Gertman, I. F., Ovchinnikov, I. M., and Y. I. Popov, Deep Convection in the Levantine Sea, Rapp. Comm. Mer Media., 32, $172,1990$.

Gibb, F. G. F. and C.M.B. Henderson Convection and Crystal Settling in Sills, Contrib. Mineral. Petrol., Vol 109, pp

538-545, 1992.

Gill, A. E., and J. S. Turner, 1969. Some new ideas about the formation of Antarctic Bottom Water. Nature, 224, 1287--1288.

Gill, A. E., Atmosphere-Ocean Dynamics, Academic Press, 662 pp. 1982.

Glansdorff, P., and I. Prigogine, Thermodynamics of structure, stability and fluctuations, Wiley-Interscience, New York,

1971.

Glendinning, P., Bifurcations near homoclinic orbits with symmetry, Phys. Lett., 10NA, 163-166, 1984.

Golubitsky, M., Stewart, I. and Shaeffer, D.G., Singularities and Groups in Bifurcation Theory. Volume II, Springer, New

York, 1988.

Gordon, A. L., 1981. South Atlantic thermocline ventiiation. Deep-Sea Research, 28, 1236--1264.

Gordon, A. L., and B. A. Huber, 1984. Thermohaline stratification below the Southern Ocean sea ice, Journal Geophysical

Research, 89, 641--648

Gosink, J. P., and G. C. Baker, 1990. Salt fingering in subsea permafrost: Some stability and and energy considerations. Journal of Geophysical Research, 95(C6), 9575--9584.

Gough, D. O., and J. Toomre, 1982. Single-mode theory of diffusive layers in thermohaline convection. Journal of Fluid

Mechanics, 125, 75--97.

Grant, H. L., A. Moilliet, and W. M. Vogel, 1968. Some observations of the occurrence of turbulence in and above the thermocline. Journal of Fluid Mechanics, 34, 443--448.

Grasshoff, K., The Hydrochemistry of Landlocked Basins and Fjords, In: Chemical Oceanography, J. P. Riley and G.

Skirrow (editors), Academic Press, New York, 2, 647 pp., 1975.

Green, T., 1984. Scales for double-diffusive fingering in porous media. Water Resources Research, 20, 1225--1229.

Greengrove, C. L. and S. E. Rennie, 1991. South Atlantic Density Ratio Distribution. Deep-Sea Research, 38, (Suppl. 1),

345-354

Gregg, M. C., 1973. The microstructure of the ocean. Scientific American, 228(2), 65--77.

Gregg, M. C., 1975. Microstructure and intrusions in the California Current. Journal of Physical Oceanography, 5, 253--278.

Gregg, M. C., 1980a. Microstructure patches in the thermocline. Journal of Physical Oceanography, 10, 915--943.

Gregg, M. C., 1980b. The three-dimensional mapping of a small thermohaline intrusion. Journal of Physical Oceanography,

$10,1468--1492$.

Gregg, M. C., 1987. Diapycnal Mixing in the Thermocline: A Review. Journal of Geophysical Research, 92(C5),

5249--5286.

Gregg, M. C., 1988. Mixing in the thermohaline staircase east of Barbados. In: Small-Scale Turbulence and Mixing in the

Ocean, J.Nihoul and B. Jamart, editors, Elsevier Oceanography Series, 46, Elsevier, New York, pp.453--470.

Gregg, M. C., 1989. Scaling turbulent dissipation in the thermocline. Journal of Geophysical Research, 94, (C7), 9686--9698.

Gregg, M. C., and C. S. Cox, 1971. Measurements of the oceanic microstructure of temperature and electrical conductivity.

Deep-Sea Research, 18, 925--934.

Gregg, M. C., and C. S. Cox, 1972. The vertical microstructure of temperature and salinity. Deep-Sea Research, 19,

355--376.

Gregg, M. C., and J. H. McKenzie, 1979. Thermohaline intrusions lie across isopycnals. Nature, 280, 310--311.

Gregg, M. C., and M. G. Briscoe, 1979. Internal waves, finestructure, microstructure, and mixing in the ocean. Reviews of

Geophysics and Space Physics, 17, 1524--1548. 
Gregg, M. C., and T. B. Sanford, 1980. Signatures of mixing from the Bermuda Slope, the Sargasso Sea and the Gulf Stream. Journal of Physical Oceanography, 10, 105--127.

Gregg, M. C., and T. B. Sanford, Shear and turbulence in thermohaline staircases, Deep-Sea Res., 34, No.1

OA.,16981696,1987.

Gregg, M. C., and T. Sanford, 1987. Shear and turbulence in a thermohaline staircase. Deep-Sea Research, 34(10), $1689--1696$.

Gregg, M. C., C. S. Cox, and P. W. Hacker, 1973. Vertical microstructure measurements in the central North pacific. Journal of Physical Oceanography, 3, 458--469.

Gregg, M. C., E. A. D'Asaro, T. J. Shay, and N. Larson, Observations of persistent mixing and near-inertial internal waves, Phys. Oceanogr., 16, 856-885, 1986.

Gregg, M. G., and J. H. McKenzie, Thermohaline instrusions lie across isopycnals, Nature, 280, 310-311, 1979.

Gregg, M. G., Small-scale mixing: a first-order process?

Gregg, M.C., and T.B. Sanford, Shear and turbulence in thermohaline staircases, Deep-Sea Res., 34, 1689-1696, 1987.

Gregg, M.C., Scaling turbulent dissipation in the thermocline, J. Geophys. Res., 94, 9686-9698, 1989.

Gregg, M.C., The three-dimensional mapping of a small thermohaline intrusion. J. Phys. Oceanogr., 10, 1468-1492, 1980.

Gresho, P. M., and Sani, R. L., The stability of a fluid layer subjected to a step change in temperature: transient vs. frozen

time analysis, Int. J. Heat Mass Transfer, 14, 207-221, 1971.

Griffiths, R. W., 1979. A note on the formation of 'salt-finger' and 'diffusive' interfaces in three-component systems.

International Journal of Heat and Mass Transfer, 22, 1687--1693.

Griffiths, R. W., 1979. The influence of a third diffusing component upon the onset of convection. Journal of Fluid

Mechanics, 92, 659--670.

Griffiths, R. W., 1979. The transport of multiple components through thermohaline diffusive interfaces. Deep-Sea Research, 26a, 383--397.

Griffiths, R. W., 1979. Transports through thermohaline interfaces in a viscous fluid and a porous medium. Ph.D.

Dissertation, Australian National University, Canberra.

Griffiths, R. W., 1981. Layered double-diffusive convection in porous media. Journal of Fluid Mechanics, 102, 221--248.

Griffiths, R. W., and B. R. Ruddick, 1980. Accurate fluxes across a salt-sugar finger interface deduced from direct density measurements. Journal of Fluid Mechanics, 99, 85--95.

Groves, G. W., 1959. Flow estimate for the perpetual salt fountain. Deep-Sea Research, 5, 209--214.

Guckenheimer, J. and Holmes, P., Nonlinear Oscillations, Dynamical Systems and Bifurcations of Vector Fields, Springer, New York, 1983.

Gupta, P. K., and Cooper, A. R. Jr., The [D] matrix for multicomponent diffusion, Physica, 54, 39-59, 1971.

Haenel, R., A Critical Review of Heat Flow Measurements in Sea and Lake Bottom Sediments, In: V. Cermak and L. Rybach (editors), Terrestrial Heat Flow in Europe, Springer-Verlag, Berlin, 1979.

Hamilton, J. M., M. R. Lewis, and B. R. Ruddick, 1989. Vertical fluxes of nitrate associated with salt fingers in the world's oceans. Journal of Geophysical Research, 94(C2), 2137--2145.

Hamilton, J. M., N. S. Oakey, and D. E. Kelley, 1993. Salt finger signatures in microstructure measurements. Journal of Geophysical Research, 98,(C2), 2453--2460.

Hansen, U. and D.A. Yuen, Dynamical influences from thermal chemical instabilities at the core-mantle boundary, Geophys.

Res. Letr., 16, 629-632, 1989a.

Hansen, U. and D.A. Yuen, Effects of depth-dependent thermal expansivity on the interaction of thermal-chemical plumes with a compositional boundary, Phys. Earth Planet. Inter., 86, 205-221, 1994.

Hansen, U. and D.A. Yuen, Evolutionary structures in double diffusive convection in magma chambers, Geophys. Res. Letr., 14, 1099-1102, 1987.

Hansen, U. and D.A. Yuen, Nonlinear physics of double-diffusive convection in geological systems, Earth Science Rev. 29, 385-399, 1990.

Hansen, U. and D.A. Yuen, Numerical simulations of thermal chemical instabilities and lateral heterogeneities at the core mantle boundary, Nature,334, 237-240, 1988.

Hansen, U. and D.A. Yuen, Subcritical double-diffusive convection at infinite Prandtl number, Geophys. Astrophys. Fluid Dyn., 47, 199-224, 1989 b.

Hansen, U. and D.A.Yuen, Potential role played by viscous heating in thermal-chemical convection in the outer core, submitted to Geochimica Cosmochitna Acta, 1995.

Hansen, U. and D. A. Yuen, 1996. Formation of Layered Structures in Double-Diffusive Convection as Applied to the Geosciences. In Double-Diffusive Convection A. Brandt and H.J.S. Fernando (eds), pp. 135-149, AGU, 1996.

Hansen, U., Yuen, D.A. and A.V. Malevsky, A comparison of steady-state and strongly chaotic thermal convection at high Rayleigh number, Phys. Rev., A46, 4742-4755,1992.

Hansen, U., Yuen, D.A., Kroening S.E. and T.B. Larsen, Dynamical consequences of depth-dependent thermal expansivity and viscosity on mantle circulations and thermal structure, Phys. Earth. Planet. Inter., 77, 205-213, 1993. 
Hansen,U., Zur Zeitabhaengigkeit der thermischen Konvektion im Erdmantel und der doppelt- diffusiven Konvektion in Magmakammern Mitteilungen aus dem Institut fuer Geophysik und Meteorologie, Koeln, 52, 155p, 1987.

Hart, J. E., 1970. Thermal convection between sloping parallel boundaries. Ph.D. Dissertation, Massachusetts Institute of Technology, Cambridge, MA.

Hart, J. E., 1971. A possible mechanism for boundary layer mixing and layer formation in a stratified fluid. Journal of Physical Oceanography, 1, 258--262.

Hart, J. E., 1971. On sideways diffusive instability. Journal of Fluid Mechanics, 49, 279--288.

Hart, J. E., 1971. Stability of the flow in a differentially heated inclined box. Journal of Fluid Mechanics, 47, 547--576.

Hart, J. E., 1973. Finite amplitude sideways diffusive convection. Journal of Fluid Mechanics, 59, 47--64.

Hart, J.E., Finite amplitude sideways diffusive convection. J. Fluid Mech. 59, 47-64, 1973.

Hay, B. J., Arthur, M. A., Dean, W. E., Neff, E. D., and S. Honjo, Sediment Deposition in the Late Holocene Abyssal Black

Sea with Climatic and Chronological Implications, Deep Sea Research, 38, Suppl. 2, S1211-S1236, 1991.

Hay, B. J., Parlicle Flux in the Western Black Sea in the Present and over the Last 5000 Years: Temporal Variability, Sources, Transport Mechanism, Ph.D. Thesis, Joint Program M.I.T./Woods Hole Oceanographic Institution, 202 pp., 1987.

Hayes, S. P., 1975. Preliminary measurements of the time-lagged coherence of vertical temperature profiles. Journal Geophysical Research, 80, 307--311.

Hayes, S. P., T. M. Joyce, and R. C. Millard, Jr., 1975. Measurements of vertical fine structure in the Sargasso sea. Journal of Geophysical Research, 80, 314--319.

Hebert, D., 1988. Estimates of salt-finger fluxes. Deep-Sea Research, 35, 1887--1901.

Hebert, D., N. Oakey and B. R. Ruddick, 1990. Evolution of a Mediterranean salt lens. Journal of Physical Oceanography, 20, 1468--83.

Hecht, A., N. Pinardi, A. R. Robinson, Currents, water masses, eddies and jets in the Mediterranean levantine basin. J. Phys. Ocean. $18,1320-1353,1988$.

Heinrich, J. C., 1984. A finite element model for double diffusive convection. International Journal of Numerical Methods

Engineering, 20, 447--464.

Hendricks, P. J., 1977. Finestructure and turbulence in the deep ocean. Ph.D. Dissertation. WHOI/MrT Joint Program in Ocean Engineering, Woods Hole, MA.

Herman, A. W., and K. L. Denman, 1979. Intrusions and vertical mixing at the shelf/slope water front south of Nova Scotia. Journal of the Fisheries Research Board of Canada, 36, 1445--1453.

Herring, J. R., 1963. Investigation of problems in thermal convection. Journal of the Atmospheric Sciences, 20, $325--338$.

Herring, J. R., 1964. Investigation of problems in thermal convection: rigid boundaries. Journal of the Atmospheric Sciences, $21,277--290$.

Hildreth, W., Gradients in silicic magma chambers: implications for lithospheric magmatism. J. Geophys. Res., 86, 10153-10192, 1981.

Hoare, R. A., 1966. Problems of heat transfer in Lake Vanda, a density stratified Antarctic lake. Nature, $210,787--789$.

Hoare, R. A., 1968. Thermohaline convection in Lake Vanda, Antarctica. Journal of Geophysical Research, 73, $607--612$.

Holloway, G., and A. Gargett, 1987. The inference of salt fingering from towed microstructure observations. Journal of Geophysical Research, 11, 1963--1965.

Holyer, J. Y., 1981. On the collective instability of salt fingers. Journal of Fluid Mechanics, 110, $195--208$.

Holyer, J. Y., 1983. Double-diffusive interleaving due to horizontal gradients. Journal of Fluid Mechanics, 137, $347--362$.

Holyer, J. Y., 1984. The stability of long, steady two-dimensional salt fingers. Journal of Fluid Mechanics, 147, $169--185$.

Holyer, J. Y., 1985. The stability of long steady three-dimensional salt fingers to long wavelength perturbations. Journal of Fluid Mechanics, 156, 495--503.

Holyer, J. Y., T. J. Jones, M. G. Priestly, and N. J. Williams, 1987. The effect of vertical temperature and salinity gradients on double-diffusive interleaving. Deep-Sea Research, 34, 517--530.

Horne, E. P. W., 1985. Ice-induced vertical circulation in an Arctic Fiord. Journal of Geophysical Research, 90, $1078--1086$. Horne, E.P.W., 1978. Interleaving at the subsurface front in the slope water off Nova Scotia. Journal of Geophysical Research, 83, 3659--3671.

Horne, E.P.W., and J. M. Toole, 1980. Sensor response mismatches and lag correction techniques for temperature-salinity profilers. Journal of Physical Oceanography, 10, 1122--1130.

Howard, J. N., Eleanor Mildred Sidgwick and the Rayleighs. Applied Optics, 3 (10), 1120-1122, $1964 \mathrm{~b}$.

Howard, J. N., The Rayleigh Notebooks. Applied Optics, 3 (10), 1129-1133, 1964a.

Howard, L. N., and G. Veronis, Stability of salt fingers with negligible salt diffusivity, J. Fluid Mech., $239,511-522,1992$.

Howard, L. N., and G. Veronis, The salt-finger zone, J. Fluid Mech., 183, 1-23, 1987.

Howard, L.N. and Krishnamurti, R., Large-scale flow in turbulent convection: a mathematical model, J. FIuid Mech., 170, 385-410, 1986.

Howard, L.N., Convection at high Rayleigh number, in Proc. 11th Int. Cong. Appl. Mech., Springer Verlag, Berlin, pp. 1109-1115, 1964. 
Howe, M. R., and R. I. Tait, 1970. Further observations of thermohaline stratification in the deep ocean. Deep-Sea Research, 17, 963--972.

Howe, M. R., and R. I. Tait, 1972. The role of temperature inversions in the mixing processes of the deep ocean. Deep-Sea Research, 19, 781--791.

Hsu, Y. S., 1974. Double diffusive instabilities with and without a weak vertical shear. Ph.D. Dissertation, Harvard University, Cambridge, MA.

Hughes, D.W. and Proctor, M.R.E., A low-order model of the shear instability of convection: chaos and the effect of noise, Nonlinearity, 5. 127-153, 1990.

Hughes, G.O. and R. I. Nokes, "Wave-Convection Coupling" in Multicomponent Convention: An Experimental Study. In Double-Diffusive Convection A. Brandt and H.J.S. Fernando (eds), pp. 49-56, AGU, 1996.

Hunt, J.C.R., Turbulence structure in thermal convection and shear-free boundary layers. J. Fluid Mech. 138, 161-184, 1984. Huppert, H. E. and R. S. J. Sparks 1984. Double-diffusive convection due to crystallization in magmas. Annual Reviews of Earth and Planetary Science, 12, 11--37.

Huppert, H. E., 1971. On the stability of a series of double diffusive layers. Deep-Sea Research, 18, 1005--1021.

Huppert, H. E., 1976. Transitions in double-diffusive convection. Nature, 263, 20--22.

Huppert, H. E., 1983. Multicomponent convection: turbulence in earth, sun and sea. Nature, 303, 478--479.

Huppert, H. E., and D. R. Moore, 1976. Nonlinear double-diffusive convection. Journal of Fluid Mechanics, 78, 821--854. Huppert, H. E., and E. G. Josberger, 1980. The melting of ice in cold stratified water. Journal of Physical Oceanography, 10, 953--960.

Huppert, H. E., and J. S. Turner, 1972. Double-diffusive convection and its implications for the temperature and salinity structure of the ocean and Lake Vanda. Journal of Physical Oceanography, 2, 456--461.

Huppert, H. E., and J. S. Turner, 1978. On melting icebergs. Nature, 271, 46--48.

Huppert, H. E., and J. S. Turner, 1980. Ice blocks melting into a salinity gradient. Journal of Fluid Mechanics, 100, 367--384.

Huppert, H. E., and J. S. Turner, 1981. Double diffusive convection. Journal of Fluid Mechanics, 106, 299--329.

Huppert, H. E., and P. C. Manins, 1973. Limiting conditions for salt fingering at an interface. Deep-Sea Research, 20, 315--323.

Huppert, H. E., and P. F. Linden, 1979. On heating a stable salinity gradient from below. Journal of Fluid Mechanics, 95 , 431--464.

Huppert, H. E., R. C. Kerr, and M. A. Hallworth, 1984. Heating or cooling a stable compositional gradient from side. Intemational Journal of Heat and Mass Transfer.

Huppert, H.E. and D.R.Moore, Non-lõnear double-diffusive convection., J. Fluid. Mech., 78, 821-854, 1976

Huppert, H.E. and M.G. Worster, Vigorous motions in magma chambers, Chaotic Processes in the Geological Sciences edited by D.A.Yuen, pp 141-173, Springer Verlag, New York, 1992.

Huppert, H.E., and J.S. Turner, A laboratory model of a replenished magma chamber. Earth Planet. Sci. Lett., 54, 144-172, 1981.

Huppert, H.E., and P. F. Linden, 1976. The spectral signature of salt fingers. Deep-Sea Research, 23, 909--914.

Huppert, H.E., J.S. Turner, and R.S.J. Sparks, Replenished magma chambers: effects of compositional zonation and input rates. Earth Planet. Sci. Lett., 57, 345-357, 1982.

Huppert, H.E., Manins, P.C., Limiting conditions for salt-fingering at an interface, Deep Sea Res., 20, 315-323 (1973).

Huppert, H.E., R.S.J. Sparks, and J.S. Turner, Laboratory investigations of viscous effects in replenished magma chambers.

Earth Planet. Sci. Lett., 65, 377-381, 1983.

Huppert, H.E., R.S.J. Sparks, The fluid dynamics of a basaltic magma chamber replenished by influx of hot, dense ultrabasic magma., Contrib. Mineral. Petrol., 75, 279-289, 1980

Huppert, H.E., The intrusion of fluid mechanics into geology. J. Fluid Mech ,173, 557-594, 1986.

Hurle, D.T.J., and E. Jakeman, 1971. Soret-driven thermosolutal convection. Journal of Fluid Mechanics, 47, 667--687.

Hyun, J. M., and H. S. Kwak, 1989. Flow of a double-diffusive stratified fluid in a differentially-rotating cylinder.

Geophysical and Astrophysical Fluid Dynamics, 46, 203--220.

Hyun, M.T. and T.L. Bergman, Direct simulation of double-diffusive layered convection, J. Heat Transfer, 117, 334-339, 1995.

Ida, Y., Slow moving deformation pulses along tectonic faults, Phys. Earth Planet. Inter., 9, 328-337, 1974.

Imhoff, P.T. and T. Green, Experimental investigation of double-diffusive groundwater fingers, J. Fluid Mech., 188, 363-382, 1988.

Ingham, M. C., 1966. The salinity extrema of the world ocean. Ph.D. Dissertation, Oregon State University, Corvallis, OR. Inoue, T. and M. V. White, Bibliography of published works by W. S. Jevons. Journal of the History of Economic Thought, $15,122-147,1993$

Iooss, G., Bifurcation and transition to turbulence in hydrodynamics, Bifurcation Theory and Applications, (L. Salvadori, ed.), Lecture Notes in Mathematics, Vol. 1057, Springer-Verlag, New York, 152-201, 1984.

Iooss, G., Existence et stabilite de la solution periodique secondaire intervenant dans les problemes d'evolution du type 
Navier-Stokes, Arch. Rational Mech. Anal., 47, 301-329, 1972.

Irvine. T.N., Magmatic infiltration metasomatism, double-diffusive fractional crystallization and adcumulus growth in the Muskox intrusion and other layered intrusions. In Physics of Magnetic Processes, ed. by R.B. Hargraves, Princeton Univ. Press, 585pp, 1980.

Ishii, M., Thermo-fluid Dynamic Theory of two-phase flow Paris: Eyrolles, 1975

Iudovich, V.I., On the origin of convection, J. Appl. Math. Mech., 30, 1193-1199, 1969.

Ivey, G. N., and G. M. Corcos, Boundary Mixing in a Stratified Fluid, J. Fluid Mech., 12I, 1-26, 1982.

Izdar, E., Konuk, T., Ittekott, V., Kempe, S., and E. T. Degens, Particle Flux in the Black Sea: Nature of Organic Matter in the Shelf Waters of the Black Sea, In: Particle Fluz in the Ocean, E. T. Degens, E. Izdar and S. Honjo (editors), Mitt.

Geol.-Paleont. Inst. Univ. Hamburg, SCOPE/UNEP Sonderband, 62, 1-18, 1987.

Jackett, D. R., and T. J. McDougall, 1985. An oceanographic variable for the characterizations of intrusions and water mass. Deep-Sea Research, 32, 1195--1207.

Jacobs, S. S., H. E. Huppert, G. Holdsworth, and D. J. Drewry, 1981. Thermohaline steps induced by melting of the Erebus Glacier tongue. Journal of Geophysical Research, 86, 6547--6555.

Jarvis, G.T. and D.P. McKenzie, Convection in a compressible fluid with infinite Prandtl number, J. Fluid Mech., 96 , 515-583, 1980.

Jaupart, C. and G. Brandeis, 1986. The stagnant bottom layer of convecting magma chambers, Earth Planet. Sci. Letr., 80, 183-199.

Jeevaraj, C. G., and J. Imberger, Experimental Study of Double Diffusive Instability in Sidewall Heating, J. Fluid Mech., $222,565-586,1991$.

Jevons, W. S., 1857. On the cirrous form of cloud. The London,Edinburgh, and Dublin Philosophical Magazine and Journal of Science,Series 4, 14(90), 22--35.

Jevons, W. S., 1858. On clouds; their various forms, and producing causes. Sydney Magazine of Science and Art, 1(8), $163-176$.

Jevons, W. S., 1858. On the forms of clouds. The London Edinburgh and Dublin Philosophical Magazine and Journal of Science, 15(100),241--255.

Jevons, W. S., On the cirrous form of cloud. London, Edinburgh and Dublin Philosophical Magazine and Journal of Science, 4th Series, 14, 22-35, 1857.

Jhaveri, B. S., and Homsy, G. M., The onset of convection in fluid layers heated rapidly in a time-dependent manner, J. Fluid Mech., 114, 251-260, 1982.

Jimenez, J., M. Cogollos, and L. P. Bemal, A perspective view of the plane mixing layer, J. Fluid Mech., 152, 125-143, 1985. Johannessen, O. M., and O. S. Lee, 1974. Thermohaline staircase structure in the Tyrrhenian Sea. Deep-Sea Research, 21, 629-639.

Johnson, C. L., C. S. Cox, and B. Gallagher, 1978. The separation of wave-induced and intrusive oceanic microstructure. Journal of Physical Oceanography, 8, 846--860.

Jones, C. A., and Moore, D. R., The stability of axisymmetric convection, Geophys. Astrophys. Fluid Dynamics, 11 , 245-270, 1979.

Jones, C. A., Moore, D. R., and Weiss, N. O., Axisymmetric convection in a cylinder, J. Fluid. Mech., 73, 353-388, 1976. Jorne, J., The effect of ionic migration on oscillations and pattern formation in chemical systems, J. Theor. Biol., 43, 375-380, 1974.

Josberger, E. G., and S. Martin, 1981. Convection generated by vertical icewalls. Journal of Fluid Mechanics, 111, $439--473$. Joseph, D. D., Stability of convection in containers of arbitrary shape, J. Fluid Mech., 47, 257-82, 1971.

Joseph, D.D., Stability of Fluid Motions, Springer Tracts in Natural Philosophy, 28, Springer-Verlag, NewYork, 1976. Joyce, T. M., 1976. Large-scale variations in small-scale temperature/salinity fine-structure in the main thermocline of the northwest Atlantic. Deep-Sea Research, 23, 1175--1186.

Joyce, T. M., 1977. A note on the lateral mixing of water masses. Journal of Physical Oceanography, 7, 626--629. Joyce, T. M., W. Zenk, and J. M. Toole, 1978. The anatomy of the Antarctic Polar Front in the Drake Passage. Journal of Geophysical Research, 83, 6093--6113.

Joyce, T.M., 1982. Marginally unstable salt fingers: limits to growth. Journal of Marine Research, 40(Suppl.), $291--306$. Kamakura, K. and H. Ozoe, Experimental and numerical analyses of double diffusive natural convection heated and cooled from opposing vertical walls with an initial condition of a vertically linear concentration gradient, Int. J. Heat Mass Transfer, 36, 2125-2134, 1993.

Kamakura, K. and Ozoe, H., Effect of the temperature dependence of fluid properties on the migration of an interface in double-diffusive natural convection. Int. J. Heat Mass Transfer, 38, 3413-3421, 1995.

Kamotani, Y., L.W. Wang, S. Ostrach and H.D. Jiang, Experimental study of natural convection in shallow enclosures with horizontal temperature and concentration gradients, Int. J. Heat Mass Transfer, 28, 165-173, 1985.

Kanamori, H., The nature of seismicity patterns before earthquakes, In Earthquake Prediction and International Review, edited by D. W. Simpson and P. G. Richards, American Geophysical Union, Washington D. C., 1-19, 1981. 
Karlin L. N., Kluikov Ye. Yu., Kut'ko V. P., Small-scale structure of hydrophysical fields of the ocean upper layer, 162 pp., Gydrometeoizdat Publishers, Moscow, 1988.

Kase, R. H., A. Beckmann, and H.-H. Hinrichsen, 1989. Observational evidence of salt lens formation in the Iberian Basin. Journal of Geophysical Research, 94(C4), 4905--4912.

Kaviany, M., 1984. Effect of a stabilizing solute gradient on the onset of thermal convection. Physics of Fluids, 27, $1108--1113$.

Kawase, M., and J. L. Sarmiento, 1986. Circulation and nutrients in mid-depth Atlantic waters. Journal of Geophysical Research, 91(C8), 9749--9770.

Kazmierczak, M. and D. Poulikakos, Numerical simulation of transient double diffusion in a composite porous/fluid layer heated from below, AIChE Symp. Ser.269, 108-114, 1989.

Kazmierczak, M. and D. Poulikakos, Transient double diffusive convection in a horizontal fluid layer situated on top of a porous substrate, Convective Mass Transfer in Porous Media, Eds. S. Kakac, B. Kilkis, F.A. Kulacki and F. Arinc, Kluwer Academic, Dordrecht, 655-672, 1991.

Kelley, D. E., 1984. Effective diffusivities within ocean thermohaline staircases. Journal of Geophysical Research, 89, 10484-10488.

Kelley, D. E., 1986. Oceanic Thermohaline Staircases. Ph.D. Dissertation, Dalhousie University, Halifax, Nova Scotia. Kelley, D. E., 1987. Interface migration in thermohaline staircases. Journal of Physical Oceanography, 17, 1633--1639.

Kelley, D. E., 1988. Explaining effective diffusivities within diffusive staircases. In: Small-Scale Turbulence and Mixing in the Ocean, J.Nihoul and B.Jamart, editors, Elsevier Oceanography Series, 46, Elsevier, New York, pp.481--502.

Kelley, D. E., 1990. Fluxes through diffusive staircases: A new formulation. Journal of Geophysical Research, 95, 3365--3371.

Kellogg, L. H., Interaction of plumes with a compositional boundary at $670 \mathrm{~km}$, Geophys. Res. Lett.. 1 S. 865-868. 1991. Kellogg. L.H. and S.D. King. Effects of mantle plumes on the growth of $D "$ by reaction between the core and mantle, Geophy. Res. Lett., 20, 379-382, 1993.

Kelly, D. 1987. The influence of planetary rotation on oceanic double-diffusive system. Journal Marine Research, 45 , 829--841.

Kempe, S., Diercks, A. R., Liebezeit, G., and A. Prange, Geochemical and Structural Aspects of the Pycnocline in the Black Sea (R/V Knorr 134-8 Leg 1, 1988), In: The Black Sea Oceanography, E. Izdar and J. M. Murray (editors), NATO/ASI Series, Dordrecht, Kluwer Academic Publishers, 89-110, 1991.

Kerpel, J., J. Tanny, and A. Tsinober, On the Secondary Layers in a Stable Solute Gradient Heated from Below, Fluid Dyn. Res., 10, 141-147, 1992.

Kerr, O. S., and J. Y. Holyer, 1986. The effect of rotation on double-diffusive interleaving. Journal of Fluid Mechanics, 162, 23-33.

Kerr, O. S., Heating a salinity gradient from a vertical sidewall: linear theory, J. Fluid Mech., 207, 323-352, 1989.

Kerr, O. S., Heating a salinity gradient from a vertical sidewall: nonlinear theory, J. Fluid Mech., 217, 529-546, 1990.

Kerr, O.S, Two-dimensional instabilities of steady double diffusive interleaving. J. Fluid Mech. 242, 99-116, 1992a.

Kerr, O.S. Horizontal effects in double-diffusive convection. Ph.D., Thesis 230 pp., University of Bristol, U. K., 1987.

Kerr, O.S., Double-diffusive instabilities at a sloping boundary. J. Fluid Mech. 225, 333-354, 1991.

Kerr, O.S., 1992. On the merging of double-diffusive convection cells at a vertical boundary. Phys. Fluids A 4, 2923-2926, 1992

Kerr, O.S. 1996. Double Diffusive Instabilities at a Vertical Boundary In Double-Diffusive Convection A. Brandt and H.J.S. Fernando (eds), AGU pp. 105-113, AGU, 1996.

Kerr, R. A., 1981. Fingers of salt help mix the sea. Science, 211, 155--157.

Kerr, R.C., and J.S. Turner, Layered convection and crystal layers in multicomponent systems. Nature, 298, 731-733, 1982. Keynes, J. M., William Stanley Jevons. in: Essays in Biography, (2nd edition), Rupert Hart-Davis, London. pp 255-309, 1951.

Kirkaldy, J.S. and Young, D. J., Diffusion in Condensed Sfare, The Institute of Metals, London 1987.

Kluikov, Ye. Yu. and L. N. Karlin, A Model of the Ocean Thermocline Stepwise Stratification Caused by Double Diffusion. In Double-Diffusive Convection A. Brandt and H.J.S. Fernando (eds), pp. 287-292, AGU, 1996.

Knightly, G.H., and D. Sather, Continua of periodic waves in rotating plane Couette flow, Euro. J. Mech. Fluids, 13, 511-526, 1994.

Knightly, G.H., and D. Sather, Langmuir circulations when the Stokes drift has a cross-wind component, Euro. J. Mech. Fluids, in press, 1995.

Knightly, G.H., and D. Sather, Periodic waves in rotating plane Couette flow, Z. anger Math. Phys., 44, 1-16, 1993.

Knightly, G.H. and Sather, D. Wind-Driven Double-Diffusive Convection in Ocean Surface Layers. In Double-Diffusive Convection A. Brandt and H.J.S. Fernando (eds), pp. 293-303, AGU, 1996.

Knobloch, E. 1986. Oscillatory convection in binary mixtures. Physical Review A, 34, 1538--1549.

Knobloch, E. and D.R. Moore, 1988 Linear stability of experimental Soret convection. Physical Review A 37, 860-870. 
Knobloch, E. et al. 1986. Transitions to chaos in two-dimensional double-diffusive convection. Journal of Fluid Mechanics, $166,409--448$.

Knobloch, E., and M.R.E. Proctor, 1981. Nonlinear periodic convection in double-diffusive systems. Journal of Fluid Mechanics, 108, 291--316.

Knobloch, E., and N. O. Weiss 1981: Bifurcations in a model of double diffusive convection. Phys. Letters, 85a, $127--130$.

Knobloch, E., Weiss, N.O. and Da Costa, L.N., 1981. Oscillatory and steady convection in a magnetic field, J. Fluid Mech., $113,153-186$.

Knobloch, E., M.R.E. Proctor and N.O. Weiss, Finite-dimensional description of doubly diffusive convection. In Turbulence in Fluid Flows: A Dynamical Systems Approach, G.R. Sell, C. Foias and R. Temam (eds), Springer (1993), pp. $59--72$.

Koyagushi, T., M. A. Hallworth, H . E. Huppert, An experimental study on the effects of phenocrysts on convection in magmas. Journ. of Volcanology and Geothermal Research, 55, pp 15-32, 1993

Koyagushi, T., M.A. Hallworth, H.E. Huppert, R.S.J. Sparks, Sedimentation of particles in convecting fluid, Nature, 343, 447-450, 1990

Kranenborg, E. J., Dijkstra, H. A., The structure of (linearly) stable double diffusive flow patterns in a laterally heated stratified liquid, Phys. Fluids A, 7, 680-682, 1995.

Kranenborg, E.J. and H. A. Dijkstra, 1996. Double Diffusive Flow Patterns in the Unicellar Flow Regime: Attractor Structure and Flow Development In Double-Diffusive Convection A. Brandt and H.J.S. Fernando (eds), pp. 89-96, AGU, 1996.

Krishnamurti, R. and Howard, L.N., Large-scale flow generation in turbulent convection, Proc. Nat. Acad. Sci. U.S.A., 78, 1981-1985, 1981.

Krishnamurti, R. and L. N. Howard, 1983. Double-diffusive instability: Measurement of heat and salt fluxes. Bulletin American Physical Society, 28, 1398--1398.

Krom, M. D., S. Bremier, N. Kress, A. Neori, L. I. Gordon, Nutrient dynamics and new production in a warm-core eddy from the Eastern Mediterranean Sea. Deep Sea Res., 39, 467480.

Kunze E., 1987. Limits on growing, finite length salt fingers: a Richardson number constraint. Journal of Marine Research, 45, 533--556.

Kunze, E., 1990. The evolution of salt fingers in inertial wave shear. Journal of Marine Research, 48, $471--504$.

Kunze, E., 1994. A proposed flux constraint for salt fingers in shear. J. Mar. Res., 52, 999-1016.

Kunze, E. Quantifying Salt-Fingering Fluxes in the Ocean. In Double-Diffusive Convection A. Brandt and H.J.S. Fernando (eds), pp. 313-320, AGU, 1995.

Kunze, E., A. J. Williams III, and R. W. Schmitt, 1987. Optical microstructure in the thermohaline staircase east of Barbados. Deep-Sea Research, 34(10), 1697--1704.

Kunze, E., A.J. Williams III, and M.G. Briscoe, Observations of shear and vertical stability from a neutrally-buoyant float, J. Geophys. Res., 95, 18,127- 18,142, 1990.

Lambert, R. B. 1982. Lateral mixing processes in the Gulf Stream. Journal Physical Oceanography, 12, 851--861.

Lambert, R. B., 1971. Experimental studies of stratified layers. In: Proceedings of the Joint Oceanographic Assembly, Tokyo, $1970,199--200$.

Lambert, R. B., and J. W. Demenkow, 1972. On the vertical transport due to fingers in double diffusive convection. Journal of Fluid Mechanics, 54, 627--640.

Lambert, R. B., and W. Sturges, 1977. A thermohaline staircase and vertical mixing in the thermocline. Deep-Sea Research, 24, 211--222.

Landau, L., E.M. Lifschitz, Lehrbuch der theoretischen PhysiL, Band VI, Akademie Verlag Berlin 1991

Landsberg, A.S. and Knobloch, E., Direction-reversing traveling waves, Phys. Lett., 159A, 17-20, 1991.

Lange, R. A., and Carmichael, I. S. E., Density of Na2O-K2OCaOMgO-FeO-

Fe203-AI203-TiO2-SiO2 liquids: New measurements and derived partial molar properties, Geochim. Cosmochim. Acta, 51, 2931-2946, 1987.

Lantz, S.R., Magnetoconvection dynamics in a stratified layer. II. A low-order model of the tilting instability, Astrophys. J., in press, 1995.

Larson, N.G., and M. C. Gregg, 1983. Turbulent dissipation and shear in thermohaline intrusions. Nature, 306(5938), 26--32. Lasheras, J. C., J. S. Cho, and T. Maxworthy, On the orgin and evolution of streamwise vertical structures in a plane, free shear layer, J. Fluid Mech., 172, 231-258, 1986.

Latif, M. A., Ozsoy, E., Oguz, T., and U. Unluata, Observations of the

Mediterranean inflow into the Black Sea, Deep Sea Research, 38, Suppl. 2, S711-S723, 1991.

Ledwell, J. R. and A. J. Watson, 1988. The use of deliberately injected tracers for the study of diapycnal mixing in the ocean. In: Small-Scale Turbulence and Mixing in the Ocean, J. Nihoul and B. Jamart, editors, Elsevier Oceanography Series, 46, Elsevier, New York, pp.11--20.

Ledwell, J.R., A.J. Watson, and C.S. Law, Evidence of slow mixing across the pycnocline from an open-ocean tracer release experiment, Nature, 364, 701-703, 1993. 
Lee, J. H., and G. Veronis, 1991. On the difference between tracer and geostrophic velocities obtained from C-SALT data. Deep-Sea Research, 38(5A), 555-568.

Lee, J. W. \& Hyun, J. M., Double diffusive convection in a cavity under a vertical solutal gradient and a horizontal temperature gradient, Int. J. Heat Mass Transfer, 34, 2423-2427, 1991 a.

Lee, J. W. \& Hyun, J. M., Time-dependent double diffusion in a stably stratified fluid under lateral heating, Int. J. Heat Mass Transfer, 34, 2409-2421, 1991b.

Lee, J.W. and Hyun, J.M., Heating or cooling a stable compositional gradient from the side. Int. J. Heat Mass Transfer, 2409-2421, 1991.

Lee, S. I., 1983. Density structure associated with salt fingers. Master's Thesis, Naval Postgraduate School, Monterey, CA.

Leibovich, S., Convective instability of stably stratified water in the ocean, J. Fluid Mech., 82, 561-581, 1977b.

Leibovich, S., Dynamics of Langmuir circulations in a stratified ocean, The Ocean Surface, (Y. Toba and H. Mitsuyasu, eds.), Reidel, 457464, 1985.

Leibovich, S., On the evolution of the system of wind drift currents and

Langmuir circulations in the ocean. Part I. Theory and averaged current, J. Fluid Mech., 79, 715-743, 1977a.

Leibovich, S., S. K. Lele, and I. M. Moroz, 1989. Nonlinear dynamics in Langmuir circulations and in thermosolutal convection. Journal of Fluid Mechanics, 198, 471--511.

Leibovich, S., The form and dynamics of Langmuir circulations, Ann. Rev. Fluid Mech., 15, 391-427, 1983.

Lewis, D. J., 1950. The instability of liquid surfaces when accelerated in a direction perpendicular to their planes. II. Proc. Roy. Soc., A202, 81--96.

Lewis, W. T., F. P. Incropera, and R. Viskanta, 1982. Interferometric study of stable salinity gradients heated from below or cooled from above. Journal of Fluid Mechanics, 116, 411--430.

Lezovatsky, I. D., and M-J. H. Lilover, Spectral structure of thermohaline inhomogeneities of cold core cyclonic ring in the ocean, Doklady USSR Acad of Sci, 291, 703-708, 1986 (in Russian).

Liang, S. F., Vidal, A. and Acrivos, A., Buoyancy-driven convection in cylindrical geometries. J. Fluid Mech., 36, 239-256, 1969.

Liang, Y., Models and experiments for multicomponent chemical diffusion in molten silicates, Ph.D. dissertation, The University of Chicago, 1994.

Liang, Y., Richter, F. M., and Watson, E. B., Convection in multicomponent silicate melts driven by coupled diffusion, Nature, 369, 390-392, 1994.

Liang, Y. 1996. Axisymmetric Double-Diffusive Convection in a Cylindrical Container: Linear Stability Analysis with Applications to Molten CaO-Al2O3-SiO2 In Double-Diffusive Convection A. Brandt and H.J.S. Fernando (eds), pp. 115-124, AGU, 1996.

Lillibridge, J. L., G. Hitchcock, T. Rossby, E. Lessard, M. Mark, and L. Golmer, 1990. Entrainment of mixing of shelf/slope waters in the near-surface Gulf Stream. Journal of Geophysical Research, 95(C8), 13,065-13,087.

Linden, P. F., 1971. Effect of turbulence and shear on salt fingers. Ph.D. Dissertation, University of Cambridge, Cambridge, U.K.

Linden, P. F., 1971. Salt fingers in the presence of grid-generated turbulence. Journal of Fluid Mechanics, 49, 611--624.

Linden, P. F., 1973. On the structure of salt fingers. Deep-Sea Research, 20, 325--340.

Linden, P. F., 1974. A note on the transport across a diffusive interface. Deep-Sea Research, 21, 283--287.

Linden, P. F., 1974. Salt fingers in a steady shear flow. Geophysical Fluid Dynamics, 6, 1--27.

Linden, P. F., 1976. The formation and destruction of fine-structure by double-diffusive processes. Deep-Sea Research, 23, 895--908.

Linden, P. F., 1978. The formation of banded salt finger structure. Journal of Geophysical Research, 83, 2902--2912.

Linden, P. F., and J. E. Weber, 1977. The formation of layers in a double diffusive system with a sloping boundary. Journal of Fluid Mechanics, 81, 757--773.

Linden, P. F., and T.G.F. Shirtcliffe, 1978. The diffusive interface in double-diffusive convection. Journal of Fluid

Mechanics, 87, 417--432.

Long, R.R., A theory of mixing in a linearly stratified fluid. J. Fluid Mech. 84, 113-124, 1978.

Lorenz, E.N., Deterministic nonperiodic flow, J. Atmos. Sci., 20, 130-141, 1963.

Lozovatsky, I.D., and V. N. Nabatov, Spectral structure of convective layers and high gradient sheets in the thermocline, Izsvestiya of USSR Acad. of Sci, ser. Atmosph. and Ocean Phys., 26, 412-420, 1990 (in Russian).

Lozovatsky, I.D. Sheet Splitting and Hierarchy of "Convective Plumes" in the North-Western Tropical Atlantic Salt Finger

Staircase. In Double-Diffusive Convection A. Brandt and H.J.S. Fernando (eds), AGU pp. 237-250, AGU, 1996.

Lueck, R., 1987. Microstructure measurements in a thermohaline staircase. Deep-Sea Research, 34(10), 1677--1688.

Lyons, T., Upper Holocene Sediments of the Black Dea: Summary of Leg 4 Box Cores (1988 Blacl; Sea Oceanographic

Expedition), in: The Black Sea Oceanography, E. Izdar and J. M. Murray (editors), NATO/ASI Series, Dordrecht, Kluwer

Academic Publishers, 401-441, 1991.

Lyubimov, D.V. and Zaks, M.A., Two mechanisms of the transition to chaos in finite-dimensional models of convection, 
Physica, 9D, 52-64, 1983.

Mack, S. A., 1985. Two-dimensional measurements of ocean microstructure: The role of double diffusion. Journal of Physical Oceanography, 15, 1581--1604.

Mack, S. A., 1989. Towed chain measurement of ocean microstructure. Journal of Physical Oceanography, 19(8), 1108-1129.

Mack, S. A., D. C. Wenstrand, J. Calman, and R. C. Burkhardt, 1982. Characteristics of thermal microstructure in the ocean. Johns Hopkins APL Technical Digest, 3, (1), 28--35.

Mack, S. A., Schoeberlein, H. C. 1993. Discriminating salt fingering from turbulence-induced microstructure: analysis of towed temperature-conductivity chain data. Journal of Physical Oceanography, 23(9), 2073-106.

Mack, S.A. and H.C. Schoberlein, 1996. Salt Fingering and Turbulence-Induced Microstructure Measured by a Towed Temperature-Conductivity Chain. In Double-Diffusive Convection A. Brandt and H.J.S. Fernando (eds), pp. 213-235, AGU, 1996.

Mackay, J. R., Lateral mixing of the Liard and Mackenzie rivers downstream from their confluence, Can. J. Ear. Sci., 7, 111-124, 1970.

Magnell, B., 1973. Oceanic microstructure observations near Bermuda using a towed sensor. Ph.D. Dissertation. WHOI/MIT Joint Program, Woods Hole, MA.

Magnell, B., 1976. Salt fingers observed in the Mediterranean outflow region ( 34 degrees N, 11 degrees W) using a towed sensor. Journal of Physical Oceanography, 6, 511--523.

Mamayev, O. I., Temperature - Salinity Analysis of World Ocean Waters, Elsevier, Amsterdam, 374 pp. 1975.

Manins, P.C., 1976. Intrusion into a stratified fluid. J. Fluid Mech., 74, 547-560.

Marmorine G. J., Caldwell D. R., 1976. Heat and salt transport through a diffusive thermohaline interface, Deep-Sea Res, 23, 59-67.

Marmorino, G. and Greenewalt, D. 1988. Inferring the nature of microstructure signals. Journal of Geophysical Research, 93, 1219--25.

Marmorino, G. O. 1987. Observations of small-scale mixing processes in the seasonal thermocline. Part I: Salt fingering. Journal of Physical Oceanography, 17, 1339--1347.

Marmorino, G. O., 1974. Equilibrium heat and salt transport through a diffusive thermohaline interface. Master's Thesis, Oregon State University, Corvallis, Oregon.

Marmorino, G. O., 1990. "Turbulent mixing'" in a salt-finger staircase. Journal of Geophysical Research, 95 , 12,983--12,994.

Marmorino, G. O., 1991. Intrusions and diffusive interfaces in a salt fingering staircase, Deep-Sea Research, 38, $1431--54$. Marmorino, G. O., and D. R. Caldwell, 1976. Heat and salt transport through a diffusive thermohaline interface. Deep-Sea Research, 23, 59--67.

Marmorino, G. O., and D. R. Caldwell, 1978. Temperature finestructure and microstructure observations in a coastal upwelling region during a period of variable winds (Oregon, summer 1974). Deep-Sea Research, 25, $1073-1106$.

Marmorino, G. O., L. J. Rosenblum, J. P. Dugan and C. V. Shen, 1985. Temperature finestructure patchiness near an upper ocean density front. Journal of Geophysical Research, 90, 11799--11810.

Marmorino, G. O., W. K. Brown, and W. D Morris, 1987. Two-dimensional temperature structure in the C-SALT

thermohaline staircase. Deep-Sea Research, 23, (10), 1667--1675.

Marmorino, G. O., W. K. Brown, and W. D. Morris, Two dimensional

temperature structure in the C-SALT thermohaline staircase, Deep-SeaRes., 34, No.1OA,1667-1676,1987.

Marmorino, G.O., 1989. Substructure of oceanic salt finger interfaces. Journal of Geophysical Research, 94(C4), 4891--4904.

Marsh, B.D., M.R.Maxey, On the distribution and the separation of crystals in convecting magma. J. Volcanol. Geotherm.

Res, 24, 95-150, 1985.

Marsh. B.D., 1989. Magma chambers, Ann. Rev. Earth Planet. Sci. 17, 439-474.

Martin, D., R.I. Nokes, 1989. A fluid dynamical study of crystal settlōng in convecting magmas. J. Petrology, 30, 1471-1500.

Martin, D., R.I. Nokes, 1988. Crystal settling in vigorously convecting magma

chambers. Nature, 332, 534-536.

Maslowe, S. A. 1985. Direct resonance in double-diffusive system. Studies in Applied Math., 73, $59--74$.

Masuda, A., 1978. Double diffusive convection in a rotating system. Joumal of the Oceanographic Society of Japan, 34, 8--16.

Matsui, E., F. Salati, I. Friedman, and W. L. F. Brinkman, Isotopic hydrology of the Amazonia, 2, Relative discharges of the Negro and Solimoes rivers through 180 concentrations, Water Resour. Res., 12, 781-785, 1976.

Matthews, P.C., Proctor, M.R.E., Rucklidge, A.M. and Weiss, N.O., Pulsating waves in nonlinear magnetoconvection, Phys. Lett. A, 183, 69-75, 1993.

Maxworthy, T., 1983. The dynamics of double-diffusive gravity currents. Journal of Fluid Mechanics, 128, $259--282$.

Mazeika, P. A., 1974. Subsurface mixed layers in the northwest tropical Atlantic. Journal of Physical Oceanography, 4, 446--453. 
McBirney, A.R., and R.M. Noyes, Crystallization and layering of the Skaergaard intrusion. J. Petrol., $20,487-554,1979$. McDougall ,T. J., Double- diffusive convection caused by coupled molecular diffusion, J. Fluid Mech. ,126, 379 - 397 (1983). McDougall, T. J, 1979. On the elimination of refractive-index variations in turbulent density-stratified liquid flows. Journal Fluid Mechanics, 93, 83--96.

McDougall, T. J. 1987. Neutral surfaces. Journal of Physical Oceanography, 17, 1950--1964.

McDougall, T. J. 1987. Thermobaricity, cabbeling, and water-mass conversion. Journal of Geophysical Research, 92 , $5448--5464$.

McDougall, T. J. and A. B. Giles 1987. Migration of intrusions across isopycnals, with examples from the Tasman Sea. Deep-Sea Research, 34, 1851--1866.

McDougall, T. J. and B. R. Ruddick, 1992. The use of ocean microstructure quantify both turbulent mixing and salt fingering. Deep-Sea Research, 39, 1931--1952

McDougall, T. J., 1981. Double diffusive convection with a non-linear equation of state. Part I: The accurate conservation of properties in a 2-layer system. Progress in Oceanography, 10, 71--89.

McDougall, T. J., 1981. Double-diffusive convection with a nonlinear equation of state. II. Laboratory experiments and their interpretation. Progress of Oceanography, 10, 91--121.

McDougall, T. J., 1981. Fluxes of properties through a series of double-diffusive interfaces with a nonlinear equation of state. Journal of Physical Oceanography, 11, 1294--1299.

McDougall, T. J., 1983. Double-diffusive convection caused by coupled molecular diffusion. Journal of Fluid Mechanics, 126, 379--397.

McDougall, T. J., 1983. Double-diffusive plumes in unconfined and confined environments. Journal of Fluid Mechanics, 133, 321--343.

McDougall, T. J., 1983. Greenland Sea Bottom Water formation: a balance between advection and double-diffusion.

Deep-Sea Research, 30, 1109--1118.

McDougall, T. J., 1984. A model of a frictionless double-diffusive gravity current on a horizontal surface. Geophysical Astrophysical Fluid Dynamics.

McDougall, T. J., 1984. Fluid dynamic implications for massive sulphide deposits of of hot saline fluid flowing into a submarine depression from below. Deep-Sea Research, 31, 145--170.

McDougall, T. J., 1984. The relative roles of diapycnal and isopycnal mixing on subsurface water mass conversion. Journal of Physical Oceanography, 14, 1577--1589.

McDougall, T. J., 1985. Convective processes caused by a dense, hot saline source flowing into a submarine depression from above. Deep-Sea Research, 29, 953--965.

McDougall, T. J., 1985. Double-diffusive interleaving. Part I: Linear stability analysis. Journal of Physical Oceanography, $15,1532--1541$.

McDougall, T. J., 1985. Double-diffusive interleaving. Part II: Finite amplitude steady state interleaving. Journal of Physical Oceanography, 15, 1542--1556.

McDougall, T. J., 1986. Oceanic intrusions: Some limitations of the Ruddick and Turner (1979) mechanism. Deep-Sea Research, 33, 1653--1664.

McDougall, T. J., 1988. Some implications of ocean mixing for ocean modelling. In: Small-Scale Turbulence and Mixing in the Ocean, J.Nihoul and B.Jamart, editors, Elsevier Oceanography Series, 46, Elsevier, New York, pp.21--36.

McDougall, T. J., 1991. Interfacial advection in the thermohaline staircase east of Barbados. Deep-Sea Research, 38(3), 367--370.

McDougall, T. J., and B. R. Ruddick, 1982. The effects on fine structure measurements of correcting for internal wave strain. Journal of Physical Oceanography, 12, 495--497.

McDougall, T. J., and J. A. Whitehead, Jr., 1984. Estimates of the relative roles of diapycnal, isopycnal and double-diffusive mixing in Antarctic Bottom Water in the North Atlantic. Journal of Geophysical Research, 89, 10,479--10,483.

McDougall, T. J., and J. R. Taylor, 1984. Flux measurements across a finger interface at low values of the stability ratio. Joumal of Marine Research, 42, 1--14.

McDougall, T. J., and J. S. Turner 1982. Influence of cross-diffusion on 'ffinger” double-diffusive convection. Nature, 299, 812--814.

McDougall, T. J., and Y. You, 1990. Implications of the nonlinear equation of state for upwelling in the ocean interior.

Journal of Geophysical Research, 95(C8), 13,263--13,276.

McIntyre, M. E., 1970. Diffusive destabilization of the baroclinic vortex. Geophysical Fluid Dynamics 1, $19--57$.

McIntyre, M.E., Diffusive destabilization of the baroclinic circular vortex. Geophys. Fluid Dyn., 3, 321-345, 1970

McKenzie, D., 1984. The Generation and Compaction of Partially Molten Rock, J.

Petrology, Vol 25, part 3, pp 713-765.

McNiven, P., 1983. Hand-list of the Jevons archives in the John Rylands University Library of Manchester. Bulletin of the John Rylands University Library of Manchester, 66, (1), 213-248.

Mellberg, L. E., O. M. Johannessen, and O. S. Lee, 1974. Acoustic effect caused by a deep thermohaline stepped structure in 
the Mediterranean Sea. Journal of the Acoustical Society of America, 55, 1081--1083.

Melling, H., R. A. Lake, D. R. Topham and D. B. Fissel, 1984. Oceanic thermal structure in the western Canadian Arctic, Continental Shelf Research, 3, 233--258.

Merceret, F. J., 1977. A possible manifestation of double-diffusive convection in the atmosphere. Boundary-Layer Met., 11, $121-123$.

Middleton, J. H., and T. D. Foster, 1980. Finestructure measurements in a temperature-compensated halocline. Journal of Geophysical Research, 85, 1107--1122.

Miller, D. G., and V. Vitagliano, 1986. Experimental test of McDougall's theory for the onset of convective instability in isothermal temary systems. Journal of Physical Chemistry, 90, 1706-1717.

Miller, D.G., Ting, A.W., Rard, J.A., Mutual diffusion coefficients of various $\mathrm{ZnC12}(0.5 \mathrm{M})$ - $\mathrm{KCl}-\mathrm{H} 2 \mathrm{O}$ mixtures at 298.15K by Rayleigh interferometry, J.Electrochem.Soc., 135, 896(1988).

Miller, D.G., Vitagliano, V., and Sartorio, R., Some comments on multicomponent diffusion. Negative main term diffusion coefficients, second law constraints, solvent choice, and reference frame transformations, J. Phys. Chem., 90,509 - 519 (1986).

Miller, L., T. H. Spurling, and E. A. Mason, 1967. Instabilities in ternary diffusion. Physics of Fluids, 10, $1809--1813$. Miller, R. R. and D. G. Browning, 1974. Thermal layering between the Galapagos Islands and South America. Deep-Sea Research, 21, 669--673.

Molcard, R., 1967. A review of the stepped structure in the Tyrrhenian Sea. Rapp. Comm. Int. Mer Medit., 23, $23--24$. Molcard, R., and A. J. Williams, 1975. Deep-stepped structure in the Tyrrhenian Sea. Mem. Soc. Roy. des Sciences de Liege, 6, 191--210.

Molcard, R., and R. I. Tait, 1977. The steady state of the step structure in the Tyrrhenian Sea. A Voyage of Discovery, George Deacon 70th Anniversary Volume, M. V. Angel, Editor, Suppl. to Deep-Sea Research, 221 - 233.

Molemaker, M.J. and H. A. Dijkstra, 1996. Layer Formation in a Salt Stratified Liquid Cooled From Above In Double-Diffusive Convection A. Brandt and H.J.S. Fernando (eds), pp. 97-104, AGU, 1996.

Monin, A. S., and A. M. Obukhov, 1954. Basic laws of turbulent mixing in the ground layer of the atmosphere. Trudy Geofiz. Inst. Akad. Nauk SSSR, 24, 163--187.

Moody, J. A. 1996. Observations of Missing Processes Downstream From the Confluence of the Mississippi and St. Croix Rivers. In Double-Diffusive Convection A. Brandt and H.J.S. Fernando (eds), pp. 275-286, AGU, 1996.

Moore, D. R., J. Toomre, E. Knobloch, and N. O. Weiss, 1983. Period doubling and chaos in partial differential equations for thermosolutal convection. Nature, 303, 663--667.

Moore, D. W., and E. A. Spiegel, 1966. A thermally excited non-linear oscillator. Astrophysics Journal, $143,871--887$.

Moore, D.R., J.Toomre, E. Knobloch, and N.O. Weiss, Period doubling and chaos in partial differential equations for thermosolutal convection, Nature, 303, 663-667, 1983.

Moore, W. S., and D. J. O'Neill, Radionuclide Distributions in recent Sea Sediments, in: The Black Sea Oceanography, E. Izdar and J. M. Murray (editors), NATO/ASI Series, Dordrecht, Kluwer Academic Publishers, 257-270, 1991.

Moses, E., and V. Steinberg, 1986. Competing patterns in a convective binary fluid. Physical Review Letters, $2018--2021$. Moses, E., and V. Steinberg, 1986. Flow patterns and nonlinear behavior of traveling waves in a convective binary fluid. Physics Review, A, 34, 693--700.

Muench, R. D., H.J.S. Fernando, and G. R. Stegan, 1990. Temperature and salinity staircases in the northwestern Weddell Sea. Journal of Physical Oceanography, 20, 295--306.

Munk, W. H., 1966. Abyssal recipes. Deep-Sea Research, 13, 707--730.

Munns, R.G., R.J. Stanley, and C.D. Densmore, Hydrographic observations of the Red Sea brines. Nature, 214, 1215-1217, 1967.

Murray, B. T., and C. F. Chen, 1989. Double-diffusive convection in a porous medium. Journal of Fluid Mechanics, 201, 147--166.

Murray, B.T. and C.F. Chen, Double-diffusive convection in a porous medium, J. Fluid Mech., 201, 147-166, 1989.

Murray, J. W., Top, Z., and E. Ozsoy, Hydrographic Properties and Ventilation of the Black Sea, Deep Sea Research, 38 , Suppl. 2, S663-S689, 1991.

Nagasaka, M., H. Nagashima and J. Yoshida, 1996. Double Diffusively Induced Intrusions into a Density Gradient. In Double-Diffusive Convection A. Brandt and H.J.S. Fernando (eds), pp. 81-87, AGU, 1996.

Nagashima, H., J. Yoshida and M. Nagasaka, The behavior of double diffusive gravity Current: laboratory and numerical experiments, In Double Diffusion in Oceanography: Proc. of a Meeting Seplember 26-29, 1989, compiled by R. W. Schmitt, pp. 193-200, Woods Hole Oceanog. Inst. Tech. Rpt, WHOI-91-20, 1990.

Nagata, Y. 1970, Detailed temperature cross section of the cold water belt along the northern edge of the Kuroshio, Journal of Marine Research, 28, 1--14.

Nagy, I.P., A. Keresztessy, J.A. Pojman, G. Bazsa and Z. Noszticzius, Chemical waves in the iodide-nitric acid system, J. Phys. Chem., 98, 6030-6037, 1994.

Narusawa, U. and Y. Suzukawa, Experimental study of double-diffusive cellular convection due to a uniform lateral heat 
flux. J. Fluid Mech. 113, 387-405, 1981.

Nauheimer, G., Untersuchungen des Modelles der Thermo Diffusiven - Sedimentiren Konvektion im schwach nichtlinearen Bereich und mogliche Anwendungen auf Konvektion in Magmakammern Dipl. Phys. Thesis, 1994

Neal, V. T., S. Neshyba, and W. Denner, 1969. Thermal stratification in the Arctic Ocean. Science, 166, 373--374.

Neshyba, S., 1977. Upwelling by icebergs. Nature, 267, 507--508.

Neshyba, S., V. T. Neal, and W. Denner, 1971b. Internal waves and thermo-solutal microstructure. In: Proceedings 8th U.S. Navy Symposium on Military Oceanography, 1, 341--348.

Neshyba, S., V. T. Neal, and W. Denner, 1972. Spectra of internal waves: In situ measurements in a multiple-layered structure. Journal of Physical Oceanography, 2, 91--95.

Neshyba, S., V. T. Neal, and W. W. Denner, 1971. Temperature and conductivity measurements under Ice Island T-3. Journal of Geophysical Research, 76, 8107--8120.

Neuymin, G. G., and V. A. Urdenko, 1979. Possible effects of "salt fingers" on measurements of the transparency of ocean waters. Oceanology, 19, 23--26.

Newell, T. A., 1984. Characteristics of a double-diffusive interface at high density stability ratios. Journal of Fluid Mechanics, 149, 385--401.

Newell, T. A., and R. F. Boehm, 1982. Gradient zone constraints in a salt-stratified solar pond. Trans. ASME: Journal of Solar Pond Engineering, 104, 280--285.

Newman, F. C., 1976. Temperature steps in Lake Kivu: a bottom-heated saline lake. Journal of Physical Oceanography, 6, 157--163.

Nicolis, G. and I. Prigogine, Self-organization in nonequilibrium systems, John Wiley \& Sons, 1977.

Nied, D.A. and A. Bejan, Convection in porous media, Springer-Verlag, N.Y., pp. 408 (TA418.9P6 N54) (See ch. 9 double-diffusive convection), 1992.

Nield, D. A., 1967. The thermohaline Rayleigh-Jeffries problem. Journal of Fluid Mechanics, 29, 545--558.

Nield, D.A., Onset of thermohaline convection in a porous medium, Water Resources Res., 11, 553-560, 1968.

Niino, H., 1986. A linear theory of double-diffusive horizontal intrusions in a temperature-salinity front. J. Fluid Mech. 171, 71-100.

Oakey, N. S., 1988. Estimates of mixing inferred from temperature and velocity microstructure. In: Small-Scale Turbulence and Mixing in the Ocean, J.Nihoul and B.Jamart, editors, Elsevier Oceanography Series, 46, Elsevier, New York, pp.239--248.

Oakey, N. S., and J. A. Elliott, 1980. Variability of temperature gradient microstructure observed in the Denmark Strait. Journal of Geophysical Research, 85, 1933--1944.

Ochoa, J. 1987. Two limiting types of oceanic finestructure. Journal of Physical Oceanography, 17, 1539--1545.

Oldenburg, C.M.. Spera, F.J., D.A. Yuen and G. Sewell. Dynamic mixing in magma bodies: theory, simulations and implications. J.Geophys. Res.94, 9215-9236, 1989.

Oldenburg, C.N. and F.J. Spera, 1992a. Hybrid model for solidification and convection, Numer. Heat Transfer, part 2, Vol. 71, 217-229.

Oldenburg. C.M. and F.J. Spera, 1992 b. Modelling transport processes in nonlinear systems in: Chaotic Processes in the Geological Sciences. edited by D.A.Yuen, pp 205-224. Springer Verlag, New York,.

Orlanski, I., and K. Bryan 1969. Formation of the thermocline step structure by large-amplitude internal waves. Journal of Geophysical Research, 74, 6975--6983.

Osborn, T. R., 1973. Temperature microstructure in Powell Lake. Journal of Physical Oceanography, 3, 302--307.

Osborn, T. R., 1980. Estimates of the local rate of vertical diffusion from dissipation measurements. Journal of Physical

Oceanography, 10, 83--89.

Osborn, T. R., 1988. Signatures of doubly diffusive convection and turbulence in an intrusive regime. Journal of Physical Oceanography, 18(1), 146--155.

Osborn, T. R., 1991. Observations of the "salt fountain." \{Atmosphere-Oceans, 29(2), 340--356.

Osborn, T. R., and R. G. Lueck, 1985. Turbulence measurements with a submarine. Journal of Physical Oceanography, 15(C11), 1502--1520.

Osborn, T., and C. S. Cox, 1972. Oceanic fine structure. Geophysical Fluid Dynamics, 3, 321--345.

Ostlund, H. G., and D. Dyrssen, Renewal Rates of the Black Sea Deep Water, In: The Chemical and Physical Oceanography of the Black Sea, Univ. of Goteborg, Rep. on the Chemistry of the Sea XXXIII. Presented in the Meeting on the Chemical and Physical Oceanography of the Black Sea, Goteborg, Sweden, 1986.

Ouchi, T., Goriki, S., and K. Ito, On the space-time pattern formation of the earthquake strain field, Tectonophysics, 113 , 31-48, 1985.

Ozmidov, R. V., 1965. On the turbulent exchange in a stably stratified ocean. Fizika Atmos. Okeana Izv. Akad. Nauk SSSR, $1,853--860$.

Ozmidov, R. V., 1982. The third voyage of the scientific R/V AKADEMIK MSTISLAV KELDYSH. Oceanology, 22, 504--506. 
Ozsoy, E., Top, Z., White, G., and J. W. Murray, Double Diffusive Intrusions, Mixing and Deep Sea Convection Processes in the Black Sea, In: The Black Sea Oceanography, E. Izdar and J. M. Murray (editors), NATO/ASI Series, Dordrecht, Kluwer Academic Publishers, 17-42, 1991

Ozsoy, E., Unluata, U., and Z. Top, The Mediterranean Water Evolution, Material Transport by Double Diffusive Intrusions, and Interior Mixing in the Black Sea, Prog. Oceanog., 31, 275-320, 1993.

Ozsoy, E and I. Besiktepe, 1996. Sources of Double Diffusive Convection and Impacts on Mixing in the Black Sea. In

Double-Diffusive Convection A. Brandt and H.J.S. Fernando (eds), pp. 261-274, AGU, 1996.

Padman, L., and T. J. Dillon, 1988. On the horizontal extent of the Canada Basin thermohaline steps. Journal of Physical Oceanography, 18, 1458--1462.

Padman, L., and T. M. Dillon, 1987. Vertical fluxes through the Beaufort sea thermohaline staircase. Journal of Geophysical Research, 92, 10799--10806.

Padman, L., and T. M. Dillon, 1989. Thermal microstructure and internal waves in the Canada Basin diffusive staircase.

Deep-Sea Research, 36, 531--542.

Paduano, L., Sartorio, R.,Vitagliano V., Albright, J.G., and Miller, D.G., Measurement of the mutual diffusion cooefficients at one composition of the four - component system: a-cyelodcxtrin L-phenylalanine monobuthyl-urea- $\mathrm{H} 2 \mathrm{O}$ at 25 degrees $\mathrm{C}$, J. Phys. Chem., 96, 7478-7483 (1992).

Paldor, N., D. Nof, Linear Instability of an anticyclonic vortex in a two-layer ocean. J. Geophys. Res., 95(c10), 18075-18079, 1990.

Paliwal, R. C., 1979. Double-diffusive convective instability in an inclined fluid layer. Ph.D. Dissertation, Department of Mechanical, Industrial and Aerospace Engineering, Rutgers University, New Brunswick, NJ.

Paliwal, R. C., and C. F. Chen 1980. Double-diffusive instability in an inclined fluid layer. Part 1. Experimental investigations. Journal of Fluid Mechanics, 98, 755--768.

Paliwal, R. C., and C. F. Chen 1980. Double-diffusive instability in an inclined fluid layer. Part 2. Stability analysis. Journal of Fluid Mechanics, 98, 769--785.

Parameterization of Small-Scale Processes, Proceedings of the 'Aha Huliko'a Hawaiian Winter Workshop, Honolulu, P. Muller and D. Henderson, editors, 117-126, 1989.

Pearlstein, A. J., R. M. Harris, and G. Terrones, 1989. The onset of convective instability in a triply diffusive fluid layer. Journal of Fluid Mechanics, 202, 443--465.

Pearlstein, Arne J., 1981. Effect of rotation on the stability of a doubly diffusive layer. Journal of Fluid Mechanics, 103, 389--412.

Pedlosky, J., Geophysical fluid dynamics. 624 pp., Springer Verlag,N.Y., 1979.

Pellew, A., and Southwell, R. V., On maintained convection motion in a fluid heated from below, Proc. Roy. Soc., 176A, 312-343, 1940.

Perkin, R. G., and E. L. Lewis, 1984. Mixing in the West Spitzbergen Current. Journal of Physical Oceanography, 14, 1315--1325.

Perkins, H., and K. Saunders, 1982. Physical oceanographic observations in the northwest tropical Atlantic. Tropical

Ocean-Atmosphere Newsletter, September 7--9.

Petrasso, R. D., Rayleigh's challenge endures. Nature, 367, 217-218, 1994.

Peyret, R., and T.D. Taylor, Computational Methods for fluid flow. Springer-Verlag, New York, 1983.

Phillips, O. M., Shyu, J-H., and H. Salmun, An Experiment on Boundary Mixing: Mean Circulation and Transport Rates, J.

Fluid Mech., 173, 473-499, 1986.

Phillips, O. M., 1966. The dynamics of upper ocean., 266 pp., Cambridge Press.

Piacsek, S. A., and J. Toomre, 1980. Nonlinear evolution and structure of salt fingers. In: Marine Turbulence, J.C.J. Nihoul, editor, Elsevier, New York, 193--219.

Piacsek, S. A., N. H. Brummell, and B. E. McDonald, 1988. Numerical experiments on thermohaline convective motions across interfaces of intrusions. In: Small-Scale Turbulence and Mixing in the Ocean, J.Nihoul and B.Jamart, editors, Elsevier Oceanography Series, 46, Elsevier, New York, pp.503--516.

Pingree, R. D., 1969. Small-scale structure of temperature and salinity near Station Cavall. Deep-Sea Research, 16, $275--295$. Pingree, R. D., 1971. Analysis of the temperature and salinity small-scale structure in the region of the Mediterranean influence in the N.E. Atlantic. Deep-Sea Research, 18, 485--491.

Pingree, R. D., 1972. Mixing in the deep stratified ocean. Deep-Sea Research, 19, 549--561.

Platten, J.K. and J.C. Legros, 1984 Convection in Liquids. Springer.

Polzin, K., J.M. Toole, and R.W. Schmitt, Finescale parameterizations of turbulent dissipation, J. Phys. Oceanogr., in press, 1995.

Poplawsky, C. J., F. P. Incropera, and R. Viskanta, 1981. Mixed-layer development in a double-diffusive, thermohaline system. Transactions of ASME, Journal of Solar Pond Engineering, 103, 351--359.

Posmentier, E. S., and A. D. Kirwan, 1985. The role of double diffusive interleaving in mesoscale dynamics: an hypothesis. Journal of Marine Research, 43, 541--552. 
Posmentier, E. S., and C. B. Hibbard, 1982. The role of tilt in double diffusive interleaving. Journal of Geophysical Research, 87, 518--524.

Posmentier, E. S., and R. W. Houghton, 1978. Fine structure instabilities induced by double diffusion in the shelf/slope water front. Journal of Geophysical Research, 83, 5135--5138.

Poulikakas, D., 1985. The effect of a third diffusing component on the onset of convection in a horizontal porous layer. Physics of Fluids, 28, 3172--3174.

Prat, J., Massaguer, J.M. and Mercader, I., 1994. Mean flow in 2D thermal convection, in: Mixing in Geophysics, edited by Redondo, J.M. and Metais, O., pp. 208-215, Universitat Politecnica de Catalunya, Barcelona.

Prigogine, I., R. Lefever, A. Goldbeter, and M. HerschkowitzKaufman, 1969. Symmetry breaking instabilities in biological systems, Nature, 233, 913-916.

Proctor, M.R.E. and Weiss, N.O., 1993. Symmetries of time dependent magnetoconvection, Geophys. Astrophys. Fluid Dynamics, 70, 137-160.

Proctor, M.R.E., 1981. Steady subcritical thermohaline convection. Journal of Fluid Mechanics, 105, 507--521.

Proctor, M.R.E., and J. Y. Holyer, 1986. Planform selection in salt fingers. Journal of Fluid Mechanics, 168, 241--253.

Proctor, M.R.E., Weiss, N.O., Brownjohn, D.P. and Hurlburt, N.E., 1994. Nonlinear compressible magnetoconvection. Part

2. Instabilities of steady convection, J. Fluid Mech., 280, 227-253.

Rayleigh, Lord, 1883. Investigation of the character of the equilibrium of an incompressible heavy fluid of variable density. Proc. London Math. Soc., 14, 170-177.

Rayleigh, Lord, 1916. On convection currents in a horizontal layer of fluid, when the higher temperature is on the under side. Philos. Mag., 32, 529--546.

Reddy, C. S., 1978. Double-diffusive convection in an infinitely tall slot -- a numerical study. American Society of Mechanical Engineers, ASME paper No.78-WA/HT-8, 8pp.

Richards, K. J., R. T. Pollard, 1991. Structure of the upper ocean in the western equatorial Pacific. Nature, 350, 48--50.

Richards, K. J., 1991. Double-diffusive interleaving at the equator. Journal of Physical Oceanography, 21(7), 933--938.

Roden, G. I., 1964. Shallow temperature inversions in the Pacific Ocean. Journal of Geophysical Research, 69(14), 2899--2914.

Ronald, J.P., Armstrong R.C., Brown R.A., Graham A.L., Abbott J.R., A constitutive equation for concentrated suspension that accounts for shear-induced particle migration Phys. Fluids A 4 (1), January 1992

Rooth, C. G. H., Comments on Circulation Diagnostics and Implications for Chemical Studies of the Black Sea, In: The Chemical and Physical Oceanography of the Black Sea, Univ. of Goteborg, Rep. on the Chemistry of the Sea XXXIII. Also presented in the Meeting on the Chemical and Physical Oceanography of the Black Sea, Goteborg, Sweden, 1986.

Rucklidge, A.M. and Matthews, P.C., Shearing instabilities in

magnetoconvection, in Solar and Planetary Dynamos, edited by Proctor,

M.R.E., Matthews, P.C. and Rucklidge, A.M., pp. 257-264, Cambridge

University Press, Cambridge, 1993.

Rucklidge, A.M. and P.C. Matthews, 1996. The Shearing Instability in Magnetoconvection. In Double-Diffusive Convection A. Brandt and H.J.S. Fernando (eds), pp. 171-184, AGU, 1996.

Rucklidge, A.M., Chaos in a low-order model of magnetoconvection, Physica, 62D, 323-337, 1993.

Ruddick, B. R. and A. S. Bennett and D. J. Lawrence, 1985. Thermohaline intrusions in the frontal zones of a ware-core ring observed by Batfish. Journal of Geophysical Research, 90, 8928--8942.

Ruddick, B. R., 1981. The 'colour polarigraph' -- a simple method for determining the two-dimensional distribution of sugar concentration. Journal of Fluid Mechanics, 109, 277--282.

Ruddick, B. R., 1985. Momentum transport in thermohaline staircase. Journal of Geophysical Research, 90, 895--902.

Ruddick, B. R., 1983. A practical indicator of the stability of the water column to double-diffusive activity, Deep-Sea Res., 30,1105 .

Ruddick, B. R., and J. S. Turner, 1979. The vertical length scale of double-diffusive intrusions. Deep-Sea Research, 26A, 903--913.

Ruddick, B. R., and T.G.L. Shirtcliffe, 1979. Data for double diffusers: Physical properties of aqueous salt-sugar solutions. Deep-Sea Research, 26, 775--787.

Ruddick, B. R., Intrusive mixing in a Mediterranean salt lens: intrusion slopes and dynamical mechanisms, J. Phys.

Oceanogr., 22, 1274-1285, 1992.

Ruddick, B. R., R. W. Griffiths, and G. Symonds, 1989. Frictional stress at a sheared double-diffusive interface. Journal of Geophysical Research, 94, 18,161--18,173.

Ruddick, B., 1984. The life of a thermohaline intrusion. Journal of Marine Research, 42, 831--852.

Ruddick, B., 1992. Intrusive mixing in a Mediterranean salt lens-intrusion slopes and dynamical mechanisms. Journal of Physical Oceanography, 22, 1274--1285.

Ruddick, B., and D. Hebert, 1988. The mixing of Meddy "Sharon." In: Small-Scale Turbulence and Mixing in the Ocean, J. Nihoul and B. Jamart, editors, Elsevier Oceanography Series, 46, Elsevier, New York, pp.249--262. 
Ruddick, B., and L. Zhang, 1989. The mythical thermohaline oscillator? Journal of Marine Research, 47(4), 717--746. Ruddick, B. and D. Walsh, 1995. Observations of the Density Perturbations which drive Thermohaline Intrusions. In Double-Diffusive Convection A. Brandt and H.J.S. Fernando (eds), pp. 329-334, AGU, 1995.

Rudraiah, N., and D. Vortmeyer, 1982. The influence of permeability and of a third diffusing component upon the onset of convection in a porous media. International Journal Heat and Mass Transfer, 25, 457--464.

Rudraiah, N., P.K. Srimani and R. Freidrich, Finite amplitude convection in a two-component fluid saturated porous layer, Int. J. Heat Mass Transfer, 25, 715-722, 1982.

Salmun, H., Killworth, P. D., and J. R. Blundell, A Two Dimensional Model of Boundary Mixing, J. Geophys. Res., 96, 18447-18474, 1991.

Salusti, E., 1978. Internal waves on a deep-stepped marine structure. Deep-Sea Research, 25, 947--956.

Sandner, H., Double diffusion effects in a cylindrical porous bed filled with salt water, Heat Transfer 1986, Hemisphere, Washington, DC, 5, 2623-2627, 1986.

Sani, R. L., 1965. On finite amplitude roll cell disturbances in a fluid layer subjected to a heat and mass transfer. American Institute of Chemical Engineering Journal, 11, 971--980.

Sarsten, J. A., 1972. LNG stratification and roll-over. Pipeline and Gas Journal, 199, 37--39.

Sartory,W.K., Instability in diffusing fluid layers, Biopolymers, 7, 251-263 (1969).

Sattinger, D., Lecture note in mathematics, 309, Berlin, Springer, 1973.

Savage, J. C., A theory of creep wave propagating along a transform fault, J. Geophys. Res., 76, 1954-1966, 1971.

Sayre, W. W., and F. M. Chang, A laboratory investigation of open channel dispersion processes for dissolved, suspended, and floating dispersants, U. S. Geological Surv. Prof: Pap., 433-E, 1968.

Schechter, R. S., I. Prigogine, and J. R. Hamm, 1972. Thermal diffusion and convective stability. Physics of Fluids, 15, 379--386.

Schladow, S.C., E. Thomas and J.R. Koseff, The dynamics of intrusions into a themohaline stratification. J. Fluid Mech. 236, 127-165, 1992.

Schladow, S.G., Simulation of dynamics of double-diffusive system, J. Hyd. Eng., 114, 1-20, 1988.

Schmitt, R. W. 1990. On the density ratio balance in the Central Water. Journal of Physical Oceanography, $20(6), 900--906$.

Schmitt, R. W., 1976. On the flux ratio of salt fingers. EOS, Transactions of the American Geophysical Union, 57, 261.

Schmitt, R. W., 1978. Time-dependent and rotational effects in Salt Finger Convection. Ph.D Dissertation, University of Rhode Island.

Schmitt, R. W., 1979a. The growth rate of super-critical salt fingers. Deep-Sea Research, 26A, 23--40.

Schmitt, R. W., 1979b. Flux measurements on salt fingers at an interface. Journal of Marine Research, 37, 419--436.

Schmitt, R. W., 1981. Form of the temperature-salinity relationship in the Central Water: evidence for double-diffusive mixing. Journal of Physical Oceanography, 11, 1015--1026.

Schmitt, R. W., 1983. The characteristics of salt fingers in a variety of fluid systems, including stellar interiors, liquid metals, oceans and magmas. Physics of Fluids, 26, 2373--2377.

Schmitt, R. W., 1987. The Caribbean Sheets And Layers Transects (C-SALT) Program. EOS, Transactions of the American Geophysical Union, 68(5), 57--60.

Schmitt, R. W., 1988. Mixing in a thermohaline staircase. In: Small-Scale Turbulence and Mixing in the Ocean, J.Nihoul and B.Jamart, editors, Elsevier Oceanography Series, 46, Elsevier, New York, pp.435--452.

Schmitt, R. W., 1994. Double Diffusion in Oceanography. Annual Review of Fluid Mechanics, 26, $255--285$.

Schmitt, R. W., 1994. Triangular and asymmetric salt fingers. Journal of Physical Oceanography, 24 (4), $855--860$.

Schmitt, R. W., 1995. The Ocean's Salt Fingers. Scientific American, 272(5), 70--75.

Schmitt, R. W., 1995. The salt finger experiments of Jevons (1857) and Rayleigh (1880). Journal of Physical Oceanography, 25(1), 8--17.

Schmitt, R. W., 1995. Why didn't Rayleigh discover salt fingers? in: Double-Diffusive Convection. A Brandt and H.

Fernando, Ed., AGU Geophysical Monograph, 94, 3--10.

Schmitt, R. W., and D. L. Evans, 1978. An estimate of the vertical mixing due to salt fingers based on observations in the North Atlantic Central Water. Journal of Geophysical Research, 83, 2913--2919.

Schmitt, R. W., and D. T. Georgi, 1982. Finestructure and microstructure in the North Atlantic Current. Journal of Marine Research, 40 (Supp1.), 659--705.

Schmitt, R. W., and R. B. Lambert, 1979. The effects of rotation on salt fingers. Journal of Fluid Mechanics, 90, $449--463$. Schmitt, R. W., H. Perkins, J. D. Boyd, and M. C. Stalcup, 1987. C-SALT: an investigation of the thermohaline staircase in the western tropical North Atlantic. Deep-Sea Research, 34, (10), 1697--1704.

Schmitt, R. W., K. L. Polzin, and J. M. Toole, 1989. Shear and Salt Fingers. Proceedings: Hawaiian winter workshop on Parameterization of small scale Processes. University of Hawail, January 17--20, 1989. pp.127--144.

Schmitt, R. W., R. G. Lueck, and T. M. Joyce, 1986. Fine- and micro-structure at the edge of a warm core ring. Deep-Sea Research, 33, 1665--1689.

Schmitz, W. J., Jr., J. R. Luyten and R. W. Schmitt, 1993. On the Florida Current T/S envelope. Bulletin of Marine Science 
of the Gulf and Caribbean, 53(1), 1048--1065.

Schoeberlein, H. C, Statistical analysis of patches of oceanic smallscale activity, Johns Hopkins APL Tech. Dig., 6(3), 194-202, 1985.

SCOR Working Group 69, 1988. Small-scale turbulence and mixing in the ocean: A glossary. In: Small-Scale Turbulence and Mixing in the Ocean, C. J.Nihoul and B. M.Jamart, editors, Elsevier Oceanography Series, 46, Elsevier, New York, pp.3-10, Elsevier.

Segal, L. A., and J. L. Jackson, Dissipative structure: an explanation and an ecological example, J. Theor. Biol., 37, 545-559, 1972.

Shen, C. and R. W. Schmitt, 1996. The wavenumber spectrum of salt fingers. in: Double-Diffusive Convection, A. Brandt

and H. Fernando, Editors, AGU Geophysical Monograph, 94, 305--312.

Shen, C. Y. and G. Veronis, 1991. Scale transition of double-diffusive finger cells, Physics Fluids A3(4) 58-69.

Shen, C. Y., 1989. The evolution of the double-diffusive instability: salt fingers. Physics of Fluids, A1(5), 829--844.

Shen, C. Y., Equilibrium salt-fingering convection, in press, 1994.

Shen, C. Y., Heat-salt finger fluxes across a density interface, Phys. Fluids A, 5, 2633-2643, 1993.

Shen, C. Y., The evolution of the double-diffusive instability: salt fingers. Physics Fluids A 1(5) 829-844, 1989.

Sherman, F. S., J. Imberger, and G. M. Corcos, 1978. Turbulence and mixing in stably stratified waters. Annual Review Fluid Mechanics, 10, 267--288.

Sherman, J. T., Observations of fine-scale vertical shear and strain, Ph.D. dissertation, University of California, San Diego, 1989.

Shirtcliffe, T. G. L., and I. M. Calhaem, 1968. Measurements of temperature and electrical conductivity in Lake Vanda, Victoria Land, Antarctica. New Zealand Journal of Geology and Geophysics, 11, 976--981.

Shirtcliffe, T.G.L, 1967. Thermosolutal convection: observation of an overstable mode. Nature, 213, 489--490.

Shirtcliffe, T.G.L., 1969. An experimental investigation of thermosolutal convection at marginal stability. Journal of Fluid Mechanics, 35, 677--688.

Shirtcliffe, T.G.L., 1969. The development of layered thermosolutal convection. International Journal of Heat and Mass Transfer, 12, 215--222.

Shirtcliffe, T.G.L., 1972. The contribution of double-diffusive processes to the vertical fluxes of salt and heat. In: R.Fraser, editor, Oceanography of the South Pacific. Wellington (New Zealand), New Zealand Committee for UNESCO, 137--139.

Shirtcliffe, T.G.L., 1973. Transport and profile measurements of the diffusive interface in double-diffusive convection with similar diffusivities. Joumal of Fluid Mechanics, 57, 27--43.

Shirtcliffe, T.G.L., and J. S. Turner, 1970. Observations of the cell structure of salt fingers. Journal of Fluid Mechanics, 41, 707-719.

Siedler, G., and W. Zenk, 1973. Variability of the thermohaline staircase. Nature (Physical Science), 244(131), 11--12.

Siegmann, W. L., and L. A. Rubenfeld, 1975. A nonlinear model for double-diffusive convection. SIAM Journal of Applied Mathematics, 29, 540--557.

Simpson, J. H., M. R. Howe, N.C.G. Morris, and J. Stratford, 1979. Velocity shear in the steps below the Mediterranean outflow. Deep-Sea Research, 26, 1381--1386.

Simpson, J.E., Gravity Currents: In the Environment and the Laboratory, 244 pp., John Wiley and Sons.

Spencer D. P., Brewer, P. G., and P. L. Sachs, Aspects of the Distribution and Trace Element Composition of Suspended Matter in the Black Sea. Geochim. et Cosmochim. Acta, 36, 71-86, 1971.

Spencer, D. W., and P. G. Brewer, 1971. Vertical advection and redox potentials as controls on the distribution of manganese and other trace metals dissolved in waters of the Black Sea. Journal of Geophysical Research, 76, 5877--5892.

Spera, F.J., Trial, A.F., Yuen, D.A. and U. Hansen, 1994. Viscous dissipation: role in magma generation and ascent. E.O.S., American Geophys. Union Trans., Vol. 75, no. 44, 703.

Spera, F.J., Yuen, D.A. and H.-J. Hong. 1986. Double-diffusive convection in magma chambers: single or multiple layers?, Geophys. Res. Let., 13, 153-156.

Srivastava, M. S., and E. M. Carter, 1983. An Introduction to Applied Multivariate Statistics, p. 39, North-Holland,.

Stallard, R. F., 1987. Cross-channel mixing and its effect of sedimentation in the Orinoco River, Water Resour. Res., 23, $1977-1986$.

Stanley, D. J., and C. Blanpied, 1980. Late Quaternary Water Exchange between the Eastern Mediterranean and the Black

Sea', Nature, 285, 537-541.

Stel, H., Cloetingh, S., 1993. Heeremans, M. and P. van der Beek, Anorogenie granites, magmatic underplating and the origin of intracratonie basins in a non-extensional setting, Tectonophysics, 225, 285-300.

Stern, M. E., 1960. The 'salt fountain' and thermohaline convection. Tellus, 12, 172--175.

Stern, M. E., 1967. Lateral mixing of water masses. Deep-Sea Research, 14, 747--753.

Stern, M. E., 1968. T--S gradients on the micro-scale. Deep-Sea Research, 15, 245--250.

Stern, M. E., 1969. Collective instability of salt fingers. Journal of Fluid Mechanics, 35, 209--218.

Stern, M. E., 1969. Salt finger convection and the energetics of the general circulation. Deep-Sea Research, 16(Suppl.), 
263--267.

Stern, M. E., 1970. Optical measurement of salt fingers. Tellus, 22, 76--81.

Stern, M. E., 1975. Ocean Circulation Physics. Academic Press.

Stern, M. E., 1976. Maximum buoyancy flux across a salt finger interface. Journal of Marine Research, 34, 95--110.

Stern, M. E., 1982. Inequalities and variational principles in double-diffusive turbulence. Journal of Fluid Mechanics, 114, $105-121$.

Stern, M. E., and J. S. Turner, 1969. Salt fingers and convecting layers. Deep-Sea Research, 16, 497--511.

Stigebrandt, A., A Model for the Vertical Circulation of the Baltic Deep Water, J. Phys. Oceanog., 17, 1772-1785, 1987.

Stommel, H. M., and G. T. Csanady, 1980. A relation between the T--S curve and global heat and atmospheric water transport. Journal of Geophysical Research, 85, 495--501.

Stommel, H., 1949. Horizontal diffusion due to oceanic turbulence. Journal of Marine Research, 8, 199--225.

Stommel, H., A. B. Arons, and D. Blanchard, 1956. An oceanographic curiosity: the perpetual salt fountain. Deep-Sea

Research, 3, 152--153.

Stommel, H., and K. N. Federov, 1967. Small-scale structure in temperature and salinity near Timor and Mindanao. Tellus, 19, 306--325.

Straus, J. M., 1972. Finite amplitude doubly diffusive convection. Journal of Fluid Mechanics, 56, 353--374.

Sur, El. I., Ozsoy, E., and U. Unluata, 1994. Boundary Current Instabilities, Upwelling, Shelf Mixing and Eutrophication

Processes In The Black Sea, Prog. Oceanog., 3S, 249-302.

Swallow, J. C., and J. Crease 1965. Hot salty water at the bottom of the Red Sea. Nature, 205, 165--166.

Swart, P. K., The Oxygen and Hydrogen Isotopic Composition of the Black Sea, Deep-Sea Res, \#8, Suppl. 2, S761-S772, 1991.

Tait, R. I., and M. R. Howe, 1968. Some observations of thermohaline stratification in the deep ocean. Deep-Sea Research, $15,275-280$.

Tait, R. I., and M. R. Howe, 1971. Thermohaline staircase. Nature, 231, 178--179.

Tait, S. and C. Jaupart, Compositional convection in a reactive cyrstalline mush and melt differentiation, J.Geophys. Res., 97, 6735-6756, 1992.

Tamai, N., T. Asaeda, and H. Ozaki 1984. Micro-structure of the mass and heat transfer near double-diffusive interface. Proceedings of the 4th Congress-Asian and Pacific Division International Association for Hydraulic Research. Chiang Mai, Thailand, 861--873.

Tang, C. L., A. S. Bennett and D. J. Lawrence 1985: Thermohaline intrusions in the frontal zones of a ware-core ring observed by Batfish. Journal Geophysical Research, 90, 8928--8942.

Tanny, J., Chen, C.C. and Chen, C.F. 1995 Effects of interaction between Marangoni and double-diffusive instabilities. \{lit J. Fluid Mech.\} \{lbf 393\}, 1.

Tanny, J. and A.B. Tsinober, 1989. On the behaviour of a system of double diffusive layers during its evolution. Phys. Fluids A $1,606-609$,

Tanny, J., and A. B. Tsinober, 1988. The dynamics and structure of double-diffusive layers in sidewall-heating experiments. Journal of Fluid Mechanics, 196, 135--156.

J. Tanny, Z. Harel, and A. Tsinober, Thermal Diffusion Phenomena in Thick Fluid Layers. In Double-Diffusive Convection A. Brandt and H.J.S. Fernando (eds), pp. 31-39, AGU, 1996.

J. Tanny, V. A. Gotlib and A. Tsinober, The Onset of Double-Diffusive Instability in a Layer with Temperature-Dependent

Fluid Properties. In Double-Diffusive Convection A. Brandt and H.J.S. Fernando (eds), pp. 41-47, AGU, 1996.

Taunton, J. W., E. N. Lightfoot, and T. Green, 1972. Thermohaline instability and salt fingers in a porous medium. Physics of Fluids, 15, 748--753.

Taylor J., and G. Veronis, 1986. Experiments on salt fingers in a Hele-Shaw cell. Science, 231, 39--41.

Taylor, G. I., 1950. The instability of liquid surfaces when accelerated in a direction perpendicular to their planes. I. Proc.

Roy. Soc., A201, 192--196.

Taylor, J., 1991. Laboratory experiments on the formation of salt fingers after the decay of turbulence. Journal of Geophysical Research, 96(C7), 12,497--12,510.

Taylor, J., and P. Bucens, 1989. Laboratory experiments on the structure of salt fingers. Deep-Sea Research, 36(11), $1675-1704$.

Terrones, G., Cross-diffusion effects on the stability criteria in a triple diffusive system, Phys. Fluid A, 5, 2172-2182, 1993.

Thangam, T., and C. F., Chen 1981. Salt-finger convection in the surface discharge of heated saline jets. Geophysical

Astrophysical Fluid Dynamics, 18, 111-146.

Thorpe, S. A., P. K. Hutt, and R. Soulsby, 1969. The effect of horizontal gradients on thermohaline convection. Journal of Fluid Mechanics, 38, 375--400.

Toole, J. M., 1980. Wintertime convection and frontal interleaving in the Southern Ocean. Sc.D. Dissertation. WHOI/MIT Joint Program in Physical Oceanography, Woods Hole, MA.

Toole, J. M., 1981. Intrusion characteristics in the Antarctic Polar Front. Journal of Physical Oceanography, 11, $780-793$. 
Toole, J. M., K. I. Polzin and R.W. Schmitt, Estimates of Diapycnal Mixing in the Abyssal Ocean. Science, 264, 1120-1123, 1994.

Toole, J.M. and D.T. Georgi, On the dynamics and effects of double diffusively driven intrusions. Prog. Oceanogr. 10, 123-145, 1981.

Toomre, J., D. O. Gough, and E. A. Spiegel, 1982. Time-dependent solutions of multimode convection equations. Journal of Fluid Mechanics, 125, 99--122.

Toor, H. L., Solution of the linearized equations of multicomponent mass transfer: I, A.l.Ch.F. J., 10, 448-455, 1964.

Trevisan, O.V. and A. Bejan, 1990. Combined heat and mass transfer by natural convection in a porous medium, Adv. Heat Transfer, 20, 315-352 (a review).

Trubitzyn, V.P., E.V. Kharybin, 1987. Convective instability of the sedimentation state in the mantle, Physics of the solid Earth (Fizika zemli), N8, pp 21-30, (in russian).

Trubitzyn, V.P., E.V. Kharybin, 1988. Hydrodynamical model of the differentiation process in the Earth's interior, Physics of the solid Earth (Fizika zemli), N4,pp 83-86, (in russian).

Trubitzyn, V.P., E.V. Kharybin, 1991. Thermoconvective instability of the two components viscous fluid, Physics of the solid Earth (Fizika zemli), N2,pp 3-17, (in russian).

Tsinober, A. B., Y. Yahalom, and D. J. Shlien, 1983. A point source of heat in a stable salinity gradient. Journal of Fluid Mechanics, 135, 199--217.

Tsitverblit, N. and E. Kit, The multiplicity of steady flows in confined double-diffusive convection with lateral heating. Phys. Fluids A 5, 1062-1064, 1993.

Turcotte, D. L., and G. Schubert, Geodynamics; Applications of Continuum Physics to Geological Problems, John Wiley \& Sons, New York, 1982.

Turing, A. M., The chemical basis for morphogeneis, Philos. Trans. R. Soc. London, 237, 37-72, 1952.

Turner, J. S. and L. B. Gustafson, 1981. Fluid motions and compositional gradients produced by crystallization or melting at vertical boundaries. Journal of Volcanology and Geothermal Research, 11, 93--125.

Turner, J. S., 1965. The behaviour of a stable salinity gradient heated from below. Journal of Fluid Mechanics, 33, 183--200.

Turner, J. S., 1965. The coupled turbulent transports of salt and heat across a sharp density interface. International Journal of Heat and Mass Transport, 8, 759--767.

Turner, J. S., 1967. Salt fingers across a density interface. Deep-Sea Research, 14, 599--611.

Turner, J. S., 1968. The behaviour of a stable salinity gradient heated from below. Journal of Fluid Mechanics, 33, 183--200.

Turner, J. S., 1968. The influence of molecular diffusivity on turbulent entrainment across a density interface. Journal of Fluid Mechanics, 33, 639-656.

Turner, J. S., 1973. Buoyancy Effects in Fluids. Cambridge University Press, Cambridge, 367pp.

Turner, J. S., 1974. Double-diffusive phenomena. Annual Reviews of Fluid Mechanics, 6, 37--56.

Turner, J. S., 1978. Double-diffusive intrusions into a density gradient. Journal of Geophysical Research, 83, 2887--2901.

Turner, J. S., 1979. Laboratory models of double diffusive processes in the ocean, In: National Academy of Sciences. Twelfth Symposium on Naval Hydrodynamics, Washington, D.C., National Academy of Sciences, 596--606.

Turner, J. S., 1981. Small-scale mixing processes. In: The Evolution of Physical Oceanography. MIT Press, Cambridge, MA. Turner, J. S., 1985. Multicomponent convection. Annual Reviews of Fluid Mechanics, 17, 11-44.

Turner, J. S., 1969. A Physical Interpretation of Hot Brine Layers in the Red Sea, In: Hot Brines and Recent Heavy Metal Deposits in the Red Sea, E. T. Degens and D. A. Ross (editors), Springer, New York, 164172,.

J. S. Turner, Laboratory Models of Double-Diffusive Processes. In Double-Diffusive Convection A. Brandt and H.J.S.

Fernando (eds), pp. 11-29, AGU, 1996.

Turner, J. S., and C.F. Chen, 1974. Two-dimensional effects in double-diffusive convection. Journal of Fluid Mechanics, 63, 577--592.

Turner, J. S., and H. Stommel, 1964. A new case of convection in the presence of combined vertical salinity and temperature gradients. Proceedings of the National Academy of Sciences, 52, 49--53.

Turner, J. S., and L. B. Gustafson, 1978. The flow of hot saline solutions from vents in the sea floor-some implications for exhalative massive sulfide and other ore deposits. Economic Geology, 73, 2082--1100.

Turner, J. S., T.G.L. Shirtcliffe, and P. G. Brewer, 1970. Elemental variations of transport coefficients across density interfaces in multiple-diffusive systems. Nature, 228, 1083--1084.

Turner, J.S. 1986 Turbulent entrainment: the development of the entrainment assumption, and its application to geophysical flows. J. Fluid Mech. 173, 431-471.

Turner, J.S. 1991 Convection and mixing in the oceans and the earth. Phys. Fluids A, 3(2), 1218-1232.

Turner, J.S. and J.H. Campbell, 1986. Convection and mixing in magma chambers, Earth Sci. Rev., 23, 255-352.

Turner, J.S. and L.B. Gustafson, 1981. Fluid motions and compositional gradients produced by crystallization or melting at vertical boundaries. J. Volcanol. and Geothermal Res., 1 1, 93-125.

Turner, J.S. and L.B. Gustafson, 1978. The flow of hot saline solutions from vents in the sea floor: some implications for exhalative sulfide and other ore deposits. Econ. Geol., 73, 1082-1100. 
Tumer, J.S., A fluid-dynamical model of differentiation and layering in magma chambers. Nature, 285, 213-215, 1980. Turner, J.S., H.E. Huppert, and R.S.J. Sparks, 1983. Experimental investigations of volatile exsolution in evolving magma chambers. J. Volcanol. and Geothermal Res., 16, 263-277.

Tumer, J.S., H.E. Huppert, and R.S.J. Sparks, 1986. Komatiites II: experimental and theoretical investigations of postemplacement cooling and crystallization. J. Petrol., 27, 397437,.

Tyrrell, H.J.V. and Harris, K.R., Diffusion in Liquids, Butterworths, London 1984

Unluata, U., Aubrey, D. G., Belberov, Z., Bologa, A., Eremeev, V. and M. Vinogradov, International Program Investigates the Black Sea, EOS, 74(36), 408-412, 1993.

Unluata, U., Oguz, T., Latif, M. A., and E. Ozsoy, On the Physical Oceanography of the Turkish Straits, In: The Physical Oceanography of Sea Siraits, L. J. Pratt (editor), NATO/ASI Series, Kluwer, Dordrecht, 2560, 1990.

Vaisaila. V., Uber die Wirkung der Windschwankungen auf die Pilotbcobachtungen. Soc. Sci. Fenn. Comnlental. Phys.-Marh., 2 (19), 19-37, 1925.

van Aken, H. M. 1982. The density ratio of frontal intrusions in the North Rockall Trough. Journal Physical Oceanography, $12,1318-1323$.

Vanderbauwhede, A., and G. Iooss, Center manifold theory in infinite dimensions, Dynamics Reported, 1, New Series, 125-163, 1992.

Varma, K. K., and L. V. Gangadhara Rao, 1978. Observations on fine structure in the northern Arabian Sea. Indian Journal of Marine Sciences, 7, 79--83.

Velarde, M. G., and R. S. Schechter, 1972. Thermal diffusion and convective stability. II. An analysis of the convected fluxes. Physics of Fluids, 15, 1707--1714.

Veronis, G., 1965. On finite amplitude instability in thermohaline convection. Journal of Marine Research, $23,1--17$.

Veronis, G., 1968. Effect of a stabilizing gradient of solute on thermal convection. Journal of Fluid Mechanics, 34, $315--336$.

Veronis, G., 1987. The role of the buoyancy layer in determining the structure of salt fingers. Journal of Fluid Mechanics, $180,327-342$.

Veronis, G., A theoretical model of Henry Stommel in: The Evolution of Physical Oceanography, B. A. Warren and C.

Wunsch, editors, Mill Press, Cambridge, MA, 1981.

Vitagliano, V. 1996. Diffusion and Double Diffusive Convection in Isothermal Liquid Boundaries. In Double-Diffusive

Convection A. Brandt and H.J.S. Fernando (eds), pp. 185-193, AGU, 1996.

Vitagliano, P.L., Della Volpe, C., and Vitagliano, V., J., 1984. Solution Chem., 13, 549 - 562.

Vitagliano, P.L.,Arnbrosone, L., and Vitagliano, V., 1992. Gravitational Instabilities in Multicomponent free diffusion boundaries, J. Phys. Chem., 96, 1431-1437.

Vitagliano, V., Borriello, G., Della Volpe, C., and Ortona, O., 1986. Instabilities in free diffusion boundaries of NaCIsucrosewater solutions at 25 degrees $C$, J. Solution Chem., 15, 811-826.

Vitagliano, V., Della Volpe, C., Ambrosone, L., and Costantino, L., 1988. Diffusion and double diffusive convection in isothermal ternary systems, Phys. Chem. Hydrodynamics, 10, $239-247$.

Vitagliano, V., Sartorio, R., Seala, S., and Spaduzzi,D., 1978. Diffusion in a ternary system and the critical mixing point, J. Solution Chem., 7, 605 - 621 .

Vitagliano, V., 1991. Some phenomenological and thermodynamic aspects of diffusion in multicomponents systems, Pure \& Appl. Chem., 63, 1441- 1448.

Vitagliano, V., Zagari, A., Sartorio, R., and Coreione, M., 1972. Dissipative structures and diffusion in ternary systems, J. Phys. Chem ., 76, $2050-2055$.

Voorhis, A. D., and D. L. Dorson, 1975. Thermal convection in the AtlantisII hot brine pool. Deep-Sea Research, 22, 167--175.

Voorhis, A. D., D. C. Webb, and R. S. Millard, 1976. Current structure and mixing in the shelf/slope water front south of New England. Journal of Geophysical Research, 81, 3695--3708.

Walin, G., 1964. Note on the stability of water stratified by both salt and heat. Tellus, 16, 389--393.

Walsh, D., and B. Ruddick, Double-diffusive interleaving: the influence of non-constant diffusivities, J. Phys. Oceanogr., 25 (3), 348-358, 1995.

Walsh, D. and B. Ruddick. 1995. An Investigation of Kunze's Salt Finger Flux Laws_Are They Stable? In Double-Diffusive Convection A. Brandt and H.J.S. Fernando (eds), pp. 321-328, AGU, 1995.

Walton, I. C., 1982. Double-diffusive convection with large variable gradients. Journal of Fluid Mechanics, 125, $123--135$.

Walton, I. C., Double Diffusive Convection with Large Variable Gradients, J. Fluid Mech., 125, 123-135, 1982.

Washburn, L., and R. H. Kase, 1987. Double diffusion and the distribution of the density ratio in the Mediterranean water from southeast of the Azores. Journal of Physical Oceanography, 17, 12--25.

Watson, B.E. and Baker, D. R., Chemical diffusion in magmas: An overview of experimental results and geochemical applications, in Physical Chemistry of Magmas, edited by L. L. Perchuk and I. Kushiro, pp. 120-151, Springer Verlag, New York.

Weaver, J.A. and R. Viskanta, 1991. Natural convection due to horizontal temperature and concentration gradients - 1. 
Variable thermophysical property effects, Int. J. Heat Mass Transfer, 34, 3107-3120.

Webel, G., and M. Schatzmann, 1984. Transverse mixing in open channel flow, J. Hdr: Eng., 110, 423-435.

Weibezalm, F. H., A. Heyvaert, and M. A. Lasi, 1989. Lateral mixing of the waters of the Oruloco, Atabapo, and Guaviare Rivers, after their confluence, in southern Venezuela, Ecologia, an, 263-270.

Weinburger, H., 1964. The physics of the solar pond. Solar Energy, 8, 45--56.

Weinstein, S.A., D.A. Yuen, P.L. Olson, Evolution of crystal settling in magma chamber convection, Earth Planet Sci. Let., 87, pp 237-248, 1988.

Welander, P., 1982. A simple heat-salt oscillator. Dynamics of Atmospheres and Oceans, 6, 233--242.

Welander, P., 1989. A new type of double-diffusive instability. Tellus, 41A, 66--72.

Weller, R. A., 1991. Overview of the frontal air-sca interaction experiment (FASINEX): A study of air-sea interaction in a region of strong oceanic gradients, J. Geophys. Res., 96, 8501-8516.

Wells, J. D., 1986. Solvent fluxes, coupled diffusion, and convection in concentrated ternary solutions. Journal of Physical Chemistry, 90, 2433--2440.

Wendt, R. P., and M. Shamim, 1970. Isothermal diffusion in the system water-magnesium chloride-sodium chloride as studied with the rotating diaphragm cell. Journal of Physical Chemistry, 74, 2770--2873.

Whitfield, D.W.A., G. Holloway, and J. Y. Holyer, 1989. Spectral transform simulations of finite amplitude double-diffusive instabilities in two dimensions, Journal of Marine Research, 47, 241--265.

Wiesenburg, D. A., 1980. Geochemistry of dissolved gases in the hypersaline Orca Basin. Ph.D. Dissertation, Texas Al\&M University, College Station, TX, 265pp.

Williams, A. J., 1974. Salt fingers observed in the Mediterranean outflow. Science, 185, 941--943.

Williams, A. J., 1975. Images of ocean microstructure. Deep-Sea Research, 22, 811--829.

Williams, A. J., 1981. The role of double-diffusion in a Gulf Stream frontal intrusion. Journal of Geophysical Research, 86, $1917--1928$.

Williams, A. J., III, 1980. Refractive microstructure from diffusive and turbulent ocean mixing. Optical Engineering, 19, 116--121.

Williams, A. J., III, The role of double diffusion in a Gulf Stream frontal intrusion, J. Geo. Res., 86, 1917-1928, 1981.

Williams, R. G., 1985. The internal tide off southern California, Ph.D. dissertation, University of California, San Diego.

Wilson, A. T., and H. W. Wellman, 1962. Lake Vanda: an Antarctic Lake. Nature, 196, 1171.

Winant, C. D., and F. K. Browand, 1974. Vortex pairing: the mechanism of turbulent mixing-layer growth at moderate Reynolds number, J. Fluid Mech., 63, 237-255.

Wirtz, R. A., D. G. Briggs, and C. F. Chen, 1972. Physical and numerical experiments on layered convection in a density stratified fluid. Geophysical Fluid Dynamics, 3, 265--288.

Woods, A. W., Boundary-Driven Mixing, J. Fluid Mech., 226, 625-654, 1991.

Woods, J. D. and R. L. Wiley, 1972. Billow turbulence and ocean microstructure, Deep-Sea Research, 19, 87--121.

Woods, J. D., 1968. Wave induced shear instability in the summer thermocline. Journal of Fluid Mechanics, 32, 791--800.

Woods, J. D., R. Onken, and J. Fischer, 1986. Thermohaline intrusions created isopycnally at oceanic fronts are inclined to isopycnals. Nature, 322, 446--449.

Worster, M.G., Huppert, H.E. and R.S.J. Sparks, 1990. Convection and crystallization in magma cooled from above, Earth Planet Sci. Lett. 101, 78-89.

Worthem, S., E. Mollo-Christensen, and F. Ostapoff, 1983. Effects of rotation and shear on doubly diffusive instability. Journal of Fluid Mechanics, 133, 297--319.

Yanase, S. 1986. The effect of time-periodic heating on the stability of a horizontal double-diffusive fluid layer. Journal of the Physical Society of Japan, 55, 1130--1138.

Yanase, S. and N. Gotoh, 1987. Super-critical state of double-diffusive convection under time-periodic heating. Journal of the Physical Society of Japan, 56, 2387--2395.

Yanase, S., and K. Kohno, 1985. The effect of a salinity gradient on the instability of natural convection in a vertical fluid layer. Journal of the Physical Society of Japan, 54, 3747--3756.

Yoshida, J., H. Nagashima, and H. Nino, 1989. The behavior of double-diffusive intrusion in a rotating system. Journal of Geophysical Research, 94(C4), 4923--4938.

Yoshida, J., H. Nagashima, and Wen-Ju Ma, 1987. A double-diffusive lock-exchange flow with small density difference.

Fluid Dynamics Research, 2, 205--215.

Yoshida, J., H. Nagashima and M. Nagasaka, 1996. Numerical Experiment on Double-Diffusive Currents. In

Double-Diffusive Convection A. Brandt and H.J.S. Fernando (eds), pp. 69-79, AGU, 1996.

Yotsukura, N., H. B. Fischer, and W. W. Sayre, Measurement of mixing characteristics of the Missouri River between Sioux City, Iowa, and Plattsmouth, Nebraska, U. S. Geol. Surv. Water Sup. Pap., 1899-G, 1970.

You, Y., and T. J. McDougall, 1990. Neutral surfaces and potential vorticity in the world's oceans. Journal of Geophysical

Research, 95(C8), 13,235--13,261.

You, Y., T. J. McDougall and R. W. Schmitt, 1995. Dianeutral motion, water-mass conversion and non-linear effects on the 
density ratio in the Pacific thermocline. Journal of Physical Oceanography, 25(8), 1891--1904.

Yuen, D.A., Hansen, U., Zhao, W., Vincent. A. P. and A.V. Malevsky, 1993. Hard turbulent thermal convection and thermal evolution of the mantle, J. Gcophys. Res., 9S, E3, 5355 -5373.

Yusa, Y. 1972. The re-evaluation of heat balance in Lake Vanda, Victoria Land, Antarctica. Contrib. Geophys. Inst. Kyoto Univ., 12, 87-100.

Yusa, Y., 1975. On the water temperature in Lake Vanda, Victoria Land, Antarctica. Mem. Natl. Polar Res., Special Issue 4, $75-89$.

Yusa, Y., 1977. A study on the thermosolutal convection in saline lakes. Mem. Fac. Sci., Kyoto Univ., Ser. A, 35, 149--183. Zangrando, F., 1979. Observation and analysis of a full-scale experimental salt gradient solar pond. Ph.D. Dissertation, University of New Mexico, Albuquerque, NM, 131pp.

Zangrando, F., and L. A. Bertram, 1985. The effect of variable stratification on linear doubly diffusive stability. Journal of Fluid of Mechanics, 151, 55--79.

Zeman, O., and J. L. Lumley, 1982. Modeling salt-fingering structures. Journal of Marine Research, 40, 315--330.

Zeman, O., and J. L. Lumley, 1983. Progress in modeling multi-layer salt-fingering structures. Mathematical Modelling, 4, 73--85.

Zenk, W., 1970. On the temperature and salinity of the Mediterranean water in the northeast Atlantic. Deep-Sea Research, 17, 627--632.

Zhang, Z. and A. Bejan, 1987. The horizontal spreading of thermal and chemical deposits in a porous medium, Int. J. Heat Mass Transfer, 30, 2289-2303.

Zhurbas, V. M., J. Laanemets, R. V. Ozmidov, and V. T. Paka, 1988. Horizontal variability of thermohaline fields with step layering in the ocean, Oceanology, 33, 903-909, (in Russian).

Zhurbas, V.M., N.P. Kuz'mina, and YE. Kul'sha, 1987. Step-Like Stratification of the ocean thermocline resulting from transformations associated with thermohaline salt finger intrusions (numerical experiment). Oceanology, 27, 277-281 (English Translation).

Zolotarev, V. G., Sochel'nikov, V. V., and Y. P. Malovitskiy, 1979. Results of Heat-Flow Measurements in the Black and Mediterranean Sea Basins, Oceanology, 19, 701-705. 


\section{DOCUMENT LIBRARY}

Distribution List for Technical Report Exchange - February 1996

University of California, San Diego

SIO Library 0175C

9500 Gilman Drive

La Jolla, CA 92093-0175

Hancock Library of Biology \& Oceanography

Alan Hancock Laboratory

University of Southern California

University Park

Los Angeles, CA 90089-0371

Gifts \& Exchanges

Library

Bedford Institute of Oceanography

P.O. Box 1006

Dartmouth, NS, B2Y 4A2, CANADA

Commander

International Ice Patrol

1082 Shennecossett Road

Groton, CT 06340-6095

NOAA/EDIS Miami Library Center

4301 Rickenbacker Causeway

Miami, FL 33149

Research Library

U.S. Army Corps of Engineers

Waterways Experiment Station

3909 Halls Ferry Road

Vicksburg, MS 39180-6199

Institute of Geophysics

University of Hawaii

Library Room 252

2525 Correa Road

Honolulu, HI 96822

Marine Resources Information Center

Building E38-320

MIT

Cambridge, MA 02139

Library

Lamont-Doherty Geological Observatory

Columbia University

Palisades, NY z10964

Library

Serials Department

Oregon State University

Corvallis, OR 97331

Pell Marine Science Library

University of Rhode Island

Narragansett Bay Campus

Narragansett, RI 02882
Working Collection

Texas A\&M University

Dept. of Oceanography

College Station, TX 77843

Fisheries-Oceanography Library

151 Oceanography Teaching Bldg.

University of Washington

Seattle, WA 98195

Library

R.S.M.A.S.

University of Miami

4600 Rickenbacker Causeway

Miami, FL 33149

Maury Oceanographic Library

Naval Oceanographic Office

Building 1003 South

1002 Balch Blvd.

Stennis Space Center, MS, 39522-5001

Library

Institute of Ocean Sciences

P.O. Box 6000

Sidney, B.C. V8L 4B2

CANADA

National Oceanographic Library

Southampton Oceanography Centre

European Way

Southampton SO14 3ZH

UK

The Librarian

CSIRO Marine Laboratories

G.P.O. Box 1538

Hobart, Tasmania

AUSTRALIA 7001

Library

Proudman Oceanographic Laboratory

Bidston Observatory

Birkenhead

Merseyside L43 7 RA

UNITED KINGDOM

IFREMER

Centre de Brest

Service Documentation - Publications BP 7029280 PLOUZANE

FRANCE 


\begin{tabular}{|c|c|c|c|}
\hline $\begin{array}{l}\text { REPORT DOCUMENTATION } \\
\text { PAGE }\end{array}$ & 1. REPORT NO. WHOI-97-10 & 2 & 3. Recipient's Accession No. \\
\hline \multirow{2}{*}{\multicolumn{2}{|c|}{$\begin{array}{l}\text { 4. Title and Subtitle } \\
\text { Double-Diffusive Processes } \\
1996 \text { Summer Study Program in Geophysical Fluid Dynamics }\end{array}$}} & & $\begin{array}{l}\text { 5. Report Date } \\
\text { July } 1997\end{array}$ \\
\hline & & & 6. \\
\hline $\begin{array}{ll}\text { 7. Author(s) } & \text { Steve Meachan } \\
& \text { Dacia Tucholke }\end{array}$ & $\begin{array}{l}\text { Director } \\
\text { Staff Assistant }\end{array}$ & & $\begin{array}{l}\text { 8. Performing Organization Rept. No. } \\
\text { WHOI-97-10 }\end{array}$ \\
\hline \multirow{2}{*}{\multicolumn{2}{|c|}{$\begin{array}{l}\text { 9. Performing Organization Name and Address } \\
\text { Woods Hole Oceanographic Institution } \\
\text { Woods Hole, Massachusetts } 02543\end{array}$}} & & 10. Project/TaskWork Unit No. \\
\hline & & & $\begin{array}{l}\text { 11. Contract(C) or Grant(G) No. } \\
\text { (C) OCE-9314484 } \\
\text { (G) }\end{array}$ \\
\hline \multirow{2}{*}{\multicolumn{2}{|c|}{$\begin{array}{l}\text { 12. Sponsoring Organization Name and Address } \\
\text { National Science Foundation }\end{array}$}} & & $\begin{array}{l}\text { 13. Type of Report \& Period Covered } \\
\text { Technical Report }\end{array}$ \\
\hline & & & 14. \\
\hline
\end{tabular}

15. Supplementary Notes

This report should be cited as: Woods Hole Oceanog. Inst. Tech. Rept., WHOI-97-10.

16. Abstract (Limit 200 words)

The physics of double diffusion and the role that it plays in the ocean provided the central theme for the 1996 summer program in Geophysical Fluid Dynamics at the Woods Hole Oceanographic Institution. Lectures and seminars given by staff and visitors surveyed observations, laboratory experiments and theoretical models of double diffusive phenomena. Several lectures dealt with the related phenomena of thermal convection in layered media, the dynamics of binary fluids, viscoelastic convection and magnetoconvection. Research projects by the fellows included experiments with double diffusion in a slot, work on the formation and evolution of staircases, double diffusion in stars and the interaction between convection and radiation. This volume includes write-ups of the principal lectures, reports of the fellows' research projects and abstracts of some of the seminars. A list of presentations and a bibliography may be found at the back of the volume.

17. Document Analysis a. Descriptors

double diffusion

thermosolutal convection

geophysical fluid dynamics

b. Identifiers/Open-Ended Terms

c. COSATI FieldJGroup

18. Availability Statement

Approved for public release; distribution unlimited.

\begin{tabular}{|l|l|}
\hline $\begin{array}{c}\text { 19. Security Class (This Report) } \\
\text { UNCLASSIFIED }\end{array}$ & $\begin{array}{c}\text { 21. No. of Pages } \\
410\end{array}$ \\
\hline 20. Security Class (This Page) & 22. Price \\
\hline
\end{tabular}

